AMES-HET 00-03

BNL-HET-00/7

ROME1-1294/00

\title{
CP Violation in Top Physics 用
}

\author{
David Atwood $^{a}$, Shaouly Bar-Shalom ${ }^{b}$, Gad Eilam $^{c}$ and Amarjit Soni ${ }^{d}$
}

\begin{abstract}
a) Department of Physics and Astronomy, Iowa State University, Ames, Iowa 50011, USA[.
b) INFN, Sezione di Roma and Dept. of Physics, University of Roma I, La Sapienza, Roma, Italy fris. $^{2}$

c) Physics Department, Technion-Institute of Technology, Haifa 32000, Israel"

d) Physics Department, Brookhaven National Laboratory, Upton, NY 11973, USA
\end{abstract}

\begin{abstract}
$\mathrm{CP}$ violation in top physics is reviewed. The Standard Model has negligible effects, consequently CP violation searches involving the top quark may constitute the best way to look for physics beyond the Standard Model. Non-standard sources of CP violation due to an extended Higgs sector with and without natural flavor conservation and supersymmetric theories are discussed. Experimental feasibility of detecting $\mathrm{CP}$ violation effects in top quark production and decays in high energy $e^{+} e^{-}, \gamma \gamma, \mu^{+} \mu^{-}, p p$ and $p \bar{p}$ colliders are surveyed. Searches for the electric, electro-weak and the chromo-electric dipole moments of the top quark in $e^{+} e^{-} \rightarrow t \bar{t}$ and in $p p \rightarrow t \bar{t} X$ are descibed. In addition, other mechanisms that appear promising for experiments, e.g., tree-level $\mathrm{CP}$ violation in $e^{+} e^{-} \rightarrow t \bar{t} h, t \bar{t} Z, t \bar{t} \nu_{e} \bar{\nu}_{e}$ and in the top decay $t \rightarrow b \tau \nu_{\tau}$ and $\mathrm{CP}$ violation driven by $s$-channel Higgs exchanges in $p p, \gamma \gamma, \mu^{+} \mu^{-} \rightarrow t \bar{t}$ etc., are also discussed.
\end{abstract}

\footnotetext{
*A 2-up version of this postscript file may be obtained at the url http://thy.phy.bnl.gov/ soni/topreview.html ${ }^{\dagger}$ Email: atwood@iastate.edu

${ }^{\ddagger}$ Email: Shaouly.BarShalom@roma1.infn.it

$\S$ Much of the work in this review was done while S. Bar-Shalom was at Physics Department, University of California, Riverside, CA, USA

^Email: eilam@physics.technion.ac.il

"Email: soni@bnl.gov
} 


\section{Contents}

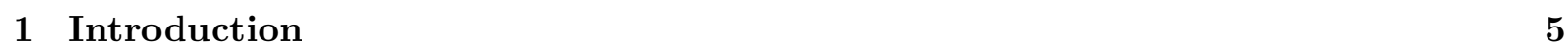

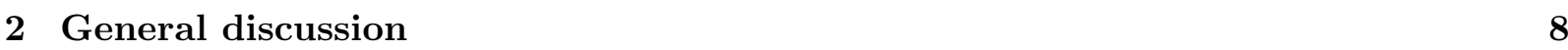

$2.1 \quad$ Definitions of discrete symmetries $\mathrm{C}, \mathrm{P} \& \mathrm{~T} \ldots \ldots \ldots$

2.2 CP-violating observables: categorizing according to $T_{N} \ldots \ldots \ldots \ldots$

2.3 Partial rate asymmetries and the CP-CPT connection . . . . . . . . . . . . . . 13

2.4 Resonant $W$ effects and CP violation in top decays $\ldots \ldots \ldots$. . . . . . . . . . . 19

2.5 Effective Lagrangians and observables . . . . . . . . . . . . . . . . . . . . . . . . . . . . . . . . . . . . . .

2.6 Optimized observables . . . . . . . . . . . . . . . . . . . . 23

2.7 The naked top $\ldots \ldots \ldots \ldots \ldots \ldots \ldots \ldots \ldots \ldots \ldots \ldots$

2.8 Elements of top polarimetry $\ldots \ldots \ldots \ldots \ldots \ldots \ldots$

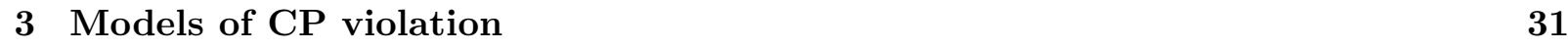

3.1 CP violation and the Standard Model $\ldots \ldots \ldots$. . . . . . . . . . . . . 31

3.1 .1 General remarks . . . . . . . . . . . . . . . . . . . 31

3.1 .2 The Jarlskog invariant $\ldots \ldots \ldots \ldots \ldots \ldots$

3.1 .3 Experimental constraints . . . . . . . . . . . . . . . . . . . . . . . . . . . . . . . . 34

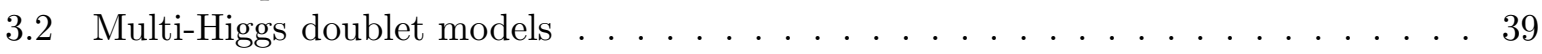

3.2 .1 Two Higgs doublet models $\ldots \ldots \ldots$. . . . . . . . . . . . . 39

$3.2 .2 \quad$ 2HDM with CP nonconservation and FCNC . . . . . . . . . . . . . . 41

3.2 .3 2HDM with CP nonconservation and no FCNC . . . . . . . . . . . . 43

3.2 .4 Three Higgs doublet model $\ldots \ldots \ldots \ldots$. . . . . . . . . . . 45

3.3 Supersymmetric models $\ldots \ldots \ldots \ldots \ldots \ldots \ldots$. . . . . . . . . . . . . 52

3.3.1 General description and the SUSY Lagrangian . . . . . . . . . . . . . . . 53

$3.3 .2 \quad$ CP violation in a general MSSM $\ldots \ldots \ldots \ldots$. . . . . . . . . . . 54

3.3.3 CP violation in a GUT-scale $N=1$ minimal SUGRA model $\ldots$. . . . . . . 57

3.3.4 A plausible low energy MSSM framework and the "SUSY-CP problem" of the NEDM $\ldots \ldots \ldots \ldots \ldots \ldots \ldots$

3.3 .5 CP and the pure Higgs sector of the MSSM $\ldots \ldots \ldots 61$

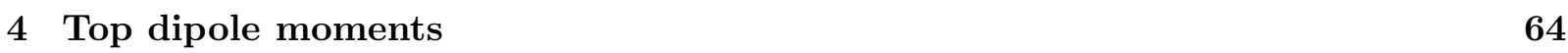

4.1 Theoretical expectations . . . . . . . . . . . . . . . 64

$4.2 \quad$ Arbitrary number of Higgs doublets and a CP-violating neutral Higgs sector . . . 65

4.3 Expectations from 2HDM's with CP violation in the neutral Higgs sector $\ldots$. . . . 68

4.4 Expectations from a CP-violating charged Higgs sector $\ldots \ldots$. . . . . . . . . 74

4.5 Expectations from the MSSM $\ldots \ldots \ldots \ldots \ldots \ldots$

4.6 Top dipole moments - summary . . . . . . . . . . . . . . . . . . . 87

\begin{tabular}{|lll}
\hline 5 & CP violation in top decays & 91 \\
\hline
\end{tabular}

5.1 Partial rate asymmetries $\ldots \ldots \ldots \ldots \ldots \ldots \ldots$

$5.1 .1 \quad$ PRA in the SM . . . . . . . . . . . . . . . . . . . . . . . . . . . . . . . . .

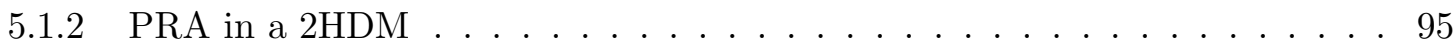

$5.1 .3 \quad \mathrm{PRA}$ in $\mathrm{a} 3 \mathrm{HDM} \ldots \ldots \ldots \ldots \ldots$

5.1 .4 PRA in the MSSM . . . . . . . . . . . . . . . . . . . 98

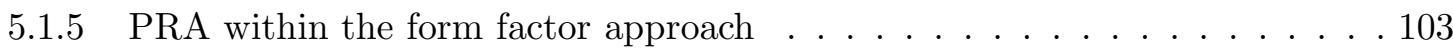

5.2 Partially integrated rate asymmetries $\ldots \ldots \ldots \ldots \ldots 11$

5.3 Energy asymmetry $\ldots \ldots \ldots \ldots \ldots \ldots \ldots \ldots \ldots$

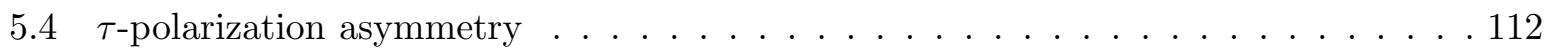

5.5 CP violation in top decays - summary $\ldots \ldots \ldots \ldots \ldots$ 
\begin{tabular}{|lll}
6 & CP violation in $e^{+} e^{-}$collider experiments & 114
\end{tabular}

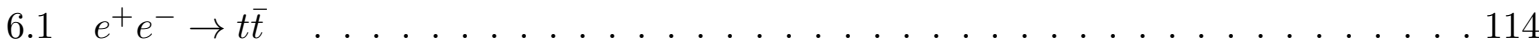

6.1 .1 Optimized observables . . . . . . . . . . . . . . . . 116

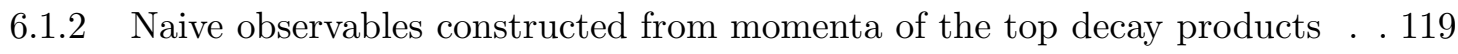

6.1.3 Improved sensitivity using energy and angular distributions of top decay products and polarized electron beams f . . . . . . . . . . . . 122

$6.2 e^{+} e^{-} \rightarrow t \bar{t} h, t \bar{t} Z$, examples of tree-level CP violation . . . . . . . . . . . . . 128

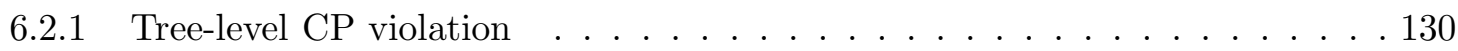

6.2.2 Generalized optimization technique and extraction of various Higgs cou-

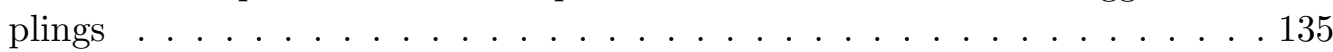

$6.2 .3 \quad$ CP asymmetries in $e^{+} e^{-} \rightarrow Z h$ and in the subsequent Higgs decay $h \rightarrow t t$

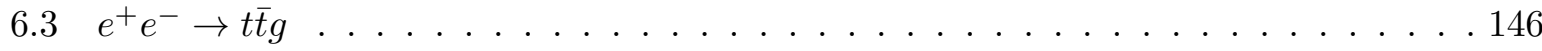

$6.3 .1 \quad 2 \mathrm{HDM}$ and CP violation in $e^{+} e^{-} \rightarrow t t g$. . . . . . . . . . . . 150

6.3 .2 Model independent constraints on top dipole moments . . . . . . . . . . 154

6.4 CP violation via $W W$ fusion in $e^{+} e^{-} \rightarrow t t \nu_{e} \bar{\nu}_{e} \ldots \ldots \ldots \ldots 1$

\begin{tabular}{|ll|}
\hline CP violation in $p p$ collider experiments & 165
\end{tabular}

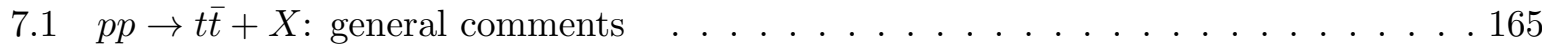

$7.2 p p \rightarrow t \bar{t}+X:$ general form factor approach and the CEDM of the top $\ldots . . .165$

$7.2 .1 \quad$ Optimal observables . . . . . . . . . . . . . . . 168

7.2 .2 Observable correlations between momenta of the top decay products . . . 168

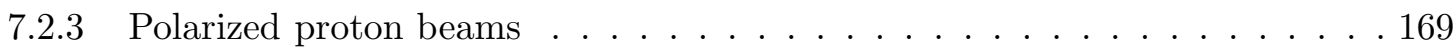

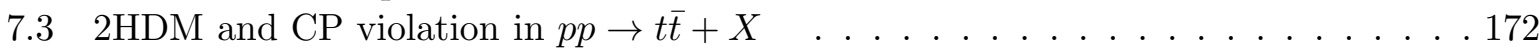

$7.3 .1 \quad$ Schmidt-Peskin signal . . . . . . . . . . . . . . . . . . . 174

7.3.2 $\hat{s}$-channel resonance Higgs effects - Bernreuther-Brandenberg approach . . 176

$7.4 \quad$ SUSY and CP violation in $p p \rightarrow t t+X \ldots \ldots \ldots 1$

\begin{tabular}{|llr}
8 & CP violation in $p \bar{p}$ collider experiments & 184
\end{tabular}

$8.1 p \bar{p} \rightarrow t \bar{t}+X \ldots \ldots \ldots \ldots \ldots \ldots \ldots \ldots$

$8.1 .1 \quad$ CP-even observables in $p \bar{p} \rightarrow t t+X$ and $p \bar{p} \rightarrow t t+j e t+X \ldots$. . . . . 184

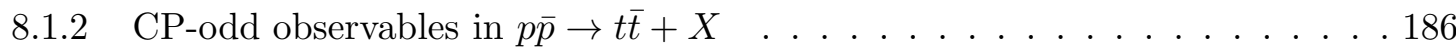

$8.1 .3 \quad$ Transverse energy asymmetry of charged leptons . . . . . . . . . . . . 186

8.1 .4 Optimal observables . . . . . . . . . . . . . . . . . . . 187

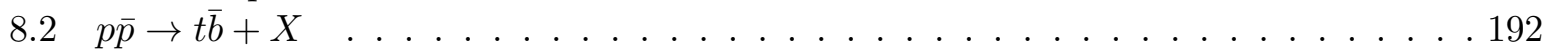

$8.2 .1 \quad 2 \mathrm{HDM}$ and the CP-violating asymmetries . . . . . . . . . . . . . 194

$8.2 .2 \quad$ SUSY and the CP-violating cross-section asymmetry . . . . . . . . . . . 197

$8.2 .3 \quad$ Feasibility of extraction from experiment $\ldots \ldots$. . . . . . . . . 203

$8.3 p \bar{p} \rightarrow t b h+X$, a case of tree-level CP violation $\ldots \ldots \ldots$. . . . . . . . . 203

\begin{tabular}{|ll|}
\hline CP violation in $\gamma \gamma$ collider experiments & 208 \\
\hline
\end{tabular}

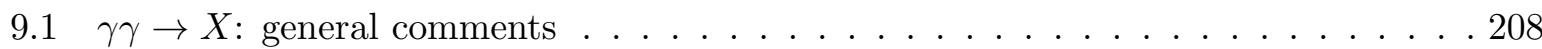

$9.2 \gamma \gamma \rightarrow t t$ and the top EDM $\ldots \ldots \ldots \ldots \ldots \ldots \ldots \ldots \ldots$

$9.3 \gamma \gamma \rightarrow t \bar{t}$ and $s$-channel Higgs exchange in a 2HDM . . . . . . . . . . . . . . 214

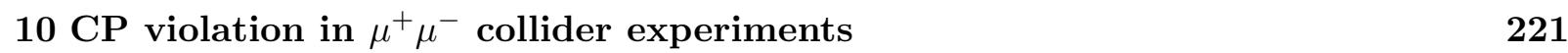

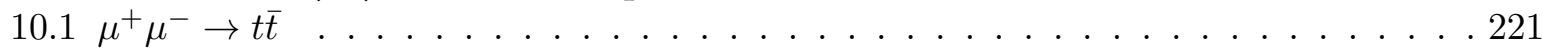

10.1 .1 A general model for the Higgs couplings . . . . . . . . . . . . 221

10.1 .2 Decay correlation asymmetry . . . . . . . . . . . . . . . . 223

10.1.3 Production asymmetry in $\mu^{+} \mu^{-} \rightarrow t t$ via polarized muons . . . . . . 226

$10.2 \mathrm{CP}$ violation in the flavor changing reaction $\mu^{+} \mu^{-} \rightarrow t \bar{c} \ldots$. . . . . . . . 229

\begin{tabular}{ll}
\hline 11 Summary and outlook & 232
\end{tabular} 
\begin{tabular}{ll}
\hline Notes & 239
\end{tabular}

\begin{tabular}{ll}
\hline References & 240
\end{tabular}

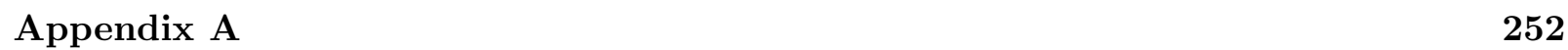

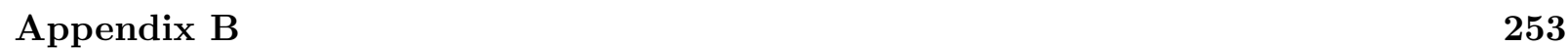




\section{Introduction}

Violations of the CP (Charge Conjugation combined with Parity) symmetry are of great interest in particle physics especially since its origin is still unclear. Better understanding of this (so far) rare phenomenon can lead to new physics which may explain both the origin of mass and the preponderance of matter over anti-matter in the present universe. Indeed, reactions that violate $\mathrm{CP}$ are such a scarce resource that in over thirty years the only confirmed examples of $\mathrm{CP}$ violation are those found in the decay of the $K_{L}$-meson!.

The first experimental observation of CP violation was in 1964 by Christenson, Cronin, Fitch and Turlay [2] who observed a non-vanishing rate for the decay $K_{L} \rightarrow 2 \pi$ [3],

$$
\operatorname{Br}\left(K_{L} \rightarrow 2 \pi\right)=3.00 \pm 0.04 \times 10^{-3} .
$$

Since the dominant decay of $K_{L}$ is to a $3 \pi$ state of $\mathrm{CP}=-1$, the above decay to a manifestly $\mathrm{CP}=+1$ state clearly violates this symmetry.

Another example of $\mathrm{CP}$ violation which is well established in $K_{L}$ is the difference between $\Gamma\left(K_{L} \rightarrow \ell^{+} \nu_{\ell} \pi^{-}\right)$and $\Gamma\left(K_{L} \rightarrow \ell^{-} \nu_{\ell} \pi^{+}\right), \ell=e, \mu[3]$ :

$$
\frac{\Gamma\left(K_{L} \rightarrow \ell^{+} \pi^{-} \nu\right)-\Gamma\left(K_{L} \rightarrow \ell^{-} \pi^{+} \bar{\nu}\right)}{\Gamma\left(K_{L} \rightarrow \ell^{+} \pi^{-} \nu\right)+\Gamma\left(K_{L} \rightarrow \ell^{-} \pi^{+} \bar{\nu}\right)}=(3.27 \pm 0.012) \times 10^{-3} .
$$

All of these observations of $\mathrm{CP}$ violation in the $K_{L}$ system can be explained by the CP violation in the mixing of the neutral $K$ mesons. Thus

$$
\begin{aligned}
& \left|K_{L}\right\rangle=\left((1+\epsilon)\left|K^{0}\right\rangle-(1-\epsilon)\left|\bar{K}^{0}\right\rangle\right) / \sqrt{2\left(1+|\epsilon|^{2}\right)}, \\
& \left|K_{S}\right\rangle=\left((1+\epsilon)\left|K^{0}\right\rangle+(1-\epsilon)\left|\bar{K}^{0}\right\rangle\right) / \sqrt{2\left(1+|\epsilon|^{2}\right)},
\end{aligned}
$$

where the experimental value of $\epsilon$ is $[3]$ :

$$
|\epsilon|=(2.263 \pm 0.023) \times 10^{-3} \quad ; \quad \arg (\epsilon)=43.49 \pm 0.08 .
$$

As is well known, this mixing can be accommodated in the Standard Model (SM) with three generations where the $\mathrm{CP}$ violation originates through a phase in the Cabibbo Kobayashi Maskawa (CKM) [4] matrix as will be discussed in some detail in section 3.1.

The SM further predicts that there is an additional CP violation in $K_{L} \rightarrow \pi \pi$ parameterized by the quantity $\epsilon^{\prime}$. The prediction is that $\epsilon^{\prime} / \epsilon=\mathcal{O}\left(10^{-4}\right)$; the theoretical difficulties in determining the hadronic matrix element prevent us from making a more precise estimate [5]. Experimentally $\Re \mathrm{e}\left(\epsilon^{\prime} / \epsilon\right)$ may be measured via [6]:

$$
\Re \mathrm{e}\left(\epsilon^{\prime} / \epsilon\right) \simeq \frac{1}{6}\left[1-\frac{\left|\eta_{00}\right|^{2}}{\left|\eta_{+-}\right|^{2}}\right],
$$

where

$$
\eta_{i j}=\frac{\left\langle\pi^{i} \pi^{j}\left|H_{W}\right| K_{L}\right\rangle}{\left\langle\pi^{i} \pi^{j}\left|H_{W}\right| K_{S}\right\rangle},
$$

and $H_{W}$ is the relevant weak interaction Hamiltonian.

After some two decades of intensive efforts, new and quite dramatic experimental developments have recently taken place that we would now briefly like to mention. First of all, let us recall that a few years ago the CERN experiment NA31 gave the result [7]:

$$
\Re \mathrm{e}\left(\epsilon^{\prime} / \epsilon\right)=(23 \pm 6.5) \times 10^{-4},
$$

\footnotetext{
${ }^{1}$ For excellent recent books on $\mathrm{CP}$ violation see ref. 酒
} 
appreciably different from zero. On the other hand, the Fermilab experiment E731 found it completely consistent with zero [8]:

$$
\Re \mathrm{e}\left(\epsilon^{\prime} / \epsilon\right)=(7.4 \pm 6) \times 10^{-4} .
$$

For the past many years improved experiments have been underway, at CERN (experiment NA48), and at FNAL (KTEV) with an expected accuracy of about $\mathcal{O}\left(10^{-4}\right)$, KTEV has recently announced their new results on $\epsilon^{\prime} / \epsilon$, based upon analysis of $20 \%$ data collected so far [9]:

$$
\Re \mathrm{e}\left(\epsilon^{\prime} / \epsilon\right)=(28 \pm 4.1) \times 10^{-4} .
$$

Combining with [7] and [8] one now finds:

$$
\Re \mathrm{e}\left(\epsilon^{\prime} / \epsilon\right)=(21.8 \pm 3) \times 10^{-4},
$$

thus conclusively establishing that $\epsilon^{\prime} / \epsilon \neq 0$. Such a non-vanishing value formally lays to rest the phenomenological superweak model [10] of CP violation as it unambiguously predicts $\epsilon^{\prime} / \epsilon=0$. However, unless the computational challenges presented by strong interactions can be overcome, it is unlikely that the measured value of $\epsilon^{\prime} / \epsilon$ would confirm or refute the SM in any reliable fashion.

Experiments involving $B$-mesons are more likely to have a quantitative bearing on the SM. Just as the SM indicates that the natural size of $\mathrm{CP}$ asymmetries in $K$ physics is $\mathcal{O}\left(10^{-3}-10^{-4}\right)$, it also strongly suggests that the effects in the $B$ system are much bigger; in many cases CP asymmetries are expected to be tens of percents. This expectation renders the $B$ system ideal for a precise extraction of the CKM phase and, indeed for a thorough quantitative test of the SM through a detailed study of the unitarity triangle [11]. The asymmetric and symmetric $B$ factories currently in the early stages of running at KEK, SLAC and Cornell and hadron machines, should have a very important role to play in confronting the experimental results with the detailed predictions of the SM. A recent CDF result 12 for CP violation in $B^{0} \rightarrow J / \Psi K_{S}$, though crude at the moment, indicates that $\mathrm{CP}$ violation may indeed be large in the $b$ system.

Experiments at FNAL have decisively [13] demonstrated that the mass of the top quark is extremely large, i.e., the D0 and CDF average is now $m_{t} \sim 174 \mathrm{GeV}$ [14]. This has some very important consequences. First the top rapidly undergoes two-body weak decay: $t \rightarrow b+W$, with a time scale of about $10^{-24}$ sec., which is shorter by an order of magnitude than the typical QCD time scale necessary for hadronic bound states to be formed [15]. Thus, unlike the other five quarks, the top does not form hadrons. It means that the dynamics of top production and decay does not get masked by the complications of non-perturbative, bound state physics, i.e., the "brown muck". All of the CP violation phenomena relevant to the top are therefore of the "direct" type.

We should think of the top quark as an elementary fermion. For example, it therefore is sensible to ask for its dipole moment [16, 17, 18, 19]. Unlike the other quarks the spin of the top quark becomes an extremely important observable. Indeed the decays of the top quark become very effective analyzers of its spin, see e.g., [20, 21, 22].

The SM predicts, however, that CP-violating effects in t-physics are very small. This is primarily due to the fact that its large mass in comparison to the other quarks renders the Glashow-Iliopoulos-Maiani (GIM) 223] cancellation particularly effective [24, 25]. This being the case, what then is the motivation for the study of CP violation in the top quark system?

There are two related reasons why one might expect to find such effects. First of all there is another important example of CP violation which the SM fails to explain, namely the excess of matter over anti-matter in the universe. It was shown by Sakharov [26] in 1967 that CP violation is one of the necessary conditions for baryon number asymmetry to appear in the early universe; baryon - anti-baryon asymmetry can be dynamically generated at early stages after the big bang even if the universe was "born" symmetric, provided that: (i) C and CP are violated, (ii) there 
are baryon number violating interactions and (iii) there is a deviation from thermal equilibrium. The basic idea is that, if $\mathrm{CP}$ is violated, then baryons and anti-baryons interact with different rates at some point in the early universe. However, the $\mathrm{CP}$ violation due to the SM appears too weak to drive such an asymmetry [27]. In many cases, extensions of the SM such as the Two Higgs Doublet Model (2HDM) or the SUperSYmmetric (SUSY) extensions of the SM are able to supply the $\mathrm{CP}$ violation required to produce such a baryon asymmetry in the early universe. In fact in some models [28, 29] it is precisely the couplings of the top quark to CP-violating phases in beyond the SM physics which drive baryogenesis. Thus, the study of CP violation in top quark interactions in the laboratory could shed light on these primordial processes.

The second motivation for investigating $\mathrm{CP}$ violation in top quark physics is that in many extensions of the SM, CP violation in the top quark can be particularly large. Indeed, because the SM contribution to $\mathrm{CP}$ violation in the top quark is so small, any observation of such effects would be a clear evidence of physics beyond the SM. The argument here parallels the search for the weak neutral current, in the 1970's, by looking for parity violation in deep-inelastic-scattering. The point is that the existing theory of the time, namely QED, could not cause parity violation in deep-inelastic-scattering. Such an effect became an unambiguous signature for the existence of the weak neutral current.

Since various extensions of the SM entail new CP-violating phase(s), we should seek the optimal strategies for searching each type of new phase. In this context we first recall, what has been emphasized on the preceding page, that the $b$-quark is very sensitive to the CKM phase of the SM. Existing literature has revealed that top physics is very sensitive to several different types of new phases. Upcoming high energy colliders of the next decade can therefore serve as excellent laboratories for searching for new physics in top quark systems, and in particular, for studying CP-violating effects associated with those new CP-odd phases. The upgraded Tevatron $p \bar{p}$ collider (runs 2 and 3) at Fermilab which will be able to produce about $10^{4}-10^{5} t \bar{t}$ /year, the CERN pp Large Hadron Collider (LHC) will produce about $10^{7}-10^{8} t \bar{t} /$ year, and about $10^{5}-10^{6} t \bar{t} /$ year are expected at a future $e^{+} e^{-}$Next Linear Collider (NLC).

A CP-odd phase due to an extended neutral Higgs sector or vertex corrections arising in other extensions of the SM can endow the top quark with a large dipole moment form factor. Such an effect could be detected both at an $e^{+} e^{-}$collider, such as the NLC or hadron colliders such as the LHC. A CP-violating phase in the neutral Higgs sector also causes large CP asymmetries in the reactions $e^{+} e^{-} \rightarrow t \bar{t} H^{0}$ and $e^{+} e^{-} \rightarrow t \bar{t} \nu_{e} \bar{\nu}_{e}$, both of which should be a prime target for the NLC. Moreover, $\mathrm{CP}$ violation in the neutral Higgs sector and in supersymmetry can have interesting effects in single top production at the upgraded Tevatron $p \bar{p}$ collider at Fermilab and in $t \bar{t}$ pair production at the LHC. The transverse polarization of the $\tau$ in the three-body top decay $t \rightarrow b \tau \nu$ is extremely sensitive to a new phase from a charged Higgs sector in Multi-Higgs Doublets Models (MHDM's). Finally, CP-odd phases in SUSY models have also interesting effects in Partial Rate Asymmetry (PRA) in $t \rightarrow W^{+} b$ versus $\bar{t} \rightarrow W^{-} \bar{b}$. These processes and others will be discussed in the subsequent chapters.

We will not consider CP violation phenomena in which the top quark is virtual rather than an external particle. It suffices to recapitulate that, in the SM, CP violation is often dominated by the virtual top quarks in the loops. Let us also comment that the discovery of the top with the measurement of $m_{t}$, and the progress in determination of the CKM matrix elements as well as considerable progress in theory, has influenced our understanding of $\mathrm{CP}$ violation in $\mathrm{K}$ and B physics within the SM [30] and beyond the SM [31]. Furthermore, for the Electric Dipole Moments (EDM's) of the electron and the neutron [32], the virtual top quark also plays a crucial role in extended Higgs sector scenarios. 


\section{General discussion}

\subsection{Definitions of discrete symmetries C, P \& T}

Let us now review the definitions of the discrete symmetries $C, P$ and $T$ and recall a few basic facts concerning their manifestation in relativistic quantum field theory.

Under the parity transformation, $P$, the spatial coordinate axes are reversed, i.e., $P \vec{x}=-\vec{x}$. Thus for an ingoing particle, $X$, in a specific momentum and spin state $|X ; \vec{P}, \vec{S}\rangle_{\text {in }}$, the action of parity is to reverse the momentum, leaving the spin fixed as angular momentum is an axial vector defined by a cross product. Hence

$$
P|X ; \vec{P}, \vec{S}\rangle_{\text {in }} \rightarrow|X ;-\vec{P}, \vec{S}\rangle_{\text {in }}
$$

Under the Wigner definition of time reversal, $T$, the sign of both momenta and spins are reversed, and also, due to the anti-unitary nature of $T,|\rangle_{\text {in }}$ and |\rangle$_{\text {out }}$ states are interchanged. Thus

$$
T|X ; \vec{P}, \vec{S}\rangle_{\text {in }} \rightarrow|X ;-\vec{P},-\vec{S}\rangle_{\text {out }}
$$

Under Charge conjugation, $C$, each particle is replaced by its anti-particle, and so

$$
C|X ; \vec{P}, \vec{S}\rangle_{\text {in }} \rightarrow|\bar{X} ; \vec{P}, \vec{S}\rangle_{\text {in }},
$$

where $\bar{X}$ means that all charges and other additive quantum numbers are reversed.

It can be shown that local relativistic quantum field theories with the usual spin-statistics relations are invariant under the combined action of all three of these symmetries where [33]:

$$
C P T|X ; \vec{P}, \vec{S}\rangle_{\text {in }}=|\bar{X} ; \vec{P},-\vec{S}\rangle_{\text {out }}
$$

Thus, such a theory violates $T$ if and only if it violates $\mathrm{CP}$, where

$$
C P|X ; \vec{P}, \vec{S}\rangle_{\text {in }} \rightarrow|\bar{X} ;-\vec{P}, \vec{S}\rangle_{\text {in }},
$$

and in this sense $\mathrm{CP}$ and $T$ violation are equivalent.

Other well known consequences of the CPT theorem are that masses of particles and antiparticles are the same, $m_{X}=m_{\bar{X}}$, and the total widths of particles and anti-particles are also equal, $\Gamma_{X}=\Gamma_{\bar{X}}$. Note that it does not follow from CPT that decay rates to specific final states are the same. In fact, partial width differences, i.e., a non-zero value of

$$
\Delta(X \rightarrow A) \equiv \Gamma(X \rightarrow A)-\Gamma(\bar{X} \rightarrow \bar{A}),
$$

is a form of $\mathrm{CP}$ violation that we will discuss in more detail in section 2.3. Clearly it follows from $\Gamma_{X}=\Gamma_{\bar{X}}$ that

$$
\sum_{A} \Delta(X \rightarrow A)=0
$$

where the sum is over all possible final states. The relationship between $\Delta(X \rightarrow A)$ and the other final states which compensate for it will also be discussed in detail in section 2.3.

In this report we are largely concerned with the violation of discrete symmetries in decay and scattering experiments. We, therefore, need to consider the implementation of $\mathrm{CP}$ and $T$ on the $S$-matrix.

For $C$ and $P$ this is straightforward. Consider the initial state of $n_{i}$ particles

$$
|i\rangle=\left|\vec{P}_{a}, \vec{P}_{b}, \ldots, \vec{S}_{a}, \vec{S}_{b}, \ldots\right\rangle_{\text {in }},
$$

and the final state of $n_{f}$ particles 


$$
|f\rangle=\left|\vec{P}_{1}, \vec{P}_{2}, \ldots, \vec{S}_{1}, \vec{S}_{2}, \ldots\right\rangle_{\text {out }}
$$

so that the $S$-matrix element is

$$
\langle f|S| i\rangle=S_{f i}
$$

The transformations $P$ and $C$ follow from the single particle transformation

$$
\begin{aligned}
\left|f_{P}\right\rangle & =\left|-\vec{P}_{1},-\vec{P}_{2}, \ldots, \vec{S}_{1}, \vec{S}_{2}, \ldots\right\rangle_{\text {out }}, \\
\left|f_{C}\right\rangle & =\left|\vec{P}_{\overline{1}}, \vec{P}_{\overline{2}}, \ldots, \vec{S}_{\overline{1}}, \vec{S}_{\overline{2}}, \ldots\right\rangle_{\text {out }},
\end{aligned}
$$

and likewise for $\left|i_{P}\right\rangle,\left|i_{C}\right\rangle$. The transformation of the $S$-matrix element under these symmetries is thus

$$
\begin{aligned}
& S_{f i} \stackrel{P}{\longrightarrow} S_{f_{P} i_{P}}, \\
& S_{f i} \stackrel{C}{\longrightarrow} S_{f_{C} i_{C}} .
\end{aligned}
$$

and thus

$$
S_{f i} \stackrel{C P}{\longrightarrow} S_{f_{C P} i_{C P}}
$$

The nature of time reversal, however, requires the interchange of |\rangle$_{\text {in }}$ and |\rangle$_{\text {out }}$ states so that the effect on the $S$-matrix will be anti-unitary. Thus

$$
\begin{aligned}
\left|f_{T}\right\rangle & =\left|-\vec{P}_{1},-\vec{P}_{2}, \ldots,-\vec{S}_{1},-\vec{S}_{2}, \ldots\right\rangle_{\text {in }} \\
\left|i_{T}\right\rangle & =\left|-\vec{P}_{a},-\vec{P}_{b}, \ldots,-\vec{S}_{a},-\vec{S}_{b}, \ldots\right\rangle_{\text {out }}
\end{aligned}
$$

such that

$$
S_{f i} \stackrel{T}{\longrightarrow} S_{i_{T} f_{T}} .
$$

Needless to say, because of the interchange of initial and final states, accelerator based experiments seldom test $T$ directly.

These symmetries, as they are defined in $S$-matrix theory are fundamental in that if the Lagrangian and the vacuum states respect $C, P$ or $T$, then the corresponding symmetry of the $S$-matrix will apply. We will also find it useful to consider the symmetry $T_{N}$ - "naive" time reversal - for which this is not true. The definition of $T_{N}$ is to apply $T$ to the initial and final states without interchanging them

$$
S_{f i} \stackrel{T_{N}}{\longrightarrow} S_{f_{T} i_{T}} .
$$

Thus, $T_{N}$ is a "symmetry" which can be tested in accelerator based scattering experiments, but, as we shall see in the following section, it only corresponds to "true" time reversal $(T)$ operation at tree-level in perturbation theory. It is nonetheless useful in categorizing the various modes of $\mathrm{CP}$ violation. 


\subsection{CP-violating observables: categorizing according to $T_{N}$}

It is useful to divide CP-violating observables into two categories (see e.g., [16, 22, 34, 35]), those that are even under "naive" time reversal $\left(T_{N}\right)$ and those that are odd. Recall that $T_{N}$ is defined as a transformation which reverses the momenta and spins of all particles without the interchange of the initial and final states. This contrasts with true time reversal, $T$, in that under $T$ initial and final states are also interchanged.

The symmetry $T_{N}$ is not a fundamental symmetry like $C, P$ and $T$ since the $S$-matrix under $T_{N}$ need not follow from the transformation properties of the Lagrangian. Nevertheless, it is a useful tool for categorization and as we shall presently show, observables which are CP-odd and

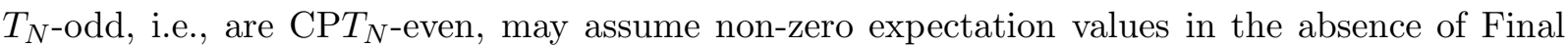
State Interaction (FSI) effects. In particular, tree-level processes in perturbation theory may lead to non-vanishing expectation values for these operators. On the other hand, CP-odd $T_{N}$-even

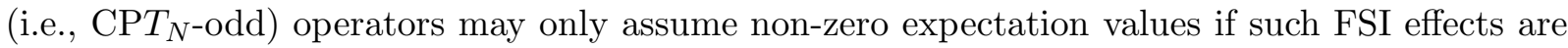
present giving a non-trivial phase to the Feynman amplitude. Such a phase, often called a strong phase or absorptive phase, may arise in several ways. It may be present in a loop diagram if the internal particle(s) can be on-shell. An interesting variation of this, which we will consider in section 2.4, is in the propagator of an unstable particle where the strong phase is the phase of the Breit-Wigner amplitude. Non-perturbative rescattering of final state particles can also give a strong phase though this is more of interest in CP studies in $B$ and $K$ physics.

Indeed, $T_{N}$ is useful in understanding when CP-conserving observables depend on an absorptive phase. In particular, a CP-even $T_{N}$-odd observable (typically a CP-even triple product correlation of momenta and/or spins) will only assume an expectation value if FSI effects are

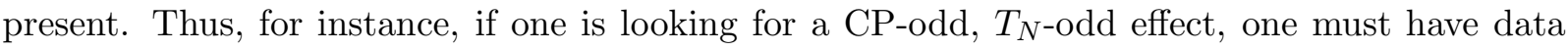
both on the process of interest and its $\mathrm{CP}$ conjugate (if they are different) in order to distinguish from the possible background of CP-even $T_{N}$-odd effects (see e.g., [36]).

In order to understand the role of $T_{N}$, let us consider the unitarity relations of the $S$-matrix (implied by conservation of probabilities). Following a derivation analogous to the optical theorem [37] we write the $S$-matrix in terms of the scattering amplitude $\mathcal{T}$

$$
S=1+i \mathcal{T}
$$

where, for a given transition $i \rightarrow f, \mathcal{T}$ is related to the "reduced scattering amplitude", $\tau$, by

$$
<f|\mathcal{T}| i>=(2 \pi)^{4} \delta^{4}\left(p_{f}-p_{i}\right)<f|\tau| i>.
$$

Substituting Eq. 2.20 into the unitarity relation $S^{\dagger} S=1$ we obtain

$$
\mathcal{T}_{f i}-\mathcal{T}_{i f}^{*}=i \sum_{n} \mathcal{T}_{n f}^{*} \mathcal{T}_{n i}
$$

where we denote $\langle a|\mathcal{T}| b\rangle \equiv \mathcal{T}_{a b}$. In terms of $\tau$ this becomes

$$
\tau_{f i}-\tau_{i f}^{*}=i(2 \pi)^{4} \sum_{n} \delta^{4}\left(p_{n}-p_{i}\right) \tau_{n f}^{*} \tau_{n i}
$$

Let us now assume that there are no rescattering effects and that (to the order of approximation considered) $i$ and $f$ are stable states so that $\tau_{i i}=\tau_{f f}=0$. Thus, for each possible intermediate state $n$, the rhs of Eq. 2.23 vanishes. Therefore, in the absence of rescattering, $\tau$ is hermitian

$$
\tau_{i f}=\tau_{f i}^{*} .
$$

Now, if $\tau$ is CP invariant, then by the CPT theorem it is also $T$ invariant. Thus

$$
\langle f|\tau| i\rangle=\left\langle i_{T}|\tau| f_{T}\right\rangle=\left\langle f_{T}|\tau| i_{T}\right\rangle^{*},
$$




\begin{tabular}{c|c|c}
\hline$T_{N}$ & CP-violating & CP-conserving \\
\hline even & $\mathrm{Y}$ & $\mathrm{N}$ \\
\hline odd & $\mathrm{N}$ & $\mathrm{Y}$ \\
\hline
\end{tabular}

Table 1: Transformation properties under $T_{N}$ and $C P$ and presence or absence of final state interactions (FSI). Here $Y \equiv F S I$ present and $N \equiv$ FSI absent.

and therefore

$$
|<f| \tau|i>|^{2}=\left|<f_{T}\right| \tau\left|i_{T}>\right|^{2} .
$$

In fact this equation means precisely that the modulus of $\langle f|\tau| i\rangle$ is invariant under $T_{N}$. Since, in the absence of rescattering, the expectation value of any operator depends only on $|\langle f|\tau| i\rangle|$, Eq. 2.26 implies that if $\mathrm{CP}$ is conserved, then only $T_{N}$-even operators can have a non-vanishing expectation value.

What we have shown therefore is that in the absence of rescattering effects (i.e., $\Im \mathrm{m}(\tau)=0$, in which case the requirement of CPT invariance leads effectively to conservation of the scattering amplitude under $\mathrm{CP} T_{N}$ ) and in the absence of $\mathrm{CP}$ violation, $T_{N}$-odd observables have zero expectation value. Thus, if such a $T_{N}$-odd observable $\mathcal{O}$ has a non-zero expectation value, either $\mathrm{CP}$ is violated and $\mathcal{O}$ is $\mathrm{CP}$-odd (i.e., $\mathrm{CP} T_{N}$-even) or there are rescattering effects present (implying $\mathrm{CPT} \neq \mathrm{CP} T_{N}$ ) and $\mathcal{O}$ is $\mathrm{CP}$-even (i.e., $\mathrm{CP} T_{N}$-odd). Conversely, let us suppose $\mathcal{O}$

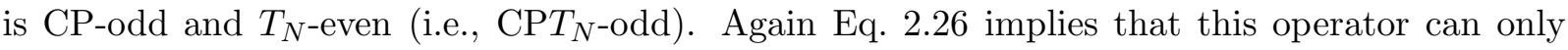
assume a non-zero expectation value if rescattering effects are present. These properties of the operators are summarized in Table 1 .

From Table 1 we see that another consequence of Eq. 2.26 is that a $T_{N}$-odd signal is only a definite signal for $T$ violation and hence of $\mathrm{CP}$ violation in the absence of rescattering effects. To confirm the $\mathrm{CP}$-even or $\mathrm{CP}$-odd nature of such a reaction one must therefore compare data from $i \rightarrow f$ with the charge conjugate channel $\bar{i} \rightarrow \bar{f}$ to explicitly verify CP violation or else rule out rescattering effects in some other way.

Recall from the definition of time reversal that the spatial components of vectors representing

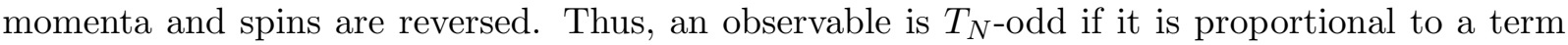
of the form $\epsilon\left(v_{1}, v_{2}, v_{3}, v_{4}\right)$, where $v_{i}$ are 4 -vectors representing spins or momenta of initial and final state particles and $\epsilon$ is the Levi-Civita tensor. Consequently, $T_{N}$-odd signals can only be observed in reactions where there are at least four independent momenta or spins that can be measured.

There are two important venues for the investigation of $\mathrm{CP}$ violation that we will deal with extensively. The first one is when $\mathrm{CP}$ nonconservation appears in decays of a particle and the second, is to search for scattering processes that can give rise to $\mathrm{CP}$ violation. The latter consist of two different possibilities: either the CP-violating effect is due to the subsequent decay of the particle which is produced in the scattering process or the $\mathrm{CP}$ nonconservation is driven by an intrinsic property of the scattering mechanism itself.

An observable which is CP-odd and $T_{N}$-even, thus requiring an absorptive phase (as was shown above), and which is widely used in the case where the CP effect appears in decays of a particle is called PRA (Partial Rate Asymmetry). This observable is non-vanishing when a particle $A$ decays to a state $B$ with a partial width $\Gamma(A \rightarrow B)$ whereas the partial width of the conjugate process, i.e., $\Gamma(\bar{A} \rightarrow \bar{B})$ is different from $\Gamma(A \rightarrow B)$. Thus, defining

$$
\alpha_{P R A} \equiv \frac{\Gamma(A \rightarrow B)-\Gamma(\bar{A} \rightarrow \bar{B})}{\Gamma(A \rightarrow B)+\Gamma(\bar{A} \rightarrow \bar{B})}
$$

it is easy to see that $\alpha_{P R A}$ is odd under $\mathrm{CP}$ and $\mathrm{CP} T_{N}$. For $\alpha_{P R A}$ to receive non-vanishing 
contributions, at least two amplitudes with different (CP-even) absorptive phases as well as with different CP-odd phases must contribute to $A \rightarrow B$. To see this explicitly, let us define $\mathcal{M}_{1}$ and $\mathcal{M}_{2}$ to be the two possible amplitudes contributing to $A \rightarrow B$

$$
\begin{aligned}
& \mathcal{M} \equiv \mathcal{M}(A \rightarrow B)=\left|\mathcal{M}_{1}\right| e^{i \varphi_{1}} e^{i \delta_{1}}+\left|\mathcal{M}_{2}\right| e^{i \varphi_{2}} e^{i \delta_{2}}, \\
& \overline{\mathcal{M}} \equiv \mathcal{M}(\bar{A} \rightarrow \bar{B})=\left|\mathcal{M}_{1}\right| e^{-i \varphi_{1}} e^{i \delta_{1}}+\left|\mathcal{M}_{2}\right| e^{-i \varphi_{2}} e^{i \delta_{2}}
\end{aligned}
$$

where $\varphi_{i}$ are CP-odd phases that change sign as one goes from $A \rightarrow B$ to $\bar{A} \rightarrow \bar{B}$, and $\delta_{i}$ are $\mathrm{CP}$-even phases that can arise due to FSI. It is then easy to see that

$$
|\mathcal{M}|^{2}-|\overline{\mathcal{M}}|^{2}=-4\left|\mathcal{M}_{1}\right|\left|\mathcal{M}_{2}\right| \sin \left(\delta_{1}-\delta_{2}\right) \sin \left(\varphi_{1}-\varphi_{2}\right)
$$

Clearly $\alpha_{P R A}$, being proportional to $\left(|\mathcal{M}|^{2}-|\overline{\mathcal{M}}|^{2}\right)$, will vanish if the two amplitudes do not have a relative absorptive phase, i.e., $\delta_{1}-\delta_{2} \neq 0$ as well as a relative CP-odd phase, i.e., $\varphi_{1}-\varphi_{2} \neq 0$. Of course $\alpha_{P R A}$ does not violate CPT [38] as the requirement of CPT applies only to total widths $\Gamma(A)=\Gamma(\bar{A})$. This equivalence (i.e., $\Gamma(\bar{A})=\Gamma(\bar{A}))$ is due to the fact that the absorptive phase of $\Gamma(A \rightarrow B)$ emanates from rescattering through an on-shell intermediate state $C$ and vice versa, i.e., the absorptive phase for $\Gamma(A \rightarrow C)$ will emanate from the on-shell intermediate state $B$. This fact is an example of the well-known "CP-CPT connection" [39, 40, 41]. The states $B$ and $C$ are referred to as compensating processes. Of course, one can in general have this compensation act between several final states.

It is sometimes useful to define a slightly different asymmetry which also requires dealing with partial rates. This asymmetry is called the Partially Integrated Rate Asymmetry (PIRA) and is defined as

$$
\alpha_{P I R A} \equiv \frac{\Gamma_{P I}(A \rightarrow B)-\Gamma_{P I}(\bar{A} \rightarrow \bar{B})}{\Gamma_{P I}(A \rightarrow B)+\Gamma_{P I}(\bar{A} \rightarrow \bar{B})}
$$

where $\Gamma_{P I}$ is the partially integrated width for $A \rightarrow B$ obtained by integrating only part of the full kinematic range of phase-space. Often such asymmetries can be larger than $\alpha_{P R A}$ since the portion of the final states not included in the integral may themselves be the compensating process. For example, in [42] it was shown that detecting CP violation effects in the process $t \rightarrow b \tau \nu_{\tau}$ through $\alpha_{P I R A}$ is more efficient than through $\alpha_{P R A}$ as the former is driven by tree-level diagrams and the latter by 1-loop diagrams.

A related observable which is also CP-odd and $T_{N}$-even is the energy asymmetry

$$
\alpha_{E} \equiv \frac{<E_{i}>-<E_{\bar{i}}>}{<E_{i}>+<E_{\bar{i}}>},
$$

where $<E_{i}>$ is the average energy of a particle $i$ in a decay of the "parent" particle and $<E_{\bar{i}}>$ is the average energy of the corresponding anti-particle $\bar{i}$ in the decay of the conjugate state of the "parent" particle. Such an asymmetry becomes relevant when the decay involves three or more particles in the final state and may be regarded as a weighted PRA.

A further generalization of the above constructions of CP-odd $T_{N}$-even observables is by considering combinations of dot products (thus being even under $T_{N}$ ) of measurable momenta or spin vectors. Examples of such CP-odd $T_{N}$-even observables will be given in the following chapters.

As an example of the various types of operators discussed above let us consider the reaction

$$
e^{-}\left(p_{e}\right)+e^{+}\left(\bar{p}_{e}\right) \rightarrow t\left(p_{t}, s_{t}\right)+\bar{t}\left(\bar{p}_{t}, \bar{s}_{t}\right),
$$

where $p_{i}$ are 4-momenta and $s_{i}$ are spins. Clearly, no $T_{N}$-odd observable can be constructed without the Levi-Civita tensor, $\epsilon$, and since the momenta satisfy $p_{e}+\bar{p}_{e}=p_{t}+\bar{p}_{t}$ one needs to use the spins of the $t$ and $\bar{t}$ to construct such observables. Indeed no non-trivial CP-odd 
observable can be constructed without knowing the spins either, so top polarimetry is essential for the study of $\mathrm{CP}$ violation in this reaction (see section 2.8).

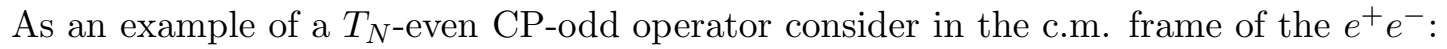

$$
\mathcal{O}_{1}=\left(p_{e}-\bar{p}_{e}\right) \cdot\left(s_{t}-\bar{s}_{t}\right)=-\left(\vec{P}_{e}-\overrightarrow{\vec{P}}_{e}\right) \cdot\left(\vec{S}_{t}-\overrightarrow{\bar{S}}_{t}\right) .
$$

Clearly this is $T_{N}$-even and also $C$-even. It is $P$-odd since $\vec{S}_{t}, \overrightarrow{\bar{S}}_{t}$ are axial vectors while $\vec{P}_{e}, \overrightarrow{\bar{P}}_{e}$ are polar vectors. Thus, since $\mathcal{O}_{1}$ is $\mathrm{CP}$-odd and $\mathrm{CP} T_{N^{-}}$odd, it will have an expectation value if both $\mathrm{CP}$ violation and rescattering effects are present. Consider now the operator

$$
\mathcal{O}_{2}=\epsilon^{\alpha \beta \gamma \delta} p_{e \alpha} \bar{p}_{e \beta} s_{t \gamma} \bar{s}_{t \delta} \propto\left(\vec{P}_{e}-\overrightarrow{\bar{P}}_{e}\right) \cdot\left(\vec{S}_{t} \times \vec{S}_{t}\right) .
$$

Clearly $\mathcal{O}_{2}$ is $T_{N}$-odd and $C$-even but also $P$-odd. This CP-odd observable can therefore give a signal of $\mathrm{CP}$ violation without the necessity of rescattering effects.

Finally the operator

$$
\mathcal{O}_{3}=\epsilon^{\alpha \beta \gamma \delta} p_{e \alpha} \bar{p}_{e \beta}\left(s_{t}-\bar{s}_{t}\right)_{\gamma}\left(p_{t}+\bar{p}_{t}\right)_{\delta} \propto\left(\vec{P}_{e}-\vec{P}_{e}\right) \cdot\left[\left(\vec{S}_{t}-\overrightarrow{\bar{S}}_{t}\right) \times\left(\vec{P}_{t}+\vec{P}_{t}\right)\right],
$$

is $T_{N}$-odd, $P$-even and $C$-even and so provides a signal of CP-conserving rescattering effects. Similarly many more operators can be constructed with the symmetries of the above. Some consideration of the physics to be tested for is helpful in selecting the operator most useful in measuring possible CP-violating effects. We will consider that in more detail in section 2.6.

\subsection{Partial rate asymmetries and the CP-CPT connection}

As mentioned in the previous section, one of the most interesting and widely studied observable for testing $\mathrm{CP}$ violation is the difference between the partial width of a reaction, $\Gamma(A \rightarrow B)$, from that of the conjugate reaction, $\Gamma(\bar{A} \rightarrow \bar{B})$. Thus, if $\Gamma(A \rightarrow B) \neq \Gamma(\bar{A} \rightarrow \bar{B})$, then CP is violated in the decay. In practice, it is better to work with the corresponding dimensionless ratio $\alpha_{\mathrm{PRA}}$ in Eq. 2.27, called the PRA. Its use, specifically in the context of heavy quarks and the SM, first appeared in 44].

Since the CPT theorem demands that particle and anti-particle have identical life-times (or total widths), that theorem imposes important restrictions on the form of CP-violating PRA's; these were first recognized by Gérard and Hou [40].

In perturbative calculations, which lead to PRA's, if all the diagrams are systematically included, the requirement of $\mathrm{CPT}$ - that the total rate and its conjugate be identical - should be manifest order by order. The compensating processes should also be evident as the internal states of loop diagrams. When simplifications are used in such calculations the constraint of the CPT theorem provides an important consistency check. Furthermore, these restrictions can be used to greatly facilitate the calculations of the PRA for a compensating process.

A general formalism for maintaining the CPT constrains in calculating PRA's was given in [41. In particular, it was shown that if one defines a partial width difference, into a particular final state, $I$, as

$$
\Delta_{I} \equiv \Gamma(P \rightarrow I)-\Gamma(\bar{P} \rightarrow \bar{I}),
$$

where $P$ and $\bar{P}$ are the decaying particle and its anti-particle, respectively, then equality of total widths $\Gamma(P)=\Gamma(\bar{P})$ implies

$$
\Delta_{I}=-\sum_{J \neq I} \Delta_{J}
$$

More specifically, in perturbation theory one can write at a given order 


$$
\Delta_{I}=\sum_{J \neq I} \Delta_{I}(J)
$$

and

$$
\Delta_{I}(J)=-\Delta_{J}(I) .
$$

Here $\Delta_{I}(J)$ denotes a contribution to the partial width difference into the final state $I$ which is driven by the final state $J$, and conversely, $\Delta_{J}(I)$ is the contribution to the particle width difference into the final state $J$ being driven by the final state $I$. Due to Eq. 2.39, summing up the partial width differences over all the final states one gets

$$
\sum_{K} \Delta_{K}=0
$$

where $K$ runs over all final states, $I, J, \ldots$ Thus the requirements of CPT are automatically satisfied. Two important conclusions that can also be drawn are:

(a) Rescattering of a state on to itself cannot give rise to PRA. This follows immediately from Eq. 2.39 by setting $J=I$.

(b) The knowledge of the PRA into some final state $I$ that is driven by the absorptive cut across a final state $J$, can be used to deduce the PRA into the final state $J$ arising over a corresponding cut across the state $I$. As mentioned before, such two processes are often called "compensating processes".

Let us first illustrate how these considerations apply to PRA's in $b$ decays. Consider, for example, the process $b \rightarrow s c \bar{c}$. The lowest order non-vanishing contribution to the PRA arises here, at order $\alpha_{s}$, from the interference of the tree graph in Fig. 1)(a) with the penguin graph in Fig. 1(b) which has an absorptive cut across the $u$ quark line. The compensating process is then $b \rightarrow s u \bar{u}$ where, for this process, the absorptive part, $\Im m(l o o p)$, is driven by a cut on the $c$ quark line in the loop. Thus

$$
\Delta_{s c \bar{c}}(s u \bar{u})+\Delta_{s u \bar{u}}(s c \bar{c})=0,
$$

where the compensating nature of these two processes is illustrated in Fig. 1 (c).

At this point it is instructive to discuss in some detail how the cancellation follows from the Feynman diagrams combined in Fig. 11(c). Let us denote by $T_{s c \bar{c}}, T_{s u \bar{u}}$ the tree-level contributions to $b \rightarrow s c \bar{c}, b \rightarrow s u \bar{u}$, respectively. Likewise, let us denote by $P_{s c \bar{c}}^{q}, P_{s u \bar{u}}^{q}$ the penguin contributions to $b \rightarrow s c \bar{c}, b \rightarrow s u \bar{u}$, respectively, with $q=u, c, t$ being the intermediate quark in the penguin. We also denote the conjugate amplitudes as $\bar{T}_{s c \bar{c}}, \bar{T}_{s u \bar{u}}, \bar{P}_{s c \bar{c}}^{q}$ and $\bar{P}_{s u \bar{u}}^{q}$.

These amplitudes may be represented in terms of their magnitude and phase as follows

$$
\begin{array}{ll}
T_{s c \bar{c}}=e^{i \phi_{c}}\left|T_{s c \bar{c}}\right| & \bar{T}_{s c \bar{c}}=e^{-i \phi_{c}}\left|T_{s c \bar{c}}\right| \\
T_{s u \bar{u}}=e^{i \phi_{u}}\left|T_{s u \bar{u}}\right| & \bar{T}_{s u \bar{u}}=e^{-i \phi_{u}}\left|T_{s u \bar{u}}\right| \\
P_{s c \bar{c}}^{q}=e^{i \phi_{q}} e^{i \lambda_{q}^{c}\left|P_{s c \bar{c}}^{q}\right|} & \bar{P}_{s c \bar{c}}^{q}=e^{-i \phi_{q}} e^{i \lambda_{q}^{c}\left|P_{s c \bar{c}}^{q}\right|} \\
P_{s u \bar{u}}^{q}=e^{i \phi_{q}} e^{i \lambda_{q}^{u}}\left|P_{s u \bar{u}}^{q}\right| & \bar{P}_{s u \bar{u}}^{q}=e^{-i \phi_{q}} e^{i \lambda_{q}^{u}}\left|P_{s u \bar{u}}^{q}\right|
\end{array}
$$

Here $\phi_{q}$ is the CP-odd weak phase which has its origin in the Lagrangian. In particular, the SM gives $\phi_{q}=\arg \left(V_{q b} V_{q s}^{*}\right)$, where $V_{q b}$ and $V_{q s}$ are the relevant CKM matrix elements. As can be seen in Eq. 2.42 this phase changes sign under CP.

The tree-level diagrams in perturbation theory have no additional phase, however the penguin diagrams will, if the internal $q \bar{q}$ pair can be on shell [43]. These strong phases do not change sign under CP and are denoted here by $\lambda_{q}^{u}$ and $\lambda_{q}^{c}$. Note that in general $\lambda_{q}^{u}, \lambda_{q}^{c}$ will depend also on the external momenta. 


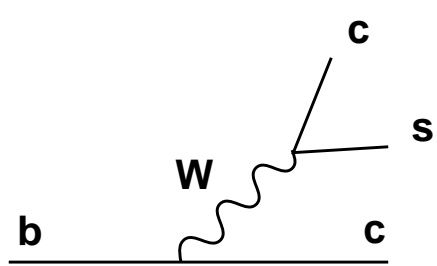

(a)

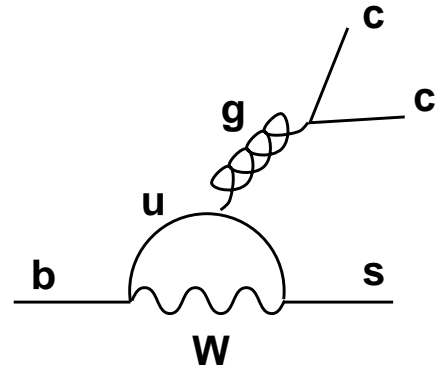

(b)

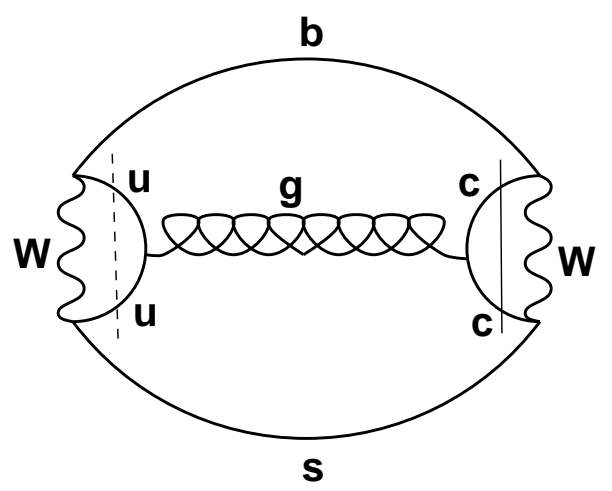

(c)

Figure 1: (a) Tree-level diagram for $b \rightarrow s c \bar{c}$, (b) 1-loop (order $\alpha_{s}$ ) penguin diagram for $b \rightarrow s c \bar{c}$, and (c) a diagrammatic description for the compensating nature of the contribution from $b \rightarrow s c \bar{c}$ and $b \rightarrow s u \bar{u}$ to the PRA's. For $b \rightarrow s c \bar{c}$ : the dashed line indicates an absorptive cut along inner particles $(u \bar{u})$ in the penguin diagram and the solid line refers to an external phase-space cut (c) $)$.

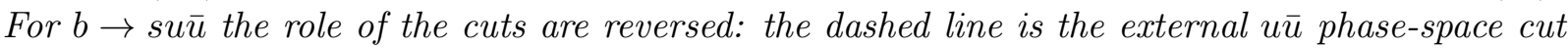
and the solid line indicates the c $\bar{c}$ absorptive cut in the penguin contribution. 
In this notation, the partial width differences that appear in Eq. 2.41 are given by the interference of the tree and penguin amplitudes

$$
\begin{aligned}
\Delta_{s c \bar{c}}(s u \bar{u}) & =\int 2\left\{\Re \mathrm{e}\left(T_{s c \bar{c}} P_{s c \bar{c}}^{u^{*}}\right)-\Re \mathrm{e}\left(\bar{T}_{s c \bar{c}} \bar{P}_{s c \bar{c}}^{u^{*}}\right)\right\} d P h(b \rightarrow s c \bar{c}) \\
& =4 \int\left|T_{s c \bar{c}}\right|\left|P_{s c \bar{c}}^{u}\right| \sin \left(\phi_{c}-\phi_{u}\right) \sin \left(\lambda_{u}^{c}\right) d P h(b \rightarrow s c \bar{c}) \\
\Delta_{s u \bar{u}}(s c \bar{c}) & =\int 2\left\{\Re \mathrm{e}\left(T_{s u \bar{u}} P_{s u \bar{u}}^{c^{*}}\right)-\Re \mathrm{e}\left(\bar{T}_{s u \bar{u}} \bar{P}_{s u \bar{u}}^{c^{*}}\right)\right\} d P h(b \rightarrow s u \bar{u}) \\
& =-4 \int\left|T_{s u \bar{u}}\right|\left|P_{s u \bar{u}}^{c}\right| \sin \left(\phi_{c}-\phi_{u}\right) \sin \left(\lambda_{c}^{u}\right) d P h(b \rightarrow s u \bar{u})
\end{aligned}
$$

where $d P h()$ indicates an integral over the phase-space of the decay.

Referring to Fig. 1( (c) we see that the expression

$$
\int\left|T_{s c \bar{c}}\right|\left|P_{s c \bar{c}}^{u}\right| \sin \left(\lambda_{u}^{c}\right) d P h(b \rightarrow s c \bar{c}),
$$

is the interference of the absorptive part of $P_{s c \bar{c}}^{u}$ (indicated by the dashed line) with the tree-level graph (shown to the right of the solid line). It is identical to

$$
\int\left|T_{\text {sū }}\right|\left|P_{s u \bar{u}}^{c}\right| \sin \left(\lambda_{c}^{u}\right) d P h(b \rightarrow u \bar{u} s),
$$

since all the couplings are the same and we have just interchanged the role of the internal phasespace of the $u \bar{u}$ cut (dashed line) with the external phase-space $c \bar{c}$ cut (solid line). That is, for the PRA in the decay $b \rightarrow s u \bar{u}$, the dashed line represents the external phase-space cut whereas the solid line indicates the absorptive $c \bar{c}$ cut. We thus conclude that

$$
\Delta_{s c \bar{c}}(s u \bar{u})=-\Delta_{s u \bar{u}}(s c \bar{c})
$$

as required.

The same kind of argument may be applied at higher orders in perturbation theory as discussed in [40] in the case of $b$ decays, and is clearly an elaboration of the discussion above. For a further discussion of this example in the $S$-matrix formalism see [41].

Let us now consider the analogous example from top decays. PRA in channels of the type $t \rightarrow u d \bar{d}, c d \bar{d}, \ldots(d=d, s$ or $b)$ arise through interference of the "tree" graph in Fig. 2(a) with the penguin in Fig. 2(b). Since $m_{t}>\left(m_{W}+m_{b}\right)$, the $W$ is on-shell and the $W$-propagator in Fig. 2(a) is complex, as the $W$ has an appreciable width. So the diagram shown in Fig. 2(a) has in fact an imaginary part (indicated by the dashed line) due to the $W$-width, which can dominate over the imaginary part of the penguin diagram depicted in Fig. 2(b). In fact, the 2-loop diagram corresponding to Fig. 2(b) with a "bubble" on the internal $W$-propagator can dominate over the 1-loop diagram.

The CP-CPT connection has two implications here, discussed by Soares [44]. Firstly, in calculating the PRA into a given channel such as $t \rightarrow c b \bar{b}$, part of the total $W$-width that arises from $W \rightarrow c \bar{b}$ must be subtracted away as it represents rescattering of the final state onto itself (see Eq. 2.38). Numerically the most important consequence here is for the channel $t \rightarrow u d \bar{d}$ as then the process $W \rightarrow u \bar{d}$ is not Cabibbo suppressed and indeed contributes about $1 / 3$ to the $W$-width.

A second interesting implication of the CP-CPT connection, pointed by Soares [44], in particular Eq. 2.39, is that the PRA of leptonic decays of the top (e.g., $t \rightarrow d e \nu_{e}$ ) can be deduced from the PRA into its hadronic decays (e.g., $t \rightarrow d c \bar{d}$ ). In the language of Eq. 2.39 these two reactions are the compensating processes of each other, i.e., $I=d c \bar{d}$ and $J=d e^{+} \nu_{e}$ in Eq. 2.39 leading to 


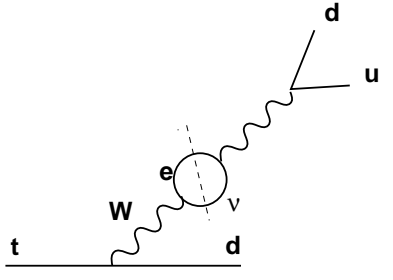

(a)

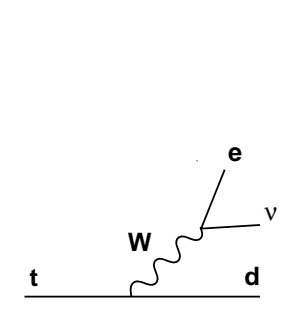

(c)

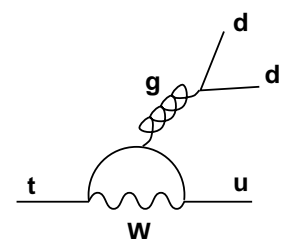

(b)

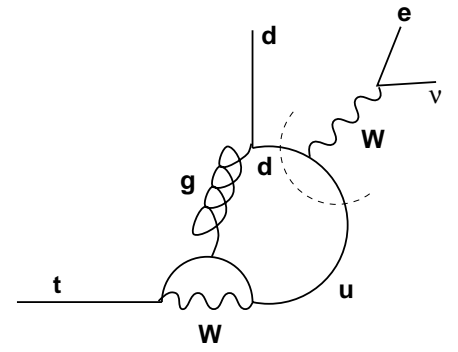

(d)

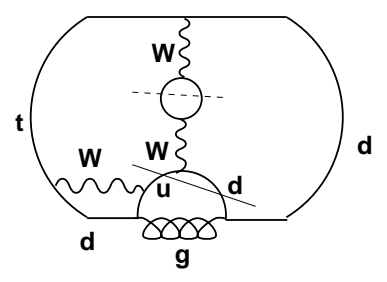

(e)

Figure 2: Feynman diagrams contributing to the decays $t \rightarrow u d \bar{d}(d=d, s$ or $b$ and $u=u$ or $c)$

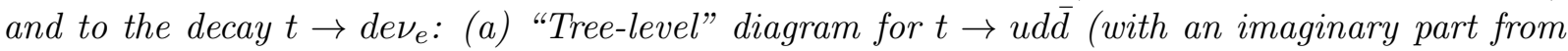
the unstable on-shell $W$ ), (b) 1-loop (order $\alpha_{s}$ ) penguin diagram for $t \rightarrow u d \bar{d}$, (c) The "real" tree-level diagram for $t \rightarrow d e \nu_{e}$, (d) 2-loop contribution to $t \rightarrow d e \nu_{e}$ with an absorptive cut along the intermediate du lines, and (e) A diagrammatic description for the compensating nature of the contribution of the 1-loop $\times 1$-loop processes to a PRA in $t \rightarrow u d \bar{d}$ and the tree $\times 2$-loop processes to a PRA in $t \rightarrow d e \nu_{e}$ (see text for discussion). 


$$
\Delta_{d e \nu_{e}}(d c \bar{d})=-\Delta_{d c \bar{d}}\left(d e \nu_{e}\right) .
$$

The relevant Feynman diagrams that contribute to a PRA in the leptonic top decay $t \rightarrow d e \nu_{e}$ are depicted in Fig. 2(c) (tree-level diagram) and Fig. 2(d) (2-loop diagram with an absorptive cut along the inner $d u$ lines). A graphic illustration of how the compensating nature between the channels $t \rightarrow d u \bar{d}$ and $t \rightarrow d e \nu_{e}$ comes about (i.e., Eq. 2.47) is shown in Fig. 2(e). In this figure the dashed line indicates the absorptive cut that, due to the Cutkosky rule, is responsible for the FSI phase in the decay $t \rightarrow d u \bar{d}$, and the solid line indicates the separation between the two contributing diagrams to the PRA in $t \rightarrow d u \bar{d}$. Similarly, for the decay $t \rightarrow d e \nu_{e}$ the roles of the dashed and solid lines are reversed such that the dashed line indicates the separation between the two contributing diagrams (shown in Figs. 2(c) and 2(d)) and the solid line represents the necessary absorptive cut for the PRA in the decay channel $t \rightarrow d e \nu_{e}$. Notice that a direct calculation of the PRA in the leptonic top decays would have required a calculation of a 2-loop graph (Fig. 2(d)). So this application of the CPT theorem is especially noteworthy as through Eq. 2.39 the necessary calculation gets simplified to dealing with two 1-loop graphs (namely Fig. 2(a) and Fig. 2(b)). Unfortunately all the PRA's resulting from such interferences between the $W$-propagator and the penguin are much too small to be of experimental interest, at least in the SM.

Before we finish this discussion on the CPT constraints on PRA's, let us emphasize two important points. First it should be very clear that these constraints are imposed only on $a$ single CP-violating observable, namely PRA. Indeed, in general, many other $\left(T_{N}\right.$-even) $\mathrm{CP}$ violating observables can be constructed (e.g., energy asymmetry, helicity asymmetry,...) which are similar to PRA in that they all require CP-conserving FSI phase(s). CPT constraints are very specific; they do not affect any of these other observables other than PRA. In particular the stricture of CPT, that the rescattering of a final state onto itself cannot give rise to an asymmetry, is specific to PRA only. Rescattering of a final state onto itself can give rise to important nontrivial experimental implications for all other CP-violating observables; rescattering graphs can certainly cause observable helicity or energy asymmetry. Another way of viewing this situation, in light of the CPT theorem, is to regard each portion of the phase-space or each polarization as a separate final state. Thus one portion of phase-space or polarization state can compensate for another.

As a specific example of this, consider the Schmidt-Peskin effect namely the helicity asymmetry [20]:

$$
\Delta N_{L R}=\frac{N\left(t_{L} \bar{t}_{L}\right)-N\left(t_{R} \bar{t}_{R}\right)}{\text { all } t \bar{t}}
$$

induced in the reaction $p p \rightarrow t \bar{t}+X$. Here $N\left(t_{L} \bar{t}_{L}\right)$ represents the number of pairs of left-handed $t$ and left-handed $\bar{t}$ produced in the inclusive reaction. In a $2 \mathrm{HDM}$ with CP-violating phase(s) in the neutral Higgs sector, a non-vanishing value of $\alpha_{h}$ arises from interference of tree-level diagrams with 1-loop diagrams involving neutral Higgs exchanges in the loops (see Fig. 47 in Chapter 0 ). In this case, the CP-conserving FSI phase required by $\alpha_{h}$ is provided by an absorptive cut which explicitly arises from $t \bar{t} \rightarrow t \bar{t}$, i.e., rescattering of a final state onto itself. So a very interesting CP-violating effect, namely the helicity asymmetry in Eq. 2.48 - a CP-odd $T_{N}$-even observable (similar to PRA with respect to this classification) - arises from self-rescattering of a final state (we will return to a more detailed discussion of the Schmidt-Peskin effect in Chapter 7). Thus, in general, in discussing $\mathrm{CP}$ violation phenomena or in calculations of $\mathrm{CP}$ violation effects, selfrescattering graphs should not automatically be discarded, unless of course one is specifically calculating PRA's. In particular, for the specific case discussed above, we may regard $t_{L} \bar{t}_{L}$ and $t_{R} \bar{t}_{R}$ as compensating final states.

An important, although somewhat obvious consequence is that even when PRA's are vanishingly small or exactly zero, other CP-odd observables can be non-vanishing and can have interesting observational consequences. 
Another interesting example arises from CP-violating phase originating from a charged Higgs sector, as in the Weinberg model [45] with three doublets of Higgs fields. Important CP-violating effects arise, for example, in the leptonic decay $t \rightarrow b \tau \nu_{\tau}$ from the interference of the SM graph (with $W$ exchange) with the tree-level charged Higgs exchange graph (see Figs. 22(a) and 22(c) in Chapter 5).

For this case, let us first consider the PRA

$$
\alpha_{\tau}=\frac{\Gamma(t \rightarrow b \tau \nu)-\Gamma(\bar{t} \rightarrow \bar{b} \bar{\tau} \bar{\nu})}{\Gamma(t \rightarrow b \tau \nu)+\bar{\Gamma}(\bar{t} \rightarrow \bar{b} \bar{\tau} \bar{\nu})} .
$$

For simplicity, let us assume that $m_{H^{+}}>m_{t}$; then a CP-conserving absorptive part required for $\alpha_{\tau}$ arises from the $W$-boson "bubble" which contains all possible states other than $\tau \nu_{\tau}$ as required by CPT. However, because of the spin zero nature of the Higgs the $W^{+}{ }_{-} H^{+}$interference has nonvanishing contributions only for the scalar part of the $W$. This argument is most readily seen if one uses the Landau gauge for calculating this interference (for more details see Chapter 5). In that case, the scalar and vector components of the $W$ propagator are cleanly separated according to their total angular momentum. Thus, graphs which pass through a vector $W$ intermediate state will not interfere with graphs that pass through a Higgs state. The Higgs must therefore interfere only with the Goldstone propagator which corresponds to the decay of longitudinal $W$ into fermion pairs $c \bar{s}, u \bar{d}, e \nu_{e} \ldots$ which are all suppressed by powers of the fermion masses. Furthermore, the Goldstone propagator shows none of the resonance enhancement associated with the vector component. Thus the $c \bar{s}$ is the most important contributor to the scalar component of the $W$-boson "bubble". The $\tau \bar{\nu}$ and $c \bar{s}$ can be thought of as the compensating processes. So, in fact, the PRA goes as

$$
\alpha_{\tau} \sim \frac{m_{c}^{2}}{m_{t}^{2}} \frac{m_{\tau}^{2}}{m_{t}^{2}} \frac{\Gamma_{W}}{m_{W}}
$$

and is extremely small [46].

However, as already mentioned before, just because PRA is vanishingly small does not, though, mean that there are no $\mathrm{CP}$ violation effects. Indeed, very important and large $\mathrm{CP}$ violating asymmetries may arise in the decay $t \rightarrow b \tau \nu_{\tau}$. First of all, one can construct an energy asymmetry:

$$
\alpha_{E}=\frac{\left\langle E_{\tau}\right\rangle-\left\langle E_{\bar{\tau}}\right\rangle}{\left\langle E_{\tau}\right\rangle+\left\langle E_{\bar{\tau}}\right\rangle} .
$$

That is, compare, e.g., the average energy of the $\tau$ in $t \rightarrow b \tau \nu$ with that of the $\bar{\tau}$ in $\bar{t} \rightarrow \bar{b} \bar{\tau} \bar{\nu}$. Then $\alpha_{E}$ will not suffer from the helicity suppression or constraints of CPT on $\alpha_{\tau}$ and one expects

$$
\frac{\alpha_{E}}{\alpha_{\tau}} \sim \frac{m_{t}^{2}}{m_{c}^{2}} \frac{m_{t}^{2}}{m_{\tau}^{2}}
$$

as explicit calculations confirm.

Indeed, a CP-violating asymmetry even bigger than the energy asymmetry, namely the transverse polarization asymmetry of the $\tau$, resides in this $W^{+}-H^{+}$interference. In fact, the transverse polarization asymmetry is enhanced by another factor of $m_{t} / m_{\tau}$ compared to the energy asymmetry as is shown in section 5.1.3.

\subsection{Resonant $W$ effects and CP violation in top decays}

The large mass of the top $\left(m_{t} \simeq 174 \mathrm{GeV}\right)$ means that it decays to a three-body final state primarily through an on-shell $W$. This fact is of particular interest in the study of CP violation in such decays since there will be a large strong (i.e., CP-even) phase inherent in this $W$-propagator. 
In particular, since the $W$-width is substantial $\left(\Gamma_{W} \sim 2 \mathrm{GeV}\right)$, the transverse modes of the $W$ are controlled by the Breit-Wigner propagator

$$
G_{T}=\frac{1}{q_{W}^{2}-m_{W}^{2}+i m_{W} \Gamma_{W}}
$$

which will have a substantial strong phase.

The enhancement of the imaginary part of $G_{T}$ is evident by considering that, at $q_{W}^{2}=m_{W}^{2}$,

$$
\left|\Im \mathrm{m}\left(G_{T}\right)\right|=\left(m_{W} \Gamma_{W}\right)^{-1} .
$$

The real part swings through 0 at this point but in the vicinity of the resonance it will also be large. For instance, if $\left|q_{W}^{2}-m_{W}^{2}\right|=m_{W} \Gamma_{W}$, then

$$
\left|\Re \mathrm{e}\left(G_{T}\right)\right|=\left(2 m_{W} \Gamma_{W}\right)^{-1} .
$$

Since $\Gamma_{W} \sim \mathcal{O}(\alpha)$ this means that near $q^{2} \rightarrow m_{W}^{2}$ both the real and imaginary parts of the amplitudes for decays such as $t \rightarrow b u \bar{d}, b c \bar{b}, \ldots$ behave as if they are $\mathcal{O}(1)$ in the gauge coupling constant. This phenomena is what we mean by "resonance enhancement". The imaginary part here then provides the needed absorptive part (i.e., FSI phase) to lead to enhancement of CP-odd $T_{N}$-even observables. Likewise, near $q^{2} \sim m_{W}^{2}$, the real part can magnify the effect of CP-odd

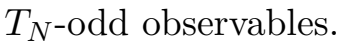

The basic idea of FSI phase driven by particle widths in decays was discussed in [47] (see also [48]). Although originally the discussion [47] took place in the context of the SM in conjunction with the CKM phase, it should be completely clear that they can be equally well used with non-standard sources of CP violation. Indeed, as dealt in section 5.1.3, the $W$-resonant effects provide a significant enhancement of $T_{N}$-even and $T_{N}$-odd CP-violating effects in top decays in the context of an extended Higgs sector.

\subsection{Effective Lagrangians and observables}

One tool that is often used to catalog the effects of new physics at an energy scale, $\Lambda$, much higher than the electroweak scale, is the effective Lagrangian $\left(\mathcal{L}_{\text {eff }}\right)$ method. If the underlying extended theory under consideration only becomes important at a scale $\Lambda$, then it makes sense to expand the Lagrangian in powers of $\Lambda^{-1}$ where the $\Lambda^{0}$ term is the SM Lagrangian and the other terms are the effective Lagrangian terms.

Simple dimensional arguments tell us that the operator which multiplies $\Lambda^{-n}$ must be of dimension $n+4$. This restriction together with symmetry considerations implies that at each order in $\Lambda^{-1}$ there are only a finite number of possible terms. Conversely, this implies that experimantal tests for the existence of specific terms in $\mathcal{L}_{\text {eff }}$ is a relatively model independent 49] way to search for new physics.

Here we are interested in top quark physics which violates $\mathrm{CP}$ and so, in the effective Lagrangian approach, the operators of interest are further restricted. For example, in Chapter 4 we discuss the top electric dipole moment (and related effects) which can arise from a dimension 5 term in the effective Lagrangian:

$$
\mathcal{L}_{e f f}^{[5]} \propto \bar{t} \sigma_{\mu \nu} \gamma_{5} t F^{\mu \nu}
$$

At dimension 6, photons can interact with the top quark via a $\mathrm{CP}$-violating operator such as

$$
\mathcal{L}_{e f f}^{[6]} \propto\left(\bar{t} \gamma_{5} t\right)\left(F^{\mu \nu} F_{\mu \nu}\right)
$$

which could arise, for instance, via a SUSY box diagram.

Let us now consider as a specific example effective Lagrangian terms which would contribute to the process $g g \rightarrow t \bar{t}$. It is useful to recall that in such an expansion, operators that are 
proportional to the QCD equations of motion for the top or the gluon fields may be eliminated by a field redefinition and are therefore redundant [49].

There are a number of requirements that an operator has to satisfy for it to be relevant to $\mathrm{CP}$ violation in top production in hadronic collisions. These are:

1. It must violate CP.

2. Its Feynman rules must include couplings to two or fewer gluons.

3. It must not be proportional to $q^{2}$ of one of the on-shell gluons in the initial state.

The need for the first requirement is clear. The second requirement is present since the events in question have two gluons in the initial state and no gluons in the final states. If one wanted to consider experiments where additional gluon jets were detected in the final state, clearly one would have to generalize this requirement.

In constructing a basis of operators which satisfy the above conditions it can be shown that [50] one can eliminate, without loss of generality, any operator which is equal to 0 modulo the equations of motion. Equivalently, if the difference of two operators is 0 modulo equations of motion, then only one need to be included.

Here is a set of operators that we choose which satisfy the requirements mentioned above and are of dimension six or less

$$
\begin{aligned}
O_{a} & =\bar{t} i\left[\frac{1}{2} f_{a}(-\square) F_{i}^{\mu \nu}\right] \sigma_{\mu \nu} \gamma_{5} T^{i} t, \\
O_{b} & =\bar{t} i\left[f_{b}(-\square) F_{i}^{\mu \nu} F_{\mu \nu}^{i}\right] \gamma_{5} t, \\
O_{c} & =\bar{t} \epsilon_{\alpha \beta \gamma \delta}\left[f_{c}(-\square) F_{i}^{\alpha \beta} F_{i}^{\gamma \delta}\right] t, \\
O_{d} & =\bar{t} i\left[f_{d}(-\square) F_{j}^{\mu \nu} F_{\mu \nu}^{k}\right] \gamma_{5} d^{i j k} T^{i} t, \\
O_{e} & =\bar{t} \epsilon_{\alpha \beta \gamma \delta}\left[f_{c}(-\square) F_{j}^{\alpha \beta} F_{k}^{\gamma \delta} d^{i j k}\right] T^{i} t,
\end{aligned}
$$

where $F$ 's are the gluon field strength tensor, $\square=D^{\mu} D_{\mu}$, the analytic functions $f_{a}, \ldots, f_{e}$ are form factors and $T^{i}=\lambda^{i} / 2, \lambda^{i}$ being the Gell-Mann color matrices. Note, that in general, higher order terms in $\square$ will imply the existence of couplings to additional numbers of gluons.

As an illustration, let us consider further the operator $O_{a}$. This operator essentially corresponds to the Chromo-Electric Dipole Moment (CEDM) form factor. The experimental implications of the static analog of this quantity were considered in 22]. We can expand the operator to obtain the Feynman rule. The vertex for the one gluon interaction is

$$
i f_{a}\left(q^{2}\right) \bar{t} \sigma^{\mu \nu} \gamma_{5} T^{j} t q_{\mu} \epsilon_{\nu}^{j}
$$

here $\epsilon_{\mu}$ is the polarization vector of the gluon. This is completely analogous to the EDM form factor. However $O_{a}$ now also gives rise to a two gluon coupling given for on-shell gluons by

$$
\begin{aligned}
& g_{s} \bar{t} \sigma^{\mu \nu} \gamma_{5} T^{i} t F^{i j k}\left[h_{a}\left(q^{2}\right) \epsilon_{1 \mu}^{j} \epsilon_{2 \nu}^{k}\right. \\
& \left.\quad+\left[\frac{h_{a}\left(q^{2}\right)-h_{a}(0)}{q^{2}}\right]\left(q_{2} \cdot \epsilon_{1}^{j} q_{2 \mu} \epsilon_{2 \nu}^{k}-q_{1} \cdot \epsilon_{2}^{k} q_{1 \mu} \epsilon_{1 \nu}^{j}\right)\right],
\end{aligned}
$$

where $q=q_{1}+q_{2}$. Note that the second term that appears is needed to maintain gauge invariance.

An important feature of $f_{a}$ (as well as of the other form factors) is that the constant piece $f_{a}(0)$ must be real while, at $q^{2} \neq 0, f_{a}$ may have an imaginary part due to the possibility of 
thresholds giving rise to absorptive pieces. Indeed these type of effects have also been considered in some particular extensions of the SM (see Chapter 田).

The phenomenology of the static CEDM (i.e., for $q^{2} \simeq 0$ ) was considered extensively in [22]. It was shown that in a hadron collider with $\sim 10^{7} t \bar{t}$ pairs, both of which decay leptonically, a precision of about $5 \times 10^{-20} g_{s}$-cm for $f_{a}(0)$ could be achieved. We can extend this consideration with a simplifying assumption that $f_{a}$ is approximately constant above the $t \bar{t}$ threshold. We can then introduce the quantity $f^{\prime}=f_{a}\left(4 m_{t}^{2}\right)-f_{a}(0)$. With this assumption and approximation we find that, under ideal conditions, $\Re \mathrm{e}\left(f^{\prime}\right)$ and $\Im \mathrm{m}\left(f^{\prime}\right)$ can also be measured to a precision of about $(2-3) \times 10^{-20} g_{s^{-}} \mathrm{cm}$.

The Weinberg model with an extended Higgs sector provides a specific example of nonstandard physics where one can study this general feature of the operator analysis above. We recall that the source of $\mathrm{CP}$ violation now are the charged Higgs exchanges (see section 3.2.4). Since $O_{a}$ is the only operator which gives a one gluon Feynman rule, the electromagnetic form factor calculated in [18] is the quantity $f_{a}$ except for the replacement of $g_{s}$ with $e$, the electric charge. The result of that reference is that thus far QCD yields a limit around $5 \times 10^{-20} \mathrm{~g}_{\mathrm{s}} \mathrm{-cm}$. In that work it is also explicitly shown (see Chapter 1 ) that the $q^{2}$ dependence is rather mild. Furthermore, above threshold the $\Im m\left(f_{a}\right)$ was also shown to have roughly the same ball park value. Thus, studies at a hadron collider could exhibit CP-violating signals although admittedly the experimental challenges are formidable.

Another useful way to characterize the amplitude for $g g \rightarrow t \bar{t}$ is to express it in terms of form factors. There are three possible color structures which such an amplitude can have. If $A, B$ are the color indices of the gluon and $i, j$ the indices of the $t$ and $\bar{t}$, these color structures are

$$
\begin{aligned}
\Delta & =\delta_{A B} \delta_{i j}, \\
D & =d^{A B C} T_{i j}^{C}, \\
F & =f^{A B C} T_{i j}^{C} .
\end{aligned}
$$

Let us define $P_{g 1}, P_{g 2}$ to be the momenta of the gluons and $P_{t 1}, P_{t 2}$ to be the momenta of the $t, \bar{t}$ quarks respectively. Let us further define the variables

$$
\begin{gathered}
s=\left(P_{g 1}+P_{g 2}\right)^{2}, \quad t=\left(P_{g 1}-P_{t 1}\right)^{2}, \quad u=\left(P_{g 1}-P_{t 2}\right)^{2} \\
z=(t-u) /\left(s-2 m_{t}^{2}\right)
\end{gathered}
$$

Let $E_{1}$ and $E_{2}$ be the polarizations of the gluons in a gauge where $E_{1} \cdot P_{2}=E_{2} \cdot P_{1}=0$.

Here, we are interested in amplitudes which violate CP. These amplitudes must also be symmetric under the interchange of the two gluons. The helicity amplitudes which satisfy these conditions are

$$
\begin{aligned}
a_{1}^{n} & =f_{1}^{n}\left(s, z^{2}\right)\left(E_{1} \cdot E_{2}\right)\left(\bar{t} \gamma^{5} t\right)[D, \Delta, F z] \\
a_{2}^{n} & =f_{2}^{n}\left(s, z^{2}\right)\left(\epsilon_{\mu \nu \sigma \rho} E_{1}^{\mu} E_{2}^{\nu} P_{g 1}^{\sigma} P_{g 2}^{\rho}\right)(\bar{t} t)[D, \Delta, F z] \\
a_{3}^{n} & =z f_{3}^{n}\left(s, z^{2}\right)\left(\epsilon_{\mu \nu \sigma \rho}\right) E_{1}^{\mu} E_{2}^{\nu} P_{t 1}^{\sigma} P_{t 2}^{\rho}(\bar{t} t)[D, \Delta, F z] \\
a_{4}^{n} & =z f_{4}^{n}\left(s, z^{2}\right) E_{1}^{\mu} E_{2}^{\nu}\left(\bar{t} \sigma_{\mu \nu} \gamma^{5} t\right)[D, \Delta, F z] \\
a_{5}^{n} & =f_{5}^{n}\left(s, z^{2}\right)\left(\epsilon_{\mu \nu \sigma \rho} E_{1}^{\mu} E_{2}^{\nu}\left(P_{g 1}+P_{g 2}\right)^{\sigma}\right)\left(\bar{t} \gamma^{\rho} t\right)[D, \Delta, F z]
\end{aligned}
$$

where $f_{i}$ is a function of $s$ and $z^{2}$ and the notation $[D, \Delta, F z]$ means that the term may be multiplied by any of these color structures, the index $n=1,2,3$ respectively depending on which of these color structures apply.

As an explicit example, at tree-level, the CEDM operator discussed above will contribute to the amplitude $a_{4}^{3}$. 


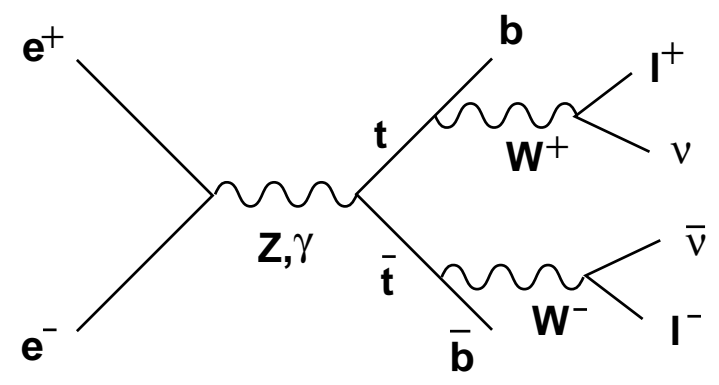

Figure 3: Feynman diagram describing the process $e^{+} e^{-} \rightarrow t \bar{t}$ followed by the $t \bar{t}$ decays $t \rightarrow$ $b W^{+} \rightarrow b \ell^{+} \nu_{\ell}$ and $\bar{t} \rightarrow \bar{b} W^{-} \rightarrow \bar{b} \ell^{-} \bar{\nu}_{\ell}$.

\subsection{Optimized observables}

Due to the exceedingly short life-time of the top quark, measurement of its couplings requires considering top production and decay simultaneously. Consider, for example, the production and decay of $t \bar{t}$ in $e^{+} e^{-}$annihilation (see Fig. 3),

$$
e^{+}\left(p_{+}\right)+e^{-}\left(p_{-}\right) \rightarrow t\left(p_{t}\right)+\bar{t}\left(p_{\bar{t}}\right)
$$

with

$$
t\left(p_{t}\right) \rightarrow b\left(p_{b}\right)+W^{+}\left(p_{W^{+}}\right)
$$

and

$$
\bar{t}\left(p_{\bar{t}}\right) \rightarrow \bar{b}\left(p_{\bar{b}}\right)+W^{-}\left(p_{W^{-}}\right) .
$$

Indeed each of the $W^{ \pm}$also decays leptonically or hadronically (i.e., into jets). Thus

$$
\begin{aligned}
W^{+}\left(p_{W^{+}}\right) & \rightarrow \ell^{+}\left(p_{\ell^{+}}\right)+\nu_{\ell}\left(p_{\nu}\right) \\
W^{-}\left(p_{W^{-}}\right) & \rightarrow \ell^{-}\left(p_{\ell^{-}}\right)+\bar{\nu}_{\ell}\left(p_{\bar{\nu}}\right), \\
W^{ \pm} & \rightarrow j_{1}\left(p_{j_{1}}\right)+j_{2}\left(p_{j_{2}}\right) .
\end{aligned}
$$

This allows one to construct a multitude of observables, involving momenta of the initial beam and various decay products, to probe the presence of anomalous vertices in the top interactions. The case of the CP violating dipole moment interactions is especially interesting to this work. Such anomalous terms could occur at the $\bar{t} t \gamma, \bar{t} t Z$ or $\bar{t} t g$ vertices corresponding to electric, weak or chromo-electric dipole moment of the top quark. Of experimental interest are the values of the corresponding form factors at $q^{2}=s$, the square of the c.m. energy rather than the moments $\left(q^{2}=0\right)$ themselves. It is possible, therefore, that the form factor is complex. The real and imaginary parts can thus lead to distinct experimental effects.

Two examples of simple (or "naive") observables that can be used to probe the presence of imaginary part of the dipole moment form factor are

$$
\begin{aligned}
& \frac{\left\langle E_{\ell^{+}}\right\rangle-\left\langle E_{\ell^{-}}\right\rangle}{\left\langle E_{\ell^{+}}\right\rangle+\left\langle E_{\ell^{-}}\right\rangle}, \\
& \frac{\left\langle E_{b}\right\rangle-\left\langle E_{\bar{b}}\right\rangle}{\left\langle E_{b}\right\rangle+\left\langle E_{\bar{b}}\right\rangle} .
\end{aligned}
$$


Examples of simple (or "naive") observables that probe the presence of the real part of the dipole moment form factor are

$$
\begin{gathered}
\frac{\left\langle\epsilon_{\mu \nu \rho \sigma} p_{\ell^{+}}^{\mu} p_{\ell^{-}}^{\nu} p_{b}^{\rho} p_{\bar{b}}^{\sigma}\right\rangle}{\left(p_{\ell^{+}} \cdot p_{\ell^{-}} p_{b} \cdot p_{\bar{b}}\right)^{1 / 2}}, \\
\frac{\left(p_{\ell^{+}}^{x} p_{\ell^{-}}^{y}-p_{\ell^{+}}^{y} p_{\ell^{-}}^{x}\right) \operatorname{sgn}\left(p_{\ell^{+}}^{z}-p_{\ell^{-}}^{z}\right)}{\left(p_{\ell^{+}} \cdot p_{\ell^{-}}\right)^{1 / 2}} .
\end{gathered}
$$

The ability to polarize the electron beams at a future $e^{+} e^{-}$collider (e.g., the NLC) allows us to construct additional observables involving beam polarization. Clearly, while many observables can be constructed to probe the dipole moment, we may ask whether it is possible to construct an "optimal observable" i.e., one which will be the most sensitive or will have the largest "resolving power". A general procedure for constructing an optimal observable was given in [16]. Here we will briefly review the method.

Let us write the differential cross-section as a sum of two terms

$$
\Sigma(\phi)=\Sigma_{0}(\phi)+\lambda \Sigma_{1}(\phi),
$$

where $\lambda$ is a parameter (e.g., dipole moment or magnetic moment form factor) and $\phi$ is some phase-space variable (including angular and polarization variables).

For an ideal detector that accurately records the value of $\phi$ for each event that occurs, any method for determining the value of $\lambda$ amounts to weighting the events with a phase-spacedependent function $f(\phi)$ which we assume is CP-odd. Let us define

$$
f^{(1)}(\lambda)=\int f(\phi) \Sigma(\phi) d \phi
$$

Thus the change due to the contribution from the presence of $\lambda$ is

$$
f^{(1)}(\lambda)=\lambda \int f(\phi) \Sigma_{1}(\phi) d \phi \equiv \lambda f^{(1)}(\lambda=1) .
$$

$f^{(1)}(\lambda)$ then has to be compared to the error in its measurement. If $n$ events are recorded, the error is

$$
\Delta f=\left[\frac{f^{(2)} \sigma-\left(f^{(1)}(\lambda)\right)^{2}}{n \sigma^{2}}\right]^{1 / 2} \approx\left[\frac{f^{(2)}}{n \sigma}\right]^{1 / 2},
$$

where

$$
\sigma=\int \Sigma d \phi=\int \Sigma_{0} d \phi
$$

is the total cross-section and

$$
f^{(2)}=\int f^{2} \Sigma d \phi=\int f^{2} \Sigma_{0} d \phi .
$$

Note that $f^{(1)}(\lambda) \propto \lambda$ but $f^{(2)}$ and $\sigma$ do not depend on $\lambda$ to first order; we will assume that $\lambda$ is sufficiently small so that the above approximation is valid as well as $f^{(2)} \sigma>>\left(f^{(1)}(\lambda)\right)^{2}$. We now introduce the "resolving power", $R$, which measures the effectiveness of an operator for determining $\lambda$. For the function $f, R$ is defined as

$$
R=\frac{1}{n \sigma^{2} \lambda^{2}} \frac{\left(f^{(1)}(\lambda)\right)^{2}}{(\Delta f)^{2}}=\frac{\left(f^{(1)}(1)\right)^{2}}{f^{(2)} \sigma} .
$$


The statistical significance $S$, with which the presence of a nonzero value of $\lambda$ may be ascertained, is given by

$$
S=\frac{f^{(1)}(\lambda)}{\sigma \Delta f}=\lambda \sqrt{n R} .
$$

Thus the larger $R$ is the more effective $f(\phi)$ is for measuring $\lambda$. Clearly one would like to choose a function $f(\phi)$ to determine $\lambda$ for which $R$ is maximal.

A special case of such observables are asymmetries, which are observables where $f(\phi)= \pm 1$. For such observables $f^{(2)}=\sigma$ so (see Eq. 2.78),

$$
R=\left(f^{(1)}(1)\right)^{2} / \sigma^{2}=\langle f\rangle^{2} / \lambda^{2} .
$$

In this case it is conventional to denote for an asymmetry $f$

$$
\langle f\rangle=\alpha_{f}
$$

so that Eq. 2.79 becomes

$$
S=\left|\alpha_{f}\right| \sqrt{n} .
$$

Thus the quantity $\lambda \sqrt{R}$ is the natural generalization of $\left|\alpha_{f}\right|$ for more general observables among which we would like to find the optimal one, i.e., that which maximizes the value of $R$ and therefore also the statistical significance with which it can be measured.

In order to define such an optimal function (i.e., $f_{\text {opt }}$ ) it is useful to decompose an arbitrary function $f$ as [16]:

$$
f=A \frac{\Sigma_{1}}{\Sigma_{0}}+\hat{f}
$$

where $A$ is defined as

$$
A=\frac{\int f \Sigma_{1} d \phi}{\int \frac{\Sigma_{1}^{2}}{\Sigma_{0}} d \phi} .
$$

Since rescaling $f$ does not change the value of $R$ we can take $A=1$. If we now expand the definition of $R$ to lowest order in $\lambda$ and use the above decomposition we get

$$
\sigma R=\frac{\left|\int f \Sigma_{1} d \phi\right|^{2}}{\int f^{2} \Sigma_{0} d \phi}=\frac{\left|\int \frac{\Sigma_{1}^{2}}{\Sigma_{0}} d \phi\right|^{2}}{\int \frac{\Sigma_{1}^{2}}{\Sigma_{0}} d \phi+\int \hat{f}^{2} \Sigma_{0} d \phi} .
$$

Since the two terms in the denominator are each positive it is clear that $R$ is maximal when $\hat{f}=0$. Thus, from Eq. 2.83,

$$
f=f_{\mathrm{opt}}=\frac{\Sigma_{1}}{\Sigma_{0}}
$$

maximizes $R$.

Another way to understand this derivation of $f_{\text {opt }}$ is to introduce the concept of a vector space as the set of all functions on which we define the scalar product

$$
g_{1} \cdot g_{2}=\int g_{1}(\phi) g_{2}(\phi) \Sigma_{0}(\phi) d \phi
$$

where $g_{1,2}$ are functions of $\phi$. If we denote $f_{0}=\Sigma_{1} / \Sigma_{0}$ then in this notation Eq. 2.85 may be rewritten as 


$$
\sigma R=\left(f \cdot f_{0}\right)^{2} / f \cdot f .
$$

Thus $R$ is maximized when $f=f_{0}=f_{\text {opt }}$.

Let us first illustrate these consideration with a toy example. Consider a $2 \rightarrow 2$ scattering process where the differential cross-section takes the form

$$
\frac{d \sigma}{d \xi}=A+B \xi+C \xi^{2}
$$

where $\xi=\cos \theta$ and $B, C \leq A$. According to the preceding discussion, the function most sensitive to $B$ is $f_{B}=\xi$. Thus explicitly we find $\sigma=2 A+\frac{2}{3} C, f_{B}^{(1)}=\frac{2}{3} B, f_{B}^{(2)}=\frac{2}{3} A+\frac{2}{5} C$. Using Eq. 2.78, the resolving power for the optimum choice $\left(f_{B}=\xi\right)$ is

$$
R\left(f_{B}\right)=\frac{1}{B^{2}} \frac{\left[\int_{-1}^{1} B \xi^{2} d \xi\right]^{2}}{\int_{-1}^{1} A d \xi \int_{-1}^{1} A \xi^{2} d \xi}=\frac{1}{3} \frac{1}{A^{2}}
$$

Suppose we consider measuring $B$ via another function, which has the same symmetry properties as $f_{B}$ and is defined as:

$$
g_{B}(\xi)=\begin{array}{ll}
+1 & \text { if } \xi>0 \\
-1 & \text { if } \xi<0
\end{array} .
$$

Then one gets $g_{B}^{(1)}=B, g_{B}^{(2)}=2 A+\frac{2}{3} C$, so that $R\left(g_{B}\right)=\frac{1}{4} \frac{1}{A^{2}}$, which is not as good as $R\left(f_{B}\right)$.

\section{$\underline{\text { Generalizations }}$}

This simple method outlined above for an optimized observable has been widely used and a useful generalization has been proposed by Gunion, Grzadkowski and He [51].

In general, we will assume that the differential cross-section can be expressed as

$$
\Sigma(\phi) \equiv \frac{d \sigma}{d \phi}=\sum_{i} c_{i} f_{i}(\phi)
$$

where $c_{i}$ are model-dependent coefficients and $f_{i}(\phi)$ are known functions of the final state phasespace variable(s), $\phi$. Thus, $c_{i}$ may be couplings; some or all of which one is trying to extract from experimental data. So, in analogy, with the discussion above, the task is to find an optimal choice for the functions $f_{i}(\phi)$ such that, with a fixed set of data, $c_{i}$ can be deduced with maximal statistical precision.

The coefficients $c_{i}$ can be extracted by using the appropriate weighting functions $w_{i}(\phi)$ such that $\int w_{i}(\phi) \Sigma(\phi) d \phi=c_{i}$. As in the special example of the electric dipole moment discussed above, in general, there are an infinite number of choices for $w_{i}(\phi)$ that satisfy this condition which is equivalent to

$$
\int w_{i}(\phi) f_{j}(\phi) d \phi=\delta_{i j}
$$

It can be shown [51] that minimizing the statistical error is equivalent to finding the stationary point of $\int w_{i} w_{j} \Sigma(\phi) d \phi$. In other words, solving

$$
\delta \int w_{i}(\phi) w_{j}(\phi) \Sigma(\phi) d \phi=0,
$$

subject to the condition in Eq. 2.93. The solution to this condition is

$$
w_{i}=\frac{\sum_{j} X_{i j} f_{j}(\phi)}{\Sigma(\phi)}
$$


where $X_{i j}=M_{i j}^{-1}$ and

$$
M_{i j} \equiv \int \frac{f_{i}(\phi) f_{j}(\phi)}{\Sigma(\phi)} d \phi
$$

The desired coefficients, $c_{i}$, are then given by

$$
c_{i}=\sum_{k} X_{i k} I_{k}=\sum_{k} M_{i k}^{-1} I_{k}
$$

where

$$
I_{k} \equiv \int f_{k}(\phi) d \phi
$$

It follows that with this method of determining $c_{i}$, the covariance matrix is

$$
V_{i j} \equiv\left\langle\Delta c_{i} \Delta c_{j}\right\rangle=M_{i j}^{-1} \sigma_{T} / N
$$

where $\sigma_{T}$ is the total cross-section and $N=L_{\text {eff }} \sigma_{T}$ is the total number of events, with $L_{e f f}$ being the effective luminosity, i.e., luminosity times the efficiencies.

It is important to note that since the entire method has to be implemented numerically utilizing experimental data, experimental cuts and efficiencies can be incorporated. Indeed the method has the additional virtue that, due to its generality, it can be applied to only a subset $\hat{\phi}$ of the kinematic variables that can be determined if some (say $\bar{\phi}$ ) among the total of $\phi$ cannot be determined. Situations such as this can occur due to experimental limitations. For example, in the case of the important reaction $e^{+} e^{-} \rightarrow t \bar{t} H^{0}, H^{0}=$ neutral Higgs, to fully define a point in phase-space one must be able to identify the momenta of the $t$ and the $\bar{t}$ and have no more than one invisible particle. This requires the use of those final states in which one top decays leptonically and the other hadronically so that the $t$ and $\bar{t}$ are reconstructible. If this can be done, then this case corresponds to all the kinematic variables $\phi$ being determined.

On the other hand, for those events in which the $t, \bar{t}$ both decay purely hadronically or both decay leptonically then the situation corresponds to only a part $(\hat{\phi})$ of the kinematic variables being determined. The technique for optimization can then be applied by using the subset of variables, $\hat{\phi}$, that can be observed. The functions to be used can now be defined as

$$
\hat{f}_{i}(\hat{\phi}) \equiv \int f_{i}(\phi) d \bar{\phi}
$$

Then the entire procedure above can be used in conjunction with these functions $\hat{f}$. In fact, the method should work even if one or more of $\hat{f}$ are zero. For example, for the reaction $e^{+} e^{-} \rightarrow t \bar{t} H^{0}$, if experimentally one is unable to distinguish $t$ and $\bar{t}$ then the $f_{k}(\phi)$ that is a CP-odd function of the variables $\phi$ reduces to $\hat{f}_{k}=0$.

The simple method, first suggested in [16], for extracting the dipole moment of the top quark has been extensively applied with appropriate modifications to deduce e.g., dipole moments of 52] $\tau$ (from $Z \rightarrow \tau^{+} \tau^{-}$), for determination of the three gauge-boson couplings in $e^{+} e^{-} \rightarrow$ $W^{+} W^{-}$[53], CP-violating phase(s) in $b$ decays [54 as well as in $t \bar{t} H^{0}$ production [55]. Many of these studies explicitly showed that optimized observables can be very effective, often yielding improvements over the "naive" operators by as much as an order of magnitude.

The generalization discussed in [51] was originally used specifically to study the reaction $e^{+} e^{-} \rightarrow t \bar{t} H^{0}$. It demonstrated extraction of the $t \bar{t} H^{0}$ and $Z Z H^{0}$ coupling $a, b$, and $c$ from the interaction terms:

$$
\begin{aligned}
t \bar{t} H^{0}: & \bar{t}\left(a+i b \gamma_{5}\right) t \\
Z_{\mu} Z_{\nu} H^{0}: & c g_{\mu \nu}
\end{aligned}
$$


respectively. An important consequence in this application is that the $\mathrm{CP}$ nature of a Higgs particle, i.e., whether it is CP-odd or CP-even, may well be deducible from studies of some momenta correlations in $e^{+} e^{-} \rightarrow t \bar{t} H^{0}$.

We will discuss in greater detail the applications of this method, i.e., optimization of observables, to the top dipole moments (see sections 6.1.1, 7.2.1 and 8.1.4) and to $e^{+} e^{-} \rightarrow t \bar{t} H^{0}$ (see section 6.2) in this review.

\subsection{The naked top}

The large mass of the top quark makes it significantly different from all the other quarks in many important ways. Since $m_{t} \sim 174 \mathrm{GeV}$ the top readily undergoes two-body weak decays

$$
t \rightarrow b+W
$$

with $\Gamma \sim 1 \mathrm{GeV}$. The top life-time, $\Gamma^{-1} \sim 1 \mathrm{GeV}^{-1}$, is therefore much shorter than the "strong interaction time scale" $\sim 1 / \Lambda_{\mathrm{QCD}} \sim 10 \mathrm{GeV}^{-1}$. As a result, the top quark, in complete contrast to all the other quarks, cannot appear in traditional bound states [15]. Studies of this "naked top" are therefore not masked by the difficult non-perturbative effects of the so-called "brown muck". Thus we should think of the top-quark as an elementary fermion; we can, for instance, try to study its anomalous magnetic moment, i.e., $(g-2)$ top and even more importantly its electric dipole moment [16, 17, 18]. The magnetic moment receives a large contribution from QCD and other SM interactions but the electric dipole moment is CP-violating and cannot arise at least to 2-loop order in the SM [56] and so is expected to be extremely small $\left(<<10^{-27} \mathrm{e}-\mathrm{cm}\right)$. Non-standard sources of CP violation can cause this to be significantly bigger to the point that it may well be measurable.

Now, generically, the electric dipole moment and its generalization to other gauge fields (i.e., the corresponding weak and chromo-electric dipole moments) is an interaction of the type $\sigma_{t} \cdot E$, i.e., the top spin with an external gauge field (photon, $Z$ or gluon). Therefore, determination of the dipole moment understandably involves tracking the top spin. Fortunately this is possible for the top quark, just because it does not bind, even though it is notoriously difficult to track the spin of $b$ or the other quarks. As we shall see spin tracking provides an extremely important tool for studying all aspects of $\mathrm{CP}$ violation in the top system, not just for probing its electric dipole moment.

\subsection{Elements of top polarimetry}

Decays of the top quark are very effective analyzers of its spin. It is indeed useful to introduce the notion of the "analyzing power" of a decay. The analyzing power $\left(\epsilon_{t}^{A}\right)$ measures the degree to which the momentum of a decay product $(A)$, in the reaction $t \rightarrow A+$ anything, is correlated with the top spin. For an angular distribution given by

$$
\frac{d \Gamma}{d \cos \theta_{A}}=a\left(1+\epsilon_{t}^{A} \cos \theta_{A}\right)
$$

we can define $\epsilon_{t}^{A}$ in terms of the expectation value of $\cos \theta_{A}$

$$
\epsilon_{t}^{A}=3\left\langle\cos \theta_{A}\right\rangle
$$

where $\theta_{A}$ is the angle between $\vec{p}_{A}$ and the spin of the top $\left(\vec{S}_{t}\right)$ in the top rest frame. Using the optimal observable introduced in section 2.6, it should be clear that with a distribution such as in Eq. 2.104, the best way to determine the polarization of the top is to experimentally measure $\left\langle\cos \theta_{A}\right\rangle$.

Using this method, it follows that the number of top decays that needs to be observed to determine the top polarization to within $1-\sigma$ is given by 


$$
N_{t}^{(1 \sigma)}=\frac{3}{\left[\epsilon_{t}^{A} \Pi_{t}\right]^{2}}
$$

where $\Pi_{t}$ is the polarization of the top quark,

$$
\Pi_{t} \equiv \frac{N(\uparrow)-N(\downarrow)}{N(\uparrow)+N(\downarrow)}
$$

$N(\uparrow)$ being the number of top quarks with spins along the positive $z$ axis. Here are three concrete handles on the top spin:

1. Leptonic decays $t \rightarrow b \ell^{+} \nu_{\ell}$ are the best analyzers, i.e., $\epsilon_{t}^{\ell}=1$ for the correlation between $\vec{p}_{\ell}$ and $\Pi_{t}$. Consider the decay chain $t \rightarrow b W^{+} \rightarrow b e^{+} \nu_{e}$. In the limit that the $b$ quark is massless, the V-A nature of the weak interaction forces all the particles in the final state to be left-handed. Thus the amplitude for the top-quark decay is proportional to factors of fermion spins times [22]:

$$
\left[\bar{u}_{b} \gamma_{\mu}\left(1-\gamma_{5}\right) u_{t}\right]\left[\bar{u}_{\nu} \gamma^{\mu}\left(1-\gamma_{5}\right) v_{e}\right]
$$

On Fierz transformation this becomes

$$
\frac{1}{2}\left[\bar{u}_{b}\left(1+\gamma_{5}\right) u_{\nu}^{c}\right]\left[\bar{v}_{e}^{c}\left(1-\gamma_{5}\right) u_{t}\right]
$$

where $u^{c}=C \bar{u}^{T}, C$ being the charge conjugate matrix. This implies that, in the rest frame of the top quark, the top spin is in the direction of the positron three-momentum. This statement is true in the same sense that in the decay $\tau^{-} \rightarrow \pi^{-} \nu_{\tau}$ the $\tau$ is polarized in the direction of the $\pi$ momentum. Thus, for a top fully polarized in the $z$-direction, the angular distribution of the positron is $\propto\left(1+\cos \theta_{e}\right)$, hence $\epsilon_{t}^{e}=1$.

2. For hadronic decays of the $W$, if the momentum of the $W$ can be determined, then it is easy to see that

$$
\epsilon_{t}^{W}=\frac{m_{t}^{2}-2 m_{W}^{2}}{m_{t}^{2}+2 m_{W}^{2}} \simeq 0.4
$$

This reflects the fact that the $W$-momentum is an imprecise indicator of the $\bar{d}$ type jet in the decay $W^{+} \rightarrow \bar{d} u$. Here the $\bar{d}$ jet plays the same role as the $e^{+}$in the previous case.

3. From the above two points, it should be clear that we can increase $\epsilon$ from 0.4 to 1 if we can identify the $\bar{d}$-type jet, which plays the role of the $e^{+}$. Since this jet is one of the two that reconstructs to the $W$ in method 2 we are approximating it with the $W$-momentum. In the limit that $m_{t}$ is much greater than $m_{W}$, the two quark jets would coincide with the $W$-momentum and $\epsilon_{t}^{W} \rightarrow 1$; however, with the given top mass, the result is far from this limit.

In some cases one could determine which jet was the $\bar{d}$-type one by detailed examination of the decay products. This may be feasible for the case of a $b$-jet. However, $b$-jets are highly suppressed in $W$ decay so we will not consider this possibility further. Probably such methods will not offer significant improvements over the above.

On the other hand, we have not exploited the full information available in the kinematics of the decay. It was suggested in [57] that the energy distribution of the two jets could give additional information concerning which is the desired $\bar{d}$-jet. In particular, in $W^{+} \rightarrow \bar{d} u$, the 
$\bar{d}$-jet is on the average less ${ }^{2}$ energetic than the $u$-jet: and so, if we take the polarization to be in the direction of the less energetic of these two jets, one would expect some improvement. Indeed, in this case we obtain

$$
\epsilon_{t}^{\text {low energy jet }} \simeq 0.5
$$

Further, if one uses the optimization methods discussed in section 2.6 but restricts the experimentally available information to quantities which are symmetric under the interchange of the two jets, then one can improve this further

$$
\epsilon_{t}^{\mathrm{opt}} \simeq 0.63
$$

which represents the best result that can be obtained without knowing the identities of the two $W$-jets.

\footnotetext{
${ }^{2}$ In passing we wish to mention that while we find the $\bar{d}$-jet to be on average less energetic then the $u$-jet, Ref. [57] found it to be the other way around.
} 


\section{Models of CP violation}

In this chapter we describe the key features of CP violation in the SM and in some popular models beyond the SM such as MHDM's (Multi-Higgs Doublet-Models) and SUSY models. In doing so, we emphasize the relevance of the new $\mathrm{CP}$-violating phases that appear in these models, to top quark physics.

\subsection{CP violation and the Standard Model}

In the $\mathrm{SM}, \mathrm{CP}$ violation emanates from a CP-odd phase in the CKM matrix [⿶] which influences directly only the quark sector [58]. In this section we will briefly describe the properties of this flavor mixing CKM matrix.

\subsubsection{General remarks}

The ElectroWeak (EW) Lagrangian of the SM can be symbolically written in the form (for notation, see e.g., [59]):

$$
\mathcal{L}=\mathcal{L}(f, G)+\mathcal{L}(f, H)+\mathcal{L}(G, H)+\mathcal{L}(G)+V(H),
$$

where: $f=$ fermions (quarks, leptons),

$G=$ gauge-bosons $(\vec{W}$ and $B)$,

$H=$ the Higgs doublet.

The Lagrangian in Eq. 3.1 is constructed so that it is invariant under the local (space time dependent) symmetry group $\mathrm{SU}(2)_{L} \times U(1)_{Y}$.

The purpose of this section in not to review the structure of this Lagrangian but rather to explore the salient features of its CP-violating part. We will therefore present a self-contained discussion only of its CP-nonconserving pieces.

All CP violation in the $\mathrm{SM}$ originates from the term $\mathcal{L}(f, H)$. The hadronic part of this term is given by

$$
\mathcal{L}^{h}(f, H)=\sum_{j, k=1}^{N}\left\{Y_{j k}^{u}{\overline{\left(q, q^{\prime}\right)}}_{j L}\left(\begin{array}{c}
\phi^{0 *} \\
-\phi^{(-)}
\end{array}\right) q_{k R}+Y_{j k}^{d}{\overline{\left(q, q^{\prime}\right)}}_{j L}\left(\begin{array}{c}
\phi^{(+)} \\
\phi^{0}
\end{array}\right) q_{k R}^{\prime}+\text { h.c. }\right\}
$$

where we introduced the multiplets of the quark weak eigenstates

$$
\left(\begin{array}{c}
q_{j L} \\
q_{j L}^{\prime}
\end{array}\right), q_{j R}, q_{j R}^{\prime}, \quad j=1,2, \ldots, N
$$

and

$$
q_{R}=\frac{1+\gamma_{5}}{2} q, \quad q_{L}=\frac{1-\gamma_{5}}{2} q
$$

Also, $j, k$ are family indices, $N$ denotes the number of families and $Y_{j k}^{u}, Y_{j k}^{d}$ are the Yukawa couplings, which are arbitrary complex numbers. In our discussion we will consider $N=3$ corresponding to the SM with the three known families of fermions. This Lagrangian has no fermion mass term; fermion masses must therefore be induced by Spontaneous Symmetry Breaking (SSB) of the $S U(2) \times U(1)$ symmetry of the scalar potential term $V(H)$. In the broken state, the scalar doublet assumes a Vacuum Expectation Value (VEV) and thus one obtains the mass terms $M^{u}$ and $M^{d}$ of the charge $2 / 3$ and charge $-1 / 3$ quarks, respectively, for the weak eigenstates of the $\mathrm{SU}(2)_{L} \times U(1)_{Y}$ gauge theory (i.e., $\bar{q}_{i R} M_{i j}^{u} q_{j L}$ and $\left.\bar{q}_{i R}^{\prime} M_{i j}^{d} q_{j L}^{\prime}\right)$. These quark mass matrices are related to the Yukawa couplings via 


$$
M_{i j}^{u}=\frac{v}{\sqrt{2}} Y_{i j}^{u}, \quad M_{i j}^{d}=\frac{v}{\sqrt{2}} Y_{i j}^{d},
$$

where $v$ is the VEV of the Higgs doublet. In general the mass matrices $M^{d, u}$ are not hermitian, and each one depends on 9 complex unknown parameters. Since an arbitrary matrix $M$ can be written as $M=H u$, with $H$ hermitian and $u$ unitary, there exists a field redefinition such that $M^{u}$ and $M^{d}$ are hermitian, i.e., that $M^{u}=M^{u \dagger}$ and $M^{d}=M^{d \dagger}$ 60]. A unitary transformation on the $u$ and $d$ quark fields gives the physical basis where the mass matrices are diagonal

$$
\begin{aligned}
U_{R}^{\dagger} M^{u} U_{L} & =\operatorname{diag}\left(m_{u}, m_{c}, m_{t}\right), \\
D_{R}^{\dagger} M^{d} D_{L} & =\operatorname{diag}\left(m_{d}, m_{s}, m_{b}\right),
\end{aligned}
$$

where $U_{R}, U_{L}, D_{R}$ and $D_{L}$ are unitary matrices that relate the weak eigenstates to the physical eigenstates. It is worth mentioning already at this point that all $\mathrm{CP}$ violation in the SM emanates from the apparent mismatch between the gauge and mass (physical) eigenstates of the quark fields.

For the physical states thus defined, there is no longer an exact $S U(2)$ identity between the left handed $d$ and $u$ quarks (since they are no longer the gauge eigenstates). To see this, write the Lagrangian in terms of the physical fields and drop the numerical factors and the coupling constants. Thus one is left with the CP-violating charge current terms

$$
X_{C}=W_{\mu}^{+} J_{C}^{\mu}+\text { h.c. }
$$

where $W^{+}$is the charged, spin $1, \mathrm{SU}(2)$ vector-boson and

$$
J_{C}^{\mu}=(\bar{u}, \bar{c}, \bar{t})_{L} \gamma^{\mu} V\left(\begin{array}{c}
d \\
s \\
b
\end{array}\right)_{L} .
$$

Here $u, c, t, d, s$ and $b$ are the quark mass eigenstates. The $3 \times 3$ unitary matrix $V$ will therefore be the product of the unitary diagonalizing matrices since

$$
V=U_{L}^{\dagger} D_{L} \equiv\left(\begin{array}{ccc}
V_{u d} & V_{u s} & V_{u b} \\
V_{c d} & V_{c s} & V_{c b} \\
V_{t d} & V_{t s} & V_{t b}
\end{array}\right)
$$

Expressing the fermions in this basis, the term $X_{C}$ is the one where all the CP violation in the $\mathrm{SM}$ resides. We will therefore consider the properties of $V$ in some detail.

$\mathrm{CP}$ conservation requires the matrix $V$ to be real up to a trivial rephasing of the quark fields. In general, for 3 families of quarks it can be specified by 18 complex parameters of a general $3 \times 3$ unitary matrix. However, 9 of these 18 parameters are eliminated by the unitarity constraints $V_{i k}^{\dagger} V_{k j}=\delta_{i j}$, therefore we are left with only 9 free parameters. Moreover, there is a freedom to absorb 5 phases into 5 out of the 6 left handed fields [61]:

$$
q_{L} \rightarrow e^{i \alpha\left(q_{L}\right)} q_{L}
$$

Thus 5 more degrees of freedom can be removed. Therefore, one is left with only 4 physically independent parameters upon which $V$ depends. These four can then be parameterized in terms of three Euler-type rotation angles and one CP-violating phase. Note that these four angles cannot be predicted within the SM and have to be deduced from experiment (see below).

In the parameterization that is called "standard" [11]:

$$
V_{C K M}=\left(\begin{array}{ccc}
c_{12} c_{13} & s_{12} c_{13} & s_{13} e^{-i \delta_{13}} \\
-s_{12} c_{23}-c_{12} s_{23} s_{13} e^{i \delta_{13}} & c_{12} c_{23}-s_{12} s_{23} s_{13} e^{i \delta_{13}} & s_{23} c_{13} \\
s_{12} s_{23}-c_{12} c_{23} s_{13} e^{i \delta_{13}} & -c_{12} s_{23}-s_{12} c_{23} s_{13} e^{i \delta_{13}} & c_{23} c_{13}
\end{array}\right) .
$$


where, as usual, $c_{i j}=\cos \theta_{i j}, s_{i j}=\sin \theta_{i j}$; the indices $1,2,3$ are "generation" labels and $\delta_{13}$ is the phase.

With the advent of the $b$-quark lifetime measurements in 1983 [62], Wolfenstein [63] made the important observation that the magnitude of the CKM elements exhibit a specific hierarchical structure. The parameterization proposed by Wolfenstein uses the Cabibbo angle, $s_{12}$, as an expansion parameter making this hierarchy manifest by rewriting the matrix in terms of the 4 parameters, $\lambda, A, \rho$ and $\eta$. These are defined as

$$
s_{12}=\lambda, \quad s_{23}=A \lambda^{2}, \quad s_{13} e^{-i \delta_{13}}=A \lambda^{3}(\rho-i \eta) .
$$

We then arrive at the Wolfenstein representation for the $3 \times 3$ matrix [64, 65]:

$$
V_{\text {Wolf }}=\left(\begin{array}{ccc}
1-\frac{\lambda^{2}}{2}-\frac{\lambda^{4}}{8} & \lambda & A \lambda^{3}(\rho-i \eta) \\
-\lambda+\frac{A^{2} \lambda^{5}}{2}(1-2 \rho)-i A^{2} \lambda^{5} \eta & 1-\frac{\lambda^{2}}{2}-\lambda^{4}\left(\frac{1}{8}+\frac{A^{2}}{2}\right) & A \lambda^{2} \\
A \lambda^{3}\left[1-\left(1-\frac{\lambda^{2}}{2}\right)(\rho+i \eta)\right] & -A \lambda^{2}\left(1-\frac{\lambda^{2}}{2}\right)\left[1+\lambda^{2}(\rho+i \eta)\right] & 1-\frac{A^{2} \lambda^{4}}{2}
\end{array}\right)+\mathcal{O}\left(\lambda^{6}\right)
$$

For most purposes, a simpler form, with truncation to order $\lambda^{4}$ suffices 63:

$$
V_{\text {Wolf }}=\left(\begin{array}{ccc}
1-\frac{\lambda^{2}}{2} & \lambda & A \lambda^{3}(\rho-i \eta) \\
-\lambda & 1-\frac{\lambda^{2}}{2} & A \lambda^{2} \\
A \lambda^{3}(1-\rho-i \eta) & -A \lambda^{2} & 1
\end{array}\right)+\mathcal{O}\left(\lambda^{4}\right) .
$$

Notice that the matrix is diagonal to a good approximation. This is due to the fact that the couplings between quarks of the same family are close to unity and the off-diagonal elements become smaller as the separation between the families gets larger. Note that all $\mathrm{CP}$ violation in the CKM matrix is proportional to $\eta$ since this parameter gives a complex phase to the CKM matrix, in particular to $V_{u b}$ and $V_{t d}$, in the above parameterization.

\subsubsection{The Jarlskog invariant}

There is a unique invariant way to parameterize $\mathrm{CP}$ nonconservation which emerges from the mixing matrix $V$ in Eq. 3.10. That is, to introduce one invariant quantity as considered in [60, 66], which will be independent of any phase convention of the quark fields and will enter in every CP-violating effect in the SM.

It was shown in 66] that in order to obtain CP nonconservation in the SM, 14 conditions must be satisfied. These conditions can be expressed as the equation

$$
\operatorname{det} C=-2 F^{u} F^{d} J \neq 0
$$

where $C$ is the commutator of the mass matrices of the up and down quarks defined by

$$
\left[M^{u}, M^{d}\right]=i C
$$

Also, $F^{u}$ and $F^{d}$ are given by

$$
\begin{aligned}
F^{u} & \equiv\left(m_{t}^{2}-m_{c}^{2}\right)\left(m_{t}^{2}-m_{u}^{2}\right)\left(m_{c}^{2}-m_{u}^{2}\right) \\
F^{d} & \equiv\left(m_{b}^{2}-m_{s}^{2}\right)\left(m_{b}^{2}-m_{d}^{2}\right)\left(m_{s}^{2}-m_{d}^{2}\right)
\end{aligned}
$$

and $J$, which is sometimes referred to as the Jarlskog invariant, is defined through [60, 66]:

$$
\Im \mathrm{m}\left(V_{\alpha j} V_{\beta k} V_{\alpha k}^{*} V_{\beta j}^{*}\right) \equiv J \sum_{l, \gamma} \epsilon_{\alpha \beta \gamma} \epsilon_{j k l}
$$


Here the indices $\gamma$ and $l$ are independently summed over while the other indices are not summed; Greek, Latin indices stand for up, down-quarks, respectively. The parameter $J$ is independent of the phase convention and, most importantly, it enters in any CP-violating effect within the SM. In the "standard" parameterization (i.e., Eq. 3.12), $J$ is given by

$$
J=s_{12}^{2} s_{13} s_{23} c_{12} c_{13} c_{23} s_{\delta_{13}} .
$$

From Eqs. 3.153 .20 the 14 conditions for obtaining CP violation in the SM can be recovered. In particular, no two quarks with the same charge are degenerate (since that will make $F^{u}$ or $F^{d}$ go to zero). Also, none of the rotation angles (i.e., $\left.\theta_{12}, \theta_{13}, \theta_{23}\right)$ and the phase $\delta_{13}$ are allowed to be 0 or $\pi$ (this condition assures that $J \neq 0$ ).

One can use the invariant parameterization of the angles of the mixing matrix $V$ to further examine the structure of CP violation in the SM by introducing the "unitarity triangles" (sometimes called "CP violation triangles") [67]. To do so, define

$$
\begin{aligned}
& a_{i} \equiv V_{i 1} V_{i 2}^{*}, b_{i} \equiv V_{i 2} V_{i 3}^{*}, c_{i} \equiv V_{1 i} V_{2 i}^{*}, \\
& d_{i} \equiv V_{2 i} V_{3 i}^{*}, e_{i} \equiv V_{i 1} V_{i 3}^{*}, f_{i} \equiv V_{1 i} V_{3 i}^{*},
\end{aligned}
$$

where $i=1-3$. The unitarity of $V$ (i.e., $\sum X_{i}=0$ for $X_{i}=a_{i}, b_{i}, c_{i}, d_{i}, e_{i}$ or $f_{i}$ ) implies that each set of the three vectors $X_{i}$ in the complex plane defines a triangle. Note that often the name "unitarity triangle" refers only to the triangle resulting from $\sum e_{i}=0$ (see below). All these 6 triangles have an equal area $S_{\Delta}=J / 2$. It is shown in 66] that the square of this area can be expressed in terms of only 4 independent moduli of the elements of $V$ as

$$
S_{\Delta}=\lambda^{1 / 2}(x, y, z),
$$

where $\lambda\left(x^{2}, y^{2}, z^{2}\right)=\left[x^{2}-(y-z)^{2}\right]\left[x^{2}-(y+z)^{2}\right]$. Here $x, y, z$ can have two sets of values

$$
\begin{aligned}
& \text { I) } x=\left|V_{\alpha j}\right|\left|V_{\alpha k}\right|, y=\left|V_{\beta j}\right|\left|V_{\beta k}\right|, z=\left|V_{\gamma j}\right|\left|V_{\gamma k}\right|, \\
& \text { II) } x=\left|V_{\alpha l}\right|\left|V_{\beta l}\right|, y=\left|V_{\alpha j}\right|\left|V_{\beta j}\right|, z=\left|V_{\alpha k}\right|\left|V_{\beta k}\right| .
\end{aligned}
$$

For set I $\alpha \neq \beta \neq \gamma$ and $j \neq k$, while for set II $\alpha \neq \beta$ and $j \neq k \neq l$. Therefore, one can compute the angles of the unitarity triangles, up to an overall sign, in terms of only 4 moduli of the elements of $V$ (note that in Eq. 3.23 only 4 independent moduli enter $x, y, z$ for any of the allowed values of $\alpha, \beta, \gamma, i, j, l)$. In other words, the existence of $\mathrm{CP}$ violation or equivalently $J^{2} \neq 0$, may be inferred in the SM with three generations if the three sides of a unitarity triangle can form a triangle with non-zero area. The moduli that enter into Eq. 3.23 may be obtained from purely CP-conserving observations. However, an experimental measurement of CP violation is needed to fix the sign of $J$.

An example of such a unitary triangle is the one commonly used in $B$ physics studies [64, 65, which is constructed out of the three vectors $e_{1}, e_{2}$ and $e_{3}$ in Eq. 3.21 corresponding to $V_{u d} V_{u b}^{*}, V_{c d} V_{c b}^{*}$ and $V_{t d} V_{t b}^{*}$, respectively, which we will discuss below.

\subsubsection{Experimental constraints}

Let us now briefly summarize the experimental constraints on the elements of the $3 \times 3 \mathrm{CKM}$ matrix. The best determined is $\left|V_{u s}\right|=\lambda \simeq s_{12}$, i.e., the sine of the Cabibbo angle. It has been determined from $K$ decays, e.g., $K^{+} \rightarrow \pi^{0} \ell^{+} \nu_{\ell}$ and from hyperon decays, e.g., $\Lambda \rightarrow p e^{-} \bar{\nu}_{e}$. In addition, it is determined from a study of charm production via neutrino beams as well as from decays of charm to non-strange final states, albeit with much less precision. The average of the two determinations gives [64] 


$$
\left|V_{u s}\right|=\lambda=0.2205 \pm 0.0018 \text {. }
$$

The next best determined element is $\left|V_{c b}\right|$ and, through it, the Wolfenstein parameter $A$ via

$$
\left|V_{c b}\right|=A \lambda^{2}
$$

$\left|V_{c b}\right|$ is deduced using semi-leptonic $B$ decays to inclusive and exclusive final states, both at LEP and at CLEO. The error in its determination is dominated by theory. In recent years, heavy quark symmetry and heavy quark effective theory [68], have had a significant impact in reducing the model dependence. The average of various techniques now gives [64]:

$$
\left|V_{c b}\right|=0.0397 \pm 0.0020
$$

thus

$$
A=0.81 \pm 0.04
$$

The other two parameters, $\rho$ and $\eta$, are poorly known. Considerable theoretical and experimental effort is being directed to improve their determinations. $B$ physics experiments at $e^{+} e^{-}$based $B$-factories as well as other facilities will surely improve our knowledge of $\rho$, and $\eta$. Indeed this will be the focus of intense theoretical and experimental activity in the near future in providing precision tests of the SM.

In the context of decays of the $b$ quark, it is very useful to consider the unitarity triangle which involves the $b \leftrightarrow d$ elements

$$
V_{u d}^{*} V_{u b}+V_{c d}^{*} V_{c b}+V_{t d}^{*} V_{t b}=0 .
$$

Using the parameterization in Eq. 3.14, and neglecting contributions of $\mathcal{O}\left(\lambda^{4}\right)$ we can write

$$
\begin{aligned}
V_{u d}^{*} V_{u b} & =A \lambda^{3}(\bar{\rho}-i \bar{\eta}) \\
V_{c d}^{*} V_{c b} & =-A \lambda^{3}, \\
V_{t d}^{*} V_{t b} & =A \lambda^{3}(1-\bar{\rho}+i \bar{\eta}) .
\end{aligned}
$$

Here we have used the parameterization introduced in [65]:

$$
\bar{\rho}=\rho\left(1-\frac{\lambda^{2}}{2}\right) \quad, \quad \bar{\eta}=\eta\left(1-\frac{\lambda^{2}}{2}\right) .
$$

Factoring out the common quantity $A \lambda^{3}$, the three elements in Eqs. 3.29 - 3.31 can be given a geometrical representation in the $(\bar{\rho}, \bar{\eta})$ plane of a triangle with apexes at $A(\bar{\rho}, \bar{\eta}), B(1,0)$ and $C(0,0)$ (see Fig. 国).

Referring to that figure we have

$$
\begin{aligned}
& |\overrightarrow{A C}|=\frac{1-\frac{\lambda^{2}}{2}}{\lambda} \frac{\left|V_{u b}\right|}{\left|V_{c b}\right|}=\sqrt{\bar{\rho}^{2}+\bar{\eta}^{2}} \\
& |\overrightarrow{A B}|=\frac{\left|V_{t d}\right|}{\lambda\left|V_{c b}\right|}=\sqrt{(1-\bar{\rho})^{2}+\bar{\eta}^{2}}
\end{aligned}
$$

The angles $\alpha, \beta, \gamma$ of this triangle (see Fig. 1 for their definition) provide a basis for testing the SM especially with regard to its description of CP violation phenomena and CKM unitarity. In particular, unitarity implies that $\alpha+\beta+\gamma=180^{\circ}$. 


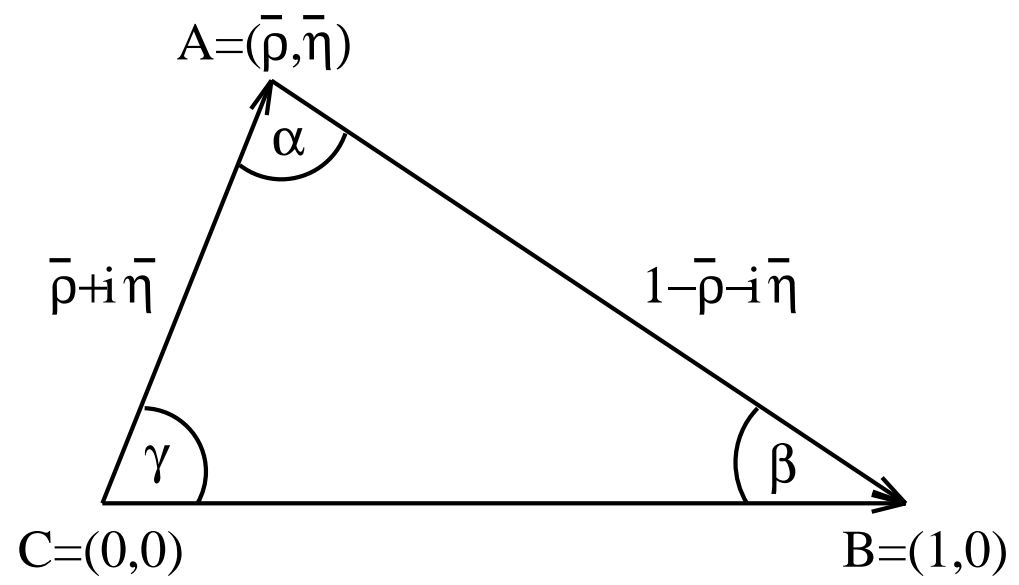

Figure 4: Unitarity triangle in the complex $(\bar{\rho}, \bar{\eta})$ plane.

Let us now briefly mention the key experimental and theoretical ingredients that enter to provide the current bounds on $\bar{\rho}, \bar{\eta}$ or alternatively on $\alpha, \beta$.

The evidence for CP violation from the $K^{0}-\bar{K}^{0}$ system through the indirect CP violation parameter, $\epsilon_{K}$, plays a crucial role in constraining $\bar{\eta}$ and $\bar{\rho}$ via [30]

$$
\begin{aligned}
\left|\epsilon_{K}\right|= & \frac{G_{F}^{2} f_{K}^{2} m_{K} m_{W}^{2}}{6 \sqrt{2} \pi^{2} \Delta m_{K}} B_{K} A^{2} \lambda^{6} \bar{\eta}\left[-\eta_{1}\left(1-\frac{\lambda^{2}}{2}\right) S\left(x_{c}\right)\right. \\
& +A^{2} \lambda^{4}\left(1-\bar{\rho}-\left(\bar{\rho}^{2}+\bar{\eta}^{2}\right) \lambda^{2}\right) \eta_{2} S\left(x_{t}\right) \\
& \left.+\eta_{3} S\left(x_{c}, x_{t}\right)\right] .
\end{aligned}
$$

The $\eta_{i}$ represent QCD corrections, evaluated to Next-to-Leading Order (NLO), the $S$ 's are functions of $x_{q}=\frac{m_{q}^{2}}{m_{t}^{2}}, f_{K}$ is the Kaon decay constant and $B_{K}$ is the so called "bag" parameter. Perhaps the best determination of $B_{K}$ comes from lattice calculations [69]

$$
B_{K} \simeq 0.9 \pm 0.1
$$

Using the experimental value of $\left|\epsilon_{K}\right|$ along with $G_{F}, m_{W}, m_{t}, B_{K}$ etc. in Eq. 3.34, leads to an allowed range for $\bar{\rho}, \bar{\eta}$ as shown in Fig. 0 .

$V_{t d}$ and therefore $\bar{\rho}, \bar{\eta}$ also enter intimately in controlling $\Delta m_{d}$, i.e., the mass difference between the two mass eigenstates of the $B_{d}^{0}-\bar{B}_{d}^{0}$ system. Thus

$$
\Delta m_{d}=\frac{G_{F}^{2} m_{W}^{2}}{6 \pi^{2}} A^{2} \lambda^{6}\left[(1-\bar{\rho})^{2}+\bar{\eta}^{2}\right] m_{B_{d}} f_{B_{d}}^{2} B_{B_{d}} \eta_{B} x_{t} F\left(x_{t}\right) .
$$

Here $F\left(x_{t}\right)$ is calculated perturbatively and is given to leading order in [70]. $\eta_{B}, f_{B_{d}}$ and $B_{B_{d}}$ have the same meaning as their values for the $K$ system, as in Eq. 3.34. Once again $f_{B_{d}}, B_{B_{d}}$ need to be calculated non-perturbatively and lattice QCD provides perhaps their best determination. The results from existing lattice calculations are best summarized as [64, 69]:

$$
f_{B_{d}}=165 \pm 20 \pm 30 \mathrm{MeV}
$$

and

$$
B_{B_{d}}(2 \mathrm{GeV})=1.0 \pm .10 \pm .15 .
$$

\footnotetext{
${ }^{3}$ The value given here is the renormalization - group - invariant - $B_{K}$ often denoted as $\hat{B}_{K}$.
} 


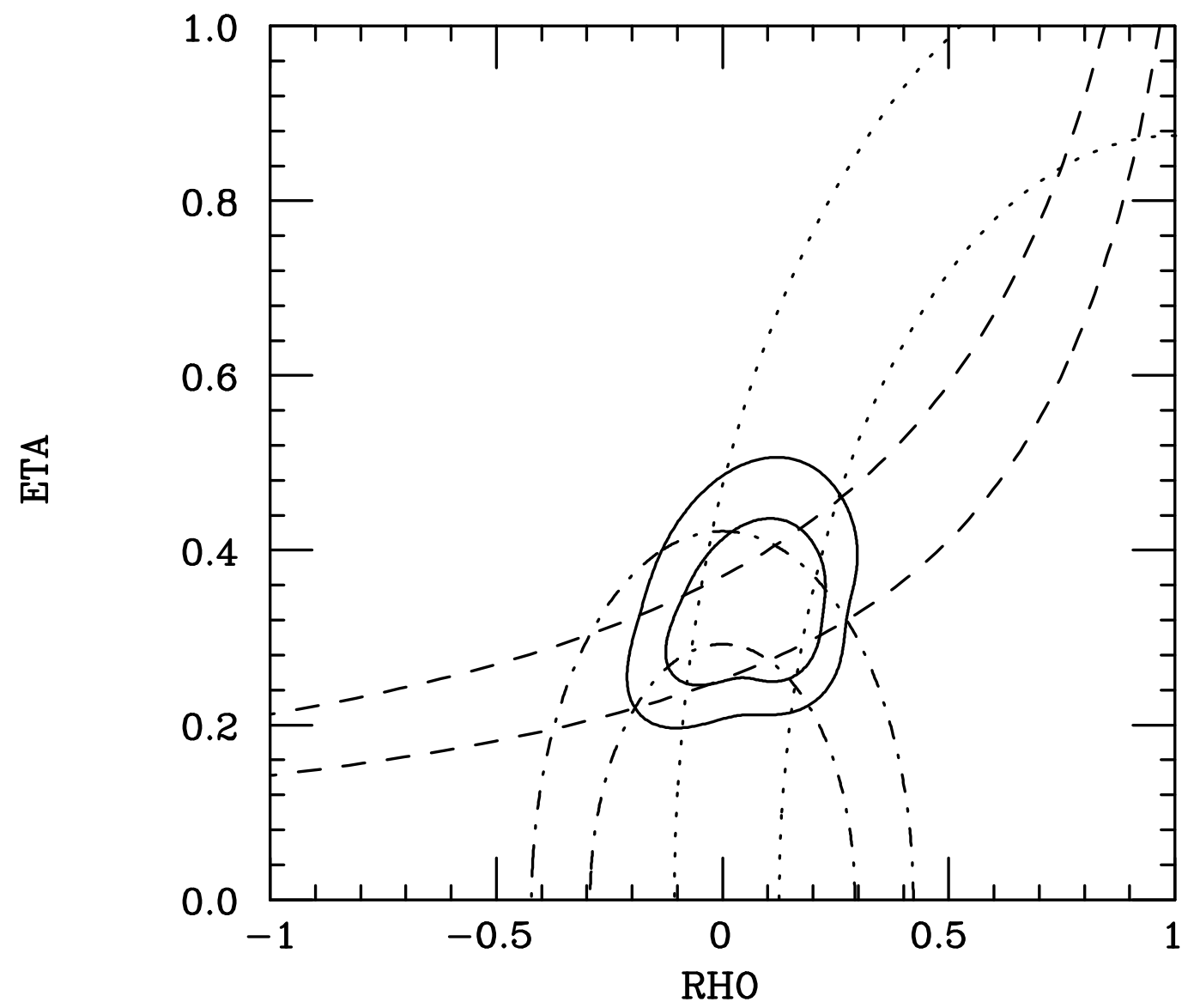

Figure 5: The allowed regions for the SM parameters $\bar{\rho}$ and $\bar{\eta}$ are shown. The solid curves show the $68 \%$ and $95 \%$ confidence regions. The 1- $\sigma$ bound originating from neutral $K_{L}$ decays is shown by the dashed curves. The 1- $\sigma$ bound originating from the rate of $b \rightarrow c$ transitions is shown with the dot-dashed curve and the 1- $\sigma$ bound originating from $B^{0}-\bar{B}^{0}$ oscillations is shown with the dotted curve. 
Using the experimental value of $\Delta m_{d}$ in conjunction with $m_{t}, G_{F}, f_{B_{d}} \sqrt{B_{B_{d}}}$ etc. in Eq. 3.36, we can represent the allowed range on the $\bar{\rho}, \bar{\eta}$ plane in the form of a hyperbola (see Fig. 5).

A non-trivial test of the CKM paradigm is obtained by examining simultaneously the allowed range of $\bar{\rho}, \bar{\eta}$ through a determination of $\left|V_{u b}\right|$ from semi-leptonic charmless $B$-decays. In this regard various techniques are used to deduce $\left|\frac{V_{u b}}{V_{c b}}\right|$ from exclusive and inclusive semi-leptonic decays. For now the uncertainties in the theoretical models of these transitions is quite substantial giving [11, 64]:

$$
\left|\frac{V_{u b}}{V_{c b}}\right|=.08 \pm .02 \Rightarrow \sqrt{\bar{\rho}^{2}+\bar{\eta}^{2}}=.35 \pm .09 .
$$

This compendium of experimental and theoretical information on $\epsilon_{K}, \Delta m_{d}$ and $b \rightarrow u \ell \nu$ is used to obtain the best fitted values [64]:

$$
\bar{\rho}=.10_{-.38}^{+.13}, \quad \bar{\eta}=.33_{-.09}^{+.06} .
$$

The corresponding $68 \%$ and $95 \%$ CL contours are shown in Fig. 5 .

In the future, the measurement of $B_{s}-\bar{B}_{s}$ oscillations will provide an extremely important test of the SM. The point is that the ratio of the mass differences

$$
\frac{\Delta m_{d}}{\Delta m_{s}}=\frac{m_{B_{d}}}{m_{B_{s}}} \frac{f_{B_{d}}^{2} B_{B_{d}}}{f_{B_{s}}^{2} B_{B_{s}}} \frac{\eta_{B_{d}}}{\eta_{B_{s}}} \frac{\left|V_{t d}\right|^{2}}{\left|V_{t s}\right|^{2}},
$$

will involve significantly less uncertainty due to hadronic matrix elements (i.e., $f^{2} B$ ). Aside from the CKM elements, the most uncertain factor on the rhs of Eq. 3.41 is

$$
r_{s d} \equiv \frac{f_{B_{s}}^{2} B_{B_{s}}}{f_{B_{d}}^{2} B_{B_{d}}} .
$$

However, theoretical uncertainties in extracting $\left|V_{t d}\right|$ from $r_{s d}$ are expected to become smaller 71] in comparison to the errors in extracting $\left|V_{t d}\right|$ from $\Delta m_{d}$ alone.

The experiments at LEP have already made significant progress in studying $B_{s}-\bar{B}_{s}$ oscillations. A combined analysis of ALEPH, DELPHI and OPAL leads to 64, 72]:

$$
\Delta m_{s}>8.0 \mathrm{ps}^{-1} \text { at } 95 \% \mathrm{CL} \text {. }
$$

Incorporating this along with $\epsilon_{K}, \Delta m_{d}$ and $b \rightarrow u \ell \nu$, into the $\bar{\rho}, \bar{\eta}$ constraints one finds 64:

$$
\bar{\rho}=.11_{-.25}^{+.13}, \quad \bar{\eta}=.33 \pm .06 .
$$

Comparing this with Eq. 3.40 we see that the LEP bound on $\Delta m_{s}$ is already reducing the negative error on $\bar{\rho}$ appreciably [64]. Further slight changes (see the update of Buras in [5]) result from improvement, obtained by combining LEP/SLD/D0 data, of the limit on $\Delta m_{s}$ to read:

$$
\Delta m_{s}>12.4 p s^{-1} \text { at } 95 \% \mathrm{CL},
$$

and from the small increase of $\left|V_{u b}\right| /\left|V_{c b}\right|$ to [73]:

$$
\left|V_{u b}\right| /\left|V_{c b}\right|=0.091 \pm 0.016 .
$$

Translated to $\beta$ and $\gamma$ (see Fig. 本) yields:

$$
\sin 2 \beta=0.71 \pm 0.13, \quad \sin \gamma=0.83 \pm 0.17 .
$$

The above value of $\sin 2 \beta$ is consistent with the recent CDF result 12, 74 $\sin 2 \beta=0.79_{-0.42}^{+0.41}$. 


\subsection{Multi-Higgs doublet models}

In the SM the interaction of the only neutral Higgs-boson with fermions is automatically $\mathrm{P}$ and $\mathrm{C}$ conserving as well as flavor conserving. This property is not valid in general in models beyond the SM. In this section we consider extensions to the SM involving the addition of extra Higgs doublets.

CP-violating effects in such models can originate in the scalar sector and be manifested in the physics of fermions, particularly the top quark. For such an effect to occur, two or more complex $\mathrm{SU}(2)$ doublets of Higgs fields are required; this was first pointed out by Lee 75 . However, the mere presence of more than one doublet does not guarantee $\mathrm{CP}$ violation in the Higgs sector. For instance, a CP-violating phase in the case of models with two Higgs doublets (2HDM) can be rotated out of the Higgs sector entirely if one imposes various discrete symmetries as will be discussed below. But if such phase(s) cannot be rotated away, this approach leads to CP violation from neutral Higgs-boson interactions, from charged Higgs-boson interactions, and perhaps in addition from the presence of a non-vanishing phase in the CKM matrix. In all cases, the various types of $\mathrm{CP}$ violation are presumably related at a fundamental level to $\mathrm{CP}$ violation in the Higgs potential, but because of our ignorance of the Higgs sector, in practice the parameters of each type of interaction are independent and should be separately measured.

Another general feature of $\mathrm{CP}$ violation in an extended Higgs sector is that larger effects are expected in heavier quarks systems (compared to the usual SM approach), because Higgs-boson couplings to fermions are proportional to the fermions masses. This makes the top quark system an especially good testing ground for such phenomena.

$\mathrm{CP}$ violation in the Higgs sector can arise in models where the Higgs potential may contain complex couplings. This might lead directly to a CP-violating interaction or to complex VEV's of the Higgs fields which can induce CP-violating effects. In addition, as we shall see in some examples below, it is also possible that a real potential can lead to a ground state with a complex $\mathrm{VEV}$, in which case $\mathrm{CP}$ is broken spontaneously. In any case, there are generally a large number of parameters in these models so that considerable experimental effort will eventually be required to determine them all. In particular, it is important to consider which predictions of such models differ from the SM, so that might lead to early signs that extra scalar fields are present. CP violation in top physics is especially useful since the $\mathrm{SM}$ contributions to $\mathrm{CP}$ violation in top quark reactions are negligible and the mass dependent coupling of the Higgs means that top quark physics is very sensitive to such effects.

It is convenient to classify CP symmetry-breaking in the scalar sector into three different categories: hard (intrinsic), soft and spontaneous. Hard or intrinsic CP violation refers to symmetrybreaking terms with dimension four, for example, terms in the Lagrangian with complex Yukawa coupling constants, or with self-coupling of scalar fields. Soft breaking is associated with terms in the Lagrangian with canonical dimension less than four. If the Lagrangian starting from the outset is $\mathrm{CP}$ invariant, $\mathrm{CP}$ violation can still be achieved by introducing complex phases from the VEV's of the scalar fields (i.e., spontaneous $\mathrm{CP}$ violation).

In the following we will consider simple versions of $2 \mathrm{HDM}$ and Three Higgs Doublets Model $(3 \mathrm{HDM})$ in which $\mathrm{CP}$ violation is manifested in the interactions of neutral and charged Higgs particles with fermions.

\subsubsection{Two Higgs doublet models}

We start with a description of the most general 2HDM. The Higgs potential for such a 2HDM is given by [76]:

$$
\begin{aligned}
V(\Phi) & =-\mu_{1}^{2} \Phi_{1}^{\dagger} \Phi_{1}-\mu_{2}^{2} \Phi_{2}^{\dagger} \Phi_{2}-\left(\mu_{12}^{2} \Phi_{1}^{\dagger} \Phi_{2}+\text { h.c. }\right) \\
& +\lambda_{1}\left(\Phi_{1}^{\dagger} \Phi_{1}\right)^{2}+\lambda_{2}\left(\Phi_{2}^{\dagger} \Phi_{2}\right)^{2}+\lambda_{3}\left(\Phi_{1}^{\dagger} \Phi_{1} \Phi_{2}^{\dagger} \Phi_{2}\right)+\lambda_{4}\left(\Phi_{1}^{\dagger} \Phi_{2}\right)\left(\Phi_{2}^{\dagger} \Phi_{1}\right)
\end{aligned}
$$




$$
+\frac{1}{2}\left[\lambda_{5}\left(\Phi_{1}^{\dagger} \Phi_{2}\right)^{2}+\text { h.c. }\right]+\left[\lambda_{6} \Phi_{1}^{\dagger} \Phi_{1} \Phi_{1}^{\dagger} \Phi_{2}+\lambda_{7} \Phi_{2}^{\dagger} \Phi_{2} \Phi_{1}^{\dagger} \Phi_{2}+\text { h.c. }\right]
$$

where $\Phi_{i}(i=1,2)$ are Higgs fields such that

$$
\Phi_{i}=\left(\begin{array}{c}
\phi_{i}^{+} \\
\phi_{i}^{0}
\end{array}\right)
$$

The scalar spectrum of a $2 \mathrm{HDM}$ consists of three neutral and two charged Higgs-bosons which we will denote by $\mathcal{H}^{k}(k=1,2,3)$ and $H^{ \pm}$, respectively.

The important parameters in $V(\Phi)$ that may drive CP violation in the Higgs sector are: $\lambda_{5}, \mu_{12}^{2}, \lambda_{6}$ and $\lambda_{7}$. It is the different choices of these parameters that will determine which type of mechanism is generating $\mathrm{CP}$ violation in the $2 \mathrm{HDM}$.

It should be noted that without the complex VEV's of the two doublets (or only one of them that can generate spontaneous CP violation), one needs at least two terms out of $\lambda_{5}, \mu_{12}^{2}, \lambda_{6}$ and $\lambda_{7}$ to be non-zero in the Higgs potential (see Eq. 3.48) in order to have CP nonconservation in the model. That is, if there is only one complex coefficient in the Higgs potential prior to SSB, then SSB leads via the minimization condition, $\partial V /\left.\partial \phi\right|_{\phi=0}=0$, to certain relations among the parameters of the Higgs potential which, in turn, forces the single complex coefficient to have a zero imaginary part if the VEV's are real. As an example, assuming that $\lambda_{6}=\lambda_{7}=0$ and only $\mu_{12}^{2}$ is complex in Eq. 3.48, $\partial V /\left.\partial \phi\right|_{\phi=0}=0$ leads to $\Im \mathrm{m}\left(\mu_{12}^{2}\right) \propto \sin \theta$, where $\theta$ is a phase associated with a complex VEV (see Eq. 3.50). Thus, if the VEV's are real (i.e., $\theta=0$ ), then $\Im \mathrm{m}\left(\mu_{12}^{2}\right)=0$ and the imaginary part of the only complex coefficient $\mu_{12}^{2}$ in the Higgs potential (prior to SSB) gets rotated away. Therefore, in general, there could be several scenarios of generating $\mathrm{CP}$ violation in a $2 \mathrm{HDM}$ emanating from $V(\Phi)$ :

1. At least two terms in the potential should violate CP when the two VEV's are chosen by a suitable field redefinition to be real.

2. Only one term in the Higgs potential violates $\mathrm{CP}$ and the two VEV's have a non-zero relative phase.

3. The Higgs potential is CP-conserving however the ground state spontaneously violates $\mathrm{CP}$ by giving a non-zero phase between the two VEV's. This scenario requires non-vanishing hard couplings of the type $\lambda_{6}$ and $\lambda_{7}$ (i.e., hard self-couplings of scalar fields).

4. $\mathrm{CP}$ violation occurs everywhere it can, i.e., explicitly in the Higgs potential and from a relative phase of the VEV's.

Let us illustrate how the above scenarios work for the Higgs potential given in Eq. 3.48. We will focus on two out of the four possible scenarios above for breaking CP symmetry that will result from two different choices of the set $\lambda_{5}, \mu_{12}^{2}, \lambda_{6}$ and $\lambda_{7}$, i.e., scenarios 2 and 3 above. In general, when there exists a relative phase $\theta$ between the two VEV's

$$
<\phi_{1}^{0}>=\frac{v}{\sqrt{2}} \cos \beta e^{i \theta}, \quad<\phi_{2}^{0}>=\frac{v}{\sqrt{2}} \sin \beta
$$

where

$$
\tan \beta=\frac{v_{2}}{v_{1}} \text { and } v=\sqrt{v_{1}^{2}+v_{2}^{2}}
$$

then with $\lambda_{5}$ non-zero and real, CP violation can arise from non-zero complex entries of one or more of $\mu_{12}^{2}, \lambda_{6}$ and $\lambda_{7}$. If $\lambda_{5}, \mu_{12}^{2}, \lambda_{6}$ and $\lambda_{7}$ are all real then $\mathrm{CP}$ violation can occur spontaneously 
(i.e., through the relative phase $\theta$ ). If one of $\mu_{12}^{2}, \lambda_{6}$ and $\lambda_{7}$ is complex then in addition to the complex VEV's above that appear after SSB, there is an explicit CP nonconservation in $V(\Phi)$. However, whether $\mathrm{CP}$ violation is spontaneous or explicit, the structure of the $\mathrm{CP}$-violating sector of the model can be redefined to depend only on the relative phase $\theta$.

Let us consider, for example, the particular choice $\lambda_{6}=\lambda_{7}=0$ which follows, for example, from type II $2 \mathrm{HDM}$, that we will describe later in this section. Here CP violation can occur only if $\Im \mathrm{m}\left[\lambda_{5} /\left(\mu_{12}^{2}\right)^{2}\right] \neq 0$ [77].

If, on the other hand, $\arg \left(\lambda_{5}\right)=2 \arg \left(\mu_{12}^{2}\right)=\psi$, then the field redefinitions $\Phi_{1} \rightarrow \exp (+i \psi / 4) \Phi_{1}$ and $\Phi_{2} \rightarrow \exp (-i \psi / 4) \Phi_{2}$ would eliminate this phase. A phase difference between $\lambda_{5}$ and $\mu_{12}^{2}$ is therefore essential in order to get $\mathrm{CP}$ nonconservation in a $2 \mathrm{HDM}$ with no hard couplings of the type $\lambda_{6}$ and $\lambda_{7}$ and, in particular, $\lambda_{5}$ cannot vanish. Let us remark at this point that in the Minimal Supersymmetric Standard Model (MSSM) with only two Higgs doublets, $\lambda_{5}=0$ is required by the supersymmetric nature of the Lagrangian. Therefore, no CP violation can arise from the pure Higgs sector in the MSSM. We will return to this point when we will describe CP violation in SUSY models (see section 3.3).

In models with extended Higgs sectors, an important experimental constraint is that the processes with Flavor Changing Neutral Currents (FCNC) are severely suppressed. To understand the extent to which such models will give rise to FCNC, consider the most general Yukawa interaction of quarks in a $2 \mathrm{HDM}[78]$ :

$$
\begin{aligned}
\mathcal{L}_{Y}= & \sum_{i, j}\left[\left(\bar{u}_{i}, \bar{d}_{i}\right)_{L}\left(U_{i j}^{1} \tilde{\Phi}_{1}+U_{i j}^{2} \tilde{\Phi}_{2}\right)\left(u_{j}\right)_{R}+\right. \\
& \left.\left(\bar{u}_{i}, \bar{d}_{i}\right)_{L}\left(D_{i j}^{1} \Phi_{1}+D_{i j}^{2} \Phi_{2}\right)\left(d_{j}\right)_{R}+\text { h.c. }\right],
\end{aligned}
$$

where $i$ and $j$ are generation indices, $u$ and $d$ stand for up and down quarks, respectively, and $\tilde{\Phi}=i \sigma_{2} \Phi^{*}$ while $U^{1}, U^{2}, D^{1}$ and $D^{2}$ are matrices in flavor space. In the general case presented above, where either both $U^{1}$ and $U^{2}$ or $D^{1}$ and $D^{2}$ are present in $\mathcal{L}_{Y}$, FCNC will appear in the model. To avoid FCNC in a 2HDM, most models which have been considered impose an ad hoc discrete symmetry on the 2HDM Lagrangian [79]; the idea being that such a symmetry may originate from physics at a more fundamental level. We will return to this point later when we discuss $\mathrm{CP}$ violation in a $2 \mathrm{HDM}$ with no FCNC at tree-level.

In the next two sections we examine two widely studied cases of the $2 \mathrm{HDM}$ where $\mathrm{CP}$ violation arises from the Higgs sector. In section 3.2.2 we consider a model where FCNC are in fact present. In particular we take $\lambda_{5}>0$ and real, and also $\mu_{12}^{2}, \lambda_{6}, \lambda_{7}$ are non-zero and real (corresponding to scenario 3 above). Since FCNC will be present, the parameters of the model will be constrained to keep them below the experimental limits. In section 3.2 .3 we consider a case which falls into scenario 2 and has flavor conservation built in at tree-level. In particular, $\lambda_{6}=\lambda_{7}=0$ and a discrete symmetry (which is only softly broken) is imposed on the model [17, 80].

\subsubsection{HDM with CP nonconservation and FCNC}

In this model, no discrete symmetry on the Yukawa couplings of any kind is imposed, thus allowing for the presence of all the couplings which appear in $V(\Phi)$ and $\mathcal{L}_{Y}$. However, in view of the low energy data on FCNC, one has to require that the Flavor Changing (FC) parameters meet those experimental constraints. One systematic way that has been suggested to achieve this without fine tuning the parameters is to impose an approximate global U(1) symmetry which acts only on fermions (see [78, 81] and references therein). This symmetry will be responsible for the smallness of the flavor changing couplings in this model and it leads to the Cheng-Sher ansatz 82, 83] which imposes a hierarchy on the terms of $\mathcal{L}_{Y}$ that can reasonably evade FCNC constraints. We choose not to concern ourselves with the technical aspects of imposing such a $\mathrm{U}(1)$ symmetry, but rather concentrate on the CP-violating consequences of a 2HDM with FCNC, 
the so called Model III. A more detailed discussion about the flavor changing parameters and their experimental constraints can be found, for example, in [84.

We can rewrite $\mathcal{L}_{Y}$ for this model in terms of the neutral and charged Higgs mass-eigenstates $\mathcal{H}^{k}$ and $H^{ \pm}$, respectively. We divide $\mathcal{L}_{Y}$ into four terms [78:

$$
\mathcal{L}_{Y}=\left(\sqrt{2} G_{F}\right)^{\frac{1}{2}}\left[\mathcal{L}_{Y}^{H^{0}}+\mathcal{L}_{Y}^{\left(H^{0}+F C\right)}+\sqrt{2}\left(\mathcal{L}_{Y}^{H^{ \pm}}+\mathcal{L}_{Y}^{\left(H^{ \pm}+F C\right)}\right)\right]
$$

where $\mathcal{L}_{Y}^{\left(H^{0}+F C\right)}\left(\mathcal{L}_{Y}^{\left(H^{ \pm}+F C\right)}\right)$ contains the FC effects for the neutral (charged) Higgs-bosons, and $\mathcal{L}_{Y}^{H^{0}}\left(\mathcal{L}_{Y}^{H^{ \pm}}\right)$has no flavor changing effects other than the ones expected from the CKM matrix of the SM part of the theory which also factors into the fermion charged Higgs coupling.

These terms are written as follows:

$$
\begin{aligned}
& \mathcal{L}_{Y}^{H^{0}}= \sum_{i, k=1}^{3}\left(\bar{u}_{L}^{i} m_{u_{i}} \eta_{u_{i}}^{(k)} u_{R}^{i}+\bar{d}_{L}^{i} m_{d_{i}} \eta_{d_{i}}^{(k)} d_{R}^{i}+\text { h.c. }\right) \mathcal{H}^{k}, \\
& \mathcal{L}_{Y}^{H^{ \pm}}= \sum_{i, j=1}^{3}\left(\bar{u}_{L}^{i} m_{d_{j}} \xi_{d_{j}} V_{i j} d_{R}^{j} H^{+}+\bar{d}_{L}^{i} m_{u_{j}} \xi_{u_{j}} V_{i j}^{\dagger} u_{R}^{j} H^{-}\right. \\
&+ \text {h.c. }), \\
& \mathcal{L}_{Y}^{\left(H^{0}+F C\right)}=\sum_{i \neq j, k=1}^{3}\left(\bar{u}_{L}^{i} \sqrt{m_{u_{i}} m_{u_{j}}} \mu_{i j}^{u(k)} u_{R}^{j}+\bar{d}_{L}^{i} \sqrt{m_{d_{i}} m_{d_{j}}} \mu_{i j}^{d(k)} d_{R}^{j}\right. \\
& \mathcal{L}_{Y}^{\left(H^{ \pm}+F C\right)}=\sum_{i, j \neq j^{\prime}=1}^{3}\left(\bar{u}_{L}^{i} V_{i j^{\prime}} \sqrt{m_{d_{j^{\prime}}} m_{d_{j}}} \mu_{j^{\prime} j}^{d} d_{R}^{j} H^{+}\right. \\
&\left.\quad+\bar{d}_{L}^{i} V_{i j^{\prime}}^{\dagger} \sqrt{m_{u_{j^{\prime}}} m_{u_{j}}} \mu_{j^{\prime} j}^{u} u_{R}^{j} H^{-}+\text {h.c. }\right) .
\end{aligned}
$$

Note that in Eqs. 3.56 and 3.57 proportionality of the FC couplings to the masses of the fermions participating in the FC vertex is imposed. This idea was originally proposed in 82, 83] and is often referred to in the literature as the Cheng-Sher ansatz. With this proportionality assumed, the severe experimental constraints on the FC couplings of light quarks can be satisfied quite naturally, i.e., without fine tuning.

The three neutral Higgs mass eigenstates, $\mathcal{H}^{k}$, are related to the real and imaginary pieces of the two Higgs doublets by a $3 \times 3$ orthogonal matrix $R$. The parameters $\xi_{f_{i}}$ in Eq. 3.55 ( $f$ stands for fermion) arise from the diagonal elements of the Yukawa couplings $U^{1}, D^{1}$ or $U^{2}, D^{2}$ in Eq. 3.52, whereas the factors $\mu_{i j}^{f}$ in Eqs. 3.56 and 3.57 arise from the off-diagonal elements. Using the notation presented in $[78]$ :

$$
\begin{aligned}
\xi_{f_{i}} & \simeq \frac{\sin \delta_{f_{i}}}{\sin \beta \cos \beta \sin \theta} e^{i \sigma_{f}\left(\theta-\delta_{f_{i}}\right)}-\cot \beta, \\
\mu_{i j}^{f} & \simeq \frac{1}{\sin \beta}\left(e^{i \sigma_{f}\left(\theta-\delta_{f_{j}}\right)}-\frac{\sin \delta_{f_{j}}}{\sin \theta}\right)\left(S_{1}^{F}\right)_{i j},
\end{aligned}
$$

and

$$
\begin{aligned}
\eta_{f_{i}}^{(k)} & =f\left(R, \xi_{f_{i}}\right), \\
\mu_{i j}^{f(k)} & =\mu_{i j}^{f} f^{\prime}(R) .
\end{aligned}
$$

Here $f$ and $f^{\prime}$ are functions of $R, \xi_{f_{i}}$ and of $R$, respectively. $S_{1}^{F}$ is an arbitrary off-diagonal real matrix. $\theta$ is the relative phase between the two VEV's as defined in Eq. 3.50 and $\delta_{f_{i}}$ is the phase associated with the mass $m_{f_{i}}$ and is defined through 


$$
\sqrt{2} m_{f_{i}} e^{i \sigma_{f} \delta_{f_{i}}} \equiv\left(g_{1}^{F_{i}} \cos \beta e^{i \sigma_{f} \theta}+g_{2}^{F_{i}} \sin \beta\right) v
$$

where $g_{1}^{F_{i}}\left(g_{2}^{F_{i}}\right)$ are diagonal elements of $U^{1}, D^{1}\left(U^{2}, D^{2}\right)$ defined in Eq. 3.52, and $\sigma_{f}=+$ or - for up or down fermion, respectively.

Evidently, $\mathcal{L}_{Y}$ manifests four patterns of $\mathrm{CP}$ violation in the $2 \mathrm{HDM}$ being considered, all of which are being driven by the relative phase $\theta$ (which appears after SSB) between the two VEV's and by some definite choice of the parameters $\lambda_{5}, \lambda_{6}, \lambda_{7}$ and $\mu_{12}^{2}$ in $V(\Phi)$ :

1. CP violation induced by the complex Yukawa couplings $\xi_{f_{i}}$ which appear both in the neutral and the charged Higgs sectors.

2. Scalar-pseudoscalar mixing in the couplings of a neutral Higgs species with fermions. That is, the mixing of the neutral imaginary and real parts of the two Higgs doublets which results from such a model generates neutral Higgs mass eigenstates that do not have a definite CP-property. Thus the couplings of neutral Higgs with fermions will have the generic form

$$
\Gamma_{\mathcal{H}^{k} f \bar{f}}=\mathcal{H}^{k} \bar{f}\left(a_{f}^{k}+i b_{f}^{k} \gamma_{5}\right) f
$$

where $a_{f}^{k}$ and $b_{f}^{k}$ are functions of $R, \tan \beta$ and $\xi_{f}$.

3. The phases in $\mu_{i j}^{f}$ which yield CP violation in FCNC interactions both in the neutral and the charged Higgs sectors.

4. The usual CKM matrix $V$ which gives $\mathrm{CP}$ violation in the charged Higgs interactions much like that of the charged $W$-boson interactions in the SM.

\subsubsection{HDM with CP nonconservation and no FCNC}

There is a natural way suggested by Glashow and Weinberg [79] to have tree-level FCNC vanish. This idea that there may be a discrete symmetry present in the 2HDM Lagrangian, also implies the vanishing of $\mathrm{CP}$ violation if this discrete symmetry is exact (see discussion below). Depending on the discrete symmetry imposed, we can then obtain different versions of a $2 \mathrm{HDM}$. The two cases usually considered are the discrete symmetries $D_{I}$ and $D_{I I}$

$$
\begin{aligned}
D_{I} & : \quad \Phi_{1} ; \Phi_{2} ;\left(d_{i}\right)_{R} ;\left(u_{i}\right)_{R} \rightarrow-\Phi_{1} ; \Phi_{2} ;-\left(d_{i}\right)_{R} ;-\left(u_{i}\right)_{R}, \\
D_{I I} & : \quad \Phi_{1} ; \Phi_{2} ;\left(d_{i}\right)_{R} ;\left(u_{i}\right)_{R} \rightarrow-\Phi_{1} ; \Phi_{2} ;-\left(d_{i}\right)_{R} ;\left(u_{i}\right)_{R} .
\end{aligned}
$$

The models with these symmetries are referred to as type I and II, respectively, depending on whether the $-1 / 3$ and $2 / 3$ charge quarks are coupled to the same or to different scalar doublets.

Let us describe now the 2HDM of type II (sometimes also referred to just as Model II) following the notation in [80]. With the discrete symmetry $D_{I I}$, we can build the Lagrangian for the $2 \mathrm{HDM}$ of type II as a special case of the general $2 \mathrm{HDM}$ Lagrangian described in the previous section, where:

(a) In order that $\mathcal{L}_{Y}$ in Eq. 3.52 be invariant under $D_{I I}, U_{i j}^{1}=D_{i j}^{2}=0$ is required. Thus

$$
\mathcal{L}_{Y}=\sum_{i, j}\left[\left(\bar{u}_{i}, \bar{d}_{i}\right)_{L} U_{i j}^{2} \tilde{\Phi}_{2}\left(u_{j}\right)_{R}+\left(\bar{u}_{i}, \bar{d}_{i}\right)_{L} D_{i j}^{1} \Phi_{1}\left(d_{j}\right)_{R}+\text { h.c. }\right]
$$

and hence only $\Phi_{2}$ gives mass to charge $+2 / 3$ quarks and only $\Phi_{1}$ is responsible for the mass generation of the charge $-1 / 3$ quarks. 
(b) If all the terms that are non-invariant under the operation of $D_{I I}$ in the Higgs potential are zero and the symmetry is exact, then there is no CP violation in the theory. Therefore, allowing only for a one non-zero value of the soft breaking term $\mu_{12}^{2} \neq 0$, we have in the Higgs potential of Eq. 3.48, $\lambda_{6}=\lambda_{7}=0$.

(c) Without loss of generality, $\lambda_{5}$ is chosen as real (this can be done by "rephasing" $\Phi_{2}$ ) and $\mu_{12}^{2}$ is chosen as complex, thus having explicit CP violation already at the Lagrangian, in addition to the relative phase between the two VEV's which arises after SSB.

With the above three points and respecting the discrete symmetry (except for the soft breaking terms which do not introduce FCNC at tree-level), the 2HDM of type II can be extracted from the general $2 \mathrm{HDM}$ of the previous section by taking [85]:

$$
\delta_{d}=\theta, \quad \delta_{u}=0,
$$

thus

$$
\xi_{d}=\tan \beta, \quad \xi_{u}=-\cot \beta
$$

Also because of point (a) above, we have $\mu_{i j}^{f}=0$ in $\mathcal{L}_{Y}$, thus

$$
\mathcal{L}_{Y}^{\left(H^{0}+F C\right)}=\mathcal{L}_{Y}^{\left(H^{ \pm}+F C\right)}=0,
$$

and we are left with only two CP-violating mechanisms in this model:

1. The scalar-pseudoscalar mixing described in the previous section (i.e., Eq. 3.63), where still $a_{f}^{k}$ and $b_{f}^{k}$ are functions of the $3 \times 3$ Higgs mixing mass matrix $R, \tan \beta$ and $\xi_{f}$. Note, however, $\xi_{f}$ is now real and is given by Eq. 3.68 above.

2. The usual CKM matrix $V$ which gives $\mathrm{CP}$ violation in the charged Higgs interactions.

For later use, let us introduce the following notation for the $\mathcal{H}^{k} f \bar{f}$ and $\mathcal{H}^{k} V V(V=W$ or $Z)$ parts of the Lagrangian in a general $2 \mathrm{HDM}$

$$
\begin{aligned}
\mathcal{L}_{\mathcal{H}^{k} f f} & =-\frac{g_{W}}{\sqrt{2}} \frac{m_{f}}{m_{W}} \mathcal{H}^{k} \bar{f}\left(a_{f}^{k}+i b_{f}^{k} \gamma_{5}\right) f, \\
\mathcal{L}_{\mathcal{H}^{k} V V} & =g_{W} m_{W} C_{V} c^{k} \mathcal{H}^{k} g_{\mu \nu} V^{\mu} V^{\nu},
\end{aligned}
$$

where $C_{W ; Z} \equiv 1 ; m_{Z}^{2} / m_{W}^{2}$. Note that in the SM the couplings in Eqs. 3.70 and 3.71 of the only neutral Higgs present are $a_{f}=1 / \sqrt{2}, b_{f}=0$ and $c=1$, and there is no phase in the $\mathcal{H}^{k} f \bar{f}$ coupling. In Model II, for up quarks for example [80]:

$$
a_{u}^{k}=R_{1 k} / \sin \beta, \quad b_{u}^{k}=R_{3 k} / \tan \beta, \quad c^{k}=R_{1 k} \sin \beta+R_{2 k} \cos \beta,
$$

where $\tan \beta \equiv v_{u} / v_{d}$ and $v_{u}\left(v_{d}\right)$ is the VEV responsible for giving mass to the up(down) quark. $R$ is the neutral Higgs rotation matrix which can be parameterized by three Euler angles $\alpha_{1,2,3}$ as follows 80]:

$$
R=\left(\begin{array}{ccc}
c_{1} & s_{1} c_{3} & s_{1} s_{3} \\
-s_{1} c_{2} & c_{1} c_{2} c_{3}-s_{2} s_{3} & c_{1} c_{2} s_{3}+s_{2} c_{3} \\
s_{1} s_{2} & -c_{1} s_{2} c_{3}-c_{2} s_{3} & -c_{1} s_{2} s_{3}+c_{2} c_{3}
\end{array}\right)
$$

where $s_{i} \equiv \sin \alpha_{i}$ and $c_{i} \equiv \cos \alpha_{i}$. 
A general feature of this class of 2HDM's, in which CP violation results from scalar-pseudoscalar mixing in the neutral Higgs interactions with fermions, is that only two out of the three neutral Higgs-bosons can simultaneously have a coupling to vector-bosons and a pseudoscalar-type of coupling to fermions. Denoting these two neutral Higgs-bosons by $h$ and $H$ with couplings $a_{f}^{h}, b_{f}^{h}, c^{h}$ and $a_{f}^{H}, b_{f}^{H}, c^{H}$ (corresponding to the light and heavy neutral Higgs, respectively), an important aspect of these 2HDM's, which has crucial phenomenological implications for CP violation, is that these couplings are subject to the constraint $b_{f}^{h} c^{h}+b_{f}^{H} c^{H}=0$. This is a general feature of $\mathrm{CP}$ violation induced by mass mixing and is due to the existence of a GIM-like cancellation, dictating that $\mathrm{CP}$-odd effects must vanish when the two Higgs-bosons $h$ and $H$ are degenerate.

To end this section let us briefly comment on the existing experimental limits on the neutral and charged Higgs masses and on the couplings $a_{f}^{k}, b_{f}^{k}, c^{k}$. There are very good reviews on this subject in the literature and we only wish to point out the highlights of those investigations. The existing limits are usually given on $\tan \beta$ and they depend on the mass of the charged Higgsboson or the neutral Higgs-boson of the theory. They can be translated to bounds on $a_{f}^{k}, b_{f}^{k}, c^{k}$ using Eq. 3.72. In particular, the limits are obtained from the experimental constraints using low energy data on $B-\bar{B}, D-\bar{D}$ and $K-\bar{K}$ mixing, $\epsilon_{K}, b \rightarrow u, b \rightarrow c$ and $b \rightarrow s \gamma$ transitions [86, 87, 88, 89, 90], on $B \rightarrow \tau \nu_{\tau} X$ decays [91, 92], on $(g-2)_{\mu}$ [93], or from high energy processes such as $e^{+} e^{-} \rightarrow Z h$ [94, the decay $t \rightarrow b H^{+}$[95, 96, 97] and from $Z$ decays [98, 99]. Typically they find that $\tan \beta<1$ is allowed if the charged Higgs mass is above several hundred $\mathrm{GeV}$ (recall that a small $\tan \beta$ enhances the Higgs coupling to the top quark). In order to have $\tan \beta \lesssim 0.5$ the charged Higgs mass is required to be typically $\gtrsim 500 \mathrm{GeV}$. If $\tan \beta$ is sufficiently large, i.e., $\tan \beta \sim \mathcal{O}\left(m_{t} / m_{b}\right)$, then a light charged Higgs-boson is possible, of the order of several tens of $\mathrm{GeV}$. Note also that there are theoretical approximate lower and upper bounds on $\tan \beta$ coming from perturbative considerations. That is, in order for a perturbative description to remain valid, $\tan \beta$ has to roughly satisfy $0.1 \lesssim \tan \beta \lesssim 100$ [87]. The lower (upper) limit corresponds to the a perturbative top (bottom) Yukawa coupling.

The inclusive decay $B \rightarrow X_{s} \gamma$, which is equivalent at the quark level to $b \rightarrow s \gamma$, plays a unique role in constraining the parameter space of 2HDM's [88]. Recall first the CLEO observation [104] $\operatorname{Br}\left(B \rightarrow X_{s} \gamma\right)=(2.32 \pm .57 \pm .35) \times 10^{-4}$ and the corresponding 95\% CL bounds of $1 \times 10^{-4}<\operatorname{Br}\left(B \rightarrow X_{s} \gamma\right)<4.2 \times 10^{-4}$. These were updated by CLEO to read $\operatorname{Br}\left(B \rightarrow X_{s} \gamma\right)<$ $(3.11 \pm 0.8 \pm 0.72) \times 10^{-4}$ where, at $95 \% \mathrm{CL}, 2.0 \times 10^{-4}<\operatorname{Br}\left(B \rightarrow X_{s} \gamma\right)<4.5 \times 10^{-4}$ 105, 106]. Furthermore, ALEPH presented the result 107] $\operatorname{Br}\left(B \rightarrow X_{s} \gamma\right)<\left(3.15 \pm 0.35_{\text {stat }} \pm 0.32_{\text {syst }} \pm\right.$ $\left.0.26_{\text {model }}\right) \times 10^{-4}$, consistent with CLEO. In Model I, the two contributions of the charged Higgs scale as $\cot ^{2} \beta$ causing significant enhancements to the decay rate for small values of $\tan \beta$. An especially interesting aspect of Model I is that the contribution of the two charged Higgs interfere destructively for some values of the parameter space. Consequently, the lower and upper bounds from CLEO enable us to place stringent constraints on the mass $\left(m_{H^{ \pm}}\right)-\tan \beta$ plane [88]. Most notably, for small value of $\tan \beta$ (especially for $\tan \beta \lesssim 0.3$ ), $m_{H^{ \pm}} \lesssim 1 \mathrm{TeV}$ gets excluded as can be seen from Fig. 60 100.

$\operatorname{Br}(b \rightarrow s \gamma)$ also places important restriction on Model II [88], again, especially for small values of $\tan \beta$ (see Fig. 1) [100, 101, 102, 103]. An important difference with Model I is that $\operatorname{Br}(b \rightarrow s \gamma)$ is now always larger than in the SM, independent of $\tan \beta$ [88. Thus, $m_{H^{ \pm}} \lesssim 300 \mathrm{GeV}$ is ruled out practically for all values of $\tan \beta$. One can relax these bounds if MSSM contributions (chargino loops) are added to the charged Higgs in the loop [88].

\subsubsection{Three Higgs doublet model}

As was mentioned above, if one chooses to adopt Natural Flavor Conservation (NFC), enforced by some discrete symmetry (see e.g., Eqs. 3.64 and 3.65), then the minimum number of Higgs doublets which allows CP violation in the gauge model with NFC is three [108, 109] (the other possibility is two Higgs doublets plus one singlet but we choose not to discuss it).

In the Weinberg 3HDM [45, 110], CP violation in the Higgs sector arises from the phase 


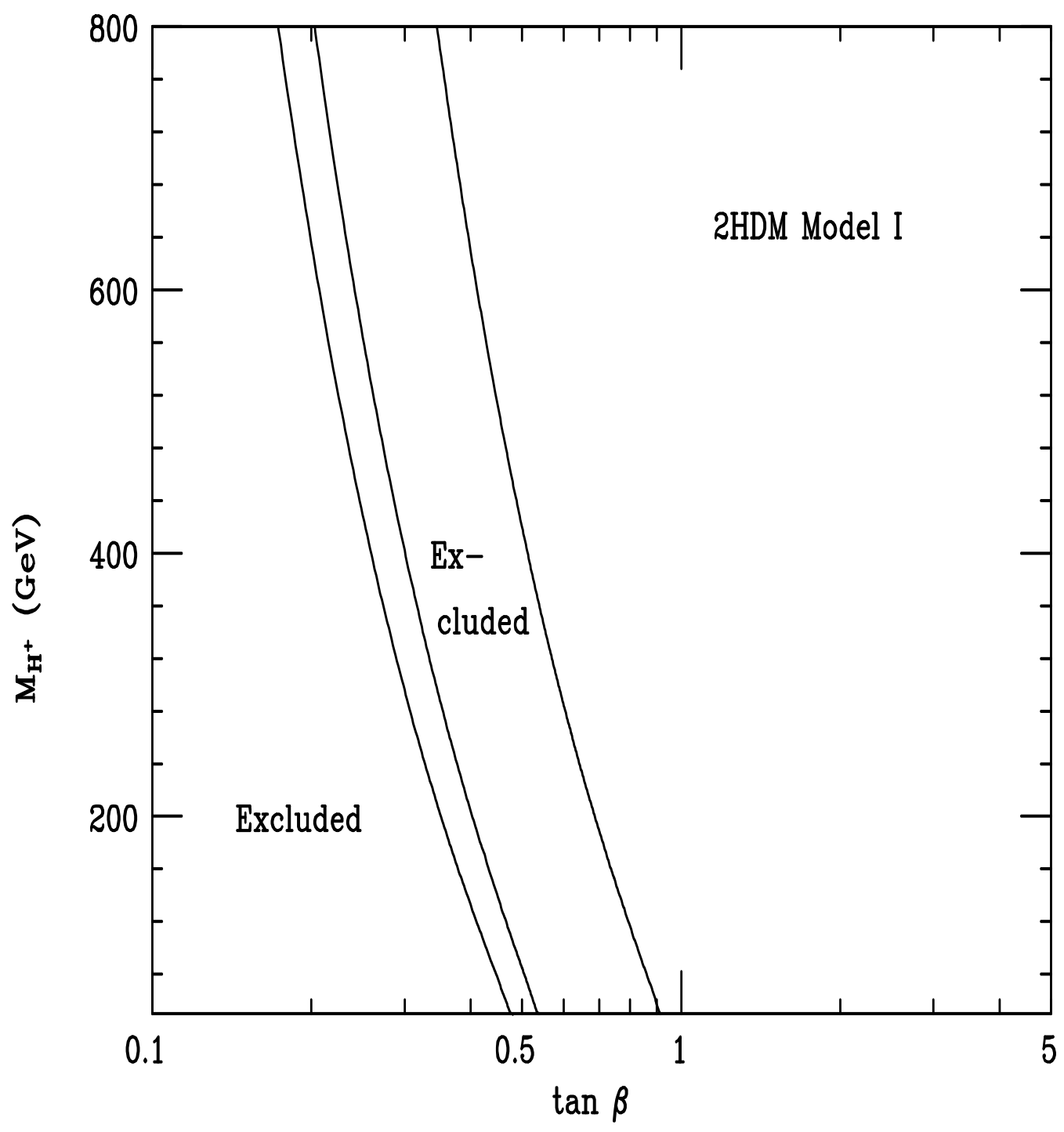

Figure 6: The excluded regions in the $m_{H^{ \pm}}-\tan \beta$ plane resulting from the present CLEO bounds in Model I and for $m_{t}=175 \mathrm{GeV}$. The excluded regions are (from left to right) to the left of the first curve and between the second and third curves. Updated figure from [88] (see [100]). 


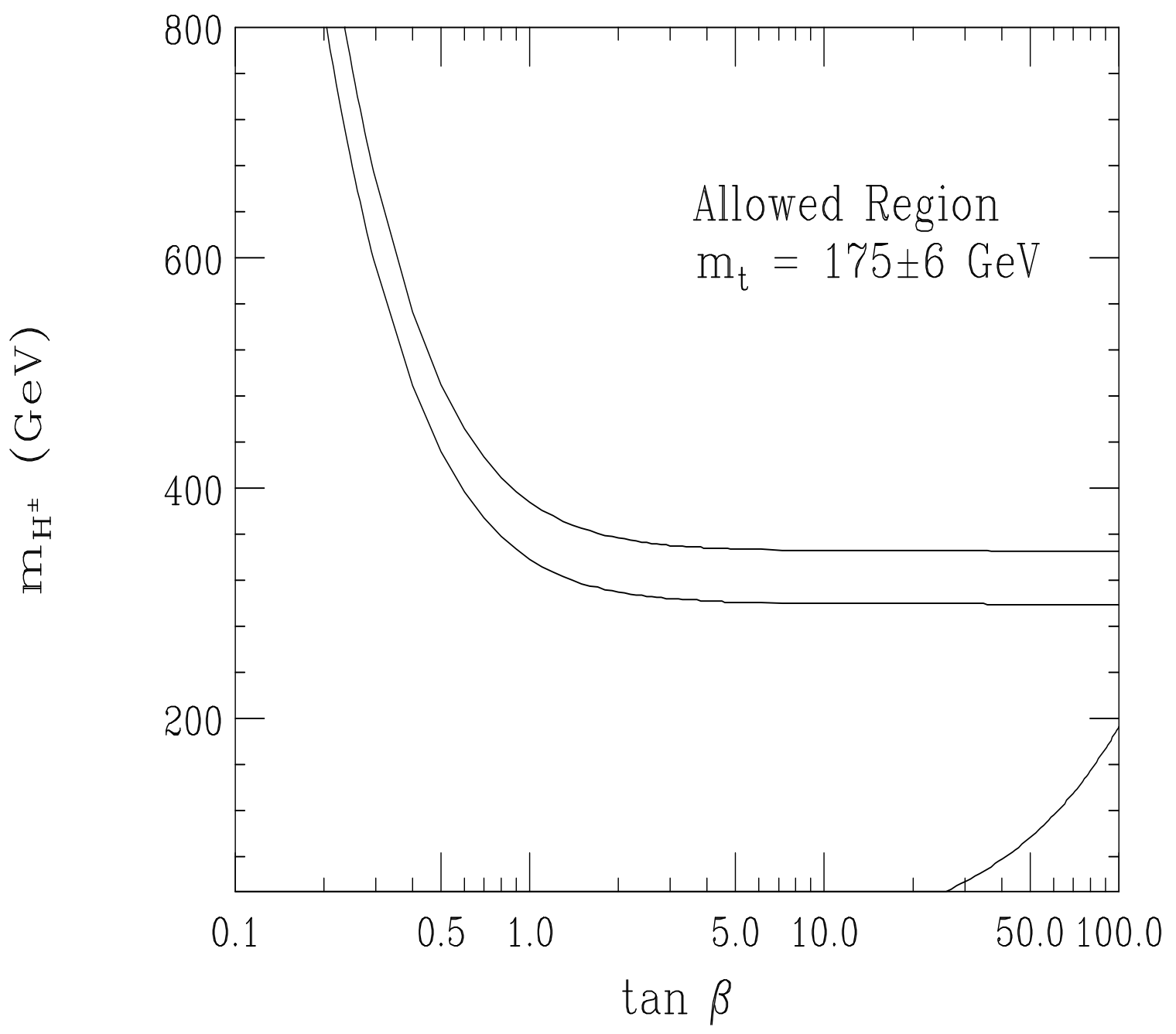

Figure 7: Constraints in the $m_{H^{ \pm}}-\tan \beta$ plane in Model II from the CLEO bound on $\operatorname{Br}(B \rightarrow$ $\left.X_{s} \gamma\right)$. The excluded region is to the left and below the curves. The upper line is for $m_{t}=181$ GeV and the lower line is for $m_{t}=169 \mathrm{GeV}$. We also display the restriction $\tan \beta / m_{H^{ \pm}}>0.52$ $\mathrm{GeV}^{-1}$ which arises from measurements of $B \rightarrow X \tau \nu$ as discussed in [101, 102]. Figure taken from 103. 
differences of the VEV's and from the complex quartic terms in the Higgs potential (as will be shown later). This model has many unknown parameters and it allows the standard CKM mechanism as one of the sources of CP nonconservation. Let us therefore present its spontaneous version which has a very attractive feature: spontaneous $\mathrm{CP}$ violation and NFC together with a real tree-level CKM matrix (for three generations) as a starting point, imply that CP violation is generated only after SSB via complex VEV's 111, 112. Thus, in such a scenario CP is a good symmetry of the Lagrangian before SSB and CP violation at tree-level comes solely from Higgs-boson exchanges once the VEV's are assigned a non-vanishing phase.

The most general scalar potential with three Higgs doublets consistent with NFC is given byt

$$
\begin{gathered}
V(\Phi)=\sum_{i} m_{i}^{2} \Phi_{i}^{\dagger} \Phi_{i}+\sum_{i \neq j} a_{i j}\left(\Phi_{i}^{\dagger} \Phi_{i}\right)\left(\Phi_{j}^{\dagger} \Phi_{j}\right)+ \\
\sum_{i \neq j} b_{i j}\left(\Phi_{i}^{\dagger} \Phi_{j}\right)\left(\Phi_{j}^{\dagger} \Phi_{i}\right)+\left[\sum_{i \neq j} c_{i j}\left(\Phi_{i}^{\dagger} \Phi_{j}\right)\left(\Phi_{i}^{\dagger} \Phi_{j}\right)+\text { h.c. }\right] .
\end{gathered}
$$

Hermiticity of the scalar potential requires that the $m_{i}^{2}$ terms, the $a_{i j}$ and the $b_{i j}$ be real while $c_{i j}$ need only be hermitian. However, if $\mathrm{CP}$ is broken spontaneously, all the parameters of $V(\Phi)$ can be chosen to be real before gauge symmetry breaking and we choose to work in the latter scheme.

The Higgs doublets can be written as

$$
\Phi_{i}=\left(\begin{array}{c}
\phi_{i}^{+} \\
\phi_{i}^{0}
\end{array}\right)=\left(\begin{array}{c}
\phi_{i}^{+} \\
\frac{1}{\sqrt{2}}\left(v_{i}+\rho_{i}+i \eta_{i}\right)
\end{array}\right),
$$

with

$$
v_{i}=\left|v_{i}\right| e^{i \theta_{i}}
$$

Assuming that the third Higgs doublet does not couple to quarks and that its VEV does participate in breaking $\mathrm{SU}(2) \times \mathrm{U}(1)$, the general Yukawa interactions consistent with $\mathrm{NFC}$ read

$$
\begin{gathered}
\mathcal{L}_{Y}=Y_{i j}^{(1)} \bar{D}_{R}^{i}\left(\phi_{1}^{+*} U_{L}^{j}+\phi_{1}^{0 *} D_{L}^{j}\right)+Y_{i j}^{(2)} \bar{U}_{R}^{i}\left(\phi_{2}^{0} U_{L}^{j}-\phi_{2}^{+} D_{L}^{j}\right)+ \\
Y_{i j}^{(3)} \bar{E}_{R}^{i}\left(\phi_{3}^{+*} N_{L}^{j}+\phi_{3}^{0 *} E_{L}^{j}\right)+\text { h.c. }
\end{gathered}
$$

where $U=(u, c, t), D=(d, s, b), E=(e, \mu, \tau)$ and $N=\left(\nu_{e}, \nu_{\mu}, \nu_{\tau}\right)$. The Yukawa couplings are chosen to be real as $\mathrm{CP}$ is assumed to be a good symmetry of $\mathcal{L}_{Y}$.

After SSB and after a phase redefinition of the quark fields in order to obtain real quark mass matrices, we can rewrite $\mathcal{L}_{Y}$ as

$$
\mathcal{L}_{Y}^{+}=\frac{\phi_{1}^{\prime+}}{v_{1}} \bar{U}_{L} K M_{D} D_{R}-\frac{\phi_{2}^{\prime+}}{v_{2}} \bar{U}_{R} M_{U} K D_{L}+\frac{\phi_{3}^{\prime+}}{v_{3}} \bar{N}_{L} M_{E} E_{R}+\text { h.c. }
$$

for charged Higgs-bosons, with $\phi_{i}^{\prime}=\phi_{i} e^{i \theta_{i}}$ and a real CKM matrix, denoted here by $K$. Also

$$
\begin{aligned}
& \mathcal{L}_{Y}^{0}= \frac{\rho_{1}}{v_{1}} \bar{D} M_{D} D+i \frac{\eta_{1}}{v_{1}} \bar{D} M_{D} \gamma_{5} D+\frac{\rho_{2}}{v_{2}} \bar{U} M_{U} U- \\
& i \frac{\eta_{2}}{v_{2}} \bar{U} M_{U} \gamma_{5} U+\frac{\rho_{3}}{v_{3}} \bar{E} M_{E} E+i \frac{\eta_{3}}{v_{3}} \bar{E} M_{E} \gamma_{5} E,
\end{aligned}
$$

\footnotetext{
${ }^{4}$ Unlike Eq. 3.48 which describes the Higgs potential for a 2HDM, we use here the more generic form of the Higgs potential applicable also for arbitrary number of Higgs doublets.
} 
for neutral Higgs-bosons, where $M_{U}, M_{D}$ and $M_{E}$ are diagonal mass matrices. The Yukawa interactions of Eqs. 3.78 and 3.79 are still $\mathrm{CP}$ invariant, however, the scalar fields $\phi_{i}, \rho_{i}$ and $\eta_{i}$ are not the physical Higgs states with definite masses.

The mass matrix in the basis of $\phi_{i}^{\prime+} / v_{i}$ is

$$
m^{2}=\left(\begin{array}{ccc}
X_{12}+X_{13} & -X_{12}-i Y & -X_{13}+i Y \\
-X_{12}+i Y & X_{12}+X_{23} & -X_{23}-i Y \\
-X_{13}+i Y & -X_{23}+i Y & X_{13}+X_{23}
\end{array}\right)
$$

where

$$
X_{i j}=\left[\frac{1}{2} b_{i j}+c_{i j} \cos \left(\theta_{i}-\theta_{j}\right)\right]\left|v_{i}\right|^{2}\left|v_{j}\right|^{2},
$$

and

$$
\begin{aligned}
Y & =-c_{12} v_{1}^{2} v_{2}^{2} \sin 2\left(\theta_{1}-\theta_{2}\right)=-c_{23} v_{2}^{2} v_{3}^{2} \sin 2\left(\theta_{2}-\theta_{3}\right) \\
& =c_{13} v_{1}^{2} v_{3}^{2} \sin 2 \theta_{1}
\end{aligned}
$$

Since the parameter $Y$ is in general non-zero, it is evident that $\mathrm{CP}$ violation in the charged Higgs-boson sector comes from the imaginary part of the off-diagonal Higgs-boson mass matrix elements.

The unitary matrix which relates the weak eigenstates $\phi_{i}^{\prime+}$ to the physical charged states $H_{i}^{+}$ is defined by

$$
\left(\begin{array}{c}
\phi_{1}^{\prime+} \\
\phi_{2}^{\prime+} \\
\phi_{3}^{\prime+}
\end{array}\right)=U^{+}\left(\begin{array}{c}
G^{+} \\
H_{1}^{+} \\
H_{2}^{+}
\end{array}\right)
$$

where $G^{+}$is the charged Goldstone-boson which is absorbed into the $W^{+} . U^{+}$, which has three arbitrary phases, of which two can be removed by a redefinition of $H_{1}^{+}$and $H_{2}^{+}$, can be parameterized exactly in the same way as the CKM matrix [110:

$$
U^{+} \equiv\left(\begin{array}{ccc}
\tilde{c}_{1} & \tilde{s}_{1} \tilde{c}_{3} & \tilde{s}_{1} \tilde{s}_{3} \\
-\tilde{s}_{1} \tilde{c}_{2} & \tilde{c}_{1} \tilde{c}_{2} \tilde{c}_{3}+\tilde{s}_{2} \tilde{s}_{3} e^{i \delta_{H}} & \tilde{c}_{1} \tilde{c}_{2} \tilde{s}_{3}-\tilde{s}_{2} \tilde{c}_{3} e^{i \delta_{H}} \\
-\tilde{s}_{1} \tilde{s}_{2} & \tilde{c}_{1} \tilde{s}_{2} \tilde{c}_{3}-\tilde{c}_{2} \tilde{s}_{3} e^{i \delta_{H}} & \tilde{c}_{1} \tilde{s}_{2} \tilde{s}_{3}+\tilde{c}_{2} \tilde{c}_{3} e^{i \delta_{H}}
\end{array}\right),
$$

with $\tilde{s}_{i} \equiv \sin \tilde{\theta}_{i}, \tilde{c}_{i} \equiv \cos \tilde{\theta}_{i}$ and $\delta_{H}$ are the charged Higgs mixing angles and phase, respectively.

From the gauge sector, it is straightforward to show that

$$
\left(2 \sqrt{2} G_{F}\right)^{-1}=\left|v_{1}\right|^{2}+\left|v_{2}\right|^{2}+\left|v_{3}\right|^{2}=v^{2},
$$

or that

$$
G^{+}=\frac{1}{v}\left(v_{1} \phi_{1}^{\prime+}+v_{2} \phi_{2}^{\prime+}+v_{3} \phi_{3}^{\prime+}\right) .
$$

It follows from Eqs. 3.83 and 3.84 that

$$
v_{1}=\tilde{c}_{1} v, \quad v_{2}=-\tilde{s}_{1} \tilde{c}_{2} v, \quad v_{3}=-\tilde{s}_{1} \tilde{s}_{2} v
$$


Therefore, the mixing angles $\tilde{\theta}_{1}, \tilde{\theta}_{2}$ are determined by the VEV's $v_{1}, v_{2}$ and $v_{3}$ whereas $\tilde{\theta}_{3}$ and $\delta_{H}$ depend on the parameters of the Higgs potential.

In terms of the Higgs mass eigenstates, the Yukawa interactions in Eq. 3.78 become

$$
\begin{aligned}
\mathcal{L}_{Y}^{+}=( & \left.2 \sqrt{2} G_{F}\right)^{1 / 2} \sum_{i=1}^{2}\left(\alpha_{i} \bar{U}_{L} K M_{D} D_{R}+\beta_{i} \bar{U}_{R} M_{U} K D_{L}\right. \\
& \left.+\gamma_{i} \bar{N}_{L} M_{E} E_{R}\right) H_{i}^{+}+\text {h.c. }
\end{aligned}
$$

with

$$
\begin{aligned}
& \alpha_{1}=\frac{\tilde{s}_{1} \tilde{c}_{3}}{\tilde{c}_{1}}, \beta_{1}=\frac{\tilde{c}_{1} \tilde{c}_{2} \tilde{c}_{3}+\tilde{s}_{2} \tilde{s}_{3} e^{i \delta_{H}}}{\tilde{s}_{1} \tilde{c}_{2}}, \gamma_{1}=\frac{\tilde{c}_{1} \tilde{s}_{2} \tilde{c}_{3}-\tilde{c}_{2} \tilde{s}_{3} e^{i \delta} H}{\tilde{s}_{1} \tilde{s}_{2}} \\
& \alpha_{2}=\frac{\tilde{s}_{1} \tilde{s}_{3}}{\tilde{c}_{1}}, \beta_{2}=\frac{\tilde{c}_{1} \tilde{c}_{2} \tilde{s}_{3}-\tilde{s}_{2} \tilde{c}_{3} e^{i \delta_{H}}}{\tilde{s}_{1} \tilde{c}_{2}}, \gamma_{2}=\frac{\tilde{c}_{1} \tilde{s}_{2} \tilde{s}_{3}+\tilde{c}_{2} \tilde{c}_{3} e^{i \delta_{H}}}{\tilde{s}_{1} \tilde{s}_{2}}
\end{aligned}
$$

and we see that

$$
\begin{aligned}
& \Im \mathrm{m}\left(\alpha_{2} \beta_{2}^{*}\right)=-\Im \mathrm{m}\left(\alpha_{1} \beta_{1}^{*}\right), \Im \mathrm{m}\left(\alpha_{2} \gamma_{2}^{*}\right)=-\Im \mathrm{m}\left(\alpha_{1} \gamma_{1}^{*}\right), \\
& \Im \mathrm{m}\left(\beta_{2} \gamma_{2}^{*}\right)=-\Im \mathrm{m}\left(\beta_{1} \gamma_{1}^{*}\right) .
\end{aligned}
$$

As in the charged Higgs case, we can write down an analogous $6 \times 6$ real mass matrix for the neutral scalar states. Then the neutral Higgs-boson Yukawa interactions in Eq. 3.79 become [109]:

$$
\begin{aligned}
\mathcal{L}_{Y}^{0}=( & \left.2 \sqrt{2} G_{F}\right)^{1 / 2} \sum_{i=1}^{5}\left(g_{1 i} \bar{D} M_{D} D+g_{2 i} \bar{D} M_{D} i \gamma_{5} D+g_{3 i} \bar{U} M_{U} U\right. \\
& \left.+g_{4 i} \bar{U} M_{U} i \gamma_{5} U+g_{5 i} \bar{E} M_{E} E+g_{6 i} \bar{E} M_{E} i \gamma_{5} E\right) H_{i}^{0}
\end{aligned}
$$

where the couplings $g_{i}$ are real. Since $\bar{\psi} \psi$ and $\bar{\psi} i \gamma_{5} \psi$ have opposite $P, T$ and CP transformation properties, $P$ and $\mathrm{CP}$ can be violated through the exchange of neutral Higgs-bosons.

We will now briefly mention some of the more notable constraints on this class of models 142, 113, 114, 115]. One class of restrictions that are of some importance follow (as in the case of the 2HDM) if we further assume that the Higgs sector of the theory is perturbative [87]. These lead to

$$
\left|\alpha_{i}\right| \lesssim 120 \quad, \quad\left|\beta_{i}\right| \lesssim 6
$$

Since these complex coupling constants arise from the diagonalization of the charged scalar mixing matrix, they obey the relation

$$
\sum_{i=1,2} \alpha_{i} \beta_{i}^{*}=1
$$

Thus [113:

$$
\Im \mathrm{m}\left(\alpha_{1} \beta_{1}^{*}\right)=-\Im \mathrm{m}\left(\alpha_{2} \beta_{2}^{*}\right)
$$

and

$$
\Im \mathrm{m}\left(\alpha_{i} \beta_{i}^{*}\right) \leq\left|\alpha_{i} \beta_{i}\right| \lesssim 720
$$

Assuming for simplicity that one of the $H^{+}$, for instance $H_{2}^{+}$, is very heavy, then $B-\bar{B}$ mixing imposes an important constraint on $\left|\beta_{1}\right|$ [87, 116]: 


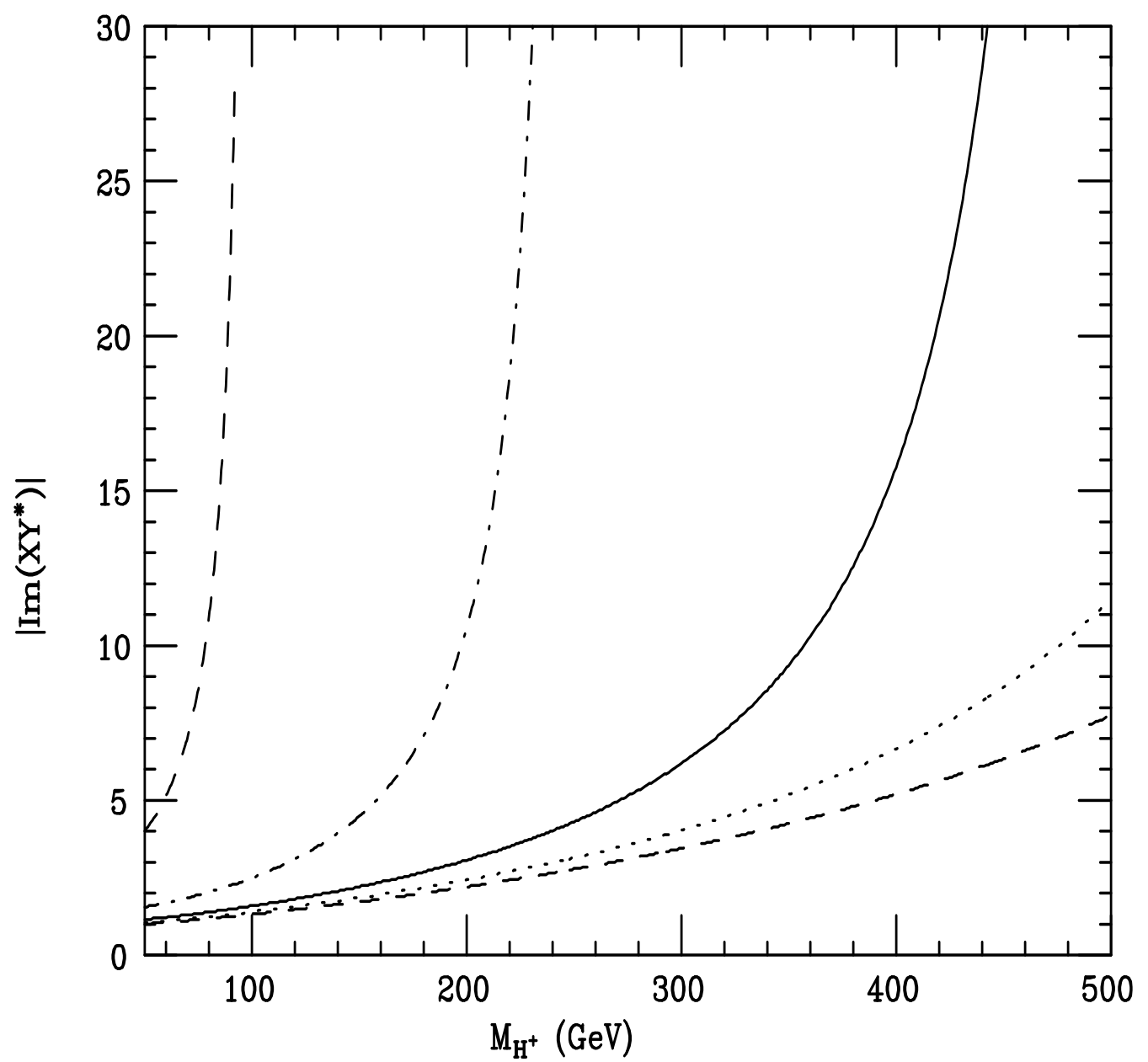

Figure 8: Constraints on $\Im \mathrm{m}\left(X Y^{*}\right) \mid\left(\equiv\left|\Im \mathrm{m}\left(\alpha \beta^{*}\right)\right|\right.$ in our notation) as a function of the lightest charged Higgs-boson mass $M_{H^{+}} \equiv m_{H_{1}}$, with $m_{H_{2}}=100$, 250, 500 and 750 GeV corresponding to (from left to right) the dashed, dashed-dotted, solid and dotted curves, respectively. The bottom dashed curve represents the case where the $\mathrm{H}_{2}^{ \pm}$contributions have been neglected. The allowed region lies to the right and below the curves. $m_{t}=175 \mathrm{GeV}$ is used. Updated figure from 88] (see 100/).

$$
\begin{aligned}
\left|\beta_{1}\right| & \lesssim 2 \text { for } m_{H_{1}} \sim \frac{1}{2} m_{Z} \\
& \lesssim 3 \text { for } m_{H_{1}} \sim 2 m_{Z} .
\end{aligned}
$$

Using $B \rightarrow \tau \nu X$, a constraint on $\left|\alpha_{1}\right|$ is deduced [101, 117]:

$$
\left|\alpha_{1}\right| \lesssim 2 m_{H_{1}} / \mathrm{GeV}
$$

A direct bound on $\Im \mathrm{m}\left(\alpha \beta^{*}\right)$ comes from the electric dipole moment of the neutron $\left(d_{n}\right)$ 118, 119:

$$
\begin{aligned}
\left|\Im \mathrm{m}\left(\alpha \beta^{*}\right)\right| & \lesssim 20 \text { for } m_{H_{1}} \sim \frac{1}{2} m_{Z} \\
& \lesssim 100 \text { for } m_{H_{1}} \sim 2 m_{Z} .
\end{aligned}
$$


Interestingly enough, the strongest constraint so far actually comes from a CP-conserving process $b \rightarrow s \gamma$ [113, 115]. The amplitude for $b \rightarrow s \gamma$ receives contributions from terms proportional to $\Im \mathrm{m}\left(\alpha_{i} \beta_{i}^{*}\right)$ that do not interfere with the other terms.

Thus these terms only enter quadratically in the expression for the rate for $b \rightarrow s \gamma$. A conservative bound on $\left|\Im \mathrm{m}\left(\alpha \beta^{*}\right)\right|$ (where $\left.\Im \mathrm{m}\left(\alpha \beta^{*}\right)=\Im \mathrm{m}\left(\alpha_{1} \beta_{1}^{*}\right)=-\Im \mathrm{m}\left(\alpha_{2} \beta_{2}^{*}\right)\right)$ is obtained by assuming that such a contribution saturates the measured rate for $b \rightarrow s \gamma$. These constraints are displayed in Fig. 8 88, 100] as a function of the light charged Higgs mass $\left(m_{H_{1}}\right)$ for various values of the heavier charged Higgs mass $\left(m_{H_{2}}\right)$, subject to the restriction $m_{H_{1}} \leq m_{H_{2}}$. On the figure, the bottom solid curve corresponds to the case when $m_{H_{2}} \gg m_{H_{1}}$ so that the contribution of the second charged Higgs is neglected. We note that the constraints depend strongly on $m_{H_{2}}$ and they essentially disappear when $m_{H_{1}} \simeq m_{H_{2}}$ due to a cancellation between the two contributions [88, 100] resulting from a GIM-like mechanism.

It is useful to note how stringent these constraints are. For example

$$
\begin{aligned}
\left|\Im \mathrm{m}\left(\alpha \beta^{*}\right)\right| & \lesssim 1.5 \text { for } m_{H_{1}} \sim \frac{1}{2} m_{Z} \\
& \lesssim 2.5 \text { for } m_{H_{1}} \sim 2 m_{Z}
\end{aligned}
$$

for $m_{H_{2}} \sim 500 \mathrm{GeV}$. A very important consequence of these tight bounds on $\Im \mathrm{m}\left(\alpha \beta^{*}\right)$ is that the charged scalar exchange can only make a negligible contribution to the $\mathrm{CP}$ violation parameters in $K \rightarrow 2 \pi$, i.e., $\epsilon$ or $\epsilon^{\prime}$. Therefore, $\mathrm{CP}$ violation in the $3 \mathrm{HDM}$ cannot be the sole source of the observed CP violation. We should note, though, that in the original Weinberg model for three Higgs doublets, CP is not assumed to be an a priori symmetry of the model. Thus CP violation arises from complex quartic terms in the Higgs potential as well as from the phase differences of the VEV's. In addition, one has the complex CKM phase as an independent source of CP violation which may be able to accommodate the $\mathrm{CP}$ violation in the Kaon system.

\subsection{Supersymmetric models}

Needless to say, the minimal SUSY extension of the SM is a very appealing theory [120]. Among its compelling features are: it allows for Radiative ElectroWeak Symmetry Breaking (REWSB), it unifies the gauge coupling constants, with masses of superpartners not much heavier then a $\mathrm{TeV}$ it gives a well-grounded explanation to the hierarchy problem and it provides a good dark matter candidate - the lightest SUSY particle.

As far as CP violation is concerned, new non-SM mechanisms are introduced in each version of such SUSY models [31, 120, 121, 122]. It is again the top quark sector in these models that may exhibit large CP-violating effects due to its very large mass. In particular, the supersymmetric partners of the top quark (these two scalar particles are often referred to as the stop and denoted by $\tilde{t}$ ), can be responsible for relatively large CP-violating phenomena. Such effects are enhanced by the possibility of having large mass splittings between the two stops which is in turn due to the relatively large top mass. These type of SUSY CP violation in top quark reactions has received considerable attention in the past few years. They are all strongly dependent on the magnitude of the low energy phase of the soft trilinear breaking term $A_{t}$ in the SUSY Lagrangian and we will describe some of these works in the following chapters.

Another possible manifestation of the CP-violating phase $\arg \left(A_{t}\right)$ is Baryogenesis in the early universe. It was shown that with $\arg (\mu) \rightarrow 0, t$ squarks can mediate the charge transport mechanism needed to generate the observed baryon asymmetry, even with squark masses $\sim$ hundred $\mathrm{GeV}$, provided that $\arg \left(A_{t}\right)$ is not much suppressed [29]. We will therefore emphasize here the phenomenological importance of possible CP-violating effects which may reside in $\tilde{t}_{L}-\tilde{t}_{R}$ mixing and are therefore proportional to $\arg \left(A_{t}\right)$. Indeed, due to experimental constraints on the Neutron Electric Dipole Moment (NEDM), the possible phase in the Higgs mass term, i.e., $\arg (\mu)$, is expected to be small (see below). Thus $\arg \left(A_{t}\right)$ should be practically the only important 
SUSY CP-odd phase observable in high-energy reactions. Of course, the most natural place to look for such effects, driven by $\arg \left(A_{t}\right)$, is high energy processes involving the top quark. Thus $\mathrm{CP}$-violating effects of the top quark observable in the laboratory may have direct bearing on Baryogenesis in the early Universe.

It was stated in 123] that using the relations obtained from the Renormalization Group Equations (RGE) of the imaginary parts in the SUSY Lagrangian, combined with the severe constraint on the low energy phase of the Higgs mass parameter, $\mu$, from the present experimental limit [124] on the NEDM, the phase in $A_{t}$ at low energy scales is likely to be very small provided one imposes some definite boundary conditions for the SUSY soft breaking terms. As a consequence, at high energies, any CP-nonconserving effect that is driven by $\arg \left(A_{t}\right)$ will then be suppressed leaving top quark reactions almost insensitive to CP-violating effects of a SUSY origin in models with these assumptions.

On the other hand we will describe below the key phenomenological features of a general MSSM and a GUT-scale N=1 minimal SUperGRAvity (SUGRA) model. We will demonstrate that the prediction made in 123 depends on the GUT-scale boundary conditions, and therefore may be significantly relaxed to yield a large $\mathrm{CP}$-violating phase in the $A_{t}$ term compatible with the existing experimental limit on the NEDM. This should encourage SUSY CP violation studies in top quark systems as they may well be the only venue for constraining $\arg \left(A_{t}\right)$ in high energy experiments at colliders in the foreseeable future.

\subsubsection{General description and the SUSY Lagrangian}

The most general low energy softly broken minimal SUSY Lagrangian which is invariant under $\mathrm{SU}(3) \times \mathrm{SU}(2) \times \mathrm{U}(1)$ consists of three generations of quarks and leptons, two Higgs doublets and the $\mathrm{SU}(3) \times \mathrm{SU}(2) \times \mathrm{U}(1)$ gauge fields, along with their SUSY partners, can be written as 120 , 125, 126, 127]:

$$
\mathcal{L}=\text { kinetic terms }+\int d^{2} \theta W+\mathcal{L}_{\text {soft }}
$$

Here $W$ is the superpotential and is given by

$$
W=\epsilon_{i j}\left(g_{U}^{I J} \hat{Q}_{I}^{i} \hat{H}_{2}^{j} \hat{U}_{J}+g_{D}^{I J} \hat{Q}_{I}^{i} \hat{H}_{1}^{j} \hat{D}_{J}^{c}+g_{E}^{I J} \hat{L}_{I}^{i} \hat{H}_{1}^{j} \hat{R}_{J}^{c}+\mu \hat{H}_{1}^{i} \hat{H}_{2}^{j}\right)
$$

$\epsilon_{i j}$ is the antisymmetric tensor with $\epsilon_{12}=1$ and the usual convention was used for the superfields $\hat{Q}, \hat{U}, \hat{L}, \hat{R}$ and $\hat{H}$ 125. $I, J=1,2$ or 3 are generation indices and $i, j$ are $\mathrm{SU}(2)$ indices.

$\mathcal{L}_{\text {soft }}$ consists of the soft breaking terms and can be divided into three pieces

$$
\mathcal{L}_{\text {soft }} \equiv \mathcal{L}_{\text {gaugino }}+\mathcal{L}_{\text {scalar }}+\mathcal{L}_{\text {trilinear }}
$$

which are the soft supersymmetry breaking gaugino, scalar mass terms and the trilinear coupling terms. These are given by 126:

$$
\begin{aligned}
\mathcal{L}_{\text {gaugino }}= & \frac{1}{2}\left(\tilde{m}_{1} \lambda_{B} \lambda_{B}+\tilde{m}_{2} \lambda_{W}^{a} \lambda_{W}^{a}+\tilde{m}_{3} \lambda_{G}^{b} \lambda_{G}^{b}\right), \\
\mathcal{L}_{\text {scalar }}= & -m_{H_{1}}^{2}\left|H_{i}^{1}\right|^{2}-m_{H_{2}}^{2}\left|H_{i}^{2}\right|^{2}-m_{L}^{2}\left|L^{i}\right|^{2}- \\
& m_{R}^{2}|R|^{2}-m_{Q}^{2}\left|Q^{i}\right|^{2}-m_{D}^{2}|D|^{2}-m_{U}^{2}|U|^{2}, \\
\mathcal{L}_{\text {trilinear }}= & \epsilon_{i j}\left(g_{U} A_{U} Q^{i} H_{2}^{j} U+g_{D} A_{D} Q^{i} H_{1}^{j} D+\right. \\
& \left.g_{E} A_{E} L^{i} H_{1}^{j} R+\mu B H_{1}^{i} H_{2}^{j}\right),
\end{aligned}
$$

\footnotetext{
${ }^{5}$ We do not include R-parity violating terms in the SUSY Lagrangian below, since we do not discuss in this review any CP-violating effect which may be driven by such terms. We only briefly mention in Chapter 11 the possible impact of R-parity violating SUSY interactions on CP violation studies in the top quark system.
} 
where we have omitted the generation indices $I$ and $J$ in the soft breaking terms. The above scalar fields correspond to the superfields which were indicated in our notation by a "hat". $\lambda_{B}$, $\lambda_{W}^{a}$ (with $a=1,2$ or 3 ) and $\lambda_{G}^{b}$ (with $b=1, \ldots, 8$ ) are the gauge superpartners of the $\mathrm{U}(1)$, $\mathrm{SU}(2)$ and $\mathrm{SU}(3)$ gauge-bosons, respectively. Also we remark that proportionality of the trilinear couplings to the Yukawa couplings (i.e., $g_{U}, g_{D}$ and $g_{E}$ ) is imposed in Eq. 3.105.

\subsubsection{CP violation in a general MSSM}

We now turn to a discussion of the CP-odd phases in the theory. In general, when no further assumptions are imposed on the pieces of the Lagrangian in Eqs. 3.101 and 3.103 - 3.105, there are several possible new sources (apart from the usual SM CKM and strong $\theta$ phases) of CP nonconservation at the scale $\Lambda_{S}$ - where the soft breaking terms are generated. These are [31, 120, 121]: the trilinear couplings $A_{F}$ (i.e., $F=U, D$ or $E$ ), the soft breaking Higgs coupling $\mu B$, the gauginos mass parameters $\tilde{m}_{a}(a=1,2$ or 3$)$ and the Higgs mass parameter $\mu$ in the superpotential. However, not all of them are physical and by a global phase change of one of the Higgs multiplets one can set $\arg (\mu B)=0$ ensuring real VEV's of the Higgs doublets and fixing the phase of $\mu$ to be $\arg (\mu)=-\arg (B)$. Moreover, in the absence of the soft breaking Lagrangian, the MSSM possesses an additional U(1) R-symmetry [128]. Thus, with an R-transformation one can remove an additional phase from the theory, say from one of the soft gaugino masses $\tilde{m}_{a}$. The remaining physical phases are: one phase for each $\arg \left(A_{f}\right)$ (corresponding to a fermion $f$ ), $\arg (B)$ and $\arg \left(\tilde{m}_{a}\right)$, say for $a=1,2$. In the most general MSSM scenario, these remaining complex parameters at the $\Lambda_{S}$-scale cannot simultaneously be made real by redefining the phases of fields without introducing phases in the other couplings.

Of course, once the above phases are set to their $\Lambda_{S}$-scale values, they feed into the SUSY parameters of the theory at the EW-scale through the RGE. Instead of studying the RGE for the full theory, one needs only consider a complete subset of the RGE of the complex parameters in the effective theory. Taking only the top and bottom Yukawa couplings and neglecting small effects from the other Yukawa couplings, such a complete subset was given in [123]:

$$
\begin{aligned}
\frac{d \tilde{m}_{a}}{d t} & =2 b_{a} \alpha_{a} \tilde{m}_{a}, \\
\frac{d A_{t}}{d t} & =2 c_{a} \alpha_{a} \tilde{m}_{a}+12 \alpha_{t} A_{t}+2 \alpha_{b} A_{b}, \\
\frac{d A_{b}}{d t} & =2 c_{a}^{\prime} \alpha_{a} \tilde{m}_{a}+12 \alpha_{b} A_{b}+2 \alpha_{t} A_{t}, \\
\frac{d A_{u, c}}{d t} & =2 c_{a} \alpha_{a} \tilde{m}_{a}+6 \alpha_{t} A_{t}, \\
\frac{d A_{d, s}}{d t} & =2 c_{a}^{\prime} \alpha_{a} \tilde{m}_{a}+6 \alpha_{b} A_{b}, \\
\frac{d B}{d t} & =2 c_{a}^{\prime \prime} \alpha_{a} \tilde{m}_{a}+6 \alpha_{b} A_{b}+6 \alpha_{t} A_{t}, \\
\frac{d \alpha_{t}}{d t} & =2 \alpha_{t}\left(-c_{a} \alpha_{a}+6 \alpha_{t}+\alpha_{b}\right), \\
\frac{d \alpha_{b}}{d t} & =2 \alpha_{b}\left(-c_{a}^{\prime} \alpha_{a}+6 \alpha_{b}+\alpha_{t}\right), \\
\frac{d \alpha_{a}}{d t} & =2 b_{a} \alpha_{a}^{2},
\end{aligned}
$$

where $t \equiv \ln \left(Q / \Lambda_{S}\right) / 4 \pi, a$ is summed from 1 to 3 and $b_{a}=(33 / 5,1,-3), c_{a}=(13 / 15,3,16 / 3), c_{a}^{\prime}=$ $(7 / 15,3,16 / 3), c_{a}^{\prime \prime}=(3 / 5,3,0)$. Also, $\alpha_{t}$ and $\alpha_{b}$ are related to the corresponding quark masses via 


$$
\alpha_{t(b)}=\frac{g_{2}^{2}}{8 \pi} \frac{m_{t(b)}^{2}}{m_{W}^{2}} \frac{1}{\sin ^{2} \beta\left(\cos ^{2} \beta\right)} .
$$

We remark that in the general MSSM framework with arbitrary CP-violating phases at $\Lambda_{S}$, the above RGE are of less importance and with such boundary conditions almost any low energy $\mathrm{CP}$-violating scenario can be generated. In particular, large CP-violating phases at the EW-scale are not excluded in this unconstrained scenario. However, in a more constrained SUSY version, one can reduce the number of the physical CP-odd phases in the theory. In this case the RGE given above are crucial for determining the SUSY CP-violating phases at the EW-scale [123]. We will return to this point later when we discuss the $N=1$ minimal low energy SUGRA - GUT model.

Let us consider now the phenomenological consequences of $\mathrm{CP}$ nonconservation in such a general SUSY model. First we need to describe briefly how these new CP-violating phases enter in reactions which are driven by supersymmetric particles. As it turns out, all $\mathrm{CP}$ violation in the low energy SUSY vertices is driven by diagonalization of the complex mass matrices of the sfermions, charginos and neutralinos. For more detailed investigations of the diagonalization procedure and extraction of the mass spectrum and $\mathrm{CP}$-violating phases from these mixing matrices we refer the reader to the existing literature, see e.g., 120, 126, 129, 130, 131, 132, 133, 134. Here we only wish to briefly describe the key features of the formulation and the definitions.

We denote by $M_{\tilde{f}}^{2}$ the mass squared matrix of the scalar partners of a fermion, and $M_{\tilde{\chi}}$ and $M_{\tilde{\chi}^{0}}$ are the mass matrices of the charginos and neutralinos, respectively. Then, with the rotation matrices $Z_{f}, Z_{N}, Z^{+}$and $Z^{-}$, we can define

$$
\begin{aligned}
Z_{f}^{\dagger} M_{\tilde{f}}^{2} Z_{f} & =\operatorname{diag}\left(m_{\tilde{f}_{1}}^{2}, m_{\tilde{f}_{2}}^{2}\right) \\
\left(Z^{-}\right)^{\dagger} M_{\tilde{\chi}} Z^{+} & =\operatorname{diag}\left(m_{\tilde{\chi}_{1}}, m_{\tilde{\chi}_{2}}\right) \\
Z_{N}^{T} M_{\tilde{\chi}^{0}} Z_{N} & =\operatorname{diag}\left(m_{\tilde{\chi}_{1}^{0}}, m_{\tilde{\chi}_{2}^{0}}, m_{\tilde{\chi}_{3}^{0}}, m_{\tilde{\chi}_{4}^{0}}\right) .
\end{aligned}
$$

$M_{\tilde{f}}^{2}$ is then given by

$$
M_{\tilde{f}}^{2}=\left(\begin{array}{cc}
m_{f}^{2}-\cos 2 \beta\left(T_{3 f}-Q_{f} \sin ^{2} \theta_{W}\right) m_{Z}^{2}+m_{\tilde{f}_{L}}^{2} & -m_{f}\left(R_{f} \mu+A_{f}^{*}\right) \\
-m_{f}\left(R_{f} \mu^{*}+A_{f}\right) & m_{f}^{2}-\cos 2 \beta Q_{f} \sin ^{2} \theta_{W} m_{Z}^{2}+m_{\tilde{f}_{R}}^{2}
\end{array}\right)
$$

where $m_{f}$ is the mass of the fermion $f, Q_{f}$ its charge and $T_{3 f}$ the third component of the weak isospin of a left-handed fermion $f . m_{\tilde{f}_{L}}^{2}\left(m_{\tilde{f}_{R}}^{2}\right)$ is the low energy mass squared parameter for the left (right) sfermion $\tilde{f}_{L}\left(\tilde{f}_{R}\right) . R_{f}=\cot \beta(\tan \beta)$ for $T_{3 f}=\frac{1}{2}\left(-\frac{1}{2}\right)$ where $\tan \beta=v_{2} / v_{1}$ is the ratio between the two VEV's of the two Higgs doublets in the model.

$M_{\tilde{\chi}}$ and $M_{\tilde{\chi}^{0}}$ are given by

$$
\begin{gathered}
M_{\tilde{\chi}}=\left(\begin{array}{ccc}
\tilde{m}_{2} & \sqrt{2} m_{W} \sin \beta \\
\sqrt{2} m_{W} \cos \beta & \mu
\end{array}\right), \\
M_{\tilde{\chi}^{0}}=\left(\begin{array}{cccc}
\tilde{m}_{1} & 0 & -m_{Z} \cos \beta \sin \theta_{W} & m_{Z} \sin \beta \sin \theta_{W} \\
0 & \tilde{m}_{2} & m_{Z} \cos \beta \cos \theta_{W} & -m_{Z} \sin \beta \cos \theta_{W} \\
-m_{Z} \cos \beta \sin \theta_{W} & m_{Z} \cos \beta \cos \theta_{W} & 0 & -\mu \\
m_{Z} \sin \beta \sin \theta_{W} & -m_{Z} \sin \beta \cos \theta_{W} & -\mu & 0
\end{array}\right),
\end{gathered}
$$

where $\tilde{m}_{1}\left(\tilde{m}_{2}\right)$ is the mass parameter for the $\mathrm{U}(1)(\mathrm{SU}(2))$ gaugino.

Because of their relatively simple form, we will discuss below the way of parameterizing the $\mathrm{CP}$-violating phases only of the sfermions and charginos diagonalizing matrices $Z_{f}$ and $Z^{+}, Z^{-}$, 
respectively. The diagonalization of the $4 \times 4$ neutralino mixing matrix with complex entries is more involved and may be estimated numerically, although in some limiting cases it may be approximated analytically (see e.g., [123]). If all elements of $Z_{N}$ are real then the diagonalization procedure can also be done analytically (see e.g., 131, 133]).

The mixing matrix of the sfermions is parameterized as

$$
Z_{f}=\left(\begin{array}{cc}
\cos \theta_{f} & -e^{-i \beta_{f}} \sin \theta_{f} \\
e^{i \beta_{f}} \sin \theta_{f} & \cos \theta_{f}
\end{array}\right),
$$

where $\theta_{f}$ is the mixing angle and $\beta_{f}$ is the phase responsible for CP-violating phenomena in sfermions interactions with other particles in the theory, and is given by

$$
\tan \beta_{f}=-\frac{|\mu| R_{f} \sin \alpha_{\mu}-\left|A_{f}\right| \sin \alpha_{f}}{|\mu| R_{f} \cos \alpha_{\mu}+\left|A_{f}\right| \cos \alpha_{f}}
$$

where we have used $A_{f}=\left|A_{f}\right| e^{i \alpha_{f}}$ and $\mu=|\mu| e^{i \alpha_{\mu}}$. Recall that $R_{f}=\cot \beta(\tan \beta)$ for $T_{3 f}=$ $\frac{1}{2}\left(-\frac{1}{2}\right)$ and $\tan \beta=v_{2} / v_{1}$. Also, the mixing angle $\theta_{f}$ is given by

$$
\tan \theta_{f}=\frac{-2 m_{f}\left|R_{f} \mu+A_{f}^{*}\right|}{\cos 2 \beta\left(2 Q_{f} \sin ^{2} \theta_{W}-T_{3 f}\right) m_{Z}^{2}+m_{\tilde{f}_{L}}^{2}-m_{\tilde{f}_{R}}^{2}} .
$$

It is obvious from Eq. 3.123 and 3.124 that in the limit where all the quark masses are small except for $m_{t}$, only the phase of $A_{t}$ leads to CP-violating effects (the limit $m_{f} \rightarrow 0, f \neq t$, is useful when considering high energy reactions). In particular, the other $A$-terms are multiplied by the light fermion masses (see also Eq. 3.119) and, therefore, they have negligible effect on any physical quantity evaluated at high enough energies. That is, the off-diagonal elements of $Z_{f}$ are zero in this limit (i.e., from Eq. 3.124 we see that $\sin \theta_{f} \rightarrow 0$ when $m_{f} \rightarrow 0$ ) and there is no mixing between the left and right components of the superpartners of light quarks. Of course, this is not the case for the NEDM which is particularly sensitive to the slight deviation from degeneracy of the supersymmetric partners of the $u$ and the $d$ quarks. We will return to a more detailed discussion on the "SUSY-CP problem" of the NEDM in the following section.

For a sfermion $\tilde{f}$, it is useful to adopt a parameterization for its $\tilde{f}_{L}-\tilde{f}_{R}$ mixing such that the sfermions of different handedness are related to their mass eigenstates through the transformation

$$
\begin{aligned}
& \tilde{f}_{L}=Z_{f}^{11} \tilde{f}_{1}+Z_{f}^{12} \tilde{f}_{2}, \\
& \tilde{f}_{R}=Z_{f}^{21} \tilde{f}_{1}+Z_{f}^{22} \tilde{f}_{2},
\end{aligned}
$$

where $\tilde{f}_{1,2}$ are the two mass eigenstates. We note that, in the case where all CP violation arises from $\tilde{f}_{L}-\tilde{f}_{R}$ mixing, i.e., from the complex entries in the sfermion mixing matrix $Z_{f}$, it has to be proportional to

$$
\Im \mathrm{m}\left(\xi_{f}^{i}\right) \equiv \Im \mathrm{m}\left(Z_{f}^{1 i *} Z_{f}^{2 i}\right)=\frac{(-1)^{i-1}}{2} \sin 2 \theta_{f} \sin \beta_{f} .
$$

Clearly, $\xi_{f}^{i} \rightarrow 0$ if there is no mixing between the left and right sfermions such that they are nearly degenerate. We will describe in the next chapters CP-nonconserving effects in top quark systems which are driven by the possibly large mass splitting between the two stop mass eigenstates and are therefore proportional to $\Im \mathrm{m}\left(\xi_{t}^{i}\right)$.

The charginos mixing matrices are given by

$$
Z^{ \pm}=P^{ \pm} O^{ \pm}
$$

where 


$$
P^{+}=\left(\begin{array}{cc}
1 & 0 \\
0 & -e^{i \alpha_{2}}
\end{array}\right), \quad P^{-}=\left(\begin{array}{cc}
e^{i \alpha_{2}} & 0 \\
0 & -1
\end{array}\right)
$$

and

$$
\begin{aligned}
O^{+} & =\left(\begin{array}{cc}
\cos \theta_{+} & -e^{-i \beta_{+}} \sin \theta_{+} \\
e^{i \beta_{+}} \sin \theta_{+} & \cos \theta_{+}
\end{array}\right), \\
O^{-} & =\left(\begin{array}{cc}
e^{i \gamma_{1}} \cos \theta_{-} & -e^{-i\left(\beta_{-}-\gamma_{2}\right)} \sin \theta_{-} \\
e^{i\left(\beta_{-}+\gamma_{1}\right)} \sin \theta_{-} & e^{i \gamma_{2}} \cos \theta_{-}
\end{array}\right) .
\end{aligned}
$$

Here $\alpha_{2}=\arg \left(\tilde{m}_{2}\right)$ and the CP-violating angles $\beta_{ \pm}$and $\gamma_{1,2}$ above are given by

$$
\begin{aligned}
\tan \beta_{+} & \equiv-\frac{\sin \theta}{\cos \theta+\frac{\left|\tilde{m}_{2}\right| \cot \beta}{|\mu|}}, \\
\tan \beta_{-} & \equiv \frac{\sin \theta}{\cos \theta+\frac{\left|\tilde{m}_{2}\right| \tan \beta}{|\mu|}}, \\
\tan \gamma_{1} & \equiv-\frac{\sin \theta}{\cos \theta+\frac{2\left|\tilde{m}_{2}\right|\left(m_{\tilde{\chi}_{1}}^{2}-|\mu|^{2}\right)}{g^{2}|\mu| v_{1} v_{2}}}, \\
\tan \gamma_{2} & \equiv \frac{\sin \theta}{\cos \theta+\frac{g^{2}\left|\tilde{m}_{2}\right| v_{1} v_{2}}{2|\mu|\left(m_{\tilde{\chi}_{2}}^{2}-\left|\tilde{m}_{2}\right|\right)}},
\end{aligned}
$$

where $\theta \equiv \alpha_{\mu}+\alpha_{2}$ and $m_{\tilde{\chi}_{i}}(i=1,2)$ are the masses of the two charginos. $\theta_{+}$and $\theta_{-}$are also functions of $\tilde{m}_{2}, \mu, v_{1}$ and $v_{2}$ (see [130]).

\subsubsection{CP violation in a GUT-scale $N=1$ minimal SUGRA model}

Let us proceed by describing a more constrained supersymmetric model. In particular, we want to consider a spontaneously broken $N=1$ SUGRA, which apart from gravitational interactions, is essentially identical at low energies to a theory with softly broken supersymmetry with GUT motivated relations at the GUT mass scale. One of the most appealing consequences of such a constrained SUSY scenario is that it allows REWSB of $\mathrm{SU}(2) \times \mathrm{U}(1)$ with the fewest number of free parameters.

According to conventional wisdom, complete universality of the soft supersymmetric parameters at the GUT-scale (or at the scale where the SUSY soft breaking terms are generated) is assumed. More explicitly, a common scalar mass $m_{0}$ and a common gaugino mass $M_{1 / 2}$ at the GUT-scale

$$
\begin{aligned}
m_{H_{1}}^{2} & =m_{H_{2}}^{2}=m_{R}^{2}=m_{L}^{2}=m_{Q}^{2}=m_{U}^{2}=m_{D}^{2}=m_{0}^{2} \\
\tilde{m}_{a} & =M_{1 / 2}, \quad a=1,2 \text { or } 3,
\end{aligned}
$$

and also universal boundary conditions for the soft breaking trilinear terms are assumed

$$
A_{E}=A_{D}=A_{U} \equiv A^{G}
$$

Of course, the above relations do not survive after renormalization effects from the GUT-scale (which is usually taken to be $M_{G} \sim 2 \times 10^{16} \mathrm{GeV}$ ) to the EW-scale are included.

It then follows that the universal parameters of the minimal SUGRA model at the GUT-scale are: $m_{0}, M_{1 / 2}, A^{G}, B^{G}, \mu^{G}$ and $\tan \beta$. This is the most general set of independent parameters 
before REWSB. However, a bonus of this economical framework is that REWSB occurs and the parameters $\mu^{G}$ and $B^{G}$ are no longer taken as independent but are set by $m_{0}, M_{1 / 2}$ and $\tan \beta$ (the magnitude of $\mu$ is adjusted to give the appropriate $Z$-boson mass but the sign of $\mu$ remains as an independent parameter). Thus, the number of independent parameters is reduced to five, namely $m_{0}, M_{1 / 2}, A^{G}, \operatorname{sign}(\mu)$ and $\tan \beta$.

In this GUT motivated SUGRA theory there are four possible new sources of CP nonconservation at the GUT-scale. These are 121]: the universal trilinear coupling $A^{G}$, the Higgs mass parameter $\mu^{G}$, the gauge mass parameter $M_{1 / 2}$ and the parameter $B^{G}$. However, as was mentioned before, $M_{1 / 2}$ can be made real by an $R$-transformation and by using one remaining phase freedom, a redefinition of the Higgs fields can set the product $B^{G} \mu^{G}$ to be real so that the VEV's of the two Higgs fields in the theory are also made real. We therefore have $\arg \left(\mu^{G}\right)=-\arg \left(B^{G}\right)$. With this choice we are left with only two new SUSY CP-odd phases at the GUT-scale which are carried by $A^{G}$ and $\mu^{G}$, apart from the usual CKM phase that originates from the Yukawa couplings in the theory for three generations.

One can proceed by choosing a more constrained CP-violating sector by setting one of these two phases to zero (we will address to this possibility in the next section), or even a "super" constrained CP-violating sector by taking $\arg \left(\mu^{G}\right)=\arg \left(A^{G}\right)=0$, thus being left (at the GUTscale) only with the usual CKM phase present as in the SM.

Note that having a universal phase for all trilinear couplings of sfermions at the GUT-scale, does not necessarily mean that all sfermions will have the same phase (driven by their trilinear coupling $\left.A_{f}\right)$ at the EW-scale. That is, the GUT-scale phases $\arg \left(A^{G}\right)$ and $\arg \left(\mu^{G}\right)$ feed into the other parameters of the theory through renormalization effects from the GUT-scale to the EWscale. In particular, these GUT-scale phases can produce different phases for different squarks and sleptons of different generations. However, irrespective of what those different phases are at the EW-scale, they can all be expressed in principle with the two new CP-odd phases of $A^{G}$ and $\mu^{G}$ through the RGE in Eqs. 3.106 3.114. Thus, it is evident from the very simple structure of the evolution equations of the gaugino masses (see Eq. 3.106) that $\tilde{m}_{a}, a=1-3$, are left with no phase at any scale. Moreover, the difference between the three real low energy gaugino mass parameters comes from the fact that they undergo a different renormalization as they evolve from the GUT-scale to the EW-scale, due to the different gauge structure of their interactions. In particular, they are related, at the EW-scale, by (see e.g., [131])

$$
\frac{3 \cos ^{2} \theta_{W}}{5} \frac{\tilde{m}_{1}}{\alpha}=\sin ^{2} \theta_{W} \frac{\tilde{m}_{2}}{\alpha}=\frac{\tilde{m}_{3}}{\alpha_{s}},
$$

where $\theta_{W}$ is the weak mixing angle and $\tilde{m}_{3}$ is the low energy gluino mass.

Notice now that with $\arg \left(\tilde{m}_{a}\right)=0$, the RGE (Eqs. 3.106-3.114) simplify to a large extent, thus we have only to consider the evolution of the $A_{f}^{G}$ 's and $B^{G}$ (or equivalently $\mu^{G}$ ) to the EW-scale. This constrained version of the MSSM has strong implications on the low energy phase in $A_{t}$ as was suggested in [123]. In particular, it was shown that with and without universal trilinear couplings at the GUT-scale and with some definite boundary conditions for them (for example, $\arg \left(\mu^{G}\right)=\arg \left(A_{f}^{G}\right)=0$ for fermion species $f$ ), the low energy CP-violating phase of $A_{t}$ induces potentially large phases in $A_{u}, A_{d}$ and $B$ at the EW-scale (through relations obtained from the above set of the RGE). This in turn gives rise to a large NEDM which is ruled out by the present experimental limit. Therefore, severe constraints on the low energy phases of $A_{f}$ and $\mu$ where obtained. We will return to this point in the next section.

\subsubsection{A plausible low energy MSSM framework and the "SUSY-CP problem" of the NEDM}

The EDM of the neutron, $d_{n}$, imposes important phenomenological constraints on SUSY models 121, 123, 130, 132, 134, 135, 136, 137]. In particular, with a low energy MSSM that originates from a GUT-scale SUGRA model (with complete universality of the soft breaking terms), keeping 
$d_{n}$ within its allowed experimental value (i.e., $\left|d_{n}\right| \lesssim 10^{-25}$ e-cm [124]) requires the "fine tuning" of the SUSY phases to be less than or of the order of $10^{-2}-10^{-3}$ for SUSY particle masses of the order of the EW symmetry breaking scale. We remark, though, that it has recently been claimed in [134], that in some regions of the SUSY parameter space, cancellations among the different components of the neutron EDM may occur and such a severe fine tuning for either the SUSY masses or the SUSY CP-violating phases (i.e., at the order of $10^{-2}$ ) may not be necessary. However, for such cancellations to occur SUSY parameters have to be suitably arranged and, also, several large SUSY phases have to be present, which renders this scenario less predictive and less attractive as well.

The NEDM can be written schematically in any low energy MSSM scenario in which the CP-phases originate from the $\mu$ and the $A_{f}$ terms as [123]:

$$
\frac{d_{n}}{10^{-25} \mathrm{e}-\mathrm{cm}}=X^{\mu} \frac{|\mu|}{M_{S}} \sin \alpha_{\mu}+X^{A_{u}} \frac{\left|A_{u}\right|}{M_{S}} \sin \alpha_{u}+X^{A_{d}} \frac{\left|A_{d}\right|}{M_{S}} \sin \alpha_{d},
$$

where $M_{S}$ is the typical SUSY mass scale which may be used to describe the typical squark masses. It was found in 123 that typical values of the $X^{i}$ s in almost every such low energy SUSY realization are: $X^{A_{u}, A_{d}}>\mathcal{O}(1)$ and $X^{\mu}>\mathcal{O}\left(10^{2}\right)$ for $M_{S} \lesssim 500 \mathrm{GeV}$. Therefore, with $\left|A_{f}\right| \approx|\mu| \approx M_{S}$, only moderate bounds can be put on $\sin \alpha_{u}$ and $\sin \alpha_{d}$. In contrast, an unambiguous severe constraint is obtained on the low energy phase of the Higgs mass term $\mu$, namely $\sin \alpha_{\mu}<\mathcal{O}\left(10^{-2}\right)$ for $M_{S} \lesssim 500 \mathrm{GeV}$ (see also [31, 130, 136] and references therein).

The important finding in 123 is that if a complete universality of the soft breaking terms is imposed at the GUT-scale and the two GUT phases are zero or very small, i.e., $\arg \left(A^{G}\right) \approx$ $\arg \left(B^{G}\right) \lesssim 0.1$, then this severe constraint on $\sin \alpha_{\mu}$ combined with the relations between $\Im \mathrm{m}\left(A_{t}\right)$ and $\Im \mathrm{m}(\mu)$, obtained from the RGE, leads to the comparable constraint $\sin \alpha_{t}<\mathcal{O}\left(10^{-2}\right)$. With a universal $A$ term at the GUT-scale this will also imply (through relations obtained from the RGE) $\sin \alpha_{u, d}<\mathcal{O}\left(10^{-2}\right)$. Moreover, the above strong constraints on the SUSY CP-violating phases hold even if the universality of the $A$ terms is relaxed at the GUT-scale as long as the GUTscale CP-violating phases are kept very small. This strong constraint on the low energy phase of $A_{t}$ would also eliminate any possible SUSY CP-nonconserving effect in top quark systems.

While this scenario with very small CP-violating phases at the GUT-scale provides an explanation of the smallness of the NEDM (in fact, the above constraints will drive the NEDM to a value of the order of $10^{-27}-10^{-28} \mathrm{e}-\mathrm{cm}$ ), an unavoidable question then arises: why do the CP-violating phases happen to be so small wherever they appear? If so, then an underlying theory that screens the CP-violating phases is required.

We therefore feel that a somewhat different phenomenological approach is needed, namely, the implication that $\sin \alpha_{t}<\mathcal{O}\left(10^{-2}\right)$ should be specially scrutinized in the top quark sector. The latter, being very sensitive to $\sin \alpha_{t}$ at high energies, may serve as a unique probe for searching for significant deviations from the above upper bound, $\sin \alpha_{t}<\mathcal{O}\left(10^{-2}\right)$ (see e.g., [138]). Indeed, if at the GUT-scale the universality of the $A$ terms is relaxed and no assumption is made on the magnitude of the CP-violating phases, then one can construct a plausible low energy MSSM framework which incorporates an $\mathcal{O}(1)$ low energy phase for $A_{t}$, while leaving the NEDM within its experimental limit. The crucial difference in assuming non-universal boundary conditions for the soft breaking trilinear $A$ terms is that, in this case, there is no a-priori reason to believe that the low energy phases associated with the different $A_{f}$ terms are related at the EW-scale. In particular, the bounds on $\sin \alpha_{u, d}$ obtained from the experimental limit of the NEDM may not be used to constrain $\sin \alpha_{t}$. In addition, when no further assumption is made on the magnitude of the CP-violating phases at the GUT-scale, a value of $\sin \alpha_{\mu} \rightarrow 0$ may be realized without contradicting any existing relation from the RGE.

\footnotetext{
${ }^{6}$ Since $M_{S}$ is the only SUSY mass scale associated with the squarks sector, it is natural to choose the soft breaking terms to be of $\mathcal{O}\left(M_{S}\right)$.
} 
As in [138] we therefore take the following phenomenological point of view in constructing a plausible low energy set of the MSSM CP-violating phases and mass spectrum:

1. $\sin \alpha_{\mu} \rightarrow 0$ as strongly implied from the analysis of the NEDM.

2. $\sin \alpha_{u}, \sin \alpha_{d}$ and $\sin \alpha_{t}$ are not correlated at the EW-scale, which implicitly assumes nonuniversal boundary conditions at the GUT-scale. In particular, $\sin \alpha_{u}, \sin \alpha_{d}$ may then be constrained only from the NEDM experimental limit, with no implications on the size of $\sin \alpha_{t}$.

3. Motivated by the theoretical prediction of the unification of the $\mathrm{SU}(3), \mathrm{SU}(2)$ and $\mathrm{U}(1)$ gauge couplings when SUSY particles with a mass scale around $1 \mathrm{TeV}$ are folded into the RGE, one may follow only the following traditional simplifying GUT assumption: there is an underlying grand unification. As mentioned before, this leads us to have a common gaugino mass parameter defined at the GUT-scale which can be made real by an R-rotation. Thus, $\tilde{m}_{a}, a=1-3$ are left with no phase at any scale. Moreover, using the relation of Eq. 3.137, once the gluino mass is set at the EW-scale, the $\mathrm{SU}(2)$ and $\mathrm{U}(1)$ gaugino masses $\tilde{m}_{2}$ and $\tilde{m}_{1}$, respectively, are determined.

4. The typical SUSY-scale is $M_{S}$ and all the squarks except for the light stop, are assumed to be degenerate with mass $M_{S}$.

Note that in this low energy framework one is only left with the phases of the various $A_{f}$ terms at the EW-scale, out of which only $A_{t}$ plays a significant role in any high energy reaction. For $m_{f} \rightarrow 0$ the superpartners of the light quarks are practically degenerate and therefore the $\mathrm{CP}$-violating effects from the phases of the other $A_{f}$ terms, corresponding to the light quarks, can be safely neglected. Note again that our approach, the EW $\rightarrow$ GUT approach, assumes a set of phases at the EW-scale, subject to existing experimental data, which implicitly assumes arbitrary phases at the scale in which the soft breaking terms are generated. As was mentioned above, this low energy CP violation scenario can naturally arise from a GUT-scale SUGRA model if only the universality of the $A$ terms is relaxed and no assumption is made on the magnitude of the GUT-scale phases.

Also, the mass matrices of the neutralinos, $M_{\tilde{\chi}^{0}}$, and charginos, $M_{\tilde{\chi}}$, depend on the low energy Higgs mass parameter $\mu$, the two gaugino masses $\tilde{m}_{2}$ and $\tilde{m}_{1}$ (which are resolved by the gluino mass) and $\tan \beta$ (see previous sections) and are therefore real in this scenario. Thus, once $\mu, \tilde{m}_{3}$ and $\tan \beta$ are set to their low energy values, the four physical neutralino species $m_{\tilde{\chi}_{n}^{0}}(n=1-4)$ and the two physical chargino species $m_{\tilde{\chi}_{m}}(m=1,2)$ are extracted by diagonalizing the real matrices $M_{\tilde{\chi}^{0}}$ and $M_{\tilde{\chi}}$.

5. Finally, the resulting SUSY mass spectrum may now be subject to the existing experimental limits, i.e., limits on the masses of squarks, gluino, neutralinos, charginos, etc. (see e.g., $[139)$.

With these assumptions, when $\arg (\mu) \rightarrow 0$, the leading contribution to a light quark EDM comes from gluino exchange, which with the approximation of degenerate $\tilde{u}$ and $\tilde{d}$ squark masses (which we will denote by $m_{\tilde{q}}$ ), can be written as [130]:

$$
d_{q}(G)=\frac{2 \alpha_{s}}{3 \pi} Q_{q} e m_{q} \frac{\left|A_{q}\right| \sin \alpha_{q}}{m_{\tilde{q}}^{3}} \sqrt{r} K(r)
$$

where $m_{q}\left(m_{\tilde{q}}\right)$ is the quark(squark) mass and $Q_{q}$ is its charge. Also, $r \equiv m_{G}^{2} / m_{\tilde{q}}^{2}$ (for the rest of this section we denote the gluino mass by $\left.m_{G}\right)$ and $K(r)$ is given by

\footnotetext{
${ }^{7}$ Note that the function $K(r)$ in Eq. 3.140 is slightly different from that obtained in 123 . However, we find that numerically the difference is insignificant and does not change our predictions below.
} 


$$
K(r)=\frac{1}{(r-1)^{3}}\left(\frac{1}{2}+\frac{5}{2} r+\frac{r(2+r)}{1-r} \ln r\right) .
$$

Then, within the naive quark model, the NEDM can be obtained by relating it to the $u$ and $d$ quarks EDM's (i.e., $d_{u}$ and $d_{d}$, respectively) as

$$
d_{n}=\left(4 d_{d}-d_{u}\right) / 3 .
$$

We now consider $\arg \left(A_{u}\right)$ and $\arg \left(A_{d}\right)$ to be free parameters of the model irrespective of $\arg \left(A_{t}\right)$. In Figs. 9(a) and 9 (b) we have plotted the allowed regions in the $\sin \alpha_{u}-\sin \alpha_{d}$ plane for $\left|d_{n}\right|$ not to exceed $1 \times 10^{-25} \mathrm{e}-\mathrm{cm}$ (for the present experimental limit see [124]) and $3 \times 10^{-25} \mathrm{e}-\mathrm{cm}$. In calculating $d_{n}$ we assumed that the above naive quark model relation holds. Although there is no doubt that it can serve as a good approximation for an order of magnitude estimate, it may still deviate from the true theoretical value which involves uncertainties in the calculation of the corresponding hadronic matrix elements. Note also that it was argued in [140] that the naive quark model overestimates the NEDM, as the strange quark may carry an appreciable fraction of the neutron spin which can partly screen the contributions to the NEDM coming from the $u$ and the $d$ quarks. To be on the safe side, we therefore slightly relax the theoretical limit on $d_{n}$ in Fig. 9(b) to be $3 \times 10^{-25} \mathrm{e}-\mathrm{cm}$.

We have used, for these plots, $m_{\tilde{d}}=m_{\tilde{u}}=M_{S}=400 \mathrm{GeV}, m_{G}=500 \mathrm{GeV}$ and for simplicity we also took $\left|A_{u}\right|=\left|A_{d}\right|=M_{S}$. As remarked before, it is only natural to choose the mass scale of the soft breaking terms according to our typical SUSY mass scale $M_{S}$. Also, we took the values for current quark masses as $m_{d}=10 \mathrm{MeV}, m_{u}=5 \mathrm{MeV}$ and $\alpha_{s}\left(m_{Z}\right)=0.118$.

From Fig. 9(a) and in particular Fig. 9(b), it is evident that $M_{S}=400 \mathrm{GeV}$ and $m_{G}=500$ $\mathrm{GeV}$ can be safely assumed, leaving "enough room" in the $\sin \alpha_{u}-\sin \alpha_{d}$ plane for $\left|d_{n}\right|$ not to exceed $1-3 \times 10^{-25}$ e-cm. We observe that while $\sin \alpha_{u}$ is basically not constrained, $-0.35 \sim$ $<\sin \alpha_{d} \lesssim 0.35$ is needed for $\left|d_{n}\right|<1 \times 10^{-25}$ e-cm and $-0.55 \lesssim \sin \alpha_{d} \lesssim 0.55$ is needed for $\left|d_{n}\right|<3 \times 10^{-25}$ e-cm. Moreover, varying $m_{G}$ between $250 \mathrm{GeV}$ to $650 \mathrm{GeV}$ has almost no effect on the allowed areas in the $\sin \alpha_{u}-\sin \alpha_{d}$ plane that are shown in Figs.9(a) and 9(b). (8) That is, keeping $M_{S}=400 \mathrm{GeV}$ and lowering $m_{G}$ down to $250 \mathrm{GeV}$, very slightly shrinks the dark areas in Figs.9(a) and 9(b), whereas, increasing $m_{G}$ up to $650 \mathrm{GeV}$ slightly widens them. Of course, $d_{n}$ strongly depends on the scalar mass $M_{S}$ - increasing $M_{S}$ enlarges the allowed regions in Figs.9(a) and 9(b) as expected from Eq. 3.139. It is also very interesting to note that, in some instances, for a cancellation between the contributions of the $u$ and $d$ quarks to occur, $\sin \alpha_{u}, \sin \alpha_{d}>0.1$ is essential rather than being just possible. For example, with $\left|\sin \alpha_{u}\right| \gtrsim 0.75,\left|\sin \alpha_{d}\right| \gtrsim 0.1$ is required in order to keep $d_{n}$ below its experimental limit.

We can therefore conclude that CP-odd phases in the $A_{u}$ and $A_{d}$ terms of the order of few $\times 10^{-1}$ can be accommodated without too much difficulty with the existing experimental constraint on the NEDM even for typical SUSY masses of $\lesssim 500 \mathrm{GeV}$. Therefore, we restate what is emphasized in 138: somewhat in contrast to the commonly held viewpoint we do not find that a "fine-tuning" at the level of $10^{-2}$ is necessarily required for the SUSY CP-violating phases for squark masses of a few hundreds $\mathrm{GeV}$ or slightly heavier.

\subsubsection{CP and the pure Higgs sector of the MSSM}

Since the superpotential is required to be a function of only left (or only right) chiral superfields, it forbids the appearance of $\hat{H}_{1}^{*}$ and $\hat{H}_{2}^{*}$ in the superpotential $W$ in Eq. 3.101. Therefore, since a $\hat{Q}_{I}^{i} \hat{H}_{1}^{j} \hat{U}_{J}$ coupling in $W$ is prohibited by gauge invariance, only $H_{2}$ is responsible for giving mass to up quarks and $H_{1}$ to down quarks [129]. As a consequence, the requirement that there will be no "hard" breaking terms of the symmetry $\Phi_{i} \rightarrow-\Phi_{i}$ in the Higgs potential is automatically

\footnotetext{
${ }^{8} \mathrm{We}$ will take $M_{S}=400 \mathrm{GeV}$ and vary $m_{G}$ in this range in some of the CP-violating effects in collider experiments to be discussed in the following chapters.
} 

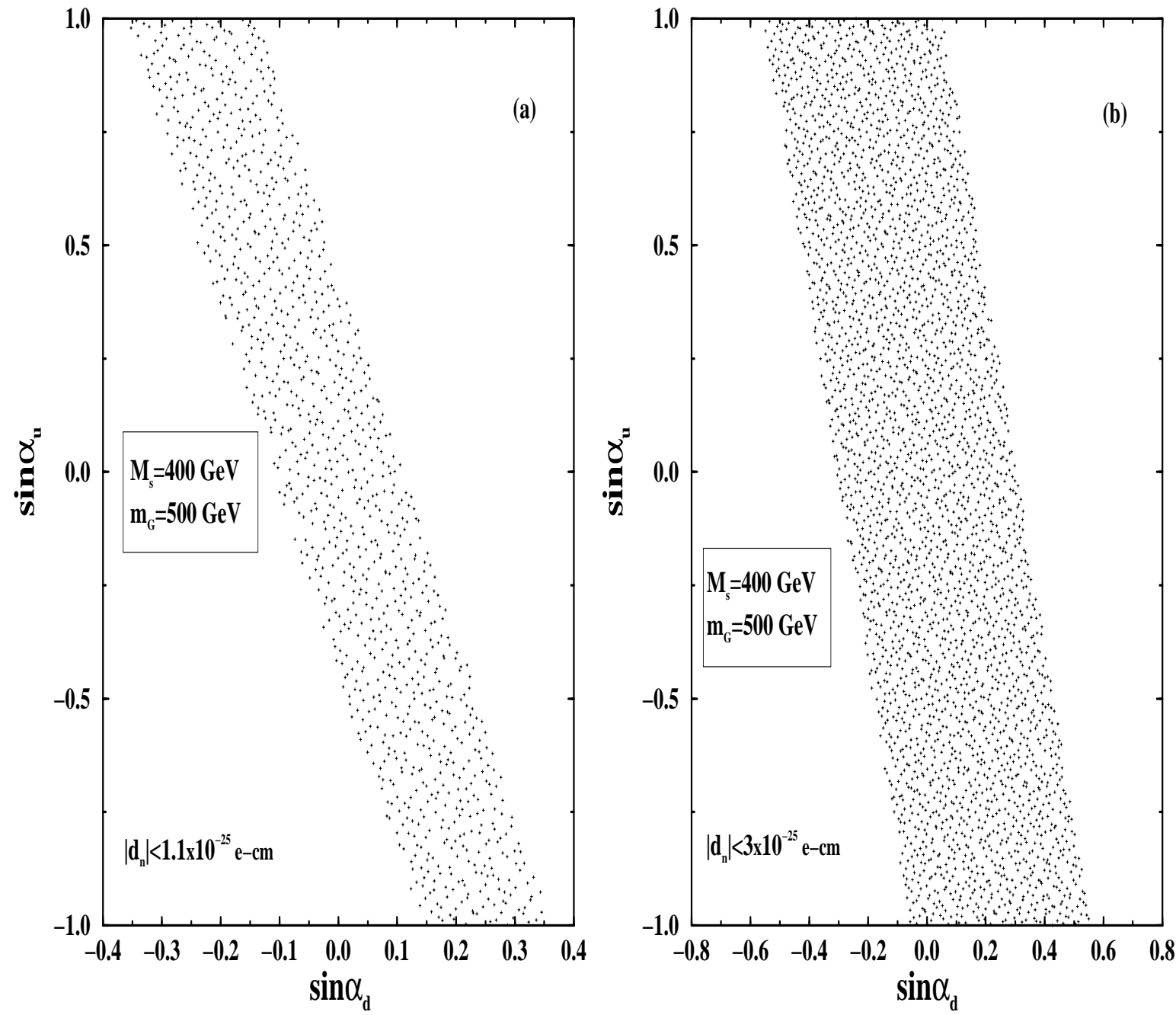

Figure 9: The allowed regions in the $\sin \alpha_{u}-\sin \alpha_{d}$ plane for the NEDM not to exceed (a) $1 \times 10^{-25}$ e-cm and (b) $3 \times 10^{-25}$ e-cm. $M_{S}=400 \mathrm{GeV}$ and $m_{G}=500 \mathrm{GeV}$ is used. The shaded areas indicate the allowed regions. Figure taken from 138]. 
satisfied in a minimal supersymmetric model. That is, $\lambda_{6}=\lambda_{7}=0$ in Eq. 3.48, for the Higgs potential in the MSSM. Moreover, no term of the form

$$
\left(\Phi_{1}^{\dagger} \Phi_{2}\right)^{2}+\text { h.c. , }
$$

appears in the Higgs potential of the MSSM [129], thus implying $\lambda_{5}=0$ in Eq. 3.48. It is then straightforward to observe that with the above constraints on the pure Higgs sector of the MSSM, any phase which may appear in a complex soft breaking parameter (i.e., proportional to $\mu_{12}^{2}$ in Eq. 3.48) can be removed by a redefinition of one of the Higgs doublet fields, thus also setting the relative phase between the two VEV's to zero. Therefore, the pure Higgs sector in the MSSM possesses no $\mathrm{CP}$ violation [129]. Of course, CP violation may emerge in interactions of the Higgs fields with the other fields in the theory due to the CP-violating phases carried by these latter fields. 


\section{Top dipole moments}

\subsection{Theoretical expectations}

A non-vanishing value for the EDM of a fermion is of special interest as it signifies the presence of CP-violating interactions. We recall that the search for the EDM of the neutron and that of the electron have intensified in recent years. Since the top is such an unusual fermion, in fact so heavy that it is very unlikely to exist as a bound state with another quark, it is clearly important to ask: What is its EDM? How can we measure it, if at all? This topic has been of interest to many for the past several years.

In the SM quarks cannot have an EDM at least to three loops [135]. For the electron the three loop contribution has been estimated to be $d_{e}^{\gamma}(0) \sim 10^{-37}$ e-cm. Simple dimensional scaling then suggest for the top the value $d_{t}^{\gamma}(0) \sim 10^{-31}-10^{-32}$ e-cm, much too small to be observable.

In contrast, in extensions of the SM, e.g., MHDM's and SUSY models, this situation changes sharply and the Top Dipole Moment (TDM) f.P can arise at the 1-loop level and as a result, the typical TDM is of the order of $10^{-18}-10^{-20} \mathrm{e}-\mathrm{cm}$ which is larger than the SM prediction by more than 10 orders of magnitude. The enhancement due to the large top mass is particularly evident in some models with an extended Higgs sector for which the dipole moments often scale as $m_{f}^{3}$. Since at 1-loop light quarks (or neutron) in these models can get dipole moment of order $10^{-26}$ e-cm, the TDM could easily reach $10^{-20} \mathrm{e}-\mathrm{cm}$ or even more. It is at that level that measurable consequences can arise.

Because of the unique importance of the top quark it should be clear that measurements of the TDM will be extremely important. However, it should also be clear that due to the extraordinary short life-time of the top quark $\left(\lesssim 10^{-24} \mathrm{sec}\right)$ it will be extremely difficult to actually measure the static (i.e., at $q^{2}=0$ ) TDM. Measurements of some of the effects driven by the presence of a dipole moment form factor may have a better chance. In fact, the TDM may be considered as a CP-odd form factor in the $\gamma t \bar{t}, Z t \bar{t}$ or $g t \bar{t}$ vertex that probes the interactions of a short-lived top quark with an off-shell $\gamma, Z$ or a gluon, respectively, and can be represented by

$$
\Gamma_{\mu}^{\gamma, Z, g}=i d_{t}^{\gamma, Z, g}(s) \bar{u}_{t}\left(p_{t}\right) \sigma_{\mu \nu} \gamma_{5} p^{\nu} v_{\bar{t}}\left(p_{\bar{t}}\right)
$$

where $s=q^{2}, q=p_{t}+p_{\bar{t}}$ and color indices for $\Gamma_{\mu}^{g}$ were suppressed. Therefore, depending on the masses in the loops, the TDM form-factor can also develop an imaginary part (contrary to the static EDM of the electron or the quark) if the energy of the off-shell $\gamma, Z$ or gluon is sufficient for the particles in the loop to be on-shell, i.e., there is an absorptive cut.

Since the dipole moment characterizes the effective coupling between the spin of the fermion and the external gauge field, to extract the dipole moment one needs information on the spin polarization of the top quark. Fortunately, the left handed nature of the weak decays of the top allows us to determine its polarization quite readily. As discussed in section 2.8, top quark decays can analyze the initial polarization of the top quark. For instance in the leptonic decay $t \rightarrow e^{+} \nu_{e} b$, in the rest frame of the top quark the top is $100 \%$ polarized in the direction of the $e^{+}$momentum. This greatly simplifies calculation of the top quark production followed by its subsequent decay. The problem is then essentially reduced to calculating the production of a polarized top quark. It is then straightforward to fold in the decay to the spin indices of the top quark. A serious limitation to be kept in mind about this procedure is that it is only valid when the decays are governed by the SM, since they assume the helicity structure of the SM. If non-standard interactions make large contributions to the decay, the decay distributions may be

\footnotetext{
${ }^{9}$ Strictly speaking, the term dipole moment refers to the static form factor (i.e., at $q^{2}=0$ ). Here we will mostly concern ourselves with the dipole moment form factor at $q^{2} \neq 0$; for simplicity, we will still use the term TDM throughout.

${ }^{10}$ We will use the abbreviation TDM in general for $d_{t}^{\gamma, Z, g}$ denoting the top quark EDM, weak-EDM (ZEDM) and Chromo-EDM (CEDM), unless we need to explicitly separate the type.
} 
modified to the point that the polarimetry we have discussed is only approximate. This point must be borne in mind when considering the effects of new physics.

A detailed discussion of the feasibility of extracting the TDM in future collider experiments such as $e^{+} e^{-}, p p \rightarrow t \bar{t}$, will be given in subsequent sections. In this section we consider the contribution to the TDM which arises in extended Higgs sectors and SUSY models.

\subsection{Arbitrary number of Higgs doublets and a CP-violating neutral Higgs sector}

It is instructive to calculate the TDM in models with an arbitrary number of Higgs doublets and singlets satisfying NFC (natural flavor conservation) constraints. CP violation arises as a result of scalar exchanges between quarks, being driven by the imaginary parts of the complex quantities (e.g., $\widetilde{Z}_{1 n}$ ) defined as [77]:

$$
\begin{aligned}
\frac{1}{\left(v_{1}\right)^{2}}\left\langle\phi_{1}^{0} \phi_{1}^{0}\right\rangle_{q} & \equiv \sum_{n} \frac{\sqrt{2} G_{F} \widetilde{Z}_{1 n}}{q^{2}-m_{H_{n}}^{2}}, \\
\frac{1}{\left(v_{1} v_{2}^{*}\right)^{2}}\left\langle\phi_{2}^{+} \phi_{1}^{*}+\right\rangle_{q} & \equiv \sum_{n} \frac{\sqrt{2} G_{F} Z_{n}}{q^{2}-m_{H_{n}^{\prime}}^{2}}
\end{aligned}
$$

where $v_{1}$ and $v_{2}$ are the VEV's of the neutral Higgs fields $\phi_{1}^{0}, \phi_{2}^{0}$ and the summation runs over all the mass eigenstates of neutral or charged scalars in the theory $\left(H_{n}\right.$ or $H_{n}^{\prime}$, respectively). Also, $\langle\chi \eta\rangle_{q}$ stands, for any pair of scalar fields, $\chi$ and $\eta$, for the momentum dependent quantity

$$
\langle\chi \eta\rangle_{q}=\int d^{4} x\langle 0|T[\chi(x) \eta(0)]| 0\rangle e^{-i q x} .
$$

$\mathrm{CP}$ violation in the neutral Higgs sector then generates the dominant 1-loop contribution to the EDM of the top, i.e., $d_{t}^{\gamma}$, through the 1-loop graph with the external photon line in Fig. 10(a) (for this discussion, $h \equiv H_{n}$ in Fig. 10(a)). This contribution is given by

$$
\begin{aligned}
d_{t}^{\gamma}(0) & =\frac{2 \sqrt{2}}{3(4 \pi)^{2}} G_{F} m_{t} e \sum_{n} \Im \mathrm{m} \widetilde{Z}_{1 n} f\left[\frac{m_{H_{n}}^{2}}{m_{t}^{2}}\right] \\
& =\left(1.4 \times 10^{-21} \mathrm{e}-\mathrm{cm}\right)\left(\frac{m_{t}}{\mathrm{GeV}}\right) \sum_{n} \Im \mathrm{m} \widetilde{Z}_{1 n} f\left[\frac{m_{H_{n}}^{2}}{m_{t}^{2}}\right],
\end{aligned}
$$

where

$$
f(r)=\left\{\begin{array}{l}
1-\frac{r}{2} \ln r+\frac{r^{2}-2 r}{\sqrt{r(4-r)}}\left[\arctan \left(\frac{2-r}{\sqrt{r(4-r)}}\right)+\arctan \left(\frac{r}{\sqrt{r(4-r)}}\right)\right], \text { if } r<4 \\
3-4 \ln 2, \text { if } r=4 \\
1-\frac{r}{2} \ln r-\frac{r^{2}-2 r}{\sqrt{r(r-4)}} \ln \left[\frac{\sqrt{r}-\sqrt{r-4}}{2}\right], \text { if } r>4
\end{array}\right.
$$

and $r=m_{H_{n}}^{2} / m_{t}^{2}$ for any value of $n$. For $r>>4, f(r)$ approaches $\frac{1}{r}\left(\ln r-\frac{3}{2}\right)$ asymptotically. The $\widetilde{Z}_{1 n}$ 's satisfy some important sum rules, for example [77]:

$$
\sum_{n} \Im \mathrm{m} \widetilde{Z}_{1 n}=0
$$

so that $d_{t}^{\gamma}$ will vanish if all the neutral Higgs-bosons were degenerate; no such degeneracy is of course expected. For illustrative purposes let us assume that the lightest neutral Higgs-boson, with mass $m_{h}$, dominates the sum in Eq. 4.5. Taking $m_{h}=100 \mathrm{GeV}$ and $m_{h}=2 m_{t}\left(m_{t}=175\right.$ 
$\mathrm{GeV}$ ) and setting $\Im \mathrm{m} Z$ 's to be of order unity, then $d_{t}^{\gamma}$ is about $1.3 \times 10^{-19} \mathrm{e}-\mathrm{cm}$ and $5.6 \times 10^{-20}$ e-cm, respectively. We note that for $m_{h}>m_{t}, d_{t}^{\gamma}(0)$ varies slowly with $m_{h}$ [18].

For experimental purposes the top EDM at high $q^{2}$ may be more relevant. This is given by 18:

$$
d_{t}^{\gamma}\left(q^{2}\right)=\frac{2 \sqrt{2}}{3(4 \pi)^{2}} G_{F} m_{t} e \sum_{n} \Im \mathrm{m} \widetilde{Z}_{1 n} f\left[\frac{m_{H_{n}}^{2}}{m_{t}^{2}}, \frac{q^{2}}{m_{t}^{2}}\right]
$$

where

$$
f(r, s)=\int_{0}^{1} d x \int_{0}^{1-x} d y \frac{x+y}{(x+y)^{2}+(1-x-y) r-x y s-i \epsilon} .
$$

For $q^{2}>4 m_{t}^{2}, d_{t}^{\gamma}\left(q^{2}\right)$ develops an imaginary part. In the following section we will present explicit numerical results for the real and the imaginary parts of $d_{t}^{\gamma}\left(q^{2}\right)$ and $d_{t}^{Z}\left(q^{2}\right)$ in a 2HDM with CP violation in neutral Higgs exchanges. In this case, the top CEDM is immediately obtained by replacing the photon with a gluon in Fig. 10(a) and, therefore, $\frac{2}{3} e$ with $g_{s}$ (the QCD coupling constant) in Eqs. 4.5 and 4.8. So, $d_{t}^{g} \sim 1.5 d_{t}^{\gamma}$ where $d_{t}^{\gamma}$ is in e-cm and $d_{t}^{g}$ in $g_{s}$-cm. In theories with such a Higgs sector many CP violation effects are driven by the top CEDM. The Schmidt-Peskin energy asymmetry in $p p \rightarrow t \bar{t}+X$ followed by top decay is one such interesting effect [20], and we will discuss it in detail in Chapter 0 . 


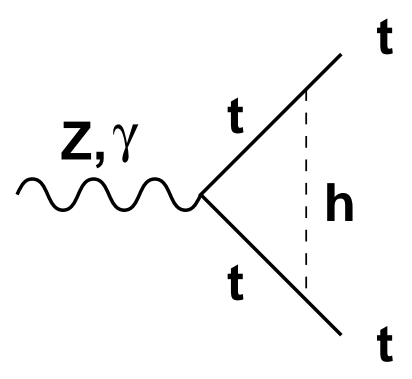

(a)

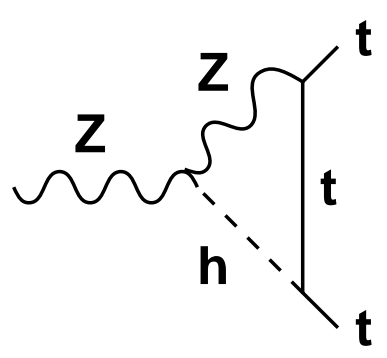

(b)

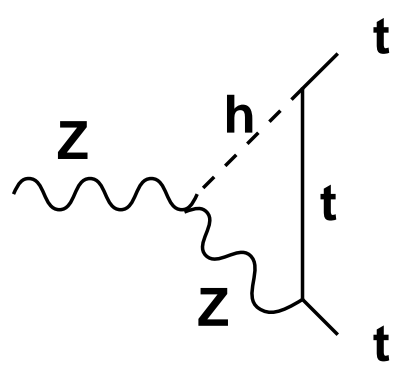

(c)

Figure 10: Feynman diagrams that contribute to the electric and weak top dipole moments in a two Higgs doublets model with CP-violating interaction of a neutral Higgs (h) with a top quark. 


\subsection{Expectations from 2HDM's with CP violation in the neutral Higgs sector}

The general analysis given in the previous section holds, of course, for any number of Higgs doublets. However, let us now focus on the simplest extension of the Higgs sector. That is, a 2HDM with $\mathrm{CP}$ violation in the neutral Higgs sector driven by a phase in the Higgs-fermionfermion interaction [17, 80]. The example that we will explicitly consider here is the type II 2HDM; however, the analysis can also be applied to type I and III models with simple redefinitions of the couplings (see also section 3.2). In this model the dipole moment form factors for the top quark start to contribute at 1-loop order via the Feynman diagrams in Fig. 10. The required CP-odd phase is provided by the $\mathcal{H}^{k} t \bar{t}$ Lagrangian piece in Eq. 3.70, where $\mathcal{H}^{k}$, for $k=1,2,3$, stands for the three neutral Higgs particles in the model. The couplings $a_{t}^{k}, b_{t}^{k}$ in Eq. 3.70 depend on the three Euler angles, i.e., $\alpha_{1,2,3}$, which parameterize the neutral Higgs mixing matrix and on $\tan \beta$ which is the ratio between the two VEV's, $v_{1}$ and $v_{2}$, corresponding to the two Higgs doublets of the model (for more details see section 3.2).

The top EDM and ZEDM within this class of 2HDM's was considered in [17, 19]. They can be written as

$$
\begin{aligned}
& d_{t}^{\gamma}(s)=\sum_{k=1}^{3} d_{k}^{\gamma}(s) g_{k}, \\
& d_{t}^{Z}(s)=\frac{3 g_{V}^{t}}{4 \sin \theta_{W} \cos \theta_{W}} d_{t}^{\gamma}(s)+\sum_{k=1}^{3} d_{t, k}^{\prime Z}(s) g_{k}^{\prime},
\end{aligned}
$$

where $g_{V}^{t}=1 / 2-4 \sin ^{2} \theta_{W} / 3$ and in Model II

$$
\begin{aligned}
& g_{k}=a_{t}^{k} b_{t}^{k}=R_{1 k} R_{3 k} \cot \beta / \sin \beta, \\
& g_{k}^{\prime}=b_{t}^{k} c^{k}=R_{3 k}\left(R_{2 k} \cos \beta+R_{1 k} \sin \beta\right) \cot \beta .
\end{aligned}
$$

The $3 \times 3$ neutral Higgs mixing mass matrix $R$ is given in Eq. 3.73. $c^{k}$ in Eq. 4.13 is the coupling constant associated with the $Z Z \mathcal{H}^{k}$ vertex, see Eqs. 3.71 and 3.72 .

From Fig. 10(a),(b) and (c) one can extract the functions $d_{k}^{\gamma}$ and $d_{t, k}^{\prime Z}$

$$
\begin{aligned}
d_{k}^{\gamma}(s) & =-\frac{e Q_{t} \sqrt{2} G_{F} m_{t}^{3}}{4 \pi^{2}} \times \frac{C_{11}^{a}}{2}, \\
d_{t, k}^{\prime Z}(s) & =\frac{e g_{V}^{t}}{8 \pi^{2} \sin \theta_{W} \cos \theta_{W}} \sqrt{2} G_{F} m_{Z}^{2} m_{t} \times \frac{C_{12}^{b}+C_{11}^{c}-C_{12}^{c}}{2},
\end{aligned}
$$

where $Q_{t}=2 / 3$. The three-point loop form factors $C_{x}^{i}, x \in\{11,12\}$ and $i=a, b, c$ corresponding to diagrams (a),(b),(c) in Fig. 10, are given in our notation by

$$
\begin{aligned}
C_{x}^{a} & =C_{x}\left(m_{\mathcal{H}^{k}}^{2}, m_{t}^{2}, m_{t}^{2}, m_{t}^{2}, s, m_{t}^{2}\right), \\
C_{x}^{b} & =C_{x}\left(m_{t}^{2}, m_{\mathcal{H}^{k}}^{2}, m_{Z}^{2}, m_{t}^{2}, s, m_{t}^{2}\right), \\
C_{x}^{c} & =C_{x}\left(m_{t}^{2}, m_{Z}^{2}, m_{\mathcal{H}^{k}}^{2}, m_{t}^{2}, s, m_{t}^{2}\right),
\end{aligned}
$$

and $C_{x}\left(m_{1}^{2}, m_{2}^{2}, m_{3}^{2}, p_{1}^{2}, p_{2}^{2}, p_{3}^{2}\right)$ is defined in Appendix A. Analytical expressions for the imaginary parts of the three-point loop form factors, $C_{x}^{i}$, may be derived through the Cutkosky rule [17]: 


$$
\begin{aligned}
\Im m G_{k}(s) \equiv & \Im \mathrm{m}\left(\frac{C_{11}^{a}}{2}\right)=\pi \frac{\Theta\left(s-4 m_{t}^{2}\right)}{m_{\mathcal{H}^{k}} \sqrt{s}}\left[Z_{k}^{-3} \ln \left(1+Z_{k}^{2}\right)-Z_{k}^{-1}\right], \\
\Im \mathrm{m} D_{k}(s) \equiv & \Im \mathrm{m}\left(\frac{C_{12}^{b}+C_{11}^{c}-C_{12}^{c}}{2}\right)=\pi \Theta\left[s-\left(m_{Z}+m_{\mathcal{H}^{k}}\right)^{2}\right] \\
& \times\left\{A_{k}(s)+\left[\frac{4 m_{t}^{2}-m_{Z}^{2}-m_{\mathcal{H}^{k}}^{2}}{2\left(4 m_{t}^{2}-s\right)}+\frac{m_{\mathcal{H}^{k}}^{2}-m_{Z}^{2}}{2 s}\right] B_{k}(s)\right\},
\end{aligned}
$$

where

$$
\begin{aligned}
& Z_{k}=\sqrt{\left(s-4 m_{t}^{2}\right) / m_{\mathcal{H}^{k}}^{2}}, \\
& A_{k}(s)=\frac{\sqrt{w_{k}}}{s\left(4 m_{t}^{2}-s\right)}
\end{aligned}
$$

and

$$
\left.\begin{array}{rlrl}
B_{k}(s) & =-a^{-1 / 2} \ln \left|\frac{b_{k}+\sqrt{a w_{k}}}{b_{k}-\sqrt{a w_{k}}}\right| & & \text {, for } a>0 \\
& =-2 \sqrt{w_{k}} / b_{k} & , \text { for } a=0 \\
& =-2(-a)^{-1 / 2} \arctan \left(\sqrt{-a w_{k}} / b_{k}\right) & , \text { for } a<0
\end{array}\right\} .
$$

also

$$
\begin{aligned}
& a=s\left(s-4 m_{t}^{2}\right), \\
& b_{k}=s\left[s-\left(m_{Z}^{2}+m_{\mathcal{H}^{k}}^{2}\right)\right], \\
& w_{k}=\left[s-\left(m_{Z}+m_{\mathcal{H}^{k}}\right)^{2}\right]\left[s-\left(m_{Z}-m_{\mathcal{H}^{k}}\right)^{2}\right] .
\end{aligned}
$$

The real parts are obtained from a dispersion relation

$$
\begin{aligned}
\Re \mathrm{e} G_{k}(s) & =\frac{1}{\pi} \mathcal{P} \int_{4 m_{t}^{2}}^{\infty} d s^{\prime} \frac{\Im \mathrm{m} G_{k}\left(s^{\prime}\right)}{s^{\prime}-s} . \\
\Re \mathrm{e} D_{k}(s) & =\frac{1}{\pi} \mathcal{P} \int_{\left(m_{Z}+m_{\mathcal{H}^{k}}\right)^{2}}^{\infty} d s^{\prime} \frac{\Im \mathrm{m} D_{k}\left(s^{\prime}\right)}{s^{\prime}-s} .
\end{aligned}
$$

Let us now assume again that the masses of the other two neutral Higgs particles are considerably larger then the lightest one and, therefore, the lightest neutral Higgs dominates the sums in Eqs. 4.10 and 4.11. Recall that the CP-violating effects would vanish if the Higgs were degenerate, i.e., $d_{t}^{\gamma}=d_{t}^{Z}=0$, due to the orthogonality properties of the neutral Higgs mixing matrix. With no loss of generality we denote the lightest neutral Higgs by $h$ with couplings $g_{1}, g_{1}^{\prime}$, corresponding to $k=1$ in Eqs. 4.12 and 4.13. Also, we scale out the couplings $g_{1}, g_{1}^{\prime}$ and plot in Figs. 11(a),(b) and 12(a),(b) the real and imaginary parts of $d_{t}^{\gamma}$ and $d_{t}^{\prime Z}$ (recall that $d_{t}^{\prime Z}$ is the contribution to the ZEDM which arises through diagrams (b) and (c) in Fig. 10) for a variety of Higgs masses, $m_{h}=100,200,300 \mathrm{GeV}$ and $m_{t}=175 \mathrm{GeV}$.

We can see from Figs. 11(a),(b) that $\Re \mathrm{e}\left(d_{t}^{\gamma}\right)$ and $\Im \mathrm{m}\left(d_{t}^{\gamma}\right)$ are typically $\sim 10^{-19}-10^{-18}$ e-cm for $m_{h}=100-300 \mathrm{GeV}$. The peak in the threshold region, shown in Fig. 11(b), originates from a Coulomb-like singularity present in diagram (a) of Fig. 10. This is more pronounced, of course, for light Higgs masses. Note that the contribution from the diagram in Fig. 10(a) to $\Re$ e $\left(d_{t}^{Z}\right)$ and $\Im \mathrm{m}\left(d_{t}^{Z}\right)$ is smaller by a factor of $3 g_{V}^{t} / 4 \sin \theta_{W} \cos \theta_{W} \simeq 0.35$ than the contribution to $\Re \mathrm{e}\left(d_{t}^{\gamma}\right)$ and $\Im \mathrm{m}\left(d_{t}^{\gamma}\right)$ as can be seen from Eq. 4.11. 
As is evident from Figs. 11 and 12, $\Re \mathrm{e}\left(d_{t}^{\prime Z}\right)$ and $\Im \mathrm{m}\left(d_{t}^{\prime Z}\right)$ are typically about one order of magnitude smaller than $\Re \mathrm{e}\left(d_{t}^{\gamma}\right)$ and $\Im \mathrm{m}\left(d_{t}^{\gamma}\right)$, respectively. Clearly, disregarding the 1-loop form factors, this difference is in part due to the different couplings in Eqs. 4.14 and 4.15. Thus one finds

$$
\frac{d_{t}^{\gamma}}{d_{t}^{\prime Z}} \approx \frac{2 Q_{t} \sin \theta_{W} \cos \theta_{W}}{g_{V}^{t}} \times \frac{m_{t}^{2}}{m_{Z}^{2}} \simeq 10.7
$$

Moreover, the difference between the contributions from Fig. 10(a) and Figs. 10(b) and (c) becomes even more pronounced once the $2 \mathrm{HDM}$ couplings $g_{1}$ and $g_{1}^{\prime}$ are included. In particular, from Eqs. 4.12 and 4.13 one finds that, for $\tan \beta<1, g_{1} \propto 1 / \tan ^{2} \beta$ and $g_{1}^{\prime} \propto 1 / \tan \beta$. Thus, as we will show below, the ratio $g_{1} / g_{1}^{\prime}$ may even become as large as $\sim 10$ for $\tan \beta \lesssim 0.5$.

In Figs. 13(a) and (b) we show the dependence of the real and imaginary parts of $d_{t}^{\gamma}$ and $d_{t}^{\prime Z}$ on the mass of the lightest Higgs-boson $m_{h}$. Evidently, the dependence of $d_{t}^{\prime Z}$ on $m_{h}$ is rather insignificant, while $d_{t}^{\gamma}$ drops as $m_{h}$ increases.

The EDM's of the neutron and electron do not constrain the neutral Higgs mixing matrix in any significant way. Thus, as already mentioned above, its matrix elements, which enters $g_{1}$ and $g_{1}^{\prime}$ in Eqs. 4.12 and 4.13 could be of $\mathcal{O}(1)$. Furthermore, for some versions of 2HDM, $\tan \beta<1$ is a viable alternative. In this case $\left|g_{1}\right|,\left|g_{1}^{\prime}\right|$ can even become larger than one, further enhancing the dipole form factors. Let us now include the factors $g_{1}$ and $g_{1}^{\prime}$ in calculating the top's EDM and ZEDM. For illustration, we choose three sets for the two Euler angles $\left\{\alpha_{1}, \alpha_{2}\right\}$ and take $\tan \beta=0.3$. Set I: $\left\{\alpha_{1}, \alpha_{2}\right\}=\{\pi / 4, \pi / 2\}$, Set II: $\left\{\alpha_{1}, \alpha_{2}\right\}=\{\pi / 4,3 \pi / 4\}$ and Set III: $\left\{\alpha_{1}, \alpha_{2}\right\}=\{\pi / 4, \pi / 4\}$. Note that in Set I $g_{1}$ is maximized, in Set II $g_{1}^{\prime}>1$ and in Set III $g_{1}$ and $g_{1}^{\prime}$ have opposite relative signs such that the contributions of $d_{t}^{\prime Z}$ and $d_{t}^{\gamma}$ to $d_{t}^{Z}$ add. In particular, in terms of $g_{1}$ and $g_{1}^{\prime}$ we have

$$
\begin{aligned}
& \text { Set I : } \quad g_{1} \simeq 5.8, g_{1}^{\prime} \simeq 0.48, \\
& \text { Set II : } \quad g_{1} \simeq 4.1, g_{1}^{\prime} \simeq 1.14, \\
& \text { Set III : } \quad g_{1} \simeq 4.1, g_{1}^{\prime} \simeq-0.46 .
\end{aligned}
$$

In Table 2 we give the real and imaginary parts of $d_{t}^{\gamma}$ and $d_{t}^{Z}$ (the total ZEDM including diagrams (a),(b) and (c) in Fig. 10). For illustration, we present numbers for $m_{h}=100,200,300 \mathrm{GeV}$ and $\sqrt{s}=500,1000 \mathrm{GeV}$. We see from Table 2 that $d_{t}^{\gamma}$ ranges from a few $\times 10^{-19}$ e-cm to a few $\times 10^{-18}$ e-cm. Also, as expected, $d_{t}^{Z}$ is typically smaller by about a factor of $\sim 3-4$. The imaginary parts tend to be bigger by factors ranging from $2-10$ for $\sqrt{s}=1000,500 \mathrm{GeV}$ respectively. In passing we also note that the weak dipole moment form factors obtained here are about an order of magnitude bigger than that found in [18]. Note that, here also, the top CEDM is immediately obtained by replacing the photon with a gluon and, therefore, $\frac{2}{3} e$ with $g_{s}$.

\footnotetext{
${ }^{11}$ note that in the parameterization of Eq. 3.73 in section $3.2 .3, g_{1}$ and $g_{1}^{\prime}$ are insensitive to $\alpha_{3}$ and it is sufficient to consider only $\alpha_{1}$ and $\alpha_{2}$
} 
Table 2: Real and Imaginary parts of $d_{t}^{\gamma}$ and $d_{t}^{Z}$ in units of $10^{-19} e-\mathrm{cm}$, for $m_{h}=100,200$ and $300 \mathrm{GeV}$ and for $\sqrt{s}=500 \mathrm{GeV}$ and $\sqrt{s}=1 \mathrm{TeV}$ (in parenthesis). $\tan \beta=0.3$ and Set I,II,III means $\left\{\alpha_{1}, \alpha_{2}\right\}=\{\pi / 4, \pi / 2\},\{\pi / 4,3 \pi / 4\},\{\pi / 4, \pi / 4\}$, respectively.

\begin{tabular}{|r||r|r|r|r|}
\hline \multicolumn{2}{|c||}{$\begin{array}{r}\text { Type of moment } \\
\left(10^{-19} \mathrm{e}-\mathrm{cm}\right) \Downarrow\end{array}$} & $m_{h}$ & \multicolumn{3}{|c|}{ The different Sets of $\left\{\alpha_{1}, \alpha_{2}\right\}, \tan \beta=0.3$} \\
\cline { 3 - 5 } & $(\mathrm{GeV}) \Downarrow$ & Set I & Set II & Set III \\
\hline \hline \multirow{3}{*}{$\Re \mathrm{e}\left(d_{t}^{\gamma}\right)$} & 100 & $1.97(3.77)$ & $1.40(2.66)$ & $1.40(2.66)$ \\
\cline { 2 - 5 } & 200 & $-3.36(2.26)$ & $-2.38(1.60)$ & $-2.38(1.60)$ \\
\cline { 2 - 5 } & 300 & $-4.75(1.27)$ & $-3.36(0.90)$ & $-3.36(0.90)$ \\
\hline \hline \multirow{3}{*}{$\Im \mathrm{m}\left(d_{t}^{\gamma}\right)$} & 100 & $-23.89(-5.44)$ & $-16.88(-3.84)$ & $-16.88(-3.84)$ \\
\cline { 2 - 5 } & 200 & $-16.56(-4.91)$ & $-11.70(-3.47)$ & $-11.70(-3.47)$ \\
\cline { 2 - 5 } & 300 & $-11.34(-4.33)$ & $-8.02(-3.06)$ & $-8.02(-3.06)$ \\
\hline \hline \multirow{3}{*}{$\Re \mathrm{e}\left(d_{t}^{Z}\right)$} & 100 & $0.62(1.25)$ & $0.36(0.83)$ & $0.52(0.93)$ \\
\cline { 2 - 5 } & 200 & $-1.17(0.74)$ & $-0.87(0.47)$ & $-0.78(0.57)$ \\
\cline { 2 - 5 } & 300 & $-1.57(0.40)$ & $-1.04(0.24)$ & $-1.18(0.33)$ \\
\hline \hline & 100 & $-7.96(-1.81)$ & $-5.41(-1.21)$ & $-5.85(-1.34)$ \\
\cline { 2 - 5 } & 200 & $-5.45(-1.62)$ & $-3.58(-1.08)$ & $-4.12(-1.22)$ \\
\cline { 2 - 5 } & 300 & $-3.64(-1.42)$ & $-2.22(-0.93)$ & $-2.91(-1.08)$ \\
\hline
\end{tabular}



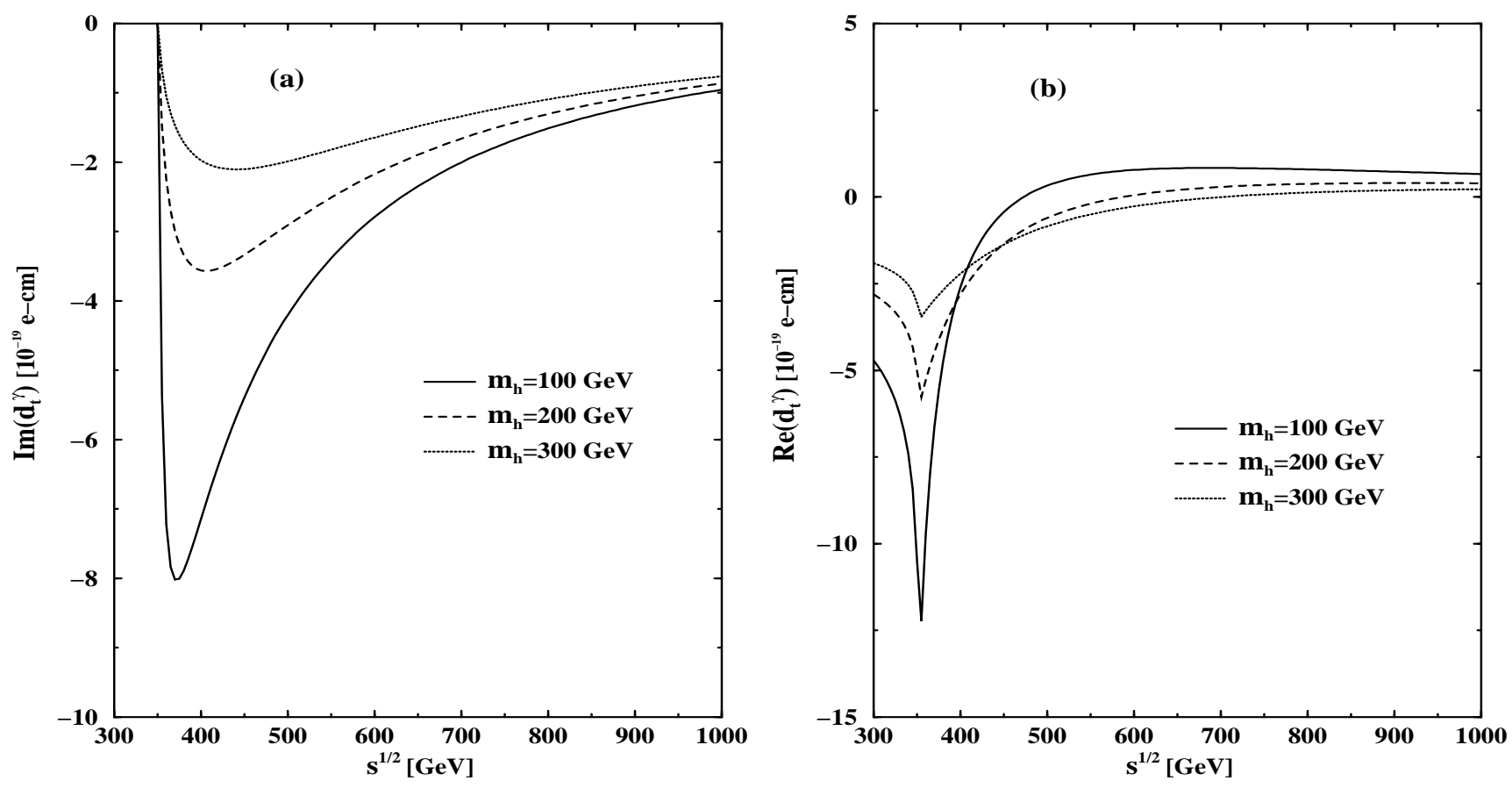

Figure 11: Imaginary (a) and Real (b) parts of $d_{t}^{\gamma}$ in units of $10^{-19}$ e-cm as a function of $\sqrt{s \text {, }}$ for various masses of the lightest neutral Higgs (h) and for $m_{t}=175 \mathrm{GeV}$. 

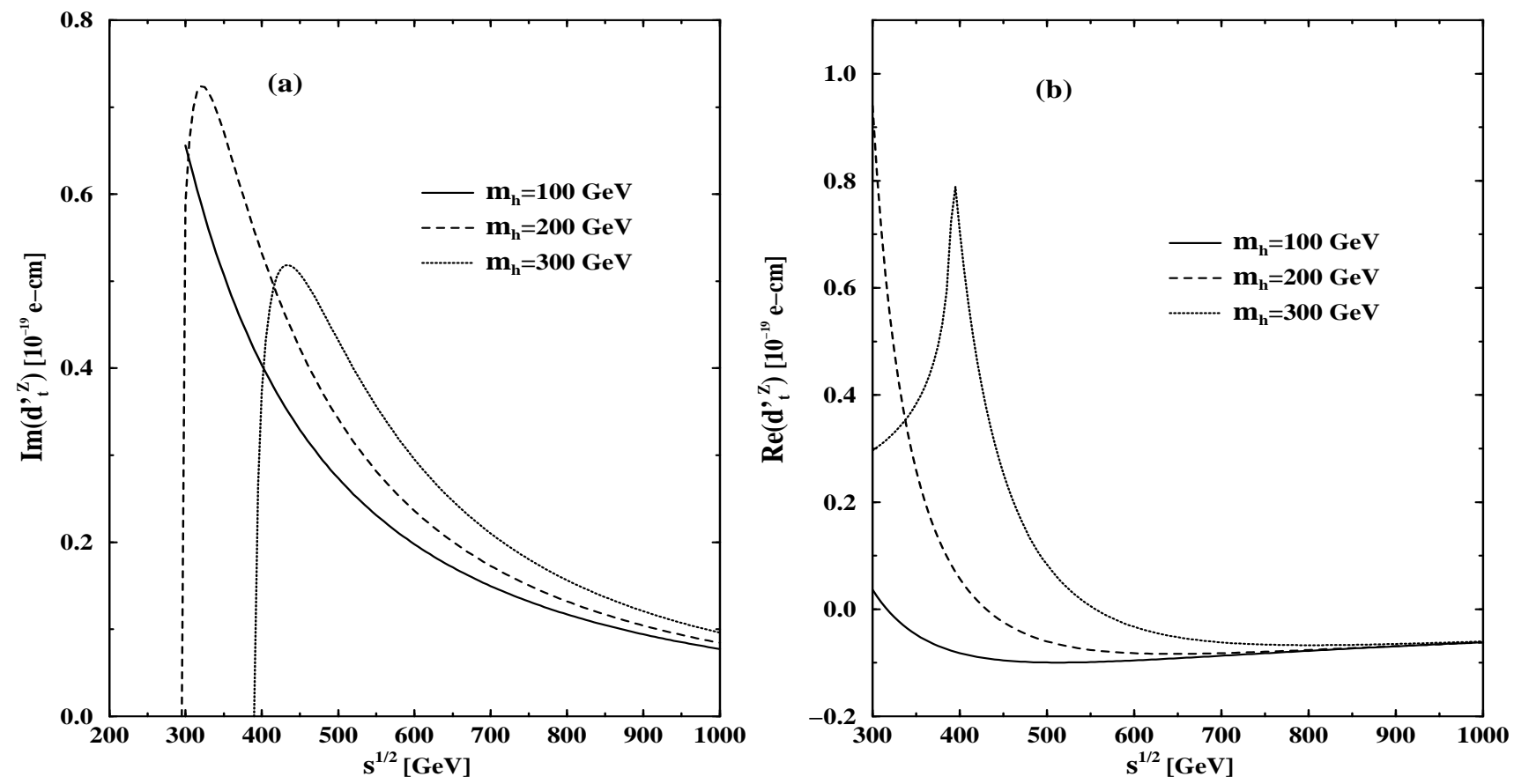

Figure 12: Imaginary (a) and Real (b) parts of $d_{t}^{\prime Z}$ in units of $10^{-19}$ e-cm as a function of $\sqrt{s \text {, }}$ for various masses of the lightest neutral Higgs (h) and for $m_{t}=175 \mathrm{GeV}$. 

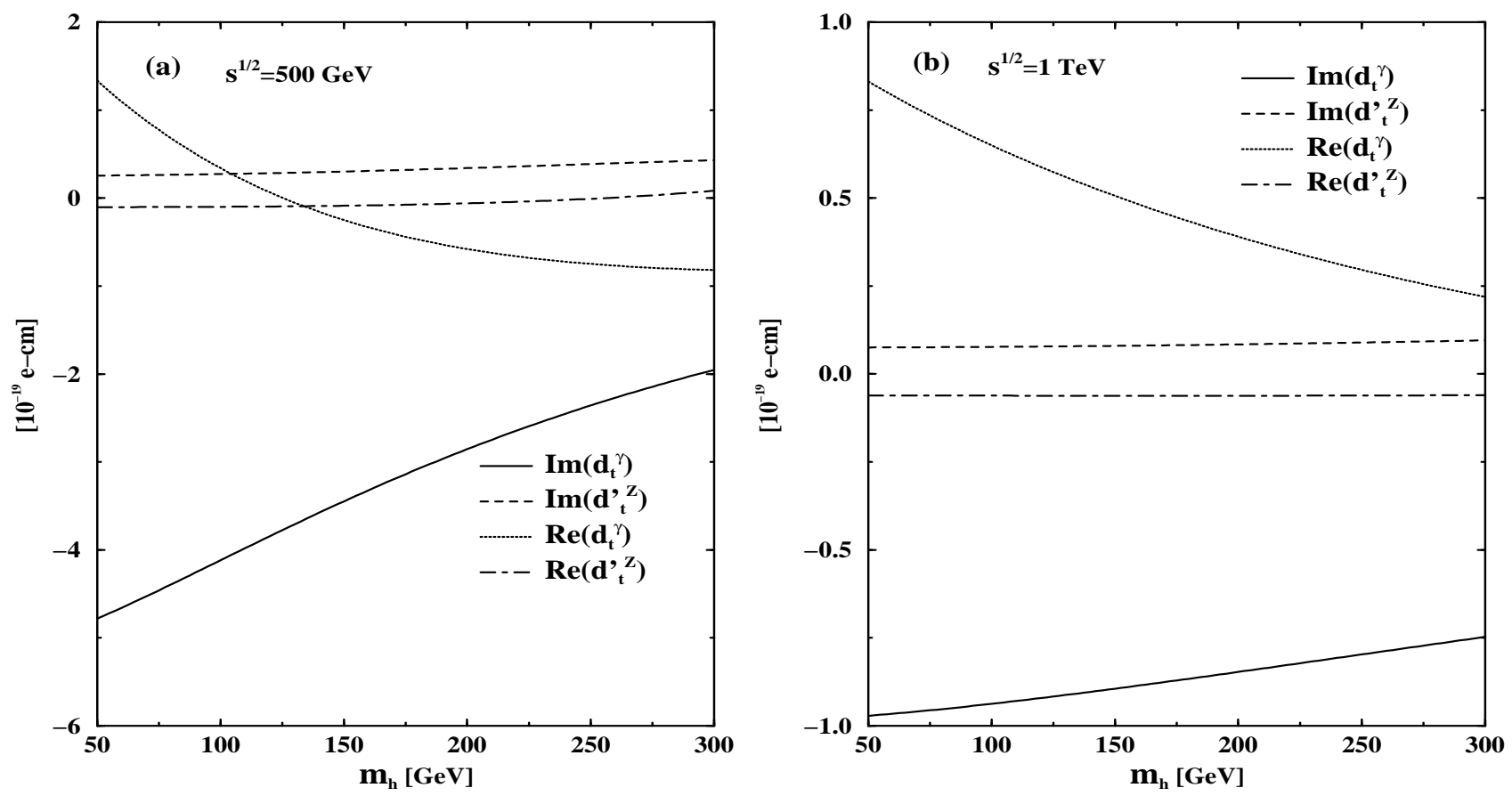

Figure 13: Imaginary and Real parts of $d_{t}^{\gamma}$ and $d_{t}^{\prime Z}$ in units of $10^{-19} \mathrm{e}-\mathrm{cm}$ as a function of $m_{h}$, for (a) $\sqrt{s}=500 \mathrm{GeV}$ and (b) $\sqrt{s}=1 \mathrm{TeV}$ and for $m_{t}=175 \mathrm{GeV}$.

\subsection{Expectations from a CP-violating charged Higgs sector}

In models with three or more Higgs doublets [45, 110], it is also possible to have CP violation in the charged Higgs sector. In this case a top EDM, ZEDM and CEDM receives contributions from diagrams in Figs. 14(a) and 14(b) with $q^{\prime}=\{d, s, b\}$. In the case of the 3 Higgs doublet model as considered for example in section 3.2.4 we may express the coupling of the lighter charged Higgs-bosons, say $\mathrm{H}_{2}^{+} \equiv H^{+}$, to the third generation of quarks as (see also Eq. 3.88 for our notation):

$$
\mathcal{L}_{t b H^{+}}=\frac{g_{W}}{\sqrt{2} m_{W}} K_{t b}\left(m_{t} \beta_{2} \bar{t}_{R} b_{L}+m_{b} \alpha_{2} \bar{t}_{L} b_{R}\right)+\text { h.c , . }
$$

where $K$ is the CKM matrix (we will assume that $K_{t b} \approx 1$ ); $\beta_{2}$ and $\alpha_{2}$ are complex parameters of the model (for a more complete description of these parameters see section 3.2.4). Also, we neglect contributions from the $H^{+} t d$ and $H^{+} t s$ couplings as those will yield a TDM smaller by a factor of $\sim\left(m_{d} / m_{b}\right)^{2}$ and $\sim\left(m_{s} / m_{b}\right)^{2}$, respectively, compared to the TDM coming from the $H^{+} t b$ Lagrangian piece.

The CP violation in this case is proportional to the quantity $\Im \mathrm{m}(V)$ where $V \equiv\left(\beta_{2} \alpha_{2}^{*}\right)$. We denote the coupling of a vector-boson to the $b$-quark by

$$
-i \gamma^{\mu}\left(A_{V}^{b}+B_{V}^{b} \gamma^{5}\right)
$$

and the coupling of a vector-boson to the pair of charged Higgs by

$$
i C_{V}^{H}\left(p_{H+}-p_{H-}\right)^{\mu},
$$

where $p_{H \pm}$ are the in-going momenta of the charged Higgs-bosons. 
In [141] we have calculated the contribution of the charged Higgs exchanges in Figs. 14(a) and 14(b) to the $b$-quark dipole moment. The top EDM, ZEDM or CEDM given by these diagrams may, therefore, be extracted from [141] by the replacement $m_{b} \leftrightarrow m_{t}$ and are thus given by

$$
d_{t}^{\gamma, Z, g}=-\frac{\alpha}{4 \pi \sin ^{2} \theta_{W}} \Im \mathrm{m}(V) \frac{m_{b}^{2}}{m_{W}^{2}} m_{t}\left[A_{\gamma, Z, g}^{b} C_{12}^{a}-C_{\gamma, Z, g}^{H}\left(C_{12}^{b}+\frac{1}{2} C_{0}^{b}\right)\right],
$$

where $C_{x}^{i}, x \in\{0,12\}$ and $i=a, b$, are the three-point loop form factors corresponding to diagrams (a),(b) in Fig. 14 such that

$$
\begin{aligned}
& C_{x}^{a}=C_{x}\left(m_{H^{+}}^{2}, m_{b}^{2}, m_{b}^{2}, m_{t}^{2}, s, m_{t}^{2}\right), \\
& C_{x}^{b}=C_{x}\left(m_{b}^{2}, m_{H^{+}}^{2}, m_{H^{+}}^{2}, m_{t}^{2}, s, m_{t}^{2}\right) .
\end{aligned}
$$

Here $s=\left(p_{t}+p_{\bar{t}}\right)^{2}$ and $C_{x}$ is defined in Appendix A. Also

$$
\begin{aligned}
& A_{\gamma}^{b}=-\frac{1}{3} e, C_{\gamma}^{H}=e, \\
& A_{Z}^{b}=\frac{e}{2 \sin \theta_{W} \cos \theta_{W}}\left(-\frac{1}{2}+\frac{2}{3} \sin ^{2} \theta_{W}\right), C_{Z}^{H}=e \cot 2 \theta_{W}, \\
& A_{g}=g_{s}, C_{g}^{H}=0,
\end{aligned}
$$

where $e$ is the electric charge, $g_{s}$ is the strong coupling constant and $\theta_{W}$ is the weak mixing angle. Note that in the case of the gluon, i.e., the CEDM, only Fig. 14(a) enters as the $g H^{+} H^{-}$coupling is absent.

Using $m_{H^{+}}=200 \mathrm{GeV}$ (also $m_{t} \simeq 175 \mathrm{GeV}$ ) and denoting $d_{t}^{\gamma, Z, g} \equiv \Im \mathrm{m}(V) \delta_{t}^{\gamma, Z, g}(s)$ we find that for $500 \mathrm{GeV}<\sqrt{s}<1000 \mathrm{GeV}$

$$
\Im \mathrm{m} \delta_{t}^{\gamma, Z, g}, \Re \mathrm{e} \delta_{t}^{\gamma, Z, g} \lesssim \text { few } \times 10^{-22} e-\mathrm{cm}, g_{s}-\mathrm{cm}
$$

where, in fact, for $\sqrt{s}=1000 \mathrm{GeV}, \Re \mathrm{e} \delta_{t}^{\gamma, Z} \lesssim 10^{-23} e-\mathrm{cm}$. Moreover, as $m_{H^{+}}$is increased the TDM drops rapidly and, for example, for $m_{H^{+}}=500 \mathrm{GeV}$, we find that, typically, $\Im \mathrm{m} \delta_{t}^{\gamma, Z, g}$ and $\Re \mathrm{e} \delta_{t}^{\gamma, Z, g}$ are smaller than $\sim 10^{-22} \mathrm{e}-\mathrm{cm}$ or $g_{s}-\mathrm{cm}$. Such small TDM, residing in charged Higgs exchanges, is expected simply by comparing $\delta_{t}^{\gamma, Z, g}$ above to the corresponding terms for neutral Higgs exchanges which were given in the previous sections. In particular, one can immediately observe that the contribution to the TDM from charged Higgs exchanges is naively suppressed by a factor of $\sim\left(m_{b} / m_{t}\right)^{2}$ with respect to the neutral Higgs exchanges wherein the TDM was found to be typically at the order of $\sim 10^{-19} \mathrm{e}-\mathrm{cm}$.

Let us briefly consider what constraints can be placed on the parameter, $\Im \mathrm{m}(V)$, by use of the experimental results on the EDM of the neutron (NEDM) and on the decay $b \rightarrow s \gamma$. For our discussion we will simply use the bound given in 124, on the NEDM

$$
d_{n}^{e} \lesssim 10^{-25} \mathrm{e}-\mathrm{cm}
$$

Using our previous results given in Eq. 4.34 one can deduce an expression for the electric dipole moment of the light $(u, d)$-quarks in the charged Higgs model (see [141]). Making the simplifying assumption that the NEDM equals that of its valence quarks one finds (as has been noted before 45]) that there is significant uncertainty from the numerical value of the mass of the light quark to be used in the above formula. For the purpose of obtaining an upper bound on $\Im \mathrm{m}(V)$ we can take the current mass to be $\sim 10 \mathrm{MeV}$. Then for $m_{t}=180 \mathrm{GeV}, m_{H^{+}}$in the range 200-500 $\mathrm{GeV}$, we find from Eq. 4.40 and using Eq. 4.34 for the light $u, d$-quarks that $\Im \mathrm{m}(V) \lesssim 10$ [141]. The experimental data on the decay $b \rightarrow s \gamma$ can also constrain this parameter. In particular, it 
was shown in 88, 113 that, for $m_{H^{+}}=200-500 \mathrm{GeV}$ and neglecting the effects of the second charged Higgs of the model, a conservative upper bound of

$$
\Im \mathrm{m}(V) \lesssim 3-9
$$

can be placed from the CLEO measurement of the decay rate of $b \rightarrow s \gamma$ (see also discussion in section 3.2.4). ${ }^{12}$ It turns out, however, that even when using $\Im \mathrm{m}(V)=10$, in conjunction with numerical values for $\delta_{t}^{\gamma, Z, g}\left(q^{2}\right)$ as given before, the TDM is expected to be $\lesssim 10^{-21}$ e-cm in this class of charged Higgs mediated CP violation. Thus, it is typically smaller by at least an order of magnitude than what one would expect from CP-violating neutral Higgs exchanges and from the MSSM (see the following section).

Finally, it should be noted that in any given MHDM with new mechanisms of CP violation in the charged Higgs sector, i.e., three and more Higgs doublets, the neutral Higgs sector will also acquire new CP-violating phases which in general cannot be screened (see section 3.2.4). Therefore, as was already mentioned before, in the top quark case, CP-violating neutral Higgs exchanges dominate the charged Higgs by a typical factor of $\sim\left(m_{t} / m_{b}\right)^{2}$. One power of $\left(m_{t} / m_{b}\right)$ originates from the ratio between the neutral and charged Higgs couplings to fermions and another power of $\left(m_{t} / m_{b}\right)$ comes in from the necessary mass insertion in the propagator of the fermion in the loop. Thus, the charged Higgs contribution is negligible compared to one from a neutral Higgs. Note, however, that in light quark systems charged Higgs exchanges are expected to yield the dominant CP-violating effects since the above argument is basically reversed [141].

\footnotetext{
${ }^{12}$ We note, however, that the limit on $\Im \mathrm{m}(V)$ gets weaker as the masses of the two charged Higgs approach the same value, due to a GIM-like cancellation of the CP-violating effect mediated by the two. For example, if $m_{H^{+}}=350 \mathrm{GeV}$ and the mass of the second charged Higgs is $\sim 500 \mathrm{GeV}$, then $\Im \mathrm{m}(V) \gtrsim 10$ does not contradict the upper bounds on $b \rightarrow s \gamma$, and, of course, no such limit exist at all if the two charged Higgs are degenerate 88].
} 


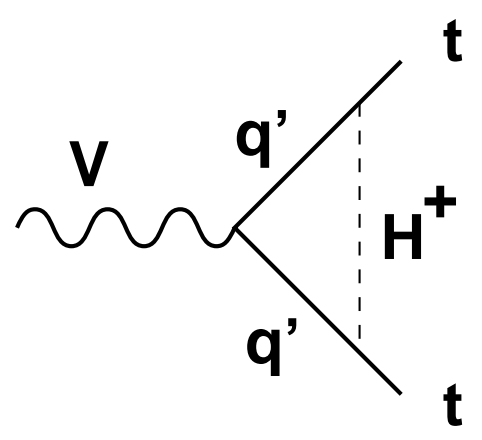

(a)

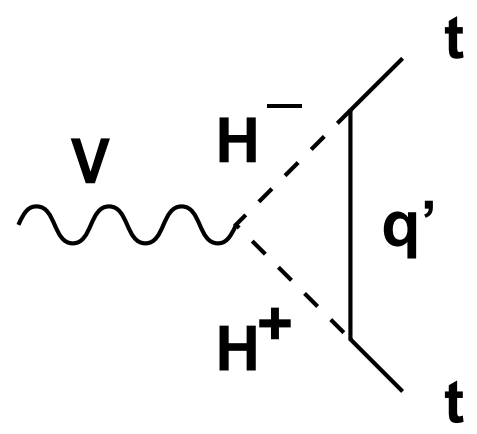

(b)

Figure 14: Feynman diagrams that contribute to the electric, weak and chromo-electric top dipole moments due to CP-violating interactions of a charged Higgs with a top quark. $V=\gamma, Z$ (also $V=$ gluon in diagram (a)) and $q^{\prime}$ stands for $d, s$ or b-quark. 


\subsection{Expectations from the MSSM}

Within the MSSM the TDM can also arise already at 1-loop even without generation mixing. As was mentioned in section 3.3, in general, the required CP-violating phases are provided by the chargino and neutralino mixing matrices as well as the squarks $\tilde{q}_{L}-\tilde{q}_{R}$ mixing matrices. If one assumes GUT-scale unification which leads to a common gaugino mass at the GUT-scale, then the phase in the gaugino mass term can be rotated away, leaving the gaugino masses phaseless at any scale. Thus one is left with only three phases (neglecting generation mixing between the squarks) relevant for the TDM at the EW-scale; $\alpha_{\mu}, \alpha_{t}$ and $\alpha_{b}$ which arise from the Higgs mass term $\mu$ and the stop and sbottom mixing matrices, respectively (for the definitions of $\alpha_{\mu}, \alpha_{t}$ and $\alpha_{b}$ see Eq. 3.123 in section 3.3.2). We recall (see Eqs. 3.122 and 3.125 in section 3.3.2) that, for a sfermion $\tilde{f}$, it is useful to adopt a parameterization for its $f_{L}-f_{R}$ mixing such that the sfermions of different handedness are related to their mass eigenstates through the transformation

$$
\begin{aligned}
& \tilde{f}_{L}=\cos \theta_{f} \tilde{f}_{1}-e^{-i \beta_{f}} \sin \theta_{f} \tilde{f}_{2} \\
& \tilde{f}_{R}=e^{i \beta_{f}} \sin \theta_{f} \tilde{f}_{1}+\cos \theta_{f} \tilde{f}_{2},
\end{aligned}
$$

where $\tilde{f}_{1,2}$ are the two mass eigenstates (i.e. physical states) and the phase $\beta_{f}$ is related to the phase $\alpha_{f}$ by Eq. 3.123. The contribution to the CP-violating TDM which arise from the above $\tilde{f}_{L}-\tilde{f}_{R}$ mixing matrix will always be proportional to the quantity (see also Eq. 3.126 )

$$
\xi_{C P}^{f} \equiv 2\left|\xi_{f}^{i}\right|=\sin 2 \theta_{f} \sin \beta_{f}
$$

Clearly, from Eq. 4.42 we see that $\xi_{C P}^{f} \rightarrow 0$ if the two sfermions are nearly degenerate.

In the MSSM the TDM can therefore acquire a non-vanishing value through the Feynman diagrams depicted in Fig. 15. One can then distinguish between the following three contributions:

1. Gluino contribution, $d_{t(\tilde{g})}^{\gamma, Z}$, with $\tilde{t} \tilde{t}^{*} \tilde{g}$ in the loop (see Fig. 15(b)).

2. Chargino contribution, $d_{t\left(\tilde{\chi}^{+}\right)}^{\gamma, Z}$, with $\tilde{\chi}^{+} \tilde{\chi}^{-} \tilde{b}$ (see Fig. 15(a)) and $\tilde{b}^{*} \tilde{\chi}^{+}$in the loop (see Fig. 15(b)).

3. Neutralino contribution, $d_{t\left(\tilde{\chi}^{0}\right)}^{\gamma, Z}$, with $\tilde{\chi}^{0} \tilde{\chi}^{0} \tilde{t}$ (see Fig. 15(a)) and $\tilde{t}^{*} \tilde{\chi}^{0}$ in the loop (see Fig. 15(b)).

The gluino contribution was considered in [142, 143, 144, 145]. It is

$$
\begin{aligned}
& d_{t(\tilde{g})}^{\gamma}= \frac{\alpha_{s}}{3 \pi} Q_{t} \xi_{C P}^{t} m_{\tilde{g}} \times\left(C_{0}^{11}+C_{11}^{11}-C_{0}^{22}-C_{11}^{22}\right), \\
& d_{t(\tilde{g})=}^{Z}=\frac{\alpha_{s}}{6 \pi \sin \theta_{W} \cos \theta_{W}} \xi_{C P}^{t} m_{\tilde{g}} \times \\
&\left\{\left(\cos ^{2} \theta_{t}-\frac{4}{3} \sin ^{2} \theta_{W}\right)\left(C_{0}^{11}+C_{11}^{11}-C_{0}^{21}-C_{11}^{21}\right)+\right. \\
&\left.\left(\sin ^{2} \theta_{t}-\frac{4}{3} \sin ^{2} \theta_{W}\right)\left(C_{0}^{12}+C_{11}^{12}-C_{0}^{22}-C_{11}^{22}\right)\right\},
\end{aligned}
$$

where the three-point 1-loop form factors, $C_{x}^{i j}, x \in\{0,11\}$ and $i, j=1,2$, are given by:

$$
C_{x}^{i j}=C_{x}\left(m_{\tilde{g}}^{2}, m_{\tilde{t}_{i}}^{2}, m_{\tilde{t}_{j}}^{2}, m_{t}^{2}, s, m_{t}^{2}\right)
$$

and $C_{x}$ is defined in Appendix A. Also, $\theta_{t}$ and $\xi_{C P}^{t}$ are defined in Eqs. 4.42 and 4.43 , respectively. 
The chargino contribution was given in 146]:

$$
\begin{aligned}
d_{t\left(\tilde{\chi}^{+}\right)}^{\gamma}= & -\frac{\alpha Y_{t}}{4 \pi \sin ^{2} \theta_{W}} \times \\
& \sum_{m, j=1}^{2} m_{\tilde{\chi}_{j}^{+}}\left(C_{11}^{m, j j}-C_{12}^{m, j j}-\frac{1}{6}\left(C_{0}^{j, m m}+C_{11}^{j, m m}\right)\right) \times \\
& \quad\left\{\left(1-(-)^{m} \cos 2 \theta_{b}\right) \Im \mathrm{m}\left[U_{j 1} V_{j 2}\right]+\right. \\
& \left.\quad Y_{b}(-)^{m} \sin 2 \theta_{b} \Im \mathrm{m}\left[U_{j 2} V_{j 2} e^{i \beta_{b}}\right]\right\}, \\
d_{t\left(\tilde{\chi}^{+}\right)}^{Z}= & \frac{\alpha}{16 \pi \sin ^{3} \theta_{W} \cos \theta_{W}} \times\left(f_{1}^{+}+f_{2}^{+}+s_{1}^{+}+s_{2}^{+}\right),
\end{aligned}
$$

where

$$
\begin{aligned}
f_{1}^{+}=- & Y_{t} \sum_{m, j, k=1}^{2} m_{\tilde{\chi}_{j}^{+}}\left(C_{11}^{m, j k}-C_{12}^{m, j k}\right) \times \\
& \left\{\left(\delta_{j k}\left(1+2 \cos 2 \theta_{W}\right)+\left|U_{k 1}\right|^{2}-\left|V_{k 2}\right|^{2}\right) \times\right. \\
& \left(\left(1-(-)^{m} \cos 2 \theta_{b}\right) \Im \mathrm{m}\left[U_{j 1} V_{j 2}\right]+Y_{b}(-)^{m} \sin 2 \theta_{b} \Im \mathrm{m}\left[U_{j 2} V_{j 2} e^{i \beta_{b}}\right]\right) \\
& \left.-Y_{b}\left(1-\delta_{j k}\right)(-)^{m} \sin 2 \theta_{b} \Im \mathrm{m}\left[U_{j 2} V_{j 2} e^{i \beta_{b}}\right]\right\}, \\
f_{2}^{+}=\quad- & m_{t} Y_{b} \sin 2 \theta_{b} \Im \mathrm{m}\left[U_{11}^{*} U_{12} e^{i \beta_{b}}\right] \times \\
& \sum_{m=1}^{2}(-)^{m}\left(C_{11}^{m, 12}+C_{21}^{m, 12}-2 C_{12}^{m, 12}-2 C_{23}^{m, 12}\right), \\
s_{1}^{+}=- & Y_{t} \sum_{k, m, n=1}^{2} m_{\tilde{\chi}_{k}^{+}}\left(C_{0}^{k, m n}+C_{11}^{k, m n}\right) \times \\
& \left(\frac{2}{3} \sin ^{2} \theta_{W} \delta_{m n}+\frac{1}{2}\left(1-(-)^{n} \cos 2 \theta_{b}\right)\right) \times \\
& \left\{\left(1-(-)^{m} \cos 2 \theta_{b}\right) \Im \mathrm{m}\left[U_{k 1} V_{k 2}\right]+\right. \\
& \left.Y_{b}(-)^{m} \sin 2 \theta_{b} \Im \mathrm{m}\left[U_{k 2} V_{k 2} e^{i \beta_{b}}\right]\right\}, \\
s_{2}^{+}=- & m_{t} Y_{b} \sin _{2} \theta_{b} \sum_{k=1}^{2}\left(C_{11}^{k, 12}+C_{21}^{k, 12}-2 C_{12}^{k, 12}-2 C_{23}^{k, 12}\right) \times \\
& \Im \mathrm{m}\left[U_{k 1}^{*} U_{k 2} e^{i \beta_{b}}\right],
\end{aligned}
$$

where $m, n$ are sbottom indices and $j, k$ are the chargino indices. Thus, the above three-point 1-loop form factors $C_{x}^{\ell, m n}$ and $C_{x}^{m, j k}$ for $m, n=1,2$ and $\ell=j$ or $k=1,2, x \in\{0,11,12,21,23\}$, are given by

$$
\begin{aligned}
& C_{x}^{\ell, m n}=C_{x}\left(m_{\tilde{\chi}_{\ell}^{+}}^{2}, m_{\tilde{b}_{m}}^{2}, m_{\tilde{b}_{n}}^{2}, m_{t}^{2}, s, m_{t}^{2}\right), \\
& C_{x}^{m, j k}=C_{x}\left(m_{\tilde{b}_{m}}^{2}, m_{\tilde{\chi}_{j}^{+}}^{2}, m_{\tilde{\chi}_{k}^{+}}^{2}, m_{t}^{2}, s, m_{t}^{2}\right),
\end{aligned}
$$

and $C_{x}$ is defined in Appendix A. Also, $Y_{t}$ and $Y_{b}$ are the top and bottom Yukawa couplings

$$
Y_{t}=\frac{m_{t}}{\sqrt{2} m_{W} \sin \beta} \quad, \quad Y_{b}=\frac{m_{b}}{\sqrt{2} m_{W} \cos \beta},
$$

and, as usual, $\tan \beta$ is the ratio between the VEV's of the two Higgs doublets in the theory. Furthermore, note that the phase $\alpha_{\mu}$, although not explicitly appearing in the above, is contained 
in $U$ and $V$ which are the $2 \times 2$ matrices that diagonalize the chargino mass matrix and, in the notation used in section 3.3.2, we have $U^{*} \equiv\left(Z^{-}\right)^{\dagger}$ and $V^{*} \equiv Z^{+}$. The definitions of $Z^{+}$and $Z^{-}$ are given in section 3.3 .2 by Eqs. 3.127 - 3.134 .

The neutralino contribution was also given in [146]:

$$
\begin{aligned}
d_{t\left(\tilde{\chi}^{0}\right)}^{\gamma}= & \frac{\alpha}{12 \pi \sin ^{2} \theta_{W}} \sum_{k=1}^{4} \sum_{m=1}^{2} m_{\tilde{\chi}_{k}^{0}}\left(C_{0}^{k, m m}+C_{11}^{k, m m}\right) \times \\
& \left\{( - ) ^ { m } \operatorname { s i n } 2 \theta _ { t } \Im \mathrm { m } \left[\left(h_{L k}^{2}-f_{L k} f_{R k}^{*}\right) e^{\left.-i \beta_{t}\right]}\right.\right. \\
& -\left(1-(-)^{m} \cos 2 \theta_{t}\right) \Im \mathrm{m}\left[h_{L k} f_{L k}^{*}\right]- \\
d_{t\left(\tilde{\chi}^{0}\right)=}^{Z}= & \frac{\left.\left(1+(-)^{m} \cos 2 \theta_{t}\right) \Im \mathrm{m}\left[h_{L k} f_{R k}\right]\right\},}{16 \pi \sin ^{3} \theta_{W} \cos \theta_{W}} \times\left(2 f_{1}^{0}+2 f_{2}^{0}+s_{1}^{0}+s_{2}^{0}\right),
\end{aligned}
$$

where

$$
\begin{aligned}
& f_{1}^{0}=\frac{1}{2} \sum_{j, k=1}^{4} \sum_{m=1}^{2} m_{\tilde{\chi}_{j}^{0}}\left(C_{11}^{m, j k}-C_{11}^{m, j k}\right) \times \\
& \left\{(-)^{m} \sin 2 \theta_{t} \Im \mathrm{m}\left[O_{j k}^{\prime \prime}\left(f_{L j} f_{R k}^{*}-f_{L k} f_{R j}^{*}\right) e^{-i \beta_{t}}\right]\right. \\
& -\left(1+(-)^{m} \cos 2 \theta_{t}\right) \Im \mathrm{m}\left[O_{j k}^{\prime \prime}\left(h_{L j}^{*} f_{R k}^{*}-h_{L k}^{*} f_{R j}^{*}\right)\right] \\
& \left.-\left(1-(-)^{m} \cos 2 \theta_{t}\right) \Im \mathrm{m}\left[O_{j k}^{\prime \prime}\left(h_{L j} f_{L k}^{*}-h_{L k} f_{L j}^{*}\right)\right]\right\} \text {, } \\
& f_{2}^{0}=\frac{1}{2} m_{t} \sum_{j<k}^{4} \sum_{m=1}^{2}\left(C_{11}^{m, j k}+C_{21}^{m, j k}-2 C_{12}^{m, j k}-2 C_{23}^{m, j k}\right) \times \\
& \left\{2(-)^{m} \cos 2 \theta_{t} \Im \mathrm{m}\left[h_{L j} O_{j k}^{\prime \prime} h_{L k}^{*}\right]\right. \\
& -2(-)^{m} \sin 2 \theta_{t} \Im \mathrm{m}\left[\left(f_{L j}^{*}-f_{R j}\right) O_{j k}^{\prime \prime} h_{L k}^{*} e^{i \beta_{t}}\right] \\
& +\left(1-(-)^{m} \cos 2 \theta_{t}\right) \Im \mathrm{m}\left[f_{L j}^{*} O_{j k}^{\prime \prime} f_{L k}\right] \\
& \left.+\left(1+(-)^{m} \cos 2 \theta_{t}\right) \Im \mathrm{m}\left[f_{R j} O_{j k}^{\prime \prime} f_{R k}^{*}\right]\right\} \text {, } \\
& s_{1}^{0}=\frac{1}{2} \sum_{k=1}^{4} \sum_{m, n=1}^{2} m_{\tilde{\chi}_{k}^{0}}\left(C_{0}^{k, m n}+C_{11}^{k, m n}\right) \times \\
& \left\{(-)^{m}\left(\frac{8}{3} \sin ^{2} \theta_{W} \delta_{m n}-\left(1-(-)^{n} \cos 2 \theta_{t}\right)\right) \Im \mathrm{m}\left[\left(h_{L k}^{2}-f_{L k} f_{R k}^{*}\right) e^{-i \beta_{t}}\right]\right. \\
& -\left(\frac{8}{3} \sin ^{2} \theta_{W} \delta_{m n}\left(1+(-)^{m} \cos 2 \theta_{t}\right)-(-)^{m+n} \sin ^{2} 2 \theta_{t}\right) \Im \mathrm{m}\left[h_{L k} f_{R k}\right] \\
& -\left(\frac{8}{3} \sin ^{2} \theta_{W} \delta_{m n}-\left(1-(-)^{n} \cos 2 \theta_{t}\right)\right)\left(1-(-)^{m} \cos 2 \theta_{t}\right) \times \\
& \left.\Im \mathrm{m}\left[h_{L k} f_{L k}^{*}\right]\right\} \text {, } \\
& s_{2}^{0}=m_{t} \sin 2 \theta_{t} \sum_{k=1}^{4}\left(C_{11}^{k, 12}+C_{21}^{k, 12}-2 C_{12}^{k, 12}-2 C_{23}^{k, 12}\right) \times \\
& \Im \mathrm{m}\left[h_{L k}^{*}\left(f_{L k}^{*}-f_{R k}\right) e^{i \beta_{t}}\right] .
\end{aligned}
$$

For the neutralino contribution above, $m, n$ are stop indices and $j, k$ are the neutralino indices. Thus, the above three-point 1-loop form factors, $C_{x}^{\ell, m n}$ and $C_{x}^{m, j k}$ for $m, n=1,2$ and $\ell=j$ or $k=$ $1,2, x \in\{0,11,12,21,23\}$, are given by

$$
\begin{aligned}
& C_{x}^{\ell, m n}=C_{x}\left(m_{\tilde{\chi}_{\ell}^{0}}^{2}, m_{\tilde{t}_{m}}^{2}, m_{\tilde{t}_{n}}^{2}, m_{t}^{2}, s, m_{t}^{2}\right), \\
& C_{x}^{m, j k}=C_{x}\left(m_{\tilde{t}_{m}}^{2}, m_{\tilde{\chi}_{j}^{0}}^{2}, m_{\tilde{\chi}_{k}^{0}}^{2},, m_{t}^{2}, s, m_{t}^{2}\right),
\end{aligned}
$$


and $C_{x}$ is again defined in Appendix A. Also, $f_{L j}, f_{R k}$ are gaugino couplings and $h_{L j}$ are higgsino couplings that contain the large Yukawa coupling $Y_{t} . O_{j k}^{\prime \prime}$ contains elements of the neutralino mixing matrices. The factors $f_{L j}, f_{R k}, h_{L j}, O_{j k}^{\prime \prime}$ are all given in [146].

In order to be able to estimate the size of the TDM one has to choose a plausible set of the SUSY masses and parameters involved, i.e., $\tilde{m}_{2}, \mu, \tan \beta, m_{\tilde{t}_{k}}, m_{\tilde{b}_{k}}, \cos \alpha_{t}, \cos \alpha_{b}$ and the phases $\alpha_{\mu}, \beta_{t}$ and $\beta_{b}$. A reference set of parameters was chosen in [146]:

\begin{tabular}{|rcc|rrrrrr|}
\hline$m_{2}$ & $=$ & $230,360 \mathrm{GeV}$ & $m_{\tilde{t}_{1}}=$ & $150 \mathrm{GeV}$ & $m_{\tilde{b}_{1}}=270 \mathrm{GeV}$ \\
$|\mu|$ & $=$ & $250 \mathrm{GeV}$ & $m_{\tilde{t}_{2}}=$ & $400 \mathrm{GeV}$ & $m_{\tilde{b}_{2}}=280 \mathrm{GeV}$ \\
$\tan \beta$ & $=$ & 2 & $\theta_{t}=$ & $\frac{\pi}{9}$ & $\theta_{b}=\frac{\pi}{36}$ \\
$\alpha_{\mu}$ & $=$ & $\frac{4 \pi}{3}$ & $\beta_{t}=$ & $\frac{\pi}{6}$ & $\beta_{b}=$ & $\frac{\pi}{3}$ \\
\hline
\end{tabular}

Note that with a common gaugino mass at the GUT-scale all the low-energy gaugino mass parameters are related and are proportional to $\tilde{m}_{2}$ - the $\mathrm{SU}(2)$ gauginos mass term at the GUTscale. For example, $m_{\tilde{g}} \approx 3 \tilde{m}_{2}$ (for more details see section 3.3).

The real and imaginary parts of the top EDM and ZEDM, including all contributions in diagrams (a) and (b) in Fig. 15 (i.e., gluino, chargino and neutralino contributions), with the above set of the relevant SUSY parameters and as a function of the c.m. energy of the collider, $\sqrt{s}$, are given in Fig. 16. We see that, typically:

$$
\Re \mathrm{e} d_{t}^{\gamma, Z}(s), \Im \mathrm{m} d_{t}^{\gamma, Z}(s) \sim 10^{-20}-10^{-19} \mathrm{e}-\mathrm{cm} .
$$

Note that this results is about one order of magnitudes smaller than what is expected in the 2HDM discussed in section 4.3 .

Consider now the low energy MSSM scenario described in section 3.3.4. There we have taken $\alpha_{\mu} \rightarrow 0$, motivated by the experimental bound on the NEDM which strongly implies that $\alpha_{\mu}<10^{-2}-10^{-3}$. Moreover, all squarks except from the light stop were assumed to be degenerate with a mass $M_{S}$. It is instructive to evaluate the TDM in this limit in which the only relevant CP-odd phase resides in $\tilde{t}_{L}-\tilde{t}_{R}$ mixing and is proportional to $\sin \beta_{t}$ (or equivalently to $\sin \alpha_{t}$ - the phase in the top trilinear soft breaking term $\left.A_{t}\right)$. In this framework $d_{t\left(\tilde{\chi}^{+}\right)}^{\gamma, Z}=0$ since the two sbottom particles are degenerate. Moreover, $d_{t\left(\tilde{\chi}^{0}\right)}^{\gamma, Z}$ gets its contribution only from terms proportional to $\sin \beta_{t}$ in Eqs. 4.56 4.61 where, in general, one finds that $d_{t\left(\tilde{\chi}^{0}\right)}^{\gamma, Z}<d_{t(\tilde{g})}^{\gamma, Z}$. Thus, in this scenario the TDM can be approximated by considering only the gluino exchange diagram in Fig. 15(b) for which only the masses $m_{\tilde{t}_{1}}, m_{\tilde{t}_{2}}$ and $m_{\tilde{g}}$ are relevant.

In Figs. 17, 18 and 19 we have plotted the imaginary and real parts of $d_{t(\tilde{g})}^{\gamma, Z}$ in the above MSSM scenario with $\xi_{C P}^{t}=1$ and with the approximation $d_{t}^{\gamma, Z} \simeq d_{t(\tilde{g})}^{\gamma, Z}$, as a function of $\sqrt{s}, m_{\tilde{g}}$ and $m_{\tilde{t}_{1}}$ (the light stop mass), respectively. Our reference set of masses for these figures are $m_{\tilde{t}_{1}}=50$ $\mathrm{GeV}$ and $m_{\tilde{t}_{2}}=m_{\tilde{g}}=400 \mathrm{GeV}$. We again see that, typically, $\Re \mathrm{e}, \Im \mathrm{m}\left(d_{t}^{\gamma, Z}\right) \sim 10^{-20}-10^{-19}$ e-cm. From Fig. 18(a) we see that there is a small enhancement in the imaginary part of the TDM as the gluino mass gets smaller and, for example, we find $\left|\Im \mathrm{m}\left(d_{t}^{\gamma}\right)\right| \simeq 3.25 \times 10^{-19} \mathrm{e}-\mathrm{cm}$ for $m_{\tilde{g}}=200 \mathrm{GeV}$. Fig. 19(b) illustrates how the TDM vanishes when the two stop mass eigenstates are degenerate, i.e., $m_{\tilde{t}_{1}}=m_{\tilde{t}_{2}}=400 \mathrm{GeV}$.

It is important to note that within the MSSM, unlike in MHDM cases, the top CEDM cannot be calculated simply by replacing the off-shell photon with an off-shell gluon. The reason is that SUSY models give rise to an additional $g \tilde{g} \tilde{g}$ coupling (i.e., gluon-gluino-gluino coupling). Thus, in addition to replacing the photon with the gluon in Fig. 15(b), a full calculation of $d_{t}^{g}$ has to include the additional diagram with $\tilde{g} \tilde{g} \tilde{t}$ in the loop. This effect was considered in the context of $\mathrm{CP}$ violation in $p p \rightarrow t \bar{t}+X$ by Schmidt [147], and we will return to it in Chapter 7 . 


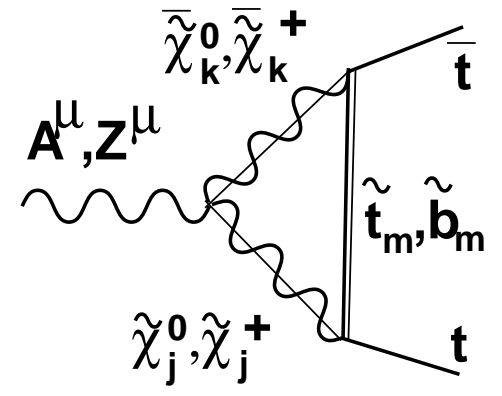

(a)

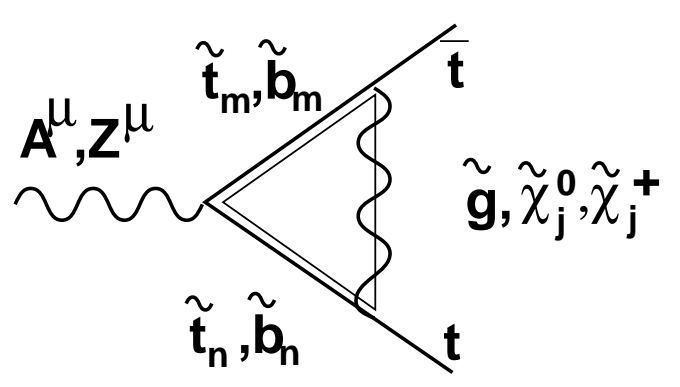

(b)

Figure 15: Feynman diagrams contributing to $d_{t}^{\gamma}$ and $d_{t}^{Z}:$ (a) with two fermions and one scalar in the loop, (b) with two scalars and one fermion in the loop. 


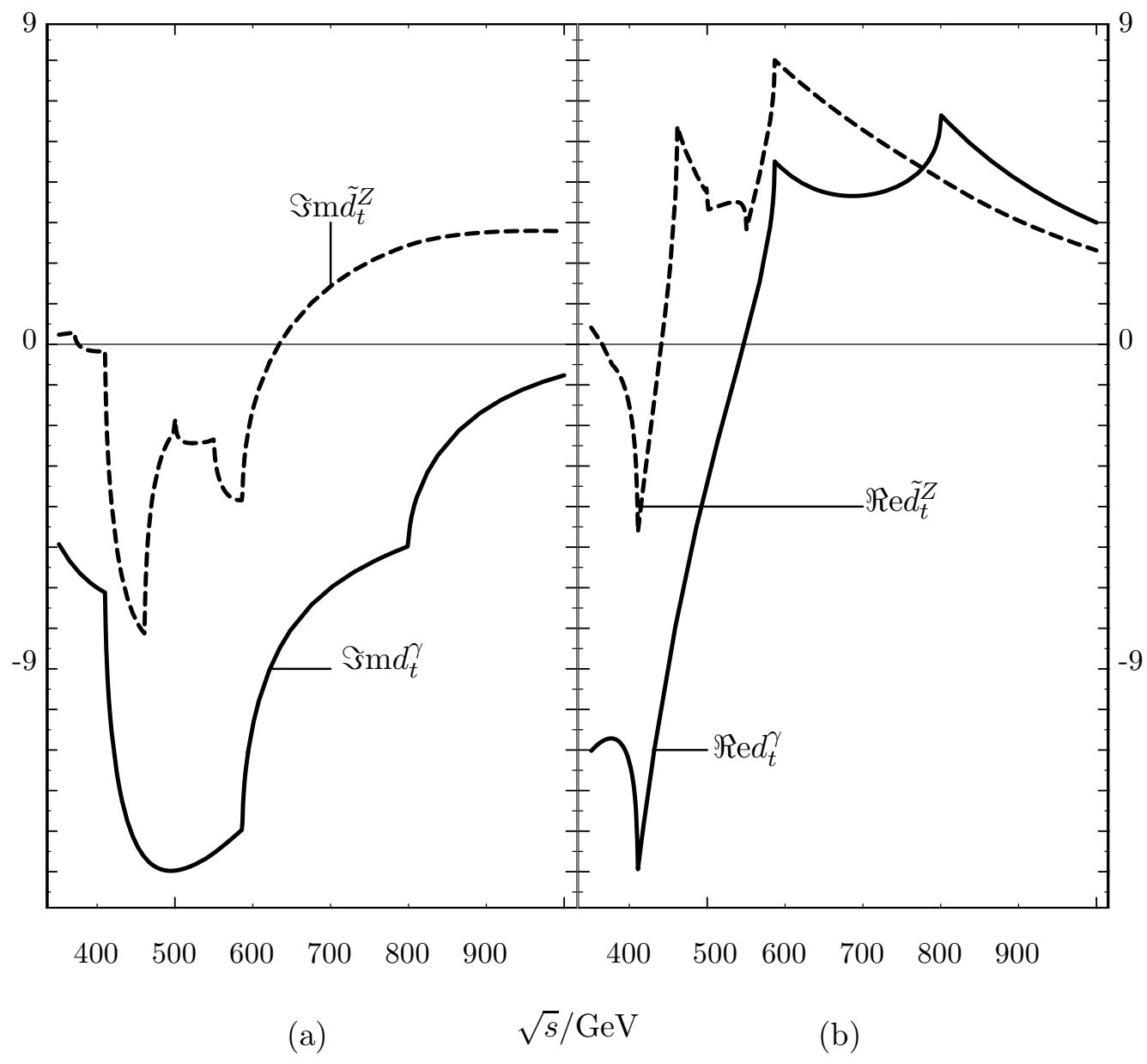

Figure 16: $d_{t}^{\gamma}(s)$ and $\tilde{d}_{t}^{Z}(s)$ (note that $d_{t}^{Z}(s) \equiv \frac{\tilde{d}_{t}^{Z}(s)}{2 \cos \theta_{W} \sin \theta_{W}}$ ) in units of $10^{-20}$ e-cm, for the reference parameter set with $\tilde{m}_{2}=230 \mathrm{GeV}$. Note that in (a) $\Im \mathrm{m} d_{t}^{\gamma}(s)$ (full line), $\Im \mathrm{m} \tilde{d}_{t}^{Z}(s)$ (dashed line), and in (b) $\Re \mathrm{e} d_{t}^{\gamma}(s)$ (full line), $\Re \mathrm{e} \tilde{d}_{t}^{Z}(s)$ (dashed line). Figure taken from [140]. 

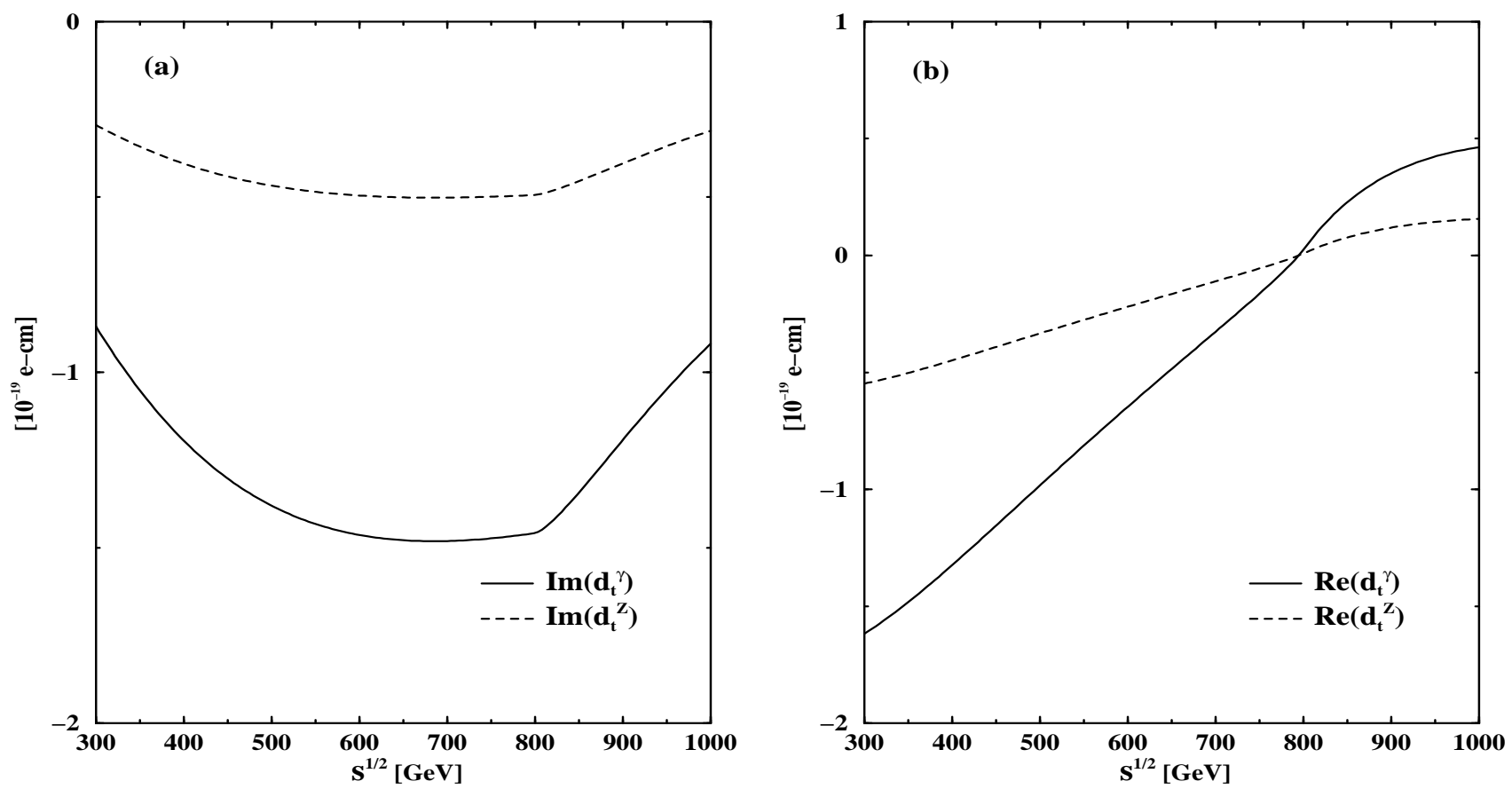

Figure 17: Imaginary (a) and Real (b) parts of $d_{t}^{\gamma}$ and $d_{t}^{Z}$, due to only the gluino exchange diagram depicted in Fig. 15(b), in units of $10^{-19}$ e-cm and as a function of $\sqrt{s}$. We use: $m_{\tilde{t}_{1}}=50$ GeV, $m_{\tilde{t}_{2}}=m_{\tilde{g}}=400 \mathrm{GeV}$ and $m_{t}=175 \mathrm{GeV}$. 

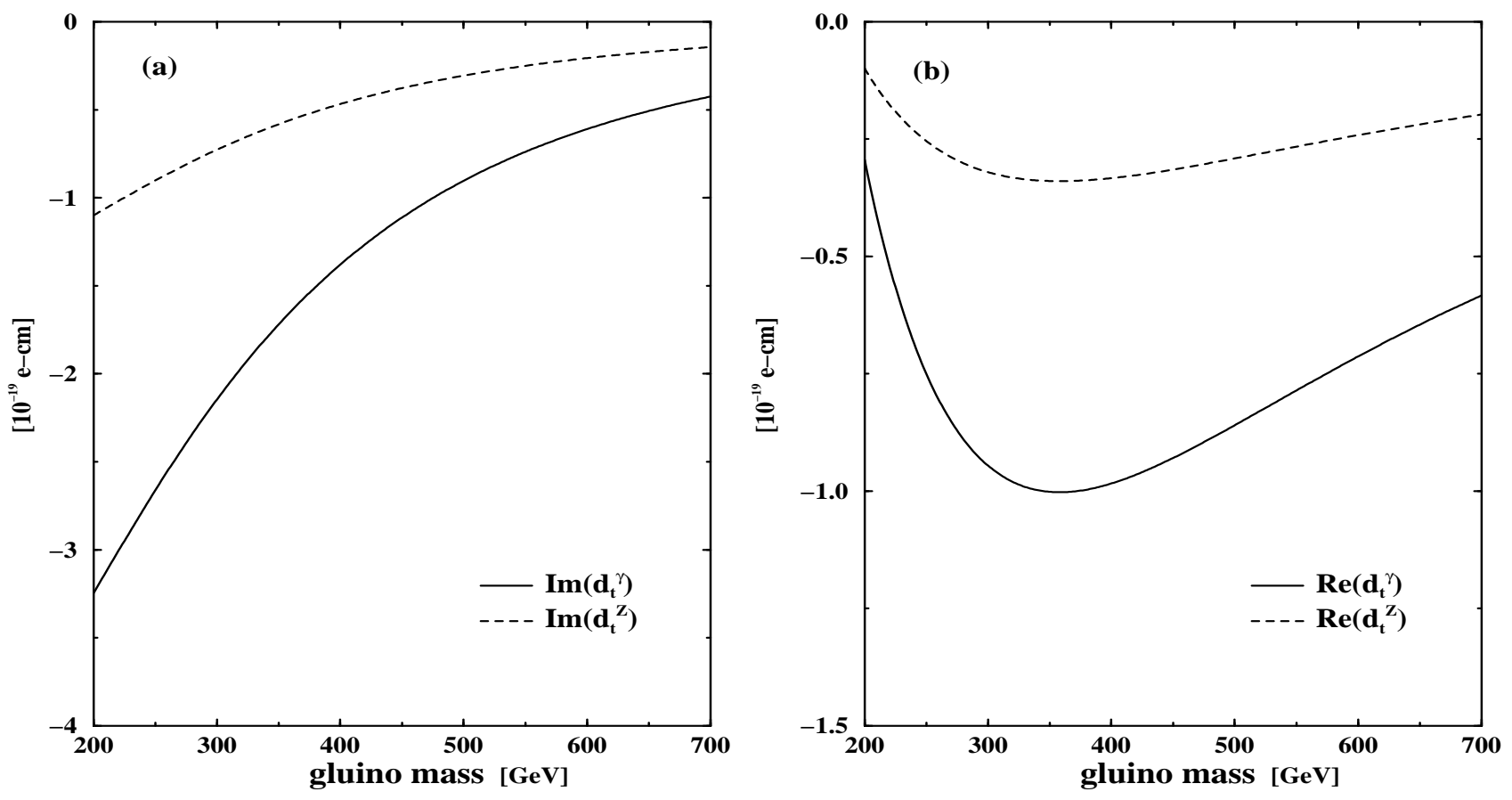

Figure 18: Imaginary (a) and Real (b) parts of $d_{t}^{\gamma}$ and $d_{t}^{Z}$, emanating from only the gluino exchange diagram depicted in Fig. $15(\mathrm{~b})$, in units of $10^{-19}$ e-cm and as a function of the gluino mass $m_{\tilde{g}}$. We use: $\sqrt{s}=500 \mathrm{GeV}, m_{\tilde{t}_{1}}=50 \mathrm{GeV}, m_{\tilde{t}_{2}}=400 \mathrm{GeV}$ and $m_{t}=175 \mathrm{GeV}$. 

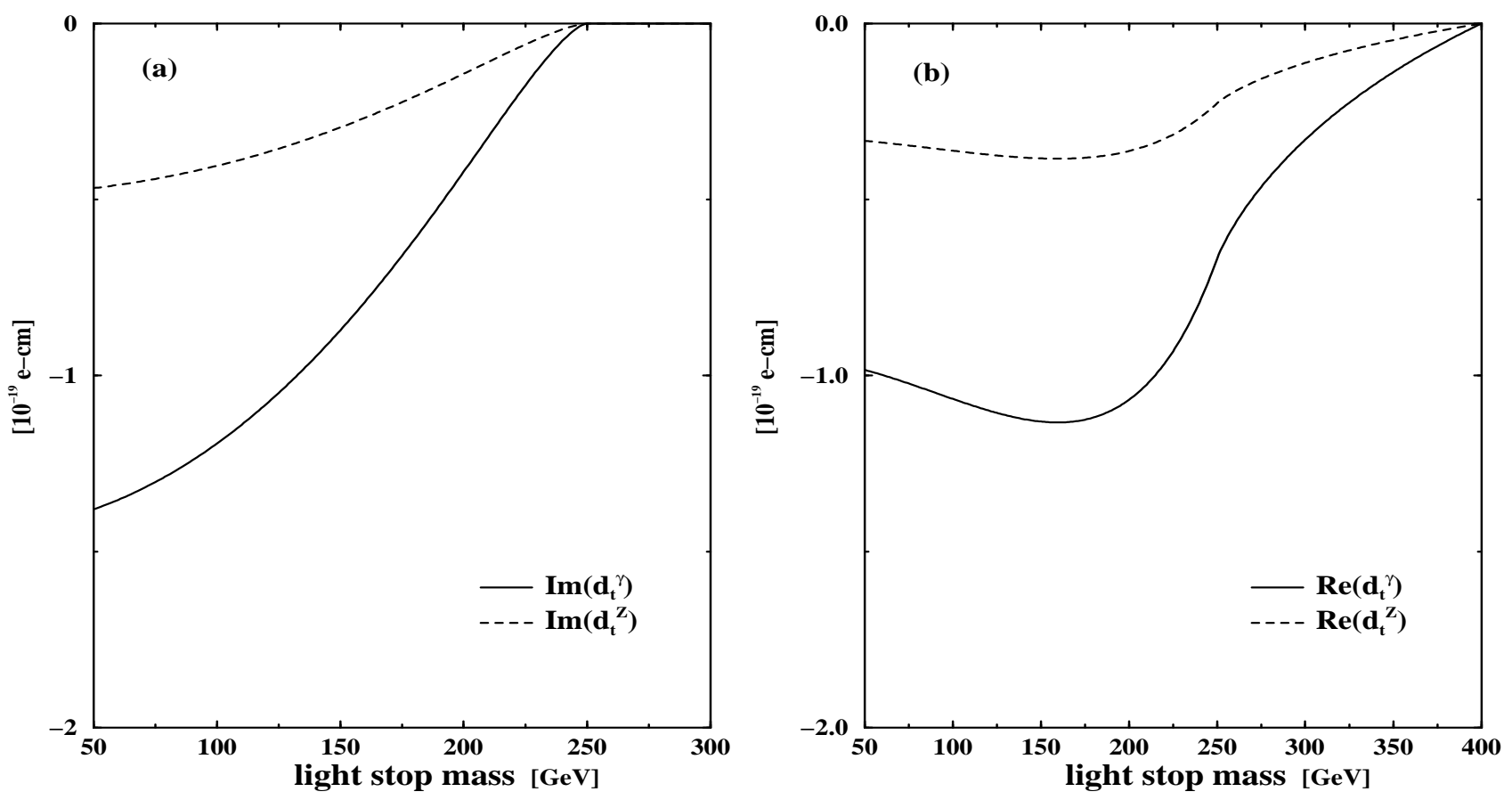

Figure 19: Imaginary (a) and Real (b) parts of $d_{t}^{\gamma}$ and $d_{t}^{Z}$, emanating from only the gluino exchange diagram depicted in Fig. 15(b), in units of $10^{-19}$ e-cm and as a function of the light stop mass $m_{\tilde{t}_{1}}$. We use: $\sqrt{s}=500 \mathrm{GeV}, m_{\tilde{t}_{2}}=m_{\tilde{g}}=400 \mathrm{GeV}$ and $m_{t}=175 \mathrm{GeV}$. 


\subsection{Top dipole moments - summary}

In this section we have performed a detailed investigation of the top quark dipole moments in models beyond the SM in which new $\mathrm{CP}$-violating phases appear rather naturally. The models that we have considered are MHDM's and the MSSM. In Table 3 we summarize our numerical results for the expected TDM within these class of models and for comparison we also write the expected size of the TDM in the SM. For illustration, for each model the TDM is evaluated by setting the corresponding $\mathrm{CP}$-violating quantities to plausible representative values, compatible with existing experimental limits if any.

Let us also summarize below the salient features of the CP-violating mechanisms of these models which give rise to a non-vanishing TDM, and specify our choice of values used in Table 3 for the CP-violating quantities of each model.

\section{- MHDM's:}

In general one can distinguish between two types of Higgs mediated CP-violating contributions to the TDM in MHDM's:

\section{TDM from neutral Higgs exchanges:}

In any MHDM with or without NFC and with new CP-violating phases in the neutral Higgs sector, the neutral Higgs-fermion-fermion interaction Lagrangian may be generically expressed as (say for $h$ - the lightest neutral Higgs)

$$
\mathcal{L}_{h f f}=-\frac{g_{W}}{\sqrt{2}} \frac{m_{f}}{m_{W}} h \bar{f}\left(a_{f}^{h}+i b_{f}^{h} \gamma_{5}\right) f
$$

The $h Z Z$ vertex which is also needed for calculating the TDM in the case of 1-loop neutral Higgs exchanges is given by (see Eq. 3.71)

$$
\mathcal{L}_{h Z Z}=g_{W} \frac{m_{Z}^{2}}{m_{W}^{2}} c^{h} h g_{\mu \nu} Z^{\mu} Z^{\nu}
$$

The CP-violating TDM then arises from the interference between the scalar, $a_{t}^{h}$, and the pseudoscalar, $b_{t}^{h}$, couplings in Eq. 4.65 and the interference between the scalar, $a_{t}^{h}$, and the $h Z Z$ coupling $\propto c^{h}$ in Eq. 4.66.

The numbers in the fourth column in Table 3 are given for masses of a neutral Higgs in the range 100-300 GeV (assuming that the masses of the other neutral Higgs particles in these models are much heavier) for

$$
a_{t}^{h}=b_{t}^{h}=c^{h}=1 \text {, }
$$

and they hold for any MHDM, i.e., a 2HDM of type I and II with NFC, a 2HDM of type III with FCNC in the neutral Higgs sector or for three or more Higgs doublets which have the generic $h t t$ interaction Lagrangian in Eqs. 4.65 and 4.66.

2. TDM from charged Higgs exchanges:

In models with three or more doublets the charged Higgs sector can acquire new CPodd phases which can give rise to the CP-violating TDM. Again, one can parameterize a generic (assumed lightest) charged Higgs-up quark-down quark CP-violating interaction Lagrangian, which will appear in such models, as 


$$
\mathcal{L}_{H^{+} u d}=\frac{g_{W}}{\sqrt{2} m_{W}} K_{u d}\left(m_{u} \beta_{2} \bar{u}_{R} d_{L}+m_{d} \alpha_{2} \bar{u}_{L} d_{R}\right)+\text { h.c. },
$$

where $K$ is the SM CKM matrix and $u$ and $d$ denote charge $+2 / 3$ and $-1 / 3$ quarks, respectively. The CP-violating TDM will then be proportional to $\Im \mathrm{m}(V) \equiv \Im \mathrm{m}\left(\beta_{2} \alpha_{2}^{*}\right)$. As mentioned before, the interaction Lagrangian in Eq 4.68 is not the only source of CP violation in this class of models and one also has to take into account the CP-violating neutral Higgs contributions arising from the $h f f$ coupling in Eq. 4.65. In fact, for the TDM we find that the CP-odd effect from a $H^{+} t b$ coupling in Eq. 4.68 is much smaller, i.e., typically by a factor of $\sim\left(m_{b} / m_{t}\right)^{2}$, than the one from the $h t t$ coupling in Eq. 4.65.

The numbers in the fifth column in Table 3 are given for masses of the charged Higgs in the range 200-500 GeV (again assuming that the masses of the other charged Higgs particles of these models are much heavier and therefore their contribution is negligible) and for

$$
\Im \mathrm{m}(V)=5,
$$

and they represent only the charged Higgs contribution to the TDM in any MHDM with three or more doublets, which have the generic $H^{+} t b$ interaction vertex in Eqs. 4.68.

\section{- MSSM:}

In the MSSM, as was shown in the previous section, if one neglects the phase in the Higgs mass parameter $\mu$ (as strongly implied from the existing limit on the NEDM) and the small mass splitting between the left and right superpartners of the light quarks, then the dominant contribution to the TDM arises from 1-loop gluino exchange. In that case the TDM emanates from $\tilde{t}_{L}-\tilde{t}_{R}$ mixing and is proportional to the CP-violating quantity

$$
\xi_{C P}^{t} \equiv \sin \left(2 \theta_{t}\right) \sin \left(\beta_{t}\right)
$$

where the angle $\beta_{t}$ represents the CP-phase in the soft trilinear breaking term associated with the top, i.e. $A_{t}$.

The numbers in the sixth column in Table 3 are given for gluino masses in the range 200-500 $\mathrm{GeV}$, for

$$
\xi_{C P}^{t}=1
$$

and for

$$
\begin{gathered}
m_{\tilde{t}_{1}}=50 \mathrm{GeV}, \\
m_{\tilde{t}_{2}}=400 \mathrm{GeV},
\end{gathered}
$$

where $m_{\tilde{t}_{1,2}}$ are the masses of the two stop mass-eigenstates. They therefore represent only the dominant gluino contribution to the TDM in any low energy supersymmetric framework in which all squarks except from the stop are degenerate and the phase in the Higgs mass parameter, $\mu$, is neglected. 
Table 3: The contribution to the top quark EDM $\left(d_{t}^{\gamma}(s)\right)$ and $Z E D M\left(d_{t}^{Z}(s)\right)$ form factors, in units of e-cm, at $s=\left(p_{t}+p_{\bar{t}}\right)^{2}=500^{2}, 1000^{2} \mathrm{GeV}^{2}$, for the $S M$ (where it is a purely guessestimate) and for some of its extensions. 4th column shows results for neutral Higgs exchanges in any MHDM's with a CP-violating htt coupling of the form $\left(g_{W} / \sqrt{2}\right)\left(m_{t} / m_{W}\right)\left(a_{t}^{h}+i b_{t}^{h} \gamma_{5}\right)$, an $h Z Z$ coupling $g_{W}\left(m_{Z}^{2} / m_{W}^{2}\right) c^{h} g_{\mu \nu}$ and with $a_{t}^{h}=b_{t}^{h}=c^{h}=1$. 5th column is for charged Higgs exchanges in any MHDM's of three or more doublets with a CP-violating $H^{+} t b$ coupling of the form $\left(g_{W} / \sqrt{2} m_{W}\right)\left[m_{t} U_{t}\left(1+\gamma_{5}\right) / 2+m_{b} U_{b}\left(1-\gamma_{5}\right) / 2\right]$ and with $\Im \mathrm{m}\left(U_{t} U_{b}^{*}\right) \equiv \Im \mathrm{m}(V)=5$. Only the contribution from the lightest neutral or charged Higgs is retained. 6th column shows the results for the MSSM where only the dominant 1-loop gluino exchange diagram with gluino masses $m_{\tilde{g}}=200-500 \mathrm{GeV}$ is considered, in which CP violation arises from $\tilde{t}_{L}-\tilde{t}_{R}$ mixing and is proportional to $\xi_{C P}^{t} \equiv \sin \left(2 \theta_{t}\right) \sin \left(\beta_{t}\right)$, where $\theta_{t}$ and $\beta_{t}$ are the angle and phase that parameterize the $\tilde{t}_{L}-\tilde{t}_{R}$ mixing matrix. The numbers are given for $\xi_{C P}^{t}=1$ and for stop masses of $50 \mathrm{GeV}$ (light stop) and $400 \mathrm{GeV}$ (heavy stop).

\begin{tabular}{|c|c|c|c|c|c|}
\hline $\begin{array}{l}\text { type of moment } \\
(e-c m) \quad \Downarrow\end{array}$ & $\begin{array}{r}\sqrt{s} \\
(\mathrm{GeV}) \Downarrow\end{array}$ & $\begin{array}{r}\text { Standard } \\
\text { Model }\end{array}$ & $\begin{array}{r}\text { neutral Higgs } \\
m_{h}=100-300\end{array}$ & $\begin{array}{r}\text { charged Higgs } \\
m_{H+}=200-500\end{array}$ & $\begin{array}{l}\text { Supersymmetry } \\
m_{\tilde{g}}=200-500\end{array}$ \\
\hline$\left|\Im \mathrm{m}\left(d_{t}^{\gamma}\right)\right|$ & $\begin{array}{r}500 \\
1000\end{array}$ & $<10^{-30}$ & $\begin{array}{l}(4.1-2.0) \times 10^{-19} \\
(0.9-0.8) \times 10^{-19}\end{array}$ & $\begin{array}{l}(29.1-2.1) \times 10^{-22} \\
(15.7-1.0) \times 10^{-22}\end{array}$ & $\begin{array}{l}(3.3-0.9) \times 10^{-19} \\
(1.2-0.8) \times 10^{-19}\end{array}$ \\
\hline$\left|\Re \mathrm{e}\left(d_{t}^{\gamma}\right)\right|$ & $\begin{array}{r}500 \\
1000\end{array}$ & $<10^{-30}$ & $\begin{array}{l}(0.3-0.8) \times 10^{-19} \\
(0.7-0.2) \times 10^{-19}\end{array}$ & $\begin{array}{c}(33.4-1.5) \times 10^{-22} \\
(0.3-2.7) \times 10^{-22}\end{array}$ & $\begin{array}{l}(0.3-0.9) \times 10^{-19} \\
(1.1-0.3) \times 10^{-19}\end{array}$ \\
\hline$\left|\Im \mathrm{m}\left(d_{t}^{Z}\right)\right|$ & $\begin{array}{r}500 \\
1000\end{array}$ & $<10^{-30}$ & $\begin{array}{l}(1.1-0.2) \times 10^{-19} \\
(0.2-0.2) \times 10^{-19}\end{array}$ & $\begin{array}{c}(15.8-2.5) \times 10^{-22} \\
(9.2-1.2) \times 10^{-22}\end{array}$ & $\begin{array}{l}(1.1-0.3) \times 10^{-19} \\
(0.4-0.3) \times 10^{-19}\end{array}$ \\
\hline$\left|\Re \mathrm{e}\left(d_{t}^{Z}\right)\right|$ & $\begin{array}{r}500 \\
1000\end{array}$ & $<10^{-30}$ & $\begin{array}{l}(1.6-0.2) \times 10^{-19} \\
(0.2-1.4) \times 10^{-19}\end{array}$ & $\begin{array}{c}(22.9-0.8) \times 10^{-22} \\
(0.6-1.9) \times 10^{-22}\end{array}$ & $\begin{array}{l}(0.1-0.3) \times 10^{-19} \\
(0.4-0.1) \times 10^{-19}\end{array}$ \\
\hline
\end{tabular}


We observe from Table 3 that the expected magnitudes of the real and imaginary parts of the top EDM and ZEDM in these models for masses in the loops of, typically, several hundreds GeV and energy scales of $500-1000 \mathrm{GeV}$ are

$$
\begin{array}{cl}
\text { Neutral Higgs : } & \Im \mathrm{m} d_{t}^{\gamma, Z} \sim \text { few } \times 10^{-20}-10^{-19} \mathrm{e}-\mathrm{cm}, \\
& \Re \mathrm{e} d_{t}^{\gamma, Z} \lesssim 10^{-19} \mathrm{e}-\mathrm{cm}, \\
\text { Charged Higgs : } \quad & \Im \mathrm{m} d_{t}^{\gamma, Z} \sim \text { few } \times 10^{-22}-10^{-21} \mathrm{e}-\mathrm{cm}, \\
& \Re \mathrm{e} d_{t}^{\gamma, Z} \sim \text { few } \times 10^{-23}-10^{-21} \mathrm{e}-\mathrm{cm}, \\
& \Im \mathrm{m} d_{t}^{\gamma, Z} \sim \text { few } \times 10^{-20}-10^{-19} \mathrm{e}-\mathrm{cm}, \\
\text { Supersymmetry : } & \Re \mathrm{e} d_{t}^{\gamma, Z} \lesssim 10^{-19} \mathrm{e}-\mathrm{cm} .
\end{array}
$$

The top CEDM $d_{t}^{g}$, given in units of $g_{s}$-cm, can be estimated within these models as follows: (i) In the neutral Higgs exchange case it is simply given by multiplying $d_{t}^{\gamma}$ by $1 / Q_{t}$, where $Q_{t}=2 / 3$ is the top quark charge. (ii) In the charged Higgs case one cannot simply replace the off-shell photon with an off-shell gluon and $d_{t}^{g}$ has to be explicitly calculated from Eq. 4.34. Nonetheless, we find that $d_{t}^{g} /\left(g_{s}-\mathrm{cm}\right) \approx d_{t}^{\gamma} /(\mathrm{e}-\mathrm{cm})$. (iii) In the MSSM case it is also not possible to extract the top CEDM from the top EDM by the simple exchange of a photon with a gluon since there is an additional graph with two gluino propagators in the loop coming from a new $g \tilde{g} \tilde{g}$ coupling. We have not estimated this additional contribution here.

To conclude this section, we have shown that in MHDM's with CP violation in the neutral or the charged Higgs sector and in the MSSM, the TDM is always bounded to be smaller then about $\sim 10^{-18} \mathrm{e}-\mathrm{cm}$. This is rather discouraging since, as we will see in the next few sections, the attainable limits on the TDM that can be obtained in future $e^{+} e^{-}$and hadronic colliders seem to fall short by about one order of magnitude compared to the above model dependent expectations for these quantities.

Basically, the strategy that we will describe in the following chapters for such investigations of the various TDM in future colliders is to incorporate an effective Lagrangian approach which elaborates new effective interactions of a dipole moment type at the $t t \gamma, Z, g$ vertices, with dimensions greater than 4 which can provide for a model independent investigation of new physics beyond the SM. The effects of such phenomenological vertices can then be studied in future $e^{+} e^{-}$ and hadron colliders. Of course, a hadron collider is appropriate for the study of the top CEDM and is not a very good environment for studying the top EDM and ZEDM couplings to a photon and a $Z$-boson; the EDM and ZEDM will obviously be masked by the gluon dynamics which will govern top quarks production in a hadronic collider. Therefore, a more natural place for such studies will be an $e^{+} e^{-}$collider. We will discuss later the feasibility of extracting information on the various TDM's in both hadron and $e^{+} e^{-}$colliders through an investigation of CP-odd and even CP-even observables, e.g., cross-sections. It should be noted that the information that can be obtained on a CP-odd quantity by studying its effect on a CP-even observable is much less than what might be learned about the various EDM's of the top by measuring a non-vanishing CP-odd observable driven by these CP-odd effective couplings. In particular, folding into a given amplitude the various CP-violating EDM interaction terms, the corresponding differential crosssection will acquire a CP-odd piece driven by the interference of the tree-level process with the EDM's interactions (to leading order only one EDM effective coupling has to enter in each diagram). Then with an appropriate CP-odd observable, which linearly depends on the EDM of the top, one can, in principle, analyze directly and separately the possible CP-violating effects that can arise from each EDM interaction in collider experiments such as $e^{+} e^{-}$or $p p \rightarrow t \bar{t}$. We will discuss CP-violating effects in these reactions in the following chapters. 


\section{CP violation in top decays}

In this chapter we discuss $\mathrm{CP}$ violation in various top decays. In particular, we will consider two-body decays, i.e., $t \rightarrow d_{k} W$, with $k=1,2,3$, the generation index, three-body decays as well as radiative decays. The following $\mathrm{CP}$-violating asymmetries will be reviewed (not for all decay modes) :

- PRA (Partial Rate Asymmetry).

- PIRA (Partially Integrated Rate Asymmetry).

- Energy asymmetry.

- $\tau$ polarization asymmetry.

Although the $\tau$ polarization asymmetry tends to be the largest effect in models with $\mathrm{CP}$ violation phase(s) in the charged Higgs exchanges, for the sake of generality and completeness, we will first discuss the other effects. Asymmetries such as top polarization asymmetry, although intimately related to the top decays, but for which most, if not all discussions in the literature are specific to the production process, are discussed in Chapters 6, 7 and 8. Models included are:

- SM (Standard Model).

- 2HDM (Two Higgs Doublet Model).

- 3HDM (Three Higgs Doublet Model).

- MSSM (Minimal Supersymmetric Standard Model).

Only PRA's are considered within the framework of all the models, while only the predictions of the $3 \mathrm{HDM}$ for $t \rightarrow b \tau \nu_{\tau}$ are presented for all the above asymmetries.

In addition, for the PRA, the Form Factor (FF) approach to the $t b W$ vertex will be presented. In this approach, the FF's can assume complex values, thus emulating physical cuts in higher order Feynman diagrams, leading to non-vanishing CP-odd, $T_{N}$-even observables such as PRA. This can be contrasted [148, 21, 149], with the effective Lagrangian approach, assuming that all new particles lie above $m_{t}$, where the coefficients are real. There, only $T_{N \text {-odd CP-violating }}$ asymmetries can emerge [150].

All CP-violating top decay asymmetries, within the SM, that have been studied so far are found to be too small to be measured. The same conclusion holds for CP-violating top production asymmetries. This results from severe GIM [23], or even double-GIM cancellations due to the fact that the masses of $d, s, b$ are too small compared to the top quark mass. The obvious conclusion is that an observation of $\mathrm{CP}$ violation in top quark decays, will serve as a very strong indication for the existence of new physics beyond the SM.

\subsection{Partial rate asymmetries}

In most models, the PRA, defined in Eq. 2.27 in section 2.2 is found to be small. This can be readily understood, in a model-independent way, from the CP-CPT connection discussed in section 2.3. Let us consider, for example, what seems to be the main decay of the top quark $t \rightarrow b W^{+}$. Due to CPT, to have a non-vanishing PRA, at least one additional decay channel should be available for the $t$. In other words, in the limit that $t \rightarrow b W$ becomes the only decay channel possible, then PRA has to vanish due to the fact that PRA then tends to become equal to the asymmetry in the total widths of $t$ and $\bar{t}$ which is constrained by CPT to vanish. In the $\mathrm{SM}$, by virtue of $V_{t b} \simeq 1$, there is very little competition to $t \rightarrow b W^{+}$, and the PRA turns out indeed to be tiny. Larger asymmetries are obtained for other decay channels, but their rates 
are too small to result in an experimentally interesting signal. In models beyond the SM, the situation is slightly better since there is a possibility for new particles to be produced in top decays, leading to the absorptive part necessary for PRA. The largest credibly possible predicted PRA, is $\sim 0.3 \%$ for $t \rightarrow b W^{+}$in the MSSM with low $\tan \beta$ which arises mainly since the top can have an appreciable decay rate into a $\tilde{t} \tilde{\chi}^{0}$ (i.e. the stop and neutralino final state) in this scenario (see below). However, once the window which allows SUSY final states in $t$ decays, such as $\tilde{t} \tilde{\chi}^{0}$ is closed, then the PRA in top quark decays become vanishingly small in this model too, i.e., the MSSM. In the following, we elaborate on some of the issues mentioned above, and more.

\subsubsection{PRA in the SM}

$\underline{t \rightarrow d_{k} W^{+}}$

$\mathrm{CP}$ violation via PRA in the process $t \rightarrow d_{k} W^{+}$, where $k=1,2,3$ is the generation index, was discussed in [148, 151, 152]. The PRA results from interference of the two diagrams in Fig. 20, i.e. from tree $\times$ loop interference, where the loop is the non-diagonal $t-u_{j}$ self-energy. By "interference" we actually mean "the difference between interferences for the process and its CP conjugated process". The loop contributes the necessary imaginary part $\Im \mathrm{m} \Sigma\left(m_{i}^{2}\right)$ through the cut on $d_{i} W$. One has to sum over $i, j$, and obviously $d_{i} \neq d_{k}$ and $u_{j} \neq t$. Before continuing to discuss the above contribution of the absorptive part to the PRA, let us digress to show that, as stated in 151], the CP-CPT connection [39, 40, 41] forbids the self-energy of the $W$ from contributing to the PRA. This will be shown within the SM; the proof holds for other models too, as can be easily generalized.

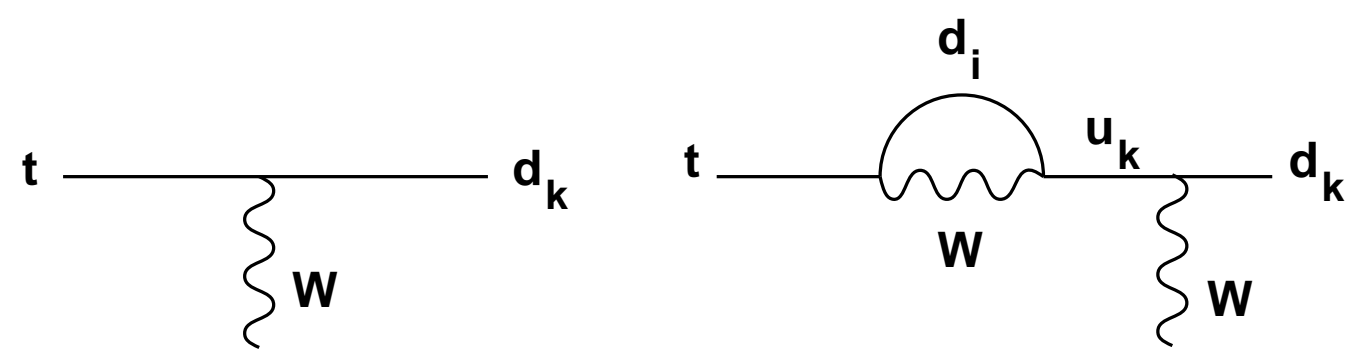

(a)

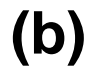

Figure 20: (a) Tree-level diagram for $t \rightarrow d_{k} W(k=1,2,3$ for $d, s, b)$. (b) Example of a 1-loop diagram for $t \rightarrow d_{k} W$.

Consider the case $d_{k}=b$ (the generalization to $k=1,2$ is trivial). The result of [151] is that the PRA in $t \rightarrow b W^{+}$comes from the interference of diagram (a) with diagram (b) in Fig. 20, where the absorptive part is provided by the $s W$ cut in diagram (b) (the $d W$ cut is negligible here). Thus we can symbolically write that the result of [151] corresponds to

$$
\Gamma\left(t \rightarrow b W^{+}\right)-\Gamma\left(\bar{t} \rightarrow \bar{b} W^{-}\right)=\Re \mathrm{e} \mathrm{a}(b W) \times \Im \mathrm{m} \mathrm{b}(b W ; s W \text { cut }),
$$

where a, b denote the contribution of diagrams (a), (b), respectively, and the arguments of their

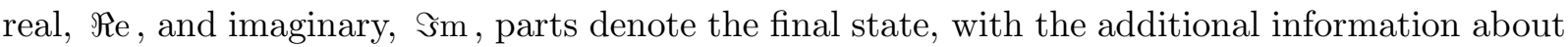
the relevant cut in $\Im \mathrm{m}$. Note that the CKM as well as numerical factors are suppressed in Eq. 5.1. Now, the rate difference in Eq. 5.1 can be written as 


$$
\begin{aligned}
\Gamma\left(t \rightarrow b W^{+}\right)-\Gamma\left(\bar{t} \rightarrow \bar{b} W^{-}\right)= & \Re \mathrm{e} 1\left(b u_{k} \bar{d}_{k}\right) \times \Im \mathrm{m} 3\left(b u_{k} \bar{d}_{k} ; s W \text { cut }\right) \\
& +\Re \mathrm{e} 1\left(b \ell \bar{\nu}_{\ell}\right) \times \Im \mathrm{m} 3\left(b \ell \bar{\nu}_{\ell} ; s W \text { cut }\right)
\end{aligned}
$$

where 1, 3 stand for diagrams (1), (3), respectively, in Fig. 21. Also $u_{k} \bar{d}_{k}$ denote summation over all quark pair states that the $W$ can decay into, and $\ell \bar{\nu}_{\ell}$ means summation over lepton generations. Other $q W$ cuts give negligible contributions to the PRA for $t \rightarrow b W^{+}$. To prove that the PRA contains no term from the interference of diagram (2) with (3), and of diagram (1) with (4), we have to show that, after summing over all final states[3]

$$
\Re \mathrm{e} 3 \times \text { sm } 2+\Im \mathrm{m} 4 \times \Re \mathrm{e} 1=0 .
$$

But for a specific final state and cut (say, the $b c \bar{s}$ final state, with a $\tau \bar{\nu}_{\tau}$ cut) in the $\Im$ m's, there is a compensating contribution (say, the $b \tau \bar{\nu}_{\tau}$ final state, with a $c \bar{s}$ cut). Thus, summing, for each final state, over all possible cuts on the $W$ line (rescattering excluded), then summing over final states, it is easy to show that Eq. 5.3 holds for $t \rightarrow b W$. We are thus left with $\Re \mathrm{e}$ a $(b W) \times$ $\Im \mathrm{m} \mathrm{b}(b W ; s W$ cut $)$, which is, by the way, compensated by $\Re \mathrm{e} \mathrm{a}(s W) \times \Im \mathrm{m} \mathrm{b}(s W ; b W$ cut $)$.

Let us now recapitulate the calculation of [151], for the CP-violating PRA for $t \rightarrow b W$, defined as

$$
\mathcal{A}_{k} \equiv \frac{\Gamma\left(t \rightarrow d_{k} W^{+}\right)-\Gamma\left(\bar{t} \rightarrow \bar{d}_{k} W^{-}\right)}{\Gamma\left(t \rightarrow d_{k} W^{+}\right)+\Gamma\left(\bar{t} \rightarrow \bar{d}_{k} W^{-}\right)}
$$

in the SM, with $k=3$. The two interfering amplitudes, Fig. 20(a) and 20(b), have a relative $\mathrm{CP}$-odd phase and Fig. 20(b) has the required absorptive phase. They are given by

$$
\begin{aligned}
A_{k}^{a} & =V_{t k}^{*} \hat{A}_{k} \\
A_{k}^{b} & =-\hat{A}_{k} \sum_{i, j} V_{t i}^{*} V_{j i} V_{j k}^{*} \frac{m_{t}^{2}}{m_{t}^{2}-m_{j}^{2}} \Sigma\left(m_{i}^{2}\right),
\end{aligned}
$$

where

$$
\hat{A}_{k}=-\frac{i g_{W}}{\sqrt{2}} \bar{u}\left(p_{k}\right) \gamma_{\mu} L u\left(p_{t}\right) \epsilon^{\mu}
$$

$\epsilon^{\mu}$ is the $W$-boson polarization vector and $L=\left(1-\gamma_{5}\right) / 2$. The PRA was then found to be

$$
\mathcal{A}_{k}=-2 \sum_{i, j} \frac{\Im \mathrm{m}\left(V_{t k}^{*} V_{t i} V_{j i}^{*} V_{j k}\right)}{\left|V_{t k}\right|^{2}} \frac{m_{t}^{2}}{m_{t}^{2}-m_{j}^{2}} \Im \mathrm{m}\left[\Sigma\left(m_{i}^{2}\right)\right] .
$$

Thus the effect is proportional to the Jarlskog invariant $J$ (recall that $J=\left|\Im \mathrm{m}\left(V_{\beta j} V_{\beta k}^{*} V_{\gamma k} V_{\gamma j}^{*}\right)\right|$, for any $\beta, \gamma, j$ and $k$ ) as expected, being the only CP-violating parameter in the $\mathrm{SM}$ (see section 3.1.2), and to the absorptive phase in the self-energy diagram of Fig. 20(b). Note that the asymmetry is doubly-GIM suppressed being summed over both $i$ and $j$. Therefore, with the Wolfenstein parameterization for the CKM elements (see Eqs. 3.13 and 3.14 in section 3.1.1), $\Im \mathrm{m}\left(V_{t k}^{*} V_{t i} V_{j i}^{*} V_{j k}\right) \sim \lambda^{6}$, one expects the leading contribution to be of the form [151]:

$$
\Gamma_{k}-\bar{\Gamma}_{k} \sim \lambda^{6} \frac{\Delta m_{u}^{2} \Delta m_{d}^{2}}{m_{W}^{4}}
$$

\footnotetext{
${ }^{13}$ Note that near resonance diagrams (2) and (4) of Fig. 21 become $\mathcal{O}\left(g^{2}\right)$ and $\mathcal{O}\left(g^{4}\right)$, respectively.
} 
where $\Delta m_{u}^{2} \equiv m_{t}^{2}-m_{u}^{2}$ and $\Delta m_{d}^{2} \equiv m_{b}^{2}-m_{d}^{2}$. It was found in 151] that

$$
\mathcal{A}_{k}=\frac{\alpha}{8 \sin ^{2} \theta_{W}} \frac{m_{b}^{2} m_{c}^{2}}{m_{W}^{4}} f_{W}(y) \eta \mathcal{C}_{k}
$$

independent of the Wolfenstein parameter $A$. $f_{W}$ originates from the imaginary part of the self-energy and is given by

$$
\begin{aligned}
& f_{W}(y)=-3 \frac{\left(1+y^{4}\right)}{y^{6}}, \\
& y \equiv m_{t} / m_{W}
\end{aligned}
$$

and

$$
\begin{aligned}
& \mathcal{C}_{d}=-\left[(1-\rho)^{2}+\eta^{2}\right]^{-1} \\
& \mathcal{C}_{s}=\lambda^{2} \\
& \mathcal{C}_{b}=-\lambda^{6} \frac{m_{s}^{2}}{m_{b}^{2}}
\end{aligned}
$$

The largest asymmetry is obtained for $d_{k}=d, \mathcal{A}_{d} \simeq 10^{-9}$, requiring at least $10^{23}$ top quarks! Even smaller asymmetries are predicted for $t \rightarrow s W$ and $t \rightarrow b W$. Thus the discussion of PRA's for $t \rightarrow d_{k} W$ in the SM is of purely an academic value. Let us note, in passing, that in the SM with four generations (a currently unfashionable approach) $\mathcal{A}_{k}$ may be substantially enhanced [151].

$\underline{t \rightarrow c V}$

The branching ratios for the rare flavor changing processes $t \rightarrow c \gamma$ [24, 153, 154, 155], $t \rightarrow c g$ [24, 156] and $t \rightarrow c Z$ [24, 157] in the SM are: $4.9 \cdot 10^{-13}, 1.4 \cdot 10^{-13}$ and $4.4 \cdot 10^{-11}$, respectively. The CP-violating PRA's, resulting from the interference of two penguin diagrams, are largest for the even rarer (by more than an order of magnitude) decays $t \rightarrow u \gamma$ [155] and $t \rightarrow u g$ [156] where they are $\simeq 0.2 \%$. A slightly larger asymmetry is obtained for $t \rightarrow u g^{*} \rightarrow u \bar{u} u$ 156.

\section{Three-body $t$ decays}

In the above we have discussed interferences of the type $\Im \mathrm{m} A_{\text {loop }} \times \Re$ e $A_{\text {tree }}$, where the loop is the off-diagonal $t-u_{j}$ self-energy (in contrast, in the MSSM, see below, "loop" stands for "vertex corrections"), and of the type $\Im \mathrm{m} A_{\text {penguin }} \times \Re \mathrm{e} A_{\text {penguin }}^{\prime}$. The prime indicates that the imaginary and real parts of the penguins must have a different weak phase. This is possible since three different quark amplitudes are in the penguin graph. PRA in the SM for the six three-body top decays of the type

$$
t \rightarrow c q \bar{q} \text { and } t \rightarrow u q \bar{q}, q=d, s, b
$$

was considered in Ref. 47, 44]. There, PRA from the interference $\Im \mathrm{m} A_{\text {tree }} \times \Re \mathrm{e} A_{\text {penguin }}$ for each of the final states in Eq. 5.15 was calculated. This interference is present in top decays due to the fact that $m_{t}>m_{W}+m_{b}$, thus endowing the tree diagram with a well-defined CP-even phase from the $W$-width [48]. Though the $W$-width turns a "tree" diagram into a "loop" diagram, we will keep loosely calling it as a "tree". The above interference is there in addition to terms of the type: $\Re$ e $A_{\text {tree }} \times \Im \mathrm{m} A_{\text {penguin, }}$, which are analogous to the interference 43 that leads to the $\mathrm{CP}$ violation in $b$ decays, such as $b \rightarrow s u \bar{u}$ (see the discussion in section 2.3). These interferences 
arise since for each specific channel $t \rightarrow q^{\prime} q \bar{q}$, where $q^{\prime}=c$ or $u$, there are two classes of possible paths:

1. The tree process $t \rightarrow q W$ followed by $W \rightarrow q^{\prime} \bar{q}$.

2. The 1-loop penguin process $t \rightarrow q^{\prime} g^{*}$ followed by $g^{*} \rightarrow q \bar{q}$.

The two Feynman amplitudes, tree and penguin, have different weak and different strong phases. Consequently, the interference of these two channels, provides the necessary condition mentioned in section 2.2 for the observability of $\mathrm{CP}$ violation. Note that penguin $\times$ penguin terms are relatively small, except in the absence of tree terms.

The PRA for the three-body decays $t \rightarrow q^{\prime} q \bar{q}$ where $q^{\prime}=c$ or $u$ and $q=d, s, b$ is [47, 44]:

$$
\begin{aligned}
\mathcal{A}= & \frac{8 g_{s} m_{W} \Gamma_{W}}{3 \pi g_{W}^{2} m_{t}^{6} Z_{V}} \sum_{j=b, s} \Im \mathrm{m}\left(V_{t q}^{*} V_{q^{\prime} q} V_{t j} V_{q^{\prime} j}^{*}\right) \\
& \times \int_{\left(m_{q^{\prime}}+m_{\bar{q})^{2}}\right.}^{\left(m_{t}-m_{q}\right)^{2}} d m_{q^{\prime} \bar{q}}^{2} \int_{\left(m_{q^{\prime} q}^{2}\right) \min }^{\left(m_{q^{\prime} q}^{2}\right) \max } d m_{q^{\prime} q}^{2} \\
& \cdot \frac{\left[\hat{\Gamma}_{W} m_{W} \Re \mathrm{e}\left(T_{j}-T_{d}\right)-K \Im \mathrm{m}\left(T_{j}-T_{d}\right)\right]}{(\bar{q}+q)^{2}\left(K^{2}+\Gamma_{W}^{2} m_{W}^{2}\right)},
\end{aligned}
$$

where $Z_{V}=\left|V_{t q}^{*} V_{q^{\prime} q}\right|^{2}(1-x)\left(1+x-2 x^{2}\right)$ with $x=m_{W}^{2} / m_{t}^{2}, K=m_{q}^{2}+m_{q^{\prime}}^{2}+2 \bar{q} \cdot q^{\prime}-m_{W}^{2} ; T_{j}$ is a lengthy expression for the resulting trace, where $j$ indicates the virtual quark exchange in the loop (for which we have used CKM unitarity). Furthermore, $\hat{\Gamma}=\Gamma_{W}-\Gamma\left(W \rightarrow q^{\prime} q\right)$, thus excluding rescattering, i.e., $q^{\prime} q \not \rightarrow q^{\prime} q$ [44]. The largest contribution to $\left(T_{j}-T_{d}\right)$ is from $j=b$, i.e., $\left(T_{b}-T_{d}\right)>>\left(T_{s}-T_{d}\right)$. As a result, the asymmetry is completely negligible for the most abundant final state, $t \rightarrow b c \bar{b}$ as $\Im \mathrm{m}\left(V_{t b}^{*} V_{q^{\prime} b} V_{t b} V_{q^{\prime} b}^{*}\right)=0$. If one resurrects $j=s$ for $t \rightarrow b c \bar{b}$, an absurdly small PRA, of the order of $10^{-11}$, emerges. Indeed, despite the resonance enhancement, the asymmetries in all the final states are very small. The largest effect is for $t \rightarrow d c \bar{d}$. Even for this mode the asymmetry is too small to be of any experimental relevance, $\mathcal{A} \sim 10^{-5}$ for $m_{t}=180 \mathrm{GeV}$.

We briefly note [44 that interferences of the type $\Re$ e (tree) $\times \Im \mathrm{m}(2$-loops $)$, where the higher order loops have both a virtual $g$ and a virtual $W$, for processes such as $t \rightarrow d e \nu_{e}$, are required [39, 40, 41] to compensate the partial width differences for the reactions in Eq. 5.15 (see Fig 2 and the discussion in section 2.3). Consequently, from such mechanisms, one gets asymmetries of the order of $10^{-7}$ for $t \rightarrow d c \bar{s}$, and there is even a tiny asymmetry in the SM for the leptonic decay $t \rightarrow d e^{+} \nu_{e}$.

\subsubsection{PRA in a 2HDM}

In a $2 \mathrm{HDM}$, there are 5 physical Higgs particles: 3 neutral, $\mathcal{H}^{k}, k=1,2,3$ and 2 charged ones, $H^{ \pm}$. If one imposes discrete symmetry [79] to avoid FCNC at tree-level, then there is no CP violation in the charged Higgs sector, (unlike the case in the 3HDM, discussed below) and CP violation then exists only in the neutral Higgs sector (see section 3.2). This class of 2HDM is further subdivided into Model I and Model II (see section 3.2.3). However, there is a version of 2HDM, the so-called Model III (see section 3.2.2), where no discrete symmetry is imposed; the model then admits tree-level FCNC. In Model III large tree-level FCNC may then be avoided for the light quarks by assuming 82] that the couplings $h q_{i} q_{j}$ are proportional to $\left(m_{i} m_{j}\right)^{1 / 2}$. Note that in the class of 2HDM's with NFC (i.e., Model I or II), CP violation resides in flavor-diagonal $\mathcal{H}^{k}$ exchanges; consequently CPT forbids PRA in $t \rightarrow d_{k} W$ to arise. In model III PRA in $t \rightarrow d_{k} W$ need not vanish, though it is expected to be very small due to the small $\mathcal{H}^{k} d_{i} d_{j} \propto \sqrt{m_{d_{i}} m_{d_{j}}} / m_{W}$ coupling $(i \neq j)$. 
The effect of a charged Higgs with the CKM phase was considered in 151 for $t \rightarrow d_{k} W^{+}$. Assuming $m_{H^{+}}+m_{i}<m_{t}$, then, since a charged Higgs exchange is added to the $W$ exchange in Fig. 20(b), one has to make the following substitution in Eq. 5.10 (where a large $1 / \tan \beta \equiv v_{d} / v_{u}$ is assumed)

$$
f_{W}(y) \longrightarrow f_{W}(y)+\frac{f_{H}(x, y)}{\tan ^{2} \beta} .
$$

$f_{W}(y)$ is defined in Eq. 5.11 and

$$
\begin{aligned}
& f_{H}(x, y)=\frac{\left(1-x^{2}\right)^{2}}{x^{4} y^{2}} \\
& x \equiv m_{t} / m_{H}
\end{aligned}
$$

Taking into account the experimental bounds on $\tan \beta$, there is no increase in the tiny PRA, $\mathcal{A}_{k}$, over what was obtained in the SM. This conclusion also holds for other observables governed by a charged Higgs exchange unless the CP-violating couplings to fermions are different from the SM.

\subsubsection{PRA in a $3 H$ HDM}

The 3HDM (Weinberg Model for CP violation [45]), as described in section 3.2.4, can cause CP violation effects, through its CP-violating couplings of a charged Higgs-boson to fermion pairs. There are no tree-level FCNC within this model. The physical charged Higgs states are $H_{1}^{ \pm}$and $H_{2}^{ \pm}$, where, for simplicity, it is usually assumed that $m_{H_{1}}>m_{H_{2}}$, thus decoupling $H_{1}$ from all predictions. It is also easy to show that any $\mathrm{CP}$ violation asymmetry vanishes for $m_{H_{1}}=m_{H_{2}}$ through a GIM-like mechanism.

In the 3HDM, the interesting new phenomenon is the existence of a CP-violating coupling $\gamma_{2}$ in the leptonic term in the Yukawa part of the Lagrangian (see Eq. 3.88)

$$
\mathcal{L}_{H_{2}^{+}}=\frac{g_{W}}{\sqrt{2} m_{W}}\left(m_{t} \beta_{2} \bar{t}_{R} b_{L}+m_{\tau} \gamma_{2} \bar{\nu}_{L} \tau_{R}\right) H_{2}^{+}+\text {h.c. },
$$

where $V_{t b}=1$ is assumed. Note the proportionality to the mass of the charged lepton, which prompted studies of CP violation in the reaction $t \rightarrow b \tau \bar{\nu}_{\tau}$. The reaction $t \rightarrow b c \bar{s}$ is not that useful in view of the difficulty in identifying $c$, and especially $s$ jets. In addition, the $\tau$ mode will enable, by following the $\tau$ decay products, measurements of spin related CP-violating observables.

It is convenient [110] to parameterize $\mathrm{CP}$ violation in the Yukawa couplings with a CKM-like matrix (the SM CKM matrix itself is assumed to be real), then $\beta_{2}$ and $\gamma_{2}$ in Eq. 5.19 are given by (see Eq. 3.89)

$$
\begin{aligned}
& \beta_{2}=\frac{\tilde{c}_{1} \tilde{c}_{2} \tilde{s}_{3}-\tilde{s}_{2} \tilde{c}_{3} e^{i \delta_{H}}}{\tilde{s}_{1} \tilde{c}_{2}}, \\
& \gamma_{2}=\frac{\tilde{c}_{1} \tilde{s}_{2} \tilde{s}_{3}+\tilde{c}_{2} \tilde{c}_{3} e^{i \delta_{H}}}{\tilde{s}_{1} \tilde{s}_{2}},
\end{aligned}
$$

where $\tilde{s}_{i} \equiv \sin \left(\tilde{\theta}_{i}\right)$ and $\tilde{c}_{i} \equiv \cos \left(\tilde{\theta}_{i}\right)$, and $\tilde{\theta}_{i}, \delta_{H}$ are parameters of the model. CP violation will be proportional to combinations such as

$$
\Im \mathrm{m}(U)=\Im \mathrm{m}\left(\beta_{2}^{*} \gamma_{2}\right) .
$$

\footnotetext{
${ }^{14}$ Note a typographical error in $\beta_{2}$ in $[110]$.
} 
Let us define the PRA

$$
\mathcal{A}_{\tau}=\frac{\Gamma\left(t \rightarrow b \tau^{+} \nu_{\tau}\right)-\Gamma\left(\bar{t} \rightarrow \bar{b} \tau^{-} \bar{\nu}_{\tau}\right)}{\Gamma\left(t \rightarrow b \tau^{+} \nu_{\tau}\right)+\Gamma\left(\bar{t} \rightarrow \bar{b} \tau^{-} \bar{\nu}_{\tau}\right)}
$$

The lowest order contribution to $\mathcal{A}_{\tau}$ arises due to the interference of the SM $W$ mediated tree diagram (see diagram (a) in Fig. 22) with the 3HDM $H^{+}$mediated tree diagram (diagram (c) in Fig. 22). We assess the contribution of the second graph making two simplifying assumptions: (i) $m_{H^{+}} \equiv m_{H_{2}}<<m_{H_{1}^{+}}$, this allows us to neglect the effect of the heavier charged Higgs, $H_{1}$. Furthermore, we also assume that (ii) $m_{H^{+}}>m_{t}$, thus the $H^{+}$width becomes irrelevant. $\mathcal{A}_{\tau}$ will then be proportional to $\Im \mathrm{m}(W-$ tree $) \times \Re \mathrm{e}(H-$ tree $)$, where, in analogy to our previous discussion in section 5.1.1, the CP-violating CKM-like angular function was factored out. It is easy to see that, because of the chirality miss match, only the longitudinal part of the $W$ propagator contributes to $\mathcal{A}_{\tau}$. In other words, decomposing the $W$-propagator in the unitary gauge as

$$
D_{\mu \nu}^{W}=i\left(-g_{\mu \nu}+\frac{q_{\mu} q_{\nu}}{q^{2}}\right) G_{T}+i \frac{q_{\mu} q_{\nu}}{q^{2}} G_{L},
$$

only $\Im \mathrm{m} G_{L}$ will appear in $\mathcal{A}_{\tau}\left[158\right.$, 159]. In fact $\mathcal{A}_{\tau}$, obtained from the interference between diagrams (a) and (c) in Fig. 22, is proportional to

$$
\mathcal{A}_{\tau} \propto \Im \mathrm{m}(U) \int_{m_{\tau}^{2}}^{\left(m_{t}-m_{b}\right)^{2}} f\left(q^{2}\right) \Im \mathrm{m} \hat{G}_{L},
$$

where $\hat{G}_{L}$ indicates that $\tau \nu$ is missing from $G_{L}$ to respect CPT invariance and $f\left(q^{2}\right)$ is a phasespace function.

Now, while the transverse part of the W-propagator in Eq. 5.24 resonates, i.e.,

$$
G_{T} \simeq \frac{1}{q^{2}-m_{W}^{2}+i \Gamma_{W} m_{W}}
$$

for $q^{2} \simeq m_{W}^{2}$, there is no such enhancement for $G_{L}$. This is one of the reasons for an extremely small asymmetry, $10^{-8}$ [159] or smaller [46], from tree $\times$ tree interference. The other reasons are the proportionality of both the Higgs coupling and $\Im \mathrm{m} \hat{G}_{L}$ to small fermion masses.15

The next logical step is to capitalize on the resonance behavior of $\Im \mathrm{m} G_{T}$ and the fact that, unlike $\Im \mathrm{m} G_{L}$, it is not proportional to small masses, by considering interferences that are higher order in the weak interaction coupling constant [42]. Thus, PRA from interferences of the type $\Im \mathrm{m}(\mathrm{a}) \times \Re \mathrm{e}(\mathrm{b})$ and $\Re \mathrm{e}(\mathrm{c}) \times \Im \mathrm{m}(\mathrm{d})$, is calculated; (a)-(d) denote the diagrams in Fig. 22, where (b) and (d) represent all box diagrams. Bremsstrahlung is included, but diagrams yielding $\mathcal{A}_{\tau} \rightarrow 0$ for $G_{L} \rightarrow 0$, see below, were neglected. The asymmetry is then given by

$$
\mathcal{A}_{\tau} \simeq \frac{\left[\Im \mathrm{m}(U) / 512 \pi^{3} m_{t}^{3}\right] \int d q^{2} d u \Im \mathrm{m} \hat{G}_{T} \Re \mathrm{e}(\text { tree } \times \text { box })}{\Gamma(t \rightarrow b W \rightarrow b \tau \nu)}
$$

where $\Im \mathrm{m}(U)$ is defined in Eq. 5.22 and

\footnotetext{
${ }^{15}$ While there is agreement in the literature as to the above facts, there is controversy - into which we do not enter here (due in part to the fact that it has no observational consequences) - regarding the form of $\Im m G_{L}$ 146, 158, 159, 160, 161 to be inserted in Eq. 5.25.
} 


$$
\begin{aligned}
u & =\left(p_{\tau}+p_{b}\right)^{2}, \quad q^{2}=\left(p_{\nu}+p_{\tau}\right)^{2}, \\
\Im \mathrm{m} \hat{G}_{T} & =-\frac{m_{W} \hat{\Gamma}_{W}}{\left(q^{2}-m_{W}^{2}\right)^{2}+\left(\Gamma_{W} m_{W}\right)^{2}},
\end{aligned}
$$

with

$$
\hat{\Gamma}_{W}=\Gamma_{W}-\Gamma_{W \rightarrow \tau \nu} .
$$

The maximal value of $\mathcal{A}_{\tau}$ turns out to be negative, and of order $10^{-5}$. Subsequently, the following contributions to $\mathcal{A}_{\tau}$, explicitly neglected in [42], were calculated in [159]:

- Since the integration in Eq. 5.25 reaches up to $\left(m_{t}-m_{b}\right)^{2}$, it includes a region with $q^{2}>m_{W}^{2}$, for which a $W-\gamma$ loop in $G_{L}$ has to be taken into account.

- Imaginary parts can also appear in box and vertex diagrams corresponding to $t \rightarrow b W$.

Both new terms are non-resonant and do not suffer small mass suppression from fermion loops in $G_{L}$. They turn out to give a large correction, of about $50 \%$ and of the same sign, as compared to the value of $\mathcal{A}_{\tau}$ calculated in [42] using only $G_{T}$. The minimal number of $t$-quarks required to observe $\mathrm{CP}$ violation in PRA within the $3 \mathrm{HDM}$ is - although many orders of magnitudes larger than the best leptonic SM result (i.e., $t \rightarrow d \ell \nu$ [47]) - of the order of $10^{10}-10^{11}$ and thus not very promising. As we will see later, one can do much better in the 3HDM by considering PIRA rather than PRA.

\subsubsection{PRA in the MSSM}

An extremely interesting possibility, investigated in [138, 151, 162, 163], is that the CP-violating PRA in two-body modes (that was found to be extremely small in the SM, 2HDM and in the 3HDM) may receive appreciable contribution from new SUSY CP-odd phases. For example, consider the PRA $\mathcal{A}_{3}$ in Eq. 5.4 for the main top decay $t \rightarrow b W$; the Feynman diagrams that can potentially contribute to $\mathcal{A}_{3}$ in the MSSM are depicted in Fig. 23 .

Recall that since $\mathcal{A}_{3}$ is $T_{N}$-even it requires an absorptive phase in the Feynman amplitude. This necessitates radiative corrections to the $t \rightarrow b W$ to at least 1-loop order and, in particular, the SUSY particles exchanged in the loops have to be light enough such that absorptive cuts will arise. Of course, in addition to the strong phase from FSI (Final State Interactions), a CP-odd phase is needed. We recall that, in the MSSM, with the most general boundary conditions for the soft breaking parameters at the scale where they are generated and ignoring generation mixing, only three places remain in the SUSY Lagrangian that can give rise to CP phases that cannot be rotated away: The superpotential contains a complex coefficient $\mu$ in the term bilinear in the Higgs superfields and the soft-supersymmetry breaking operators introduce two further complex terms, the gaugino masses $\tilde{m}$ and the left and right-handed squark mixing terms. The latter, being proportional to the trilinear soft breaking terms (i.e., the $A_{q}$ terms) and to $\mu$, may be complex in general (for more details see section 3.3). It is clear then that, in general, there are many sources of CP-violating phases. Therefore, reliable predictions cannot be made unless we make some simplifying assumptions.

Let us first describe a convenient way to derive the PRA $\mathcal{A}_{3}$. Following [138], the $t \rightarrow b W^{+}$ and $\bar{t} \rightarrow \bar{b} W^{-}$decay vertices can be parameterized as follows

$$
\begin{aligned}
J_{k}^{\mu(t)} & \equiv i \frac{g_{W}}{\sqrt{2}} \sum_{P=L, R} \bar{u}_{b}\left(\frac{\mathcal{D}_{1(k)}^{P} p_{t}^{\mu}}{m_{t}}+\mathcal{D}_{2(k)}^{P} \gamma^{\mu}\right) P u_{t}, \\
J_{k}^{\mu(\bar{t})} & \equiv i \frac{g_{W}}{\sqrt{2}} \sum_{P=L, R} \bar{v}_{t}\left(\frac{\overline{\mathcal{D}}_{1(k)}^{P} p_{t}^{\mu}}{m_{t}}+\overline{\mathcal{D}}_{2(k)}^{P} \gamma^{\mu}\right) P v_{b},
\end{aligned}
$$


where $\mathcal{D}_{1(k)}^{L, R}$ and $\mathcal{D}_{2(k)}^{L, R}$ defined in Eqs. 5.31 and 5.32, contain the CP-violating phases as well as the absorptive phases of the decay diagram $(k)(k=a, b, c$ or $d$ corresponding to diagrams (a),(b),(c) or (d) in Fig. 23). The important contributions to the $\mathcal{D}$ 's above are likely to come from those diagrams in which one of the two on-shell superparticle is the Lightest Supersymmetric Particle (LSP), e.g., the neutralino in our case. Such is the case for diagrams (b) and (d) in Fig. 23. Also, with a very light stop (i.e., $\sim 50 \mathrm{GeV}$ ) an absorptive cut can arise from diagram (a) in Fig. 23 if the gluino mass is below $\lesssim 130 \mathrm{GeV}$. The current experimental bounds on the superparticles involved in the loop of diagram (c) in Fig. 23 are already stringent enough that they are unlikely to have absorptive parts for $m_{t} \sim 175 \mathrm{GeV}$ [139].

Let us now write $\mathcal{A}_{3}$ in the most general case with no assumptions on the masses of the SUSY particles and taking into account all four diagrams in Fig. 23 [16. In terms of the scalar $\left(\mathcal{D}_{1(k)}\right)$ and vector $\left(\mathcal{D}_{2(k)}\right)$ form factors, the PRA $\mathcal{A}_{3}$ is given by

$$
\mathcal{A}_{3}=\sum_{k}\left[\frac{(x-1)}{2(x+2)} \Re \mathrm{e}\left(\mathcal{D}_{1(k)}^{R}+\overline{\mathcal{D}}_{1(k)}^{L}\right)+\Re \mathrm{e}\left(\mathcal{D}_{2(k)}^{L}-\overline{\mathcal{D}}_{2(k)}^{L}\right)\right],
$$

where $x \equiv m_{t}^{2} / m_{W}^{2}$ and the sum is carried out over all decay diagrams in Fig. 23 (i.e., $k=\mathrm{a}, \mathrm{b}, \mathrm{c}$ and $d)$. It is easy to show that if one defines

$$
\begin{aligned}
& \mathcal{D}_{1(k)}^{R} \sim e^{i \delta_{s}^{1(k)}} \times e^{i \delta_{w}^{1(k)}}, \\
& \mathcal{D}_{2(k)}^{L} \sim e^{i \delta_{s}^{2(k)}} \times e^{i \delta_{w}^{2(k)}},
\end{aligned}
$$

where $\delta_{s}^{1(k)}, \delta_{s}^{2(k)}$ are the CP-even absorptive phases (i.e., FSI phases) and $\delta_{w}^{1(k)}, \delta_{w}^{2(k)}$ are the CP-odd phases associated with diagrams (a)-(d) in Fig. 23, then

$$
\begin{aligned}
& \overline{\mathcal{D}}_{1(k)}^{L} \sim-e^{i \delta_{s}^{1(k)}} \times e^{-i \delta_{w}^{1(k)}}, \\
& \overline{\mathcal{D}}_{2(k)}^{L} \sim e^{i \delta_{s}^{2(k)}} \times e^{-i \delta_{w}^{2(k)}} .
\end{aligned}
$$

We then get for the scalar form factors in Eq. 5.33

$$
\begin{aligned}
\Re \mathrm{e}\left(\mathcal{D}_{1(a)}^{R}+\overline{\mathcal{D}}_{1(a)}^{L}\right)= & -\frac{8}{3} \frac{\alpha_{s}}{\pi} m_{t} m_{\tilde{g}} \mathcal{O}_{a}^{1} \Im \mathrm{m} C_{12}^{a}, \\
\Re \mathrm{e}\left(\mathcal{D}_{1(b)}^{R}+\overline{\mathcal{D}}_{1(b)}^{L}\right)= & -\frac{\alpha}{\pi \sin ^{2} \theta_{W}} m_{t}\left[m_{t} \mathcal{O}_{b}^{1} \Im \mathrm{m}\left(C_{22}^{b}-C_{23}^{b}\right)\right. \\
& \left.+m_{\tilde{\chi}_{n}^{0}} \mathcal{O}_{b}^{2} \Im \mathrm{m} C_{12}^{b}\right] \\
\Re \mathrm{e}\left(\mathcal{D}_{1(c)}^{R}+\overline{\mathcal{D}}_{1(c)}^{L}\right)= & \frac{\alpha}{\pi \sin ^{2} \theta_{W}} m_{t}\left[m_{t} \mathcal{O}_{c}^{1} \Im \mathrm{m}\left(C_{23}^{c}-C_{22}^{c}\right)\right. \\
& -m_{\tilde{\chi}_{m}} \mathcal{O}_{c}^{2} \Im \mathrm{m}\left(C_{11}^{c}-C_{12}^{c}\right) \\
& \left.+m_{\tilde{\chi}_{n}^{0}} \mathcal{O}_{c}^{3} \Im \mathrm{m}\left(C_{0}^{c}+C_{11}^{c}\right)\right], \\
\Re \mathrm{e}\left(\mathcal{D}_{1(d)}^{R}+\overline{\mathcal{D}}_{1(d)}^{L}\right)= & \Re \mathrm{e}\left(\mathcal{D}_{1(c)}^{R}+\overline{\mathcal{D}}_{1(c)}^{L}\right)\left(m_{\tilde{\chi}_{n}^{0}} \rightarrow-m_{\tilde{\chi} m}, m_{\tilde{\chi}_{m}} \rightarrow m_{\tilde{\chi}_{n}^{0}},\right. \\
& \left.\mathcal{O}_{c}^{i} \rightarrow \mathcal{O}_{d}^{i}, \Im \mathrm{m} C_{i j}^{c} \rightarrow \Im^{c} C_{i j}^{d}\right),
\end{aligned}
$$

while the vector form factors in Eq. 5.33 are given by

\footnotetext{
${ }^{16}$ In pages to follow we will evaluate $\mathcal{A}_{3}$ within a plausible set of the low energy SUSY parameter space.

${ }^{17}$ We note that in 138 there is a misprint in one of the terms proportional to $m_{W}^{2}$ in the form factor $\Re \mathrm{e}\left(\mathcal{D}_{2(c)}^{L}-\right.$ $\left.\overline{\mathcal{D}}_{2(c)}^{L}\right)$. The correct form of this term is given in Eq. 5.44.
} 


$$
\begin{aligned}
\Re \mathrm{e}\left(\mathcal{D}_{2(a)}^{L}-\overline{\mathcal{D}}_{2(a)}^{L}\right)= & 0, \\
\Re \mathrm{e}\left(\mathcal{D}_{2(b)}^{L}-\overline{\mathcal{D}}_{2(b)}^{L}\right)= & -\frac{\alpha}{\pi \sin ^{2} \theta_{W}} \mathcal{O}_{b}^{1} \Im \mathrm{m} C_{24}^{b}, \\
\Re \mathrm{e}\left(\mathcal{D}_{2(c)}^{L}-\overline{\mathcal{D}}_{2(c)}^{L}\right)= & \frac{1}{2} \frac{\alpha}{\pi \sin ^{2} \theta_{W}}\left[\mathcal { O } _ { c } ^ { 1 } \left(m_{t}^{2} \Im \mathrm{m}\left(C_{22}^{c}-C_{23}^{c}\right)\right.\right. \\
& \left.+m_{W}^{2} \Im \mathrm{m}\left(C_{11}^{c}+C_{21}^{c}-C_{12}^{c}-C_{23}^{c}\right)+2 \Im \mathrm{m} C_{24}^{c}\right) \\
& +m_{t} m_{\tilde{\chi}_{m}} \mathcal{O}_{c}^{2} \Im \mathrm{m}\left(C_{11}^{c}-C_{12}^{c}\right) \\
& -m_{t} m_{\tilde{\chi}_{n}^{0}} \mathcal{O}_{c}^{3} \Im \mathrm{m}\left(C_{0}^{c}+C_{11}^{c}-C_{12}^{c}\right) \\
& \left.-m_{\tilde{\chi}_{m}} m_{\tilde{\chi}_{n}^{0}} \mathcal{O}_{c}^{4} \Im \mathrm{m} C_{0}^{c}\right], \\
\Re \mathrm{e}\left(\mathcal{D}_{2(d)}^{L}-\overline{\mathcal{D}}_{2(d)}^{L}\right)= & \Re \mathrm{e}\left(\mathcal{D}_{2(c)}^{L}-\overline{\mathcal{D}}_{2(c)}^{L}\right)\left(m_{\tilde{\chi}_{n}^{0}} \rightarrow-m_{\tilde{\chi}_{m}}, m_{\tilde{\chi}_{m}} \rightarrow m_{\tilde{\chi}_{n}^{0}},\right. \\
& \left.\mathcal{O}_{c}^{i} \rightarrow \mathcal{O}_{d}^{i}, \Im \mathrm{m} C_{i j}^{c} \rightarrow \Im \mathrm{m} C_{i j}^{d}\right) .
\end{aligned}
$$

Here $\Im \mathrm{m} C_{x}^{k}, x \in\{0,11,12,21,22,23,24\}$ and $k=a-d$, are the imaginary parts, i.e., absorptive parts, of the three-point form factors associated with the 1-loop integrals in diagrams (a)-(d) in Fig. 23. The $C_{x}^{k}$ are given by 138 :

$$
\begin{aligned}
C_{x}^{a} & =C_{x}\left(m_{\tilde{b}_{j}}^{2}, m_{\tilde{t}_{i}}^{2}, m_{G}^{2}, m_{W}^{2}, m_{t}^{2}, m_{b}^{2}\right), \\
C_{x}^{b} & =C_{x}\left(m_{\tilde{b}_{j}}^{2}, m_{\tilde{t}_{i}}^{2}, m_{\tilde{\chi}_{n}^{0}}^{2}, m_{W}^{2}, m_{t}^{2}, m_{b}^{2}\right), \\
C_{x}^{c} & =C_{x}\left(m_{\tilde{\chi}_{n}^{0}}^{2}, m_{\tilde{\chi}_{m}}^{2}, m_{\tilde{b}_{j}}^{2}, m_{W}^{2}, m_{t}^{2}, m_{b}^{2}\right), \\
C_{x}^{d} & =C_{x}\left(m_{\tilde{\chi}_{m}}^{2}, m_{\tilde{\chi}_{n}^{0}}^{2}, m_{\tilde{t}_{i}}^{2}, m_{W}^{2}, m_{t}^{2}, m_{b}^{2}\right),
\end{aligned}
$$

and $C_{x}\left(m_{1}^{2}, m_{2}^{2}, m_{3}^{2}, p_{1}^{2}, p_{2}^{2}, p_{3}^{2}\right)$ is defined in appendix A. The indices $i, j=1,2$ stand for the two stop, sbottom mass eigenstates, respectively, and $m=1,2$ and $n=1-4$ correspond to the two charginos and four neutralinos mass eigenstates, respectively; also, $m_{G}$ is the gluino mass.

The $\mathcal{O}_{k}^{i}$ 's in Eqs. 5.385.45 contain the SUSY CP-odd phases for the decay diagrams and they were given in [138]. There, also the required Feynman rules for calculating the above PRA were given. For example, $\mathcal{O}_{d}^{i}$, for $i=1-4$, containing the SUSY CP-odd phases which appear in diagram $(d)^{18}$ are

$$
\begin{aligned}
\mathcal{O}_{d}^{1} & =-\Im \mathrm{m}\left(K^{-} M^{1}\right), \\
\mathcal{O}_{d}^{2} & =\Im \mathrm{m}\left(K^{-} M^{2}\right), \\
\mathcal{O}_{d}^{3} & =\Im \mathrm{m}\left(K^{+} M^{2}\right), \\
\mathcal{O}_{d}^{4} & =-\Im \mathrm{m}\left(K^{+} M^{1}\right) .
\end{aligned}
$$

Here we have defined

$$
\begin{aligned}
K^{+} & \equiv Z_{N}^{2 n *} Z_{1 m}^{-}+\frac{1}{\sqrt{2}} Z_{N}^{3 n *} Z_{2 m}^{-}, \\
K^{-} & \equiv Z_{N}^{2 n} Z_{1 m}^{+*}-\frac{1}{\sqrt{2}} Z_{N}^{4 n} Z_{2 m}^{+*},
\end{aligned}
$$

\footnotetext{
${ }^{18}$ As will be shown below, in our case this diagram will give rise to the leading contribution to $\mathcal{A}_{3}$.
} 


$$
\begin{aligned}
M^{1} \equiv & \frac{1}{\sqrt{2}} \frac{m_{t}}{M_{W} \sin \beta}\left(Z_{2 m}^{+} L^{+*} \xi_{t}^{i *}-\sqrt{2} Z_{1 m}^{+} Z_{N}^{4 n *} \xi_{t}^{i}\right) \\
& +\frac{1}{\sqrt{2}}\left(\left(\frac{m_{t}}{M_{W} \sin \beta}\right)^{2}\left|Z_{t}^{2 i}\right|^{2} Z_{2 m}^{+} Z_{N}^{4 n *}-\sqrt{2}\left|Z_{t}^{1 i}\right|^{2} Z_{1 m}^{+} L^{+*}\right), \\
M^{2} \equiv & \frac{1}{\sqrt{2}} \frac{m_{t}}{M_{W} \sin \beta}\left(\frac{4}{3} \tan \theta_{W}\left|Z_{t}^{2 i}\right|^{2} Z_{2 m}^{+} Z_{N}^{1 n}+\sqrt{2}\left|Z_{t}^{1 i}\right|^{2} Z_{1 m}^{+} Z_{N}^{4 n}\right) \\
& -\left(\frac{1}{\sqrt{2}}\left(\frac{m_{t}}{M_{W} \sin \beta}\right)^{2} Z_{2 m}^{+} Z_{N}^{4 n} \xi_{t}^{i *}+\frac{4}{3} \tan \theta_{W} Z_{1 m}^{+} Z_{N}^{1 n} \xi_{t}^{i}\right),
\end{aligned}
$$

and

$$
\begin{aligned}
\xi_{t}^{i} & \equiv Z_{t}^{1 i *} Z_{t}^{2 i} \\
L^{ \pm} & \equiv \frac{1}{3} \tan \theta_{W} Z_{N}^{1 n} \pm Z_{N}^{2 n}
\end{aligned}
$$

In Eqs. 5.54 5.59, $Z_{t}, Z_{N}$ and $Z^{-}, Z^{+}$are the mixing matrices of the stops, neutralinos and charginos, respectively (i.e., with indices $i, n$ and $m$ ), which are defined in section 3.3.2.

Obviously, to obtain an estimate of the numerical value of the asymmetry, one needs to know the definite form for the mixing matrices and various other parameters. Not knowing these makes it very difficult to give a reliable quantitative prediction for the asymmetry. Therefore, one has to choose a reference set of the SUSY spectrum subject to theoretically motivated assumptions as well as experimental data. Such a reference set which constructs a plausible low energy MSSM framework was described in 138 (and is also described in section 3.3.4). The key assumptions made there are:

- There is an underlying grand unification which leads to the relation in Eq. 3.137 between $U(1)$ and $S U(2)$ gaugino masses and the gluino mass.

- All squarks except the lighter stop (with a mass denoted hereafter by $m_{l}$ ) are degenerate with a mass $M_{S}$; in the analysis below we set $M_{S}=400 \mathrm{GeV}$.

- The gluino mass is varied subject to $m_{G}>250 \mathrm{GeV}$ 139.

- The parameters are chosen subject to the upper limit on the NEDM, $d_{n}<1.1 \times 10^{-25}$ e-cm [3]. In particular, the Higgs parameter $\mu$ is chosen to be real as strongly implied from this upper bound on the NEDM when the squark masses are below $\sim 1 \mathrm{TeV}$.

With the above criteria one is left with only one CP-odd phase arising from $\tilde{t}_{L}-\tilde{t}_{R}$ mixing. That is, when $\mu$ is real all the elements in $\mathcal{O}_{k}^{i}$ above except from $\xi_{t}^{i}$, defined in Eq. 5.58, are real. Recall that the stop squarks of different handedness are related to their mass eigenstates $\tilde{t}_{+}, \tilde{t}_{-}$through the following transformations (see section 3.3.2)

$$
\begin{aligned}
\tilde{t}_{L} & =\cos \theta_{t} \tilde{t}_{-}-e^{-i \beta_{t}} \sin \theta_{t} \tilde{t}_{+}, \\
\tilde{t}_{R} & =e^{i \beta_{t}} \sin \theta_{t} \tilde{t}_{-}+\cos \theta_{t} \tilde{t}_{+} .
\end{aligned}
$$

The asymmetry is thus proportional to the quantity (see also Eqs. 3.126 and 4.43)

$$
\xi_{C P}^{t} \equiv 2\left|\xi_{t}^{i}\right|=\sin \left(2 \theta_{t}\right) \sin \left(\beta_{t}\right),
$$

where $\xi_{t}^{i}$ is defined in Eq. 5.58. 
Although the CP-odd phases in the squarks sector generate the NEDM, the resulting restrictions on the CP-phases in the $\tilde{t}_{L}-\tilde{t}_{R}$ mixing are rather weak. As we have demonstrated in section 3.3.4, the main contribution to the NEDM (when $\mu$ is real) comes from the mixing of the superpartners of the lighter squarks. Therefore, if the trilinear soft breaking terms $A_{u}, A_{d}$ and $A_{t}$ are not correlated at the EW-scale, as is the case in our low energy MSSM framework described in section 3.3.4, then it is not unreasonable to study the effects of maximal CP violation in the stop sector, i.e., $\xi_{C P}^{t}=1$ without contradicting the current limit on the NEDM.

With no further assumptions, the reference parameter set consists of $M_{S}, m_{l}, m_{G}, \mu, \tan \beta$ and $\xi_{C P}^{t}$. The neutralinos and charginos masses are extracted by diagonalizing the corresponding mass matrices which are functions of $\mu, m_{G}$ and $\tan \beta$ (see section 3.3.2). Note that the consequences of such a low energy MSSM scenarios on the various diagrams in Fig. 23 that can potentially contribute to the PRA, $\mathcal{A}_{3}$, are:

- For $m_{G} \gtrsim 250 \mathrm{GeV}$ diagram (a) does not have the needed absorptive cut and thus does not contribute to the PRA.

- Diagram (c) does not have a CP-violating phase when $\arg (\mu)=0$.

This simplifies our discussion to a great extent and we are therefore left with only two diagrams that can contribute to $\mathcal{A}_{3}$. These are diagrams (b) and (d), where in fact we find that, by far, the leading contribution comes from diagram (d). In particular, we have calculated the PRA effect, $\mathcal{A}_{3}$, arising from diagrams (b) and (d), for $\arg (\mu)=0, m_{\tilde{q}}=M_{S}=400 \mathrm{GeV}$ and subject to $m_{l}>50 \mathrm{GeV}, m_{G}>250 \mathrm{GeV}$, the LSP (in our case the neutralino) mass to be above $20 \mathrm{GeV}$ and the mass of the lighter chargino to be above $65 \mathrm{GeV}$.

In Figs. 24 and 25 we plot $\mathcal{A}_{3}$ for two values of $\tan \beta$ which correspond to a low $(\tan \beta=1.5)$ and high $(\tan \beta=35) \tan \beta$ scenarios, where the SUSY mass parameters are varied subject to all the above constraints and maximal $\mathrm{CP}$ violation is taken in the sense that $\xi_{C P}^{t}=1$, thus presenting $\mathcal{A}_{3}$ in units of $\sin 2 \theta_{t} \sin \beta_{t}$. In particular, in Figs. 24(a) and 24(b) we plot the asymmetry as a function of $\mu$ for several values of $m_{G}$ and for $\tan \beta=1.5$ and $\tan \beta=35$, respectively. In Figs. 25(a) and 25(b) the asymmetry is plotted as a function of the gluino mass $m_{G}$ for several values of $\mu$ and for $\tan \beta=1.5$ and $\tan \beta=35$, respectively. In both figures we set $M_{S}=400 \mathrm{GeV}$ and $m_{l}=50 \mathrm{GeV}$. Evidently, from Figs. 24 and 25 we see that a PRA in $t \rightarrow b W$ is very small over the whole range of our SUSY parameter space. In particular, we always find

$$
\left|\mathcal{A}_{3}\right|<0.3 \% \text {. }
$$

Of course the asymmetry further drops as the mass of the lighter stop, $m_{l}$, is increased and vanishes when $m_{l} \gtrsim 130 \mathrm{GeV}$ since in that case there is no absorptive cut in the relevant contributing diagrams. Also, we find that the PRA is almost insensitive to $\tan \beta$ in the range $\tan \beta \gtrsim 10$ and that $\mathcal{A}_{3} \sim 0.3 \%$ become possible only for $\tan \beta \sim \mathcal{O}(1)$.

The asymmetry we find is therefore somewhat small compared to the estimates of Grzadkowski and Keung (GK) [151] and of Christova and Fabbrichesi (CF) [162. In the GK limit only the gluino exchange of diagram (a) was considered. They utilized the CP-violating, quarksquark-gluino interaction, occurring with coupling strength $g_{s}$ (the QCD coupling) and the $W \tilde{t} \tilde{b}$ interaction

$$
\begin{aligned}
\mathcal{L}_{\tilde{q} q \lambda}= & i \sqrt{2} g_{s}\left[\tilde{t}_{L}^{*} T^{a}\left(\bar{\lambda}^{a} t_{L}\right)+\tilde{t}_{R}^{*} T^{a}\left(\bar{\lambda}^{a} t_{R}\right)\right]+(t \leftrightarrow b) \\
& -\frac{i g_{W}}{\sqrt{2}} V_{t b} \tilde{b}_{L}^{+} \stackrel{\leftrightarrow}{\partial}_{\mu} \tilde{t}_{L} W^{-\mu}+\text { h.c. }
\end{aligned}
$$

As in our case, the most important source of CP violation is then the phase in the $\tilde{t}_{L}-\tilde{t}_{R}$ mixing and, therefore, their effect is also proportional to $\xi_{C P}^{t}$ defined above. However, the GK limit is 
applicable only if $m_{t}>m_{G}+m_{\tilde{t}}$, so that an absorptive cut can occur in diagram (a). In the best case, GK found a $\sim 1 \%$ asymmetry for $m_{G}=m_{\tilde{b}}=100 \mathrm{GeV}$.

On the other hand, in the CF limit, numerical results were given only for the neutralino exchange diagram (i.e., diagram (b)) wherein the CP-odd phase was chosen to be proportional to $\arg (\mu)$ and maximal $\mathrm{CP}$ violation with regard to $\arg (\mu)$ was taken. This can be parameterized by introducing a single CP-violating phase [162]:

$$
f_{n}^{b} N_{n 4}^{*} \simeq \frac{1}{2} \sin \delta_{C P}
$$

where $f_{k}^{b}$ and $N_{k 4}$ appear in the $\tilde{q} q \tilde{\chi}_{n}^{0}$ Lagrangian

$$
\begin{aligned}
L_{\tilde{q} q \tilde{\chi}^{0}}= & \frac{1}{2} g_{W} \sum_{n, f} \bar{q}_{f}\left[f_{n}^{f}\left(1+\gamma_{5}\right)-\frac{\sqrt{2} m_{f}}{2 m_{W} B_{f}} N_{n, 5-f}^{*}\left(1-\gamma_{5}\right)\right] \tilde{\chi}_{n}^{0} \tilde{q}_{f L} \\
& +\frac{1}{2} g_{W} \sum_{n, f} \bar{q}_{f}\left[g_{n}^{f}\left(1-\gamma_{5}\right)-\frac{\sqrt{2} m_{f}}{2 m_{W} B_{f}} N_{n, 5-f}^{*}\left(1+\gamma_{5}\right)\right] \tilde{\chi}_{n}^{0} \tilde{q}_{f R} \\
& + \text { h.c. },
\end{aligned}
$$

and are defined, together with $g_{n}^{f}$ and $B_{f}$, in 162. For maximal CP violation, i.e., $\sin \delta_{C P}=1$, and with $m_{\tilde{q}}=m_{\tilde{\chi}^{+}}=100 \mathrm{GeV}$ and $m_{\tilde{\chi}^{0}}=18 \mathrm{GeV}$, CF find $\mathcal{A}_{3} \simeq 2 \%$. So an asymmetry in the main two-body mode, $t \rightarrow b W$, of a few percent can occur in their limit.

However, these relatively large PRA's, reported by GK and CF in 151, 162 suffer from the following drawbacks:

- For the GK limit, $m_{G}+m_{\tilde{t}}<m_{t}$ is now essentially disallowed by the current experimental bounds.

- For the CF limit, $\arg (\mu) \gtrsim 10^{-2}$ is an unnatural choice in view of the stringent constraints on this phase coming from the experimental limits on the NEDM as discussed in section 3.3 .4 .

- For both the GK and CF limits, the large asymmetry arises once the masses of the superpartners of the light quarks are set to $100 \mathrm{GeV}$. Again, this is a rather unnatural choice as it is theoretically very hard, if at all possible, to meet the NEDM experimental limits when the masses of the squarks (except for the lighter stop) are of the order of $100 \mathrm{GeV}$. Besides, the current experimental limits disfavor down squarks lighter than about $200 \mathrm{GeV}$.

We also remark that PRA in $t \rightarrow b W$ within the more constrained $\mathrm{N}=1$ SUGRA model was investigated in [164], where similar numerical results (i.e. $\mathcal{A}_{3}<0.3 \%$ ) for $\mathcal{A}_{3}$ were obtained.

To conclude this section, although PRA's in the range of $\sim 10^{-3}-10^{-2}$ in the main two-body decays of $t, \bar{t}$ are appreciable, their measurements is likely to be very hard. Presumably an $e^{+} e^{-}$ collider (NLC) could be suitable due to its cleanliness. However, there may be about 10,000 to 50,000 t $\bar{t}$ events a year. Therefore, bearing experimental efficiency factors, under the best of circumstances only an asymmetry of the order a few percent could be measured in the NLC. The LHC, being able to produce $10^{7}-10^{8} t \bar{t}$ pairs, might seem more appropriate for a measurement of such a small PRA. However, for a measurement of a $\sim 0.3 \%$ asymmetry, experimental systematics can pose serious limitations.

\subsubsection{PRA within the form factor approach}

$\underline{t \rightarrow b W}$ 
The basic idea in the form factor approach is to write a model independent coupling, then investigate the dependence of various asymmetries on the form factors involved [148, 21, 149, 165, 166, 167. Thus one can write the amplitude for $t \rightarrow b W^{+}$as the sum

$$
\mathcal{M}_{t b W} \equiv \mathcal{M}_{t b W}^{0}+\mathcal{M}_{t b W}^{1},
$$

where $\mathcal{M}_{t b W}^{0}$ is the amplitude at the lowest order in the SM which is given by

$$
\mathcal{M}_{t b W}^{0}=-\frac{g_{W}}{\sqrt{2}} V_{t b} \epsilon_{\mu}^{*}\left(p_{W^{+}}\right) \bar{u}_{t}\left(p_{b}\right) \gamma^{\mu} L u_{t}\left(p_{t}\right) .
$$

In this equation $\epsilon_{\mu}\left(p_{W^{+}}\right)$is the polarization vector of $W^{+}$with four momentum $p_{W^{+}}$and $p_{b}, p_{t}$ are the four momenta of $b, t$, respectively. $\mathcal{M}_{t b W}^{1}$ contains the new CP-violating interactions and can be written in general (for on-shell $W^{+}$and in the limit $m_{e}=0$ ) as follows

$$
\mathcal{M}_{t b W}^{1}=-\frac{g_{W}}{\sqrt{2}} V_{t b} \epsilon_{\mu}^{*}\left(p_{W^{+}}\right) \bar{u}_{b}\left(p_{b}\right)\left\{\sum_{P=L, R}\left(f_{1}^{P} \gamma^{\mu} P+i \frac{f_{2}^{P}}{m_{W}} \sigma_{\nu}^{\mu} p_{W^{+}}^{\nu} P\right)\right\} u_{t}\left(p_{t}\right)
$$

where $P=L$ or $R, L(R)=\left(1-(+) \gamma_{5}\right) / 2$ and the form factors $f_{1}^{P}$ and $f_{2}^{P}$ are complex in general; they can both have an absorptive phase and a CP-violating phase. Note also that, in the SM, $f_{1}^{L}=1 ; f_{1}^{R}=f_{2}^{L}=f_{2}^{R}=0$, at tree level.

Similarly, the non-standard part of the amplitude for $\bar{t} \rightarrow \bar{b} W^{-}$is defined as

$$
\overline{\mathcal{M}}_{t b W}^{1}=-\frac{g_{W}}{\sqrt{2}} V_{t b}^{*} \epsilon_{\mu}\left(p_{W^{-}}\right) \bar{v}_{t}\left(p_{\bar{t}}\right)\left\{\sum_{P=L, R}\left(\bar{f}_{1}^{P} \gamma^{\mu} P+i \frac{\bar{f}_{2}^{P}}{m_{W}} \sigma_{\nu}^{\mu} p_{W^{-}}^{\nu} P\right)\right\} v_{b}\left(p_{\bar{b}}\right)
$$

In general the form factors $f_{i}^{P}$ and $\bar{f}_{i}^{P}$ can be further simplified to the form

$$
\begin{aligned}
f_{i}^{P} & \equiv f_{i, C P C}^{P} \times f_{i, C P V}^{P}, \\
\bar{f}_{i}^{P} & \equiv \bar{f}_{i, C P C}^{P} \times \bar{f}_{i, C P V}^{P},
\end{aligned}
$$

where the indices $C P C$ and $C P V$ stand for the CP-conserving and CP-violating parts in the above form factors. In particular, $f_{i, C P C}^{P}, \bar{f}_{i, C P C}^{P}$ can be complex due to an absorptive phase (FSI phase), and $f_{i, C P V}^{P}, \bar{f}_{i, C P V}^{P}$ are complex in the presence of a non-zero CP-violating phase.

In terms of the $\mathrm{CP}$-conserving and $\mathrm{CP}$-violating parts of these form factors in Eqs. 5.70 and 5.71, it is useful to note that the following relations exist (between the form factors associated with $t \rightarrow b W^{+}$and those related to $\bar{t} \rightarrow \bar{b} W^{-}$)

$$
\begin{array}{rll}
f_{1, C P C}^{L}=\bar{f}_{1, C P C}^{L} \quad \text { and } & f_{1, C P C}^{R}=\bar{f}_{1, C P C}^{R}, \\
f_{1, C P V}^{L}=\left(\bar{f}_{1, C P V}^{L}\right)^{*} \quad \text { and } & f_{1, C P V}^{R}=\left(\bar{f}_{1, C P V}^{R}\right)^{*}, \\
f_{2, C P C}^{L}=\bar{f}_{2, C P C}^{R} \quad \text { and } & f_{2, C P C}^{R}=\bar{f}_{2, C P C}^{L}, \\
f_{2, C P V}^{L}=\left(\bar{f}_{2, C P V}^{R}\right)^{*} \quad \text { and } & f_{2, C P V}^{R}=\left(\bar{f}_{2, C P V}^{L}\right)^{*} .
\end{array}
$$

Using the relations above it is then easy to show that any CP-violating observable must always be proportional to any one of the combinations: $\left(f_{1}^{L}-\bar{f}_{1}^{L}\right),\left(f_{1}^{R}-\bar{f}_{1}^{R}\right),\left(f_{2}^{L}-\bar{f}_{2}^{R}\right)$ or $\left(f_{2}^{R}-\bar{f}_{2}^{L}\right)$. In particular, a CP-odd, $T_{N}$-even quantity (like the PRA) will be proportional to the real parts of these combinations, e.g., $\Re \mathrm{e}\left(f_{2}^{L}-\bar{f}_{2}^{R}\right)$, but a CP-odd, $T_{N^{-}}$odd quantity will be proportional to their imaginary parts, e.g., $\Im \mathrm{m}\left(f_{2}^{L}-\bar{f}_{2}^{R}\right)$. 
Assuming these form factors to be purely CP-violating, i.e., $\Re \mathrm{e}\left(f_{i, C P V}^{P}\right)=0$ and $\Re \mathrm{e}\left(\bar{f}_{i, C P V}^{P}\right)=$ 0, the PRA defined in Eq. 5.4 for $t \rightarrow b W$ can be expressed as

$$
\mathcal{A}_{3}=\sum_{i=1}^{2} \sum_{P=L, R} a_{i}^{P} \Re \mathrm{e}\left(f_{i}^{P}\right) .
$$

In this context it was found that $\left[\right.$ [148] $a_{1}^{L} \simeq 0.7, a_{1}^{R} \simeq-0.04, a_{2}^{L} \simeq 0.04$ and $a_{2}^{R} \simeq-0.7$. We thus see that the PRA is more sensitive to $f_{1}^{L}$ and $f_{2}^{R}$ than to $f_{1}^{R}$ and $f_{2}^{L}$.

\footnotetext{
${ }^{19}$ Note the slight difference between our definition of the form factors $f_{i}^{P}, \bar{f}_{i}^{P}$ and the definition presented in [148].
} 


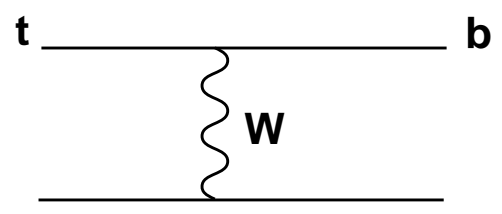

(1)

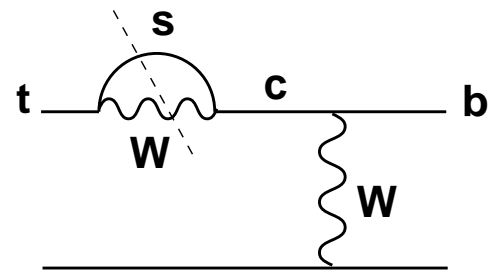

(3)

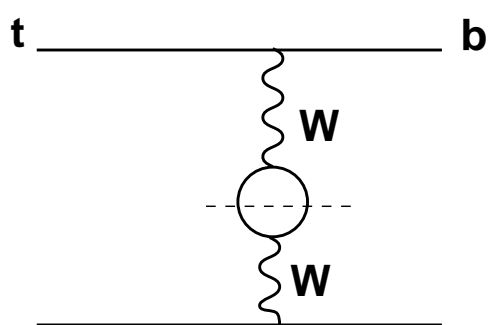

(2)

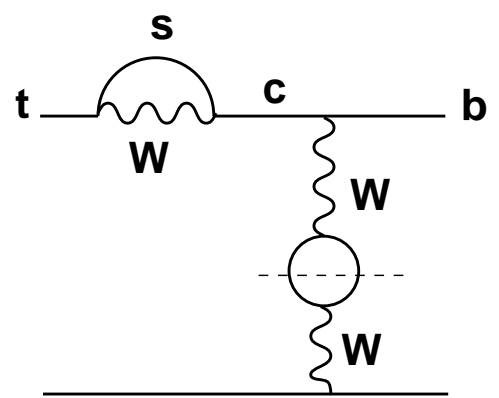

(4)

Figure 21: The four diagrams considered in the proof that the $W$ self-energy diagram does not contribute to $C P$ violation for $t \rightarrow d_{k} W$, within the SM. Dashed lines indicate cuts. Similar considerations hold for other models. 


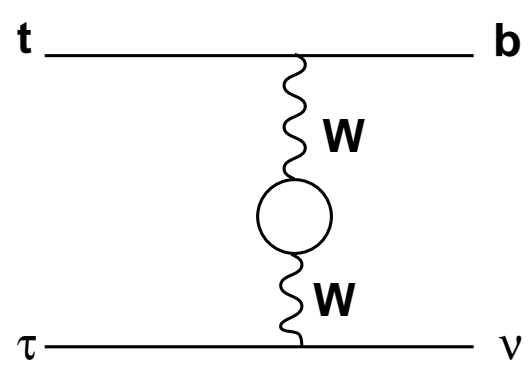

(a)

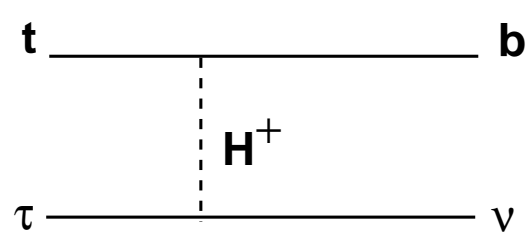

(c)

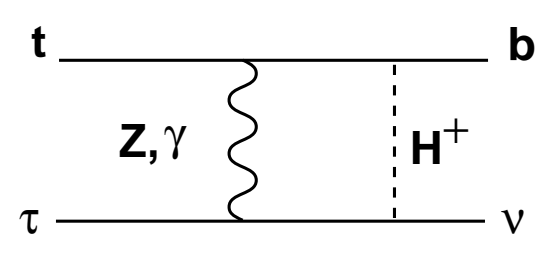

(b)

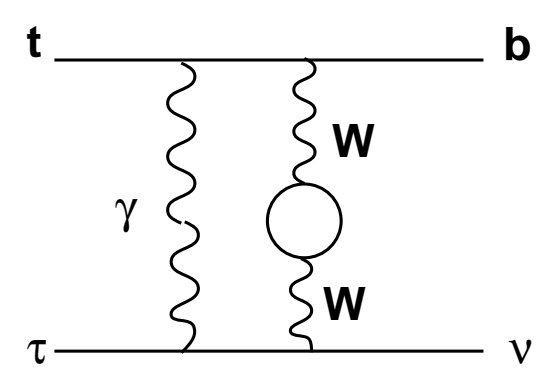

(d)

Figure 22: Tree-level diagrams and a representative set of box diagrams considered for PRA in $t \rightarrow b \tau \nu$, within the 3HDM. 


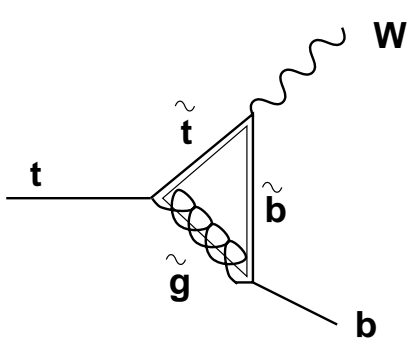

(a)

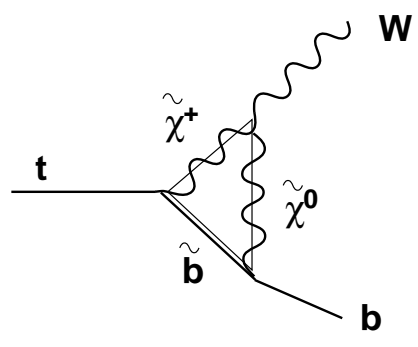

(c)

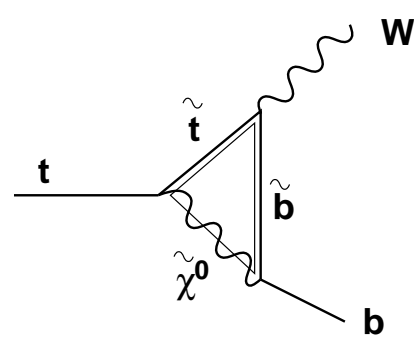

(b)

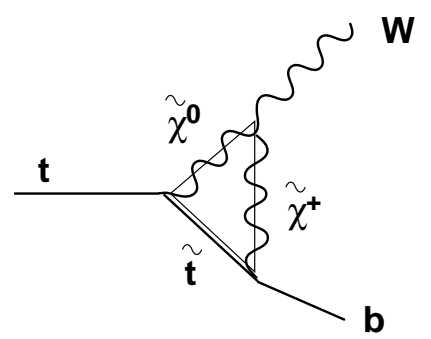

(d)

Figure 23: The SUSY induced 1-loop Feynman diagrams that contribute to CP violation in the main top decay $t \rightarrow b W$. $\tilde{\chi}$ is the chargino, $\tilde{\chi}^{0}$ is the neutralino, $\tilde{g}$ is the gluino and $\tilde{t}, \tilde{b}$ are the stop and sbottom particles, respectively. 

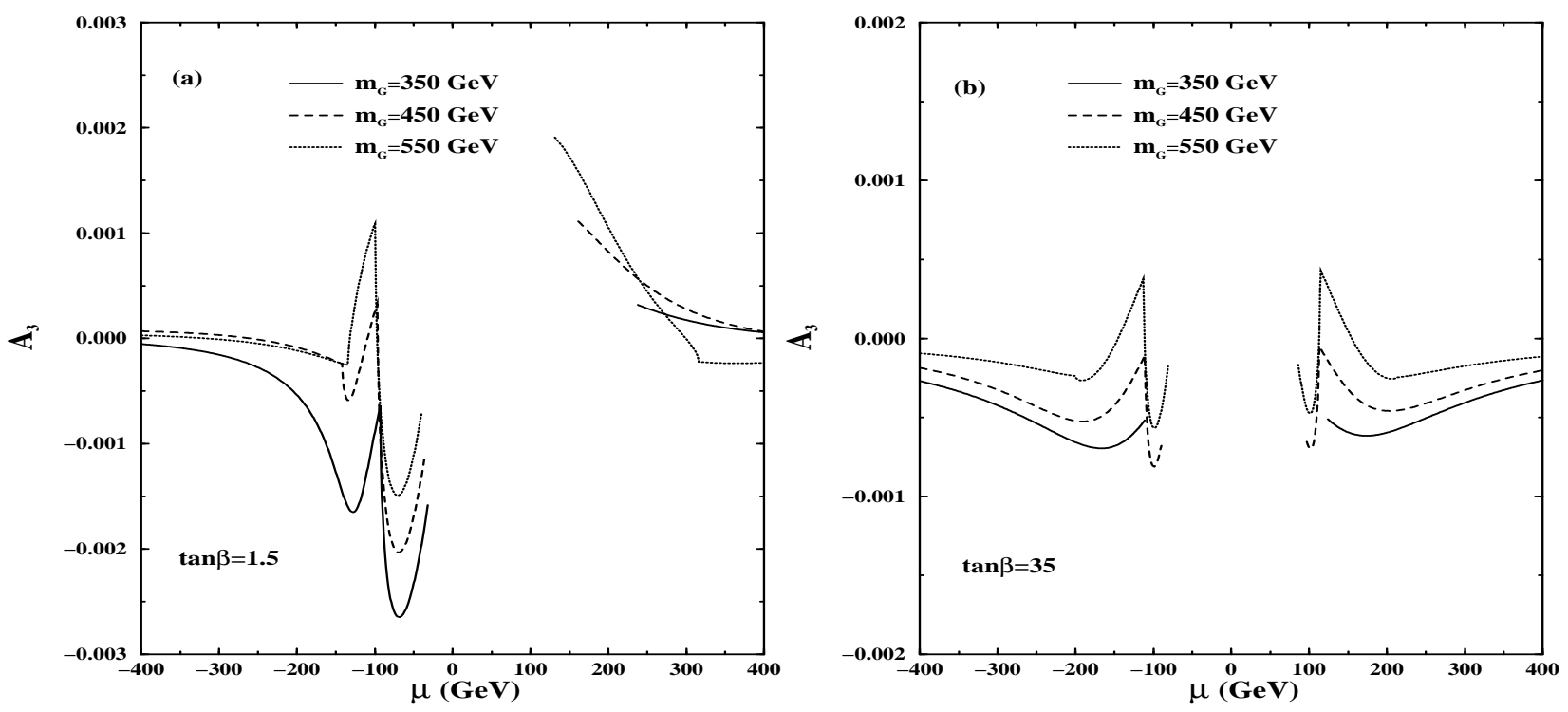

Figure 24: The SUSY induced PRA $\mathcal{A}_{3}$ in the main top decay $t \rightarrow b W$, as a function of $\mu$, for several values of $m_{G}$ and for (a) $\tan \beta=1.5$ and (b) $\tan \beta=35 . M_{S}=400 \mathrm{GeV}, m_{l}=50 \mathrm{GeV}$ is used. Figure taken from [138]. 

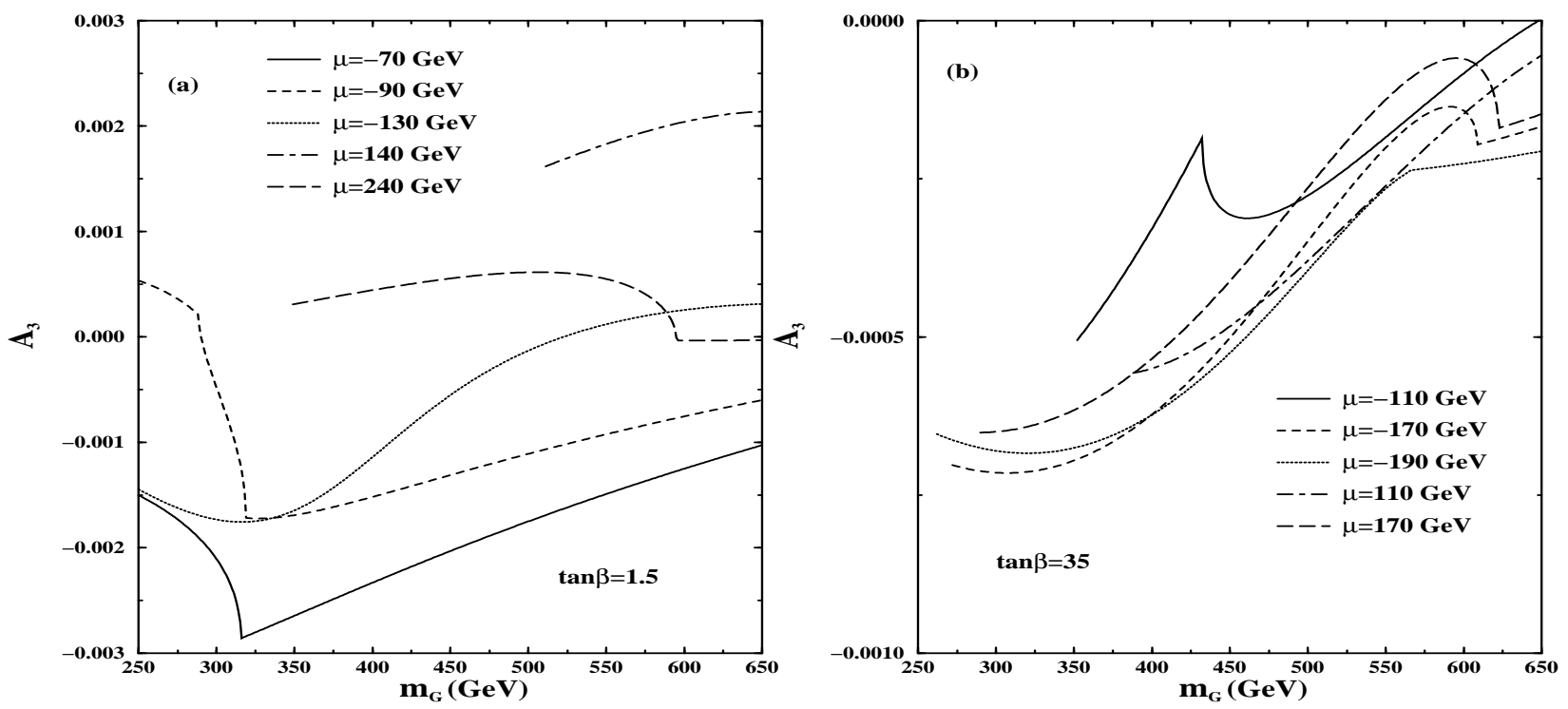

Figure 25: The SUSY induced PRA $\mathcal{A}_{3}$ in the main top decay $t \rightarrow b W$, as a function of $m_{G}$, for several values of $\mu$ and for (a) $\tan \beta=1.5$ and $(b) \tan \beta=35 . M_{S}=400 \mathrm{GeV}, m_{l}=50 \mathrm{GeV}$ is used. Figure taken from [138]. 


\begin{tabular}{cccccc}
\hline \hline & & & & & \\
$m_{H^{+}}$ & $\tilde{s}_{1}$ & $\tilde{s}_{2}$ & $\tilde{s}_{3}$ & $\mathcal{A}_{+}$ & $\left(\mathcal{A}_{+}^{2} \mathcal{B}_{+}\right)^{-1}$ \\
\hline 200 & 0.252 & $8.29 \times 10^{-3}$ & 0.707 & $2.9 \times 10^{-3}$ & $3.0 \times 10^{6}$ \\
300 & 0.210 & $9.99 \times 10^{-3}$ & 0.707 & $1.5 \times 10^{-3}$ & $1.2 \times 10^{7}$ \\
\hline \hline
\end{tabular}

Table 4: Results for the PIRA $\mathcal{A}_{+}$, see Eq. 5.78, and $\left(\mathcal{A}_{+}^{2} \mathcal{B}_{+}\right)^{-1}$, in $t \rightarrow b \tau \nu_{\tau}$ within the Weinberg model for CP violation. $\tilde{s}_{i} \equiv \sin \left(\tilde{\theta}_{i}\right)$, where $\tilde{\theta}_{i}, \delta_{H}=\pi / 2$, are CKM-like angles chosen to maximize the charged Higgs coupling $\Im \mathrm{m}(U)$ (see Eq. 5.22). Note $\left(\mathcal{A}_{+}^{2} \mathcal{B}_{+}\right)^{-1}$ is the number of $t \bar{t}$ pairs required to observe the asymmetry to $1-\sigma . \mathcal{B}_{+} \simeq 0.04$ is the appropriate branching fraction. Table taken from [49], updated to $m_{t}=180 \mathrm{GeV}$.

\subsection{Partially integrated rate asymmetries}

In the rest of the chapter we will discuss $\mathrm{CP}$ asymmetries for the $t \rightarrow b \tau \nu_{\tau}$ decay within the 3HDM, starting with PIRA. This asymmetry is defined as follows

$$
\mathcal{A}_{P I R A} \equiv \frac{\Gamma_{P I}\left(t \rightarrow b \tau^{+} \nu_{\tau}\right)-\Gamma_{P I}\left(\bar{t} \rightarrow \bar{b} \tau^{-} \bar{\nu}_{\tau}\right)}{\Gamma_{P I}\left(t \rightarrow b \tau^{+} \nu_{\tau}\right)+\Gamma_{P I}\left(\bar{t} \rightarrow \bar{b} \tau^{-} \bar{\nu}_{\tau}\right)},
$$

where $\Gamma_{P I}$ stands for the partially integrated width, i.e., the width obtained by integrating over only a part of phase-space, rather than over the full kinematic range available to $u$ and $q^{2}$, defined in Eq. 5.28. It is easy to see that, unlike the PRA, the PIRA is non-vanishing even for $m_{f} \rightarrow 0(f \neq \tau)$. The reason is that in the calculation of the PRA, when the integration over the full range of $u$ is performed, $p_{\tau}^{\mu}$ from the $\tau \nu_{\tau}$ loop sandwiched between the $W$ and the $H^{+}$, necessarily gets replaced by $q^{\mu}$. But then, the contribution of the transverse part of the $W$-propagator (i.e., $\left.G_{T}\right)$ vanishes since $q^{\mu} \rho_{T}^{\mu \nu}=0\left(\rho_{T}^{\mu \nu} \equiv-g^{\mu \nu}+q^{\mu} q^{\nu} / q^{2}\right)$. On the other hand, when we calculate the PIRA, then the relevant integration is over only a part of the full kinematic range of $u$ which allows $G_{T}$ to contribute even to the $W^{+}$-tree $\times H^{+}$-tree interference.

Let us consider the integration over $u$ for a fixed $q^{2}$ in the rest frame of the $W$-boson, i.e., $\vec{q}=0$. The integration over $u$ is now equivalent to that over the angle $\theta$ between $\left(-\vec{p}_{\tau}\right)$ and $\vec{p}_{b}$. Define the PIRA over positive values of $\cos \theta$ to be $\mathcal{A}_{+}$. Then, explicit calculation of the $W^{+}$-tree $\times H^{+}$-tree interference (i.e., diagrams (a) and (c) in Fig. 22] yields [42]:

$$
\mathcal{A}_{+}=\frac{\sqrt{2}}{4 \pi} \frac{G_{F} m_{\tau}^{2} r_{W H} \Im \mathrm{m}(U)}{\left(2+r_{W t}\right)\left(1-r_{W H}\right) \mathcal{B}\left(W \rightarrow \tau \nu_{\tau}\right)},
$$

where $\Im \mathrm{m}(U)$ is defined in Eq. 5.22, $r_{W t}=m_{W}^{2} / m_{t}^{2}$ and $r_{W H}=m_{W}^{2} / m_{H}^{2}$. For $200 \lesssim m_{H} \lesssim 300$ $\mathrm{GeV}, \mathcal{A}_{+} \sim$ a few $\times 10^{-3}$ (see Table $\mathbb{1}$ ) so it is enhanced by two orders of magnitude over the PRA, $\mathcal{A}_{\tau}$, in Eq. 5.23. Even larger asymmetries are likely for $m_{H}<m_{t}$.

A related PIRA was investigated in [168]. There, a PRA for $t \rightarrow b \tau \nu_{\tau}$ was defined, specifically for $g g \rightarrow \bar{t} t$ in a hadron collider. The imposition of experimental cuts (for details see [168]), turns the asymmetry into PIRA. Looking for the maximal CP-violating effect by choosing the most favorable values for the three CKM-like angles and $\delta_{H}=\pi / 2$, subject to experimental constraints, asymmetries of the order of a few $\times 10^{-4}$ were obtained from tree $\times$ tree interference with a resonant $W$.

\section{$5.3 \quad$ Energy asymmetry}

Another explicit example of how an interesting CP-violating asymmetry can be sizable even when the PRA is vanishingly small is the energy asymmetry. Specifically, let us define [42]: 


$$
\mathcal{A}_{E}=\frac{\left\langle E_{\tau^{+}}\right\rangle-\left\langle E_{\tau^{-}}\right\rangle}{\left\langle E_{\tau^{+}}\right\rangle+\left\langle E_{\tau^{-}}\right\rangle}
$$

where $\left\langle E_{\tau^{+}}\right\rangle$is the average of the $\tau^{+}$energy in $t \rightarrow b \tau^{+} \nu$, etc. In the calculation of $\left\langle E_{\tau^{+}}\right\rangle$the integrand is of course equal to that for the PRA with just an additional factor of $E_{\tau^{+}}$. Now $G_{T}$ does contribute even when the integration is over the full range of $u$. Explicitly one finds

$$
\mathcal{A}_{E}=\frac{\sqrt{2}}{12 \pi} \frac{G_{F} m_{\tau}^{2} r_{W H}\left(1-r_{W t}\right) \Im \mathrm{m}(U)}{\left(1+3 r_{W t}^{2}+2 r_{W t}\right)\left(1-r_{W H}\right)\left[B\left(W \rightarrow \tau \nu_{\tau}\right)\right]} .
$$

The energy asymmetry is closely related to the PIRA and in fact numerically [42]:

$$
\mathcal{A}_{E} \sim \mathcal{A}_{+} / 3
$$

Indeed, both are weighted CP-odd observables constructed from the outgoing momenta. Observables constructed in this way have the drawback that they are proportional to $m_{\tau}$. This factor of $m_{\tau}$ is in addition to a factor of $m_{\tau}$ in the Yukawa coupling. The latter cannot be dispensed with, as long as we are dealing with $t \rightarrow b \tau \nu$. However, the additional power of $m_{\tau}$ entering these asymmetries can be overcome by examining the transverse polarization of the $\tau$ as we will discuss next.

\section{$5.4 \tau$-polarization asymmetry}

The advantage of using a polarization asymmetry over an energy or a rate asymmetry is that the latter asymmetries go as $m_{\tau}^{2} / m_{H}^{2}$, where one power of $m_{\tau}$ comes from the Yukawa coupling at the $H \tau \nu_{\tau}$ vertex. The second power of $m_{\tau}$ comes from the trace over the lepton loop in $W^{+}{ }_{-} H^{+}$ interference, i.e.,

$$
\operatorname{Tr}\left[\gamma^{\mu}\left(\not p_{\tau}+m_{\tau}\right)\left(1-\gamma_{5}\right) \not p_{\nu}\right]=4 m_{\tau} p_{\nu}^{\mu}
$$

The only way to avoid this power of $m_{\tau}$ is to avoid summing over the spin $\left(s_{\tau}\right)$ of $\tau$ in the preceding trace. Then the trace will take the form

$$
\operatorname{Tr}\left[\gamma^{\mu}\left(\not p_{\tau}+m_{\tau}\right)\left(1+\gamma_{5} \not \phi_{\tau}\right)\left(1-\gamma_{5}\right) \not p_{\nu}\right] \stackrel{m_{\tau} \rightarrow 0}{\longrightarrow} 4 i \epsilon\left(\mu, s_{\tau}, p_{\tau}, p_{\nu}\right) \ldots
$$

Thus the $W^{+}$-tree $\times H^{+}$-tree interference will make a contribution to the transverse polarization of the $\tau$, i.e., to $\vec{s}_{\tau} \cdot\left(\vec{p}_{\tau} \times \vec{p}_{\nu}\right)$ without suffering a suppression by an additional power of $m_{\tau}$ (i.e. in addition to the Yukawa coupling) so this asymmetry will be enhanced over the PIRA and energy asymmetries by a factor of about $m_{t} / m_{\tau} \sim 100$ !

We will consider the following $\mathrm{CP}$ asymmetries that involve the $\tau$ polarization [114]:

$$
\begin{aligned}
& \mathcal{A}_{y} \equiv \frac{\tau^{+}(\uparrow)-\tau^{+}(\downarrow)+\tau^{-}(\uparrow)-\tau^{-}(\downarrow)}{\tau^{+}(\uparrow)+\tau^{+}(\downarrow)+\tau^{-}(\uparrow)+\tau^{-}(\downarrow)}, \\
& \mathcal{A}_{z} \equiv \frac{\tau^{+}(\uparrow)-\tau^{+}(\downarrow)-\tau^{-}(\uparrow)+\tau^{-}(\downarrow)}{\tau^{+}(\uparrow)+\tau^{+}(\downarrow)+\tau^{-}(\uparrow)+\tau^{-}(\downarrow)},
\end{aligned}
$$

where for $\mathcal{A}_{y}\left(\mathcal{A}_{z}\right)$ the arrows indicate the spin up or down in the direction $y(z)$. The reference frame is defined to be the $\tau$ rest frame, such that the $t$ momentum is in the $-x$ direction (i.e. the $x$ axis is the boost axis from the top to the $\tau$ frame), the $y$ axis is defined to be in the decay plane with a positive $y$ component for the $b$ momentum. The $z$ axis is defined by the right-hand rule. Also, $\mathcal{A}_{y}\left(\mathcal{A}_{z}\right)$ is $\mathrm{CP}$-odd, $T_{N}$-even (CP-odd, $T_{N}$-odd), and is therefore proportional to the absorptive (dispersive) part of the bubble in the $W$-propagator. When integrated over the entire phase-space, $\mathcal{A}_{y}$ and $\mathcal{A}_{z}$ give 


$$
\begin{gathered}
\mathcal{A}_{y}=-\frac{9}{64} \frac{g_{W}^{2} \Im \mathrm{m}(U) \sqrt{x_{\tau} x_{W}}}{\left(1+2 x_{W}\right)\left(x_{H}-x_{W}\right)} \\
\mathcal{A}_{z}=\frac{9}{64 \pi} \frac{g_{W}^{2} \Im \mathrm{m}(U) \sqrt{x_{l}}}{\left(1-x_{W}\right)^{2}\left(1+2 x_{W}\right) x_{H}} f\left(x_{W}, y_{W}, x_{H}\right) .
\end{gathered}
$$

Recall that $\Im \mathrm{m}(U)$ is given by Eqs. 5.20, 5.21 and 5.22, $x_{j} \equiv m_{j}^{2} / m_{t}^{2}$ and $y_{W} \equiv \Gamma_{W}^{2} / m_{t}^{2}$. Also, $f$ is defined as the integral

$$
f\left(x_{W}, y_{W}, x_{H}\right) \equiv \int_{0}^{1} \frac{\left(\lambda-x_{W}\right) x_{H}(1-\lambda) \sqrt{\lambda}}{\left[\left(\lambda-x_{W}\right)^{2}+x_{W} y_{W}\right]\left(x_{H}-\lambda\right)} d \lambda,
$$

and $\lambda \equiv\left(p_{e}+p_{\nu}\right)^{2} / m_{t}^{2}$.

A fully integrated polarization asymmetry as in Eq. 5.87, but weighting events differently for different ranges of the $\tau \nu_{\tau}$ invariant mass, was also given in 114:

$$
\mathcal{A}_{z}^{\prime}=\frac{9}{64 \pi} \frac{g_{W}^{2} \Im \mathrm{m}(U) \sqrt{x_{l}}}{\left(1-x_{W}\right)^{2}\left(1+2 x_{W}\right) x_{H}} f^{\prime}\left(x_{W}, y_{W}, x_{H}\right),
$$

where $f^{\prime}$ is the integral of Eq. 5.88 except that $\lambda-x_{W}$ is replaced by $\left|\lambda-x_{W}\right|$.

The results for the above asymmetries, where experimental constraints were imposed on the relevant 3DHM parameters (for details see [114]), are a few percents for $\mathcal{A}_{y}$ and $\mathcal{A}_{z}$, and a few tens of percents for $\mathcal{A}_{z}^{\prime}$. As expected, the $\tau$-polarization asymmetries are much larger and, therefore, perhaps better suited to look for $\mathrm{CP}$ violation within the $3 \mathrm{HDM}$, than any other asymmetry.

\subsection{CP violation in top decays - summary}

As discussed in Chapter 2, CP violation can manifest in decays of particles. Such CP-violating signals may be driven by new physics containing new heavy, particles. Thus, the large mass of the top may cause enhancments of $\mathrm{CP}$ violation in top decays as compared to the situation in light quarks decays. CP-odd signals in top decays, therefore, are attractive venues for such studies.

In this chapter we have discussed several types of CP-violating asymmetries in two and threebody top decays. In particular, PRA, PIRA, energy asymmetry in the top decay products and $\tau$-polarization asymmetries in the three-body decay $t \rightarrow b \tau \nu$. In the $\mathrm{SM}$, the CP violation in the top decays is found to be vanishingly small. This fact makes $\mathrm{CP}$ violation in top decays an extremly useful place for searching for new physics. We have, of course, also considered CP violation in top decays in extensions of the SM such as MHDM's and SUSY.

We found that a sizable CP-violating PRA can arise in the main top decay, $t \rightarrow b W$, in SUSY models. In particular, a stop-neutralino-chagrino loop in the $t b W$ vertex can give rise to a PRA of the order of $0.1 \%$ if the SUSY parameter space turns out to be favorable. Such a PRA is, in principle, within the reach of the LHC provided that detector systematics can be kept sufficiently under control.

A much bigger CP-violating signal is expected in the three-body decay, $t \rightarrow b \tau \nu$, in a MHDM's with new CP-odd phases in the charged Higgs sector, e.g., a 3HDM. Indeed such CP violation may arise already at tree-level and is best observed through a CP-violating transverse $\tau$-polarization asymmetry. In a favorable scenario this asymmetry may be as large as a few tens of percents, requiring $\sim 1000$ top quarks for its detection. This is a particularly gratifying result since over $10^{4}$ top quark pairs are expected to be produced in the future colliders. The decay $t \rightarrow b \tau \nu$ is therefore a very promising place to look for new signals of $\mathrm{CP}$ violation in top decays. 


\section{CP violation in $e^{+} e^{-}$collider experiments}

The energy of circular $e^{+} e^{-}$machines cannot be increased beyond the energy of LEP-II, due to heavy losses to the synchrotron radiation. Therefore, the next step in $e^{+} e^{-}$physics will involve linear colliders only, the "existence proof" of which has been demonstrated at the $100 \mathrm{GeV}$ scale by SLC. For recent reviews on linear colliders see [169]. The luminosity of future $e^{+} e^{-}$colliders is projected to be $\mathcal{L} \approx 10^{34} \mathrm{~cm}^{-2} \mathrm{sec}^{-1}$, corresponding to a yearly integrated luminosity of $\mathcal{L} \approx 100$ $\mathrm{fb}^{-1}$. The working assumption usually is to take it as tens of $\mathrm{fb}^{-1}$ at the lower end of the scale of c.m. energies, and as hundreds of $\mathrm{fb}^{-1}$ at its upper scale, corresponding to higher c.m. energies, to compensate for the decreasing cross-sections. In the first stage, the c.m. energy will cover the range approximately between LEP-II and $500 \mathrm{GeV}$, eventually reaching perhaps $1.6 \mathrm{TeV}$ and hopefully even $2 \mathrm{TeV}$. Furthermore, beam polarization - which can help in clarifying many of the physics issues - is an interesting option. In this context recall that the SLC achieved polarization as high as $70-80 \%$.

\section{1 $e^{+} e^{-} \rightarrow t \bar{t}$}

In an high energy $e^{+} e^{-}$collider running with c.m. energies of $500-2000 \mathrm{GeV}$ and an integrated luminosity of $\mathcal{L} \sim \mathcal{O}(100) \mathrm{fb}^{-1}, 10^{4}-10^{5}$ pairs of $t \bar{t}$ will be produced mainly through the simple reaction $e^{+} e^{-} \rightarrow \gamma, Z \rightarrow t \bar{t}$. This facility, especially due to its relatively clean environment, may therefore be thought of as a very efficient "top factory" and it is expected that many of the rare phenomena associated with top quark systems will be intensely studied there. Here we will focus on $\mathrm{CP}$ violation in the overall reaction

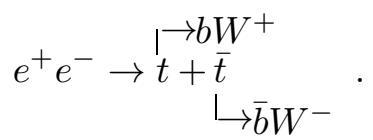

Decays of the $W$ also need to be included; the leptonic channels $\left(W \rightarrow \ell \nu_{\ell}, \ell=e, \mu\right)$ are perhaps the cleanest although experimental simulations suggest that $W$ 's could be detected through jet topologies as well [170]. In what follows we will not entertain the theoretical possibility that there is additional CP violation in $W^{+}, W^{-}$decays and will focus only on effects directly related to the top quark.

In general, in the limit $m_{e}=0$, the $\gamma($ or $Z) t \bar{t}$ vertex can be modified to include the top magnetic and electric(or weak) dipole moments

$$
-i\left[\gamma^{\mu}\left(A_{t}^{V}+B_{t}^{V} \gamma_{5}\right)+\sigma^{\mu \nu} q_{\nu}\left(i c_{t}^{V}+d_{t}^{V} \gamma_{5}\right)\right],
$$

where $c_{t}^{V}$ and $d_{t}^{V}$, for $V=\gamma$ or $Z$, are the magnetic and electric dipole moment form factors of the top quark at $q^{2}=s$, assuming that they are deduced by the use of the reaction in Eq. 6.1. The tree-level SM values for these parameters are

$$
\begin{aligned}
A_{t}^{\gamma} & =\frac{2}{3} e \\
B_{t}^{\gamma} & =0 \\
A_{t}^{Z} & =\frac{e}{\sin \theta_{W} \cos \theta_{W}}\left(\frac{1}{4}-\frac{2}{3} \sin ^{2} \theta_{W}\right) \\
B_{t}^{Z} & =-\frac{e}{4 \sin \theta_{W} \cos \theta_{W}} \\
c_{t}^{V} & =d_{t}^{V}=0 .
\end{aligned}
$$

Note also that, in the SM, the $V e^{+} e^{-}$couplings in the notation of Eq. 6.2 are 


$$
\begin{aligned}
A_{e}^{\gamma} & =-e, \\
B_{e}^{\gamma} & =0, \\
A_{e}^{Z} & =\frac{e}{\sin \theta_{W} \cos \theta_{W}}\left(-\frac{1}{4}+\sin ^{2} \theta_{W}\right), \\
B_{e}^{Z} & =\frac{e}{4 \sin \theta_{W} \cos \theta_{W}} .
\end{aligned}
$$

The magnetic form factor, which is CP-conserving, has a significant SM contribution at 1-loop due to QCD corrections and therefore is of lesser interest. Since in $e^{+} e^{-} \rightarrow t \bar{t}$ we have $q^{2}=s>4 m_{t}^{2}$, these form factors are in general complex. In particular, with regard to the EDM form factors: $\Re$ e $d_{t}^{\gamma, Z}\left(q^{2}\right)$ is $T_{N^{-o d d}}$, and $\Im \mathrm{m} d_{t}^{\gamma, Z}\left(q^{2}\right)$ is $T_{N^{-}}$even and, of course, all of these four quantities are CP-odd.

Similar to the production vertex, the $t b W^{+}$and $\bar{t} \bar{b} W^{-}$decay amplitudes may have CPviolating pieces. In order to take into account this possibility, for on-shell $W^{+}$and in the limit $m_{e}=0$, the decay amplitudes for $t \rightarrow b W^{+}$may be decomposed with the most general form factors as (see also section 5.1.5)

$$
\mathcal{M}_{t b W}=-\frac{g_{W}}{\sqrt{2}} V_{t b} \epsilon_{\mu}^{*}\left(p_{W^{+}}\right) \bar{u}_{b}\left(p_{b}\right)\left\{\gamma_{\mu} L+\sum_{P=L, R}\left(f_{1}^{P} \gamma^{\mu} P+i \frac{f_{2}^{P}}{m_{W}} \sigma_{\nu}^{\mu} p_{W^{+}}^{\nu} P\right)\right\} u_{t}\left(p_{t}\right)
$$

where $\epsilon_{\mu}^{*}\left(p_{W^{+}}\right)$is the polarization vector of $W^{+}$with four momentum $p_{W^{+}}$and $p_{t}, p_{b}$ are the four momenta of the $t, b$ respectively. $P=L$ or $R$ where $L(R)=\left(1-(+) \gamma_{5}\right) / 2$ and the form factors $f_{1}^{P}$ and $f_{2}^{P}$ are complex in general.

Similarly the amplitude for $\bar{t} \rightarrow \bar{b} W^{-}$is defined as

$$
\overline{\mathcal{M}}_{t b W}=-\frac{g_{W}}{\sqrt{2}} V_{t b}^{*} \epsilon_{\mu}\left(p_{W^{-}}\right) \bar{v}_{t}\left(p_{\bar{t}}\right)\left\{\gamma_{\mu} L+\sum_{P=L, R}\left(\bar{f}_{1}^{P} \gamma^{\mu} P+i \frac{\bar{f}_{2}^{P}}{m_{W}} \sigma_{\nu}^{\mu} p_{W^{-}}^{\nu} P\right)\right\} v_{b}\left(p_{\bar{b}}\right) .
$$

Furthermore, some useful relations exist (see Eqs. 5.70 5.75) between pairs of $\left(f_{i}^{P}, \bar{f}_{i}^{P}\right)$ in terms of their CP-conserving and CP-violating parts. In particular, CP-violating observables associated with top decays must always be proportional to any one of the combinations: $\left(f_{1}^{L}-\bar{f}_{1}^{L}\right),\left(f_{1}^{R}-\bar{f}_{1}^{R}\right)$, $\left(f_{2}^{L}-\bar{f}_{2}^{R}\right)$ or $\left(f_{2}^{R}-\bar{f}_{2}^{L}\right)$, such that a CP-odd, $T_{N}$-even quantity will be proportional to the real parts of these combinations, but a CP-odd, $T_{N}$-odd quantity will be proportional to their imaginary parts (for details see section 5.1.5).

$\mathrm{CP}$ violation effects in $e^{+} e^{-} \rightarrow t \bar{t} \rightarrow b W^{+} \bar{b} W^{-}$may thus enter in both the production and the decay vertices of the top and the anti-top ${ }^{20}$. To leading order in the CP-violating form factors present in the production or the decay of the top, one has to include interferences of diagrams (b)-(d) with the SM diagram (a) in Fig. 26.

In principle, in order to experimentally separate $\mathrm{CP}$-nonconserving effects in the production vertex from the decay vertex, one has to construct appropriate observables with sensitivity to only one CP-violating vertex, i.e., either production or decay (see e.g., [172]), or alternatively some simplifying assumptions have to be made.

It is important to note that the description of the $V t \bar{t}$ vertex for $e^{+} e^{-} \rightarrow t \bar{t}$ in terms of Eq. 6.2 is not necessarily sufficient. For example, in the MSSM, 1-loop box diagrams with exchanges of SUSY particles may contribute to $\mathrm{CP}$ violation in $e^{+} e^{-} \rightarrow t \bar{t}$. In these type of diagrams a $V t \bar{t}$ vertex is obviously absent (such effects were investigated in [142, 173]). However, note that in 2HDM's with CP violation in the neutral Higgs sector, the general parameterization in Eq. 6.2 holds as there are no 1-loop box diagrams contributing to $e^{+} e^{-} \rightarrow t \bar{t}$, in the limit $m_{e}=0$.

\footnotetext{
${ }^{20}$ For a comprehensive treatment of the helicity amplitudes for $e^{+} e^{-} \rightarrow t \bar{t}$ and for the subsequent top decays $t \rightarrow b \ell \nu_{l}$ in the presence of the CP-violating couplings in Eqs. 6.2, 6.5 and 6.6, see e.g., 21, 171].
} 


\subsubsection{Optimized observables}

As mentioned before, in the reaction $e^{+} e^{-} \rightarrow t \bar{t} \rightarrow b W^{+} \bar{b} W^{-}$, CP violation can arise from both the production (see Eq. 6.2) and the decay (see Eqs. 6.5 and 6.6) of the top. In this problem many

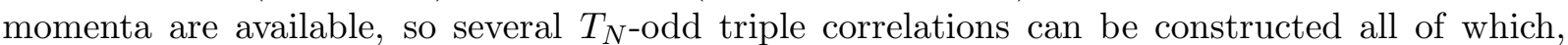
in principle, can have non-vanishing expectation values that are proportional to $\Re$ e $d_{t}^{\gamma, Z}\left(q^{2}\right)$.

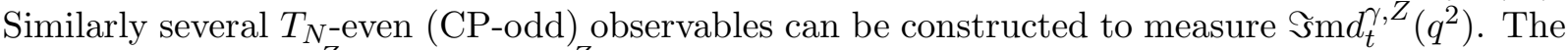
sensitivity to $\Re \mathrm{e} d_{t}^{\gamma, Z}\left(q^{2}\right)$ or $\Im \mathrm{m} d_{t}^{\gamma, Z}\left(q^{2}\right)$ can vary considerably amongst the observables. It is, therefore, useful to devise a general procedure that represents a rough measure of the sensitivity of the observables in such situations. Thereby, one is lead to consider the possibility of constructing "optimized observables", i.e., observables that have the maximum statistical sensitivity. Recall that for a given number of $t \bar{t}$ events, the optimized observables will yield the smallest attainable limit on the real and the imaginary parts of $d_{t}^{\gamma, Z}\left(q^{2}\right)$. The basic idea of the optimized observables was first outlined in [16]; the general recipe for construction of such observables is given in section 2.6 .

Optimal observables have by now been used extensively in [16, 174, 175, 176, 177]. In [16, 174, 175 CP violation in the top decays was ignored and the CP-odd effect was attributed solely to the EDM $\left(d_{t}^{\gamma}\right)$ and ZEDM $\left(d_{t}^{Z}\right)$ of the top in the $\gamma t \bar{t}$ and $Z t \bar{t}$ production vertex. Indeed, in [143] it was shown that, in model calculations such as 2HDM and MSSM, the dipole moment in the $t \bar{t}$ production leads to larger CP-nonconserving effects than what might be expected in the top decays.

In 176, 177 the optimization technique was employed to the overall reaction $e^{+} e^{-} \rightarrow t \bar{t} \rightarrow$ $b W^{+} \bar{b} W^{-}$, where $\mathrm{CP}$ violation from both the production and the decay vertices of the top were investigated.

Using the general $V t t$ vertex in Eq. 6.2, the differential cross-section for $e^{+} e^{-} \rightarrow t \bar{t}$ may be expressed as

$$
\Sigma(\phi) d \phi=\Sigma_{0}(\phi) d \phi+\sum_{V=\gamma, Z}\left(\Re \mathrm{e} d_{t}^{V}(s) \Sigma_{\Re \mathrm{e}\left(d_{t}^{V}\right)}(\phi)+\Im \mathrm{m} d_{t}^{V}(s) \Sigma_{\Im \mathrm{m}\left(d_{t}^{V}\right)}(\phi)\right) d \phi .
$$

As was explained in [16], the simplest optimized observables for the real and imaginary parts of $d_{t}^{\gamma, Z}\left(q^{2}\right)$ are

$$
\mathcal{O}_{R}^{\gamma, Z}=\Sigma_{\Re \mathrm{e}\left(d_{t}^{\gamma, Z}\right)} / \Sigma_{0}, \quad \mathcal{O}_{I}^{\gamma, Z}=\Sigma_{\Im \mathrm{m}\left(d_{t}^{\gamma, Z}\right)} / \Sigma_{0}
$$

These optimal observables are constructed simply from the available four momenta in $e^{+} e^{-} \rightarrow t \bar{t}$ and the subsequent decays. It was found in [16] that, for example, with $10^{4} t \bar{t}$ events in an NLC running at c.m. energies of $\sqrt{s}=500 \mathrm{GeV}, \Re \mathrm{e}\left(d_{t}^{\gamma, Z}\right)$ and $\Im \mathrm{m}\left(d_{t}^{\gamma, Z}\right)$ of about $\sim$ few $\times 10^{-17}$ e-cm become accessible at the 1- $\sigma$ significance level. Recall that in model calculations, such as MHDM's and SUSY, the size of top EDM and ZEDM are typically at the level of $\lesssim 10^{-18} \mathrm{e}-\mathrm{cm}$ (see Chapter A) if one pushes the CP-violating phases of these models to their largest allowed values. Thus, the 1- $\sigma$ limit obtained in [16] is at least one order of magnitude above the theoretical expectation for these dipole form factors within extensions of the SM.

Consider now the reaction $e^{+} e^{-} \rightarrow t \bar{t} \rightarrow b W^{+} \bar{b} W^{-}$where the $W^{+}, W^{-}$further decay leptonically via $W \rightarrow \ell \nu_{l}$ or hadronically, i.e., to up and down quark jets. Of course, as is well known, the top quark decay occurs in an extremely short time and one measures directly only the momenta of the decay products of the top. The optimization technique may be therefore improved to include all available 4-momenta in a given decay scenario of the $t \bar{t}$ as, for example, was done in 174, 175. The basic idea there was to translate the CP-odd top spin correlations generated by the dipole moments in $e^{+} e^{-} \rightarrow t \bar{t}$ to correlations among momenta of the decaying products of the $t$ and $\bar{t}$. For this purpose, the most promising decay scenario is the single-leptonic decay channels, i.e., when one, say the $t$, decays leptonically and the other, i.e., $\bar{t}$, decays hadronically or vice versa 
Table 5: Attainable 1- $\sigma$ sensitivities to the CP-violating dipole moment form factors in units of $10^{-18}$ e-cm, with $\left(P_{e}= \pm 1\right)$ and without $\left(P_{e}=0\right)$ beam polarization. $m_{t}=180 \mathrm{GeV}$. Table taken from 179].

\begin{tabular}{|c|c|c|c||c|c|c|}
\hline & \multicolumn{2}{|c||}{$20 \mathrm{fb}^{-1}, \sqrt{s}=500 \mathrm{GeV}$} & \multicolumn{3}{c|}{$50 \mathrm{fb}^{-1}, \sqrt{s}=800 \mathrm{GeV}$} \\
& $P_{e}=0$ & $P_{e}=+1$ & $P_{e}=-1$ & $P_{e}=0$ & $P_{e}=+1$ & $P_{e}=-1$ \\
\hline$\delta\left(\Re \mathrm{e} d_{t}^{\gamma}\right)$ & 4.6 & 0.86 & 0.55 & 1.7 & 0.35 & 0.23 \\
\hline$\delta\left(\Re \mathrm{e} d_{t}^{Z}\right)$ & 1.6 & 1.6 & 1.0 & 0.91 & 0.85 & 0.55 \\
\hline$\delta\left(\Im \mathrm{m} d_{t}^{\gamma}\right)$ & 1.3 & 1.0 & 0.65 & 0.57 & 0.49 & 0.32 \\
\hline$\delta\left(\Im \mathrm{m} d_{t}^{Z}\right)$ & 7.3 & 2.0 & 1.3 & 4.0 & 0.89 & 0.58 \\
\hline
\end{tabular}

$$
\begin{array}{ll}
t \bar{t} & \rightarrow \quad \ell^{+}\left(q_{+}\right)+\nu_{\ell}+b+\bar{X}_{\mathrm{had}}\left(q_{\bar{X}}\right), \\
t \bar{t} & \rightarrow \quad X_{\mathrm{had}}\left(q_{X}\right)+\ell^{-}\left(q_{-}\right)+\bar{\nu}_{\ell}+\bar{b} .
\end{array}
$$

The decay scenarios in Eqs. 6.9 and 6.10 (each of which has a branching ratio, $B_{\ell} \sim 0.15$, if $\ell=e, \mu)$ allow for the reconstruction of the $\bar{t}$ and $t$ momentum, respectively, which, in turn, gives the rest frames of these quarks. The fact that the rest frames of $t$ and $\bar{t}$ may be accessible in these decay modes allows one to use CP-odd observables in terms of lepton unit momenta $\hat{q}_{ \pm}^{*}$ in the corresponding top rest frames, instead of $\hat{q}_{ \pm}$- defined in the $e^{+} e^{-}$c.m. frame.

The optimal observables that they used are, again, simply the ratio between the CP-odd and CP-even differential cross-sections and are given in [174]. However, in their optimal observables, the differential cross-section corresponds to the overall production and decay of the $t \bar{t}$ and the leptonic momenta are taken in the corresponding $t, \bar{t}$ rest frames. With the optimal observables they [175] calculated the best 1- $\sigma$ sensitivity to the CP-violating dipole moments form factors $\Re \mathrm{e}\left(d_{t}^{\gamma}\right), \Re \mathrm{e}\left(d_{t}^{Z}\right), \Im \mathrm{m}\left(d_{t}^{\gamma}\right), \Im \mathrm{m}\left(d_{t}^{Z}\right)$ assuming $100 \%$ tagging efficiency of the single-leptonic decay modes of $t \bar{t}$ in Eqs. 6.9 and 6.10; these are given in Table 5. We thus see that beam polarization (of the incoming electrons), denoted here by $P_{e}$, may increase the sensitivity to $\Re \mathrm{e}\left(d_{t}^{\gamma}\right)$ and $\Im \mathrm{m}\left(d_{t}^{Z}\right)$ by almost an order of magnitude. Evidently, the results in Table 5 imply that a NLC running with a c.m. energy of $\sqrt{s}=500 \mathrm{GeV}$ and an integrated luminosity of $20 \mathrm{fb}^{-1}$, or $\sqrt{s}=800 \mathrm{GeV}$ and an integrated luminosity of $50 \mathrm{fb}^{-1}$, will be able to probe, at $1-\sigma$ and in the best cases, real and imaginary parts of the TDM, typically of the order of a few $\times 10^{-19}-10^{-18}$. As in their analysis, this may be achieved by investigating the single-leptonic decay mode of $t \bar{t}$. This improves the limits obtained in [16] by about one order of magnitude. However, at the 3- $\sigma$ significance level, the corresponding sensitivities are typically few $\times 10^{-18}-10^{-17}$ and so are still about an order of magnitude above the expectations from the models such as MHDM's and SUSY.

It should be noted again that a CP-violating dipole moment at the $t \bar{t} \gamma$ and $t \bar{t} Z$ vertices may not, in general, account for the entire $\mathrm{CP}$ violation effect in $t \bar{t}$ production. As mentioned before, this will, for example, be the case in the MSSM where 1-loop CP-violating box diagrams can cause an additional CP-odd effect [173]. We note, however, that specifically in the MSSM, these box contributions to $\mathrm{CP}$ violation in $t \bar{t}$ cannot significantly enhance the $\mathrm{CP}$-violating signal. In fact, in some ranges of the relevant SUSY parameter space the contribution of the box graphs comes with an opposite sign relative to the top dipole moments, such that cancellations may occur, thus decreasing the net $\mathrm{CP}$ violation effect in $t \bar{t}$ production $[173]$.

A more complete investigation was carried out in [176, 177]. There the CP-violating form factors from both the production and the decay amplitudes of the $t$ and $\bar{t}$ were included. For this purpose, they used the single-leptonic energy spectrum 


$$
\frac{1}{\sigma^{ \pm}} \frac{d \sigma^{ \pm}}{d x}=\sum_{i=1}^{3} c_{i}^{ \pm} f_{i}(x),
$$

where

$$
c_{1}^{ \pm}=1, \quad c_{2}^{ \pm}=\mp \xi, \quad c_{3}^{+}=-\Re \mathrm{e}\left(f_{2}^{R}\right), \quad c_{3}^{-}=-\Re \mathrm{e}\left(\bar{f}_{2}^{L}\right) .
$$

The functions $f_{i}(x)$ are all given in [176] and $f_{2}^{R}, \bar{f}_{2}^{L}$ are defined in Eqs. 6.5 and 6.6. Also, \pm indicates the charge of the lepton and

$$
x(\bar{x})=2 \frac{E^{\ell^{+}}\left(E^{\ell^{-}}\right)}{m_{t}} \sqrt{\frac{(1-\beta)}{(1+\beta)}},
$$

$E^{\ell^{+}}\left(E^{\ell^{-}}\right)$being the energy of $\ell^{+}\left(\ell^{-}\right)$in the $e^{+} e^{-}$c.m. frame and $\beta=\sqrt{1-4 m_{t}^{2} / s}$. Specifically, for $m_{t}=180 \mathrm{GeV}$ and $\sqrt{s}=500 \mathrm{GeV}$

$$
\xi \simeq-\frac{1.76 \times 10^{16}}{[\mathrm{e}-\mathrm{cm}]} \times\left(1.06 \Im \mathrm{m}\left(d_{t}^{\gamma}\right)+0.18 \Im \mathrm{m}\left(d_{t}^{Z}\right)\right)
$$

In [176], optimal observables that may be used to separately measure the CP-violating form factors in the production or the decay vertex were given. Their optimal observables utilize the single-leptonic energy spectrum in Eq. 6.11. With the optimization technique they showed that $\xi$ and $\Re \mathrm{e}\left(f_{2}^{R}-\bar{f}_{2}^{L}\right)$ may be extracted individually from the difference in the $\ell^{+}$versus $\ell^{-}$ energy spectra by convoluting the differential energy spectrum with approximately chosen kernel functions

$$
\begin{aligned}
& \xi=\frac{1}{2} \int d x\left[\frac{1}{\sigma^{+}} \frac{d \sigma^{+}}{d x}-\frac{1}{\sigma^{-}} \frac{d \sigma^{-}}{d x}\right] \Omega_{\xi}, \\
& \Re e\left(f_{2}^{R}-\bar{f}_{2}^{L}\right)=\int d x\left[\frac{1}{\sigma^{+}} \frac{d \sigma^{+}}{d x}-\frac{1}{\sigma^{-}} \frac{d \sigma^{-}}{d x}\right] \Omega_{f},
\end{aligned}
$$

where $\Omega_{f}, \Omega_{\xi}$ are functions of $f_{i}(x)$ defined in Eq. 6.11 and are given in [177]. The minimal values of $|\xi|$ and $\left|\Re \mathrm{e}\left(f_{2}^{R}-\bar{f}_{2}^{L}\right)\right|$ that can be obtained with a statistical significance $N_{S D}^{t t V}$ and $N_{S D}^{t b W}$, respectively, at the NLC with $\sqrt{s}=500 \mathrm{GeV}$ and $m_{t}=180 \mathrm{GeV}$, can then be computed [176]:

$$
\begin{aligned}
& |\xi|^{\mathrm{min}}=11.3 \frac{N_{S D}^{t t V}}{\epsilon_{L} \times \mathrm{pb}^{1 / 2}}, \\
& \left|\Re \mathrm{e}\left(f_{2}^{R}-\bar{f}_{2}^{L}\right)\right|=13.1 \frac{N_{S D}^{t b W}}{\epsilon_{L} \times \mathrm{pb}^{1 / 2}} .
\end{aligned}
$$

Here $\epsilon_{L}$ represents the square root of the effective luminosity for the single-leptonic $t \bar{t}$ pairs at the NLC. Thus, $\epsilon_{L} \equiv \sqrt{\epsilon_{t t} \mathcal{L}}$, where $\mathcal{L}$ is the integrated luminosity at the NLC and $\epsilon_{t t}$ is the tagging efficiency for the single-leptonic mode. Note that, in the best case, $\epsilon_{t t}=B_{\ell} \approx 15 \%$, if one assumes $100 \%$ efficiency in measuring the single leptons from $t \bar{t}$.

We see from Eqs. 6.17 and 6.18 that, for example, with $\mathcal{L}=100 \mathrm{fb}^{-1}$ and $\epsilon_{t t}=0.15$ we have $\epsilon_{L} \approx 122.5 \mathrm{pb}^{-1 / 2}$ (note that with these values one has $\sim 9000$ single-leptonic $t \bar{t}$ events). Therefore, with this number, a $3-\sigma$ detection of $|\xi|$ will be possible for $|\xi| \approx 0.28$. Using the relation between $\xi$ and $\Im \mathrm{m}\left(d_{t}^{\gamma, Z}\right)$ in Eq. 6.14 we then get the following 3- $\sigma$ equality

\footnotetext{
${ }^{21}$ Notice the difference in our notation (Eq. 6.2) and the one used in $[176]$ for the top dipole moments. The translation is $D_{\gamma, Z}=\left(4 m_{t} \sin \theta_{W} / e\right) \times i d_{t}^{\gamma, Z}$.
} 


$$
1.06 \Im \mathrm{m}\left(d_{t}^{\gamma}\right)+0.18 \Im \mathrm{m}\left(d_{t}^{Z}\right) \simeq 1.6 \times 10^{-17} \mathrm{e}-\mathrm{cm} .
$$

Eq. 6.19 implies that $\Im \mathrm{m}\left(d_{t}^{\gamma, Z}\right)$ of the order of $\sim 10^{-17}$ e-cm may be detected at the 3- $\sigma$ level at the NLC, running with c.m. energy of $\sqrt{s}=500 \mathrm{GeV}$ and with an integrated luminosity of $\mathcal{L}=100 \mathrm{fb}^{-1}$, using the optimal observables suggested in [176]. This result is again about one order of magnitude better compared to the results obtained in [16] and it is comparable to the results shown in Table 5 which were obtained in [175].

As for the CP-violating form factors in the decay amplitude, we can use Eq. 6.18 to get the $3-\sigma$ limit on $\left|\Re \mathrm{e}\left(f_{2}^{R}-\bar{f}_{2}^{L}\right)\right|$. For $\epsilon_{L} \approx 122.5 \mathrm{pb}^{-1 / 2}$

$$
\left|\Re \mathrm{e}\left(f_{2}^{R}-\bar{f}_{2}^{L}\right)\right| \approx 0.32 .
$$

In Chapter 5 we have discussed the theoretical expectations for couplings such as $f_{2}^{R}$ and $\bar{f}_{2}^{L}$ in the SM and its extensions. The SM prediction for such form factors, induced by the CKM matrix, is much too small to be observed. Moreover, even within MHDM's and the MSSM the resulting 3- $\sigma$ limit in Eq. 6.20 falls short by at least one order of magnitude.

Finally, in [177] the single-leptonic channel was compared to the double-leptonic mode, i.e., when both $t$ and $\vec{t}$ decay leptonically, using the optimization technique. It was found there that the single-leptonic mode comes out favorable by about a factor of 2 .

\subsubsection{Naive observables constructed from momenta of the top decay products}

Various types of "naive" observables to deduce the real and imaginary parts of the non-standard form factors in top production and decay were considered in [17, 16, 143, 165, 174, 175, 178, 179, 180. These observables are constructed simply from correlations between momenta of the decaying products of the top quark. The basic idea again utilizes the fact that weak decays of the top quark act as very efficient analyzer of the top spin. So the momenta of the decay products (via $t \rightarrow b \ell \nu)$ can be used to construct the observables with the right transformation properties. In general, as expected, the "naive" operators are less effective than the optimal ones, sometimes by as much as an order of magnitude. However, it is important to bear in mind that this advantage pertains only with respect to statistical errors; in actual experimental considerations, systematic errors will also need to be taken into account and that could offset some of the advantage of the optimized observables.

In [16], amongst the various possible correlations, the best simple operators that they found are

$$
\begin{aligned}
\epsilon_{\mu \nu \sigma \rho} P_{b}^{\mu} Q_{Z}^{\nu} H^{+\sigma} H^{-\rho} & \text { for } \Re \mathrm{e} d_{t}^{\gamma}, \\
\epsilon_{\mu \nu \sigma \rho} P_{e}^{\mu} Q_{Z}^{\nu} H^{+\sigma} H^{-\rho} & \text { for } \Re \mathrm{e} d_{t}^{Z}, \\
H^{-} \cdot Q_{Z} & \text { for } \Im \mathrm{m} d_{t}^{\gamma} \text { and } \Im \mathrm{m} d_{t}^{Z},
\end{aligned}
$$

where the momenta above are given by

$$
\begin{aligned}
P_{b} & =p_{b}-p_{\bar{b}}, \\
P_{e} & =p_{e}^{+}-p_{e}^{-}, \\
Q_{Z} & =p_{e}^{+}+p_{e}^{-}, \\
H^{ \pm} & =2\left(E_{W}^{+} \cdot p_{t}\right) E_{W}^{+} \pm 2\left(E_{W}^{-} \cdot p_{t}\right) E_{W}^{-},
\end{aligned}
$$

and $E_{W}$ is the $W$-boson polarization for the reaction $W\left(p_{W}\right) \rightarrow \ell\left(p_{\ell}\right) \nu_{\ell}\left(p_{\nu}\right)$. Because of the left-handed nature of the coupling of $W$ to leptons its polarization can be constructed from the momenta of the decay products as 


$$
E_{W}^{\mu}=\frac{\operatorname{Tr}\left[\not p_{\nu} \omega_{0} \not p_{\ell} \gamma^{\mu}\left(1-\gamma_{5}\right)\right]}{4 \sqrt{p_{\nu} \cdot \omega_{0} p_{\ell} \cdot \omega_{0}}}
$$

Here $\omega_{0}$ is an arbitrary light-like vector that determines the phase convention for the polarization. The expression above requires the $\nu$ momenta. Recall that the final state consists of six particles $b \ell^{+} \nu_{\ell} \bar{b} \ell^{-} \bar{\nu}_{\ell}$. Of these only four are directly observable as the neutrinos escape detection. However, by imposing the following conditions, the momenta of the missing neutrinos may in fact be inferred. The conditions that need to be imposed are: (1) conservation of four-momentum together with the conditions that (2) the lepton and a neutrino reconstruct to the $W^{ \pm}$mass, (3) the $b$-quark together with a lepton and neutrino reconstruct the $t, \bar{t}$ mass and (4) the neutrinos are massless.

It was then found in [16] that the use of the $W$ polarization in the operators of Eq. 6.21 can easily improve the sensitivity to the dipole moments by factors of 10-50 when compared to simple correlations which do not involve the $W$ polarization vector. As compared to the optimal observables discussed in their work, the observables in Eq. 6.21 are less effective, typically, by about a factor of $2-10$.

In 117, 165], the following CP-odd and $T_{N}$-odd correlations were considered

$$
\begin{aligned}
& \hat{T}_{i j}=\left(\hat{q}_{-}-\hat{q}_{+}\right)_{i} \frac{\left(\hat{q}_{-} \times \hat{q}_{+}\right)_{j}}{\left|\hat{q}_{-} \times \hat{q}_{+}\right|}+(i \leftrightarrow j), \\
& \hat{A}_{1}=\hat{p}_{+} \cdot \frac{\left(\hat{q}_{-} \times \hat{q}_{+}\right)}{\left|\hat{q}_{-} \times \hat{q}_{+}\right|},
\end{aligned}
$$

where $\hat{p}_{+}$is the unit momentum of the incoming positron and $\hat{q}^{ \pm}$are the unit momenta of a charged decay product from $t \rightarrow A, \bar{t} \rightarrow \bar{B}$ in the overall c.m. system. Thus, $\hat{q}_{ \pm}$are the directions of a charged lepton or a $b$ jet. An interesting property of these correlations is that they are not sensitive to CP-violating effects in the $t$ and $\bar{t}$ decays and, therefore, they can be expressed in terms of only the real part of the EDM and ZEDM form factors, $\Re$ e $\left(d_{t}^{\gamma, Z}\right)$. The mean values of $\hat{T}_{i j}$ and $\hat{A}_{1}$ plus the conjugate ones are given by 165 :

$$
\begin{aligned}
\left\langle\hat{T}_{i j}\right\rangle_{A \bar{B}}+\left\langle\hat{T}_{i j}\right\rangle_{B \bar{A}} & =2 \frac{\sqrt{s}}{e}\left(c_{\gamma, A B} \Re \mathrm{e} d_{t}^{\gamma}+c_{Z, A B} \Re \mathrm{e} d_{t}^{Z}\right) s_{i j} \\
\left\langle\hat{A}_{1}\right\rangle_{A \bar{B}}+\left\langle\hat{A}_{1}\right\rangle_{B \bar{A}} & =2 \frac{\sqrt{s}}{e}\left(r_{\gamma, A B} \Re \mathrm{e} d_{t}^{\gamma}+r_{Z, A B} \Re \mathrm{e} d_{t}^{Z}\right)
\end{aligned}
$$

where, identifying the $z$-axis with the $e^{+}$beam axis

$$
s_{i j}=\frac{1}{2}\left(\hat{p}_{+i} \hat{p}_{+j}-\frac{1}{3}\right)=\operatorname{diag}\left(-\frac{1}{6},-\frac{1}{6}, \frac{1}{3}\right) .
$$

The coefficients $c_{\gamma, Z}$ and $r_{\gamma, Z}$ depend on the specific decay channel and were calculated as a function of $s$ in [165] for correlations among $A B=\ell^{+} \ell^{-}, b \bar{b}$ and $\ell^{+} b+\ell^{-} \bar{b}$.

Possible CP-odd, $T_{N}$-even correlations that use the momenta of the decay products of $t$ and $\bar{t}$ were also examined in 165:

$$
\begin{aligned}
\hat{Q}_{i j} & =\left(\hat{q}_{+}+\hat{q}_{-}\right)_{i}\left(\hat{q}_{-}-\hat{q}_{+}\right)_{j}+(i \leftrightarrow j), \\
\hat{A}_{2} & =\hat{p}_{+} \cdot\left(\hat{q}_{+}+\hat{q}_{-}\right) .
\end{aligned}
$$

In contrast to the $T_{N}$-odd observables in Eqs. 6.24 and 6.25, the $T_{N}$-even observables $\hat{Q}_{i j}$ and $\hat{A}_{2}$, which acquire absorptive phases, are sensitive also to the combinations $\Re \mathrm{e}\left(f_{i}^{P}-\bar{f}_{i}^{P}\right.$ ) (recall that $P=L$ or $R$ ), e.g., $\Re$ e $\left(f_{2}^{R}-\bar{f}_{2}^{L}\right)$, of the form factors in the decay amplitudes of Eqs. 6.5 
and 6.6. In [165], CP-odd effects in the decay process were neglected when evaluating the two CP-odd, $T_{N}$-even observables in Eqs. 6.29 and 6.30. In terms of the imaginary parts of the EDM and ZEDM of the top they obtained

$$
\begin{aligned}
\left\langle\hat{Q}_{i j}\right\rangle_{A \bar{B}}+\left\langle\hat{Q}_{i j}\right\rangle_{B \bar{A}} & =2 \frac{\sqrt{s}}{e}\left(q_{\gamma, A B} \Im \mathrm{m} d_{t}^{\gamma}+q_{Z, A B} \Im \mathrm{m} d_{t}^{Z}\right) s_{i j} \\
\left\langle\hat{A}_{2}\right\rangle_{A \bar{B}}+\left\langle\hat{A}_{2}\right\rangle_{B \bar{A}} & =2 \frac{\sqrt{s}}{e}\left(p_{\gamma, A B} \Im \mathrm{m} d_{t}^{\gamma}+p_{Z, A B} \Im \mathrm{m} d_{t}^{Z}\right) .
\end{aligned}
$$

Here, again, the coefficients $q_{\gamma, Z}$ and $p_{\gamma, Z}$ depend on the specific channel and were given as a function of $s$ in 165] for correlations among $A B=\ell^{+} \ell^{-}, b \bar{b}$ and $\ell^{+} b+\ell^{-} \bar{b}$.

From the simultaneous measurement of the pairs $\left\langle\hat{T}_{i j}\right\rangle,\left\langle\hat{A}_{1}\right\rangle$ and $\left\langle\hat{Q}_{i j}\right\rangle,\left\langle\hat{A}_{2}\right\rangle$ and for a given c.m. energy, $\Re \mathrm{e}\left(d_{t}^{\gamma, Z}\right)$ and $\Im \mathrm{m}\left(d_{t}^{\gamma, Z}\right)$ can be disentangled, respectively. Assuming $10^{4}$ available $t \bar{t}$ events at each c.m. energy, the $1-\sigma$ statistical sensitivity to $\Re$ e $d_{t}^{\gamma}$ and $\Re$ e $d_{t}^{Z}$, using only the 33 component of $\hat{T}_{i j}$, are

$$
\begin{aligned}
\delta\left(\Re \mathrm{e} d_{t}^{\gamma}\right) & =\frac{e}{\sqrt{s N_{\mathrm{eff}}}} \frac{\sqrt{\left\langle\left(3 r_{Z} \hat{T}_{33}-c_{Z} \hat{A}_{1}\right)^{2}\right\rangle}}{\left|c_{\gamma} \cdot r_{Z}-c_{Z} \cdot r_{\gamma}\right|} \\
\delta\left(\Re \mathrm{e} d_{t}^{Z}\right) & =\frac{e}{\sqrt{s N_{\mathrm{eff}}}} \frac{\sqrt{\left\langle\left(3 r_{\gamma} \hat{T}_{33}-c_{\gamma} \hat{A}_{1}\right)^{2}\right\rangle}}{\left|c_{Z} \cdot r_{\gamma}-c_{\gamma} \cdot r_{Z}\right|}
\end{aligned}
$$

where $N_{\text {eff }}=10^{4} \times \operatorname{Br}(t \rightarrow A) \times \operatorname{Br}(\bar{t} \rightarrow \bar{B})$. Similar relations can be obtained for $\Im \mathrm{m} d_{t}^{\gamma}$ and $\Im m d_{t}^{Z}$ using $\hat{Q}_{33}$ and $\hat{A}_{2}$.

Using this formalism, Ref. 165 presents the one standard deviation accuracies in measuring the real and imaginary parts of $d_{t}^{\gamma, Z}$, again assuming $10^{4} t \bar{t}$ events at c.m. energy $\sqrt{s}=500 \mathrm{GeV}$ and with $m_{t}=175 \mathrm{GeV}$

$$
\begin{aligned}
& \Re \mathrm{e} d_{t}^{\gamma} \approx 1 \times 10^{-17}, \quad \Im \mathrm{m} d_{t}^{\gamma} \approx 1.2 \times 10^{-17}, \\
& \Re \mathrm{e} d_{t}^{Z} \approx 5 \times 10^{-18}, \quad \Im \mathrm{m} d_{t}^{Z} \approx 7.5 \times 10^{-17},
\end{aligned}
$$

where the best sensitivity was obtained for correlations between the $\ell^{+} \ell^{-}$momenta. We note that the sensitivity to $\Re$ e $d_{t}^{\gamma, Z}$ is slightly better than to $\Im \mathrm{m} d_{t}^{\gamma, Z}$.

Cuypers and Rindani [181 have investigated the effect of polarized incoming electron beams. They found that the sensitivity of observables of the type $A_{1}$ and $A_{2}$ in Eqs. 6.25 and 6.30 to the real and imaginary parts of $d_{t}^{\gamma, Z}$, respectively, can be enhanced if the incoming electron beam is longitudinally polarized. Note also [174, the type of observables in Eqs. 6.24, 6.25, 6.29 and 6.30 may be improved (with respect to their sensitivity to the dipole moments) if one replaces the momentum of the charged lepton in the $e^{+} e^{-}$c.m. frame by its momentum in the top (or anti-top) rest frame, in the single-leptonic $t \bar{t}$ channel.

As was shown in the previous section, the CP-violating effects in the top decay $t \rightarrow b W^{+}$and its conjugate may be isolated using the optimization procedure. This may also be achieved in some limiting cases using naive observables. In [143, 165] a naive observable that projects onto $\mathrm{CP}$ violation in the top decay vertex was suggested

$$
\mathcal{O}_{1}=\hat{p}_{+} \cdot\left[\frac{\left(\hat{q}_{\bar{\ell}} \times \hat{q}_{b}\right)}{\left|\hat{q}_{\bar{\ell}} \times \hat{q}_{b}\right|}-\frac{\left(\hat{q}_{\ell} \times \hat{q}_{\bar{b}}\right)}{\left|\hat{q}_{\ell} \times \hat{q}_{\bar{b}}\right|}\right] .
$$

Although, in general, the expectation value of $\mathcal{O}_{1}$ above receives contributions both from the $\mathrm{CP}$ violation in $t \bar{t}$ production and in the decay amplitude, it was shown in 165 that close to 
threshold, i.e., $\sqrt{s} \simeq 2 m_{t}$, the contribution from the $t \bar{t}$ production vertex vanishes. For example, with the correlation $\mathcal{O}_{1}$, for an appropriate $e^{+} e^{-}$collider with $m_{t}=150 \mathrm{GeV}$ and c.m. energy $\sqrt{s} \simeq 2 m_{t}$, they find

$$
\left\langle\mathcal{O}_{1}\right\rangle \approx 0.15 \times \Im \mathrm{m}\left(f_{2}^{R}-\bar{f}_{2}^{L}\right),
$$

where $f_{2}^{R}, \bar{f}_{2}^{L}$ are form factors in the decay amplitudes defined in Eqs. 6.5 and 6.6. Assuming $3 \times 10^{3} t \bar{t}$ events in which $t \rightarrow b \ell^{+} \nu_{\ell}$ and $\bar{t} \rightarrow \bar{b} \ell^{-} \bar{\nu}_{\ell}$, they found that, to $1-\sigma$,

$$
\delta\left(\Im \mathrm{m}\left(f_{2}^{R}-\bar{f}_{2}^{L}\right)\right) \approx 0.1
$$

can be determined from a measurement of $\mathcal{O}_{1}$. Again, the limit in Eq. 6.39 falls short from model predictions for these form factors (see Chapter 5 ). This is easily understood from Eq. 6.38 which implies an asymmetry of the order of $\sim 10^{-3}$ for $\Im \mathrm{m}\left(f_{2}^{R}-\bar{f}_{2}^{L}\right) \sim 0.1$. Recall that the typical asymmetries in extensions of the SM that we have described in Chapter 5 are $\lesssim$ a few times $10^{-3}$ and in the SM they are a lot smaller than that.

A related $\mathrm{CP}$-odd and $T_{N}$-odd asymmetry that projects only onto $\mathrm{CP}$-violating effects in the decay processes of the $t$ and $\bar{t}$ was suggested by Grzadkowski and Keung, 167. This asymmetry was defined by partially integrating over the azimuthal angle of $\ell^{+}\left(\ell^{-}\right)$in the $W^{+}\left(W^{-}\right)$rest frame and subtracting the integration in the range $\{-\pi, 0\}$ from the integration in the range $\{0, \pi\}$ and it is essentially proportional to the triple product $p_{\ell} \cdot\left(p_{b} \times p_{W}\right)$. When evaluated within the MSSM, the asymmetry was found to be $\sim 10^{-4}-10^{-3}$ which again falls short by at least one order of magnitude from the limits that are anticipated to be obtainable, through the study of $e^{+} e^{-} \rightarrow t \bar{t} \rightarrow \ell^{+} \ell^{-} \nu_{\ell} \bar{\nu}_{\ell} b \bar{b}$ in a future $e^{+} e^{-}$high energy collider.

\subsubsection{Improved sensitivity using energy and angular distributions of top decay products and polarized electron beams}

Several differential leptonic asymmetries with respect to the charged lepton in $t \rightarrow b \ell \nu_{\ell}$ and for longitudinal electron (positron) beam polarizations, $P_{e}\left(P_{\bar{e}}\right)$, have been suggested in [16, 176, 177, 182, 183.

Consider the CP-violating $t-\bar{t}$ spin correlation $\hat{p}_{t} \cdot\left(s_{t}-s_{\bar{t}}\right)$. This spin correlation simply translates to the asymmetry

$$
\Delta N_{L R}=\frac{\left[N\left(t_{L} \bar{t}_{L}\right)-N\left(t_{R} \bar{t}_{R}\right)\right]}{\text { all } t \bar{t}},
$$

suggested first by Schmidt and Peskin (SP) in [20] in the context of $t \bar{t}$ production in hadron colliders (the SP effect will be discussed in more detail in Chapter 7).

Now, $\Delta N_{L R}$ is related to the asymmetry in the energy spectrum defined as [19, 182, 183]:

$$
A_{E}(x)=\frac{1}{\sigma}\left[\frac{d \sigma}{d x\left(l^{+}\right)}-\frac{d \sigma}{d x\left(l^{-}\right)}\right]
$$

through a simple multiplication by kinematic functions present in the lepton energy distribution functions (see [176]). The energy asymmetry in Eq. 6.41 is between distributions of $l^{+}$and $l^{-}$at the same value of $x=x\left(l^{+}\right)=x\left(l^{-}\right)=4 E\left(l^{ \pm}\right) / \sqrt{s}$. Note that when CP violation is present in the top production and not in the top decay, then $A_{E}(x) \propto \Delta N_{L R} \propto \xi$, where $\xi$ is defined in Eq. 6.14.

An up-down asymmetry, $A_{u d}$, was also studied in [19, 182]:

$$
A_{u d}=\int_{-1}^{+1} A_{u d}(\theta) d \cos \theta
$$

where 


$$
A_{u d}(\theta)=\frac{1}{2 \sigma}\left[\frac{d \sigma\left(l^{+}, \mathrm{up}\right)}{d \cos \theta}-\frac{d \sigma\left(l^{+}, \mathrm{down}\right)}{d \cos \theta}+\frac{d \sigma\left(l^{-}, \mathrm{up}\right)}{d \cos \theta}-\frac{d \sigma\left(l^{-}, \mathrm{down}\right)}{d \cos \theta}\right],
$$

and up/down refers to $\left(p_{l^{ \pm}}\right)_{y} \gtrless 0, \quad\left(p_{l^{ \pm}}\right)_{y}$ being the $y$ component of $\vec{p}_{l^{ \pm}}$with respect to a coordinate system chosen in the $e^{+} e^{-}$c.m. frame so that the $z$-axis is along $\vec{p}_{t}$, and the $y$-axis is along $\vec{p}_{e} \times \vec{p}_{t}$. The $t \bar{t}$ production plane is thus the $x z$ plane. $\theta$ refers to the angle between $\vec{p}_{t}$ and $\vec{p}_{e}$ in the c.m. frame. Note that the asymmetry $A_{u d}$ is related to spin components of the top

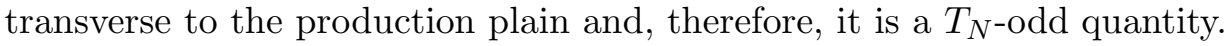

Three additional asymmetries were considered in [182]. The combined up-down and forwardbackward asymmetry

$$
A_{u d}^{\mathrm{fb}}=\int_{0}^{1} A_{u d}(\theta) d \cos \theta-\int_{-1}^{0} A_{u d}(\theta) d \cos \theta,
$$

with $A_{u d}(\theta)$ given in Eq. 6.43. The left-right asymmetry

$$
A_{l r}=\int_{-1}^{+1} A_{l r}(\theta) d \cos \theta
$$

where

$$
A_{l r}(\theta)=\frac{1}{2 \sigma}\left[\frac{d \sigma\left(l^{+}, \text {left }\right)}{d \cos \theta}-\frac{d \sigma\left(l^{+}, \text {right }\right)}{d \cos \theta}+\frac{d \sigma\left(l^{-}, \text {left }\right)}{d \cos \theta}-\frac{d \sigma\left(l^{-}, \text {right }\right)}{d \cos \theta}\right],
$$

and left/right refers to $\left(p_{l^{ \pm}}\right)_{x} \gtrless 0$. The combined left-right and forward-backward asymmetry

$$
A_{l r}^{\mathrm{fb}}=\int_{0}^{1} A_{l r}(\theta) d \cos \theta-\int_{-1}^{0} A_{l r}(\theta) d \cos \theta,
$$

with $A_{l r}(\theta)$ given in Eq. 6.46.

To leading order in the dipole form factors and on ignoring CP violation in the $t$ and $\bar{t}$ decays, all the above five CP-odd asymmetries are linear functions of $d_{t}^{\gamma}$ and $d_{t}^{Z}$. The asymmetries $A_{E}, A_{l r}, A_{l r}^{\mathrm{fb}}$ are $T_{N}$-even and are therefore proportional to $\Im \mathrm{m} d_{t}^{\gamma, Z}$, while $A_{u d}, A_{u d}^{\mathrm{fb}}$ are $T_{N}$-odd and are proportional to $\Re$ ed $d_{t}^{\gamma, Z}$. The $T_{N}$-even asymmetries can be symbolically written as

$$
\mathcal{A}_{i} \equiv a_{i}^{\gamma}\left(P_{e}, P_{\bar{e}}\right) \Im \mathrm{m} d_{t}^{\gamma}+a_{i}^{Z}\left(P_{e}, P_{\bar{e}}\right) \Im \mathrm{m} d_{t}^{Z}
$$

Similarly, for the $T_{N}$-odd observables, one obtains

$$
\mathcal{B}_{i} \equiv b_{i}^{\gamma}\left(P_{e}, P_{\bar{e}}\right) \Re \mathrm{e} d_{t}^{\gamma}+b_{i}^{Z}\left(P_{e}, P_{\bar{e}}\right) \Re \mathrm{e} d_{t}^{Z} .
$$

where $\mathcal{A}_{i}=A_{E}, A_{l r}$ or $A_{l r}^{\mathrm{fb}}$ and $\mathcal{B}_{i}=A_{u d}$ or $A_{u d}^{\mathrm{fb}}$. The functions $a^{\gamma, Z}, b^{\gamma, Z}$ depend, among other parameters, on the polarizations of the incoming electron and positron beams, $P_{e}$ and $P_{\bar{e}}$, respectively, and are explicitly given in [182].

It is evident from Eqs. 6.48 and 6.49 that, without beam polarization, by measuring only one asymmetry of the type $\mathcal{A}_{i}$ and/or $\mathcal{B}_{i}$, one can extract information only on one combination of $\left\{\Im \mathrm{m} d_{t}^{\gamma}, \Im \mathrm{m} d_{t}^{Z}\right\}$ and/or $\left\{\Re \mathrm{e} d_{t}^{\gamma}, \Re \mathrm{e} d_{t}^{Z}\right\}$, respectively. However, any two asymmetries with the same $T_{N}$ property can be used to determine two independent combinations of the corresponding real or imaginary parts of $d_{t}^{\gamma}$ and $d_{t}^{Z}$, thus, giving $\Im \mathrm{m} d_{t}^{\gamma}$ and $\Im \mathrm{m} d_{t}^{Z}$ or $\Re \mathrm{e} d_{t}^{\gamma}$ and $\Re \mathrm{e} d_{t}^{Z}$ independently. Such an analysis was described in the previous section where the observable pairs $\hat{T}_{33}, \hat{A}_{1}$ and $\hat{Q}_{33}, \hat{A}_{2}$ were used to find the sensitivity of a high energy $e^{+} e^{-}$collider to $\Re \mathrm{e} d_{t}^{\gamma}, \Re \mathrm{e} d_{t}^{Z}$ and $\Im m d_{t}^{\gamma}, \Im m d_{t}^{Z}$, respectively. However, it was suggested in [182] that if, in addition, beam polarization is included, then one $T_{N}$-even $\left(T_{N}\right.$-odd $)$ asymmetry is sufficient to determine $\Im$ m $d_{t}^{\gamma}$ and 
Table 6: 90\% confidence limits on the real and imaginary parts of the top dipole form factors $d_{t}^{\gamma}$ and $d_{t}^{Z}$, in units of $10^{-17} \mathrm{e}-\mathrm{cm}$, from different asymmetries. In the unpolarized case, the asymmetries $A_{u d}, A_{u d}^{\mathrm{fb}}$ are together used to get limits on $\Re \mathrm{e} d_{t}^{\gamma, Z}$ and $A_{l r}$, $A_{l r}^{\mathrm{fb}}$ to obtain the limits on $\Im \mathrm{m} d_{t}^{\gamma, Z}$. In the polarized case, the limits obtained from $A_{u d}$ and $A_{l r}$ are denoted by (a) and the ones from $A_{u d}^{\mathrm{fb}}$ and $A_{l r}^{\mathrm{fb}}$ are denoted by (b). The numbers are for the single-leptonic $t \bar{t}$ mode, for $m_{t}=174 \mathrm{GeV}, \sqrt{\mathrm{s}}=500 \mathrm{GeV}$ and an integrated luminosity of $\mathcal{L}=10 \mathrm{fb}^{-1}$.

\begin{tabular}{|l|c|c|c|c|}
\hline Case & $\left|\Re \mathrm{e} d_{t}^{\gamma}\right|$ & $\left|\Re \mathrm{e} d_{t}^{Z}\right|$ & $\left|\Im \mathrm{m} d_{t}^{\gamma}\right|$ & $\left|\Im \mathrm{m} d_{t}^{Z}\right|$ \\
\hline unpolarized & 54.4 & 15.9 & 7.9 & 62.4 \\
(a) polarized $\left(P_{e}= \pm 0.5\right)$ & 2.3 & 2.3 & 2.3 & 9.1 \\
(b) polarized $\left(P_{e}= \pm 0.5\right)$ & 12.5 & 9.1 & 2.3 & 7.9 \\
\hline
\end{tabular}

$\Im m d_{t}^{Z}\left(\Re \mathrm{e} d_{t}^{\gamma}\right.$ and $\left.\Re \mathrm{e} d_{t}^{Z}\right)$ independently by measuring this asymmetry for different polarizations. Both approaches were adopted in [182]. Their best results are summarized in Table 6 6 where $90 \%$ confidence level limits are given for $\sqrt{s}=500 \mathrm{GeV}$ and $m_{t}=174 \mathrm{GeV}$. We can see from Table 6 that the second approach, of incorporating beam polarization, increases the sensitivity to both the real and imaginary parts of the EDM and ZEDM of the top, in some cases, by about one order of magnitude. With $50 \%$ beam polarization, the $90 \%$ confidence level limits for $\Im \mathrm{m} d_{t}^{\gamma}, \Im \mathrm{m} d_{t}^{Z}, \Re \mathrm{e} d_{t}^{\gamma}$ and $\Re \mathrm{e} d_{t}^{Z}$ are again at best around $\sim$ few $\times 10^{-17} \mathrm{e}-\mathrm{cm}$.

An interesting differential asymmetry that combines information from both the production and decay vertices of the top was suggested in [177]:

$$
A_{\ell \ell} \equiv \frac{\left(\iint_{x<\bar{x}} d x d \bar{x} \frac{d^{2} \sigma}{d x d \bar{x}}-\iint_{x>\bar{x}} d x d \bar{x} \frac{d^{2} \sigma}{d x d \bar{x}}\right)}{\iint d x d \bar{x} \frac{d^{2} \sigma}{d x d \bar{x}}} .
$$

This asymmetry utilizes the double-leptonic energy distribution in $e^{+} e^{-} \rightarrow t \bar{t} \rightarrow \ell^{+} \ell^{-} \nu_{\ell} \bar{\nu}_{\ell} b \bar{b}$

$$
\frac{1}{\sigma} \frac{d^{2} \sigma}{d x d \bar{x}}=\sum_{i=1}^{3} c_{i} f_{i}(x, \bar{x}),
$$

where $x, \bar{x}$ are defined in Eq. 6.13 and

$$
c_{1}=1 \quad, \quad c_{2}=\xi \quad, \quad c_{3}=-\frac{1}{2} \Re \mathrm{e}\left(f_{2}^{R}-\bar{f}_{2}^{L}\right) .
$$

$f_{2}^{R}, \bar{f}_{2}^{L}$ and $\xi$ are defined in Eqs. 6.5, 6.6 and 6.14, respectively. In terms of $\xi$ (or equivalently of $\left.\Im \mathrm{m} d_{t}^{\gamma, Z}\right)$ and $\Re \mathrm{e}\left(f_{2}^{R}-\bar{f}_{2}^{L}\right)$ and with $\sqrt{s}=500 \mathrm{GeV}, m_{t}=180 \mathrm{GeV}$ and the SM parameters they obtained the simple relation

$$
A_{\ell \ell}=-0.34 \xi-0.31 \Re \mathrm{e}\left(f_{2}^{R}-\bar{f}_{2}^{L}\right) .
$$

Given a number of available double-leptonic $t \bar{t}$ events in the NLC and using the relation in Eq. 6.53, one can now plot the 1- $\sigma, 2-\sigma$ and $3-\sigma$ detectable regions in the $\Im \mathrm{m} d_{t}^{\gamma, Z}-\Re \mathrm{e}\left(f_{2}^{R}-\bar{f}_{2}^{L}\right)$ plane. These regions are shown in Fig. 27 for $\sim 700$ double-leptonic $t \bar{t}$ events in a $500 \mathrm{GeV}$ collider. We see from Fig. 27 that a $3-\sigma$ detection of CP violation through $A_{\ell \ell}$ is possible in a wide range of the parameters $\xi$ and $f_{2}^{R}, \bar{f}_{2}^{L}$. However, if one parameter is very small, then 
it requires the other to be relatively large. Thus, for example, if $\Re \mathrm{e}\left(f_{2}^{R}-\bar{f}_{2}^{L}\right)=0$ then, at $3-\sigma,\left|\Im \mathrm{m}\left(d_{t}^{\gamma, Z}\right)\right| \gtrsim 1.7 \times 10^{-17} \mathrm{e}-\mathrm{cm}$ (or, with the notation used in $\|177\|,\left|\Re \mathrm{e}\left(D_{\gamma, Z}\right)\right| \gtrsim 0.3$, see also Fig. 27). This is again comparable to obtainable limits from other observables described in this section and, thus, falls short by about one order of magnitude when compared to model dependent predictions for the top dipole moment.

Finally, interesting CP-violating asymmetries which involve correlations among $b$-quarks from $t \bar{t} \rightarrow b W^{+} \bar{b} W^{-}$were suggested by Bartl et al. in [178, 179, 180]. They utilized the angular 178] and energy 179 distributions of $b$ and $\bar{b}$ with initial beam polarization, in $e^{+} e^{-} \rightarrow t \bar{t}$ followed by $t \rightarrow b W^{+}$and $\bar{t} \rightarrow \bar{b} W^{-}$, to construct CP-violating asymmetries that can disentangle $\mathrm{CP}$ violation in the $t \bar{t}$ production mechanism from $\mathrm{CP}$ violation in the top decay. Unfortunately, their asymmetries, when evaluated within the MSSM, range from $10^{-4}$ to $10^{-3}$ at best, and therefore also seem to be too small to be detectable at a future NLC. 


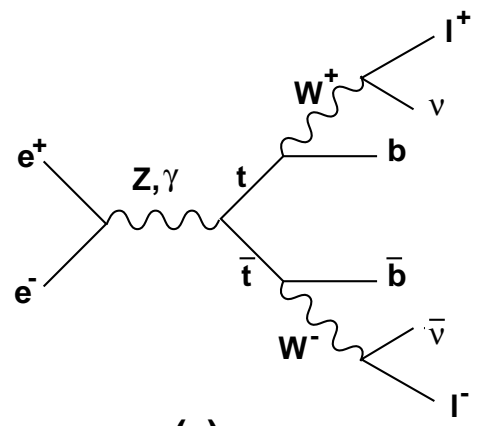

(a)

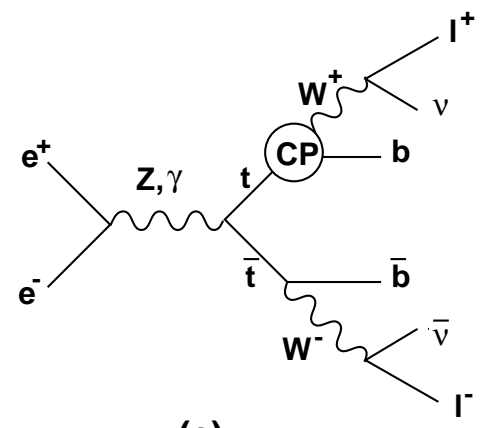

(c)

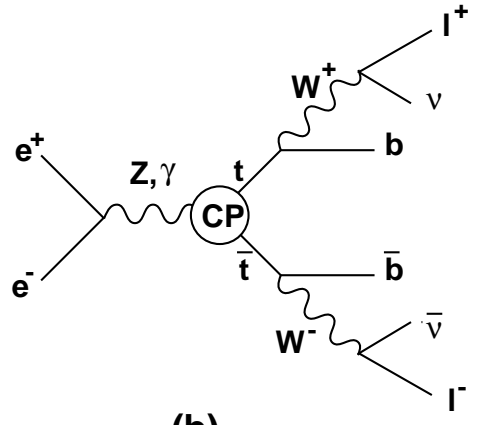

(b)

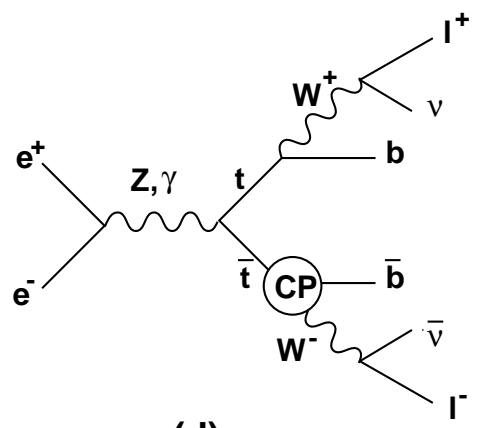

(d)

Figure 26: Tree-level (a) and CP-violating amplitudes (b),(c),(d) to leading order in the SM couplings and in CP-violating form factors. 


\section{$6.2 e^{+} e^{-} \rightarrow t \bar{t} h, t \bar{t} Z$, examples of tree-level CP violation}

Let us now turn our attention to the reactions

$$
\begin{aligned}
& e^{+}\left(p_{+}\right)+e^{-}\left(p_{-}\right) \rightarrow t\left(p_{t}\right)+\bar{t}\left(p_{\bar{t}}\right)+h\left(p_{h}\right), \\
& e^{+}\left(p_{+}\right)+e^{-}\left(p_{-}\right) \rightarrow t\left(p_{t}\right)+\bar{t}\left(p_{\bar{t}}\right)+Z\left(p_{Z}\right),
\end{aligned}
$$

which may exhibit large $\mathrm{CP}$ violation asymmetries in a $2 \mathrm{HDM}$ [55, 184, 185, 186]. Note that for reasons discussed below, for the CP-violating effects in $e^{+} e^{-} \rightarrow t \bar{t} h$ as well as in $e^{+} e^{-} \rightarrow t \bar{t} Z$, only two out of the three neutral Higgs of the $2 \mathrm{HDM}$ are relevant. We denote these two neutral Higgs particles by $h$ and $H$ corresponding to the lighter and heavier Higgs-boson, respectively. In some instances we denote a neutral Higgs by $\mathcal{H}$, then $\mathcal{H}=h$ or $H$ is to be understood.

A novel feature of these reactions is that the effect arises at the tree-graph level. As a consequence, one can construct new type of asymmetries which are $\propto$ (tree $\times$ tree) $/($ tree $\times$ tree) and are therefore a-priori of $\mathcal{O}(1)$. This stands in contrast to loop induced CP-violating effects in $t \bar{t}$ production for which the $\mathrm{CP}$ asymmetries, in general, are $\propto$ (tree $\times$ loop $) /($ tree $\times$ tree $)$ and are therefore suppressed by additional small couplings to begin with, i.e., at 1-loop, typically, by $\alpha$ - the fine structure constant.

Indeed, we will show below that $\mathrm{CP}$ violation at the level of tens of a percent is possible in the reactions in Eqs. 6.54 and 6.55. Basically, for the $t \bar{t} h(t \bar{t} Z)$ final states, $\operatorname{Higgs}(Z)$ emission off the $t$ and $\bar{t}$ interferes with the $\operatorname{Higgs}(Z)$ emission off the $s$-channel $Z$-boson (see Fig. 28) [55, 184, 185]. We find that the processes $e^{+} e^{-} \rightarrow t \bar{t} h$ and $e^{+} e^{-} \rightarrow t \bar{t} Z$ provide two independent, but analogous, promising probes to search for the signatures of the same CP-odd phase, residing in the $t \bar{t}$-neutral Higgs coupling, if the value of $\tan \beta$ (the ratio between the two VEV's in a $2 \mathrm{HDM}$ ) is in the vicinity of 1 . In particular, they serve as good examples for large CP-violating effects that could emanate from $t$ systems due to the large mass of the top quark and, thus, they might illuminate the role of a neutral Higgs particle in $\mathrm{CP}$ violation.

Although these reactions are not meant (necessarily) to lead to the discovery of a neutral Higgs, they will, no doubt, be intensely studied at the NLC since they stand out as very important channels for a variety of reasons. In particular, they could perhaps provide a unique opportunity to observe the top-Higgs Yukawa couplings directly [51, 187, 188, 189, 190, 191]. In [51, 189], using a generalization of the optimal observables technique outlined below (see also section 2.6), Gunion et al. have extended the initial work [55] on $\mathrm{CP}$ violation in $e^{+} e^{-} \rightarrow t \bar{t} h$ to include a detailed cross-section analysis such that all Higgs Yukawa couplings combinations are extracted (see below). A similar analysis which also uses the optimized observable technique for $e^{+} e^{-} \rightarrow t \bar{t} Z$ is given in [186]. A detailed cross-section analysis of the reaction $e^{+} e^{-} \rightarrow t \bar{t} Z$ in the SM was performed by Hagiwara et al. [190]. There, it was found that the Higgs exchange contribution of diagram (b) on the right hand side of Fig. 28 will be almost invisible in a $\mathrm{TeV} e^{+} e^{-}$collider for neutral Higgs masses in the range $m_{h}<2 m_{t}$. Interestingly, we will show here that, if the scalar sector is doubled, then the lightest neutral Higgs $(h)$ may reveal itself through CP-violating interactions with the top quark even if $m_{h}<2 m_{t}$. Obviously, a non-SM (e.g., larger) top-Higgs Yukawa coupling can cause an enhancement in the rates for both the $t \bar{t} h$ and $t \bar{t} Z$ final states. Thus, a "simple" cross-section study for these reactions may also come in handy for searching for new physics. However, one should keep in mind that, from the experimental point of view, asymmetries, i.e., ratios of cross-section, are easier to handle and, in particular, CP-violating signals are very distinctive evidence for new physics.

This section will be divided to three parts. In the first part we present a detailed analysis of the tree-level $\mathrm{CP}$ violation in the reactions $e^{+} e^{-} \rightarrow t \bar{t} h$ and $e^{+} e^{-} \rightarrow t \bar{t} Z$ which manifests itself as a $T_{N}$-odd correlation of momenta. In the second part we will consider the generalized optimization technique developed by Gunion et al. and its application to the reaction $e^{+} e^{-} \rightarrow t \bar{t} h$. In the last part we will discuss CP violation in the Higgs decay $h \rightarrow t \bar{t}$, where we take the Higgs to be produced through the Bjorken mechanism $e^{+} e^{-} \rightarrow Z h$. 


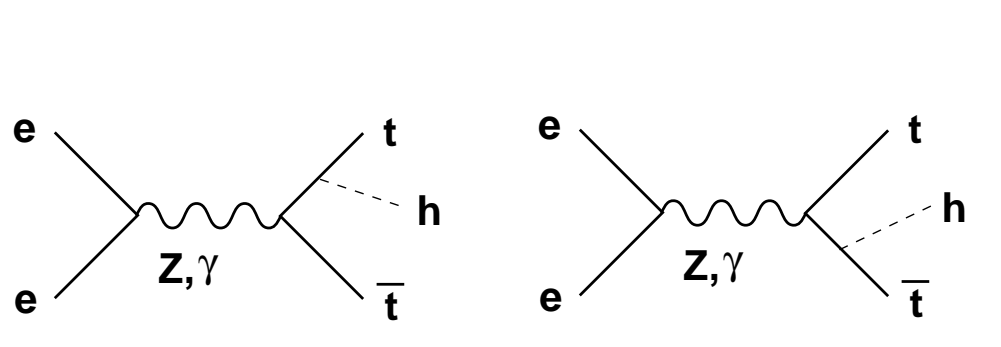

(a)

(b)

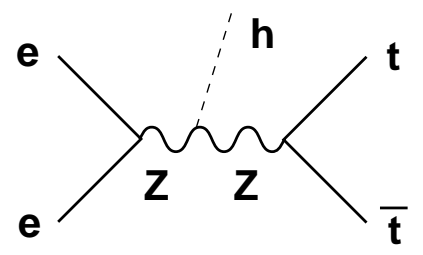

(c)

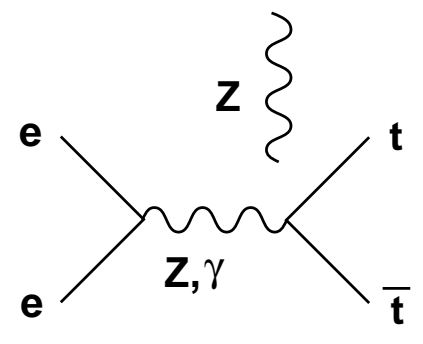

(a)

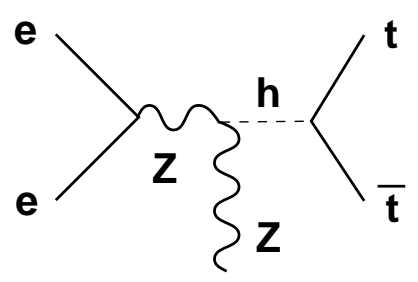

(b)

Figure 28: Tree-level Feynman diagrams contributing to $e^{+} e^{-} \rightarrow t \bar{t} h$ (left hand side) and $e^{+} e^{-} \rightarrow$ $t \bar{t} Z$ (right hand side) in the unitary gauge, in a 2HDM. For $e^{+} e^{-} \rightarrow t \bar{t} Z$, diagram (a) on the right hand side represents 8 diagrams in which either $Z$ or $\gamma$ are exchanged in the s-channel and the outgoing $Z$ is emitted from $e^{+}, e^{-}, t$ or $\bar{t}$. 


\subsubsection{Tree-level CP violation}

In the unitary gauge the reactions in Eqs. 6.54 and 6.55 can proceed via the Feynman diagrams depicted in Fig. 28. We see that for $e^{+} e^{-} \rightarrow t \bar{t} Z$, diagram (b) on the right hand side of Fig. 28, in which $Z$ and $\mathcal{H}$ are produced $\left(\mathcal{H}=h\right.$ or $H$ is either a real or a virtual particle, i.e. $m_{\mathcal{H}}>2 m_{t}$ or $m_{\mathcal{H}}<2 m_{t}$, respectively) followed by $\mathcal{H} \rightarrow t \bar{t}$, is the only place where new CP-nonconserving dynamics from the Higgs sector can arise, being proportional to the CP-odd phase in the $t \bar{t} \mathcal{H}$ vertex. As mentioned above, in both the $t \bar{t} h$ and the $t \bar{t} Z$ final state cases, $\mathrm{CP}$ violation arises due to interference of the diagrams where the neutral Higgs is coupled to a $Z$-boson with the diagrams where it is radiated off the $t$ or $\bar{t}$. We note that in the $t \bar{t} Z$ case there is no CP-violating contribution coming from the interference between the diagrams with the $Z Z \mathcal{H}$ coupling and the diagrams where the $Z$-boson is emitted from the incoming electron or positron lines (not shown in Fig. 28).

The relevant pieces of the interaction Lagrangian involve the $t \bar{t} \mathcal{H}^{k}$ Yukawa and the $Z Z \mathcal{H}^{k}$ couplings and are given in Eqs. 3.70 and 3.71. There, $\mathcal{H}^{k}(k=1,2$ or 3$)$ are the three neutral Higgs scalars in the theory. As usual the three couplings $a_{t}^{k}, b_{t}^{k}$ and $c^{k}$ in Eqs. 3.70 and 3.71 are functions of $\tan \beta \equiv v_{2} / v_{1}$ (the ratio of the two VEV's) and of the three mixing angles $\alpha_{1}, \alpha_{2}$, $\alpha_{3}$ which characterize the Higgs mass matrix in Eq. 3.73 (for details see section 3.2.3).

As was also mentioned in section 3.2.3, only two out of the three neutral Higgs can simultaneously have a coupling to vector-bosons and a pseudoscalar coupling to fermions. Therefore, only those two neutral Higgs particles are relevant for the present discussion and, without loss of generality we denote them as $\mathcal{H}^{1}=h$ and $\mathcal{H}^{2}=H$ with couplings $a_{t}^{h}, b_{t}^{h}, c^{h}$ and $a_{t}^{H}, b_{t}^{H}, c^{H}$, corresponding to the light, $h$, and heavy, $H$, neutral Higgs, respectively. This implies the existence of a "GIM-like" cancellation, namely, when both $h$ and $H$ contribute to CP violation, then all CP-nonconserving effects, being proportional to $b_{t}^{h} c^{h}+b_{t}^{H} c^{H}$, must vanish when the two Higgs states $h$ and $H$ are degenerate. In the following we set the mass of the heavy Higgs, $H$, to be $m_{H}=750 \mathrm{GeV}$ or $1 \mathrm{TeV}$.

In the process $e^{+} e^{-} \rightarrow t \bar{t} h$, a Higgs particle is produced in the final state, therefore, the heavy Higgs-boson, $H$, is not important and this "GIM-like" mechanism is irrelevant. Note that there is an additional diagram contributing to $e^{+} e^{-} \rightarrow t \bar{t} h$, which involves the $Z h H$ coupling and is not shown in Fig. 28. This diagram is, however, negligible compared to the others for the large $m_{H}$ values used here. In contrast, in the process $e^{+} e^{-} \rightarrow t \bar{t} Z$, the Higgs is exchanged as a virtual or a real particle and the effect of $H$ is, although small compared to $h$, important in order to restore the "GIM-like" cancellation discussed above.

For both the $t \bar{t} h$ and $t \bar{t} Z$ final states processes, we denote the tree-level polarized Differential Cross-Section (DCS) by $\Sigma_{(j) f}$, where $f=t \bar{t} h$ or $f=t \bar{t} Z$ corresponding to the $t \bar{t} h$ or $t \bar{t} Z$ final states, respectively, and $j=1(-1)$ for the left(right) polarized incoming electron beam. $\Sigma_{(j) f}$ can be subdivided into its CP-even $\left(\Sigma_{+(j) f}\right)$ and CP-odd $\left(\Sigma_{-(j) f}\right)$ parts

$$
\Sigma_{(j) f}=\Sigma_{+(j) f}+\Sigma_{-(j) f} .
$$

The CP-even and CP-odd DCS's can be further subdivided into different terms which correspond to the various Higgs coupling combinations and which transform as even or odd (denoted by the letter $n$ ) under $T_{N}$. For both final states, $f=t \bar{t} h$ and $f=t \bar{t} Z$, we have

$$
\begin{aligned}
& \Sigma_{+(j) f}=\sum_{i} g_{+f}^{i(n)} F_{+(j) f}^{i(n)}, \quad \mathrm{CP}-\text { even } \\
& \Sigma_{-(j) f}=\sum_{i} g_{-f}^{i(n)} F_{-(j) f}^{i(n)}, \mathrm{CP}-\text { odd }
\end{aligned}
$$

where $g_{+f}^{i(n)}, g_{-f}^{i(n)}, n=+$ or - , are different combinations of the Higgs couplings $a_{t}^{\mathcal{H}}, b_{t}^{\mathcal{H}}, c^{\mathcal{H}}$ and $F_{+(j) f}^{i(n)}, F_{-(j) f}^{i(n)}$, again with $n=+$ or - , are kinematical functions of phase space which transform 
like $n$ under $T_{N}$.

Let us first write the Higgs coupling combinations for the CP-even part. In the case of $e^{+} e^{-} \rightarrow t \bar{t} h$, neglecting the imaginary part in the s-channel $Z$-propagator, we have four relevant coupling combinations [51, 55, 185:

$$
g_{+t \bar{t} h}^{1(+)}=\left(a_{t}^{h}\right)^{2}, g_{+t \bar{t} h}^{2(+)}=\left(b_{t}^{h}\right)^{2}, g_{+t \bar{t} h}^{3(+)}=\left(c^{h}\right)^{2}, g_{+t \bar{t} h}^{4(+)}=a_{t}^{h} c^{h} .
$$

In the case of $e^{+} e^{-} \rightarrow t \bar{t} Z$, apart from the SM contribution, which corresponds to interference terms among the four SM diagrams represented by diagram (a) on the right hand side of Fig. 28, and keeping terms proportional to both the real and imaginary parts of the Higgs propagator, $\Pi_{\mathcal{H}}$, we get [184, 185]:

$$
\begin{aligned}
g_{+t \bar{t} Z}^{1(+)} & =\left(a_{t}^{\mathcal{H}} c^{\mathcal{H}}\right) \Re \mathrm{e}\left(\Pi_{\mathcal{H}}\right), g_{+t t \bar{Z} Z}^{2(-)}=\left(a_{t}^{\mathcal{H}} c^{\mathcal{H}}\right) \Im \mathrm{m}\left(\Pi_{\mathcal{H}}\right), \\
g_{+t \bar{t} Z}^{3(+)} & =\left(a_{t}^{\mathcal{H}} c^{\mathcal{H}}\right)^{2} \Re \mathrm{e}\left(\Pi_{\mathcal{H}}\right), g_{+t \bar{t} Z}^{4(+)}=\left(a_{t}^{\mathcal{H}} c^{\mathcal{H}}\right)^{2} \Im \mathrm{m}\left(\Pi_{\mathcal{H}}\right), \\
g_{+t \bar{t} Z}^{5(+)} & =\left(b_{t}^{\mathcal{H}} c^{\mathcal{H}}\right)^{2} \Re \mathrm{e}\left(\Pi_{\mathcal{H}}\right), g_{+t \bar{t} Z}^{6(+)}=\left(b_{t}^{\mathcal{H}} c^{\mathcal{H}}\right)^{2} \Im \mathrm{m}\left(\Pi_{\mathcal{H}}\right),
\end{aligned}
$$

where

$$
\Pi_{\mathcal{H}} \equiv\left(s+m_{Z}^{2}-m_{\mathcal{H}}^{2}-2 p \cdot p_{Z}+i m_{\mathcal{H}} \Gamma_{\mathcal{H}}\right)^{-1},
$$

$p \equiv p_{-}+p_{+}$and $\Gamma_{\mathcal{H}}$ is the width of $\mathcal{H}=h$ or $H$.

The CP-odd coupling combinations are

$$
g_{-t \bar{t} h}^{1(-)}=b_{t}^{h} c^{h}
$$

for the $t \bar{t} h$ final state and

$$
g_{-t \bar{t} Z}^{1(-)}=b_{t}^{\mathcal{H}} c^{\mathcal{H}} \Re \mathrm{e}\left(\Pi_{\mathcal{H}}\right), g_{-t \bar{t} Z}^{2(+)}=b_{t}^{\mathcal{H}} c^{\mathcal{H}} \Im \mathrm{m}\left(\Pi_{\mathcal{H}}\right),
$$

for $t \bar{t} Z$ final state. $t \bar{t} Z)$

The CP-even pieces, $\Sigma_{+(j) f}$, yield the corresponding cross-sections (recall that $f=t \bar{t} h$ or

$$
\sigma_{(j) f}=\int \Sigma_{+(j) f}(\Phi) d \Phi,
$$

where $\Phi$ stands for the phase-space variables. In Fig. 29(a) and 29(b) we plot the unpolarized cross-sections, $\sigma_{t \bar{t} h}$ and $\sigma_{t \bar{t} Z}$ as a function of $m_{h}$ and $\sqrt{s}$, for Model II (i.e., 2HDM of type 2 as defined in section 3.2.3), with $m_{H}=750 \mathrm{GeV}$ and the set of values $\left\{\alpha_{1}, \alpha_{2}, \alpha_{3}\right\}=\{\pi / 2, \pi / 4,0\}$ which we denote as set II. Set II is also adopted later when discussing the CP-violating effect. Furthermore, for the $t \bar{t} h$ final state we choose $\tan \beta=0.5$ while for $t \bar{t} Z$ we choose $\tan \beta=0.3$. Afterwards, we will discuss the dependence of the CP-violating effect on $\tan \beta$ in the $t \bar{t} h$ and $t \bar{t} Z$ cases. One can observe the dissimilarities in the two cross-sections $\sigma_{t \bar{t} h}$ and $\sigma_{t \bar{t} Z}$ : while $\sigma_{t \bar{t} h}$ is at most $\sim 1.5 \mathrm{fb}, \sigma_{t \bar{t} Z}$ can reach $\sim 7 \mathrm{fb}$ at around $\sqrt{s} \approx 750 \mathrm{GeV}$ and $m_{h} \gtrsim 2 m_{t} . \sigma_{t \bar{t} h}$ drops with $m_{h}$ while $\sigma_{t \bar{t} Z}$ grows in the range $m_{h} \lesssim 2 m_{t} \cdot \sigma_{t \bar{t} Z}$ peaks at around $m_{h} \gtrsim 2 m_{t}$ and drops as $m_{h}$ grows further. Moreover, $\sigma_{t \bar{t} h}$ peaks at around $\sqrt{s} \approx 1(1.5) \mathrm{TeV}$ for $m_{h}=100(360) \mathrm{GeV}$, while $\sigma_{t \bar{t} Z}$ peaks at around $\sqrt{s} \approx 750 \mathrm{GeV}$ for both $m_{h}=100$ and $360 \mathrm{GeV}$. As we will see later, these different features of the two cross-sections are, in part, the cause for the different behavior of the $\mathrm{CP}$ asymmetries discussed below.

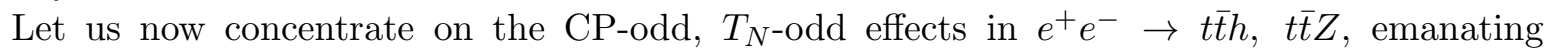

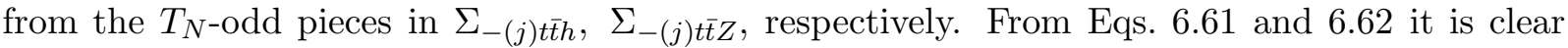


that the CP-violating pieces $\Sigma_{-(j) t \bar{t} h}, \Sigma_{-(j) t \bar{t} Z}$ have to be proportional to $b_{t}^{h} c^{h}$ (in the $t \bar{t} Z$ case there is an additional similar piece corresponding to the heavy Higgs $H$ ). The corresponding CP-odd kinematic functions, $F_{-(j) t \bar{t} h}^{1(-)}, F_{-(j) t \bar{t} Z}^{1(-)}$, being $T_{N^{-o d d}}$, are pure tree-level quantities and are proportional to the only non-vanishing Levi-Civita tensor present, $\epsilon\left(p_{-}, p_{+}, p_{t}, p_{\bar{t}}\right)$, when the spins of the top are disregarded. The explicit expressions for $F_{-(j) f}^{1(-)}$ are (recall that $j=1(-1)$ for left(right) polarized incoming electron beam)

$$
\begin{aligned}
F_{-(j) t \bar{t} h}^{1(-)}= & -\frac{1}{\sqrt{2}}\left(\frac{g_{W}^{3}}{c_{W}^{3}}\right)^{2} \frac{m_{t}^{2}}{m_{Z}^{2}} \Pi_{Z h} \Pi_{Z} T_{t}^{3} c_{j}^{Z} \epsilon\left(p_{-}, p_{+}, p_{t}, p_{\bar{t}}\right) \times \\
& \left\{j\left(\Pi_{t}^{h}+\Pi_{\bar{t}}^{h}\right)\left[\left(s-s_{t}-m_{h}^{2}\right)\left(3 w_{j}^{-}-w_{j}^{+}\right)+m_{Z}^{2}\left(w_{j}^{-}-w_{j}^{+}\right)\right]+\right. \\
& \left.T_{t}^{3} c_{j}^{Z} \Pi_{Z}\left(\Pi_{t}^{h}-\Pi_{\bar{t}}^{h}\right) f\right\} \\
F_{-(j) t \bar{t} Z}^{1(-)}= & -\sqrt{2}\left(\frac{2 g_{W}^{3}}{c_{W}^{3}}\right)^{2} \frac{m_{t}^{2}}{m_{Z}^{2}} \Pi_{Z} T_{t}^{3} c_{j}^{Z} \epsilon\left(p_{-}, p_{+}, p_{t}, p_{\bar{t}}\right) \times \\
& \left\{j\left(\Pi_{t}^{Z}+\Pi_{\bar{t}}^{Z}\right)\left[m_{Z}^{2} w_{j}^{-}+\left(s_{t}-s\right) w_{j}^{+}\right]+\right. \\
& \left.T_{t}^{3} c_{j}^{Z} \Pi_{Z}\left(\Pi_{t}^{Z}-\Pi_{\bar{t}}^{Z}\right) f\right\}
\end{aligned}
$$

where $s \equiv 2 p_{-} \cdot p_{+}$is the c.m. energy of the colliding electrons, $s_{t} \equiv\left(p_{t}+p_{\bar{t}}\right)^{2}$ and $f \equiv\left(p_{-}-\right.$ $\left.p_{+}\right) \cdot\left(p_{t}+p_{\bar{t}}\right)$. Also

$$
\begin{aligned}
\Pi_{t(\bar{t})}^{h} & \equiv\left(2 p_{t(\bar{t})} \cdot p_{h}+m_{h}^{2}\right)^{-1}, \Pi_{t(\bar{t})}^{Z} \equiv\left(2 p_{t(\bar{t})} \cdot p_{Z}+m_{Z}^{2}\right)^{-1}, \\
\Pi_{Z} & \equiv\left(s-m_{Z}^{2}\right)^{-1}, \Pi_{\gamma} \equiv s^{-1}, \Pi_{Z h} \equiv\left(\left(p-p_{h}\right)^{2}-m_{Z}^{2}\right)^{-1} .
\end{aligned}
$$

and

$$
w_{j}^{ \pm} \equiv\left(s_{W}^{2} Q_{t}-\frac{1}{2} T_{t}^{3}\right) c_{j}^{Z} \Pi_{Z} \pm Q_{t} s_{W}^{2} c_{W}^{2} \Pi_{\gamma},
$$

where $s_{W}\left(c_{W}\right)$ is the $\sin (\cos )$ of the weak mixing angle $\theta_{W}, Q_{f}$ and $T_{f}^{3}$ are the charge and the $z$-component of the weak isospin of a fermion, respectively, and $c_{-1}^{Z}=1 / 2-s_{W}^{2}, c_{1}^{Z}=-s_{W}^{2}$.

Since at tree-level there cannot be any absorptive phases, CP-violating asymmetries only of

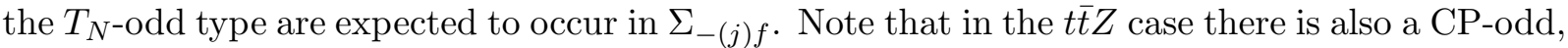
$T_{N}$-even piece, $b_{t}^{\mathcal{H}} c^{\mathcal{H}} \operatorname{Im}\left(\Pi_{\mathcal{H}}\right) \times F_{-(j) t \bar{t} Z}^{2(+)}$ (see Eq. 6.62), in the DCS. However, being proportional to the absorptive part coming from the Higgs propagator, it is not a pure tree-level quantity.

Simple examples of observables that can trace the tree-level CP-odd effect in $e^{+} e^{-} \rightarrow t \bar{t} h ; t \bar{t} Z$ are [55]:

$$
O=\frac{\vec{p}_{-} \cdot\left(\vec{p}_{t} \times \vec{p}_{\bar{t}}\right)}{s^{3 / 2}}, \quad O_{\text {opt }}(t \bar{t} h)=\frac{\Sigma_{-(j) t \bar{t} h}}{\Sigma_{+(j) t \bar{t} h}} ; \quad O_{\text {opt }}(t \bar{t} Z)=\frac{\Sigma_{-(j) t \bar{t} Z}}{\Sigma_{+(j) t \bar{t} Z}} .
$$

Here $O_{\text {opt }}(t \bar{t} h ; t \bar{t} Z)$ are optimal observables in the sense that the statistical error in the measured asymmetry is minimized [16]. Note also that only the $T_{N}$-odd part of $\Sigma_{-(j) t \bar{t} Z}$ is involved. As mentioned before, since the final state consists of three particles, using only the available momenta, there is a unique antisymmetric combination of momenta that can be formed. Thus, both observables are proportional to $\epsilon\left(p_{-}, p_{+}, p_{t}, p_{\bar{t}}\right)$. Furthermore, $O_{\mathrm{opt}}(t \bar{t} h ; t \bar{t} Z)$ are related to $O$ through a multiplication by a CP-even function. In the following we focus only on the CP-odd effects coming from the optimal observables. We remark, however, that the results for the simple observable $O$ exhibit the same behavior, though slightly smaller then those for $O_{\text {opt }}$. 
The theoretical statistical significance, $N_{S D}$, in which an asymmetry can be measured in an ideal experiment is $N_{S D}=A \sqrt{L} \sqrt{\sigma}$ ( $\sigma=\sigma_{t \bar{t} h}, \sigma_{t \bar{t} Z}$ for the $t \bar{t} h, t \bar{t} Z$ final states, respectively), where for the observables $O$ and $O_{\text {opt }}$, the CP-odd quantity $A$, defined above, is

$$
A_{O} \approx\langle O\rangle / \sqrt{\left\langle O^{2}\right\rangle}, A_{\mathrm{opt}} \approx \sqrt{\left\langle O_{\mathrm{opt}}\right\rangle} .
$$

Also, $L$ is the effective luminosity for fully reconstructed $t \bar{t} h$ or $t \bar{t} Z$ events. In particular, we take $L=\epsilon \mathcal{L}$, where $\mathcal{L}$ is the total yearly integrated luminosity and $\epsilon$ is the overall efficiency for reconstruction of the $t \bar{t} h$ or $t \bar{t} Z$ final states.

In the following numerical analysis we have used set II defined before for the angles $\alpha_{1,2,3}$, i.e., $\left\{\alpha_{1}, \alpha_{2}, \alpha_{3}\right\}=\{\pi / 2, \pi / 4,0\}$ 22. Figs. 30(a) and 30(b) show the expected asymmetry and statistical significance in the unpolarized case, corresponding to $O_{\text {opt }}$ in Model II for the $t \bar{t} h$ and $t \bar{t} Z$ final states, respectively. The asymmetry is plotted as a function of the mass of the light Higgs $\left(m_{h}\right)$ where again, $m_{H}=750 \mathrm{GeV}$ in the $t \bar{t} Z$ case. We plot $N_{S D} / \sqrt{L}$, thus scaling out the luminosity factor from the theoretical prediction 23 .

We remark that set II corresponds to the largest CP effect, though not unique since we are dealing with angles, i.e., $\alpha_{1,2,3}$, which may be rotated by $\pi$ or $\pi / 2$ leaving the relevant combinations of angles with the same value (e.g., $b_{t}^{h} \propto \sin \alpha_{1} \sin \alpha_{2}$ ). In the $t \bar{t} h$ case $\tan \beta=0.5$ is favored, however, the effect mildly depends on $\tan \beta$ in the range $0.3 \lesssim \tan \beta \lesssim 1$ [55, 185]. In the $t \bar{t} Z$ case, the effect is practically insensitive to $\alpha_{3}$ and is roughly proportional to $1 / \tan \beta$, it therefore drops as $\tan \beta$ is increased. Nonetheless, we find that $N_{S D} / \sqrt{L}>0.1$, even in the unpolarized case for $\tan \beta \lesssim 0.6$ [184, 185]; note that $N_{S D} / \sqrt{L}$ here is dimensionless if $L$ is in $\mathrm{fb}^{-1}$.

From Fig. 30(a) we see that, in the $t \bar{t} h$ case, as $m_{h}$ grows the asymmetry increases while the statistical significance drops, in part because of the decrease in the cross-section. Evidently, the asymmetry can become quite large; it ranges from $\sim 15 \%$, for $m_{h} \lesssim 100 \mathrm{GeV}$, to $\sim 35 \%$ for $m_{h} \sim 600 \mathrm{GeV}$. Indeed, the CP-effect is more significant for smaller masses of $h$, wherein $A_{\text {opt }}$ is smaller. In contrast, Fig. 30(b) shows that, in the $t \bar{t} Z$ case, $A_{\text {opt }}$ stays roughly fixed at around $7-8 \%$ for $m_{h} \lesssim 2 m_{t}$, and then drops till it totally vanishes at $m_{h}=m_{H}=750 \mathrm{GeV}$, due to the "GIM-like" mechanism discussed above. The scaled statistical significance $N_{S D} / \sqrt{L}$ behaves roughly as $A_{\text {opt }}$. That is, $N_{S D} / \sqrt{L} \approx 0.1-0.2$ in the mass range $50 \mathrm{GeV} \lesssim m_{h} \lesssim 350 \mathrm{GeV}$, for both $\sqrt{s}=1$ and $1.5 \mathrm{TeV}$.

Figs. 31(a) and 31(b) show the dependence of $A_{\mathrm{opt}}$ and $N_{S D} / \sqrt{L}$ on the c.m. energy, $\sqrt{s}$, for the $t \bar{t} h$ and $t \bar{t} Z$ cases, respectively. We see that, in the case of $t \bar{t} h$, the CP-effect peaks at $\sqrt{s} \approx 1.1(1.5) \mathrm{TeV}$ for $m_{h}=100(360) \mathrm{GeV}$ and stays roughly the same as $\sqrt{s}$ is further increased to $2 \mathrm{TeV}$. In the case of $t \bar{t} Z$, the statistical significance is maximal at around $\sqrt{s} \approx 1 \mathrm{TeV}$ and then decreases slowly as $\sqrt{s}$ grows for both $m_{h}=100$ and $360 \mathrm{GeV}$. Contrary to the $t \bar{t} h$ case, where a light $h$ is favored, in the $t \bar{t} Z$ case, the effect is best for $m_{h} \gtrsim 2 m_{t}$. In that range, on-shell $Z$ and $h$ are produced followed by the $h$ decay $h \rightarrow t \bar{t}$, thus, the Higgs exchange diagram becomes more dominant.

In Tables 7 and 8 we present $N_{S D}$ for $O_{\text {opt }}$, for the $t \bar{t} h$ and $t \bar{t} Z$ cases, respectively, in Model II with set II, and we also compare the effect of beam polarization with the unpolarized case. As before, we take $\tan \beta=0.5$ and $\tan \beta=0.3$ for the $t \bar{t} h$ and $t \bar{t} Z$ cases, respectively, where for the $t \bar{t} Z$ case we present numbers for both $m_{H}=750 \mathrm{GeV}$ (shown in the parentheses) and $m_{H}=1 \mathrm{TeV}$, to demonstrate the sensitivity of the CP-effect to the mass of the heavy Higgs. For illustrative purposes, we choose $m_{h}=100,160$ and $360 \mathrm{GeV}$ and show the numbers for $\sqrt{s}=1$ $\mathrm{TeV}$ with $\mathcal{L}=200[\mathrm{fb}]^{-1}$ and for $\sqrt{s}=1.5 \mathrm{TeV}$ with $\mathcal{L}=500[\mathrm{fb}]^{-1}$ (see [192]). In both cases we take $\epsilon=0.5$ assuming that there is no loss of luminosity when the electrons are polarized ${ }^{24}$

\footnotetext{
${ }^{22}$ Recall that for the $t \bar{t} h$ final state we $\operatorname{choose} \tan \beta=0.5$ while for the $t \bar{t} Z$ final state we $\operatorname{take} \tan \beta=0.3$

${ }^{23}$ as a reference value, we note that for $L=100 \mathrm{fb}^{-1}, N_{S D} / \sqrt{L}=0.1$ will correspond to a $1-\sigma$ effect

${ }^{24}$ Clearly if the efficiency for $t \bar{t} h$ and/or $t \bar{t} Z$ reconstruction is $\epsilon=0.25$, then our numbers would correspondingly require 2 years of running.
} 
Evidently, for both reactions, left polarized incoming electrons can probe CP violation slightly better than unpolarized ones. We see that in the $t \bar{t} h$ case the CP-violating effect drops as the mass of the light Higgs $(h)$ grows, while in the $t \bar{t} Z$ case it grows with $m_{h}$. In particular, we find that with $\sqrt{s}=1.5 \mathrm{TeV}$ and for $m_{h} \gtrsim 2 m_{t}$ the effect is comparable for both the $t \bar{t} h$ and the $t \bar{t} Z$ cases where it reaches above $3-\sigma$ for negatively polarized electrons. With a light Higgs mass in the range $100 \mathrm{GeV} \lesssim m_{h} \lesssim 160 \mathrm{GeV}$, the $t \bar{t} h$ case is more sensitive to $O_{\text {opt }}$ and the $\mathrm{CP}$-violating effect can reach $\sim 4-\sigma$ for left polarized electrons. In that light Higgs mass range, the CP-violating effect reaches slightly below $2.5-\sigma$ for the $t \bar{t} Z$ case. For a c.m. energy of $\sqrt{s}=1$ $\mathrm{TeV}$ and $m_{h}=360 \mathrm{GeV}$, the $t \bar{t} Z$ case is much more sensitive to $O_{\text {opt }}$ and the effect can reach $2.2-\sigma$ for left polarized electron beam. However, with that c.m. energy, the $t \bar{t} h$ mode gives a larger CP-odd effect in the range $m_{h} \sim 100-160 \mathrm{GeV}$.

Table 7: The statistical significance, $N_{S D}$, in which the CP-nonconserving effects in $e^{+} e^{-} \rightarrow t \bar{t} h$ can be detected in one year of running of a future high energy collider with either unpolarized or polarized incoming electron beam. We have used $\tan \beta=0.5$, a yearly integrated luminosity of $\mathcal{L}=200$ and $500[\mathrm{fb}]^{-1}$ for $\sqrt{s}=1$ and $1.5 \mathrm{TeV}$, respectively, and an efficiency reconstruction factor of $\epsilon=0.5$ for both energies. Recall that $j=1(-1)$ stands for right(left) polarized electrons. Set II means $\left\{\alpha_{1}, \alpha_{2}, \alpha_{3}\right\} \equiv\{\pi / 2, \pi / 4,0\}$. Table taken from [185].

\begin{tabular}{|r||r|r|r|r|}
\cline { 3 - 5 } \multicolumn{2}{c|}{} & \multicolumn{3}{c|}{$e^{+} e^{-} \rightarrow t \bar{t} h$ (Model II with Set II) } \\
\hline \multirow{2}{*}{$\begin{array}{r}\sqrt{s} \\
(\mathrm{TeV}) \Downarrow\end{array}$} & $\mathrm{j}$ & \multicolumn{3}{|c|}{$O_{\text {opt }}$} \\
\cline { 3 - 5 } & $(\mathrm{GeV}) \Rightarrow$ & $m_{h}=100$ & $m_{h}=160$ & $m_{h}=360$ \\
\cline { 2 - 5 } 1 & -1 & 2.2 & 2.0 & 1.1 \\
\cline { 2 - 5 } & unpol & 2.0 & 1.9 & 1.0 \\
\hline \hline & 1 & 1.8 & 1.7 & 0.9 \\
\cline { 2 - 5 } 1.5 & unpol & 3.0 & 3.9 & 3.2 \\
\cline { 2 - 5 } & 1 & 3.2 & 3.5 & 2.9 \\
\hline \hline
\end{tabular}

Table 8: The same as Table 0 but for $e^{+} e^{-} \rightarrow t \bar{t} Z$, with $\tan \beta=0.3$. In this reaction, effects of the heavy Higgs, $H$, are included and $N_{S D}$ is given for both $m_{H}=750 \mathrm{GeV}$ (in parentheses) and $m_{H}=1 \mathrm{TeV}$. Table taken from 185].

\begin{tabular}{|r||r|r|r|r|}
\cline { 3 - 5 } \multicolumn{2}{c|}{} & \multicolumn{4}{c|}{$e^{+} e^{-} \rightarrow t \bar{t} Z$ (Model II with Set II) } \\
\cline { 3 - 5 }$(\mathrm{TeV}) \Downarrow$ & $\mathrm{j}$ & \multicolumn{3}{|c|}{$O_{\text {opt }}$} \\
\cline { 3 - 5 }$(\mathrm{GeV}) \Rightarrow$ & $m_{h}=100$ & $m_{h}=160$ & $m_{h}=360$ \\
\hline \hline \multirow{3}{*}{1} & -1 & $(1.8) 1.7$ & $(1.8) 1.8$ & $(2.2) 2.2$ \\
\cline { 2 - 5 } & unpol & $(1.6) 1.6$ & $(1.7) 1.6$ & $(2.0) 2.0$ \\
\cline { 2 - 5 } & 1 & $(1.5) 1.5$ & $(1.5) 1.5$ & $(1.8) 1.8$ \\
\hline \hline & -1 & $(2.3) 2.9$ & $(2.4) 3.0$ & $(2.8) 3.3$ \\
\cline { 2 - 5 } 1.5 & unpol & $(2.1) 2.6$ & $(2.1) 2.7$ & $(2.5) 3.0$ \\
\cline { 2 - 5 } & 1 & $(1.8) 2.3$ & $(1.8) 2.3$ & $(2.1) 2.6$ \\
\hline
\end{tabular}

Let us now summarize the above results and add some concluding remarks. We have shown that an extremely interesting CP-odd signal may arise at tree-level in the reactions $e^{+} e^{-} \rightarrow t \bar{t} h$ and $e^{+} e^{-} \rightarrow t \bar{t} Z$. The asymmetries that were found are $\sim 15 \%-35 \%$ in the $t \bar{t} h$ case and $\sim 5 \%^{-}$ 
$10 \%$ for the $t \bar{t} Z$ final state. These asymmetries may give rise in the best cases, i.e., for a favorable set of the relevant $2 \mathrm{HDM}$ parameters, to $\sim 3-4-\sigma$, CP-odd, signals in a future $e^{+} e^{-}$collider running with c.m. energies in the range $1 \mathrm{TeV} \lesssim \sqrt{s} \lesssim 2 \mathrm{TeV}$.

Note, however, that the simple observable, $O$, as well as the optimal one, $O_{\text {opt }}$, require the identification of the $t$ and $\bar{t}$ and the knowledge of the transverse components of their momenta in each $t \bar{t} h$ or $t \bar{t} Z$ event. Thus, for the main top decay, $t \rightarrow b W$, the most suitable scenario is when either the $t$ or the $\bar{t}$ decays leptonically and the other decays hadronically. Distinguishing between $t$ and $\bar{t}$ in the double-hadronic decay case will require more effort and still remains an experimental challenge. If, for example, the identification of the charge of the $b$-jets coming from the $t$ and the $\bar{t}$ is possible, then the difficulty in reconstructing the transverse components of the $t$ and $\bar{t}$ momenta may be surmountable by using the momenta of the decay products in the processes $e^{+} e^{-} \rightarrow t \bar{t} h \rightarrow b W^{+} \bar{b} W^{-} h$ and $e^{+} e^{-} \rightarrow t \bar{t} Z \rightarrow b W^{+} \bar{b} W^{-} Z$. For example, the observable

$$
O_{b}=\frac{\epsilon\left(p_{-}, p_{+}, p_{b}, p_{\bar{b}}\right)}{s^{2}},
$$

may then be used. We have considered this observable for the reaction $e^{+} e^{-} \rightarrow t \bar{t} h \rightarrow b W^{+} \bar{b} W^{-} h$ in [55]. We found there that, close to threshold, this observable is not very effective. However, at higher energies, $O_{b}$ is about as sensitive as the simple triple product correlation $O$ defined in Eq. 6.69 and, therefore, only slightly less sensitive then $O_{\text {opt }}$.

Note also that for the light Higgs mass, $m_{h}=100 \mathrm{GeV}$, the most suitable way to detect the Higgs in $e^{+} e^{-} \rightarrow t \bar{t} h \rightarrow b W^{+} \bar{b} W^{-} h$ is via $h \rightarrow b \bar{b}$ with branching ratio $\sim 1$. For $m_{h} \gtrsim 2 m_{t}$,

and specifically with set II used above, there are two competing Higgs decays, $h \rightarrow t \bar{t}$ and $h \rightarrow W^{+} W^{-}$, depending on the value of $\tan \beta$. For example, for $\tan \beta=0.5$, as was chosen above, one has $\operatorname{Br}(h \rightarrow t \bar{t}) \approx 0.77$ and $\operatorname{Br}\left(h \rightarrow W^{+} W^{-}\right) \approx 0.17$, thus, the $h \rightarrow t \bar{t}$ mode is more suitable. Of course, $h \rightarrow t \bar{t}$ will dominate more for smaller values of $\tan \beta$ and less if $\tan \beta>0.5$. In particular, for $\tan \beta=0.3(1)$ one has $\operatorname{Br}(h \rightarrow t \bar{t}) \approx 0.89(0.57)$ and $\operatorname{Br}\left(h \rightarrow W^{+} W^{-}\right) \approx 0.08(0.32)$.

Finally, as emphasized before, the final states $t \bar{t} h$ and $t \bar{t} Z$, in particular the $t \bar{t} h$, are expected to be the center of considerable attention at a linear collider. Extensive studies of these reactions are expected to teach us about the details of the couplings of the neutral Higgs to the top quark 193. Thus, it is gratifying that the same final states promise to exhibit interesting effects of $\mathrm{CP}$ violation. It would be very instructive to examine the effects in other extended models. Numbers emerging from the $2 \mathrm{HDM}$ that was used, especially with the specific value of the parameters, should be viewed as illustrative examples. The important point is that the reactions $e^{+} e^{-} \rightarrow t \bar{t} h \rightarrow b W^{+} \bar{b} W^{-} h$ and $e^{+} e^{-} \rightarrow t \bar{t} Z \rightarrow b W^{+} \bar{b} W^{-} Z$ appear to be very powerful and very clean tools for extracting valuable information on the parameters of the underlying model for CP violation.

\subsubsection{Generalized optimization technique and extraction of various Higgs couplings}

An optimization technique was employed by Gunion et al. in [51] for the process $e^{+} e^{-} \rightarrow t \bar{t} h$. It was shown that this reaction may provide a powerful tool for extracting the $t \bar{t} h$ Yukawa couplings and the $Z Z h$ couplings. A similar analysis for the reaction $e^{+} e^{-} \rightarrow t \bar{t} Z$ was likewise considered in [186]. This technique is outlined in section 2.6. The basic idea is that the nature of the Higgs particle, i.e., whether it is CP-odd or CP-even, may well be distinguishable through studies of momentum correlations in $e^{+} e^{-} \rightarrow t \bar{t} h$. In particular, greater information on the detailed dependence of $\Sigma_{(j) t \bar{t} h}(\Phi)$ on the variable $\Phi$ is extracted to deduce limits that can be obtained on the different Higgs couplings combinations in Eqs. 6.58 and 6.61. As described before, the differential $e^{+} e^{-} \rightarrow t \bar{t} h$ cross-section contains five distinct terms which are explicitly written in Eqs. 6.58 and 6.61. The only CP-violating component is $b_{t}^{h} c^{h}$, while the others enter into the total cross-section as in Eq. 6.63. 
Gunion et al. have investigated two issues. For a given c.m. energy and integrated luminosity at the NLC, they have examined:

1. The 1- $\sigma$ error in the determination of the couplings $a_{t}^{h}, b_{t}^{h}, c^{h}$, by fixing $\chi^{2}(t \bar{t} h)=1$ for a given input model with couplings $g_{ \pm t \bar{t} h}^{0, i(n)}$.

2. To what degree of statistical significance can a model be ruled out, given a certain input model.

Let us now elaborate more on how these two points were studied in [51]. With the optimal technique outlined in [51], Gunion et al. used unique weighting functions such that the statistical error in the determination of the various $g_{ \pm t \bar{t} h}^{i(n)}$ in Eqs. 6.58 and 6.61 is minimized. They write

$$
g_{ \pm t \bar{t} h}^{i(n)}=\sum_{k} M_{i k}^{-1} I_{k}
$$

where $I_{k}, M_{i k}$ and the appropriate weighting functions are given in [51] (see also section 2.6). Then, given an input model, for which the couplings are denoted with the superscript 0 as $g_{ \pm t \bar{t} h}^{0, i(n)}$, one can compute the confidence level at which parameters of choice, different from the input model, can be ruled out

$$
\chi^{2}(t \bar{t} h)=\sum_{i, j=1}^{5}\left(g_{ \pm t \bar{t} h}^{i(n)}-g_{ \pm t \bar{t} h}^{0, i(n)}\right)\left(g_{ \pm t \bar{t} h}^{j(n)}-g_{ \pm t \bar{t} h}^{0, j(n)}\right) V_{i j}^{-1}
$$

where

$$
V_{i j} \equiv<\Delta g_{ \pm t \bar{t} h}^{i(n)} \Delta g_{ \pm t \bar{t} h}^{j(n)}>=\frac{M_{i j}^{-1} \sigma_{t \bar{t} h}}{N_{t \bar{t} h}}
$$

is the covariance matrix. $N_{t \bar{t} h}=L \sigma_{t \bar{t} h}$ is the total number of $t \bar{t} h$ events, with $L$ the effective luminosity defined previously (after the efficiency factor, $\epsilon$, is included). Therefore, the sensitivity of $\chi^{2}(t \bar{t} h)$ to the couplings $a_{t}^{h}, b_{t}^{h}, c^{h}$ is determined by the covariance matrix directly.

Three input models were considered in [51]. These correspond to different choices of the set of parameters $a_{t}^{h}, b_{t}^{h}$ and $c^{h}$ (see Eqs. 3.70 and 3.71 in section 3.2.3), as follows:

(I) A SM neutral Higgs, with $a_{t}^{h}=1 / \sqrt{2}, b_{t}^{h}=0, c^{h}=1$.

(II) A pure CP-odd neutral Higgs, with $a_{t}^{h}=0, b_{t}^{h}=1 / \sqrt{2}, c^{h}=0$.

(III) A CP-mixed neutral Higgs, with $a_{t}^{h}=b_{t}^{h}=1 / 2, c^{h}=1 / \sqrt{2}$.

Given the couplings $g_{ \pm t \bar{t} h}^{0, j(n)}$ in the above input models, they calculated $\chi^{2}(t \bar{t} h)$ as a function of the location in $a_{t}^{h}, b_{t}^{h}, c^{h}$ parameter space, from which the 1- $\sigma$ error in any one of these parameters was determined. Their results are shown in Table 9 .

We see that $a_{t}^{h}$ is well determined in all input models; in input models I and III, where $a_{t}^{h} \neq 0$, the 1- $\sigma$ error is at the few percent level. In the same manner, $b_{t}^{h}$ is best determined in input model II, where $\Delta b \approx 10-15 \%$, at $1-\sigma$. The error in $c^{h}$ is above the $50 \%$ level in all three input models. However, this can be improved by considering the reaction at hand, i.e., $e^{+} e^{-} \rightarrow t \bar{t} h$, combined with information extracted from the $e^{+} e^{-} \rightarrow Z h$ cross-section as was done in [189]. Assuming real Higgs production and disregarding the subsequent $h$ decay in the reaction $e^{+} e^{-} \rightarrow Z h$, the crosssection $\sigma\left(e^{+} e^{-} \rightarrow Z h\right)$ contains one useful Higgs coupling combination, $g_{+t \bar{t} h}^{3(+)}=\left(c^{h}\right)^{2}$. Therefore, the sensitivity to $\left(c^{h}\right)^{2}$ is increased when the above technique is also applied to $\sigma\left(e^{+} e^{-} \rightarrow Z h\right)$.

Let us continue with a discussion of the work of Ref. 51 in which only the $t \bar{t} h$ final state was considered. The ability to distinguish between different models was furthermore investigated by using the optimization technique. This is shown in Table 10. We see from this table, for 
Table 9: The 1- $\sigma$ errors in $a_{t}^{h}, b_{t}^{h}$, and $c^{h}$ are given, for the three Higgs coupling cases I, II and III. Results are given for unpolarized beams and for $100 \%$ negative $e^{-}$polarization $\left(P_{e}=-1\right)$ also $\sqrt{s}=1 \mathrm{TeV}, m_{h}=100 \mathrm{GeV}, m_{t}=176 \mathrm{GeV}$ and $L=50 \mathrm{fb}^{-1}$. Table taken from 51.

\begin{tabular}{|c|c|c|c|c|c|c|}
\hline Case & \multicolumn{3}{|c|}{ Unpolarized $e^{-}$} & \multicolumn{3}{|c|}{$P_{e}=-1$} \\
\hline I & $a_{t}^{h} \pm \Delta a_{t}^{h}$ & $b_{t}^{h} \pm \Delta b_{t}^{h}$ & $c^{h} \pm \Delta c^{h}$ & $a_{t}^{h} \pm \Delta a_{t}^{h}$ & $b_{t}^{h} \pm \Delta b_{t}^{h}$ & $c^{h} \pm \Delta c^{h}$ \\
\hline II & $\frac{1}{2}_{-0.047}^{+0.030}$ & $0_{-0.76}^{+0.76}$ & $1_{-0.82}^{+0.51}$ & $\frac{1}{\sqrt{2}}+0.026$ & $0_{-0.70}^{+0.70}$ & $1_{-0.77}^{+0.51}$ \\
\hline III & $0_{-0.19}^{+0.19}$ & $\frac{1}{\sqrt{2}}{ }_{-0.099}^{+0.066}$ & $0_{-0.58}^{+0.58}$ & $0_{-0.18}^{+0.18}$ & $\frac{1}{\sqrt{2}}{ }_{-0.0085}^{+0.056}$ & $0_{-0.55}^{+0.55}$ \\
\hline
\end{tabular}

Table 10: The number of standard deviations, $\sqrt{\chi^{2}}$, at which a given input model (I, II or III) can be distinguished from the other two models, are tabulated, for $\sqrt{s}, m_{h}, m_{t}$ and $L$ as in Table 9 . Table taken from [51].

\begin{tabular}{|c|c|c|c|c|c|c|}
\hline & \multicolumn{3}{|c|}{ Unpolarized $e^{-}$} & \multicolumn{4}{|c|}{$P_{e}=-1$} \\
Trial Model & \multicolumn{3}{c|}{ Trial Model } \\
Input Model & I & II & III & I & II & III \\
\hline I & - & 9.5 & 4.8 & - & 11 & 5.5 \\
II & 34 & - & 17 & 40 & - & 20 \\
III & 6.3 & 6.3 & - & 7.3 & 7.3 & - \\
\hline
\end{tabular}

example, that if the Higgs is the SM one, then the pure CP-odd case (input model II) and the equal CP-mixture case (input model III) are ruled out at the 9.5- $\sigma$ and 4.8- $\sigma$ level, respectively. Note also that negative beam polarization slightly improves the results.

Finally, the ability for determining a non-zero CP-violating component, $b_{t}^{h} c^{h}$, was also investigated in [51]. They found that with $m_{h}=100 \mathrm{GeV}, L=100 \mathrm{fb}^{-1}$ 25, a non-zero $b_{t}^{h} c^{h}$ coupling can be established at a level better then $1-\sigma$ in a $1 \mathrm{TeV} e^{+} e^{-}$collider.

\subsubsection{CP asymmetries in $e^{+} e^{-} \rightarrow Z h$ and in the subsequent Higgs decay $h \rightarrow t \bar{t}$}

We now consider the process $e^{+} e^{-} \rightarrow Z h$ followed by $h \rightarrow t \bar{t}$. As we have discussed above, in general, one cannot ignore the SM-like diagrams of class (a) on the right hand side of Fig. 28 when analyzing $\mathrm{CP}$ violation in the reaction $e^{+} e^{-} \rightarrow t \bar{t} Z$. Moreover, inclusion of those diagrams and interfering them with diagram (b) on the right hand side of Fig. 28, gives a bonus in the appearance of tree-level $\mathrm{CP}$ violation in $e^{+} e^{-} \rightarrow t \bar{t} Z$. However, let us assume that the Higgs has already been discovered, with a mass of $m_{h}>2 m_{t}$, by the time a high energy $e^{+} e^{-}$collider starts its first run. In such a scenario, one should in principle be able to separate the contribution of the Higgs exchange graph in $e^{+} e^{-} \rightarrow t \bar{t} Z$ from the rest of the SM-like diagrams which lead to the same final state, by imposing a suitable cut on the invariant $t \bar{t}$ mass. Taking this viewpoint, we will consider Higgs production via $e^{+} e^{-} \rightarrow h Z$ and $\mathrm{CP}$ violation in the subsequent Higgs decay

\footnotetext{
${ }^{25}$ this is the value considered by us in the previous section 6.2 .1 for which the results in Tables 7 and 8 are given
} 
$h \rightarrow t \bar{t}$.

A general method for tracing CP-odd and CP-even $t, \bar{t}$ spin-correlations, in $h \rightarrow t \bar{t}$, was introduced in [194]. There, it was assumed that an on-shell Higgs, with $m_{h}>2 m_{t}$, is produced through, for example, $e^{+} e^{-} \rightarrow Z h, \ell^{+} \ell^{-} h$ or even $\mu^{+} \mu^{-} \rightarrow h$, and that its rest system can be reconstructed. In [195], a helicity asymmetry in $h \rightarrow t \bar{t}$ was suggested, where $e^{+} e^{-} \rightarrow Z h$ was explicitly assumed as the Higgs production mechanism. We will describe below these two works. Other related works can be found in [57, 196, 197, 198, 199, 200, 201, 202].

In the method suggested in [194], the decay $h \rightarrow t \bar{t}$ stands out as an independent decay process, in which top spin-asymmetries can be formed. Consider, for example, the observables

$$
\begin{aligned}
& \mathcal{S}_{1}=\hat{\mathbf{k}_{\mathbf{t}}} \cdot\left(\mathbf{s}_{t}-\mathbf{s}_{\bar{t}}\right), \\
& \mathcal{S}_{2}=\hat{\mathbf{k}_{\mathbf{t}}} \cdot\left(\mathbf{s}_{t} \times \mathbf{s}_{\bar{t}}\right), \\
& \mathcal{S}_{3}=\mathbf{s}_{t} \cdot \mathbf{s}_{\bar{t}},
\end{aligned}
$$

where $\mathbf{s}_{t}\left(\mathbf{s}_{\bar{t}}\right)$ is the spin operator of $t(\bar{t})$ and $\mathbf{k}_{\mathbf{t}}$ is the top quark 3-momentum in the $t \bar{t}$ c.m. frame.

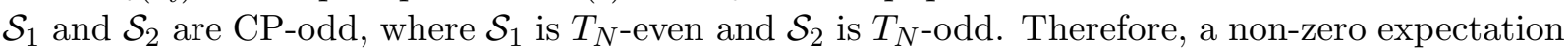
value of $\mathcal{S}_{1}$ will also require absorptive parts, while $\left\langle\mathcal{S}_{2}\right\rangle \neq 0$ can be generated already at the tree-level. $\mathcal{S}_{3}$ is CP-even and is also generated at the lowest order (i.e., tree-level). For a general $t \bar{t} h$ Yukawa interaction Lagrangian as in Eq. 3.70, it was found in 194 that the spin-asymmetries, $\mathcal{S}_{1,2,3}$ in Eqs. 6.75 6.77, depend only on one combination of the couplings $a_{t}^{h}$ and $b_{t}^{h}$

$$
r_{t}=\frac{b_{t}^{h}}{a_{t}^{h}+b_{t}^{h}}
$$

which takes values between 0 to 1 , i.e., $0 \leq r_{t} \leq 1$, where the lower limit corresponds to $b_{t}^{h}=0$ and the upper one to $a_{t}^{h}=0$.

These observables can be translated to correlations between momenta of the $t$ and $\bar{t}$ decay products. To do so, one can define decay scenarios of the $t$ and $\bar{t}$, through which both the $t$ and $\bar{t}$ momenta and spins can be reconstructed in the most efficient way [194]:

$$
\mathcal{A}:\left\{\begin{array}{l}
t \rightarrow W^{+}+b \rightarrow \ell^{+}+\nu_{\ell}+b \\
\bar{t} \rightarrow W^{-}+\bar{b} \rightarrow q+\bar{q}^{\prime}+\bar{b}
\end{array} .\right.
$$

The sample $\overline{\mathcal{A}}$ is defined by the charge conjugate decay channels of the $t \bar{t}$ pairs

$$
\overline{\mathcal{A}}:\left\{\begin{array}{l}
t \rightarrow W^{+}+b \rightarrow \bar{q}+q^{\prime}+b \\
\bar{t} \rightarrow W^{-}+\bar{b} \rightarrow \ell^{-}+\bar{\nu}_{\ell}+\bar{b}
\end{array} .\right.
$$

In these decay samples, either the $t$ or $\bar{t}$ decays leptonically while the other decays hadronically. Each of these samples has a branching fraction of about $2 / 9$ of all $t \bar{t}$ pairs.

With these decay scenarios Ref. [194] found the momentum correlations $\mathcal{O}_{1,2,3}$ which trace the spin correlations $\mathcal{S}_{1,2,3}$, respectively,

$$
\begin{aligned}
\left\langle\mathcal{O}_{1}\right\rangle & =\left\langle\hat{\mathbf{k}}_{t} \cdot \hat{\mathbf{p}}_{\ell^{+}}^{*}\right\rangle_{\mathcal{A}}+\left\langle\hat{\mathbf{k}}_{t} \cdot \hat{\mathbf{p}}_{\ell^{-}}^{*}\right\rangle_{\overline{\mathcal{A}}}=\frac{2}{3}\left\langle\mathcal{S}_{1}\right\rangle, \\
\left\langle\mathcal{O}_{2}\right\rangle & =\left\langle\hat{\mathbf{k}}_{t} \cdot\left(\hat{\mathbf{p}}_{\ell^{+}}^{*} \times \hat{\mathbf{p}}_{\bar{b}}^{*}\right)\right\rangle_{\mathcal{A}}-\left\langle\hat{\mathbf{k}}_{t} \cdot\left(\hat{\mathbf{p}}_{\ell^{-}}^{*} \times \hat{\mathbf{p}}_{b}^{*}\right)\right\rangle_{\overline{\mathcal{A}}}=\frac{8}{9}\left(\frac{1-2 x}{1+2 x}\right)\left\langle\mathcal{S}_{2}\right\rangle, \\
\left\langle\mathcal{O}_{3}\right\rangle & =\left\langle\hat{\mathbf{p}}_{\ell^{+}}^{*} \cdot \hat{\mathbf{p}}_{\bar{b}}^{*}\right\rangle_{\mathcal{A}}+\left\langle\hat{\mathbf{p}}_{\ell^{-}}^{*} \cdot \hat{\mathbf{p}}_{b}^{*}\right\rangle_{\overline{\mathcal{A}}}=\frac{8}{9}\left(\frac{1-2 x}{1+2 x}\right)\left\langle\mathcal{S}_{3}\right\rangle,
\end{aligned}
$$


where $\hat{\mathbf{p}}_{\ell^{+}}^{*}\left(\hat{\mathbf{p}}_{\ell^{-}}^{*}\right)$ is the flight direction of $\ell^{+}\left(\ell^{-}\right)$in the $t(\bar{t})$ quark rest system. Similarly, $\hat{\mathbf{p}}_{b}^{*}\left(\hat{\mathbf{p}}_{\bar{b}}^{*}\right)$ is the unit momentum of the $b(\bar{b})$ in the $t(\bar{t})$ rest system. Also, $x \equiv m_{W}^{2} / m_{t}^{2}$ and the factor $(1-2 x) /(1+2 x) \approx 0.41$ measures the spin analyzing quality of the $b(\bar{b})$ (see section 2.8 ).

The number $N_{t \bar{t}}^{1,2,3}$ of $h \rightarrow t \bar{t}$ events that is required to establish a nonzero correlation $\left\langle\mathcal{O}_{1,2,3}\right\rangle$ at the $N_{S D}$ - standard deviations - significance level are given by

$$
N_{t \bar{t}}^{1,2,3}=\frac{N_{S D}^{2}}{\operatorname{Br}(\mathcal{A}) \times A_{1,2,3}^{2}}
$$

where

$$
A_{1,2,3}=\frac{\left\langle\mathcal{O}_{1,2,3}\right\rangle}{\sqrt{\left\langle\mathcal{O}_{1,2,3}^{2}\right\rangle}},
$$

and $\operatorname{Br}(\mathcal{A})=\operatorname{Br}(\overline{\mathcal{A}})$ is the branching ratio of the decay samples $\mathcal{A}$ or $\overline{\mathcal{A}}$. In particular, disregarding the $\tau$ leptons we have $\operatorname{Br}(\mathcal{A})=4 / 27$. The number of events needed for a $3-\sigma$ (i.e., $N_{S D}=3$ ) observation of the spin-correlations, $\mathcal{S}_{1,2,3}$, of Eqs. 6.75 6.77 are given in Fig. 32, for $m_{h}=400$ $\mathrm{GeV}, \operatorname{Br}(\mathcal{A})=4 / 27$ and as a function of the parameter $r_{t}$ defined in Eq. 6.78. These can be simply obtained from $N_{t \bar{t}}^{1,2,3}$ in Eq. 6.84, by using the relations between $\mathcal{O}_{1,2,3}$ and $\mathcal{S}_{1,2,3}$ given in Eqs. 6.816.83.

It should be noted again that, while $\mathcal{O}_{2,3}$ are non-zero already at the tree-level, $\mathcal{O}_{1}$, being $T_{N}$-even, requires an absorptive phase and, therefore, its non-zero contribution first arises only at the 1-loop level in perturbation theory. Thus, $\mathcal{O}_{1}$ is expected to be less effective (as can be seen from Fig. 32). In [194] the 1-loop QCD corrections to $\mathcal{O}_{1,2,3}$ were computed. For the operators, $\mathcal{O}_{2,3}$, the QCD corrections were found to be of the order of a few percent compared to the leading tree-level contribution, and were therefore neglected.

We see from Fig. 32 that, for example, values of $r_{t}$ in the range, $0.18 \lesssim r_{t} \lesssim 0.52$, would give rise to a 3- $\sigma$ CP-odd effect in $\mathcal{O}_{2}$ with a data sample of $N_{t \bar{t}}^{2} \approx 1500$, i.e., $\sim 1500(h \rightarrow t \bar{t})$ events. Note also that a simultaneous measurement of $\mathcal{O}_{2}$ and $\mathcal{O}_{3}$, with these $\sim 1500$ events, would have a $3-\sigma$ sensitivity to $r_{t}$ from 0.18 to its maximal value of 1 . Furthermore, production of a few thousands of $h \rightarrow t \bar{t}$ events through the Bjorken mechanism, $e^{+} e^{-} \rightarrow h Z$, is indeed feasible. For example, if a CP-mixed neutral Higgs (with both scalar and pseudoscalar couplings $a_{t}^{h}$ and $b_{t}^{h}$ ) with a mass $m_{h}=400 \mathrm{GeV}$, has a $Z Z h$ coupling $c^{h}$ of a SM strength, then the cross-section for $e^{+} e^{-} \rightarrow h Z$ is of the order of a few fb's for $e^{+} e^{-}$c.m. energies in the range $500 \mathrm{GeV} \lesssim \sqrt{s} \lesssim 1000$ $\mathrm{GeV}$. ${ }^{20}$ Therefore, with a yearly integrated luminosity of $\mathcal{L} \sim 10^{2} \mathrm{fb}^{-1}$, hundreds of $h Z$ pairs can be produced and, thus, a $\sim 2-\sigma$ limit on $r_{t}$ may be achievable.

An interesting CP-violating helicity asymmetry in $h \rightarrow t \bar{t}$ was suggested in [195:

$$
\mathcal{O}_{t t}^{h}=\frac{\Gamma(++)-\Gamma(--)}{\Gamma(++)+\Gamma(++)},
$$

where $\Gamma(++)$ and $\Gamma(--)$ are the decay widths of the lightest Higgs-boson $h$, into a pair of $t \bar{t}$ with the indicated helicities. Since under CP: $(++) \leftrightarrow(--)$, non-zero $\mathcal{O}_{t t}^{h}$ would be a signal of $\mathrm{CP}$ violation.

$\mathcal{O}_{t t}^{h}$ is CP-odd but $T_{N}$-even, therefore, it requires a CP-odd as well as a CP-even absorptive phase (i.e., FSI phase). As mentioned several times before, in a 2HDM with a CP-mixed neutral Higgs, the CP-odd phase is provided by the simultaneous presence of the scalar and pseudoscalar $t \bar{t} h$ couplings in the $t \bar{t} h$ interaction Lagrangian. The FSI absorptive phase is generated at the 1-loop level from the diagrams in Fig. 33. The expressions for the different contributions to $\mathcal{O}_{t t}^{h}$ corresponding to the different diagrams in Fig. 33 are given in [195]. There, it was found that with $m_{t}=180 \mathrm{GeV}$ and $m_{h} \gtrsim 2 m_{t}, \mathcal{O}_{t t}^{h}$ of the order of $50 \%$ is possible. Explicitly, assuming the

\footnotetext{
${ }^{26}$ The cross-section for $e^{+} e^{-} \rightarrow h Z$ is given, for example, in 61]. With $m_{h}=400 \mathrm{GeV}$ and for $c^{h}=1$, i.e., the SM $Z Z h$ coupling, $\sigma\left(e^{+} e^{-} \rightarrow h Z\right) \sim 3.5(8.5)$ fb for $\sqrt{s}=500(1000) \mathrm{GeV}$.
} 
Higgs to be produced through the Bjorken mechanism, $e^{+} e^{-} \rightarrow h Z$, the statistical significance, $N_{S D}$, with which this asymmetry can potentially be detected is

$$
N_{S D}=\sqrt{L} \sqrt{\sigma\left(e^{+} e^{-} \rightarrow h Z\right)} \times \mathcal{O}_{t t}^{h} .
$$

In [195] an effective integrated luminosity of $L=85 \mathrm{fb}^{-1}$ was assumed and a scan for maximal $N_{S D}$ was performed as a function of the $2 \mathrm{HDM}$ parameters $\tan \beta$ and $\alpha_{1,2,3}$. It was found that with $\tan \beta=0.5$, for example, and with $m_{t}=180 \mathrm{GeV}, m_{h} \gtrsim 2 m_{t}$, up to a $7-\sigma$ detection of a CP-violating signal from $\mathcal{O}_{t t}^{h}$ is feasible, if the Higgs is produced via $e^{+} e^{-} \rightarrow h Z$. It should be emphasized, however, that there is a potential background to the $t \bar{t}$ pairs (coming from the subsequent Higgs decay in $e^{+} e^{-} \rightarrow h Z$ ) from the SM-like diagrams included in Fig. 28. Therefore, as mentioned before, in order for such a study to be practical one has to know the mass of the Higgs prior to the actual experiment and, with a sufficient mass resolution, demand that the invariant $t \bar{t}$ mass reconstructs the Higgs. 

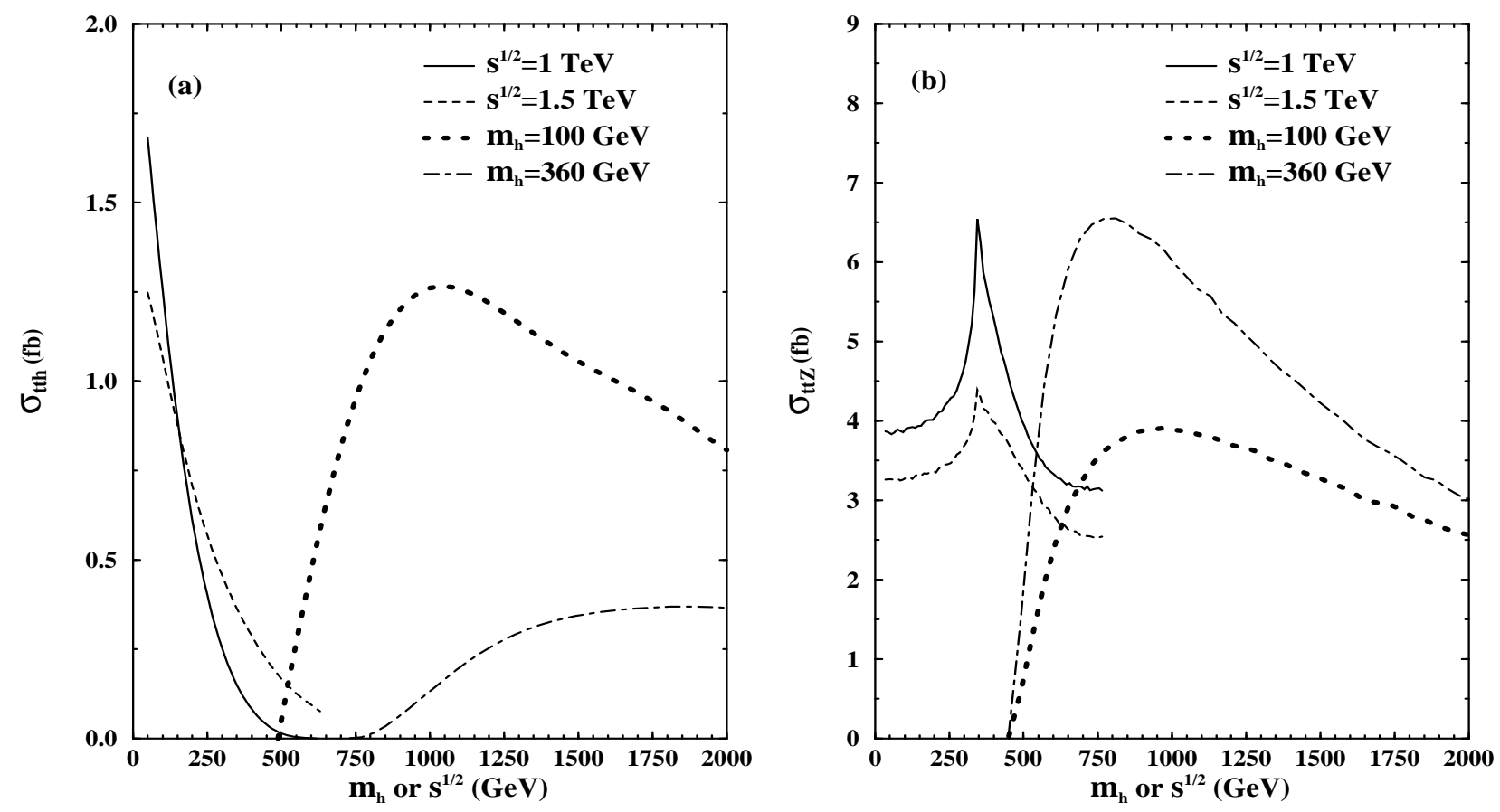

Figure 29: The cross-sections (in fb) for: (a) the reaction $e^{+} e^{-} \rightarrow t \bar{t} h$ with $\tan \beta=0.5$ and (b) the reaction $e^{+} e^{-} \rightarrow t \bar{t} Z$ with $\tan \beta=0.3$, assuming unpolarized electron and positron beams, for Model II with set II and as a function of $m_{h}$ (solid and dashed lines) and $\sqrt{s}$ (dotted and dotted-dashed lines). Set II means $\left\{\alpha_{1}, \alpha_{2}, \alpha_{3}\right\} \equiv\{\pi / 2, \pi / 4,0\}$. Figure taken from [185]. 

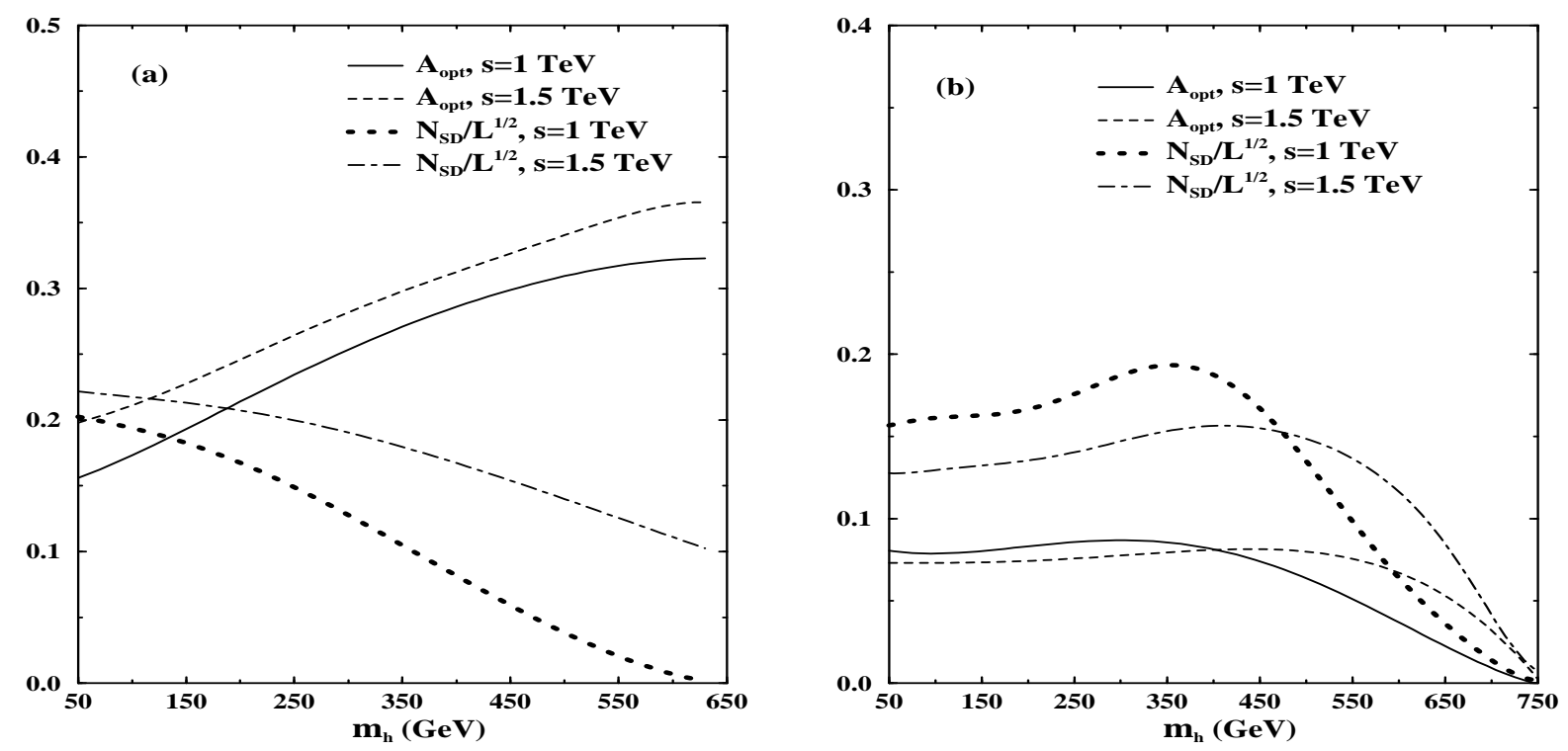

Figure 30: The asymmetry, $A_{\mathrm{opt}}$, and scaled statistical significance, $N_{S D} / \sqrt{L}$, for the optimal observable $O_{\mathrm{opt}}$ for: (a) the reaction $e^{+} e^{-} \rightarrow t \bar{t} h$ with $\tan \beta=0.5$ and (b) the reaction $e^{+} e^{-} \rightarrow$ $t \bar{t} Z$ with $\tan \beta=0.3$, as a function of the light Higgs mass $m_{h}$, for $\sqrt{s}=1$ TeV and 1.5 TeV. All graphs are with set II of the parameters, as in Fig. 29, figure taken from [185]. 

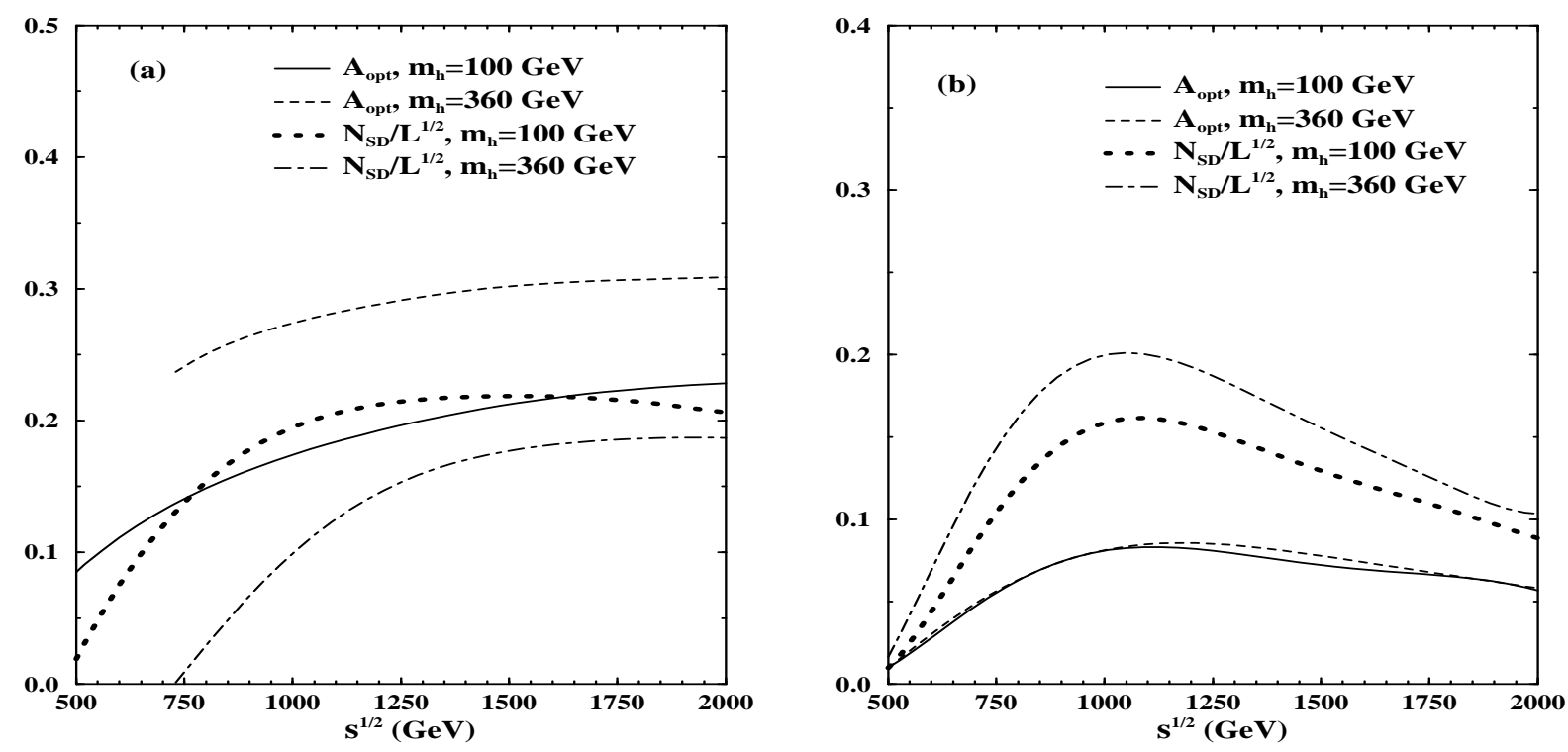

Figure 31: The asymmetry, $A_{\mathrm{opt}}$, and scaled statistical significance, $N_{S D} / \sqrt{L}$, for the optimal observable $O_{\mathrm{opt}}$ for: (a) the reaction $e^{+} e^{-} \rightarrow t \bar{t} h$ with $\tan \beta=0.5$ and (b) the reaction $e^{+} e^{-} \rightarrow$ $t \bar{t} Z$ with $\tan \beta=0.3$, as a function of the c.m. energy $\sqrt{s}$, for $m_{h}=100$ GeV and $m_{h}=360$ GeV. All graphs are with set II of the parameters, as in Fig. 29. Figure taken from 185]. 


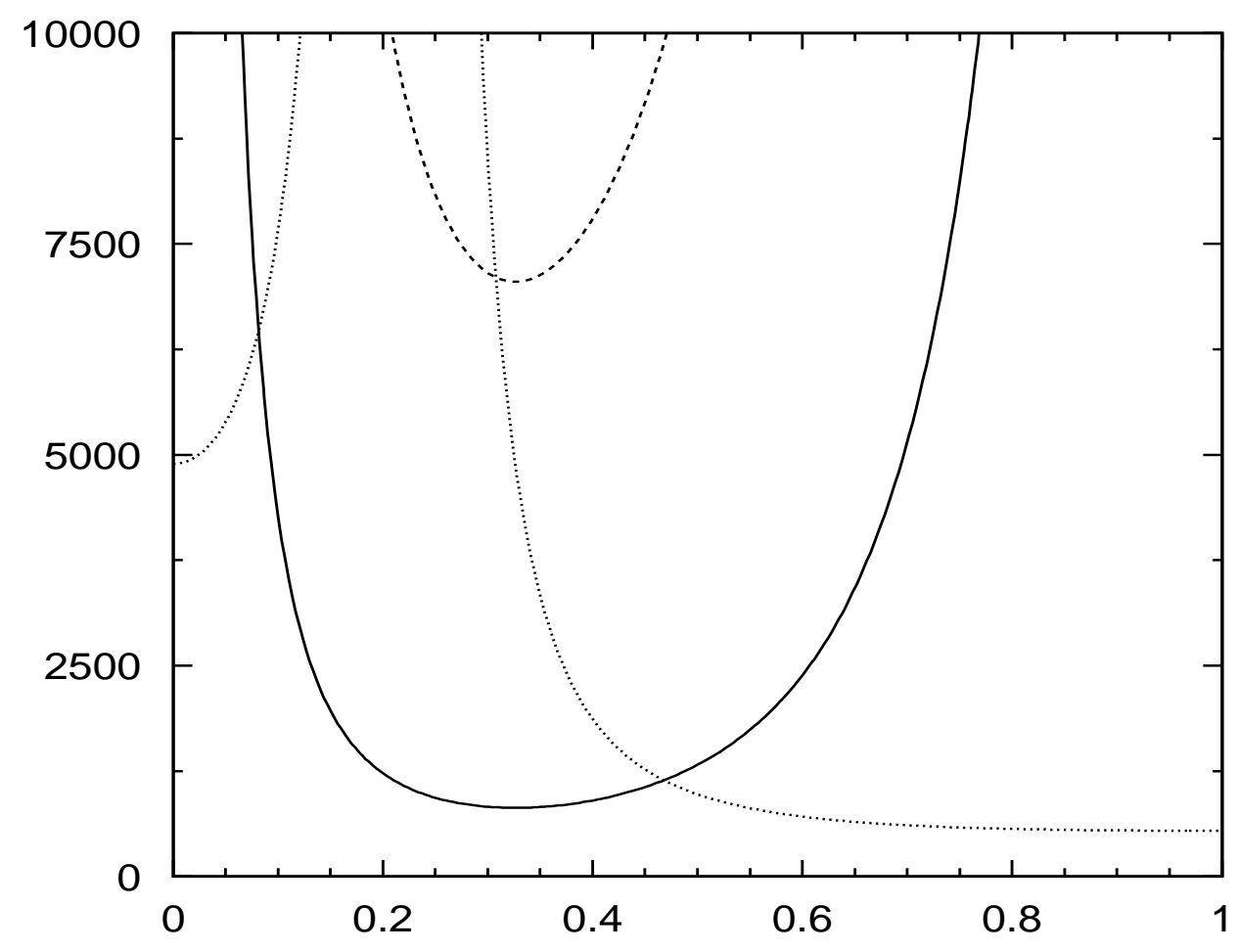

Figure 32: Number of events $h \rightarrow t \bar{t}$ to establish a non-zero correlation $\left\langle\mathcal{S}_{1,2,3}\right\rangle$ with 3 standard deviation significance, as a function of $r_{t}$ (see Eq. 6.78) and for a fixed Higgs mass of $m_{h}=400$ $G e V$. The dashed line represents the result for $N_{t \bar{t}}^{1}$, the solid line is the result for $N_{t \bar{t}}^{2}$ and the dotted line is the result for $N_{t \bar{t}}^{3}$. $m_{t}=175 \mathrm{GeV}$, figure taken from [194]. 

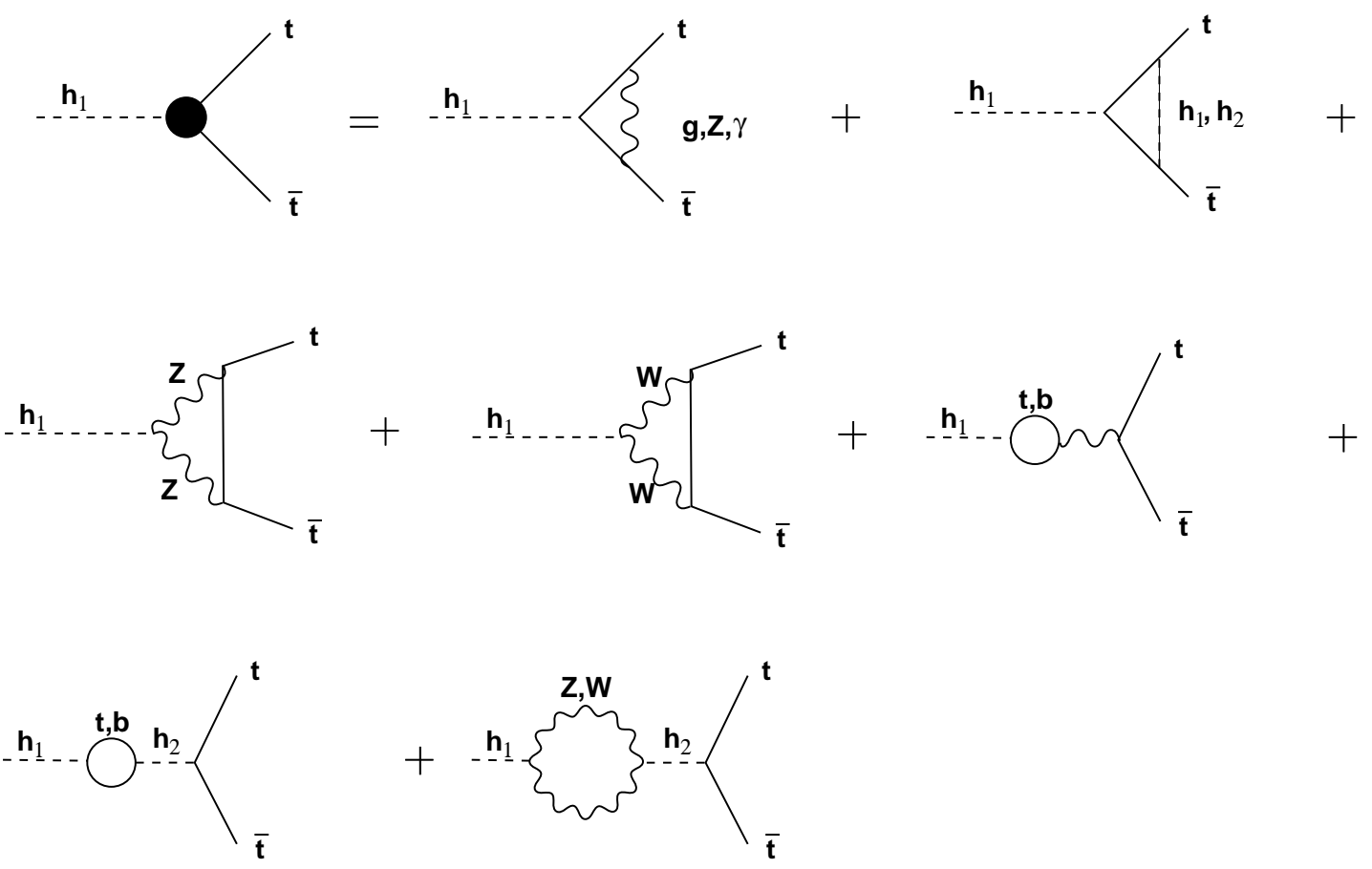

Figure 33: The 1-loop diagrams contributing to the asymmetry $\mathcal{O}_{t t}^{h}$ in a $2 H D M$. 


\section{$6.3 \quad e^{+} e^{-} \rightarrow t \bar{t} g$}

Given the importance of the top pair production at the NLC, it should be clear that the associated gluon emission will also receive considerable attention. Of course, the gluons will be radiated off top quarks quite readily once the threshold for top pair production will be reached. One important advantage of the reaction $e^{+} e^{-} \rightarrow t \bar{t} g$ is that it is rich in exhibiting several different types of CP asymmetries which can be driven by 1-loop effects induced by extensions of the SM. For example, exchanges of neutral Higgs from MHDM's with CP violation in the scalar sector, or exchanges of SUSY particles which carry a CP-odd phase in their interaction vertices, could give rise to both $T_{N \text {-odd }}$ and $T_{N}$-even type CP-violating dynamics. Therefore, both CP-odd, $T_{N}$-odd and CP-odd, $T_{N}$-even type observables can be used to extract information on the real and imaginary parts of the amplitude. With three particles in the final state there are enough linearly independent momenta available so that the construction of CP-odd, $T_{N}$-odd observables is straightforward; there is no need to involve the spins of the top.

Following our work in [203], we give below the full analytical formulae of the tree-level DCS (Differential Cross-Section) as well as a description for extracting the 1-loop CP-violating DCS that can be used for any given model. The SM tree-level diagrams, are depicted in Fig. 34.

The incoming polarized electron-positron current can be written as

$$
J_{e}^{\mu(j)}=\bar{v}_{e}\left(p_{+}\right) \gamma^{\mu} P_{j} u_{e}\left(p_{-}\right),
$$

where $P_{j}=\frac{1}{2}\left(1+j \times \gamma_{5}\right)$ and $j=-1(1)$ for left (right) handed incoming electrons. $p_{+}\left(p_{-}\right)$are the 4 -momentum of the positron (electron) and $p=\left(p_{+}+p_{-}\right)$is the 4-momentum of the $s$-channel gauge-boson (the contribution from an $s$-channel Higgs vanishes for $m_{e} \rightarrow 0$ ).

We also define the following constants

$$
\mathcal{C}_{Z}=\frac{(4 \pi \alpha)}{2 c_{W}^{2} s_{W}^{2}} T^{a} g_{s} \Pi_{Z} C_{j}^{e} \quad, \quad \mathcal{C}_{\gamma}=(4 \pi \alpha) T^{a} g_{s} \Pi_{\gamma} Q_{q},
$$

where $T^{a}$ is the appropriate $\mathrm{SU}(3)$ generator, $g_{s}$ is the strong coupling constant, $Q_{q}$ is the charge of the quark and $c_{W}\left(s_{W}\right)$ stands for $\cos \theta_{W}\left(\sin \theta_{W}\right)$, respectively. Also $C_{j}^{e}=C_{L}^{e}\left(C_{R}^{e}\right)$ for $j=-1(1)$ with $C_{L}^{f}=-2 I_{3}^{f}+2 Q_{f} s_{W}^{2}$ and $C_{R}^{f}=2 Q_{f} s_{W}^{2} . \Pi_{Z}$ and $\Pi_{\gamma}$ are the gauge-boson propagators given by

$$
\Pi_{\gamma}=\frac{1}{p^{2}}, \quad \Pi_{Z}=\frac{1}{p^{2}-m_{Z}^{2}} .
$$

Then the tree-level matrix element is given by

$$
\mathcal{M}^{0} \equiv \mathcal{M}_{a 1}+\mathcal{M}_{a 2}+\mathcal{M}_{b 1}+\mathcal{M}_{b 2}
$$

where $\mathcal{M}_{a 1}, \mathcal{M}_{a 2}, \mathcal{M}_{b 1}$ and $\mathcal{M}_{b 2}$ are obtained from diagrams $\left(a_{1}\right),\left(a_{2}\right),\left(b_{1}\right)$ and $\left(b_{2}\right)$ in Fig. 34, respectively, all emanating from the SM. We thus get

$$
\begin{aligned}
\mathcal{M}^{0}= & \frac{1}{2} J_{e}^{\mu(j)} \bar{u}\left(p_{q}\right)\left\{\mathcal{C}_{Z}\left[\Pi_{q} T_{(a 1) \mu}-\Pi_{\bar{q}} T_{(a 2) \mu}\right]+\right. \\
& \left.2 \mathcal{C}_{\gamma}\left[\Pi_{\bar{q}} T_{(b 1) \mu}-\Pi_{q} T_{(b 2) \mu}\right]\right\} v\left(p_{\bar{q}}\right)
\end{aligned}
$$

Here $p_{q}\left(p_{\bar{q}}\right)$ is the 4-momentum of the outgoing quark (anti-quark), $p_{g}$ is the gluon's 4-momentum and the quark and anti-quark propagators are given by

$$
\Pi_{q}=\frac{1}{2 p_{q} \cdot p_{g}}, \quad \Pi_{\bar{q}}=\frac{1}{2 p_{\bar{q}} \cdot p_{g}} .
$$

Furthermore, the hadronic vector elements in Eq. 6.92 are 


$$
\begin{aligned}
T_{(a 1)_{\mu}} & =\not h\left(p_{q}+\not p_{g}+m_{q}\right) \gamma_{\mu} C_{L R}^{+}, \\
T_{(a 2)_{\mu}} & =\gamma_{\mu} C_{L R}^{+}\left(p_{\bar{q}}+\not p_{g}-m_{q}\right) \not h \\
T_{(b 1) \mu} & =T_{(a 1)_{\mu}}\left(C_{L R}^{+} \rightarrow 1\right) \\
T_{(b 2) \mu} & =T_{(a 2)_{\mu}}\left(C_{L R}^{+} \rightarrow 1\right)
\end{aligned}
$$

$\eta_{\alpha}$ being the polarization vector of the gluon and $C_{L R}^{+}=C_{L}^{q} L+C_{R}^{q} R$, where $L(R)=P_{j=-1}\left(P_{j=1}\right)$.

In Fig. 35 we have plotted the tree-level cross-section as a function of the c.m. energy in an $e^{+} e^{-}$collider for polarized and unpolarized incoming electron beam. To facilitate experimental identification as well as to avoid infrared singularities we have imposed a cut on the invariant mass of the jet pairs so that $\left(p_{g}+p_{t}\right)^{2}$ and $\left(p_{g}+p_{\bar{t}}\right)^{2} \geq\left(m_{t}+m_{0}\right)^{2}$ where we have taken $m_{0}=25$ $\mathrm{GeV}$. This cut, which effectively cuts the gluon energy, also removes soft gluon emission from the secondary $b$-quarks of the top decays. We see from Fig. 35 that with an integrated luminosity of $\mathcal{L} \sim 200 \mathrm{fb}^{-1}$, a $1 \mathrm{TeV}(500 \mathrm{GeV}) e^{+} e^{-}$collider will be able to produce about $\sim 3 \times 10^{4}$ $\left(\sim 1 \times 10^{4}\right) t \bar{t} g$ events.

In a given model, the $\mathrm{CP}$-violating corrections for the reaction

$$
e^{-}\left(p_{-}\right) e^{+}\left(p_{+}\right) \rightarrow q\left(p_{q}\right) \bar{q}\left(p_{\bar{q}}\right) g\left(p_{g}\right)
$$

requires the calculation of the corresponding 1-loop diagrams. Let us write the general form of the 1-loop matrix elements that violate $\mathrm{CP}$ as $\mathcal{M}_{\sigma}^{\rho}$. For a given underlying model, the subscript indicates the diagram and the superscript indicates which gauge particle is exchanged in the $s$-channel. Thus

$$
\mathcal{M}_{\sigma}^{\rho}=J_{e}^{\mu(j)} \bar{u}\left(p_{q}\right) H_{\sigma \mu}^{\rho} v\left(p_{\bar{q}}\right),
$$

where $H_{\sigma \mu}^{\rho}$ is the "hadronic vector" corresponding to each diagram and exchanged quanta. Denoting the complete $\mathrm{CP}$-violating 1-loop contribution by

$$
\mathcal{M}^{V}=\sum_{\rho} \sum_{\sigma} \mathcal{M}_{\sigma}^{\rho}
$$

the $\mathcal{M}^{V}$ can be calculated within a given model, and the polarized CP-nonconserving DCS to 1-loop is then obtained from the interference terms between the 1-loop and the Born amplitudes

$$
\sum\left(\mathcal{M}^{V} \mathcal{M}^{0 *}+\mathcal{M}^{0} \mathcal{M}^{V *}\right)
$$

Here the sum is carried over the polarizations of $e^{+}, t, \bar{t}$ and $g$. 


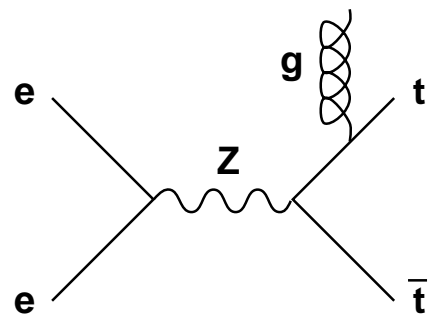

$a_{1}$

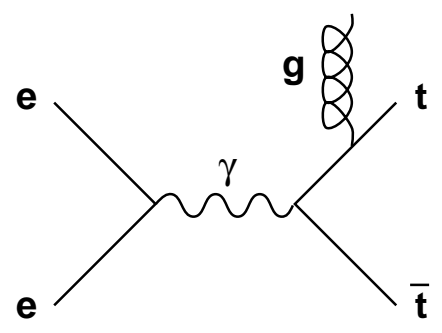

$b_{1}$

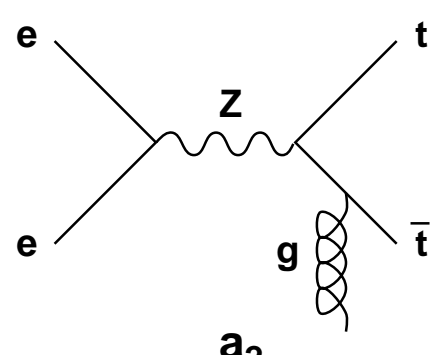

$a_{2}$

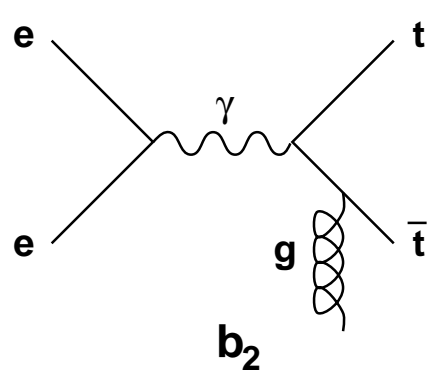

Figure 34: Tree-level Feynman diagrams contributing to $e^{+} e^{-} \rightarrow t \bar{t} g($ for $q=t)$. 


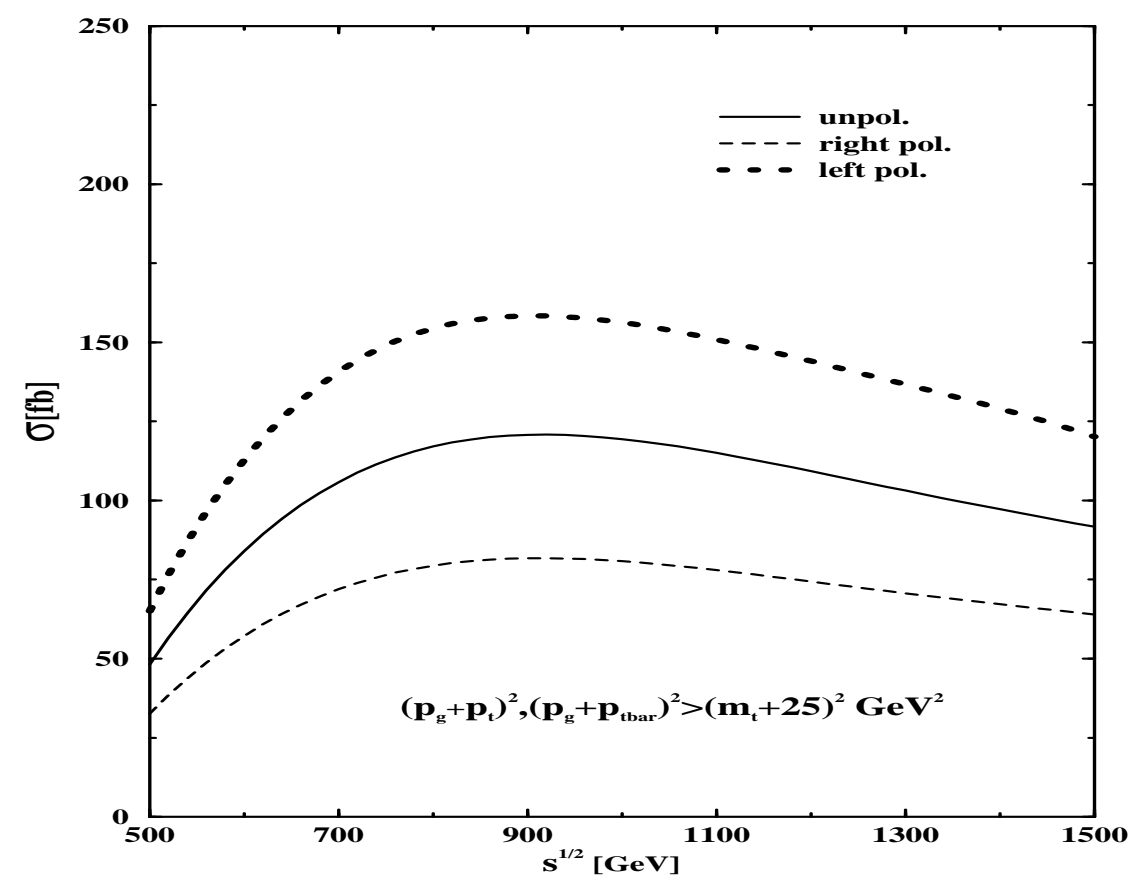

Figure 35: The cross-section for the reaction $e^{+} e^{-} \rightarrow t \bar{t} g$ (in fb) as a function of the c.m. energy $\sqrt{s}$, for unpolarized (solid line), negatively polarized (dotted line) and positively polarized (dashed line) incoming electron beam. The cuts $\left(p_{g}+p_{t}\right)^{2} \geq\left(m_{t}+m_{0}\right)^{2}$ and $\left(p_{g}+p_{\bar{t}}\right)^{2} \geq\left(m_{t}+m_{0}\right)^{2}$, $m_{0}=25 \mathrm{GeV}$, are imposed. Figure taken from [203. 


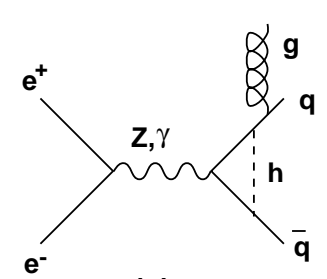

(a)

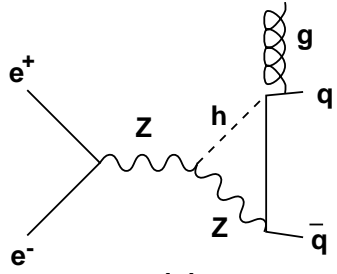

(c)

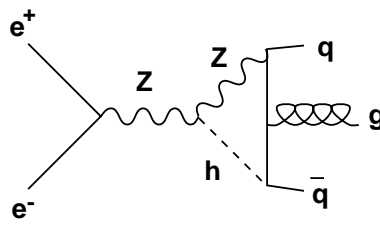

(e)

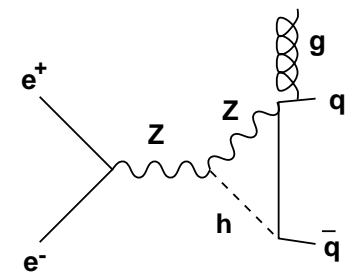

(b)

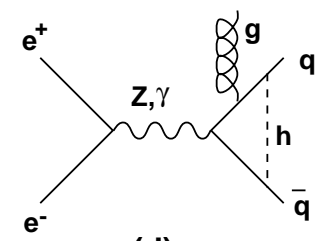

(d)

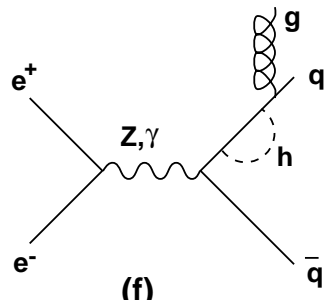

(f)

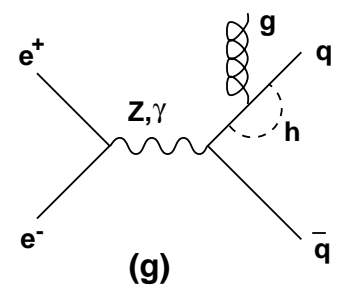

Figure 36: $C P$ violating Feynman diagrams contributing to $e^{+} e^{-} \rightarrow q \bar{q} g$ to 1-loop order in a 2HDM (h is a neutral Higgs-boson). Diagrams with permuted vertices (i.e., $q \rightarrow \bar{q}$ ) are not shown.

\subsubsection{HDM and CP violation in $e^{+} e^{-} \rightarrow t \bar{t} g$}

In a 2HDM, CP-violating neutral Higgs exchanges, at 1-loop order, can give rise to the Feynman diagrams depicted in Fig. 36 [203]. We take the limit $m_{e} \rightarrow 0$, thus neglecting all the diagrams that are proportional to the electron mass. This includes any diagram that involves electron coupling to the Goldstone modes, hence proportional to $m_{e}$.

The relevant Feynman rules for the diagrams in Fig. 36 can be extracted from parts of the Lagrangian involving the $f \bar{f} \mathcal{H}^{k}$ and $Z Z \mathcal{H}^{k}$ couplings $a_{f}^{k}, b_{f}^{k}$ and $c^{k}$ defined in Eqs. 3.70 and 3.71 , respectively.27 Again, for simplicity, we will consider only one light neutral Higgs, $h$, assuming that the remaining two are considerably heavier. Furthermore, for simplicity, whenever necessary we set the masses $\left(m_{H}\right)$ of the remaining two neutral Higgs particles to be $1 \mathrm{TeV}$, i.e., assume that they are degenerate.

All the CP-violating terms in the 1-loop amplitudes corresponding to the diagrams in Fig. 36 emerge through interference of the scalar coupling $a_{q}^{h}$ (for a quark $q$ ) with the pseudo-scalar

\footnotetext{
${ }^{27}$ Recall that $\mathcal{H}^{k}$ stand for any one of the three, i.e., $k=1,2$ or 3 , neutral Higgs in a 2HDM.
} 
coupling $b_{q}^{h}$ in any exchange of a neutral Higgs. In the diagrams where the Higgs exchange is generated at the $Z Z h$ vertex, the CP-violating terms will be proportional to $b_{q}^{h} \times c^{h}$. The CPviolating 1-loop amplitude can then be calculated (for details see 203]) and the DCS can be schematically written as

$$
\Sigma(\phi)=\Sigma_{0}(\phi)+\Sigma_{1}^{\Re \mathrm{e}}(\phi)+\Sigma_{1}^{\Im \mathrm{m}}(\phi) .
$$

Here $\Sigma_{0}(\phi)$ is a CP-even piece and $\Sigma_{1}$ is a CP-odd piece which is further subdivided into two parts that depend on the real and imaginary components of the amplitude. Thus, CP-odd, $T_{N}$-odd and CP-odd, $T_{N}$-even effects will emanate from $\Sigma_{1}^{\Re e}(\phi)$ and $\Sigma_{1}^{\Im \mathrm{m}}(\phi)$, respectively.

To estimate the CP-violating effects of both the $T_{N}$-even and $T_{N}$-odd types, the following two CP-odd observables were considered in [203] for the reaction $e^{+} e^{-} \rightarrow t \bar{t} g$

$$
\begin{array}{ccc}
\mathrm{T}_{\mathrm{N}}-\text { even } & : & O_{i 1} \equiv \frac{\vec{p}_{-} \cdot\left(\vec{p}_{t}+\vec{p}_{\bar{t}}\right)}{s} \\
\mathrm{~T}_{\mathrm{N}}-\text { odd } & : & O_{r 1} \equiv \frac{\vec{p}_{-} \cdot\left(\vec{p}_{t} \times \vec{p}_{\bar{t}}\right)}{s^{3 / 2}} .
\end{array}
$$

A non-vanishing expectation value of any one of these would signal CP violation so that experimental searches for them can be performed without recourse to any model. However, as was mentioned in section 2.6, within the context of any given model one can also construct optimal observables i.e., those observables which will be the most sensitive to $\mathrm{CP}$ violation effects in that model [16],

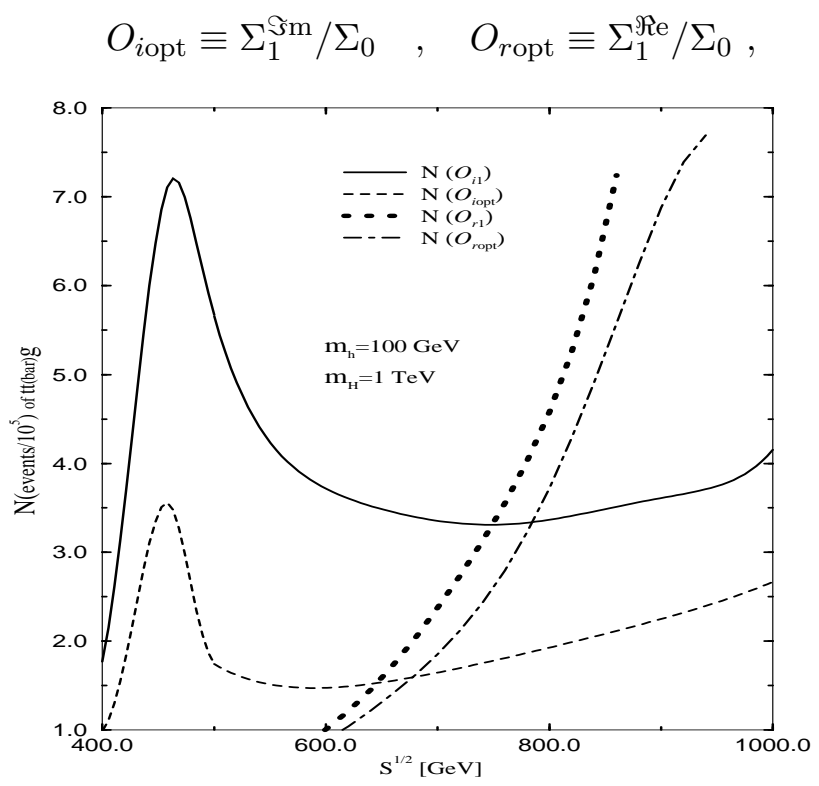

Figure 37: Number of events (in units of $10^{5}$ ) needed to detect $C P$ violation via $\left\langle O_{i 1}\right\rangle,\left\langle O_{r 1}\right\rangle$, $\left\langle O_{i \mathrm{opt}}\right\rangle$ and $\left\langle O_{\text {ropt }}\right\rangle$ to $1-\sigma$ level, as a function of the total beam energy, $\sqrt{s}$, for left-handed polarized incoming electron beam. $m_{h}=100 \mathrm{GeV}, m_{H}=1 \mathrm{TeV}$ and $a_{t}^{h}=b_{t}^{h}=c^{h}=1$ are used. Also, the cuts $\left(p_{g}+p_{t}\right)^{2} \geq\left(m_{t}+m_{0}\right)^{2}$ and $\left(p_{g}+p_{\bar{t}}\right)^{2} \geq\left(m_{t}+m_{0}\right)^{2}, m_{0}=25$ GeV, are imposed. Figure taken from [203].

The number of events needed in order to detect a CP-odd signal at the 1- $\sigma$ level via each of the above four CP-violating observables, is shown in Figs. 37 and 38 for $m_{h}=100$ and $200 \mathrm{GeV}$, respectively. In Fig. 39 we have magnified the range $\sqrt{s}=400-600 \mathrm{GeV}$ using the same Higgs masses. In these figures we have focused on the case of left polarized incoming electrons while in Table 11 we give a brief comparison of the left, right and unpolarized electron beam cases. Also, the following assumptions are made: 


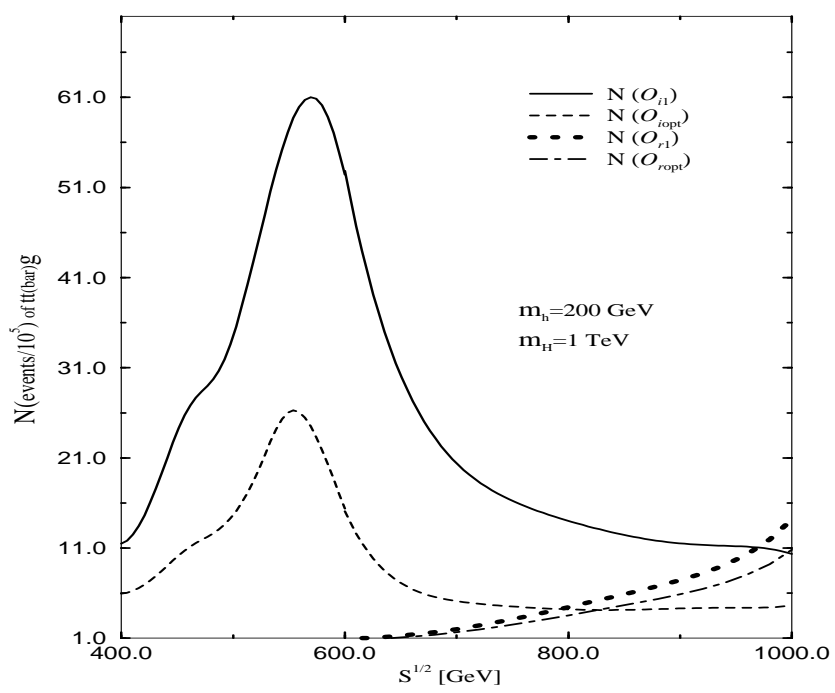

Figure 38: Same as Fig. 37 except $m_{h}=200 \mathrm{GeV}$. Figure taken from [203].

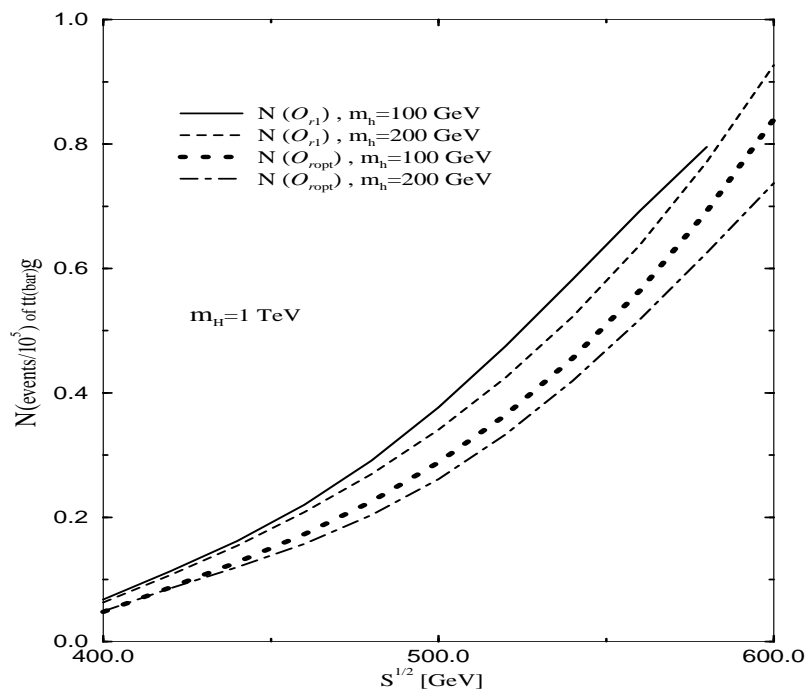

Figure 39: Number of events (in units of $10^{5}$ ) needed to detect CP violation via $\left\langle O_{r 1}\right\rangle$ and $\left\langle O_{r o p t}\right\rangle$ to $1-\sigma$ level as a function of total beam energy in the range $\sqrt{s}=400-600 \mathrm{GeV}$ for $m_{H}=1$ $\mathrm{TeV}, m_{h}=100$ and $200 \mathrm{GeV}$. The rest of the parameters are as in Figs. 37 and 38. Figure taken from [203]. 
1) As mentioned before a cut on the invariant mass of the jet pairs was imposed, so that $\left(p_{g}+p_{t}\right)^{2}$ and $\left(p_{g}+p_{\bar{t}}\right)^{2} \geq\left(m_{t}+m_{0}\right)^{2}$, where we have taken $m_{0}=25 \mathrm{GeV}$.

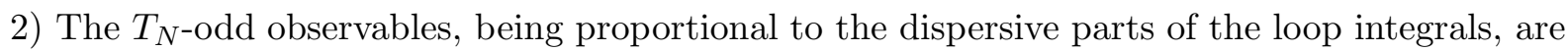
sensitive also to the mass of the two heavier neutral Higgs particles, $m_{H}$. For simplicity, we have chosen these two Higgs particles to be degenerate with a mass of $1 \mathrm{TeV}$.

3) We set the relevant Higgs couplings to unity. That is, $a_{t}^{h}=b_{t}^{h}=c^{h}=1$ (for $q=t$ ) which serves our purpose of finding the order of magnitude of the CP-odd signal that can arise in this reaction. In fact, $\mathrm{CP}$ violation in $e^{+} e^{-} \rightarrow t \bar{t} g$ is found to be dominated by the terms proportional to $a_{t}^{h} \times b_{t}^{h}$. With regard to that, we note that, for low values of $\tan \beta$, i.e., $\tan \beta \lesssim 0.5$, the product $a_{t}^{h} \times b_{t}^{h}$ can reach values above $\sim 5$ and, therefore, our choice above of $a_{t}^{h} \times b_{t}^{h}=1$ is rather conservative.

\begin{tabular}{|c|c|c|c|c|c|c|c|c|c|}
\hline \multirow[b]{2}{*}{$\sqrt{s}$} & \multirow[b]{2}{*}{$j$} & \multicolumn{2}{|c|}{$O_{i 1}$} & \multicolumn{2}{|c|}{$O_{r 1}$} & \multicolumn{2}{|c|}{$O_{i \mathrm{opt}}$} & \multicolumn{2}{|c|}{$O_{\text {ropt }}$} \\
\hline & & $\begin{array}{l}m_{h}= \\
100\end{array}$ & $\begin{array}{l}m_{h}= \\
200\end{array}$ & $\begin{array}{l}m_{h}= \\
100\end{array}$ & $\begin{array}{l}m_{h}= \\
200\end{array}$ & $\begin{array}{l}m_{h}= \\
100\end{array}$ & $\begin{array}{l}m_{h}= \\
200\end{array}$ & $\begin{array}{l}m_{h}= \\
100\end{array}$ & $\begin{array}{l}m_{h}= \\
200\end{array}$ \\
\hline \multirow{3}{*}{400} & -1 & 1.8 & 11.5 & 0.07 & 0.05 & 1.0 & 6.0 & 0.05 & 0.05 \\
\hline & unpol. & 22.5 & 134.8 & 0.05 & 0.05 & 6.5 & 37.0 & 0.05 & 0.04 \\
\hline & 1 & 2.3 & 17.1 & 0.05 & 0.04 & 1.3 & 8.4 & 0.04 & 0.03 \\
\hline & & & & & & & & & \\
\hline \multirow{3}{*}{700} & -1 & 3.4 & 20.0 & 2.2 & 2.1 & 1.7 & 5.1 & 1.9 & 1.7 \\
\hline & unpol. & 48.6 & 263.9 & 2.2 & 1.9 & 12.2 & 38.6 & 1.8 & 1.4 \\
\hline & 1 & 4.5 & 30.8 & 1.9 & 1.8 & 2.0 & 5.9 & 1.5 & 1.2 \\
\hline & & & & & & & & & \\
\hline \multirow{3}{*}{1000} & -1 & 4.0 & 10.5 & 14.4 & 14.1 & 2.6 & 4.5 & 11.6 & 10.8 \\
\hline & unpol. & 63.4 & 158.2 & 14.3 & 10.3 & 20.5 & 35.3 & 10.8 & 8.4 \\
\hline & 1 & 5.0 & 14.0 & 14.1 & 10.3 & 3.1 & 5.4 & 10.7 & 8.3 \\
\hline
\end{tabular}

Table 11: The unpolarized case is compared with left polarization $(j=-1)$ and right polarization $(j=+1)$ of the $e^{-}$. The number of events in units of $10^{5}$ needed for detection of asymmetries, to 1- $\sigma$ level are given. The values of $\sqrt{s}$ and $m_{h}$ are given in GeV. The results for the $T_{N}$-odd observables are given for $m_{H}=1 \mathrm{TeV}$, where $m_{H}$ is the mass of the two heavy Higgs (see also text). Table taken from [203].

Summarizing briefly the numerical results presented in Figs. 3739 and in Table 11, we can see that for the optimal observable $O_{i \mathrm{opt}}$, for both a $500 \mathrm{GeV}$ and a $1 \mathrm{TeV} e^{+} e^{-}$collider, the number of needed $t \bar{t} g$ events in order to detect a 1- $\sigma$ CP-odd signal is comparable and is around few $\times 10^{5}$ with neutral Higgs masses in the range $100 \mathrm{GeV}<m_{h}<200 \mathrm{GeV}$. With $O_{\text {ropt }}$ the number of needed $t \bar{t} g$ events at c.m. energies around $1 \mathrm{TeV}$ is few $\times 10^{6}$. However, we see from Fig. 39 that, at a c.m. energy of $500 \mathrm{GeV}$ and for $100 \mathrm{GeV}<m_{h}<200 \mathrm{GeV}$, a $1-\sigma$ measurement of $O_{\text {ropt }}$ will require few $\times 10^{4} t \bar{t} g$ events. From Table 11 we see that for the $T_{N^{-}}$-even (i.e., $O_{i 1}$ and $\left.O_{i \text { opt }}\right)$ cases the polarization makes a significant difference and improves their effectiveness by about an order of magnitude or even more. For these it seems that the left-polarized case is marginally better than the right one.

Bearing in mind that with an integrated luminosity of $\mathcal{L} \sim 200 \mathrm{fb}^{-1}$, about $\sim 10^{4} t \bar{t} g$ will be produced in a $500 \mathrm{GeV} \mathrm{NLC}$, and few $\times 10^{4}$ in a $1 \mathrm{TeV}$ NLC (see Fig. 35), the observability of a non-vanishing value for $O_{r o p t}$, to the $1-\sigma$ level, in a NLC with c.m. energies of $500 \mathrm{GeV}$ is marginal, while $O_{i \text { opt }}$ falls short by about an order of magnitude. Also, with a $1 \mathrm{TeV}$ NLC that can produce up to $3 \times 10^{4} t \bar{t} g$ 's a year, the CP-odd signal from $O_{r o p t}$ falls short by almost two 
orders of magnitude, while the number of events needed to detect a CP-odd effect through $O_{i \text { opt }}$ is one order of magnitude away from the expected number of available events in such a collider.

Clearly, although the CP-violating effects driven by neutral Higgs exchanges that were found in 203] fall short by at least one order of magnitude for a $3-\sigma$ detection, this does not rule out the possibility of larger effects in other extensions of the SM (e.g., SUSY). Therefore, theoretical and experimental studies of $\mathrm{CP}$ violation in the process $e^{+} e^{-} \rightarrow t \bar{t} g$ may still be worthwhile.

\subsubsection{Model independent constraints on top dipole moments}

The effects of anomalous EDM $\left(d_{t}^{\gamma}\right), \mathrm{ZEDM}\left(d_{t}^{Z}\right)$ and CEDM $\left(d_{t}^{g}\right)$ couplings of the top quark to a photon, $Z$-boson and a gluon, respectively, in $e^{+} e^{-} \rightarrow t \bar{t} g$ were considered in [204, 205].

Let us write an effective top quark interaction with a neutral gauge-bosons $V=\gamma, Z$ or $g$, which involves the top magnetic and electric dipole moments (see also section 2.5)

$$
\mathcal{L}_{V}=\frac{i g_{V}}{2 m_{t}} \bar{t} \sigma_{\mu \nu} q^{\nu}\left(\kappa_{V}-i \tilde{\kappa}_{V} \gamma_{5}\right) t F_{V}^{\mu}
$$

Here $g_{\gamma}=g_{W} s_{W}=e, g_{Z}=g_{W} / 2 c_{W}$ and $g_{g}=g_{s}$, where $g_{W}\left(g_{s}\right)$ is the weak(strong) coupling constant, $c_{W} \equiv \cos \theta_{W}, q$ is the gauge-boson 4-momentum, and $F_{V}, V=A, Z$ or $G$, is the appropriate gauge field (color index is suppressed). Moreover, in Eq. 6.106 we have introduced the CP-conserving(violating) dimensionless effective anomalous couplings $\kappa_{V}\left(\tilde{\kappa}_{V}\right)$ of the top quark to a gauge-boson $V=\gamma, Z$ or $g$. Note that $\kappa_{V}$ and $\tilde{\kappa}_{V}$ are related to $c_{t}^{V}$ (the magnetic-like dipole moments) and to $d_{t}^{V}$ (the electric-like dipole moments), defined in Eq. 6.2, via

$$
\begin{aligned}
\kappa_{V} & \equiv \frac{2 m_{t}}{g_{V}} \times c_{t}^{V} \\
\tilde{\kappa}_{V} & \equiv \frac{2 m_{t}}{g_{V}} \times d_{t}^{V}
\end{aligned}
$$

and that $\kappa_{V}$ and $\tilde{\kappa}_{V}$ are, in general, complex. In particular, for the convenience of the reader we note that $\tilde{\kappa}_{V} \sim 0.1(1)$ corresponds to a top electric-like dipole moment coupling of $d_{t}^{V} \sim$ $0.55(5.5) \times 10^{-17} g_{V}$-cm.

The implications of the effective top couplings in Eq. 6.106 can be studied by either considering CP-even or CP-odd observables in the reaction $e^{+} e^{-} \rightarrow t \bar{t} g$. Of course, it should be clear that an analysis of CP-even quantities like cross-sections and the shape of the gluon energy spectrum [204], or CP-even combination of polarization asymmetry [205, can place rather mild constraints on the absolute values of various EDM's as only $\tilde{\kappa}_{V}^{2}$ enters into such quantities. Let us summarize below the limits that can be obtained on the various EDM couplings of Eq. 6.106 by analyzing the effects of CP-even and CP-odd quantities on the reaction $e^{+} e^{-} \rightarrow t \bar{t} g$.

\subsection{2a CP-even observables}

In [204] it was suggested that the process $e^{+} e^{-} \rightarrow t \bar{t} g$ can be used to obtain limits on the anomalous dipole-like couplings of the top to $\gamma, g$ and $Z$ through the analysis of the associated gluon energy spectrum.

If the couplings of the top to the neutral gauge-bosons $\gamma, Z$ and $g$ are altered by the effective magnetic and electric-like interactions in Eq. 6.106, then by allowing one or more of the different $\kappa_{V}$ 's and $\tilde{\kappa}_{V}$ 's to be non-zero, the shape of the gluon energy spectrum in the process $e^{+} e^{-} \rightarrow t \bar{t} g$ can be modified. In [204], Monte Carlo data samples (assuming that the SM is correct) were generated and then a fit to the general expressions for the $\kappa_{V}-\left|\tilde{\kappa}_{V}\right|$ dependent spectrum were performed with which a 95\% CL allowed region in the $\kappa_{V}-\left|\tilde{\kappa}_{V}\right|$ was obtained. This procedure is done for each gauge-boson separately. That is, in analyzing the limits that can be placed on the various dipole moment couplings, only one pair of $\kappa_{V}, \tilde{\kappa}_{V}$ corresponding to one neutral 
gauge-boson, $V=\gamma, Z$ or $g$, was allowed to have a non-zero value. As we have mentioned before the usefulness of the bound on the magnetic moment is rather limited as it receives significant contribution from QCD.

Summarizing now the limits obtained in [204], Fig. 40 shows the 95\% CL allowed region in the $\kappa_{g}-\left|\tilde{\kappa}_{g}\right|$ plane for both a $500 \mathrm{GeV}$ and a $1 \mathrm{TeV} e^{+} e^{-}$NLC. We see that the CEDM coupling, $\tilde{\kappa}_{g}$, can be bounded in a $500 \mathrm{GeV}$ NLC to $\left|\tilde{\kappa}_{g}\right| \lesssim 0.6-0.8$. For the CMDM coupling, for whatever it is worth, the allowed values are $\kappa_{g} \sim \pm$ few $\times 10^{-2}$, with integrated luminosities of $50-100$ $\mathrm{fb}^{-1}$ and with a cut on the gluon energy of $E_{g}>25 \mathrm{GeV}$. In a $1 \mathrm{TeV}$ NLC, the limit on the CEDM coupling is approximately twice as strong as what can be achieved in a $500 \mathrm{GeV}$ NLC.

It should be noted that in 205 two other CP-even quantities were considered: the crosssection itself and a CP-even combination of the top and anti-top polarizations. The limits obtained there for the CEDM of the top, $\tilde{\kappa}_{g}$, are somewhat weaker then those shown in Fig. 40.

In analyzing the anomalous EDM's of the top to a photon and a $Z$-boson, the "normalized" gluon energy distribution was used in [204:

$$
\frac{d \mathcal{R}}{d z}=\frac{1}{\sigma\left(e^{+} e^{-} \rightarrow t \bar{t}\right)} \frac{d \sigma\left(e^{+} e^{-} \rightarrow t \bar{t} g\right)}{d z},
$$

It was then found that the EDM coupling of the top quark to a photon, $\tilde{\kappa}_{\gamma}$, can be constrained at the NLC by studying the reaction $e^{+} e^{-} \rightarrow t \bar{t} g$. From Fig. 41(a) we see that at a $500 \mathrm{GeV}$ NLC with integrated luminosity of $50 \mathrm{fb}^{-1}$, only long narrow bands around $\kappa_{\gamma} \sim-1$ or 0 are allowed which then gives $\left|\tilde{\kappa}_{\gamma}\right| \lesssim 0.4-0.6$. In a $1 \mathrm{TeV}$ NLC with integrated luminosity of $100 \mathrm{fb}^{-1}$, one circular narrow band between $-0.4 \lesssim \kappa_{\gamma} \lesssim 0$ is allowed giving $0 \lesssim\left|\tilde{\kappa}_{\gamma}\right| \lesssim 0.2$.

The anomalous EDM coupling of the top quark to the $Z, \tilde{\kappa}_{Z}$, is much less constrained. In particular, from Fig. 41(b) we see that with a $500 \mathrm{GeV}$ NLC and integrated luminosity of 50 $\mathrm{fb}^{-1}, 0 \lesssim\left|\tilde{\kappa}_{Z}\right| \lesssim 0.5$ is allowed if $-0.5 \lesssim \kappa_{Z} \lesssim 0.1$. However, with a $1 \mathrm{TeV}$ NLC and integrated luminosity of $100 \mathrm{fb}^{-1}$, the allowed region in the $\kappa_{Z}-\tilde{\kappa}_{Z}$ plane is considerably reduced. Namely, $0 \lesssim\left|\tilde{\kappa}_{Z}\right| \lesssim 0.1$ can be achieved if $-0.2 \lesssim \kappa_{Z} \lesssim 0$. Also, as was shown in [204], doubling the integrated luminosity does not increase the sensitivity of the NLC to these anomalous couplings of the top quark.

To conclude, note that while the process $e^{+} e^{-} \rightarrow t \bar{t}$ will presumably be more appropriate for the exploration of CP-odd effects driven by the top dipole moment couplings to $\gamma$ and $Z$ (see section 6.1), the reaction $e^{+} e^{-} \rightarrow t \bar{t} g$ might be the only place for searching for the CEDM of the top quark at the NLC. In that sense, an investigation of the effects of $\tilde{\kappa}_{g}$ on CP-odd quantities in the $t \bar{t} g$ final state at an $e^{+} e^{-}$collider, is worthwhile. This was done in [205] by constructing a genuinely CP-odd observable out of the top and anti-top polarizations and is described below.

\subsection{2b CP-odd observables}

An interesting CP-odd observable was suggested in [205]. This observable involves the top polarization and is defined as

$$
\Delta \sigma^{(-)}=\frac{1}{2}[\sigma(\uparrow)-\sigma(\downarrow)+\bar{\sigma}(\uparrow)-\bar{\sigma}(\downarrow)],
$$

where $\sigma(\uparrow), \bar{\sigma}(\uparrow)$ refer respectively to the cross-sections for top and anti-top with a positive spin component in its direction of flight, and $\sigma(\downarrow), \bar{\sigma}(\downarrow)$ are the same quantities with a corresponding negative spin component.

In 205] the sensitivity of $\Delta \sigma^{(-)}$to the CEDM of the top, $\tilde{\kappa}_{g}$, was studied. Note that $\Delta \sigma^{(-)}$ is CP-odd and $T_{N}$-even and therefore depends on the imaginary parts of the combinations of couplings $\Im \mathrm{m}\left(\kappa_{g}^{*} \tilde{\kappa}_{g}\right)$ and $\Im \mathrm{m}\left(\tilde{\kappa}_{g}\right)$ in Eq. 6.106. The $90 \%$ CL limits on the values of $\Im \mathrm{m}\left(\kappa_{g}^{*} \tilde{\kappa}_{g}\right)$ and $\Im \mathrm{m}\left(\tilde{\kappa}_{g}\right)$ were obtained from [205]:

$$
\epsilon \mathcal{L}\left|\Delta \sigma^{(-)}\left(\kappa_{g}, \tilde{\kappa}_{g}\right)-\Delta \sigma_{S M}^{(-)}\right|=2.15 \sqrt{\mathcal{L}\left|\sigma_{S M}(\uparrow)+\bar{\sigma}_{S M}(\uparrow)\right|}
$$


where in the above expressions, the subscript "SM" denotes the value expected in the standard model, with $\kappa_{g}=\tilde{\kappa}_{g}=0 ; \epsilon$ is the top detection efficiency and $\mathcal{L}$ is the integrated luminosity.

Eq. 6.111 gives contours in the $\Im \mathrm{m}\left(\kappa_{g}^{*} \tilde{\kappa}_{g}\right)-\Im \mathrm{m}\left(\tilde{\kappa}_{g}\right)$ plane which are shown in Figs. 42(a) and 42 (b) for c.m. energies of $\sqrt{s}=0.5$ and $1 \mathrm{TeV}$, respectively, for an integrated luminosity of $\mathcal{L}=50 \mathrm{fb}^{-1}$ and for $\epsilon=0.1$. Also different polarizations of the incoming electron beam, $P_{e}$, are considered in Figs. 42(a), (b). The allowed regions in Figs. 42(a), (b) are the bands lying between the upper and lower straight lines. We see that the dependence of $\Delta \sigma^{(-)}$on the electron beam polarization is rather mild.

It was also suggested in 205] that a measurement of $\Delta \sigma^{(-)}$at two different c.m. energy may improve the limits on $\Im \mathrm{m}\left(\kappa_{g}^{*} \tilde{\kappa}_{g}\right)$ and $\Im \mathrm{m}\left(\tilde{\kappa}_{g}\right)$. This is demonstrated in Fig. 43 where it is shown that from measurements of $\Delta \sigma^{(-)}$at $\sqrt{s}=0.5$ and $1 \mathrm{TeV}$, the possible limits are

$$
-0.8<\Im \mathrm{m}\left(\kappa_{g}^{*} \tilde{\kappa}_{g}\right)<0.8, \quad-11<\Im \mathrm{m}\left(\tilde{\kappa}_{g}\right)<11 .
$$

Although dealing with a genuine CP-odd observable, the limits in Eq. 6.112 are still weaker by about an order of magnitude than those obtained through the study of the gluon jet energy distribution. Note however, that those limits are placed on the imaginary part of $\tilde{\kappa}_{g}$ while the limits obtained through the study of the gluon jet energy distribution are set on the absolute value of the top CEDM. 

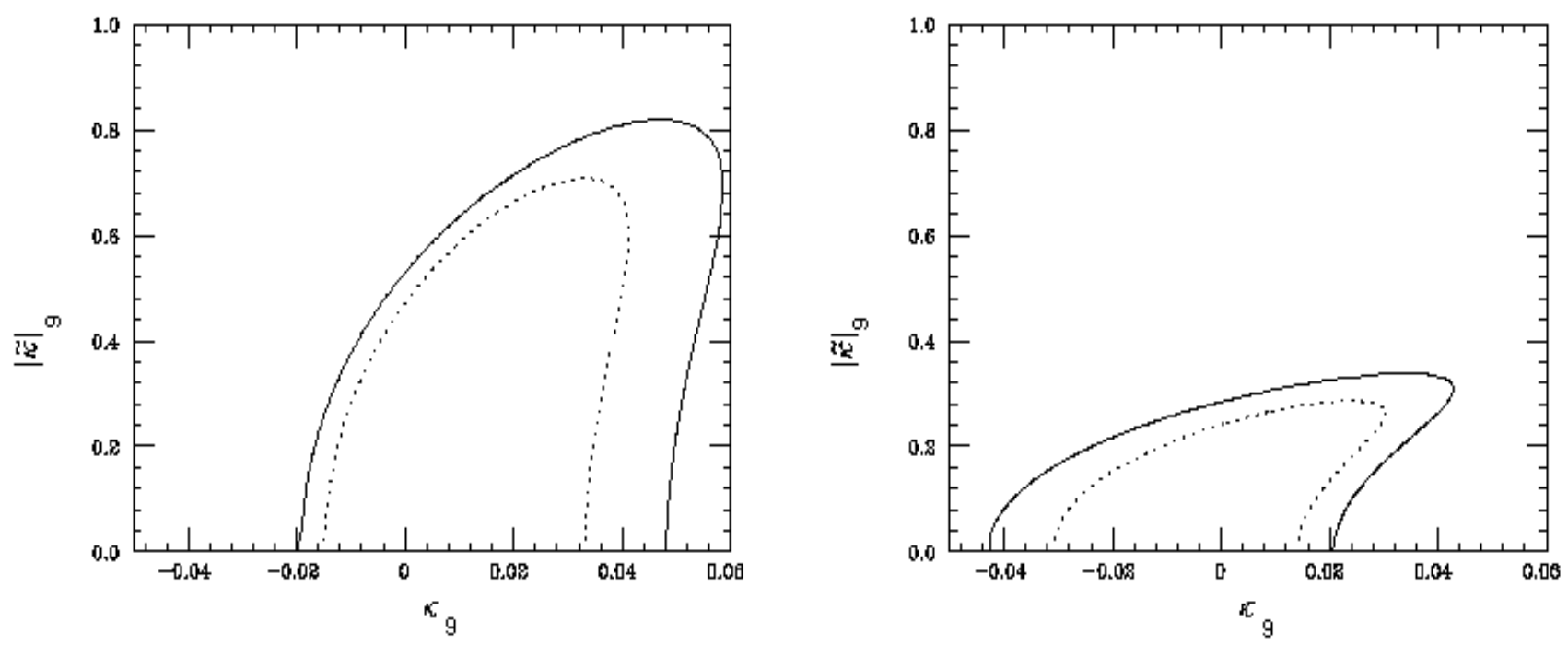

Figure 40: 95\% CL allowed region in the $\kappa_{g}-\left|\tilde{\kappa}_{g}\right|\left(\right.$ recall that $\left.\tilde{\kappa}_{g} \equiv\left(2 m_{t} / g_{s}\right) \times d_{t}^{g}\right)$ plane obtained from fitting the gluon spectrum. On the left above: $E_{g}^{\text {min }}=25 \mathrm{GeV}$ at a $500 \mathrm{GeV} \mathrm{NLC}$ assuming an integrated luminosity of 50(solid) or 100 (dotted) $\mathrm{fb}^{-1}$. On the right above: for a $1 \mathrm{TeV}$ collider with $E_{g}^{\text {min }}=50 \mathrm{GeV}$ and luminosities of 100(solid) and 200(dotted) $\mathrm{fb}^{-1}$. Figure taken from 204 . 

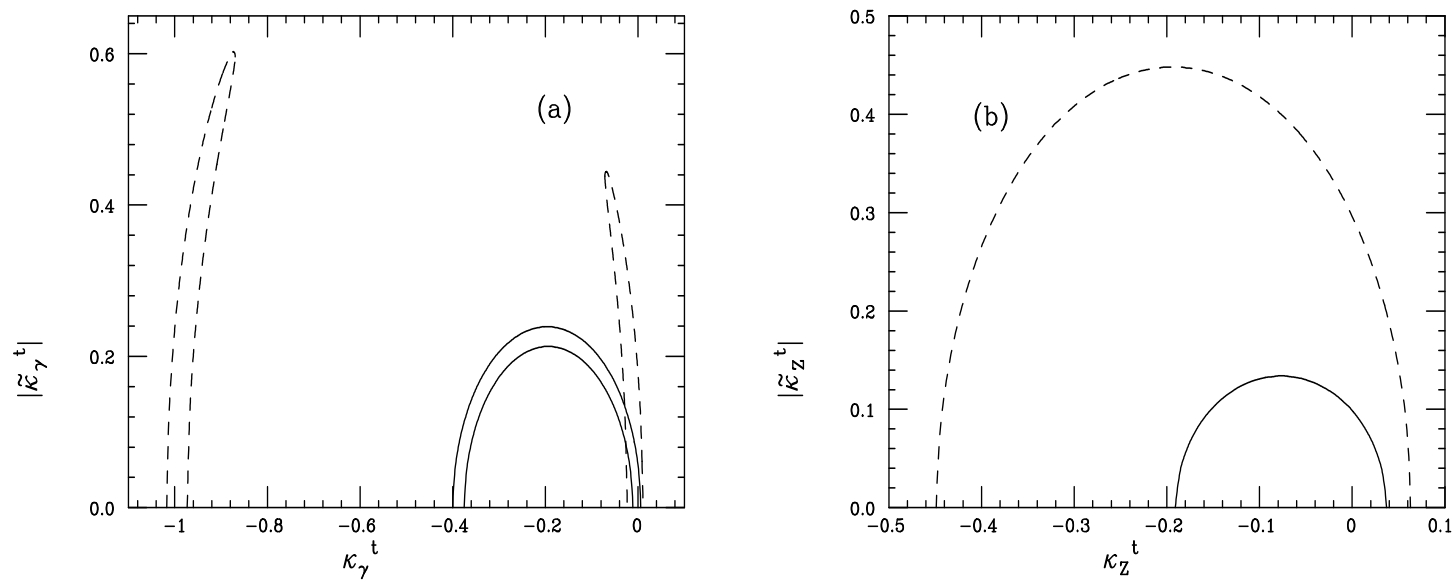

Figure 41: 95\% CL allowed regions obtained for the anomalous couplings (a) $\kappa_{\gamma}, \tilde{\kappa}_{\gamma}$ and (b) $\kappa_{Z}, \tilde{\kappa}_{Z}$ at a 500(1000) GeV NLC, assuming a luminosity of 50(100) $\mathrm{fb}^{-1}$, lie within the dashed(solid) curves (recall that $\tilde{\kappa}_{\gamma} \equiv\left(2 m_{t} / e\right) \times d_{t}^{\gamma}$ and $\left.\tilde{\kappa}_{Z} \equiv\left(2 m_{t} / g_{W} / 2 c_{W}\right) \times d_{t}^{Z}\right)$. The gluon energy range $z \equiv 2 E_{g} / \sqrt{s} \geq 0.1$ was used in the fit. Only two anomalous couplings are allowed to be non-zero at a time. Figure taken from [204]. 


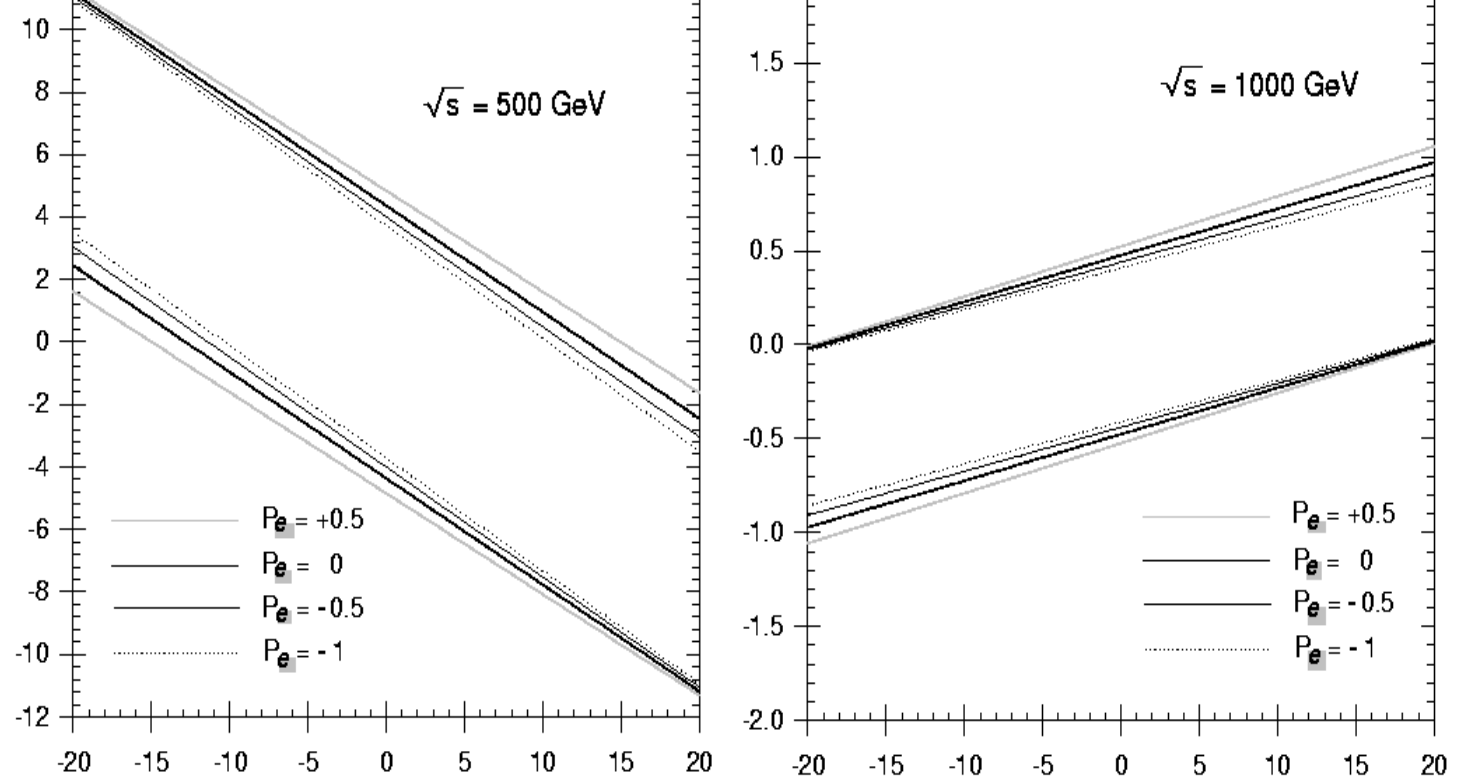

Figure 42: $\Delta \sigma^{(-)}$contour plots in the $\Im \mathrm{m}\left(\kappa_{g}^{*} \tilde{\kappa}_{g}\right)$ (vertical axis)- $\Im \mathrm{m}\left(\tilde{\kappa}_{g}\right)$ (horizontal axis) plane, with 90\% confidence level at c.m. energies $\sqrt{s}=500 \mathrm{GeV}$ (left side) and $\sqrt{s}=1000 \mathrm{GeV}$ (right side), and for different values of the electron beam polarization: $P_{e}=+0.5,0,-0.5,-1$. Figure taken from 205. 


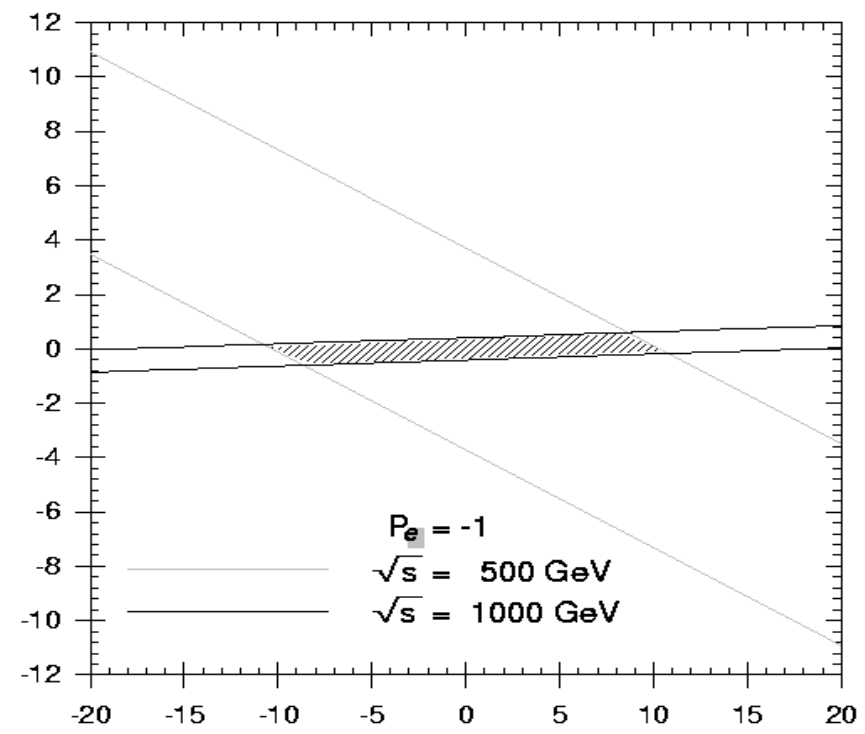

Figure 43: Intersecting area in the $\Im \mathrm{m}\left(\kappa_{g}^{*} \tilde{\kappa}_{g}\right)-\Im \mathrm{m}\left(\tilde{\kappa}_{g}\right)$ plane resulting from two independent $\Delta \sigma^{(-)}$ measurements at $\sqrt{s}=500 \mathrm{GeV}$ and $\sqrt{s}=1000 \mathrm{GeV} . P_{e}=-1$ is used. See also caption to Fig. 49. Figure taken from 205. 


\subsection{CP violation via $W W$ fusion in $e^{+} e^{-} \rightarrow t \bar{t} \nu_{e} \bar{\nu}_{e}$}

At the NLC with a very high c.m. energy, above $1 \mathrm{TeV}$, the t-channel $W^{+} W^{-}$fusion subprocesses $W^{+} W^{-} \rightarrow t \bar{t}$, where the two $W$-bosons are emitted from the initial $e^{+} e^{-}$beams, starts to dominate over the simple $s$-channel production mechanism of a pair of $t \bar{t}$, i.e., $e^{+} e^{-} \rightarrow \gamma, Z \rightarrow t \bar{t}$. As it turns out [206], the reaction

$$
e^{+} e^{-} \rightarrow W^{+} W^{-} \nu_{e} \bar{\nu}_{e} \rightarrow t \bar{t} \nu_{e} \bar{\nu}_{e}
$$

can potentially exhibit large $\mathrm{CP}$-violating phenomena, driven by $\mathrm{CP}$-odd phases in the neutral Higgs sector in MHDM's.

To lowest order there are four Feynman graphs, shown in Fig. 44, relevant to the reaction in Eq. 6.113. Indeed, at large c.m. energies, i.e., as $s / m_{W}^{2}$ becomes very large, the cross-section for the reaction in Eq. 6.113 is dominated by collisions of longitudinally polarized $W$ 's and the subprocess $W^{+} W^{-} \rightarrow t \bar{t}$ shown in Fig. 44, when calculated in the Effective $W$-boson Approximation [207], serves as a good approximation to the reaction $e^{+} e^{-} \rightarrow t \bar{t} \nu_{e} \bar{\nu}_{e}$.

The key point here, as suggested in [206], is again to construct CP-odd observables utilizing the top polarization, which in turn can be traced through the top decays. Following [206], in the rest frame of the $t$ one defines the basis vectors: $-e_{z} \propto\left(\vec{p}_{W^{+}}+\vec{p}_{W^{-}}\right), e_{y} \propto \vec{p}_{W^{+}} \times \vec{p}_{W^{-}}$and $e_{x}=e_{y} \times e_{z}$. For the anti-top one uses a similar set of the definitions in the $\bar{t}$ rest frame related by charge conjugation: $-\bar{e}_{z} \propto\left(\vec{p}_{W^{-}}+\vec{p}_{W^{+}}\right), \bar{e}_{y} \propto \vec{p}_{W^{-}} \times \vec{p}_{W^{+}}$and $\bar{e}_{x}=\bar{e}_{y} \times \bar{e}_{z}$. Now let $P_{j}$ (for $j=x, y$ or $z$ ) be the polarization of $t$ along $e_{x}, e_{y}, e_{z}$ and similarly, $\bar{P}_{j}$ the polarization of $\bar{t}$ along $\bar{e}_{x}, \bar{e}_{y}, \bar{e}_{z}$. One can then combine information from the $t$ and $\bar{t}$ systems and define the following asymmetries

$$
\begin{aligned}
& A_{x}=\frac{1}{2}\left(P_{x}+\bar{P}_{x}\right) \quad, \quad B_{x}=\frac{1}{2}\left(P_{x}-\bar{P}_{x}\right), \\
& A_{y}=\frac{1}{2}\left(P_{y}-\bar{P}_{y}\right) \quad, \quad B_{y}=\frac{1}{2}\left(P_{y}+\bar{P}_{y}\right), \\
& A_{z}=\frac{1}{2}\left(P_{z}+\bar{P}_{z}\right) \quad, \quad B_{z}=\frac{1}{2}\left(P_{z}-\bar{P}_{z}\right),
\end{aligned}
$$

where it is easy to verify that, within the above coordinate systems, the $A$ 's are CP-odd and the

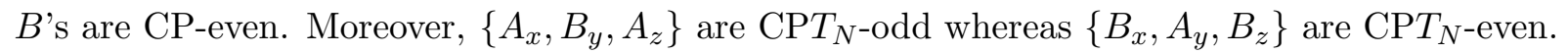

We note here that the CP-even spin observable $B_{y}$, being proportional to the imaginary part of the Higgs propagator in Fig. 44(a), is also useful for experimentally measuring the Higgs width [206]. However, since here we are only interested in CP non-conservation effects in the reaction $e^{+} e^{-} \rightarrow t \bar{t} \nu_{e} \bar{\nu}_{e}$, we will focus below on results obtained for the CP-odd observables, i.e., the $A$ 's in Eq. 6.114.

Let us consider a 2HDM with the $t \bar{t} \mathcal{H}^{k}$ and $W^{+} W^{-} \mathcal{H}^{k}$ Lagrangian pieces of Eqs. 3.70 and 3.71 , respectively. Here also, the simultaneous presence of the scalar, $a_{t}^{k}$, and pseudoscalar couplings, $b_{t}^{k}$, in Eq. 3.70 is required for a non-zero expectation value of the CP-violating asymmetries $A_{x}, A_{y}, A_{z}$. Therefore, since only two out of the three neutral Higgs particles, i.e., $k=1,2$ or 3, (say, $h \equiv \mathcal{H}^{1}$ and $H \equiv \mathcal{H}^{2}$ for the lighter and heavier ones, respectively) have a simultaneous scalar and pseudoscalar couplings to $t \bar{t}$ (see section 3.2.3), the third neutral Higgs need not be considered. $A_{z}$ is expected to receive significant contributions from loop corrections. Therefore, we focus below on $A_{x}$ and $A_{y}$ only (see discussion in [206]).

The two asymmetries $A_{x}, A_{y}$ are shown in Fig. 45, for a NLC with a c.m. energy of $\sqrt{s}=1.5$ $\mathrm{TeV}$, as a function of the lighter Higgs mass $m_{h}$. The heavier Higgs mass is fixed to $m_{H}=1$ $\mathrm{TeV}$. Also, for illustration, we use $\tan \beta=0.5$ and choose $\left\{\alpha_{1}, \alpha_{2}, \alpha_{3}\right\}=\{-\pi / 2, \beta,-\pi / 2\}$, where $\alpha_{1}, \alpha_{2}$ and $\alpha_{3}$ are the three Euler angles that specify the $3 \times 3$ orthogonal mixing matrix of the three neutral Higgs-bosons (see Eq. 3.73). With this set of parameters the $t \bar{t} h, t \bar{t} H, W^{+} W^{-} h$ and $W^{+} W^{-} H$ couplings are fixed according to Eqs. 3.70, 3.71, 3.72 and 3.73 in section 3.2.3. 
We observe from Fig. 45 that for a wide range of the lighter Higgs mass the asymmetries are appreciable. In particular, $A_{x}$ is about $10 \%$ for $m_{h} \sim 400-800 \mathrm{GeV}$ whereas $A_{y}$ is around $30 \%$ for $m_{h} \sim 100-300 \mathrm{GeV}$. Although not shown in Fig. 45, the asymmetries vanish when $m_{h}=m_{H}$ due to a GIM-like cancellation as explained in section 3.2.3.

As mentioned above, in order to measure those top polarization asymmetries, one needs the momentum of the $t$ and $\bar{t}$ decay products in a given decay scenario. In [206] two such decay scenarios useful for top polarimetry were considered:

1. The decay $t \rightarrow W^{+} b$ followed by $W^{+} \rightarrow \ell^{+} \nu$, where $\ell=e, \mu$; in this case only the hadronic decays of $\bar{t}$ are included. This case occurs with a branching ratio of $B_{1} \approx(2 / 9)(2 / 3)=4 / 27$.

2. The decay $t \rightarrow W^{+} b$ followed by $W^{+} \rightarrow$ hadrons. Now the decay of $\bar{t}$ to a $\tau^{-}$is excluded. This case occurs with a branching ratio of $B_{2} \approx(2 / 3)(8 / 9)=16 / 27$.

In the case of the leptonic decay of $t$ (or equivalently $\bar{t}$ ), the angular distribution of the lepton is $\propto\left(1+R_{1} P \cos \eta_{\ell}\right)$, where $P$ is its polarization, $\eta_{\ell}$ is the angle between the polarization axis and the momentum of the lepton in the top rest frame and $R_{1}=1$ in the SM. Thus, the optimal method to obtain the value of $P$ is to use $P=3\left\langle\cos \eta_{\ell}\right\rangle / R_{1}$ (see section 2.8). Similarly, in the case of the hadronic $t$ decay (or equivalently $\bar{t}$ ), one uses the distribution of the $W$ momentum in the top frame which is $\propto\left(1+R_{2} P \cos \eta_{W}\right)$ to extract the top polarization, where $R_{2}=\left(m_{t}^{2}-2 m_{W}^{2}\right) /\left(m_{t}^{2}+2 m_{W}^{2}\right)$. Therefore, in this case $P=3\left\langle\cos \eta_{W}\right\rangle / R_{2}$.

Hence, bearing in mind that the leptonic decay of the top is self polarizing, the number of events needed to obtain a $3-\sigma$ signal in the $t, \bar{t}$ decays of case 1 above is 206]:

$$
N_{t \bar{t}}^{3 \sigma}=(27 / 2)\left(R_{1}^{2} B_{1}+R_{2}^{2} B_{2}\right)^{-1} \times a^{-2},
$$

where $a$ is the asymmetry in question (either $A_{x}$ or $A_{y}$ ). Therefore, given the above numbers for $R_{1}, R_{2}, B_{1}$ and $B_{2}$, numerically $N_{t \bar{t}}^{3 \sigma} \approx 52 a^{-2}$, thus requiring some 5200 events for an asymmetry of $10 \%$. In fact, this can be further improved in the case of the hadronic decays of $t$ (case 2 above) by observing that [57 the less energetic of the two jets from the decay of the $W$ is more likely to be the $\bar{d}$-type quark as noted in section 2.8 . In particular, in case 2 one obtains $N_{t \bar{t}}^{3 \sigma} \approx 32 a^{-2}$, thus reducing the requirement to 3200 events for an asymmetry of $10 \%$ [206]. For an asymmetry of about $30 \%$, which was found possible in the case of $A_{y}$ (see Fig. 45), only a few hundred events will be needed.

Indeed, the cross-section for the reaction in Eq. 6.113 was calculated in [206] for the case of a $2 \mathrm{HDM}$ with the couplings described above and it was found to be at the level of a few fb, reaching $\gtrsim 10 \mathrm{fb}$ for $350 \mathrm{GeV} \lesssim m_{h} \lesssim 550 \mathrm{GeV}$. Thus, given that at $\sqrt{s}=1.5 \mathrm{TeV}$ the projected luminosity could be about $5 \times 10^{34} \mathrm{~cm}^{-2} \mathrm{~s}^{-1}$ [192], a cross-section of $10 \mathrm{fb}$ would yield about 5000 events rendering it feasible to detect asymmetries $\gtrsim 10 \%$. The conclusion is therefore that the top polarization asymmetries for the reaction $e^{+} e^{-} \rightarrow t \bar{t} \nu_{e} \bar{\nu}_{e}$ are accessible to the NLC and can serve as a powerful probe of CP violation driven by the neutral Higgs sector of a $2 \mathrm{HDM}$. However, this last statement must be taken with some caution since, the two neutrinos in the final state, carry a substantial amount of missing energy and may therefore pose a problem in reconstructing the $t$ and $\bar{t}$ rest frames, as required for measuring the polarization asymmetries in question when the $t$ or $\bar{t}$ decays leptonically. No such problem arises if both the $t$ and the $\bar{t}$ decay purely hadronically but, in that case, it remains to be seen if it will be possible to distinguish $t$ from $\bar{t}$ which is also required for measuring $A_{x}$ and $A_{y}$.

An interesting generalization of this work [206] is to consider instead the reaction $e^{+} e^{-} \rightarrow$ $t \bar{t} e^{+} e^{-}$. Now the fusion takes place via neutral gauge-bosons $(\gamma, Z)$. Although there may be some loss of the cross-section, to compensate that, there is also the advantage that the difficulties in reconstructing the rest frames of $t, \bar{t}$ may be far less formidable. 


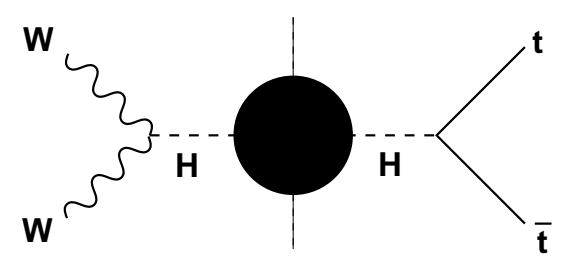

(a)

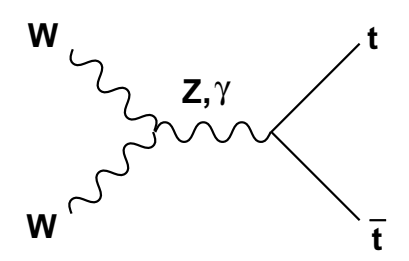

(b)

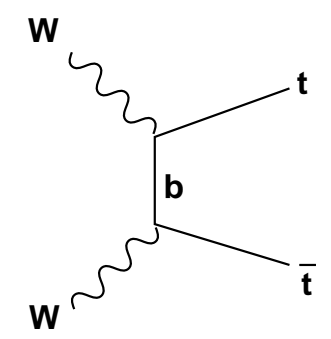

(c)

Figure 44: The Feynman diagrams that participate in the subprocess $W^{+} W^{-} \rightarrow t \bar{t}$. The blob in diagram (a) represents the width of the Higgs resonance and the cut across the blob is to indicate the imaginary part. 


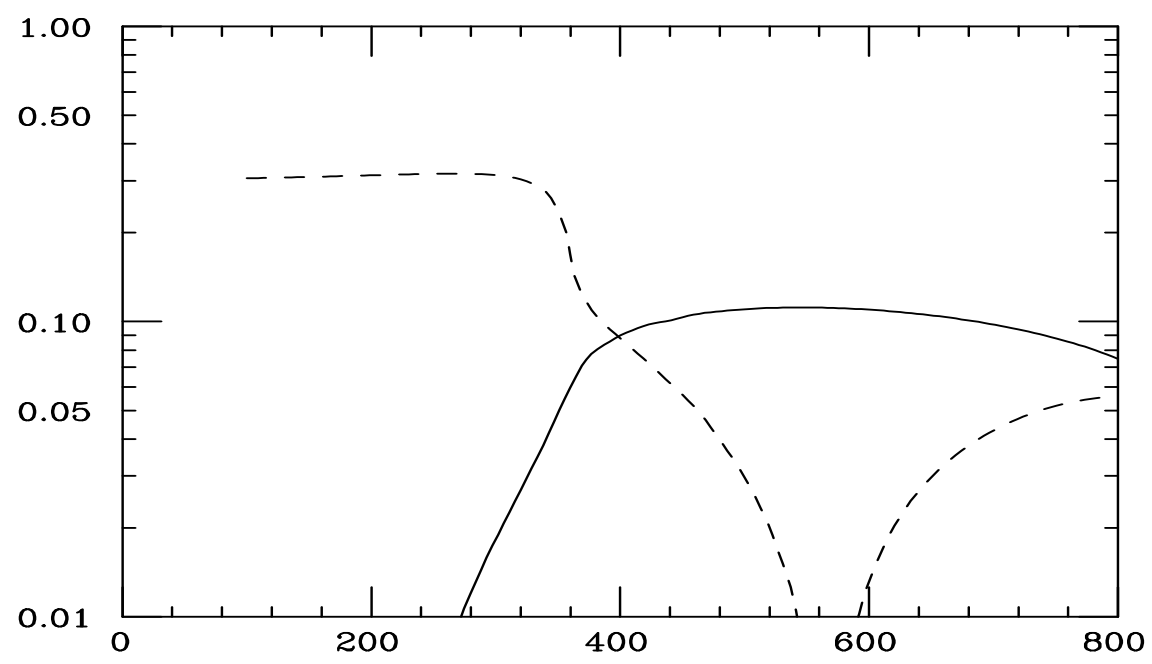

Figure 45: The asymmetries $A_{x}$ (solid) and $A_{y}$ (dashes) integrated over $\hat{s}$ as a function of $m_{h}$ for $\sqrt{s}=1.5 \mathrm{TeV}$ and $m_{H}=1 \mathrm{TeV}$. The coupling parameters are for $\tan \beta=0.5$ and $\left\{\alpha_{1}, \alpha_{2}, \alpha_{3}\right\}=$ $\{-\pi / 2, \beta,-\pi / 2\}$ as described in the text. Figure taken from [200]. 


\section{$7 \quad \mathrm{CP}$ violation in $p p$ collider experiments}

The LHC is a $p p$ collider at CERN, with c.m. energy of $14 \mathrm{TeV}$, scheduled to start running around 2005 (For a recent review on machine parameters see [208].) Its design luminosity is $\mathcal{L}=10^{34}$ $\mathrm{cm}^{-2} \mathrm{sec}^{-1}$, corresponding to a yearly integrated luminosity of $100 \mathrm{fb}^{-1}$. A low luminosity first stage of $10 \mathrm{fb}^{-1}$ is usually assumed in articles discussing physics at the LHC. The issues discussed in the following section, will be relevant for the future CMS and ATLAS experiments (for a review see [209]); heavy ions and LHC-B will not be discussed in the present work. For recent reviews on the physics at LHC, see 209] and 2210].

\section{1 $p p \rightarrow t \bar{t}+X:$ general comments}

In hadronic collisions $t \bar{t}$ pairs are produced through the parton level subprocesses $q \bar{q} \rightarrow t \bar{t}$ and $g g \rightarrow t \bar{t}$. The latter, $g g$ fusion process, dominates over the quark-anti-quark annihilation in a multi-TeV $p p$ collider. For example, at the LHC, $\sigma(g g \rightarrow t \bar{t}) \sim 90 \%$ and $\sigma(q \bar{q} \rightarrow t \bar{t}) \sim 10 \%$ are expected. It is therefore important to investigate the expected $\mathrm{CP}$ violation effects in $p p \rightarrow t \bar{t}+X$ that can arise from CP non-conservation in the subprocess $g g \rightarrow t \bar{t}$.

Note that the simple $q \bar{q}$ fusion process is the analog of the $e^{+} e^{-} \rightarrow t \bar{t}$ production mechanism where an s-channel gauge-boson is exchanged. In the case of $q \bar{q} \rightarrow g \rightarrow t \bar{t}$, the CP-odd effect can therefore be attributed to the CEDM $\left(d_{t}^{g}\right)$ of the top present at the $g t \bar{t}$ vertex. In contrast, the $g g$ production process gives rise to a much richer possibility of $\mathrm{CP}$-violating interactions and the resulting asymmetries in $g g \rightarrow t \bar{t}$ need not be related merely to the CEDM of the top quark. This fact can be readily seen in model calculations (such as 2HDM and MSSM to be discussed below), where additional CP-violating 1-loop box diagrams as well as $\hat{s}$-channel resonant neutral Higgs exchange become relevant.

We will first discuss an effective Lagrangian approach in which all CP-violating effects are assumed to originate only from the CEDM of the top. We will then present model dependent analysis of $\mathrm{CP}$ non-conservation in $g g \rightarrow t \bar{t}$ where all possible CP-violating operators are taken into account.

As will be shown, the typical size of the CP-violating asymmetries in $p p \rightarrow t \bar{t}+X$ is $\sim 10^{-3}$. Although, naively one may expect such asymmetries to be within the experimental reach of the LHC, which is expected to produce $\sim 10^{7}-10^{8} t \bar{t}$ pairs, there are at least two types of hurdles that make this objective very difficult to attain. First there is the detector dependent systematics which are expected to present serious limitations for asymmetries at the $\sim 10^{-3}$ level. Another serious difficulty is that the initial state $(p p)$ is not an eigenstate of CP. Therefore one expects fake asymmetries to arise at some level even though the underlying interactions do not violate $\mathrm{CP}$. These backgrounds are process dependent and the fake asymmetries that they produce needs to be much smaller in comparison to the CP-violating signal that is of interest. In some cases, e.g., an $\hat{s}$-channel resonant Higgs exchange within a 2HDM, as will be described in section 7.3.2, by employing clever cuts on the $t \bar{t}$ invariant mass one can obtain asymmetries at the percent level. In these cases, the CP signal is more robust and may be within the reach of the LHC if the 2HDM parameter space turns out favorable.

\section{2 $p p \rightarrow t \bar{t}+X$ : general form factor approach and the CEDM of the top}

As already mentioned in previous sections, in close analogy to the EDM and the weak $(Z)$-EDM of the top, one can generalize the top quark-gluon effective Lagrangian to include terms of dimension 5 which can give rise to a CEDM for the top quark (see Eq. 6.106 in section 6.3.2). In general, the CEDM coupling, $d_{t}^{g}$, may be considered as a form factor. Its momentum dependence is generated by effective Lagrangian operators of dimension greater than 5 . In model dependent calculations, this from factor may acquire momentum dependent imaginary parts as well as real parts. In momentum space, similar to the EDM and weak-EDM cases, the CEDM modifies the ttg 
interaction to read (we will not concern ourselves here with the CP-conserving Chromo-magnetic dipole moment of the top)

$$
-i T_{a}\left(g_{s} \gamma^{\mu}+d_{t}^{g} \sigma^{\mu \nu} \gamma_{5} k_{\nu}\right)
$$

where $k=p_{t}+p_{\bar{t}}$ is the gluon four-momentum and $p_{t}\left(p_{\bar{t}}\right)$ is the $t(\bar{t})$ four momentum.

The subprocess $g g \rightarrow t \bar{t}$ then proceeds through diagrams (a)-(d) in Fig. 46 where the heavy dots indicate the vertices modified by the CEDM of the top defined in Eq. 7.1. Diagram (d) involves an additional dimension $5 \mathrm{ttg}$ contact term and is needed to preserve gauge invariance (see section 2.5). Assuming that $d_{t}^{g}$ is small enough such that one can expand the matrix element squared to first order in $d_{t}^{g}$, the differential cross-section for the subprocess $g g \rightarrow t \bar{t}$ can be written, similar to the $e^{+} e^{-} \rightarrow t \bar{t}$ case, as [22]:

$$
\Sigma(\phi) d \phi=\Sigma_{0}(\phi) d \phi+\left[\Re \mathrm{e} d_{t}^{g}(\hat{s}) \Sigma_{\Re \mathrm{e}}(\phi)+\Im \mathrm{m} d_{t}^{g}(\hat{s}) \Sigma_{\Im \mathrm{m}}(\phi)\right] d \phi,
$$

where $\hat{s}=x_{1} x_{2} s$ and $x_{1}, x_{2}$ are the gluons momentum fractions. In Eq. 7.2 above, the gluon structure functions are included and thus $\phi$ represents the final state phase space including the gluon momentum fraction variables. Also, with no summation over the $t$ and $\bar{t}$ spins $s_{t}$ and $s_{\bar{t}}$, respectively, the CP-odd differential cross-sections $\Sigma_{\Re \mathrm{e}}(\phi)$ and $\Sigma_{\Im \mathrm{m}}(\phi)$ are functions of $s_{t}, s_{\bar{t}}$. Therefore, because of the correlation between the top spin and the momentum of the charged lepton from the top decay $t \rightarrow b W^{+} \rightarrow b \ell^{+} \nu_{\ell}$, Eq. 7.2 with the $s_{t}$ and $s_{\bar{t}}$ dependence, gives in fact the differential cross-section for the complete process $g g \rightarrow t \bar{t}$ including the subsequent leptonic decay chains of the tops. 


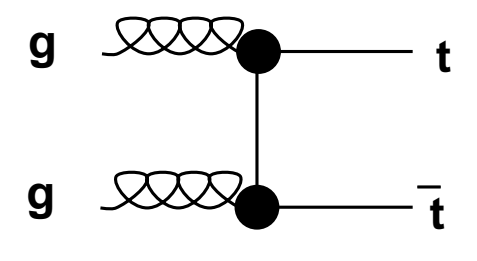

(a)

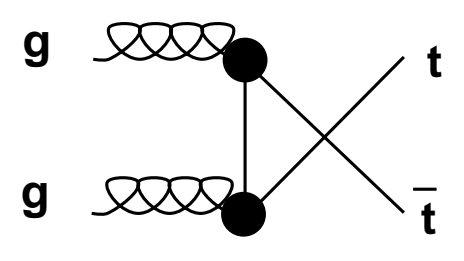

(c)

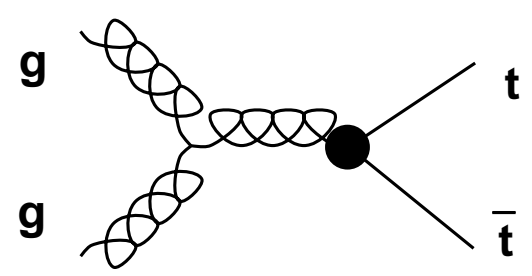

(b)

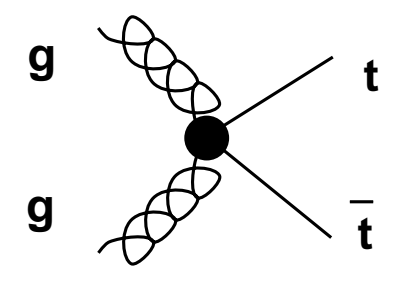

(d)

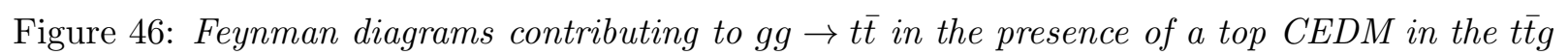
vertex which is denoted by the heavy dot. 


\subsubsection{Optimal observables}

With the effective Lagrangian in Eq. 6.106 in section 6.3.2 and by ignoring operators of dimension greater than 5, only the effect of a constant real $d_{t}^{g}$ was investigated in [22]. Indeed, in model calculations to be described below, the real part of the CEDM form factor is a constant to a good approximation. Similar to the $e^{+} e^{-} \rightarrow t \bar{t}$ case, an optimal $T_{N^{-}}$odd, CP-violating observable for $g g \rightarrow t \bar{t}$ was defined in 22] as

$$
\mathcal{O}=\frac{\Sigma_{\Re \mathrm{e}}}{\Sigma_{0}}
$$

In a realistic hadronic collider however, not all momenta which enter into the problem are immediately observable. For example, with leptonic decays of both $t$ and $\bar{t}$, the momenta of the neutrinos and the longitudinal momenta of the initial gluons are not observed. As was shown in [22], this leads to a twofold or fourfold ambiguity (depending on the number of solutions to the kinematics which results in a quartic equation) in determining the neutrinos momenta. To bypass this difficulty an "improved" optimal observable, that averages over the reconstruction ambiguity, was suggested in [22]:

$$
\mathcal{O}^{\prime} \equiv \frac{\sum_{i} \Sigma_{\Re \mathrm{e}}\left(\phi_{i}\right)}{\sum_{i} \Sigma_{0}\left(\phi_{i}\right)}
$$

where the sum is over the different possible reconstructions of the neutrino and anti-neutrino momenta from the leptonic $t$ and $\bar{t}$ decays, respectively.

Using the optimal observables $\mathcal{O}$ and $\mathcal{O}^{\prime}$, the attainable $1-\sigma$ limits on $\Re$ e $d_{t}^{g}$, assuming $10^{7}$ dilepton $t \bar{t}$ decays, were given in [22]. Note that one can consider also leptonic-hadronic and purely hadronic decays of the $t \bar{t}$ pairs. Due to the branching ratios of the $W$-boson, $10^{7}$ leptonic $t \bar{t}$ pairs implies a sample of $\sim 6 \times 10^{7}$ leptonic-hadronic $t \bar{t}$ pairs and $\sim 9 \times 10^{7}$ hadronic $t \bar{t}$ pairs. With $m_{t}=175 \mathrm{GeV}$, for the "simple" optimal observable $\mathcal{O}$, with dilepton $t \bar{t}$ pairs, it was found that the $1-\sigma$ limit is $\Re$ e $d_{t}^{g} \sim 2.8 \times 10^{-20} g_{s}$-cm. For the "improved" optimal observable $\mathcal{O}^{\prime}$, $\Re$ e $d_{t}^{g} \sim 3.0 \times 10^{-20} g_{s}$-cm with dilepton or hadronic $t \bar{t}$ pairs and $\Re$ e $d_{t}^{g} \sim 2.0 \times 10^{-20} g_{s}$-cm with leptonic-hadronic $t \bar{t}$ pairs. Comparing the 1- $\sigma$ limits on $\Re$ e $d_{t}^{g}$ attainable with dileptonic $t \bar{t}$ pairs and through the use of the optimal observables $\mathcal{O}$ and $\mathcal{O}^{\prime}$ in Eqs. 7.3 and 7.4, respectively, we see that the reconstruction ambiguity does not cause any significant changes. Evidently, with these optimal observables, $\Re$ e $d_{t}^{g}$ may be measured to a precision of $\sim 10^{-20} g_{s}$-cm. $\mathcal{O}^{\prime}$ with the leptonic-hadronic $t \bar{t}$ channel seems to be the most sensitive to $\Re$ e $d_{t}^{g}$. Comparable results for $\Re$ e $d_{t}^{g}$ were found in [211] by using the same type of optimal observables. Ref. [211] has also extended the analysis of [22] by including effects of the imaginary part of $d_{t}^{g}$. They found that the attainable limit at the LHC for $\Im \mathrm{m} d_{t}^{g}$ is of the same order, i.e., $\Im \mathrm{m} d_{t}^{g} \sim 10^{-20} g_{s}$-cm, although slightly better than the one for $\Re$ e $d_{t}^{g}$.

This result is encouraging since, as we have discussed in Chapter 1 the CEDM of the top may be $\gtrsim 10^{-20} g_{s}$-cm in some extensions of the SM, e.g., MHDM's and the MSSM.

\subsubsection{Observable correlations between momenta of the top decay products}

It is also instructive to consider simple observables constructed exclusively out of momenta which are directly observed. With the decays $t \rightarrow b \ell \nu_{\ell}$ and $\bar{t} \rightarrow \bar{b} \bar{\nu}_{\ell}$, the momenta $p_{\ell}, p_{\bar{\ell}}, p_{b}$ and $p_{\bar{b}}$ will be directly observed and observables which involve correlations between those momenta are

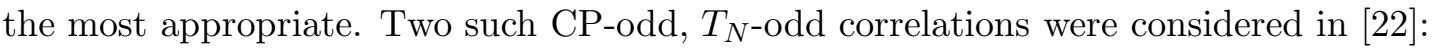

$$
\begin{aligned}
f_{1} & =\frac{\epsilon_{\mu \nu \sigma \rho} p_{e}^{\mu} p_{\bar{e}}^{\nu} p_{b}^{\sigma} p_{\bar{b}}^{\rho}}{\left(p_{e} \cdot p_{\bar{e}} p_{b} \cdot p_{\bar{b}}\right)^{1 / 2}} \\
f_{2} & =\left(p_{e}^{x} p_{\bar{e}}^{y}-p_{e}^{y} p_{\bar{e}}^{x}\right) \cdot \operatorname{sgn}\left(p_{e}^{z}-p_{\bar{e}}^{z}\right)\left(p_{e} \cdot p_{\bar{e}}\right)^{1 / 2}
\end{aligned}
$$


where $\operatorname{sgn}(X)=+1$ for $X \geq 0$ and -1 for $X<0$.

The attainable $1-\sigma$ limits on $\Re$ e $d_{t}^{g}$ for the observables $f_{1}$ and $f_{2}$, with $m_{t}=175 \mathrm{GeV}$ and assuming $10^{7}$ dileptonic $t \bar{t}$ pairs, were also given in [22]. The findings were for $f_{1}$ : $\Re$ e $d_{t}^{g} \sim$ $5.3 \times 10^{-20} g_{s}-\mathrm{cm}$ and for $f_{2}$ : $\Re$ e $d_{t}^{g} \sim 3.0 \times 10^{-19} g_{s}-\mathrm{cm}$. We see that the limit that might be achieved with $f_{1}$ is about an order of magnitude smaller than that from $f_{2}$. However, $f_{2}$ depends only on the lepton momenta and is, therefore, easiest to determine experimentally. Also, the limit from $f_{1}$ is about 2 times weaker then the one obtained from the "improved" optimal observable discussed previously.

In 212 CP-odd $T_{N}$-even observables which might be used to probe the imaginary part of the CEDM, i.e., $\Im m d_{t}^{g}$, were considered

$$
\begin{aligned}
A_{E} & =E_{\bar{\ell}}-E_{\ell}, \\
Q_{33} & =2\left(p_{\bar{\ell}}^{z}+p_{\ell}^{z}\right)\left(p_{\bar{\ell}}^{z}-p_{\ell}^{z}\right)-\frac{2}{3}\left(\vec{p}_{\bar{\ell}}^{2}-\vec{p}_{\ell}^{2}\right) .
\end{aligned}
$$

$A_{E}$ is the energy asymmetry between $\ell$ and $\bar{\ell}$ and $Q_{33}$ is an asymmetry originally suggested by Bernreuther et al. in 165] (see section 6.1.2). In a $p p$ collider with $\sqrt{s}=14 \mathrm{TeV}$, an integrated luminosity of $10 \mathrm{fb}^{-1}$ and an acceptance efficiency of $\epsilon=10 \%$, taking only leptonic $(\ell=e, \mu) t \bar{t}$ pairs and assuming $m_{t}=175 \mathrm{GeV}$ the following $1-\sigma$ limits on $\Im \mathrm{m} d_{t}^{g}$ were obtained through the observables $A_{E}$ and $Q_{33}$

$$
\begin{aligned}
& A_{E} \quad: \quad\left|\Im \mathrm{m} d_{t}^{g}\right|=8.58 \times 10^{-19} g_{s}-\mathrm{cm}, \\
& Q_{33}: \quad\left|\Im \mathrm{m} d_{t}^{g}\right|=2.05 \times 10^{-18} \quad g_{s}-\mathrm{cm} .
\end{aligned}
$$

Thus the limits on the imaginary part of the top CEDM are weaker by about an order of magnitude than those that might be obtained on the real part of the top CEDM, using the optimal observables discussed before.

\subsubsection{Polarized proton beams}

A very interesting $\mathrm{CP}$-violating polarization rate asymmetry was originally suggested by Gunion et al. in [213], for Higgs production through $g g$ fusion in a $p p$ collider. This asymmetry was applied to $p p \rightarrow t \bar{t}+X$ in [212]. The basic idea is that, if the gluons in a polarized proton are polarized, then the initial CP-odd gluon-gluon configuration allows to probe CP-violating effects without requiring full reconstruction of the $t \bar{t}$ final state. The polarization rate asymmetry is defined as

$$
A_{p r} \equiv \frac{\sigma_{+}-\sigma_{-}}{\sigma_{+}+\sigma_{-}}
$$

where $\sigma_{ \pm}$, in the subprocess $g g \rightarrow t \bar{t}$, is the cross-section for $t \bar{t}$ production in collisions of an unpolarized proton with a proton of helicity \pm . Clearly, $A_{p r}$ is CP-odd and $T_{N}$-even and therefore can only probe the imaginary part of the top CEDM. Of course, a crucial point for such an analysis is the degree of polarization that can be achieved for gluons in the $p p$ collider. The amount of gluon polarization in a positively polarized proton beam is defined by the structure functions difference $\Delta g(x)=g_{+}(x)-g_{-}(x)$. The structure functions of polarized gluons, $g_{ \pm}$, are not well known and depend on the amount of the proton's spin carried by the gluons. In [212] the following parameterization was adopted ( $g$ is the unpolarized gluon distribution)

$$
\Delta g(x)=\left\{\begin{array}{ll}
g(x) & \left(x>x_{c}\right) \\
\left(x / x_{c}\right) g(x) & \left(x<x_{c}\right)
\end{array},\right.
$$


Table 12: The number of $t \bar{t}$ events $N$, the ratio $\Delta \hat{N} / N$ (see text), and the attainable 1- $\sigma$ limits on $\left|\Im \mathrm{m} d_{t}^{g}\right|$, for various $p_{T}$-cuts with $\sqrt{s}=14 \mathrm{TeV}, m_{t}=175 \mathrm{GeV}$ and $\mathcal{L}=10 \mathrm{fb}^{-1}$. Table taken from 219].

\begin{tabular}{c|ccc}
\hline \hline$p_{T}$-cuts $(\mathrm{GeV})$ & $N\left(\times 10^{6}\right)$ & $\Delta \hat{N} / N$ & $\left|\Im m d_{t}^{g}\right|\left(\times 10^{-20} g_{s}-\mathrm{cm}\right)$ \\
\hline 0 & 2.62 & 1.44 & 0.766 \\
20 & 2.55 & 1.42 & 0.788 \\
40 & 2.36 & 1.37 & 0.847 \\
60 & 2.08 & 1.30 & 0.951 \\
80 & 1.74 & 1.22 & 1.107 \\
100 & 1.41 & 1.14 & 1.313 \\
\hline \hline
\end{tabular}

where $x_{c} \sim 0.2$ yields a value of $\Delta g \sim 2.5$ at $Q^{2}=10 \mathrm{GeV}^{2}$. The above distribution was actually evaluated at $Q^{2}=100 \mathrm{GeV}^{2}$ disregarding any scale evolution.

The 1- $\sigma$ attainable limits on $\left|\Im m d_{t}^{g}\right|$ were calculated in [212] and are given in Table 12, for various transverse-momentum cuts and for $\sqrt{s}=14 \mathrm{TeV}, m_{t}=175 \mathrm{GeV}, \mathcal{L}=10 \mathrm{fb}^{-1}$ and an efficiency acceptance of 10\%. Also, in Table $12 N=N_{+}+N_{-}$is the total number of $t \bar{t}$ events, $\Delta \hat{N}=N_{+}-N_{-}$and $N_{+}\left(N_{-}\right)$is the number of $t \bar{t}$ events predicted for positively(negatively) polarized proton. They have included all possible $t$ decay modes so that the net branching ratio was taken as unity. We see that, even with high $p_{T}$-cuts, it is possible to put a $1-\sigma$ limit on $\left|\Im \mathrm{m}\left(d_{t}^{g}\right)\right|$ up to the order of $10^{-20} \mathrm{~g}_{s}$-cm in the LHC with polarized incoming protons. This limit is more stringent than the ones obtained in Eqs. 7.9 and 7.10 through the leptonic correlations $A_{E}$ and $Q_{33}$, respectively, and is of the same order as that obtained on the real part of the top CEDM with the optimal observables discussed before. 


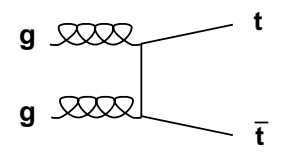

(a)

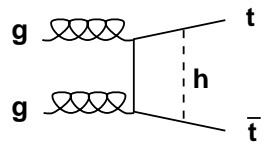

(c)

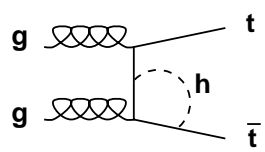

(e)

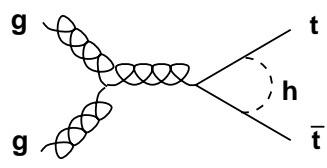

(g)

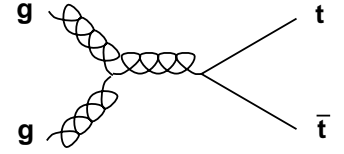

(b)

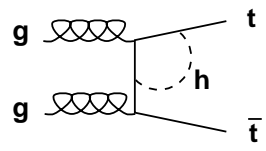

(d)

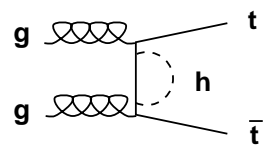

(f)

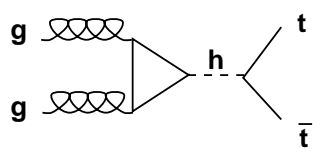

(h)

Figure 47: Feynman diagrams for the tree level $Q C D$ and neutral Higgs exchanges (denoted by the dashed lines) which contribute to the production density matrix for $g g \rightarrow t \bar{t}$. Diagrams with crossed gluons are not shown. 


\subsection{HDM and CP violation in $p p \rightarrow t \bar{t}+X$}

In a $2 \mathrm{HDM}$ with the $\mathrm{CP}$-violating $t \bar{t} \mathcal{H}$ couplings in Eq. 3.70, neutral Higgs exchanges can give rise to $\mathrm{CP}$ violation in $g g \rightarrow t \bar{t}$ and $q \bar{q} \rightarrow t \bar{t}$ at the 1-loop order in perturbation theory. In Fig. 47(c)(h) all possible 1-loop CP-violating Higgs exchanges in $g g \rightarrow t \bar{t}$ are drawn and in Fig. 48(b) the only CP-violating 1-loop diagram for $q \bar{q} \rightarrow t \bar{t}$ is shown. Interference of diagrams (c)-(h) with the SM tree-level diagrams (a) and (b) in Fig. 47 and interference of diagram (b) with diagram (a) in Fig. 48 can then give rise to $\mathrm{CP}$ non-conservation effects in $g g$ and $q \bar{q}$ fusion, respectively. One can then identify various $\mathrm{CP}$-violating correlations to trace the resulting $\mathrm{CP}$-odd quantities which appear in the corresponding differential cross-sections.

Here also we assume that two out of the three neutral Higgs particles in the 2HDM model are very heavy or have very small CP-violating couplings, such that either way their effects decouple. Thus, only the couplings of the lightest neutral Higgs (denoted by $h$ ) are important and there will be only one dimensionless CP-odd quantity relevant for the study of CP violation in $q \bar{q}, g g \rightarrow t \bar{t}$. Using the notation in 196, 198, 214 in conjunction with our parameterization in Eq. 3.70, this quantity is

$$
\gamma_{C P} \equiv-2 a_{t}^{h} b_{t}^{h}
$$

where $a_{t}^{h}$ and $b_{t}^{h}$ are defined by the $t \bar{t} h$ (say $h=\mathcal{H}^{1}$ ) coupling in Eq. 3.70 and are functions of $\tan \beta$ - the ratio between the two VEV's in the Higgs potential and of the three Euler angles which parameterize the Higgs mixing matrix (for details see section 3.2.3).

Below we present two very interesting approaches of probing CP violation in $p p \rightarrow t \bar{t}+X$. The first is the Schmidt and Peskin (SP) approach [20], which utilizes the distribution of the leptonic decay products of the top. The second is the Bernreuther and Brandenburg (BB) approach [196, 198, 214], which studies the CP-violating effect in the resonant $\hat{s}$-channel Higgs shown in Fig. 47(h). 


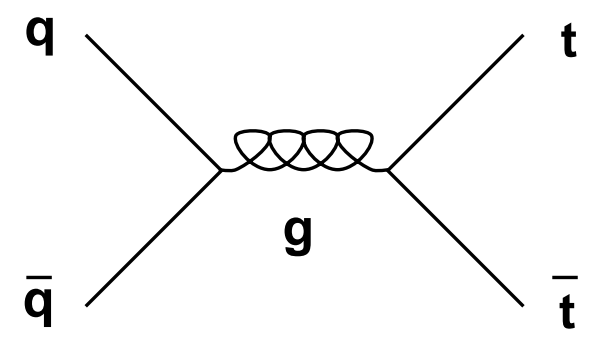

(a)

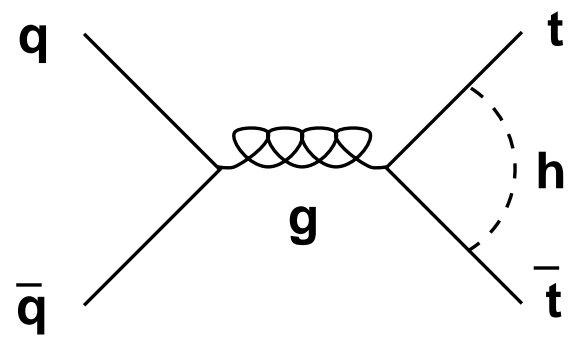

(b)

Figure 48: Born level QCD and relevant neutral Higgs exchange (denoted by the dashed line) Feynman diagrams for $q \bar{q} \rightarrow t \bar{t}$. 


\subsubsection{Schmidt-Peskin signal}

Schmidt-Peskin (SP) proposed [20] a signature for CP violation in production and decays of $t \bar{t}$ pairs for hadron colliders, namely via the reaction

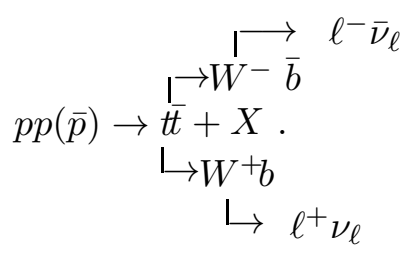

Despite the complexity of the reaction and the hadronic environment, the signal for CP violation that they suggest, i.e., the lepton energy asymmetry

$$
\alpha_{E}=\frac{\left\langle E_{\ell^{+}}\right\rangle-\left\langle E_{\ell^{-}}\right\rangle}{\left\langle E_{\ell^{+}}\right\rangle+\left\langle E_{\ell^{-}}\right\rangle} .
$$

is very simple and robust. Such an asymmetry can only arise from non-SM sources such as an extended Higgs sector or supersymmetry. The size of the asymmetry is unfortunately rather small $\sim 10^{-3}$.

Since this asymmetry is CP-odd and $T_{N}$-even, it requires an absorptive part to the Feynman amplitude. Such an absorptive part is already present (see Figs. 47(c),(g),(h) and 48(b)) as $t \bar{t}$ pair production requires the kinematic threshold

$$
\hat{s}>4 m_{t}^{2},
$$

where $\hat{s}$ is the square of the energy in the subprocess $q \bar{q}$ or $g g$ c.m. frame. In particular, when a neutral Higgs exchange leads to the CP-violating phase (as in their study), then the absorptive part due to the threshold condition in Eq. 7.16 arises even if $m_{h}^{2}>\hat{s}$.

Note again that for the subprocess $q \bar{q} \rightarrow t \bar{t}$, the underlying cause of CP violation in extended Higgs models is the CEDM of the top quark. Of course given the extremely short life time of the top quark $\left(\sim 10^{-24} \mathrm{sec}\right)$ the CEDM as such (i.e., at $\left.q^{2}=0\right)$ is extremely difficult to be seen. Consider, however, the asymmetry [20]:

$$
\Delta N_{L R}=\frac{\left[N\left(t_{L} \bar{t}_{L}\right)-N\left(t_{R} \bar{t}_{R}\right)\right]}{\text { all } t \bar{t}}
$$

where $N\left(t_{L} \bar{t}_{L}\right)$ is the number of $t_{L} \bar{t}_{L}$ pairs produced via $q \bar{q}(g g) \rightarrow t \bar{t}$, etc. \&8. Clearly $\Delta N_{L R}$ is CP-odd and $T_{N}$-even. The $q \bar{q}$ contribution to $\Delta N_{L R}$ arises from interference of Fig. 48(b) with the lowest order graph for $q \bar{q} \rightarrow t \bar{t}$ depicted in Fig. $48(\mathrm{a})$. They found

$$
\Delta N_{L R}=\frac{2 \beta}{3-\beta^{2}} \Re \mathrm{e}\left(F_{2 A}\right),
$$

where $\beta=\left(1-4 m_{t}^{2} / \hat{s}\right)^{1 / 2}$ and, in their notation, $F_{2 A}(\hat{s})$ is the CEDM form factor and $\Re$ e $\left(F_{2 A}\right)$ involves the absorptive part of the Feynman integral

$$
\begin{aligned}
\Re \mathrm{e}\left(F_{2 A}\right)= & \frac{1}{8 \pi}\left(\frac{m_{t}}{v}\right)^{2} \frac{4 m_{t}^{2}}{\hat{s} \beta} \gamma_{C P} \\
& \times\left[1-\frac{m_{h}^{2}}{\hat{s} \beta^{2}} \ln \left(1+\frac{\hat{s} \beta^{2}}{m_{h}^{2}}\right)\right] .
\end{aligned}
$$

Here $m_{h}$ is the mass of the lightest neutral Higgs and $\gamma_{C P}$ is defined in Eq. 7.13. It is easy to understand [20] the effect intuitively: for $\hat{s}>>m_{t}^{2}$, the gluon will predominantly couple to

\footnotetext{
${ }^{28}$ Note that in this notation, $\bar{t}_{L}$ means an anti-top quark with momentum, for instance, along $+z$ and spin along $-z$
} 
$t_{L} \bar{t}_{R}$ or $t_{R} \bar{t}_{L}$. However, when $\beta \rightarrow 0, t_{L} \bar{t}_{L}$ and $t_{R} \bar{t}_{R}$, which are related to each other via CP, are dominantly produced, which may thus lead to $\Delta N_{L R} \neq 0$. The resulting asymmetry at the parton level, $\Delta N_{L R}$, for the subprocess $q \bar{q} \rightarrow t \bar{t}$ for $m_{t}=175 \mathrm{GeV}, \gamma_{C P}=1 / \sqrt{2}$ and for different values of $m_{h}$ and $\sqrt{\hat{s}}$, is found to be of order $10^{-3}$.

For $g g \rightarrow t \bar{t}$ the calculation is more involved. In particular, in addition to the $\hat{t}$-channel $h$ exchange, now an $\hat{s}$-channel Higgs exchange graph is also present (see Fig. 47(h)). There is in fact constructive interference between these two channels for $m_{h}<2 m_{t}$. The result for the asymmetry in the $g g$ fusion case, but without the $\hat{s}$-channel Higgs exchange (see Fig. 48(b)), was also given in [20]. Near threshold, i.e., $\sqrt{\hat{s}} \gtrsim 2 m_{t}$, the asymmetry in the $g g$ fusion case is about twice as big as that of the $q \bar{q}$ fusion case. However, although larger than the $q \bar{q}$ fusion subprocess, it is again at the level of $10^{-3}$. Adding the $q \bar{q}$ and $g g$ subprocesses, then $\Delta N_{L R}$ can reach optimistically $\sim 10^{-2}$, for low values of $m_{h}$ and $\tan \beta$. In any case, the $g g$ initial state gives rise to a much richer possibility of $\mathrm{CP}$-violating operators and, as was mentioned before, the resulting asymmetry cannot be attributed merely to the CEDM of the top quark. Indeed, as noted in 196, 198, 214, the $\hat{s}$-channel neutral Higgs exchange that was ignored in [20], can give rise to larger asymmetries in $g g \rightarrow t \bar{t}$ and may be attainable at the LHC. We will return to this effect in the next section.

As has been emphasized at several places in this review, the fact that top decays are a powerful spin analyzer comes in extremely handy here too in leading to a detectable signature. The CP violation in the production process causes the polarization asymmetry above, which leads to an asymmetry in the energies of the charged leptons emerging from $t$ and $\bar{t}$ decays.

The distribution of the charged lepton in the $t$-rest frame is given by

$$
\frac{d^{2} \Gamma}{d E_{\ell} d \cos \psi}=\frac{d \Gamma}{d E_{\ell}} \frac{1+\cos \psi}{2},
$$

where $\psi$ is the angle between the top spin and the lepton momentum. When the top quark is boosted to the $q \bar{q}(g g)$ c.m. frame, Eq. 7.20 provides the correlation between the helicity of the top and the energy of the decay lepton. The resulting energy spectrum for $t_{L}\left(\bar{t}_{R}\right)$ and $t_{R}\left(\bar{t}_{L}\right)$ is significantly different from each other as was shown in [20]. Clearly, their findings indicate that the energy spectrum of the leptons, serves as a useful spin analyzer.

The asymmetry in $p p$ collision is calculated by folding in as usual the parton distributions. For this purpose SP used the parton density functions proposed in [215]. The effects of the longitudinal boost of the parton-parton collision are eliminated by considering the transverse energy $\left(E_{T}\right)$ of the leptons. The resulting asymmetry is [20]:

$$
\Delta N\left(E_{T}\right)=\frac{d \sigma / d E_{T, \ell^{+}}-d \sigma / d E_{T, \ell^{-}}}{d \sigma / d E_{T, \ell^{+}}+d \sigma / d E_{T, \ell^{-}}}
$$

and it was calculated in [20] for $m_{h}=100 \mathrm{GeV}, m_{t}=150 \mathrm{GeV}$ and $\gamma_{C P}=1 / \sqrt{2}$. Unfortunately, numerically it is again only of order $10^{-3}$.

Let us briefly discuss the background for these type of CP-violating asymmetries in $p p \rightarrow$ $t \bar{t}+X$. As was mentioned at the beginning of this chapter, the initial state $(p p)$ at hadron Supercolliders, such as the LHC, is not an eigenstate of CP. Consequently energy asymmetry in the decay lepton spectrum are not necessarily due to $\mathrm{CP}$ violation. The point is that the protons in the initial state produce more energetic quarks than anti-quarks. Also the reaction $q \bar{q} \rightarrow t \bar{t}$ has a small forward-backward asymmetry induced by $\alpha_{s}$ corrections. Thus the top quarks produced by this reaction tend to have a slightly higher energy than $\bar{t}$, leading to an asymmetry in the energy of the decay lepton. Such an effect, originating from higher order QCD corrections, causes an irreducible background.

Fortunately this background is very small. First of all, $q \bar{q}$ annihilation is subdominant at such $p p$ collider energies and the leading reaction $g g \rightarrow t \bar{t}$ is free from such a forward-backward asymmetry. Also, as mentioned before, the background to the asymmetry arises from higher 
order (QCD) radiative corrections. Furthermore, since the forward-backward asymmetry mainly affects longitudinal variables, its effect on the transverse energy asymmetry in Eq. 7.21 would cancel if there were no lepton acceptance cuts.

This background can be crudely estimated from the electromagnetic analog of the forwardbackward asymmetry for $e^{+} e^{-} \rightarrow \mu^{+} \mu^{-}$. The analogous asymmetry is crudely estimated by the replacement $\alpha \rightarrow\left[\left(d^{a b c}\right)^{2} / 32\right] \alpha_{s}=(5 / 12) \alpha_{s}$. SP in [20] used the approximate formula in [216] which allows them to get an estimate for massless $t \bar{t}$ pairs. This approximation tends to overestimate this background. For numerical estimates SP also impose a cutoff on the gluon energy of $\Delta E / E=0.3$. The resulting background was found to be of the order of $10^{-4}$. Therefore, it is much smaller than the desired CP-violating effect and also it is essentially independent of the lepton energy.

\subsection{2 $\hat{s}$-channel resonance Higgs effects - Bernreuther-Brandenberg approach}

For $m_{h}>2 m_{t}$, as noted before, there is an interesting $\hat{s}$-channel Higgs contribution to $g g \rightarrow t \bar{t}$ shown by diagram (h) in Fig. 47. This was explored in some detail in 1196, 198] who recently improved their analysis in [214]. For the simple on-shell decay $h \rightarrow t \bar{t}$, a large $t \bar{t}$ spin-spin correlation can be induced already at the tree-level if $h$ is not a CP eigenstate, as happens in a class of $2 \mathrm{HDM}$ 's. 29 In a $2 \mathrm{HDM}$, with the $t \bar{t} h$ coupling of Eq. $3.70 \mathrm{BO}$, this spin-spin correlation is given by 196:

$$
\left\langle\hat{k}_{t} \cdot\left(\vec{s}_{t} \times \vec{s}_{\bar{t}}\right)\right\rangle=\frac{\gamma_{C P} \beta_{t}}{\left(b_{t}^{h}\right)^{2}+\left(a_{t}^{h}\right)^{2} \beta_{t}^{2}},
$$

where $\beta_{t}=\left(1-4 m_{t}^{2} / m_{h}^{2}\right)^{1 / 2}, s_{t}, s_{\bar{t}}$ are the spin operators of $t$ and $\bar{t}$, respectively, $\hat{k}_{t}$ is the unit vector of the momentum of the top quark and $\gamma_{C P}$ is defined in Eq. 7.13. It is remarkable that this CP-violating spin-spin correlation can, in principle, be as large as 0.5. In practice, though, this decay has to be coupled to some particular production process and the final asymmetry can vary significantly between different processes. Moreover, for $p p$ collisions, there is an interference between the continuum and the resonant $t \bar{t}$ production which tends to diminish the spin-spin correlation. For the gluon-gluon fusion, the CP-violating expectation value of $\left\langle\hat{k} \cdot\left(\vec{s}_{t} \times \vec{s}_{\bar{t}}\right)\right\rangle$ was calculated in [198]. The resulting asymmetry was found to be at best only a few percent and falls significantly short compared to the value of 0.5 mentioned above. In fact, when this is translated to an asymmetry that utilizes the $t$ and $\bar{t}$ decay products, as was done in [196, 198], the signal to noise ratio for such an asymmetry was found to be at best $\sim 10^{-3}$.

The same non-vanishing spin-spin correlation of Eq. 7.22 can arise in $q \bar{q} \rightarrow t \bar{t}$. The asymmetry for the $q \bar{q}$ fusion subprocess was also calculated in [198] for the same set of parameters as in the $g g$ fusion case. As expected, in the case of $q \bar{q}$ fusion, the asymmetry is about one order of magnitude smaller than $g g$ fusion, since in this channel the resonant Higgs graph is absent. Furthermore, the asymmetry gets smaller with growing Higgs-boson masses as opposed to the $g g$ fusion case which we now discuss in some detail.

Let us now focus on an improved analysis of the results mentioned above. This was recently suggested by Bernreuther, Brandenburg and Flesch (BBF) in [214]. In their analysis the basic idea was to include new cuts on the $t \bar{t}$ invariant mass which significantly improved their previous results in 198 .

For the case when both $t$ and $\bar{t}$ decay leptonically, consider the CP-violating observables [214]:

$$
Q_{1}=\hat{k}_{t} \cdot \hat{q}_{+}-\hat{k}_{\bar{t}} \cdot \hat{q}_{-},
$$

\footnotetext{
${ }^{29} \mathrm{~A}$ more detailed analysis of the possible spin-spin correlations in $h \rightarrow t \bar{t}$ is given in section 6.2.3.

${ }^{30}$ recall that the lightest neutral Higgs is assumed to be $h=\mathcal{H}^{1}$, i.e., $k=1$ in Eq. 3.70
} 
and

$$
Q_{2}=\left(\hat{k}_{t}-\hat{k}_{\bar{t}}\right) \cdot\left(\hat{q}_{-} \times \hat{q}_{+}\right) / 2,
$$

where $\hat{k}_{t}, \hat{k}_{\bar{t}}$ are the $t, \bar{t}$ momentum directions in the parton c.m. system and $\hat{q}_{-}, \hat{q}_{+}$are the $\ell^{+}$, $\ell^{-}$momentum directions (from $t \rightarrow b \ell^{+} \nu_{\ell}$ and $\bar{t} \rightarrow \bar{b} \ell^{-} \bar{\nu}_{\ell}$ ) in the $t$ and $\bar{t}$ rest frames, respectively. Note that the decay channels to $\ell^{+}, \ell^{-}$(disregarding the $\tau$ lepton) may include all different combinations of $e$ and $\mu$ and, in 214, all possible combinations were summed over. It is useful to note that $Q_{2}$ in Eq. 7.24 is in fact a transcription of the spin-spin correlation $\hat{k}_{t} \cdot\left(\vec{s}_{t} \times \vec{s}_{\vec{t}}\right)$ defined in Eq. 7.22 above, and is a $T_{N}$-odd quantity. Also, $Q_{1}$ traces the spin-spin correlation $\hat{k}_{t} \cdot\left(\vec{s}_{t}-\vec{s}_{\bar{t}}\right)$ which corresponds to the CP asymmetry $\Delta N_{L R}$ defined in Eq. 7.17 and was suggested originally in [20]. Clearly it is $T_{N}$-even, thus requiring an absorptive phase.

For the leptonic-hadronic decay channel of the $t \bar{t}$ pairs, it is useful to consider the two possible decay scenarios: sample $\mathcal{A}$ in which the $t$ decays leptonically and $\bar{t}$ decays hadronically , and sample $\overline{\mathcal{A}}$ in which the $t$ decays hadronically and $\bar{t}$ decays leptonically (see Eqs. 6.79 and 6.80 , respectively, in section 6.2.3). One can then define the following CP-violating quantities with respect to samples $\mathcal{A}$ and $\overline{\mathcal{A}}$ [214]:

$$
\begin{aligned}
& \mathcal{E}_{1}=\left\langle\mathcal{O}_{1}\right\rangle_{\mathcal{A}}-\left\langle\overline{\mathcal{O}}_{1}\right\rangle_{\overline{\mathcal{A}}}, \\
& \mathcal{E}_{2}=\left\langle\mathcal{O}_{2}\right\rangle_{\mathcal{A}}+\left\langle\overline{\mathcal{O}}_{2}\right\rangle_{\overline{\mathcal{A}}},
\end{aligned}
$$

where

$$
\begin{array}{rll}
\mathcal{O}_{1} \equiv \hat{k}_{t} \cdot \hat{q}_{+} & , & \overline{\mathcal{O}}_{1} \equiv \hat{k}_{\bar{t}} \cdot \hat{q}_{-}, \\
\mathcal{O}_{2} \equiv \hat{k}_{t} \cdot\left(\hat{q}_{+} \times \hat{q}_{\bar{b}}\right) & , \quad & \overline{\mathcal{O}}_{2} \equiv \hat{k}_{\bar{t}} \cdot\left(\hat{q}_{-} \times \hat{q}_{b}\right) .
\end{array}
$$

Here $\hat{q}_{b}$ and $\hat{q}_{\bar{b}}$ denote the momentum direction of the $b$ and $\bar{b}$ jets in the $t$ and $\bar{t}$ rest frames, respectively. Again, $\mathcal{E}_{1}$ effectively corresponds to the spin-spin correlation $\hat{k}_{t} \cdot\left(\vec{s}_{t}-\vec{s}_{\bar{t}}\right)$ and is a $T_{N}$-even observable, and $\mathcal{E}_{2}$ traces the spin-spin correlation $\hat{k}_{t} \cdot\left(\vec{s}_{t} \times \vec{s}_{\bar{t}}\right)$ and is therefore a $T_{N \text {-odd }}$ quantity.

In [214] it was shown that, in the region $m_{h}>2 m_{t}$, the magnitude of the asymmetry increases significantly and the dominant contribution comes from the interference of the CP-violating terms in the amplitude of the neutral Higgs resonant diagram in Fig. 47(h) with the Born amplitude. They have evaluated the dependence of the differential expectation values of $Q_{1}$ and $Q_{2}$ on the $t \bar{t}$ invariant mass, $M_{t \bar{t}}$. An example of such a dependence for the $T_{N}$-odd observable $Q_{2}$ and in the dilepton decay channels of the $t \bar{t}$ pairs is shown in Fig. 49. In this figure the resonant contribution in $g g \rightarrow h \rightarrow t \bar{t}$ is compared with the resonant + the remaining $h$ contribution (i.e., all the non-resonant graphs), for different values of $m_{h}$, for $\sqrt{s}=14 \mathrm{TeV}$ and setting the $\mathrm{CP}$-violating quantity, $\gamma_{C P}$, from the $t \bar{t} h$ vertex to be equal to 1 . Also, since the CP-violating effect is sensitive to the neutral Higgs total width, it is therefore sensitive to the $Z Z h, W W h$ "reduced" coupling $c^{h}$ (defined in Eq. 3.71 for $h=\mathcal{H}^{1}$, i.e., $k=1$ ), which determines the decay rates $\Gamma(h \rightarrow Z Z)$ and $\Gamma(h \rightarrow W W)$ [214]. In Fig. 49, $c^{h}=0$ was chosen, in which case the above decay channels of a neutral Higgs to the massive gauge-bosons are forbidden at tree-level.

Clearly, looking at Fig. 49, the non-resonant contributions are negligible with respect to the $\hat{s}$-channel $h$ contribution which in turn gives rise to a CP asymmetry at the level of a percent when $M_{t \bar{t}}$ is in the vicinity of $m_{h}$. 

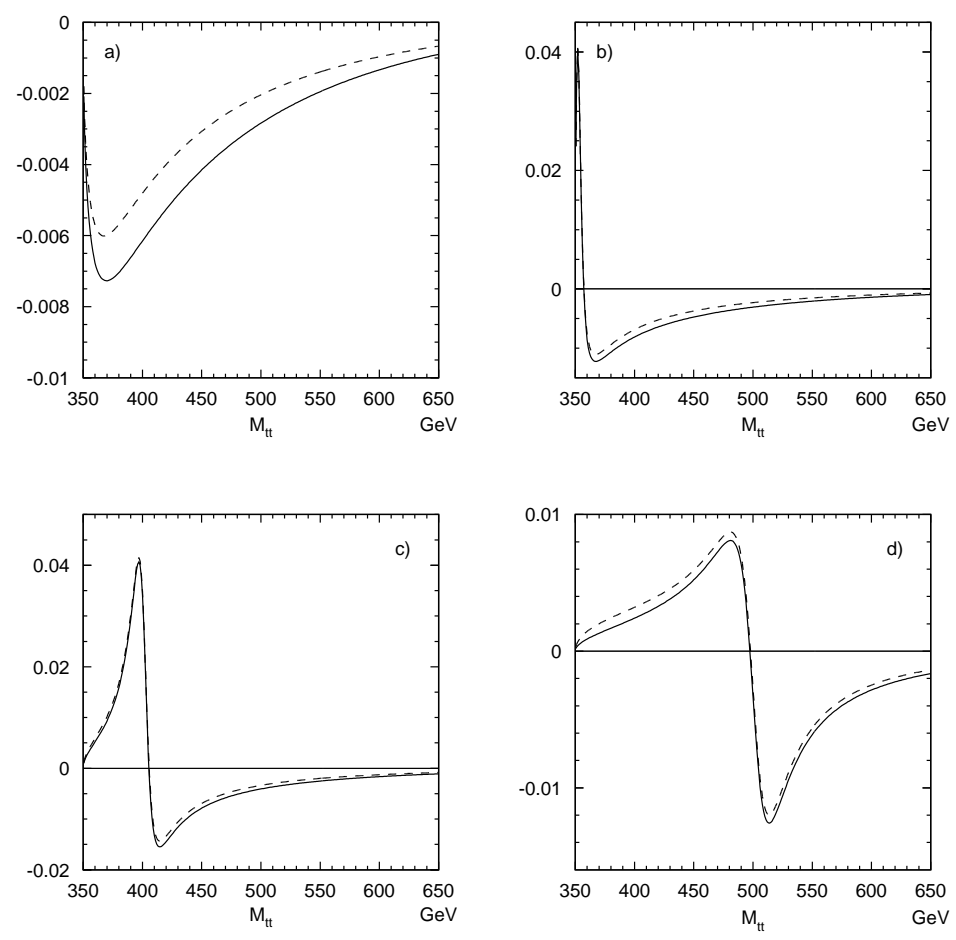

Figure 49: $d<Q_{2}>/ d M_{t t}$ as a function of $M_{t t}\left(Q_{2}\right.$ is defined in $E q$. 7.24) at $\sqrt{s}=14$ TeV, for reduced Yukawa couplings $c^{h}=0, a_{t}^{h}=1 / \sqrt{2}, b_{t}^{h}=-1 \sqrt{2}$, i.e., $\gamma_{C P}=1$ (see text), and Higgsboson masses: (a) $m_{h}=320 \mathrm{GeV}$, (b) $350 \mathrm{GeV}$, (c) $400 \mathrm{GeV}$, and (d) $500 \mathrm{GeV}$, in the dilepton channel. The dashed line represents the resonant and the solid line the sum of the resonant and non-resonant $h$ contributions. Figure taken from [214]. 
Table 13: The expectation value of $Q_{2}$ and its sensitivity at the $L H C$ with $\sqrt{s}=14$ TeV and an integrated luminosity of $100 \mathrm{fb}^{-1}$, for the dilepton $t \bar{t}$ decay channels. The $M_{t \bar{t}}$ intervals are chosen below $m_{h}$ such that: for $m_{h}=370,400,500 \mathrm{GeV},\left\langle Q_{2}\right\rangle$ was integrated over $\Delta M_{t \bar{t}}=15,40,80$ $\mathrm{GeV}$, in the $M_{t \bar{t}}$ ranges 355-370, 360-400 and 420-500 GeV, respectively. For each pair $\left(m_{h}\right.$, $\left.c^{h}\right)$ the first column is $\left\langle Q_{2}\right\rangle$ in percent and the second column is the sensitivity in standard deviations. The rows correspond, in descending order, to $\left(a_{t}^{h}, b_{t}^{h}\right)=(1,-1) / \sqrt{2},(1,-0.3) / \sqrt{2}$ and $(0.3,-0.3) / \sqrt{2}$, i.e., to $\gamma_{C P}=1,0.3$ and 0.09 , respectively. Numbers for $m_{h}$ are in GeV. The non-resonant $h$ contributions have been neglected for these values of $m_{h}$. Table taken from 214.

\begin{tabular}{|c|c|c|c|c|c|c|}
\hline \multirow{3}{*}{$m_{h}$} & \multicolumn{2}{|c|}{0.0} & \multicolumn{2}{c|}{0.2} & \multicolumn{2}{c|}{0.4} \\
\hline \multirow{5}{*}{370} & 4.4 & 29.8 & 4.1 & 27.4 & 3.3 & 20.9 \\
& 3.9 & 23.4 & 2.9 & 16.7 & 1.6 & 9.0 \\
& 1.2 & 6.6 & 0.75 & 4.1 & 0.39 & 2.1 \\
\hline \multirow{5}{*}{400} & 2.3 & 24.4 & 2.1 & 22.8 & 1.8 & 18.7 \\
& 1.3 & 13.4 & 1.1 & 11.1 & 0.75 & 7.5 \\
& 0.49 & 4.9 & 0.35 & 3.5 & 0.21 & 2.1 \\
\hline \multirow{5}{*}{500} & 0.65 & 8.6 & 0.59 & 7.9 & 0.46 & 6.0 \\
& 0.31 & 4.1 & 0.26 & 3.5 & 0.18 & 2.4 \\
& 0.14 & 1.9 & 0.10 & 1.4 & 0.06 & 0.77 \\
\hline
\end{tabular}


The sharp peaks observed in the range $M_{t \bar{t}} \sim m_{h}$ give an extra handle in an attempt to enhance the CP signal. Indeed, Figs. 49(b), (c) and (d) show that, in the case of $m_{h}>2 m_{t}$, $\left\langle Q_{2}\right\rangle$ changes sign as one goes from $M_{t \bar{t}} \lesssim m_{h}$ to $M_{t \bar{t}} \gtrsim m_{h}$, such that integrating over $M_{t \bar{t}}$ will diminish the CP-violating effect. Therefore, choosing appropriate $M_{t \bar{t}}$ mass bins below or above $m_{h}$, allows for a significant enhancement of the CP-odd signal. This is demonstrated in Table 13, where the three values $c^{h}=0,0.2$ and 0.4 were considered (see discussion above). Also, in Table 13, the left column gives the expectation value of $Q_{2}$ in percent and the right column shows the statistical significance in which this CP effect can be measured at the LHC with an integrated luminosity of $100 \mathrm{fb}^{-1}$. The $M_{t \bar{t}}$ intervals (i.e., mass bins) in Table 13 where chosen below $m_{h}$ (see caption of Table 13). Also the rows in Table 13 correspond in descending order to $\gamma_{C P}=1,0.3,0.09$.

The numbers for the statistical significance of the CP-violating signal that were found in 214] and are shown in Table 13 are quite remarkable. In most cases the CP-violating signal is well above the 3- $\sigma$ level, perhaps even a lot better than 5- $\sigma$ in the best case. As an example, note that with $m_{h}=370 \mathrm{GeV}$ and $\gamma_{C P}$ as low as 0.09 , the observable $Q_{2}$ can yield a $7-\sigma$ effect with an appropriately chosen interval for $M_{t \bar{t}}$. Recall that values as large as $\gamma_{C P} \sim 4$, corresponding to $\tan \beta \lesssim 0.5$, are still allowed by present experimental data (see section 3.2.3).

A few additional remarks are in order regarding the analysis in [214]:

1. The expected statistical significance for a CP-odd signal from the observable $\mathcal{E}_{2}$ in Eq. 7.26 corresponding to the leptonic-hadronic channel (i.e., lepton + jet from the $t \bar{t}$ pairs) was found to be comparable to that of $Q_{2}$ discussed above. Moreover, for the $T_{N}$-even observables $Q_{1}$ (see Eq. 7.23) and the corresponding one for the leptonic-hadronic channel $\mathcal{E}_{1}$ (see Eq. 7.25) the CP-violating signal, although somewhat smaller, may yield more than a $3-\sigma$ effect as long as $\gamma_{C P} \gtrsim 0.3$.

2. Apart from the cuts on the $M_{t \bar{t}}$ invariant mass, i.e., the chosen intervals/mass bins, Ref. 214] employed additional cuts on the rapidities of the $t$ and $\bar{t}$ and on the transverse momenta of the final state charged leptons and quarks in both the dilepton channel and leptonichadronic channel samples.

3. It is important to note that the $T_{N}$-odd observables $Q_{2}$ and $\mathcal{E}_{2}$ are insensitive to CP violation from the subsequent $t, \bar{t}$ decays to leading order in the CP-violating couplings. This is ensured by $C P T$ invariance [214]. Moreover, the $T_{N}$-even observables $Q_{1}$ and $\mathcal{E}_{1}$, although may acquire contributions from CP-violating absorptive parts in the $t, \bar{t}$ decays, at least in the $2 \mathrm{HDM}$ case, these absorptive parts are absent in the limit of vanishing $b$-quark mass (see also related discussion in section 5.1.2). Therefore, for the $2 \mathrm{HDM}$ case, both the $T_{N^{-}}$

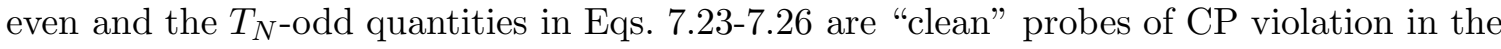
production mechanism of $t \bar{t}$ at the LHC.

Refs. [196, 198] considered possible contaminations to an asymmetry of the type $Q_{2}$ (or equivalently the spin-spin correlation in Eq. 7.22). Again, the key point is that the dominant $g g$ fusion subprocess is free from undesired CP-conserving background to $Q_{2}$. Therefore, background considerations are relevant only for the case of $q \bar{q} \rightarrow t \bar{t}$. Refs. [196, 198] estimated the CPconserving background to $Q_{2}$ to be of order $10^{-6}$ which is about 3 orders of magnitude smaller than the actual asymmetry. The reason for that is that $T_{N}$-odd observables such as $Q_{2}$ do not receive contributions from $\mathrm{CP}$-invariant interactions at the Born level but only from absorptive parts. Thus, in the case of $q \bar{q} \rightarrow t \bar{t}$, the main background comes from order $\alpha_{s}^{3}$ and $\alpha_{s}^{2} \alpha_{\text {weak }}$ absorptive parts [196, 198].

Finally, let us note that the optimization technique (with no additional cuts), i.e., the use of optimal observables, employed in [211] for CP violation in $g g \rightarrow t \bar{t}$, yield roughly the same results as those obtained in [214]. That is, optimal observables can be sensitive to values down to $\gamma_{C P} \sim 0.1$ with no cuts on the $t \bar{t}$ invariant mass. 


\subsection{SUSY and CP violation in $p p \rightarrow t \bar{t}+X$}

As we have discussed in previous sections, 1-loop exchanges of SUSY particles may give rise to CP-violating phenomena in top systems which are driven by SUSY CP-odd phases in the supersymmetric vertices. Such SUSY CP-violating 1-loop effects in $g g \rightarrow t \bar{t}$ were investigated by Schmidt [147]. In the case of $g g \rightarrow t \bar{t}$, only exchanges of gluinos and stops are relevant and are shown in Fig. 50. The only CP-odd phase arises then from the off diagonal elements of the $\tilde{t}_{L}-\tilde{t}_{R}$ mixing matrix. Writing again (see also sections 3.3 .2 and 4.5 ) the top squarks of different handedness in terms of their mass eigenstates, $\tilde{t}_{+}, \tilde{t}_{-}$, as

$$
\begin{aligned}
& \tilde{t}_{L}=\cos \theta_{t} \tilde{t}_{-}-e^{-i \beta_{t}} \sin \theta_{t} \tilde{t}_{+}, \\
& \tilde{t}_{R}=e^{i \beta_{t}} \sin \theta_{t} \tilde{t}_{-}+\cos \theta_{t} \tilde{t}_{+},
\end{aligned}
$$

the asymmetry will then be proportional to the quantity

$$
\xi_{C P}^{t}=\sin \left(2 \theta_{t}\right) \sin \left(\beta_{t}\right) .
$$

Schmidt neglected possible CP-nonconserving effects in the subdominant process $q \bar{q} \rightarrow t \bar{t}$ and considered the asymmetry $\Delta N_{\mathrm{LR}}$ defined in Eq. 7.17 only for $g g \rightarrow t \bar{t}$. As mentioned before, $\Delta N_{\mathrm{LR}}$ being CP-odd and $T_{N^{-}}$-even, requires both a CP-odd phase and an absorptive phase. Such absorptive phases are present in diagrams (a), (b) of Fig. 50 if the c.m. energy is large enough to produce on-shell gluino $(\tilde{g})$ pairs and in diagrams (c)-(e) in Fig. 50 if the c.m. energy of the colliding gluons is sufficient to produce on-shell top squark $(\tilde{t})$ pairs. Obviously, if SUSY particles have masses of $O(1 \mathrm{TeV})$, then this condition is satisfied at the LHC in which the c.m. energy of the colliding protons is $14 \mathrm{TeV}$. Thus, $\Delta N_{\mathrm{LR}}$ is a sum of the two contributions

$$
\Delta N_{\mathrm{LR}} \equiv \frac{\int d \cos \theta\left[A^{\tilde{g}}(\cos \theta)+A^{\tilde{t}}(\cos \theta)\right]}{(\text { all } t \bar{t})},
$$

where $\theta$ is the production angle of the top quark in the $g g$ c.m. frame and $A^{\tilde{g}}\left(A^{\tilde{t}}\right)$ is the contribution from on-shell gluino (stop) pairs. Schmidt found

$$
\begin{aligned}
A^{\tilde{g}}(\cos \theta) & =\frac{9 \pi \alpha_{s}^{2} \beta_{t} \delta}{64 \hat{s}} \sum_{\sigma=1}^{2}(-1)^{\sigma} \Theta\left(\sqrt{\hat{s}}-2 m_{\tilde{g}}\right) \\
& \times\left\{\frac{1}{1-\beta_{t} \cos \theta}\left[\frac{\beta_{t}}{\beta_{\tilde{g}}}\left(1-\beta_{\tilde{g}}^{2}\right) K_{0}^{-}-\left(1-\beta_{t}^{2}\right) K_{1}^{-}+\beta_{t} \beta_{\tilde{g}} \sin ^{2} \theta\left(K_{2}^{-}-K_{3}^{-}\right)\right]\right. \\
& +\frac{1}{1+\beta_{t} \cos \theta}\left[\frac{\beta_{t}}{\beta_{\tilde{g}}}\left(1-\beta_{\tilde{g}}^{2}\right) K_{0}^{+}-\left(1-\beta_{t}^{2}\right) K_{1}^{+}\right. \\
& \left.\left.+\beta_{t} \beta_{\tilde{g}} \sin ^{2} \theta\left(K_{2}^{+}-K_{3}^{+}\right)\right]\right\}
\end{aligned}
$$

and

$$
\begin{aligned}
A^{\tilde{t}}(\cos \theta) & =\frac{9 \pi \alpha_{s}^{2} \beta_{t} \delta}{64 \hat{s}} \sum_{\sigma=1}^{2}(-1)^{\sigma} \Theta\left(\sqrt{\hat{s}}-2 m_{\sigma}\right) \\
& \times\left\{\left(\frac{1 / 81}{1-\beta_{t} \cos \theta}+\frac{10 / 81}{1+\beta_{t} \cos \theta}\right)\left[\frac{\beta_{t}}{\beta_{\sigma}}\left(1-\beta_{\sigma}^{2}\right) \bar{K}_{0}^{-}-\beta_{t} \beta_{\sigma} \sin ^{2} \theta \bar{K}_{3}^{-}\right]\right. \\
& +\left(\frac{1 / 81}{1+\beta_{t} \cos \theta}+\frac{10 / 81}{1-\beta_{t} \cos \theta}\right)\left[\frac{\beta_{t}}{\beta_{\sigma}}\left(1-\beta_{\sigma}^{2}\right) \bar{K}_{0}^{+}\right. \\
& \left.\left.-\beta_{t} \beta_{\sigma} \sin ^{2} \theta \bar{K}_{3}^{+}\right]\right\},
\end{aligned}
$$


where $\beta_{i}=\left(1-4 m_{i}^{2} / \hat{s}\right)^{1 / 2}$ and the index $\sigma$ refers to the two mass eigenstates of the stop. Also, here

$$
\delta \equiv \alpha_{s} \frac{m_{\tilde{g}} m_{t}}{\hat{s} \beta_{t}} \xi_{C P}^{t}
$$

and the form factors $K_{i}^{ \pm}(i=0-3)$ are given in 147.

The asymmetry $\Delta N_{\mathrm{LR}}$ was calculated for several values of $m_{\tilde{t}_{-}}, m_{\tilde{t}_{+}}$(the masses of the two stop eigenstates) and $m_{\tilde{g}}$, and for $m_{t}=150 \mathrm{GeV}, \xi_{C P}^{t}=-1$ (i.e., its maximal negative value). In general, the asymmetry was found to be dominated by the amplitudes which contain the intermediate gluinos (Fig. 50(a) and 50(b)) even if the intermediate stops in Fig. 50(c)-(e) can go on their mass shell. For example, with $m_{\tilde{g}}=210 \mathrm{GeV}, m_{\tilde{t}_{-}}=100 \mathrm{GeV}$ and $m_{\tilde{t}_{+}}=500 \mathrm{GeV}$, Schmidt found that the asymmetry at the parton (i.e. gluons) level can reach $\sim 2 \%$ if the c.m. energy of the colliding gluons is around $450 \mathrm{GeV}$. Note that, in this c.m. energy, and with the above stops masses, $\Delta N_{\mathrm{LR}}$ receives contributions only from diagrams (a) and (b) in Fig. 50 since there is no absorptive cut along the stops lines in diagrams (c)-(e) in Fig. 50. These results for $\Delta N_{\mathrm{LR}}$ are about an order of magnitude larger than what was found in the 2HDM case [20] and it is roughly comparable to the $\hat{s}$-channel Higgs resonant effect [196, 198, 214.

However, in a more realistic study, one will have to integrate over the structure functions of the incoming gluons and present asymmetries in the overall reaction $p p \rightarrow t \bar{t}+X$. Unfortunately, folding in the gluons structure functions, the CP-violating asymmetry drops again to the level of $10^{-3}$. For this purpose Schmidt again considered the asymmetry in the transverse energy of the leptons defined in Eq. 7.21. He [147] found that, similar to the 2HDM case when no additional cuts are made (like the ones suggested in [214] and were described in the previous section), at the LHC with $\sqrt{s}=14 \mathrm{TeV}$ and with typical SUSY masses of a few hundreds GeV, the asymmetry $\Delta N\left(E_{T}\right)$ of Eq. 7.21 is again typically of the order of $\sim$ few $\times 10^{-3}$. Schmidt also examined the non-CP-violating background, and again found it to be negligible compared to the CP-violating effect.

Clearly, the Schmidt SUSY CP-violating effect in $g g \rightarrow t \bar{t}$ as it stands, is smaller than that of Ref. 214, i.e. the signal caused by the resonant Higgs contribution. This is mainly due to the appreciable improvement that can be achieved with appropriate cuts on the $t \bar{t}$ invariant mass, as was discussed in the previous section. Note however, that by using optimal observables for extracting information on $\mathrm{CP}$ violation in $g g \rightarrow t \bar{t}$, it was shown in [211] that the signal to noise ratio for the CP-violating effect in $g g \rightarrow t \bar{t}$ (driven by the diagrams in Fig. 50 that were considered by Schmidt) can reach the percent level after folding in the gluons structure functions. This allows a 1- $\sigma$ detection of the CP-odd effect even for smaller values of $\xi_{C P}^{t} \sim 0.1$. 


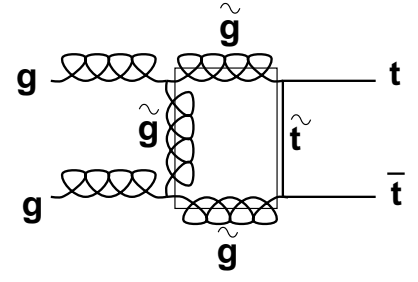

(a)

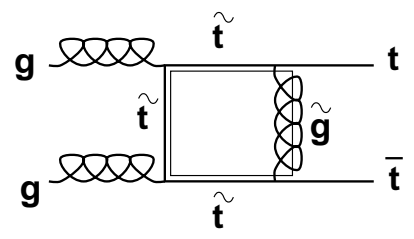

(c)

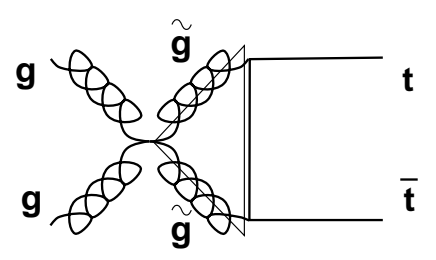

(b)

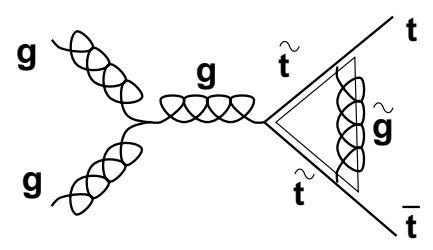

(d)

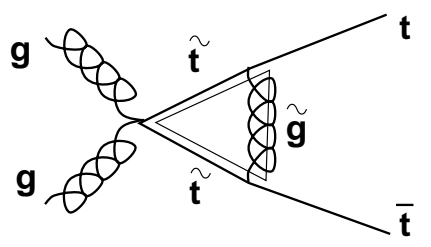

(e)

Figure 50: 1-loop Feynman diagrams contributing to CP violation in $g g \rightarrow t \bar{t}$ in supersymmetry. Diagrams with crossed gluons are not shown. 


\section{$8 \mathrm{CP}$ violation in $p \bar{p}$ collider experiments}

Shortly after the demise of the SSC, it was suggested to upgrade the energy of the Tevatron. More recently, substantial, two stage, upgrades in luminosity without a factor of two or so increase in energy remain as viable options. For a review see [217]. In the previous run at the Tevatron, at c.m. energy of $1.8 \mathrm{TeV}$, the $\mathrm{D} \emptyset$ and $\mathrm{CDF}$ experiments accumulate more than $0.2 \mathrm{fb}^{-1}$ of integrated luminosity. In the first upgrade, called Run II, $\mathcal{L}$ will be increased from its current peak value of $2 \times 10^{31} \mathrm{~cm}^{-2} \mathrm{sec}^{-1}$ to $10^{32} \mathrm{~cm}^{-2} \mathrm{sec}^{-1}$ (or even to twice this value). In the second stage, so called Run III (or TeV-33), the luminosity will be further increased to $\mathcal{L} \approx 10^{33} \mathrm{~cm}^{-2} \mathrm{sec}^{-1}$. The working hypotheses are that in Runs II and III the integrated luminosity will be $2 \mathrm{fb}^{-1}$ and 30 $\mathrm{fb}^{-1}$, respectively, with a modest increase of c.m. energy to $2 \mathrm{TeV}$. In addition, the $\mathrm{D} \emptyset$ and CDF detectors are also being upgraded.

\section{$8.1 p \bar{p} \rightarrow t \bar{t}+X$}

Contrary to the LHC $p p$ collider, where $t \bar{t}$ pairs are produced predominantly through the $g g$ fusion process $g g \rightarrow g \rightarrow t \bar{t}$, in the Tevatron $p \bar{p}$ collider with c.m. energy of $\sqrt{s} \simeq 2 \mathrm{TeV}$ the main production mechanism of $t \bar{t}$ pairs is the $q \bar{q}$ fusion, $q \bar{q} \rightarrow g \rightarrow t \bar{t}$. In particular, the $q \bar{q}$ fusion process is responsible for about $90 \%$ of the cross-section $p \bar{p} \rightarrow t \bar{t}+X$. Being so, the processes $p \bar{p} \rightarrow t \bar{t}, t \bar{t} g+X$, where $g$ stands for an extra gluon jet in $t \bar{t}$ production, will presumably be sensitive to the CEDM and CMDM of the top quark, which can be incorporated as effective interactions at the $t t g$ vertex. As already mentioned in previous chapters, being a $\mathrm{CP}$-odd quantity, a non-vanishing CEDM coupling might give rise to observable $\mathrm{CP}$ violation in top systems in such a hadron collider. If so, this will be a clue for new physics, as in the SM the CP-nonconserving effects in the reactions $p \bar{p} \rightarrow t \bar{t}, t \bar{t} g+X$ are extremely small.

In principle, CP-nonconserving effects due to the CEDM can be searched for through a study of either $\mathrm{CP}$-even or $\mathrm{CP}$-odd correlations in the reactions $p \bar{p} \rightarrow t \bar{t}, t \bar{t} g+X$. Of course, CP-odd correlations are expected to be more sensitive to the CEDM than CP-even observables as the former are linear in CEDM whereas the latter are proportional to the square of the CEDM form factor.

We will first present a study of the sensitivity of some CP-even observables to the CEDM, and then describe some interesting CP-odd correlations that may be applied to $p \bar{p} \rightarrow t \bar{t}+X$.

\subsubsection{CP-even observables in $p \bar{p} \rightarrow t \bar{t}+X$ and $p \bar{p} \rightarrow t \bar{t}+j e t+X$}

The effective Lagrangian for the interaction between the top quark and a gluon, that includes the CEDM and CMDM form factors of the top, is given in Eq. 6.106 (for $V=g$ ). Recall that the effective interaction at the ttgg vertex, absent in the SM, is also required to ensure gauge invariance (see discussion in section 2.5).

The CMDM and CEDM dimensionless form factors $\kappa_{g}$ and $\tilde{\kappa}_{g}$, which are defined in Eq. 6.106, can develop an imaginary part.\$1 However, these imaginary parts vanish at zero momentum transfer, and are only present if an on-shell intermediate state exists. Using form factors as a probe for new physics is most useful when the novel states can only be produced virtually and so here we consider the case where $\kappa_{g}$ and $\tilde{\kappa}_{g}$ are purely real.

As mentioned above, the process $p \bar{p} \rightarrow t \bar{t}+X$ can proceed at the parton level via: (a) $q \bar{q} \rightarrow t \bar{t}$, or (b) $g g \rightarrow t \bar{t}$. With the effective interactions in Eq. 6.106 and the additional ggtt effective vertex, the Feynman diagrams contributing to these two processes are shown in Fig. 51. The parton level cross-sections for $p \bar{p} \rightarrow t \bar{t}+X$ are then given by [22, 218]:

\footnotetext{
${ }^{31}$ we will loosely refer here to $\kappa_{g}$ and $\tilde{\kappa}_{g}$ as the CMDM and CEDM form factors, respectively. They are related to the dimensionful CMDM and CEDM via Eqs. 6.107 and 6.108 .
} 


$$
\begin{aligned}
\frac{d \hat{\sigma}_{q \bar{q} \rightarrow t \bar{t}}}{d \hat{t}} & =\frac{8 \pi \alpha_{s}^{2}}{9 \hat{s}^{2}}\left[\frac{1}{2}-v+z-\kappa_{g}+\frac{1}{4}\left(\kappa_{g}^{2}-\tilde{\kappa}_{g}^{2}\right)+\frac{v}{4 z}\left(\kappa_{g}^{2}+\tilde{\kappa}_{g}^{2}\right)\right], \\
\frac{d \hat{\sigma}_{g g \rightarrow t \bar{t}}}{d \hat{t}} & =\frac{\pi \alpha_{s}^{2}}{12 \hat{s}^{2}}\left[\left(\frac{4}{v}-9\right)\left(\frac{1}{2}-v+2 z\left(1-\frac{z}{v}\right)-\kappa_{g}\left(1-\frac{\kappa_{g}}{2}\right)\right)\right. \\
& +\frac{1}{4}\left(\kappa_{g}^{2}+\tilde{\kappa}_{g}^{2}\right)\left(\frac{7}{z}\left(1-\kappa_{g}\right)+\frac{1}{2 v}\left(1+\frac{5 \kappa_{g}}{2}\right)\right) \\
& \left.+\frac{1}{16}\left(\kappa_{g}^{2}+\tilde{\kappa}_{g}^{2}\right)^{2}\left(-\frac{1}{z}+\frac{1}{v}+\frac{4 v}{z^{2}}\right)\right],
\end{aligned}
$$

where

$$
z=\frac{m_{t}^{2}}{\hat{s}}, \quad v=\frac{\left(\hat{t}-m_{t}^{2}\right)\left(\hat{u}-m_{t}^{2}\right)}{\hat{s}^{2}} .
$$

The process $p \bar{p} \rightarrow t \bar{t} j+X$, where $j$ stands for a jet, can proceed via the following parton level subprocesses: (a) $q \bar{q} \rightarrow t \bar{t} g$, (b) $g g \rightarrow t \bar{t} g$, or (c) $q(\bar{q}) g \rightarrow t \bar{t} q(\bar{q})$ where an extra light quark jet is radiated. The number of diagrams for these subprocesses is large. A detailed description of the calculation of the cross-section for the reaction $p \bar{p} \rightarrow t \bar{t} j+X$ is given in [218].

A plot of the dependence of the total cross-section for $t \bar{t}$ production on $\kappa_{g}$ and $\tilde{\kappa}_{g}$ was given in [218] and is shown in Fig. 52. Of course, as expected, the cross-section is symmetric about $\tilde{\kappa}_{g}=0$ as only $\tilde{\kappa}_{g}^{2}$ appears in the cross-section, being a CP-even observable. The SM point is given by $\kappa_{g}=\tilde{\kappa}_{g}=0$ and the SM cross-section at this point is $\sigma(t \bar{t}) \sim 5 \mathrm{pb}$. Therefore, a measurement of $\sigma(t \bar{t})>5 \mathrm{pb}$ will indicate the existence of a non-zero $\kappa_{g}$ or $\tilde{\kappa}_{g}$.

In 219] an "anomalous cross-section" was defined as

$$
\Delta \sigma \equiv \sigma\left(\kappa_{g}, \tilde{\kappa}_{g}\right)-\sigma(0,0)
$$

and a plot of $\Delta \sigma$ in the $\left(\kappa_{g}, \tilde{\kappa}_{g}\right)$ plane was given. From the current experimentally allowed region of $\Delta \sigma$, not surprisingly, they found that a rather large CEDM coupling is permitted. For example, for certain values of $\kappa_{g}$, they found that $0.5 \lesssim\left|\tilde{\kappa}_{g}\right| \lesssim 1.5$ is allowed, within $1 \sigma$. This corresponds to $2.8 \times 10^{-17} g_{s}-\mathrm{cm} \lesssim\left|\Re \mathrm{e} d_{t}^{g}\right| \lesssim 8.3 \times 10^{-17} g_{s}-\mathrm{cm}$, at $1-\sigma$.

Considering again an associated extra jet, $j$, in top pair production at the $2 \mathrm{TeV}$ Tevatron, it was shown in [218] that not much will be gained from a simple cross-section analysis of the $t \bar{t} j$ final state compared to the $t \bar{t}$ final state. However, a more interesting CP-conserving quantity was further suggested in [218]; it was shown that the ratio of the two cross-sections, $\mathcal{R}=\sigma(t \bar{t} j) / \sigma(t \bar{t})$, can be used to further constrain the CEDM and CMDM couplings. Fig. 53(a), (b) and (c) show the main behavior of the ratio $\mathcal{R}$ in the $\left(\kappa_{g}, \tilde{\kappa}_{g}\right)$ plane for $p_{T}(j)>5,10,20 \mathrm{GeV}$, respectively, where $p_{T}(j)$ is the transverse momentum of the jet $j$. The most interesting feature is the one depicted in Fig. 53(a); we see that for the cut $p_{T}(j)>5 \mathrm{GeV}$, at some regions in the $\left(\kappa_{g}, \tilde{\kappa}_{g}\right)$ plane, $\mathcal{R}>1$. This happens as a consequence of the new $t t g$ and ttgg vertices (absent in the SM) in $t t+$ jet production.

A discussion on the possible limits that can be put on the CEDM and CMDM of the top by analyzing CP-even observables in single top production at the Tevatron was presented in [220]. There, single top production can occur either via the $q \bar{q} \rightarrow t \bar{b}$ or the $W$-gluon fusion subprocess $W g \rightarrow t \bar{b}$. An analysis of the cross-section for the reaction $W g \rightarrow t \bar{b}$ was performed [220], trying to further constrain the CEDM and CMDM of the top quark which may enter the ttg vertex present in this reaction. However, it was found that, for both the Tevatron and the LHC, the sensitivity of the $W$-gluon fusion subprocess leading to a single top quark for the CEDM and CMDM couplings is more than an order of magnitude smaller than the usual top pair production channel. 


\subsubsection{CP-odd observables in $p \bar{p} \rightarrow t \bar{t}+X$}

As already mentioned before, if all non-standard effects reside in the $t \bar{t} g$ coupling, ${ }^{\mathbf{B}}$ then they can be parameterized by the effective $t t g$ and $t t g g$ interaction Lagrangian. However, upon neglecting the $g g$ fusion process (which is a good approximation under the conditions of the Tevatron upgrade with c.m. energy of $\sqrt{s}=2 \mathrm{TeV}$ ), the ttgg contact term plays no role and the only relevant effective interaction for the reaction $q \bar{q} \rightarrow t \bar{t}$ is the effective $t t g$ CEDM interaction in Eq. 6.106. Similarly, the $t b W$ vertex (with an on-shell $W$-boson) may give rise to additional nonstandard couplings as in Eqs. 6.5 and 6.6, which may cause CP violation in the top and anti-top decays. Therefore, in the overall $t \bar{t}$ production and decays, $\mathrm{CP}$ violation is parameterized by non-zero values of the CEDM, $d_{t}^{g}$, in the production vertex, and/or by the quantities $f_{2}^{L, R}-\bar{f}_{2}^{R, L}$ (defined in Eqs. 6.5 and 6.6) emanating from the $t, \bar{t}$ decays.

In order to detect such $\mathrm{CP}$-violating couplings one has to construct appropriate $\mathrm{CP}$-odd observables. Following [221], let us again consider two decay scenarios for $t$ and $\bar{t}$. In the first one, both $t$ and $\bar{t}$ decay leptonically

$$
p \bar{p} \rightarrow t \bar{t} \rightarrow \ell^{+} \ell^{-} X,
$$

while in the second scenario only one of the $t$ or $\bar{t}$ decays leptonically (see also Eqs. 6.79 and 6.80 in section 6.2.3

$$
p \bar{p} \rightarrow t \bar{t} \rightarrow \ell^{+} X \quad, \quad p \bar{p} \rightarrow t \bar{t} \rightarrow \ell^{-} X .
$$

The processes in Eq. 8.6 have better statistics than the one in Eq. 8.5 and give the best signature for the top quark identification. Within these decay scenarios two possible CP-odd observables were considered in [221] which we will describe below.

\subsubsection{Transverse energy asymmetry of charged leptons}

The transverse energy asymmetry of the charged leptons was originally suggested by Peskin and Schmidt in 20] for $t \bar{t}$ production in a $p p$ collider (see Eq. 7.21) and was discussed in detail in sections 7.3 and 7.4. Recall that in the case where both $t$ and $\bar{t}$ decay leptonically it can be defined as (using here the notation in [221])

$$
A_{T}=\frac{\sigma\left(E_{T}^{-}>E_{T}^{+}\right)-\sigma\left(E_{T}^{+}>E_{T}^{-}\right)}{\sigma\left(E_{T}^{-}>E_{T}^{+}\right)+\sigma\left(E_{T}^{+}>E_{T}^{-}\right)} .
$$

The result for the expected CP-violating asymmetry in the transverse energy of the muons $\left(A_{T}^{\mu}\right)$ was given in [221]. They considered $A_{T}^{\mu}$ as a function of the imaginary part of the top CEDM, $\Im \mathrm{m} d_{t}^{g}$ (recall that $A_{T}$ is $T_{N}$-even thus requiring an absorptive phase), and of $\Re \mathrm{e}\left(f_{2}^{R}-\bar{f}_{2}^{L}\right)$. Also, the usual CDF cuts were applied. They found that CP violation from the production mechanism, i.e., $\propto \Im \mathrm{m} d_{t}^{g}$, is larger then that arising from the decay process, i.e., $\propto \Re \mathrm{e}\left(f_{2}^{R}-\bar{f}_{2}^{L}\right)$. For example, they found that with $\Im \mathrm{m} d_{t}^{g} \sim 10^{-17} g_{s}$-cm and $\Re \mathrm{e}\left(f_{2}^{R}-\bar{f}_{2}^{L}\right) \sim 0.2$ one can obtain an asymmetry around the $\sim 10 \%$ level.

In order to understand the feasibility of extracting such values for the CP-violating couplings in production and decays of the $t \bar{t}$, it is useful to decompose $A_{T}^{\mu}$ as follows [221]:

$$
A_{T}^{\mu} \equiv c_{P} A_{P}+c_{D} A_{D}
$$

where the dimensionless couplings $c_{P}$ and $c_{D}$ are

$$
c_{P} \equiv \frac{m_{t}}{g_{s}} \Im \mathrm{m} d_{t}^{g} \quad, \quad c_{D} \equiv \frac{1}{2} \Re \mathrm{e}\left(f_{2}^{R}-\bar{f}_{2}^{L}\right) .
$$

\footnotetext{
${ }^{32}$ Recall that this is not necessarily true in model calculations, e.g., in the MSSM - see discussion in section 7.4
} 
Then, in terms of $c_{P}$ and $c_{D}$, the statistical significance for $A_{T}^{\mu}$ determination is given by

$$
N_{S D}^{T} \equiv\left|c_{P} A_{P}+c_{D} A_{D}\right| \sqrt{N_{\ell \ell}},
$$

where $N_{\ell \ell}$ is the number of dilepton events expected to be $\sim 80$ and $\sim 1200$ at an integrated luminosity $L=2$ and $30 \mathrm{fb}^{-1}$, respectively [222]. $A_{P}$ and $A_{D}$ can be calculated and, thus, it was found in 221] that a $3-\sigma$ effect will require the following relations to be satisfied

$$
\begin{aligned}
& \left|2.5 c_{P}+0.9 c_{D}\right| \geq 1 \quad \text { for } \quad L=2 \mathrm{fb}^{-1} \\
& \left|9.8 c_{P}+3.3 c_{D}\right| \geq 1 \text { for } \quad L=30 \mathrm{fb}^{-1}
\end{aligned}
$$

Clearly, $A_{T}^{\mu}$ is more sensitive to CP violation in the production mechanism than in the $t \bar{t}$ decays. So, for example, $L=30 \mathrm{fb}^{-1}$ allows for an observation of $c_{P}=c_{D}=0.08$ at $\sqrt{s}=2 \mathrm{TeV}$. Note that $c_{P}=0.08$ corresponds to $\Im m d_{t}^{g}=8.8 \times 10^{-18}$ which, again, is more than an order of magnitude larger then what is expected for the CEDM in beyond the SM scenarios such as MHDM's and SUSY (see Chapter 团). Similarly, the resulting 3- $\sigma$ limit $\Re \mathrm{e}\left(f_{2}^{R}-\bar{f}_{2}^{L}\right)=0.16$ (corresponding to $c_{D}=0.08$ ) falls short by about one order of magnitude from model predictions for this quantity (see discussion in Chapter 司).

A related asymmetry which can be used in the case when only one top decays leptonically was also suggested in 221]:

$$
A_{\text {cut }}^{\mu}\left(E_{\text {Tcut }}\right)=\frac{\sigma^{-}\left(E_{T}^{-}>E_{T c u t}\right)-\sigma^{+}\left(E_{T}^{+}>E_{T c u t}\right)}{\sigma} .
$$

The $\sigma$ in Eq. 8.13 denotes the integrated cross-section with no cuts except for the standard experimental cuts. We note that, with the CP-violating coupling $c_{P}$ and $c_{D}$ of the order of 0.1 , which tends to be somewhat optimistic, this asymmetry in Eq. 8.13 can also reach the $\sim 10 \%$ level.

\subsubsection{Optimal observables}

It is useful to be able to experimentally separate $\mathrm{CP}$ violation in the production from that in the decay. The optimization method outlined in section 2.6 can provide for such a detection and was also used in [221].

Consider the transverse lepton energy spectrum in the single leptonic (say $\ell^{+}$) and the dileptonic final states

$$
\begin{gathered}
\frac{1}{\sigma} \frac{d \sigma}{d E_{T}^{+}}=f_{1}^{+}\left(E_{T}^{+}\right)+c_{P} f_{P}^{+}\left(E_{T}^{+}\right)+c_{D} f_{D}^{+}\left(E_{T}^{+}\right), \\
\frac{1}{\sigma} \frac{d^{2} \sigma}{d E_{T}^{+} d E_{T}^{-}}=f_{1}^{ \pm}\left(E_{T}^{+}, E_{T}^{-}\right)+c_{P} f_{P}^{ \pm}\left(E_{T}^{+}, E_{T}^{-}\right)+c_{D} f_{D}^{ \pm}\left(E_{T}^{+}, E_{T}^{-}\right),
\end{gathered}
$$

where in Eqs. 8.14 and $8.15 \sigma$ denotes the cross-section for the process $p \bar{p} \rightarrow \ell^{+}+$jets and $p \bar{p} \rightarrow \ell^{+} \ell^{-}+$jets, respectively. Also, $c_{P}, c_{D}$ are defined in Eq. 8.9 and $f^{ \pm}$are known functions of $E_{T}^{+}$and $E_{T}^{-}$.

As can be seen from Eqs. 8.14 and 8.15, the transverse lepton energy spectrums, in both the single and double-leptonic channels, are sensitive to $c_{P}$ and $c_{D}$. Using the above transverse lepton energy spectrum, the optimal weighting functions can be obtained. This was done in [221] where in both cases the statistical significances for the experimental determination of $c_{P}$ and $c_{D}$, i.e., $N_{S D}^{P, D} \equiv\left|c_{P, D}\right| / \Delta c_{P, D}$, were calculated. For the single leptonic events they obtained

$$
N_{S D}^{P}=\frac{\left|c_{P}\right|}{2.37} \sqrt{N_{\ell}}, \quad N_{S D}^{D}=\frac{\left|c_{D}\right|}{18.43} \sqrt{N_{\ell}}
$$




\begin{tabular}{||c|c|c||}
\hline \hline$L\left[\mathrm{fb}^{-1}\right]$ & 2 & 30 \\
\hline$\left|c_{P}\right|$ & 0.20 & 0.05 \\
\hline$\left|c_{D}\right|$ & 1.50 & 0.40 \\
\hline \hline
\end{tabular}

Table 14: The minimal values of $c_{P}$ and $c_{D}$ necessary to observe CP violation in the single-lepton mode at the 3- $\sigma$ level for $L=2,30 \mathrm{fb}^{-1}$. As a reference value, recall that $c_{P}=1$ corresponds to $\Im \mathrm{m} d_{t}^{g}=1.1 \times 10^{-16} \mathrm{~g}_{\mathrm{s}} \mathrm{cm}$. Table taken from 221.

\begin{tabular}{||c|c|c||}
\hline \hline$L\left[\mathrm{fb}^{-1}\right]$ & 2 & 30 \\
\hline$\left|c_{P}\right|$ & 0.39 & 0.10 \\
\hline$\left|c_{D}\right|$ & 1.93 & 0.50 \\
\hline \hline
\end{tabular}

Table 15: The minimal values of $c_{P}$ and $c_{D}$ necessary to observe $C P$ violation in the dilepton mode at the 3- $\sigma$ level for $L=2,30 \mathrm{fb}^{-1}$. See also caption to Table 14. Table taken from 221].

and for the dileptonic events

$$
N_{S D}^{P}=\frac{\left|c_{P}\right|}{1.17} \sqrt{N_{\ell \ell}}, \quad N_{S D}^{D}=\frac{\left|c_{D}\right|}{5.76} \sqrt{N_{\ell \ell}},
$$

where $N_{\ell}$ and $N_{\ell \ell}$ are the expected number of single and double-leptonic events, respectively. $N_{\ell} \sim 1300(20,000)$ and $N_{\ell \ell} \sim 80(1200)$ for $L=2(30) \mathrm{fb}^{-1}$, respectively [222]. The minimal values of $c_{P}$ and $c_{D}$ necessary to observe a $3-\sigma \mathrm{CP}$-violating effect with the optimization technique are listed in Tables 14 and 15 for the single and double-leptonic channels, respectively. We see that the single-leptonic modes are more sensitive to the non-standard couplings. Thus, for example, the Tevatron upgrade Run III with $L=30 \mathrm{fb}-1$ will be able to probe $\Im m d_{t}^{g}$ down to values of $\sim 5 \times 10^{-18} \mathrm{~g}_{\mathrm{s}}$-cm in the single-leptonic channel. Note that, as expected, this result is somewhat better than what can be achieved with "naive" observables such as $A_{\text {cut }}^{\mu}$ in Eq. 8.13 .

We note in passing that comparable limits for $\Im \mathrm{m} d_{t}^{g}$ but also for $\Re$ e $d_{t}^{g}$, i.e., $\Im \mathrm{m}, \Re \mathrm{e} d_{t}^{g} \sim$ few $\times 10^{-18} g_{s}$-cm, were found also in [211] using optimal observables for the reaction $p \bar{p} \rightarrow t \bar{t}+X$ and with the Tevatron upgrade parameters.

To summarize, as was noted in [221], it is not inconceivable that non-standard CP-violating couplings of the top quark to a gluon may be discovered at the Tevatron before precision measurements at the LHC are done. 


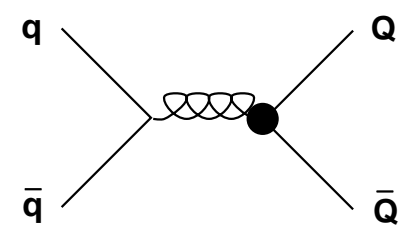

(a)
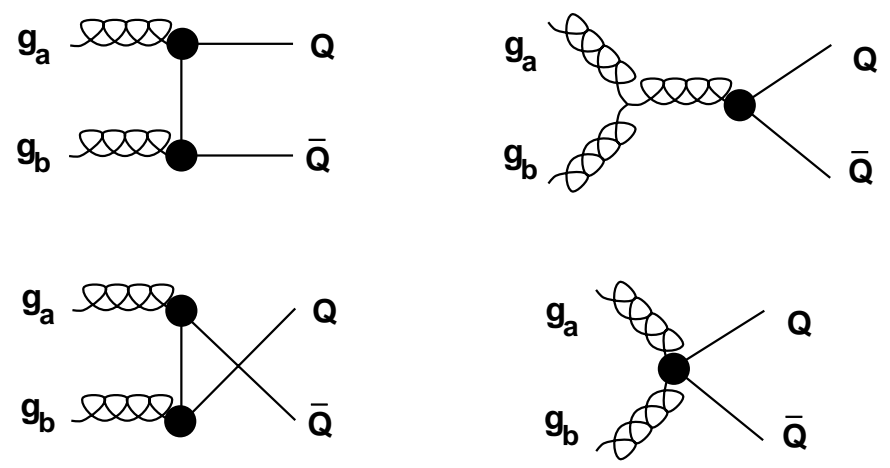

(b)

Figure 51: Feynman diagrams for the process (a) $q \bar{q} \rightarrow Q \bar{Q}$, and (b) $g g \rightarrow Q \bar{Q}$ with $Q=t$. The heavy dots represent the effective vertices involving $\kappa_{g}$ and $\tilde{\kappa}_{g}$ (see also text). 


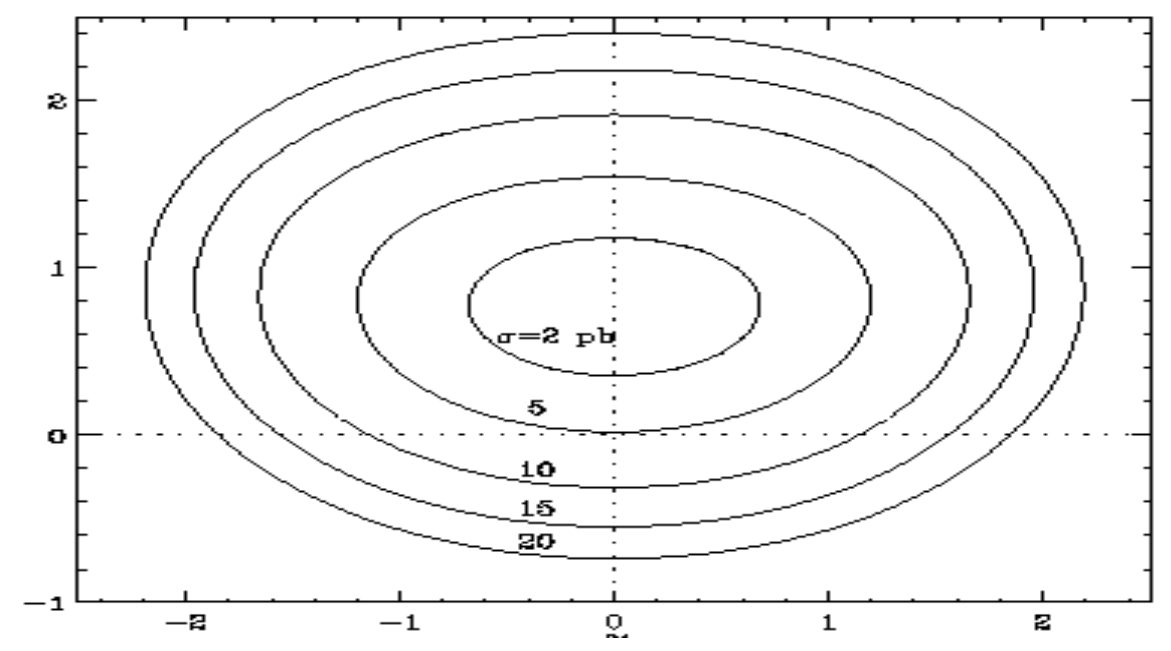

Figure 52: Contours of the $p \bar{p} \rightarrow t \bar{t}+X$ cross-sections, in $p b$, in the $\tilde{\kappa}_{g}$ (horizontal axis) $-\kappa_{g}$ (vertical axis) plane. Figure taken from [218]. 


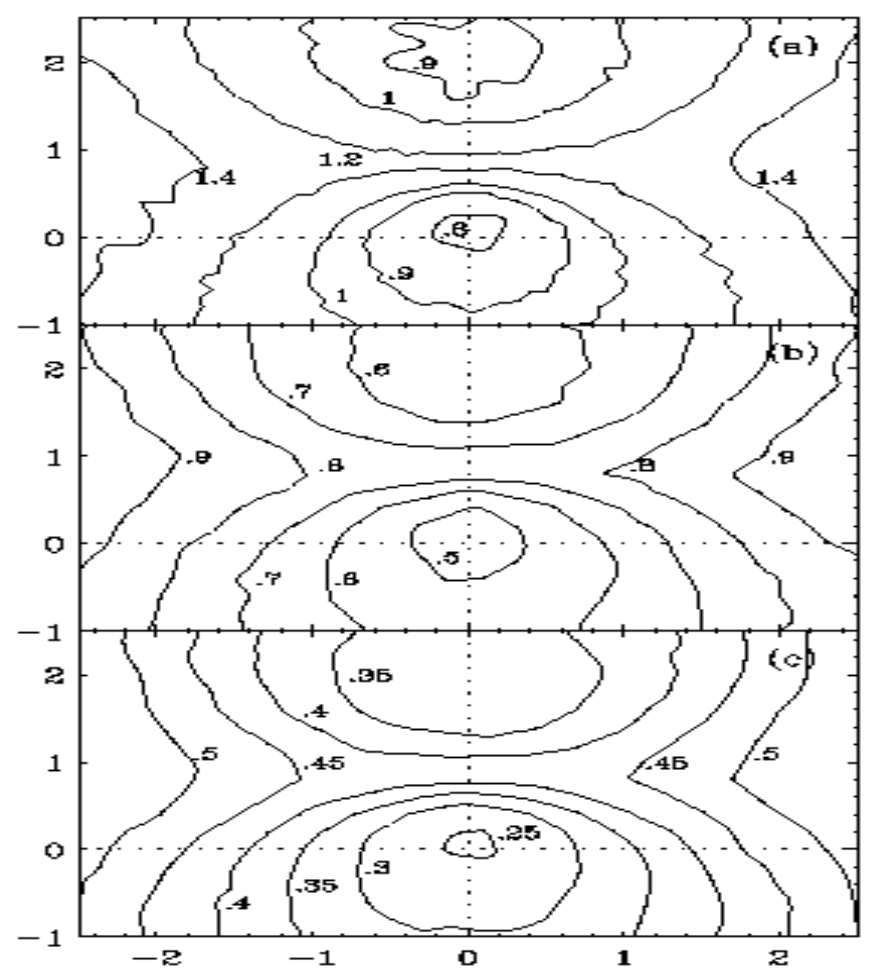

Figure 53: Contours of the ratio $\mathcal{R}=\sigma(t \bar{t} j) / \sigma(t \bar{t})$ in the $\tilde{\kappa}_{g}$ (horizontal axis) $\kappa_{g}$ (vertical axis) plane, with $p_{T}(j)>5,10$ and $20 \mathrm{GeV}$ in (a), (b), and (c), respectively. Figure taken from [218]. 


\section{$8.2 p \bar{p} \rightarrow t \bar{b}+X$}

In spite of the fact that at the Tevatron $p \bar{p}$ collider, top quarks are mainly produced as $t \bar{t}$ pairs via an s-channel gluon exchange [223], the subleading electroweak production mechanism of a single top forms a significant fraction of the $t \bar{t}$ pair production. It will therefore be closely scrutinized in the next runs of the Tevatron [224]. The production rate of $t \bar{b}$ through an s-channel off-shell $W$-boson, $p \bar{p} \rightarrow W^{*} \rightarrow t \bar{b}+X$, (the corresponding partonic reaction, $u \bar{d} \rightarrow W^{*} \rightarrow t \bar{b}$, is depicted in Fig. 54) is expected to yield about $10 \%$ of the $t \bar{t}$ production rate 224.

In this section we examine $\mathrm{CP}$ violation asymmetries in top quark production and its subsequent decay via the basic quark level reactions [138, 172, 225]:

$$
u \bar{d} \rightarrow t \bar{b} \rightarrow b W^{+} \bar{b} \quad, \quad \bar{u} d \rightarrow \bar{t} b \rightarrow \bar{b} W^{-} b .
$$

Indeed this reaction is rich for CP violation studies as it exhibits many different types of asymmetries. Some of these, which we consider below, involve the top spin. Therefore, the ability to track the top spin through its decays becomes important and top decays have to be examined as well (see e.g. 226]).

The asymmetries in $t \bar{b}$ production can be appreciably larger than those in $t \bar{t}$ pair production wherein they tend to be about a few tenths of percent (see Chapters 6 and 7). Moreover, while in the SM, CP-odd effects in $p \bar{p} \rightarrow W^{*} \rightarrow t \bar{b}+X$ are expected to be extremely small since they are severely suppressed by the GIM mechanism (see e.g., [47, 151]), it is shown below that, in extensions of the SM, CP asymmetries can be sizable - in some cases at the level of a few percent. Therefore, since the number of events needed to observe an asymmetry scales as (asymmetry) ${ }^{-2}$, the enhanced CP-violating effects in $t \bar{b}(\bar{t} b)$ may make up for the reduced production rates for $t \bar{b}$ compared to $t \bar{t}$. In fact larger asymmetries could be essential as detector systematics can be a serious limitation for asymmetries less than about $1 \%$.

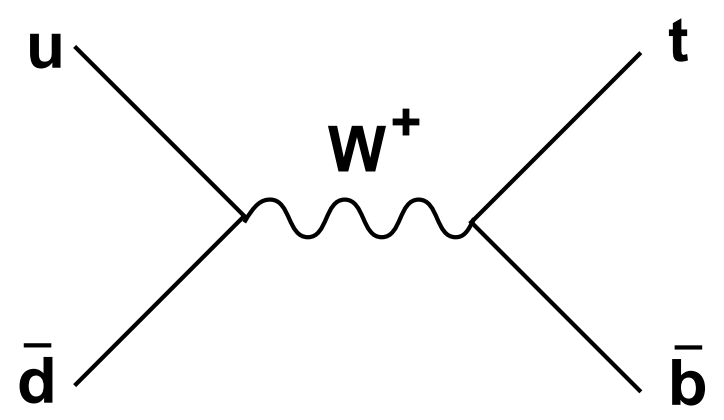

Figure 54: The tree-level Feynman diagram contributing to $u \bar{d} \rightarrow t \bar{b}$.

Let us discuss now the asymmetries in the $u \bar{d}(\bar{u} d)$ subprocess. We consider four types of asymmetries that may be present [172]. First, is the CP-violating asymmetry in the cross-section

$$
A_{0}=\left(\sigma_{t \bar{b}}-\sigma_{\bar{t} b}\right) /\left(\sigma_{t \bar{b}}+\sigma_{\bar{t} b}\right)
$$

where $\sigma_{t \bar{b}}$ and $\sigma_{\bar{t} b}$ are the cross-sections for $u \bar{d} \rightarrow t \bar{b}$ and $\bar{u} d \rightarrow \bar{t} b$, respectively, at $\hat{s}=\left(p_{t}+p_{\bar{b}}\right)^{2}$.

The spin of the top allows us to define three additional types of CP-violating polarization asymmetries. For these it is convenient to introduce the coordinate system in the top quark (or anti-top) rest frame where the unit vectors are $\vec{e}_{z} \propto-\vec{p}_{b}, \vec{e}_{y} \propto \vec{p}_{u} \times \vec{p}_{b}$ and $\vec{e}_{x}=\vec{e}_{y} \times \vec{e}_{z}$. Here $\vec{p}_{b}$ and $\vec{p}_{u}$ are the 3 -momenta of the $\bar{b}$-quark and the initial $u$-quark in that frame. We denote the longitudinal polarization or helicity asymmetry as

$$
A(\hat{z})=\left(N_{R}-N_{L}-\bar{N}_{R}+\bar{N}_{L}\right) /\left(N_{R}+N_{L}+\bar{N}_{R}+\bar{N}_{L}\right)
$$


where $N_{L}$ is the number of left-handed top quarks produced in $u \bar{d} \rightarrow t \bar{b}$ and $\bar{N}_{R}$ is the number of right-handed $\bar{t}$ produced in $\bar{u} d \rightarrow \bar{t} b$, etc. Therefore, in the frame introduced above righthanded tops have spin up along the $z$-axis and left-handed ones spin down. We further define the CP-violating spin asymmetries in the $x$ and $y$ directions as follows

$$
\begin{aligned}
& A(\hat{x})=\left(N_{x+}-N_{x-}+\bar{N}_{x+}-\bar{N}_{x-}\right) /\left(N_{x+}+N_{x-}+\bar{N}_{x+}+\bar{N}_{x-}\right), \\
& A(\hat{y})=\left(N_{y+}-N_{y-}-\bar{N}_{y+}+\bar{N}_{y-}\right) /\left(N_{y+}+N_{y-}+\bar{N}_{y+}+\bar{N}_{y-}\right),
\end{aligned}
$$

where, for example, $N_{j+}\left(\bar{N}_{j+}\right)$ represent the number of $t(\bar{t})$ with spin up with respect to $\hat{j}$-axis, for $j=x, y$, etc.

While all these four asymmetries are manifestly CP-violating, $A_{0}, A(\hat{z})$ and $A(\hat{x})$ are even under naive time reversal $\left(T_{N}\right)$ whereas $A(\hat{y})$ is $T_{N}$-odd. So the first three, unlike $A(\hat{y})$, require a complex amplitude, i.e., absorptive phases.

These asymmetries are related to form factors arising from radiative corrections of the $W^{*} \rightarrow$ $t b$ production vertex due to non-standard physics. To see this, it is useful to parameterize the 1-loop $t \bar{b}$ and $\bar{t} b$ currents of the production amplitude as follows [138]:

$$
\begin{aligned}
J^{\mu(t \bar{b})} & \equiv i \frac{g_{W}}{\sqrt{2}} \sum_{P=L, R} \bar{u}_{t}\left(\frac{\mathcal{P}_{1}^{P} p_{b}^{\mu}}{m_{t}}+\mathcal{P}_{2}^{P} \gamma^{\mu}\right) P v_{b}, \\
J^{\mu(\bar{t} b)} & \equiv i \frac{g_{W}}{\sqrt{2}} \sum_{P=L, R} \bar{u}_{b}\left(\frac{\overline{\mathcal{P}}_{1}^{P} p_{b}^{\mu}}{m_{t}}+\overline{\mathcal{P}}_{2}^{P} \gamma^{\mu}\right) P v_{t},
\end{aligned}
$$

where $P=L$ or $R$ and $L(R) \equiv\left(1-(+) \gamma_{5}\right) / 2 . \mathcal{P}_{1}^{L, R}$ and $\mathcal{P}_{2}^{L, R}$, defined in Eqs. 8.22 and 8.23 , contain the necessary absorptive phases as well as the CP-violating phases in a given model. It is easy to show that if one defines

$$
\begin{aligned}
& \mathcal{P}_{1}^{L} \sim e^{i \delta_{s}^{1}} \times e^{i \delta_{w}^{1}} \\
& \mathcal{P}_{2}^{L} \sim e^{i \delta_{s}^{2}} \times e^{i \delta_{w}^{2}}
\end{aligned}
$$

where $\delta_{s}^{1}, \delta_{s}^{2}$ are the CP-even absorptive phases (i.e., FSI phases) and $\delta_{w}^{1}, \delta_{w}^{2}$ are the CP-odd phases, then

$$
\begin{aligned}
& \overline{\mathcal{P}}_{1}^{R} \sim-e^{i \delta_{s}^{1}} \times e^{-i \delta_{w}^{1}} \\
& \overline{\mathcal{P}}_{2}^{L} \sim e^{i \delta_{s}^{2}} \times e^{-i \delta_{w}^{2}} .
\end{aligned}
$$

In terms of these form factors, the two $T_{N}$-even asymmetries $A_{0}$ and $A(\hat{z})$ are given by [172]:

$$
\begin{array}{r}
A_{0}=\frac{(x-1)}{2(x+2)} \Re \mathrm{e}\left(\mathcal{P}_{1}^{L}+\overline{\mathcal{P}}_{1}^{R}\right)-\Re \mathrm{e}\left(\mathcal{P}_{2}^{L}-\overline{\mathcal{P}}_{2}^{L}\right), \\
A(\hat{z})=\frac{(x-1)}{2(x+2)} \Re \mathrm{e}\left(\mathcal{P}_{1}^{L}+\overline{\mathcal{P}}_{1}^{R}\right)-\frac{x-2}{x+2} \Re \mathrm{e}\left(\mathcal{P}_{2}^{L}-\overline{\mathcal{P}}_{2}^{L}\right),
\end{array}
$$

where $x \equiv m_{t}^{2} / \hat{s}$. Notice that the three quantities $\left\{A_{0}, A(\hat{z}), A(\hat{x})\right\}$ are linear combinations of only two form factors. Thus one can show that

$$
A(\hat{x})=-3 \pi x^{-\frac{1}{2}}\left[(2+x) A_{0}+(2-x) A(\hat{z})\right] / 32 .
$$


which will therefore hold if the new $\mathrm{CP}$ violating physics takes place through such a vertex correction of $W^{*} \rightarrow t b$.

Similarly, the asymmetry $A(\hat{y})$ is proportional to the real parts of the 1-loop integrals and may therefore be obtained from the corresponding imaginary parts through the use of dispersion relations. In particular, since the $T_{N}$-even asymmetries are proportional to the absorptive phases in the above form factors, one can express $A(\hat{y})$ in terms of $A_{0}$ and $A(\hat{z})$,

$$
\begin{aligned}
A(\hat{y})[\hat{s}]= & -\frac{3}{32}\left[\frac{1-x}{(2+x) \sqrt{x}}\right] \Re \mathrm{e}\left\{\int_{0}^{\infty} \frac{2 \xi+x}{(\xi-x)(\xi-1+i \epsilon) \xi}\right. \\
& \left.\times\left[(2 \xi-x) A_{0}[\xi \hat{s}]+(2 \xi+x) A(\hat{z})[\xi \hat{s}]\right] d \xi\right\} .
\end{aligned}
$$

Note that the integrand is 0 if $\xi \hat{s}$ is below the threshold to produce an imaginary part since then $A_{0}$ and $A(\hat{z})$ will vanish.

Let us now evaluate the form factors defined in Eqs. 8.22 and 8.23 in two extensions of the SM: the 2HDM of type II and the MSSM. As was pointed above, once these form factors are calculated in a given model, the asymmetries $A_{0}, A(\hat{z}), A(\hat{x})$ and $A(\hat{y})$ can be readily obtained.

\subsubsection{HDM and the CP-violating asymmetries}

As emphasized through out this article, in the 2HDM of type II, a CP-odd phase can reside in neutral Higgs exchanges and there is only one Feynman diagram that contributes to CP violation in $u \bar{d} \rightarrow W^{*} \rightarrow t \bar{b}$ to 1-loop order. This diagram is shown in Fig. 55. The relevant Feynman rules for this diagram, required for calculating the asymmetries of interest, can be extracted from the parts of the 2HDM Lagrangian involving the $t \bar{t} \mathcal{H}^{k}$ and $W W \mathcal{H}^{k}$ couplings in Eqs. 3.70 and 3.71, with $k=1,2,3$ for the three neutral Higgs fields. Recall again that the coupling constants, $a_{t}^{k}$, $b_{t}^{k}$ and $c^{k}$ are functions of $\tan \beta$, which is the ratio between the two VEV's in this model, and of the three mixing angles $\alpha_{1,2,3}$ which diagonalize the $3 \times 3$ Higgs mass matrix (see section 3.2.3). As usual, for simplicity, we have assumed that two of the three neutral Higgs-bosons are much heavier compared to the third one which we denote by $h$. Thus, the CP-violating effect will be dominated by the lightest neutral Higgs, $h$, and is proportional to $b_{t}^{h} c^{h}$. Choosing the angles $\alpha_{1}=\alpha_{2}=\pi / 2$ and $\alpha_{3}=0$ which give maximal effects [172], one obtains $b_{t}^{h} c^{h} \propto \cos \beta \cot \beta$ so the asymmetries are now functions of $\tan \beta$ and $m_{h}$ only.

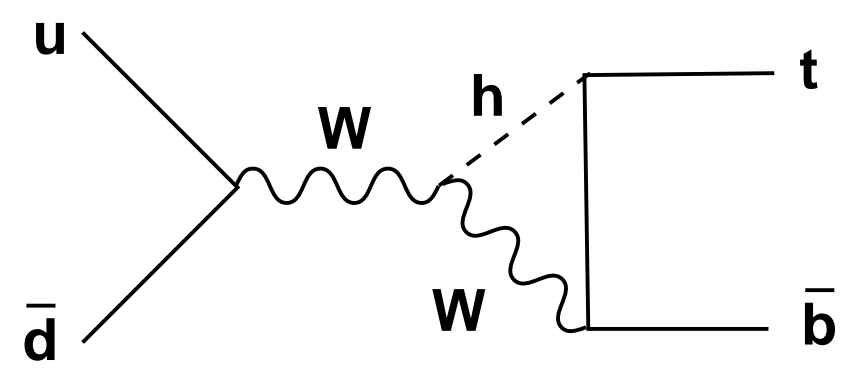

Figure 55: The CP-violating 1-loop graph in 2HDM's with CP violation from neutral Higgs exchanges; $h$ denotes the lightest neutral Higgs.

Using the Lagrangian in Eqs. 3.70 and 3.71, the form factors $\Re$ e $\left(\mathcal{P}_{1}^{L}+\overline{\mathcal{P}}_{1}^{R}\right)$ and $\Re$ e $\left(\mathcal{P}_{2}^{L}-\overline{\mathcal{P}}_{2}^{L}\right)$ can be readily calculated and we get

$$
\Re \mathrm{e}\left(\mathcal{P}_{1}^{L}+\overline{\mathcal{P}}_{1}^{R}\right)=-\left(\frac{\alpha}{\sqrt{2}}\right) \frac{b_{t}^{h} c^{h}}{2 \pi \sin ^{2} \theta_{W}} \frac{m_{t}^{2}}{m_{W}^{2}} \Im \mathrm{m}\left[2 m_{W}^{2}\left(C_{11}-C_{12}\right)\right.
$$




$$
\begin{aligned}
& \left.-m_{t}^{2}\left(C_{12}+C_{23}\right)-\tilde{C}_{0}-\tilde{C}_{11}\right], \\
\Re \mathrm{e}\left(\mathcal{P}_{2}^{L}-\overline{\mathcal{P}}_{2}^{L}\right)= & \left(\frac{\alpha}{\sqrt{2}}\right) \frac{b_{t}^{h} c^{h}}{4 \pi \sin ^{2} \theta_{W}} \frac{m_{t}^{2}}{m_{W}^{2}} \Im \mathrm{m}\left[2 m_{W}^{2}\left(C_{0}+C_{12}\right)\right. \\
& \left.-2 C_{24}\right],
\end{aligned}
$$

where the $C_{x}$ above, $x \in\{0,11,12,21,22,23,24\}$, are the three-point form factor associated with the 1-loop diagram in Fig. 55, and are given via [172, 225]:

$$
C_{x}=C_{x}\left(m_{t}^{2}, m_{W}^{2}, m_{h}^{2}, m_{b}^{2}, \hat{s}, m_{t}^{2}\right)
$$

where $\hat{s}=\left(p_{t}+p_{\bar{b}}\right)^{2}$ and $C_{x}\left(m_{1}^{2}, m_{2}^{2}, m_{3}^{2}, p_{1}^{2}, p_{2}^{2}, p_{3}^{2}\right)$ is defined in appendix A.

The quark level asymmetries of interest can be converted to the hadron (i.e., $p \bar{p}$ ) level by folding in the structure functions in the standard manner [61]. The results for the 2HDM case are shown in Fig. 56, for $\tan \beta=0.3$ and as a function of the lightest Higgs-boson mass, $m_{h}$. For the asymmetry $A(\hat{y})$ we apply a cut of $\hat{s}>\left(m_{h}+m_{W}\right)^{2}$. We can see that $A_{0}$ and $A(\hat{x})$ can reach above the percent level for $m_{h} \lesssim 200 \mathrm{GeV}$. The measurable consequences for such an asymmetry are discussed in section 8.2 .3 below.

It is interesting to note that in the $2 \mathrm{HDM}$ (to the 1-loop order) the $T_{N}$-even asymmetries $A_{0}, A(\hat{z})$ and $A(\hat{x})$ do not receive any contribution from the decay vertex in $t \rightarrow b W$. The only diagram that can potentially drive $\mathrm{CP}$ violation in $t \rightarrow b W$ is the same one as shown in Fig. 55 with the momenta of the $t$ and the $W$ reversed. Thus, an important necessary condition for $A_{0}, A(\hat{z}), A(\hat{x}) \neq 0$, that there is an imaginary part in the decay amplitude, is not satisfied. Moreover, as it turns out, the observed value of $A(\hat{y})$ is not affected by $\mathrm{CP}$ violation in the decay process. The key point is that the measurement of $A(\hat{y})$ through the decay chain $u\left(p_{u}\right) \bar{d}\left(p_{d}\right) \rightarrow$ $\bar{b}\left(p_{b 1}\right) t\left(p_{t}\right)$ followed by $t\left(p_{t}\right) \rightarrow b\left(p_{b 2}\right) e^{+}\left(p_{e}\right) \nu\left(p_{\nu}\right)$ is equivalent to a measurement of a term proportional to $\epsilon\left(p_{e}, p_{d}, p_{t}, p_{b 1}\right)$ ( $\epsilon$ being here the Levi-Civita tensor). On the other hand, CP violation arising from the decay process is proportional to $\epsilon\left(p_{e}, p_{d}, p_{t}, p_{b 2}\right)$. It is easy to see that an observable related to the first of these will be insensitive to the second. So, in this way asymmetries in the production can be separated from those in the decay. 


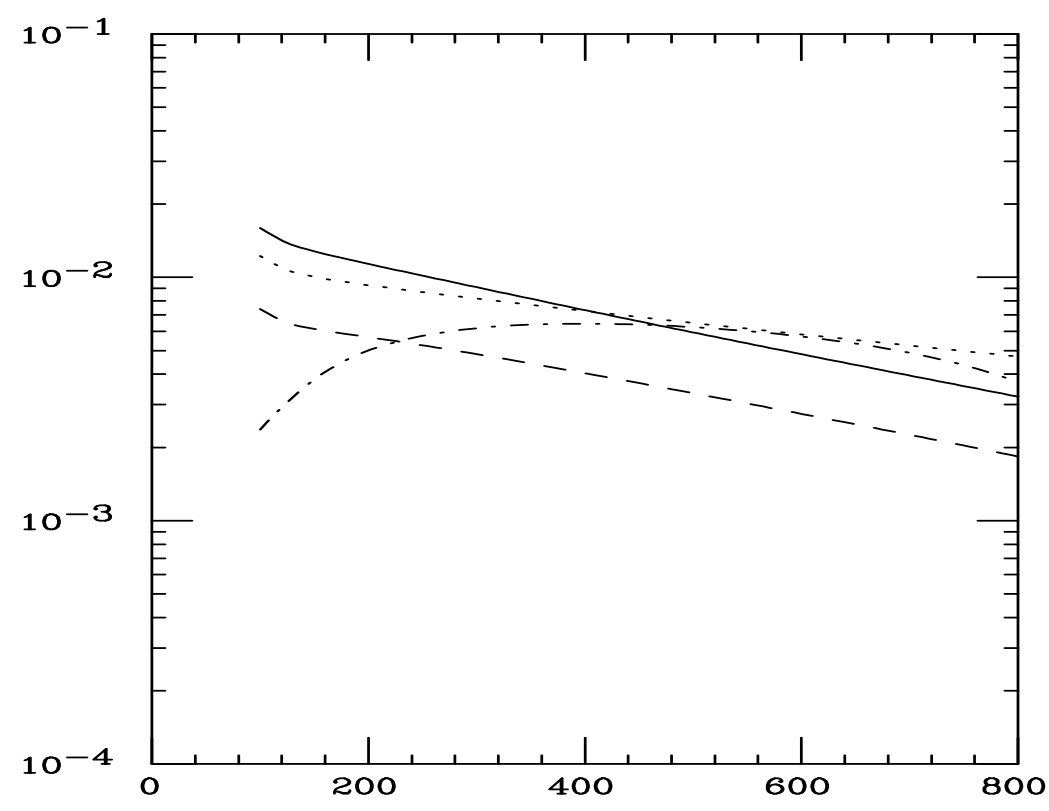

Figure 56: The CP-violating asymmetries in $p \bar{p} \rightarrow t \bar{b}+X$ for the 2HDM case and in the p $\bar{p}$ c.m. frame for $\sqrt{s}=2 \mathrm{TeV}$, as a function of $m_{h}$ (horizontal axis); $A_{0}$ (solid), $A(\hat{z})$ (dashed), $A(\hat{x})$ (dotted) and $A(\hat{y})$ (dot-dashed). Figure taken from [179]. 


\subsubsection{SUSY and the CP-violating cross-section asymmetry}

The MSSM possesses several CP-odd phases that can give rise to CP violation in $p \bar{p} \rightarrow t \bar{b}+X$ and in the subsequent top decay $t \rightarrow b W$ (see section 5.1.4). In [138] we have constructed a plausible low-energy MSSM framework in which there are only five relevant free parameters needed to evaluate the cross-section asymmetry $A_{0}$ (see also sections 3.3.4 and 5.1.4). These are: $M_{S}-\mathrm{a}$ typical SUSY mass scale that characterizes the mass of the heavy squarks, $m_{G}$ - the mass of the gluino, $\mu$ - the Higgs mass parameter in the superpotential, $m_{l}$ - the mass of the lighter stop and $\tan \beta$ - the ratio between the VEV's of the two Higgs fields in the theory. In this framework there are two sources of $\mathrm{CP}$ violation that can potentially contribute to $A_{0}$ [138. The first may arise from the Higgs mass parameter $\mu$ which may be complex in general. The second CP-violating phase arises from $\tilde{t}_{L}-\tilde{t}_{R}$ mixing (see e.g., Eq. 5.60) and may be parameterized by a single quantity $\xi_{C P}^{t}$ defined in Eq. 5.61.

In this scenario, when no further assumptions are made, there are 12 Feynman diagrams that can give rise to $\mathrm{CP}$ violation at the parton level process $u \bar{d} \rightarrow t \bar{b}$ which are depicted in Fig. 57. However, if we assume that $\arg (\mu)=0$ as implied from the existing experimental limit on the NEDM (see discussion in section 3.3.4) and take $m_{u}=m_{d}=m_{b}=0$, then only diagrams (a), (b), (d), (e) and (g) have the necessary CP-violating phase, being proportional to one quantity $\xi_{C P}^{t}$. In [138] we have shown that with $M_{S}$ and $m_{G}$ of about several hundred GeV, diagram (d) is in fact responsible for $\sim 90 \%$ of the total CP violation effect.

Let us, therefore, present the results for the asymmetry $A_{0}$ corresponding only to diagram (d) in Fig. 57. The Feynman rules needed to calculate $A_{0}$ can be derived from the following parts of the SUSY Lagrangian [126]:

$$
\begin{aligned}
\mathcal{L}_{\tilde{u}_{i} d \tilde{\chi}_{m}}= & \tilde{u}_{i}^{+} \bar{d}\left[\left(-g_{W} Z_{u}^{1 i} Z_{1 m}^{+*}+\frac{\sqrt{2} m_{u}}{v_{2}} Z_{u}^{2 i} Z_{2 m}^{+*}\right) R\right. \\
& \left.+\frac{\sqrt{2} m_{d}}{v_{1}} Z_{u}^{1 i} Z_{2 m}^{-} L\right] V^{u d} \tilde{\chi}_{m}^{c}+h . c . \\
\mathcal{L}_{\tilde{u}_{i} u \tilde{\chi}_{n}^{0}}= & \tilde{u}_{i}^{-} \overline{\tilde{\chi}}_{n}^{0}\left[\left(-\frac{g_{W}}{\sqrt{2}} Z_{u}^{1 i *} L^{+}-\frac{\sqrt{2} m_{u}}{v_{2}} Z_{u}^{2 i *} Z_{N}^{4 n}\right) L\right. \\
& \left.+\left(\frac{2 \sqrt{2}}{3} g_{W} \tan \theta_{W} Z_{u}^{2 i *} Z_{N}^{1 n *}-\frac{\sqrt{2} m_{u}}{v_{2}} Z_{u}^{1 i *} Z_{N}^{4 n *}\right) R\right] u+\text { h.c. }, \\
\mathcal{L}_{W \tilde{\chi}_{m} \tilde{\chi}_{n}^{0}=} & g_{W} \overline{\tilde{\chi}}_{m} \gamma^{\mu}\left(K^{-} L+K^{+} R\right) \tilde{\chi}_{n}^{0} W_{\mu}^{+}+h . c .
\end{aligned}
$$

where $L(R)=\frac{1}{2}\left(1-(+) \gamma_{5}\right), \tilde{u}_{i}$ and $u\left(\tilde{d}_{j}\right.$ and $\left.d\right)$ stand for up squark and up quark (down squark and down quark), respectively, $\tilde{\chi}_{m}(m=1,2)$ and $\tilde{\chi}_{n}^{0}(n=1-4)$ are the charginos and neutralinos, respectively. Also, in Eqs. 8.37 and 8.36 we have defined

$$
\begin{aligned}
L^{ \pm} & \equiv \frac{1}{3} \tan \theta_{W} Z_{N}^{1 n} \pm Z_{N}^{2 n} \\
K^{+} & \equiv Z_{N}^{2 n *} Z_{1 m}^{-}+\frac{1}{\sqrt{2}} Z_{N}^{3 n *} Z_{2 m}^{-} \\
K^{-} & \equiv Z_{N}^{2 n} Z_{1 m}^{+*}-\frac{1}{\sqrt{2}} Z_{N}^{4 n} Z_{2 m}^{+*},
\end{aligned}
$$

and the mixing matrices $Z_{u}, Z_{d}, Z_{N}, Z^{-}$and $Z^{+}$were given in [138] (also given in section 3.3.2).

Using the Lagrangian in Eqs. 8.35 - 8.37, the form factors $\Re \mathrm{e}\left(\mathcal{P}_{1}^{L}+\overline{\mathcal{P}}_{1}^{R}\right)$ and $\Re \mathrm{e}\left(\mathcal{P}_{2}^{L}-\overline{\mathcal{P}}_{2}^{L}\right)$ (corresponding to diagram (d) in Fig. 57) can be evaluated within the MSSM and are given by 


$$
\begin{aligned}
\Re \mathrm{e}\left(\mathcal{P}_{1}^{L}+\overline{\mathcal{P}}_{1}^{R}\right)= & -\frac{\alpha}{\pi \sin ^{2} \theta_{W}} m_{t}\left[m_{t} \mathcal{O}_{d}^{1} \Im \mathrm{m}\left(C_{12}^{d}+C_{23}^{d}\right)+m_{\tilde{\chi}_{n}^{0}} \mathcal{O}_{d}^{2} \Im \mathrm{m} C_{12}^{d}\right. \\
& \left.-m_{\tilde{\chi}_{m}} \mathcal{O}_{d}^{3} \Im \mathrm{m}\left(C_{12}^{d}-C_{11}^{d}\right)\right] \\
\Re \mathrm{e}\left(\mathcal{P}_{2}^{L}-\overline{\mathcal{P}}_{2}^{L}\right)= & \frac{1}{2} \frac{\alpha}{\pi \sin ^{2} \theta_{W}}\left[\mathcal { O } _ { d } ^ { 1 } \left(\left(\hat{s}-m_{t}^{2}\right) \Im \mathrm{m} C_{23}^{d}-\hat{s} \Im \mathrm{m} C_{22}^{d}\right.\right. \\
& \left.-2 \Im \mathrm{m} C_{24}^{d}-m_{t}^{2} \Im \mathrm{m} C_{12}^{d}\right) \\
& -m_{t} m_{\tilde{\chi}_{n}^{0}} \mathcal{O}_{d}^{2} \Im \mathrm{m} C_{12}^{d}+m_{t} m_{\tilde{\chi}_{m}} \mathcal{O}_{d}^{3} \Im \mathrm{m}\left(C_{0}^{d}+C_{12}^{d}\right) \\
& \left.+m_{\tilde{\chi}_{m}} m_{\tilde{\chi}_{n}^{0}} \mathcal{O}_{d}^{4} \Im \mathrm{m} C_{0}^{d}\right]
\end{aligned}
$$

where $\hat{s}=\left(p_{t}+p_{\bar{b}}\right)^{2}$ and $\mathcal{O}_{d}^{i}$ contain the SUSY CP-weak phases associated with diagram (d) in Fig. 57. In fact, the same CP-violating phases occur also in the decay $t \rightarrow b W$ (see section 5.1.4) and, therefore, the $\mathcal{O}_{d}^{i}$ above are the same as the ones given in Eqs. 5.50-5.53. The $\Im \mathrm{m} C_{x}^{d}$, $x \in\{0,11,12,21,22,23,24\}$, in Eqs. 8.41 and 8.42 are the imaginary parts of the three-point form factors associated with diagram (d) in Fig. 57. Thus, $C_{x}^{d}$ are given via 138]:

$$
C_{x}^{d}=C_{x}\left(m_{\tilde{t}_{i}}^{2}, m_{\tilde{\chi}_{m}}^{2}, m_{\tilde{\chi}_{n}^{0}}^{2}, m_{b}^{2}, \hat{s}, m_{t}^{2}\right)
$$

and $C_{x}\left(m_{1}^{2}, m_{2}^{2}, m_{3}^{2}, p_{1}^{2}, p_{2}^{2}, p_{3}^{2}\right)$ is defined in appendix A.

In [138], instead of calculating the cross-section asymmetry $A_{0}$, we considered a partially integrated cross-section asymmetry, $A_{0}^{\mathrm{PICA}}$, in which we have imposed a cut on the $t b$ invariant mass, $m_{t b}<350 \mathrm{GeV}$. Such a cut on $m_{t b}$ may help to remove the $t \bar{t}$ "background" from a measurement of a cross-section asymmetry in $p \bar{p} \rightarrow t \bar{b}+X$. The results in the SUSY case are shown in Figs. 58 and 59 as a function of $\mu$ and $m_{G}$, respectively, for $M_{S}=400 \mathrm{GeV}, m_{l}=50$ $\mathrm{GeV}\left(m_{l}\right.$ is the mass of the lighter stop particle) and for $\tan \beta=1.5$, 35. Maximal CP violation was chosen in the sense that $\xi_{C P}^{t}=1$, thus the asymmetry plotted in Figs. 58 and 59 is in fact given in units of $\xi_{C P}^{t}$. Evidently, for some values of $\mu$ around $100 \mathrm{GeV}$ and with $m_{G} \sim 450$ $\mathrm{GeV}$ the asymmetry can almost reach the $3 \%$ level. The asymmetry is above the $1 \%$ level for several other choices of $\mu$. It was also shown in [138] that, in general, in order for the asymmetry to be above the percent level the mass of the lighter stop is required to be below $\sim 75 \mathrm{GeV}$. Furthermore, the asymmetry tends to drop as $\tan \beta$ is increased in the range $1 \lesssim \tan \beta \lesssim 10$, and it is almost insensitive to $\tan \beta$ for $\tan \beta \gtrsim 10$.

In the MSSM, 1-loop radiative corrections to the amplitude of top decay $t \rightarrow b W$ that can violate $\mathrm{CP}$ are also present. In fact, disregarding the incoming $u$ and $d$ lines, diagrams (a)-(d) in Fig. 57 with the $t$ and $W$ momenta reversed, can give rise to a CP-violating $t b W$ decay vertex. This was discussed in some detail in section 5.1.4. To 1-loop order in perturbation theory, where the CP-violating virtual corrections enter only either the production or the decay vertices of the top in the overall reaction $p \bar{p} \rightarrow t \bar{b}+X \rightarrow W^{+} b \bar{b}+X$, and in the narrow width approximation for the decaying top, an overall $\mathrm{CP}$ asymmetry, $A$, can be broken into

$$
A=A_{P}+A_{D}
$$

In Eq. 8.44 $A_{P}$ and $A_{D}$ are the CP asymmetries emanating from the production and decay of the top, respectively, and are defined by

$$
\begin{aligned}
A_{P} & \equiv \frac{\sigma(p \bar{p} \rightarrow t \bar{b}+X)-\bar{\sigma}(p \bar{p} \rightarrow \bar{t} b+X)}{\sigma(p \bar{p} \rightarrow t \bar{b}+X)+\bar{\sigma}(p \bar{p} \rightarrow \bar{t} b+X)}, \\
A_{D} & \equiv \frac{\Gamma\left(t \rightarrow W^{+} b\right)-\bar{\Gamma}\left(\bar{t} \rightarrow W^{-} \bar{b}\right)}{\Gamma\left(t \rightarrow W^{+} b\right)+\bar{\Gamma}\left(\bar{t} \rightarrow W^{-} \bar{b}\right)}
\end{aligned}
$$


The PRA $A_{D}$, defined in Eq. 8.46, does not depend on the specific production mechanism of the top and was calculated in section 5.1.4. We have shown there that, with the low-energy MSSM parameters described above, one gets $\left|A_{D}\right|<0.3 \%$ where, in most instances, it tends to be even smaller $-\left|A_{D}\right|<0.1 \%$. Therefore, it is about one order of magnitude smaller than the asymmetry in the production of $t b$ and its relevance to the overall asymmetry in $p \bar{p} \rightarrow t \bar{b}+X \rightarrow W^{+} b \bar{b}+X$ is negligible.

As a final remark here, let us recall that in the $2 \mathrm{HDM}$ case discussed before, the PRA $A_{D}$ in Eq. 8.46 is forbidden at 1-loop order because of CPT invariance, i.e., no rescattering of final states as shown in section 2.3. This was also discussed in section 5.1.2. 


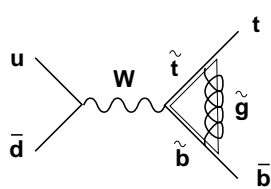

(a)

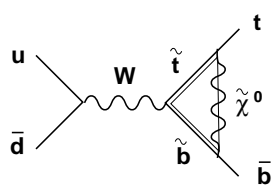

(b)

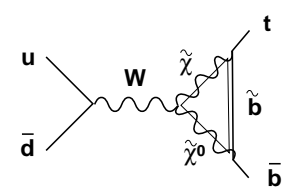

(c)

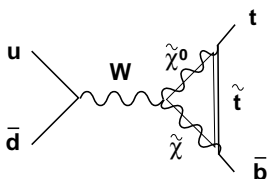

(d)

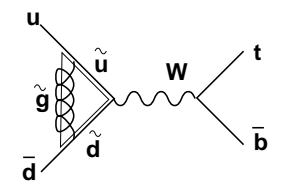

(a')

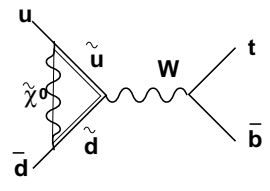

(b')

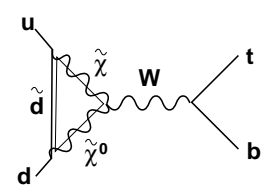

(c')

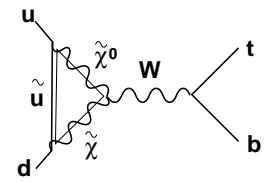

(d')

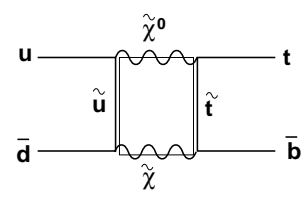

(e)

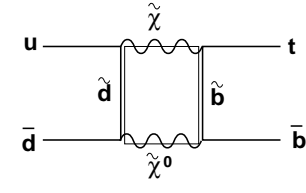

(f)

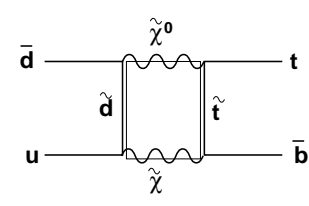

(g)

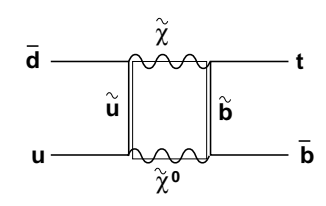

(h)

Figure 57: CP-violating, SUSY induced, 1-loop diagrams for the processes $u \bar{d} \rightarrow t \bar{b}$. $\tilde{g}$ is the gluino, $\tilde{\chi}$ is a chargino, $\tilde{\chi}^{0}$ is a neutralino and $\tilde{t}$ and $\tilde{b}$ are top and bottom squarks, respectively. 

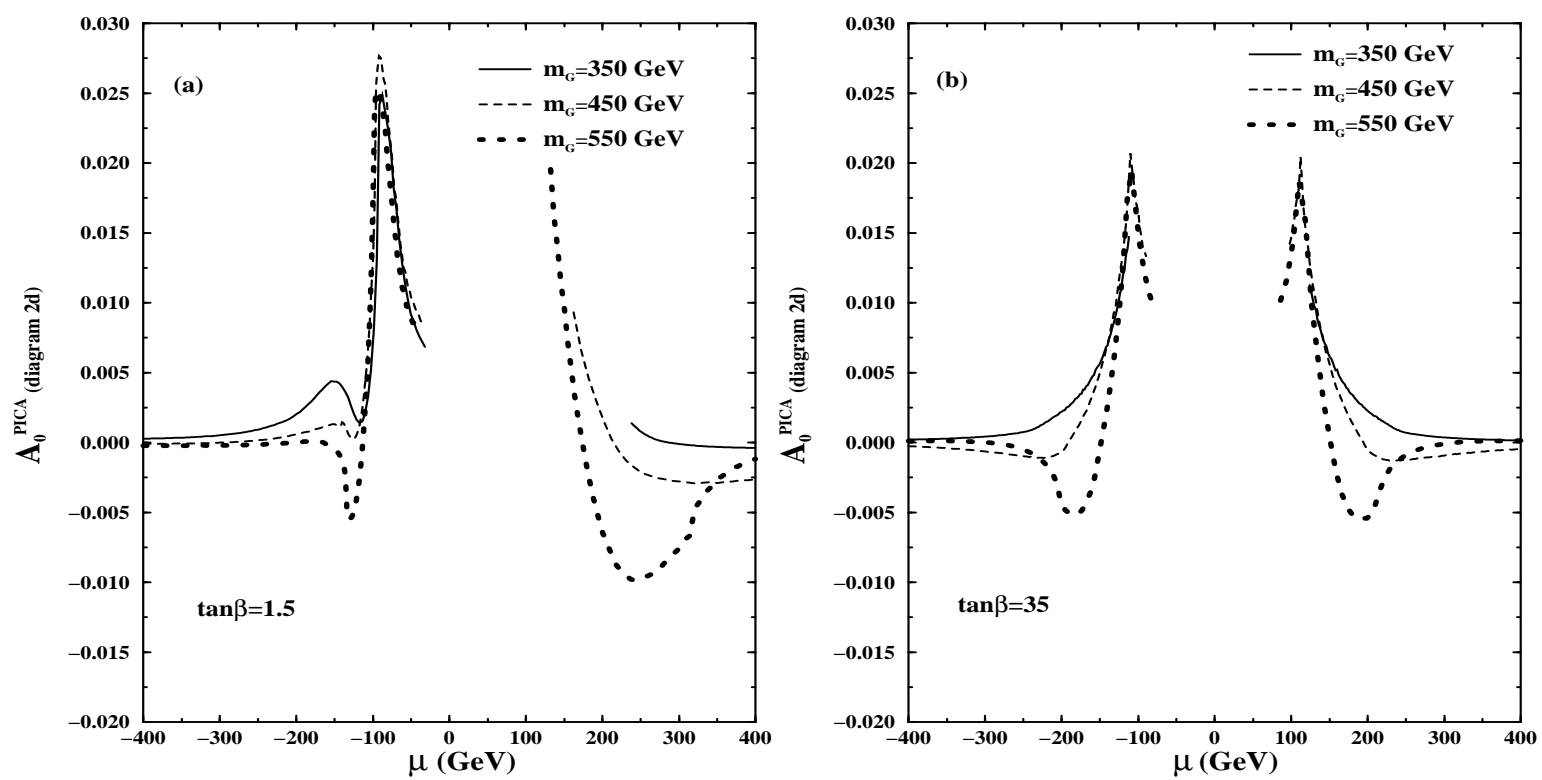

Figure 58: The SUSY induced partially integrated cross-section asymmetry $A_{0}^{\mathrm{PICA}}$ defined in the text, as a function of $\mu$, for $M_{S}=400 \mathrm{GeV}, m_{l}=50 \mathrm{GeV}$ and for $\sqrt{s}=2 \mathrm{TeV}$. With (a) $\tan \beta=1.5$ and (b) $\tan \beta=35$. Figure taken from [138]. 

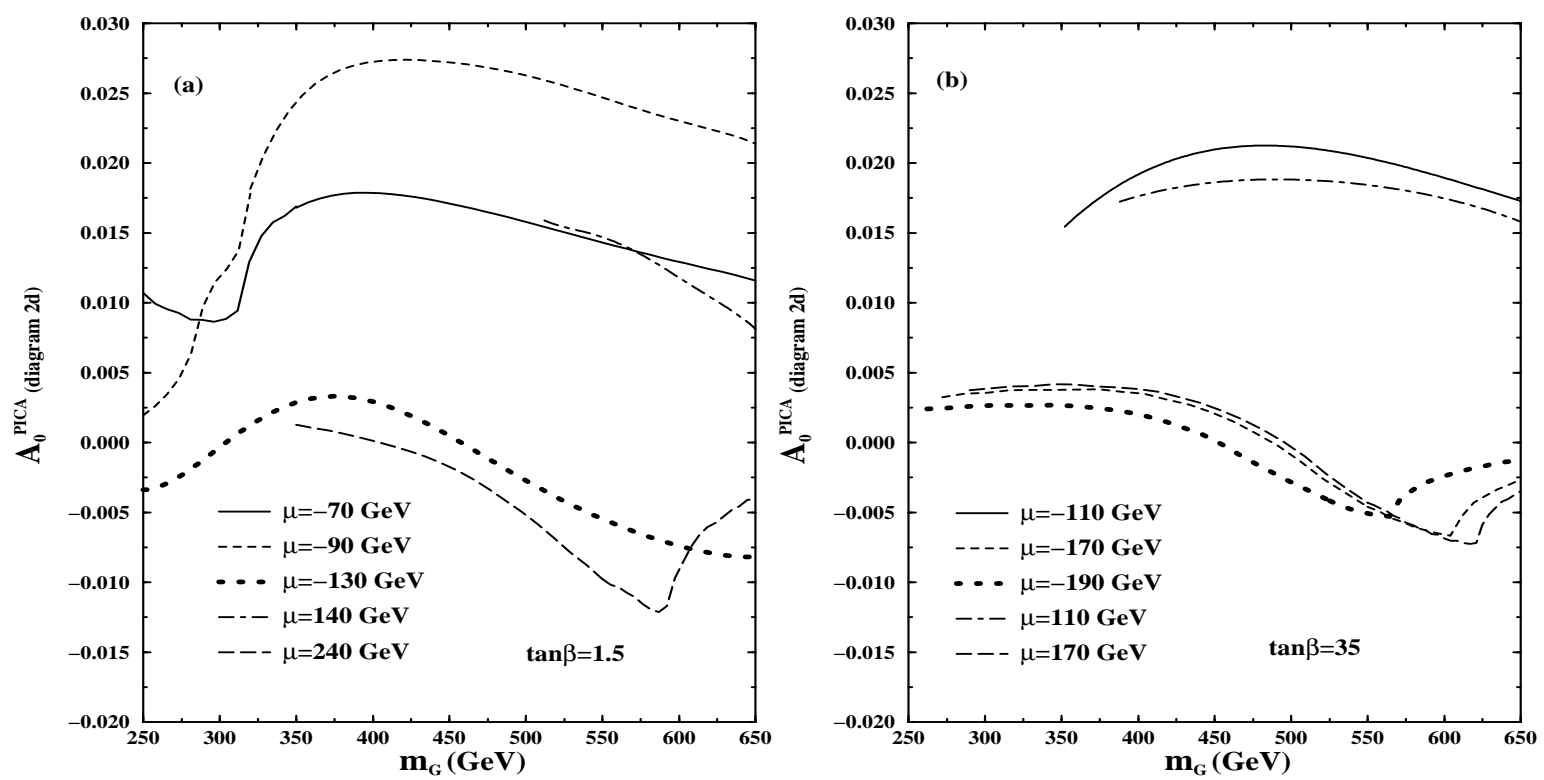

Figure 59: The SUSY induced partially integrated cross-section asymmetry $A_{0}^{\mathrm{PICA}}$ defined in the text, as a function of $m_{G}$, for several values of $\mu, M_{S}=400 \mathrm{GeV}, m_{l}=50 \mathrm{GeV}$ and for $\sqrt{s}=2$ TeV. With (a) $\tan \beta=1.5$ and (b) $\tan \beta=35$. Figure taken from [138]. 


\subsubsection{Feasibility of extraction from experiment}

To summarize the results of sections 8.2.1 and 8.2.2, CP-violating asymmetries in single top production and decay at the Tevatron through $p \bar{p} \rightarrow t \bar{b}+X \rightarrow W^{+} b \bar{b}+X$ may optimistically reach a few percent in extensions of the SM such as SUSY and 2HDM's. In future upgrades of the Tevatron to $\sqrt{s}=2 \mathrm{TeV}$, the cross-section for $p \bar{p} \rightarrow t \bar{b}+X$ is expected to be about $300 \mathrm{fb}$, if a cut of $m_{t b}<350 \mathrm{GeV}$ is applied on the invariant mass of the $t \bar{b}$ [138]. Therefore, with an integrated luminosity of $\mathcal{L}=30 \mathrm{fb}^{-1}$ [224], an asymmetry of $\sim 3 \%$ can be naively detected with a statistical significance of $3-\sigma$. Therefore, a percent level CP-violating signal in the reaction $p \bar{p} \rightarrow t \bar{b}+X \rightarrow W^{+} b \bar{b}+X$ is especially notable as it may become accessible at the near future $2 \mathrm{TeV} p \bar{p}$ collider. In particular, based on the results presented in this section, such a measurement may impose limits on the CP-violating parameters $\arg \left(A_{t}\right)$ and $b_{t}^{h} c^{h}$ of the MSSM and the 2HDM, respectively. However, it should be noted that such a detection at the Tevatron will require the identification of all $t \bar{b}$ pairs, which, in principle, can be achieved only if the top can be reconstructed even when the $W$ decays hadronically.

It will be also useful to explore SUSY or 2HDM mediated CP-violating effects that can originate from the $W$-gluon fusion subprocess which contributes to the same final state (i.e., $W g \rightarrow t \bar{b} d)$ and which has a comparable production rate to that of the simple $u \bar{d} \rightarrow t \bar{b}$ in the $2 \mathrm{TeV}$ Tevatron. While in the MSSM various 1-loop triangle and box corrections can give rise to $\mathrm{CP}$ nonconservation in the $W$-gluon fusion subprocess, in the $2 \mathrm{HDM} \mathrm{CP}$-violating radiative corrections to $W g$ fusion, at the 1-loop order, do not yield absorptive parts in the limit $m_{b}=0$. Therefore, it will not contribute to $\mathrm{CP}$ asymmetries of the $T_{N}$-even type in single top production. Note, however, that the $W$-gluon fusion subprocess has its own characteristics, e.g., the extra light jet in the final state, which may be used in order to experimentally distinguish it from the "simple" ud fusion process (see e.g., Heinson et al. in [224]).

\section{3 $p \bar{p} \rightarrow t \bar{b} h+X$, a case of tree-level $\mathrm{CP}$ violation}

Motivated by the large, tree-level, CP-violating effects found in the the reaction $e^{+} e^{-} \rightarrow t \bar{t} h$ (see section 6.2), we were led to consider an analogous reaction in the Tevatron $p \bar{p}$ collider with a $t \bar{b} h$ final state [225]. Thus, in this section we focus on CP violation, driven by $2 \mathrm{HDM}$ in the process $p \bar{p} \rightarrow t \bar{b} h+X$, where $h$ is the lightest neutral Higgs in the 2HDM of type II. From the outset we remark that a statistically significant $\mathrm{CP}$ study in the reaction $p \bar{p} \rightarrow t \bar{t} h+X$ in a future Tevatron upgrade with c.m. energy of $2 \mathrm{TeV}$ and even $4 \mathrm{TeV}$, is unlikely due to the low $t \bar{b} h$ event rate.

As in the case of $e^{+} e^{-} \rightarrow t \bar{t} h$, a very interesting feature of the reaction $u \bar{d} \rightarrow t \bar{b} h$ (at the parton level) is that it exhibits a CP asymmetry at the tree graph level. Such an effect arises from interference of the Higgs emission from the $t$ (but not from the $\bar{b}$ in the limit $m_{b} \rightarrow 0$ ) with the Higgs emission from the $W$-boson. Being a tree-level effect the resulting asymmetry is quite large. This asymmetry may be measurable in principle, through a CP-odd, $T_{N}$-odd observable.

Let us now discuss the tree-level cross-section and CP violation effects in our reactions,

$$
u\left(p_{u}\right) \bar{d}\left(p_{\bar{d}}\right) \rightarrow t\left(p_{t}\right) \bar{b}\left(p_{\bar{b}}\right) h\left(p_{h}\right), \bar{u}\left(p_{\bar{u}}\right) d\left(p_{d}\right) \rightarrow \bar{t}\left(p_{\bar{t}}\right) b\left(p_{b}\right) h\left(p_{h}\right)
$$

In the limit $m_{b}=0$ (and also $m_{u}=m_{d}=0$ ), and to lowest order, the only two diagrams that can contribute to $\mathrm{CP}$ violation in the reactions of Eq. 8.47 are depicted in Fig. 60. The relevant Feynman rules needed to calculate the tree-level CP asymmetry are extracted from the Lagrangian in Eqs. 3.70 and 3.71. Here also, we assume that two of the three neutral Higgs particles are much heavier than the remaining one, i.e. $h$. We, therefore, omit the index $k$ in Eqs. 3.70 and 3.71, and denote the couplings for the lightest neutral Higgs as: $a_{t}^{h}, b_{t}^{h}$ and $c^{h}$. The tree-level differential cross-section $\hat{\Sigma}^{0}$ at the parton level, is a sum of two terms: the CP-even and CP-odd terms $\hat{\Sigma}_{+}^{0}$ and $\hat{\Sigma}_{-}^{0}$, respectively, 


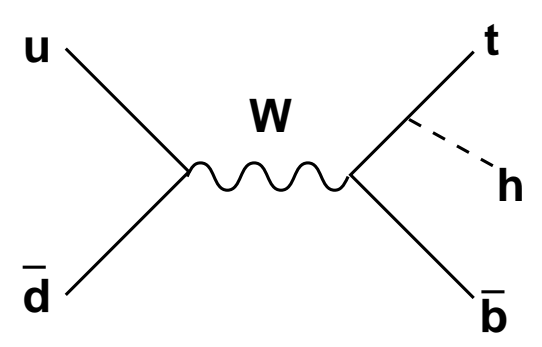

(a)

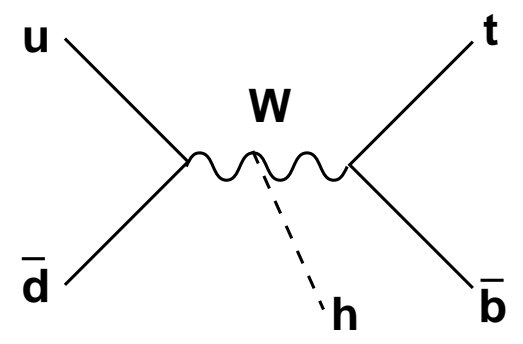

(b)

Figure 60: Tree-level Feynman diagrams contributing to $u \bar{d} \rightarrow t \bar{b} h$ in the limit $m_{d}=m_{u}=m_{b}=$ $0 ; \mathrm{h}$ stands for the lightest neutral Higgs in a $2 H D M$.

$$
\hat{\Sigma}^{0} \equiv \hat{\Sigma}_{+}^{0}+\hat{\Sigma}_{-}^{0}
$$

where $\hat{\Sigma}_{ \pm}^{0}$ are calculated from the tree-level diagrams in Fig. 60. The expression for the CP-even part, i.e., $\hat{\Sigma}_{+}^{0}$, can be parameterized as

$$
\begin{aligned}
\hat{\Sigma}_{+}^{0}= & \left(\frac{2 \pi \alpha}{\sin ^{2} \theta_{W}}\right)^{3}\left(\frac{\Pi_{W}}{2}\right)^{2} \frac{m_{t}^{2}}{m_{W}^{2}} \times\left[\left(a_{t}^{h}\right)^{2} \Pi_{t}^{2} \mathcal{A}+\left(b_{t}^{h}\right)^{2} \Pi_{t}^{2} \mathcal{B}\right. \\
& \left.+2\left(c^{h}\right)^{2} \frac{m_{W}^{2}}{m_{t}^{2}} \Pi_{W h}^{2} \mathcal{C}+2^{3 / 2} a_{t}^{h} c^{h} \Pi_{t} \Pi_{W h} \mathcal{D}\right]
\end{aligned}
$$

where the terms $\mathcal{A}, \mathcal{B}, \mathcal{C}$ and $\mathcal{D}$ are quite involved and were calculated in [225]. $\Pi_{W}$ is the $W$-boson propagator, and together with $\Pi_{t}$ and $\Pi_{W h}$ are given by

$$
\begin{aligned}
\Pi_{W} & \equiv \frac{1}{\hat{s}-m_{W}^{2}}, \Pi_{t} \equiv \frac{1}{2 p_{t} \cdot p_{h}+m_{h}^{2}} \\
\Pi_{W h} & \equiv \frac{1}{m_{t}^{2}-m_{W}^{2}+2 p_{t} \cdot p_{\bar{b}}} .
\end{aligned}
$$

Furthermore, $p \equiv p_{u}+p_{\bar{d}}$ is the $\hat{s}$-channel 4-momentum at the quark level, and $\hat{s}$ is defined to be $\hat{s}=p^{2}$.

The CP-violating piece of the tree-level differential cross-section is [225]:

$$
\hat{\Sigma}_{-}^{0}=2^{3 / 2}\left(\frac{2 \pi \alpha}{\sin ^{2} \theta_{W}}\right)^{3} \frac{m_{t}^{2}}{m_{W}^{2}} \Pi_{W}^{2} \Pi_{W h} \Pi_{t} b_{t}^{h} c^{h} \times \epsilon\left(p_{\bar{b}}, p_{\bar{d}}, p_{t}, p_{u}\right) \times\left(f-s_{t}+w\right),
$$

where $s_{t} \equiv\left(p_{t}+p_{\bar{b}}\right)^{2}, f \equiv\left(p_{u}-p_{\bar{d}}\right) \cdot\left(p_{t}+p_{\bar{b}}\right), w \equiv\left(p_{u}+p_{\bar{d}}\right) \cdot\left(p_{t}+p_{\bar{b}}\right)$ and $\epsilon$ is the Levi-Civita tensor.

For illustration, we adopt here also the value $\tan \beta=0.3$. We fold in the structure functions of the $u$ and the $\bar{d}$ inside the $p$ and $\bar{p}$, respectively, and plot in Fig. 61 the tree-level crosssection for $p \bar{p} \rightarrow t \bar{b} h+X$, with c.m. energies of $\sqrt{s}=2$ and $\sqrt{s}=4 \mathrm{TeV}$. Four possible sets of the Higgs coupling constants $a_{t}^{h}, b_{t}^{h}$ and $c^{h}$ were chosen. For illustrative purposes, the first two sets of parameters which are chosen for a $2 \mathrm{TeV}$ collider are: set I with $\tan \beta=0.3$, $\left\{\alpha_{1}, \alpha_{2}, \alpha_{3}\right\}=\{\pi / 4, \pi / 2,3 \pi / 4\}$ and set II with $\tan \beta=0.3,\left\{\alpha_{1}, \alpha_{2}, \alpha_{3}\right\}=\{\pi / 2, \pi / 2,0\}$. The other two, chosen for a $4 \mathrm{TeV}$ collider are: set III with $\tan \beta=0.3,\left\{\alpha_{1}, \alpha_{2}, \alpha_{3}\right\}=\{\pi / 4, \pi / 2, \pi / 2\}$ 
and set IV with $\tan \beta=0.3,\left\{\alpha_{1}, \alpha_{2}, \alpha_{3}\right\}=\{\pi / 2,3 \pi / 4,3 \pi / 4\}$. The general feature of these sets is that sets I and III give rise to a large CP asymmetry but "small" cross-section, while sets II and IV increase the event rate but decrease the asymmetry. Also, note that each set by itself is not unique, as there are other values of the angles $\alpha_{1}, \alpha_{2}$ and $\alpha_{3}$ for each set which lead to the same effect.

As in the reaction $e^{+} e^{-} \rightarrow t \bar{t} h$ discussed in section 6.2, we are dealing here with a tree-level $\mathrm{CP}$-violating effect. Thus the CP-violating term $\hat{\Sigma}_{-}^{0}$ can probe only CP asymmetries of the $T_{N^{-}}$ odd type. In this case the final state is not a $\mathrm{CP}$ eigenstate. Therefore, one has to construct a $T_{N}$-odd, triple correlation product (or equivalently, a Levi-Civita) which takes into account the conjugate reaction as well $(\bar{u} d \rightarrow \bar{t} b h)$, thus endowing the observable with definite CP properties. This led us to consider the following CP-odd, $T_{N}$-odd observable

$$
O=\left(\epsilon\left(p_{u}, p_{\bar{b}}, p_{t}, p_{h}\right)+\epsilon\left(p_{\bar{u}}, p_{b}, p_{\bar{t}}, p_{h}\right)\right) / s^{2} .
$$

Fig. 62 shows the results for the signal to noise ratio, i.e., the asymmetry $A_{O} \equiv\langle O\rangle$ $/ \sqrt{\left\langle O^{2}\right\rangle}$, for $\sqrt{s}=2 \mathrm{TeV}$ with sets I and II and for $\sqrt{s}=4 \mathrm{TeV}$ with sets III and IV. Evidently, the asymmetry $A_{O}$ is of the order of $10-15 \%$ for a light Higgs particle in the mass range $50 \mathrm{GeV}<m_{h}<100 \mathrm{GeV}$ and of the order of $20-30 \%$ for a heavy Higgs particle with mass in the range $200 \mathrm{GeV}<m_{h}<250 \mathrm{GeV}$, for both set II (which corresponds to a $2 \mathrm{TeV}$ collider) and set IV (which we chose for the $4 \mathrm{TeV}$ collider). Sets I and III give asymmetries of the order of a few percent. Although with these sets, i.e., sets I and III, the cross-section can be 10 times larger than that corresponding to sets II and IV, the statistical significance of the CP-violating effect that can be achieved when the free parameters of the 2HDM are chosen according to sets I and III is much smaller than that with sets II and IV. This is simply due to the fact that the number of events needed to detect the CP-violating effect scale as (asymmetry) ${ }^{-2}$. Therefore, the enhanced effect for sets II and IV makes up for the reduced production rate in those scenarios.

Let us proceed by analyzing the two scenarios which give the large asymmetries. For the reaction at hand, the statistical significance $N_{S D}$ of the CP-odd signal in the collider is

$$
N_{S D}=\sqrt{\mathcal{L}} \sqrt{\sigma(p \bar{p} \rightarrow t \bar{b} h+X)} \times A_{O}
$$

where $\mathcal{L}$ is the collider luminosity. From Fig. 61 we see that $\sigma(p \bar{p} \rightarrow t \bar{b} h+X) \sim 0.1-10$ $\mathrm{fb}$, depending on the parameters $a_{t}^{h}, b_{t}^{h}$ and $c^{h}$ and the neutral Higgs mass. Thus, since $A_{O} \sim$ $0.1-0.3$ in the best cases, it is evident from Eq. 8.53 that, typically, an integrated luminosity of about $\sim 100 \mathrm{fb}^{-1}$ will be required to be able to observe a statistically significant effect in this reaction. Therefore, although the CP asymmetry in this process could reach the $10-30 \%$ level, it is, unfortunately, not likely to be able to produce a CP-violating signal in the next runs of the Tevatron with $\mathcal{L}=2 \mathrm{fb}^{-1}$ and even with $30 \mathrm{fb}^{-1}$. 

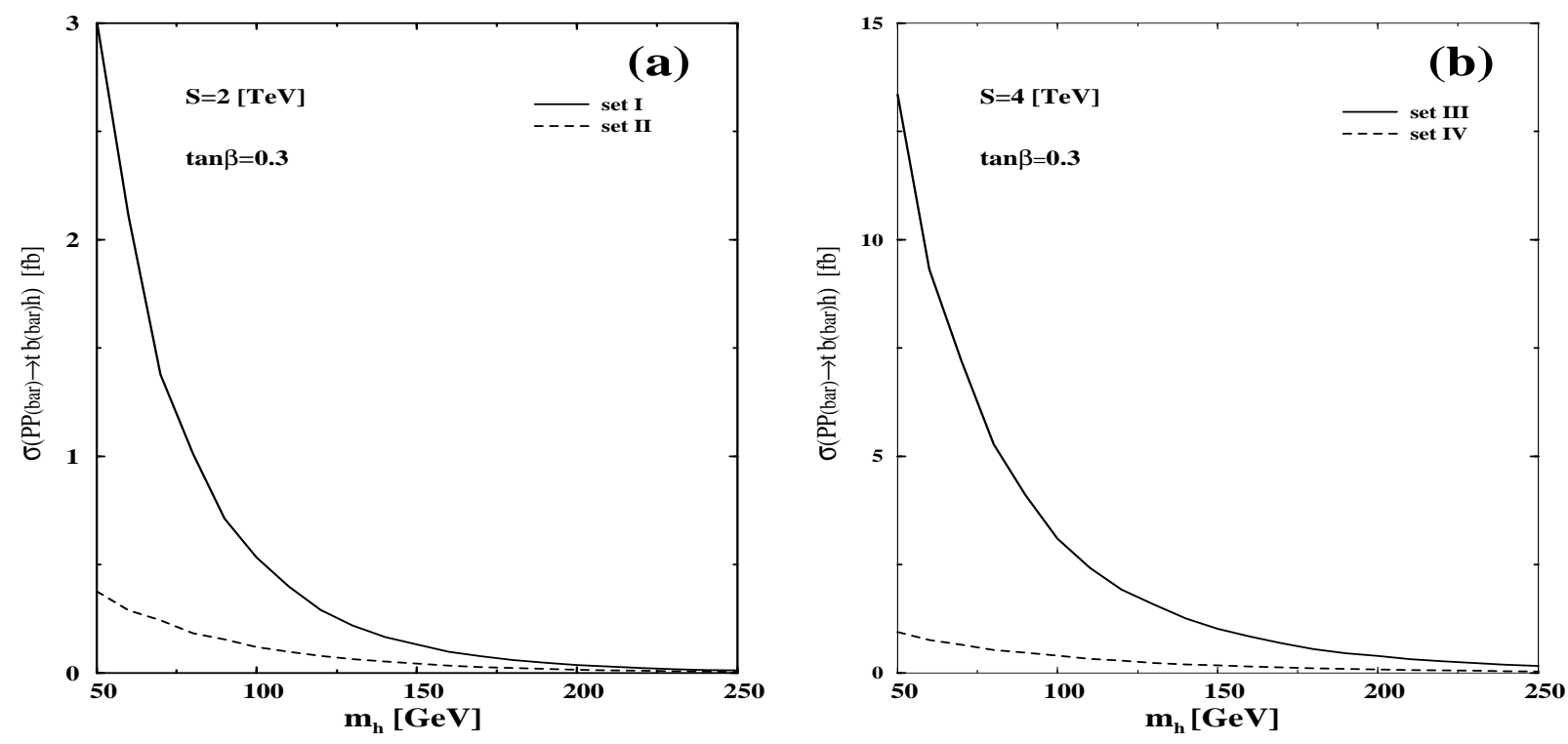

Figure 61: The cross-section for the reaction $p \bar{p} \rightarrow t \bar{b} h+X$ (in fb), for (a): $\sqrt{s}=2$ TeV and for sets I (solid line) and II (dashed line), (b): $\sqrt{s}=4$ TeV and for sets III (solid line) and IV (dashed line). For the definition of the sets I,II,III and IV, see text. 

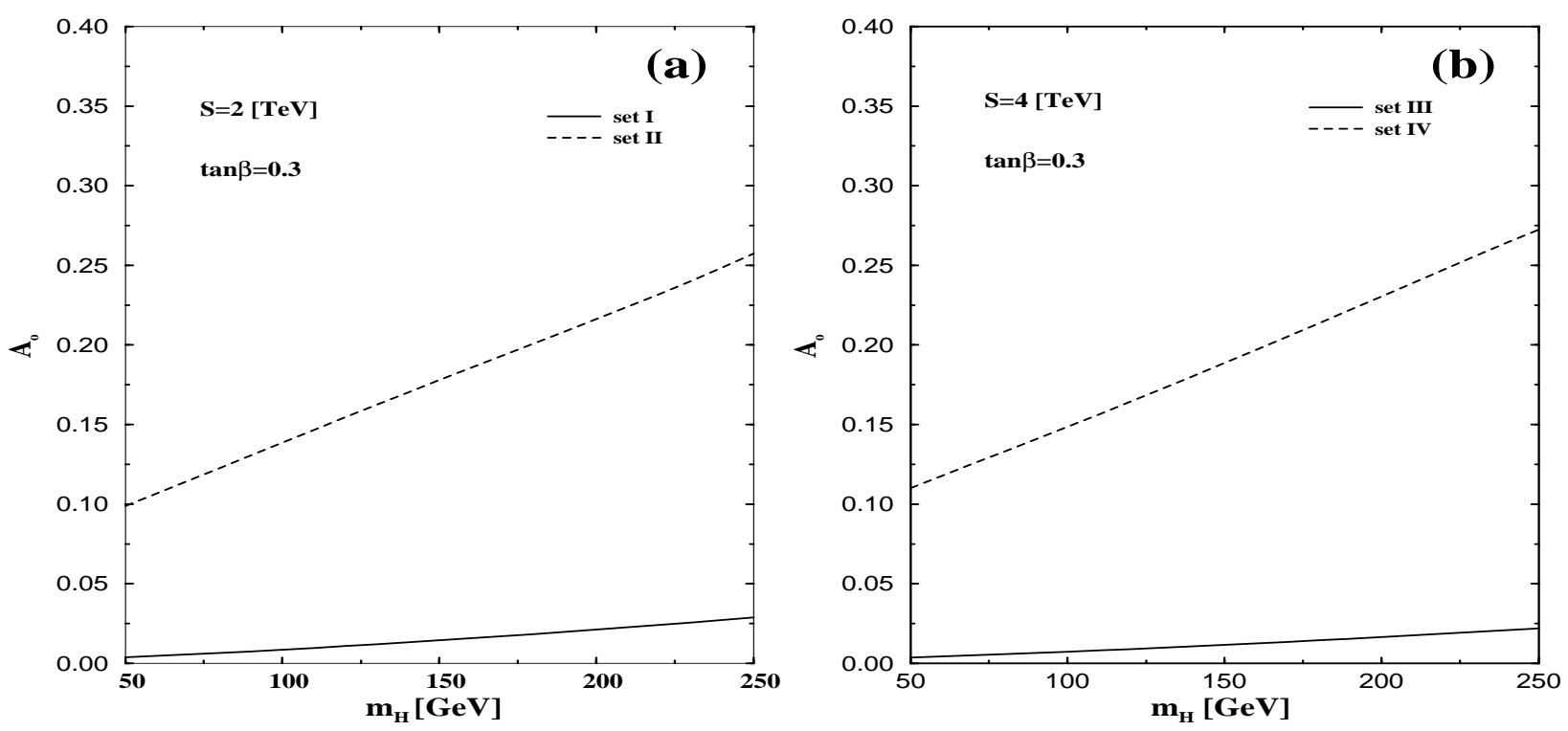

Figure 62: The asymmetry, $A_{O}$ (see text) as a function of $m_{h}$ for $(a): \sqrt{s}=2$ TeV with sets $I$ (solid line) and II (dashed line), (b): $\sqrt{s}=4 \mathrm{TeV}$ with sets III (solid line) and IV (dashed line). For the definition of the sets I,II,III and IV, see text. 


\section{CP violation in $\gamma \gamma$ collider experiments}

Future electron-positron colliders include the attractive option of a linear $\gamma \gamma$ collider, where each beam of photons is produced by Compton backscattering of laser light on an electron or positron beam. The peak energies and luminosities of the $\gamma \gamma$ are expected to be slightly smaller than those of the corresponding $e^{+} e^{-}$collider. The idea was originally suggested in [227, 228]; for recent reviews see [229]. The attractive option of obtaining polarized photon beams is also being considered. Note also that $\gamma \gamma$ collisions have been discussed in the context of heavy ion colliders (for a review see [230]) as well. Unfortunately, the invariant $\gamma \gamma$ mass reach for the LHC (running in its heavy ion mode), will only be about $100 \mathrm{GeV}$, with lower values attainable at RHIC [230]. Therefore, this option will not be discussed here.

\section{1 $\gamma \gamma \rightarrow X:$ general comments}

In a $\gamma \gamma$ collider there are two distinguishable modes: unpolarized and polarized incoming photons. In the unpolarized case, in order to be able to detect $\mathrm{CP}$ violation in the reaction $\gamma \gamma \rightarrow t \bar{t}$ one needs information on the spins of the $t$ and the $\bar{t}$, or equivalently, one needs to construct asymmetries involving the decay products of the top quark. In this case, including the subsequent decays of the $t$ and $\bar{t}$, one can break the differential cross-section for the process

$$
\gamma_{1}\left(k_{1}, \lambda_{1}\right) \gamma_{2}\left(k_{2}, \lambda_{2}\right) \rightarrow t\left(p_{t}, s_{t}\right) \bar{t}\left(p_{\bar{t}}, s_{\bar{t}}\right)
$$

to its CP-odd and CP-even parts as

$$
d \sigma \equiv d \sigma^{o d d}+d \sigma^{e v e n}
$$

In Eq. 9.1 $\lambda_{1}, \lambda_{2}$ denote the helicities of the incoming photons $\gamma_{1}, \gamma_{2}$, respectively, and $s_{t}, s_{\bar{t}}$ are the covariant spins of $t, \bar{t}$, respectively.

In general, the CP-odd terms in Eq. 9.2 has the form

$$
d \sigma^{\text {odd }} \propto \delta_{-} \vec{q} \cdot\left(\vec{s}_{t} \times \vec{s}_{\vec{t}}\right)+\delta_{+} \vec{q} \cdot\left(\vec{s}_{t}-\vec{s}_{\bar{t}}\right)
$$

where $\vec{q}$ is a three momentum of any of the particles in the final or the initial state (i.e., $\vec{q}=$ $\vec{k}_{1}, \quad \vec{k}_{2}, \vec{p}_{t}$ or $\left.\vec{p}_{\bar{t}}\right)$ and $\vec{s}_{t}\left(\vec{s}_{\bar{t}}\right)$ is the spin three vectors of the $t(\vec{t}) . \delta_{-}$and $\delta_{+}$are non-zero only if there is CP violation in the underlying dynamics of the process $\gamma_{1} \gamma_{2} \rightarrow t \bar{t}$. Furthermore, $\delta_{-}$ is proportional to the dispersive, CP-odd, $T_{N}$-odd contributions, while $\delta_{+}$gets its contribution from absorptive, CP-odd, $T_{N}$-even terms. Of course, as mentioned above, the CP-odd polarization correlations of the top and the anti-top in Eq. 9.3 will lead to CP-odd correlations among the momenta of the decay products of the $t$ and the $\bar{t}$. Asymmetries which involve the top decay products in the case of the unpolarized photons were investigated in [175, 231].

If the incoming photons are polarized, then one can construct CP-odd correlations by using linearly polarized photons where no information on the momenta and polarization of the top quark decay products are needed. The amplitude squared for a general final state $X$, i.e., $\gamma \gamma \rightarrow X$, in the case where the two photons are linearly polarized is given by [171]:

$$
\begin{aligned}
d \Sigma(\eta, \bar{\eta} ; \chi, \phi) & =\Sigma_{\text {unpol }}-\frac{1}{2}[\eta \cos (\phi+\chi)+\bar{\eta} \cos (\phi-\chi)] \Re \mathrm{e}\left(\Sigma_{02}\right) \\
& +\frac{1}{2}[\eta \sin (\phi+\chi)-\bar{\eta} \sin (\phi-\chi)] \Im \mathrm{m}\left(\Sigma_{02}\right) \\
& -\frac{1}{2}[\eta \cos (\phi+\chi)-\bar{\eta} \cos (\phi-\chi)] \Re \mathrm{e}\left(\Delta_{02}\right)
\end{aligned}
$$




$$
\begin{aligned}
& +\frac{1}{2}[\eta \sin (\phi+\chi)+\bar{\eta} \sin (\phi-\chi)] \Im \mathrm{m}\left(\Delta_{02}\right) \\
& +\quad \bar{\eta} \cos (2 \phi) \Re \mathrm{e}\left(\Sigma_{22}\right)+\eta \bar{\eta} \sin (2 \phi) \Im \mathrm{m}\left(\Sigma_{22}\right) \\
& +\quad \eta \bar{\eta} \cos (2 \chi) \Re \mathrm{e}\left(\Sigma_{00}\right)+\eta \bar{\eta} \sin (2 \chi) \Im \mathrm{m}\left(\Sigma_{00}\right) .
\end{aligned}
$$

Here $\eta, \bar{\eta}$ are the degrees of linear polarization of the two initial photons, $\chi$ and $\phi$ are the azimuthal angle difference and sum, respectively, and the invariant functions are defined as [171]:

$$
\begin{aligned}
\Sigma_{\text {unpol }} & =\frac{1}{4} \sum_{X}\left[\left|M_{++}\right|^{2}+\left|M_{+-}\right|^{2}+\left|M_{-+}\right|^{2}+\left|M_{--}\right|^{2}\right], \\
\Sigma_{02} & =\frac{1}{2} \sum_{X}\left[M_{++}\left(M_{+-}^{*}+M_{-+}^{*}\right)+\left(M_{+-}+M_{-+}\right) M_{--}^{*}\right], \\
\Delta_{02} & =\frac{1}{2} \sum_{X}\left[M_{++}\left(M_{+-}^{*}-M_{-+}^{*}\right)-\left(M_{+-}-M_{-+}\right) M_{--}^{*}\right], \\
\Sigma_{22} & =\frac{1}{2} \sum_{X}\left(M_{+-} M_{-+}^{*}\right), \\
\Sigma_{00} & =\frac{1}{2} \sum_{X}\left(M_{++} M_{--}^{*}\right) .
\end{aligned}
$$

The subscripts 0 and 2 in Eqs. 9.6 - 9.9 represent the magnitude of the sum of the initial photon helicities and the notation for the helicity amplitudes for the reaction $\gamma_{1}\left(\lambda_{1}\right) \gamma_{2}\left(\lambda_{2}\right) \rightarrow X$ using Eqs. $9.5-9.9$ is

$$
M_{\lambda_{1} \lambda_{2}}=\left\langle X|M| \lambda_{1} \lambda_{2}\right\rangle
$$

Furthermore, the event rate of any final state production through $\gamma \gamma$ fusion can be written in general as [232]:

$$
d N=d \mathcal{L}_{\gamma \gamma} \sum_{i, j=0}^{3}<\xi_{1}^{(i)} \xi_{2}^{(j)}>d \sigma^{i j}
$$

where $\xi_{1}^{(i)}\left(\xi_{2}^{(j)}\right)$ are the so called Stokes polarization parameters for $\gamma_{1}\left(\gamma_{2}\right)$ with $\xi_{1}^{(0)}=\xi_{2}^{(0)}=1$. In particular, $\xi_{1}^{(2)}$ and $\xi_{2}^{(2)}$ are the mean helicities of $\gamma_{1}$ and $\gamma_{2}$, respectively, and $\sqrt{\left(\xi_{i}^{(1)}\right)^{2}+\left(\xi_{i}^{(3)}\right)^{2}}$ are their degrees of linear polarization. Also, $d \mathcal{L}_{\gamma \gamma}$ is the luminosity of the two photons and $\sigma^{i j}$ are the corresponding cross-sections.

There are only three CP-odd functions out of the nine invariant functions in Eqs. 9.5 - 9.9: $\Im \mathrm{m}\left(\Sigma_{02}\right)$, ऽm $\left(\Sigma_{00}\right)$ and $\Re \mathrm{e}\left(\Delta_{02}\right)$. While $\Im \mathrm{m}\left(\Sigma_{02}\right)$ and $\Im \mathrm{m}\left(\Sigma_{00}\right)$ are $T_{N^{-}}$odd, $\Re$ e $\left(\Delta_{02}\right)$ is $T_{N^{-}}$ even. A CP-odd asymmetry can be formed at a $\gamma \gamma$ collider if, for example, the $J=0$ amplitudes of two photons in the CP-even and CP-odd states are both non-vanishing:

$$
\begin{aligned}
& \mathcal{M}[\gamma \gamma \rightarrow X(\mathrm{CP}=+)] \propto \vec{\epsilon}_{1} \cdot \vec{\epsilon}_{2}, \\
& \mathcal{M}[\gamma \gamma \rightarrow X(\mathrm{CP}=-)] \propto\left(\vec{\epsilon}_{1} \times \vec{\epsilon}_{2}\right) \cdot \vec{k}_{1},
\end{aligned}
$$

where $\vec{\epsilon}_{1}$ and $\vec{\epsilon}_{2}$ are the polarizations of the two colliding photons and $\vec{k}_{1}$ is the momentum vector of one photon in the $\gamma \gamma$ c.m. frame. Such an asymmetry can be constructed, for example, by taking the difference of distributions at $\chi= \pm \pi / 4$ [171, 233]: 


$$
A_{00} \equiv \frac{\Im \mathrm{m}\left(\Sigma_{00}\right)}{\Sigma_{\text {unpol }}}=\frac{\int_{0}^{4 \pi} \mathrm{d} \phi\left[\left(\frac{\mathrm{d} \sigma}{\mathrm{d} \phi}\right)_{\chi=\frac{\pi}{4}}-\left(\frac{\mathrm{d} \sigma}{\mathrm{d} \phi}\right)_{\chi=-\frac{\pi}{4}}\right]}{\int_{0}^{4 \pi} \mathrm{d} \phi\left[\left(\frac{\mathrm{d} \sigma}{\mathrm{d} \phi}\right)_{\chi=\frac{\pi}{4}}+\left(\frac{\mathrm{d} \sigma}{\mathrm{d} \phi}\right)_{\chi=-\frac{\pi}{4}}\right]} .
$$

Alternatively, in terms of event rates which correspond to the $(0,0)$ and the unpolarized initial photon-photon states this reads

$$
A_{00}=\frac{N_{00}}{N_{\text {unpol }}}
$$

\section{2 $\gamma \gamma \rightarrow t \bar{t}$ and the top EDM}

Recall that a top EDM, i.e., $d_{t}^{\gamma}$, modifies the SM $t \bar{t} \gamma$ coupling to read

$$
\Gamma_{\mu}=i e \frac{2}{3} \gamma_{\mu}+i d_{t}^{\gamma} \sigma_{\mu \nu} \gamma_{5}\left(p_{t}+p_{\bar{t}}\right)^{\nu}
$$

and CP violation arising due to this EDM of the top can be studied in the reaction $\gamma_{1} \gamma_{2} \rightarrow t \bar{t}$ of Eq. 9.1.

The relevant lowest order Feynman diagrams for $\gamma \gamma \rightarrow t \bar{t}$ are shown in Fig. 63 wherein the top EDM can be folded into any of the $t \bar{t} \gamma$ vertices in those two diagrams. We note however again that, in general, the CP-violating effects in $\gamma \gamma \rightarrow t \bar{t}$ can not necessarily be all attributed to the top quark EDM. For example, in a $2 \mathrm{HDM}$ (see next section) additional box diagrams can give rise to $\mathrm{CP}$-nonconserving terms in the amplitude of the reaction $\gamma \gamma \rightarrow t \bar{t}$.
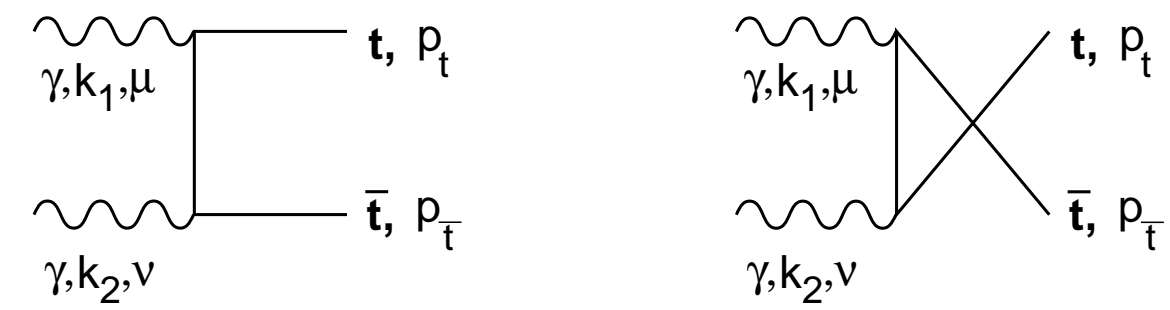

Figure 63: Lowest order Feynman diagrams for the process $\gamma_{1}^{\mu}\left(k_{1}\right) \gamma_{2}^{\nu}\left(k_{2}\right) \rightarrow t\left(p_{t}\right) \bar{t}\left(p_{\bar{t}}\right)$. $\mu$ and $\nu$ are Lorenz indices

With the notation $M\left(\lambda_{1}, \lambda_{2}, \lambda_{t}, \lambda_{\bar{t}}\right)$ for the amplitude, where $\lambda_{1}, \lambda_{2}, \lambda_{t}$ and $\lambda_{\bar{t}}$ correspond to the helicities of the two incoming photons, the top quark and the top anti-quark, respectively, the non-vanishing helicity amplitudes for the process in Eq. 9.1, obtained for combinations such as $\lambda_{2}=\lambda_{1}=\lambda_{\gamma}$ or $\lambda_{2}=-\lambda_{1}=\lambda_{\gamma}$ and $\lambda_{\bar{t}}=\lambda_{t}$ or $\lambda_{\bar{t}}=-\lambda_{t}$, are given by [171, 233, 234]:

$$
\begin{aligned}
M\left(\lambda_{\gamma}, \lambda_{\gamma}, \lambda_{t}, \lambda_{t}\right)= & -4 C_{t} \frac{m_{t}}{\sqrt{s}}\left\{\left(\lambda_{\gamma}+\lambda_{t} \beta_{t}\right)\right. \\
& -i d_{t}^{\gamma} 2 m_{t}\left[2+\frac{s}{4 m_{t}^{2}} \beta_{t}\left(\beta_{t}-\lambda_{t} \lambda_{\gamma}\right) \sin ^{2} \theta_{t}\right] \\
& \left.+\left(d_{t}^{\gamma}\right)^{2} \frac{s \lambda_{\gamma}}{2}\left[\frac{4 m_{t}^{2}}{s}+\beta_{t}\left(\beta_{t}-\lambda_{\gamma} \lambda_{t}\right) \sin ^{2} \theta_{t}\right]\right\},
\end{aligned}
$$




$$
\begin{aligned}
M\left(\lambda_{\gamma}, \lambda_{\gamma}, \lambda_{t},-\lambda_{t}\right)= & -4 m_{t} C_{t} \\
& \times \beta_{t} \sin \theta_{t} \cos \theta_{t}\left[\lambda_{\gamma} i d_{t}^{\gamma}-m_{t}\left(d_{t}^{\gamma}\right)^{2}\right] \\
M\left(\lambda_{\gamma},-\lambda_{\gamma}, \lambda_{t}, \lambda_{t}\right)= & 4 C_{t} \frac{m_{t}}{\sqrt{s}} \\
& \times\left[\lambda_{t} \beta_{t}+i d_{t}^{\gamma} \frac{s}{2 m_{t}} \beta_{t}^{2}-\left(d_{t}^{\gamma}\right)^{2} \frac{s}{2} \lambda_{t} \beta_{t}\right] \sin ^{2} \theta_{t} \\
M\left(\lambda_{\gamma},-\lambda_{\gamma}, \lambda_{t},-\lambda_{t}\right)= & 2 C_{t} \beta_{t} \sin \theta_{t}\left\{\left(\lambda_{\gamma} \lambda_{t}+\cos \theta_{t}\right)\right. \\
- & \left.\left(d_{t}^{\gamma}\right)^{2} \frac{s}{2}\left[\frac{4 m_{t}^{2}}{s} \cos \theta_{t}+\lambda_{\gamma} \lambda_{t}\left(1-\beta_{t}^{2} \cos ^{2} \theta_{t}\right)\right]\right\}
\end{aligned}
$$

where $\theta_{t}$ is the scattering angle in the c.m. frame and $\beta_{t}$ is the top quark velocity and $C_{t}=$ $e^{2} Q_{t}^{2} /\left(1-\beta_{t}^{2} \cos ^{2} \theta_{t}\right)$.

The CP-odd $T_{N}$-odd distribution $\Im \mathrm{m}\left(\Sigma_{00}\right)$ defined in Eq. 9.9 depends linearly on $d_{t}^{\gamma}$ and is given by [171]:

$$
\Im \mathrm{m}\left(\Sigma_{00}\right)=24\left(1-\beta_{t}^{2} \cos ^{2} \theta_{t}\right) \Re \mathrm{e}\left(d_{t}^{\gamma}\right)
$$

and the asymmetry $A_{00}$ defined in Eq. 9.14 can then be calculated [171, 233]. After extracting the top EDM from $A_{00}$ and defining $A_{00} \equiv \Re \mathrm{e}\left(\tilde{\kappa}_{\gamma}\right) \tilde{A}_{00}$, where $\tilde{\kappa}_{\gamma} \equiv 2 m_{t} d_{t}^{\gamma} / e$ is a dimensionless EDM form factor, as in Eq. 6.108, one obtains the allowed sensitivity (i.e., $N_{S D}=$ number of standard deviations) to the dispersive part, $\Re \mathrm{e}\left(\tilde{\kappa}_{\gamma}\right)$, in the case that no asymmetry is found

$$
\operatorname{Max}\left(\left|\Re \mathrm{e}\left(\tilde{\kappa}_{\gamma}\right)\right|\right)=\frac{\sqrt{2} N_{S D}}{\left|\tilde{A}_{00} \sqrt{\varepsilon N_{\text {unpol }}}\right|},
$$

where $\varepsilon$ is the detection efficiency which was taken to be $10 \%$ in [171]. The kinematics of the Compton backscattering process at hand is characterized in part by the dimensionless parameter $x \equiv 2 p_{e} \cdot p_{\gamma} / m_{e}^{2}$. Larger $x$ values are favored to produce highly energetic photons but the degree of linear polarization is larger for smaller $x$ values (for more details see [171]). In particular, the denominator on the r.h.s. of Eq. 9.21 depends on $x$, which for a given c.m. energy squared, $s$, is bounded by

$$
\frac{2 m_{t}}{\sqrt{s}-2 m_{t}} \leq x \leq 2(1+\sqrt{2})
$$

for the process $\gamma \gamma \rightarrow t \bar{t}$ where the upper bound is required to prevent $e^{+} e^{-}$pair production in the scattering of the photon while the lower bound is required to have photons energetic enough to produce top pairs.

In [171] the $x$ dependence of the $\Re$ e $\left(\tilde{\kappa}_{\gamma}\right)$ upper bound, i.e. $\operatorname{Max}\left(\left|\Re \mathrm{e}\left(\tilde{\kappa}_{\gamma}\right)\right|\right)$, was given which is shown in Fig. 64 for two c.m. energies $\sqrt{s}=0.5$ and $1 \mathrm{TeV}$. We see that $\operatorname{Max}\left(\left|\Re \mathrm{e}\left(\tilde{\kappa}_{\gamma}\right)\right|\right)$ can reach below 0.1 where the optimal sensitivities are obtained with $x=3.43$ and $x=0.85$ for $\sqrt{s}=0.5$ and $1 \mathrm{TeV}$, respectively. For these $x$ values, and to 1- $\sigma$, the upper bounds that can be achieved are: $\Re \mathrm{e}\left(\tilde{\kappa}_{\gamma}\right)=0.16$ and $\Re \mathrm{e}\left(\tilde{\kappa}_{\gamma}\right)=0.02$ for $\sqrt{s}=0.5$ and $1 \mathrm{TeV}$, respectively. This corresponds to $\Re \mathrm{e}\left(d_{t}^{\gamma}\right) \approx 0.9 \times 10^{-17}$ and $0.1 \times 10^{-17} \mathrm{e}-\mathrm{cm}$ for $\sqrt{s}=0.5$ and $1 \mathrm{TeV}$, respectively.

Different type of asymmetries which involve the polarization of both the initial photons beams and the decay products of the $t$ and $\bar{t}$ (e.g., $t \rightarrow b \ell \nu_{\ell}$ ) in the reaction $\gamma \gamma \rightarrow t \bar{t}$, were suggested in [234]. The first one is a charge asymmetry, $A_{\mathrm{ch}}$, which measures the difference between the number 
of leptons and anti-leptons produced as decay products of the top and anti-top, respectively. The second, $A_{\mathrm{FB}}$, is a sum of the forward-backward asymmetries of the leptons and anti-leptons and requires polarized laser beams. In terms of the differential cross-sections these asymmetries are given by

$$
A_{\mathrm{ch}}\left(\theta_{0}\right)=\frac{\int_{\theta_{0}}^{\pi-\theta_{0}} d \theta_{\ell}\left(\frac{d \sigma^{+}}{d \theta_{\ell}}-\frac{d \sigma^{-}}{d \theta_{\ell}}\right)}{\int_{\theta_{0}}^{\pi-\theta_{0}} d \theta_{\ell}\left(\frac{d \sigma^{+}}{d \theta_{\ell}}+\frac{d \sigma^{-}}{d \theta_{\ell}}\right)}
$$

and

$$
A_{\mathrm{FB}}\left(\theta_{0}\right)=\frac{\int_{\theta_{0}}^{\frac{\pi}{2}} d \theta_{\ell}\left(\frac{d \sigma^{+}}{d \theta_{\ell}}+\frac{d \sigma^{-}}{d \theta_{\ell}}\right)-\int_{\frac{\pi}{2}}^{\pi-\theta_{0}} d \theta_{\ell}\left(\frac{d \sigma^{+}}{d \theta_{\ell}}+\frac{d \sigma^{-}}{d \theta_{\ell}}\right)}{\int_{\theta_{0}}^{\pi-\theta_{0}} d \theta_{\ell}\left(\frac{d \sigma^{+}}{d \theta_{\ell}}+\frac{d \sigma^{-}}{d \theta_{\ell}}\right)} .
$$

where $\frac{d \sigma^{+}}{d \theta_{\ell}}$ and $\frac{d \sigma^{-}}{d \theta_{\ell}}$ refer to the $\ell^{+}$and $\ell^{-}$distributions in the c.m. frame, respectively, and $\theta_{0}$ is a cutoff on the polar angle of the lepton. It is important to note that if there is no CP violation in the top decays, then the charge asymmetry is zero in the absence of the cutoff $\theta_{0}$.

Both $A_{\mathrm{ch}}$ and $A_{\mathrm{FB}}$ are $T_{N}$-even asymmetries, thus they probe the imaginary part of the top $\mathrm{EDM}, \Im \mathrm{m}\left(d_{t}^{\gamma}\right)$. In [234], the case where one of the $t$ or $\bar{t}$ decays leptonically and the other decays hadronically, was studied [3. Also, it was assumed that no $\mathrm{CP}$ violation enters these top decays. The asymmetries $A_{\mathrm{ch}}$ and $A_{\mathrm{FB}}$ were then evaluated in the $\gamma \gamma$ c.m. frame and $90 \%$ C.L. limits on the top EDM, in the case that no asymmetry is found in the experiment, were obtained. The $90 \%$ C.L. limits were evaluated for different electron and laser beam energies as well as for different cutoff angles. Also, different helicity combinations of the initial beam and different values of the dimensionless parameter $x$ defined before (see Eq. 9.22 and the discussion above), were analyzed. They found that for an electron beam energy of $250 \mathrm{GeV}$, and for a suitable choice of circular polarizations of the laser photons and longitudinal polarizations for the electron beams, and assuming a luminosity of $20 \mathrm{fb}^{-1}$ for the electron beam, in the best cases and in an ideal experiment, it is possible to obtain limits on the imaginary part of the top EDM, again, of the order of $10^{-17} \mathrm{e}-\mathrm{cm}$. However, an order of magnitude improvement may be possible if the beam energy is increased to $500 \mathrm{GeV}$ [234].

To conclude this section, the reaction $\gamma \gamma \rightarrow t \bar{t}$ can serve to limit both the real and the imaginary parts of the top EDM. However, it is worth mentioning that the limits that may be placed on the top EDM through a CP study in $\gamma \gamma \rightarrow t \bar{t}$ are roughly comparable to those which might be obtained through a study of the reaction $e^{+} e^{-} \rightarrow t \bar{t}$ at the NLC (for comparison see section 6.1). Therefore, the motivation for going to a $\gamma \gamma$ collider in order to study effects of the top EDM is somewhat arguable. On the other hand, model calculations of CP violation in $\gamma \gamma \rightarrow t \bar{t}$, such as the one described below, i.e., a 2HDM, show that CP-nonconserving signals in this reaction, which are not necessarily associated with the top EDM, may be sizable; i.e., at the detectable level in a future photon collider.

\footnotetext{
${ }^{33}$ then the asymmetries correspond to samples $\mathcal{A}$ and $\overline{\mathcal{A}}$ defined in Eqs. 6.79 and 6.80 in section 6.2 .3
} 


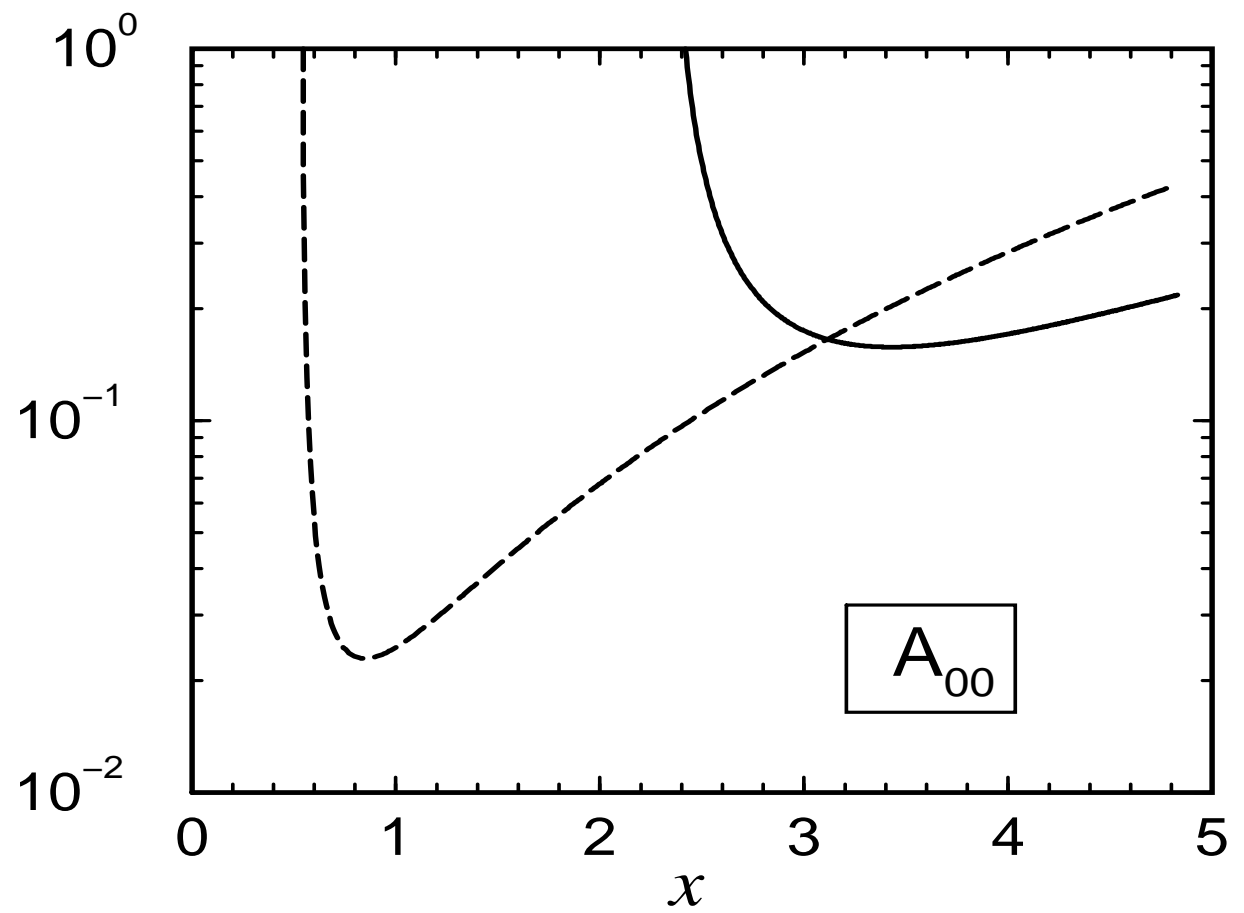

Figure 64: The $x$ dependence of the $\Re \mathrm{e}\left(\tilde{\kappa}_{\gamma}\right)$ upper bound, i.e., $\operatorname{Max}\left(\left|\Re \mathrm{e}\left(\tilde{\kappa}_{\gamma}\right)\right|\right)$, at $\sqrt{s}=0.5 \operatorname{TeV}$ (solid line) and $1 \mathrm{TeV}$ (dashed line), from the asymmetry $A_{00}$. Figure taken from [171]. 


\section{3 $\gamma \gamma \rightarrow t \bar{t}$ and $s$-channel Higgs exchange in a 2HDM}

A $\gamma \gamma$ collider can also provide an interesting possibility for producing an s-channel neutral Higgsboson, via $\gamma \gamma \rightarrow h$, which can then decay to a pair of fermions, $h \rightarrow f \bar{f}$ (recall that a related process was considered in the context of a $p p$ collider in section 7.3.2). Once again, $h$ stands for the lightest neutral Higgs-boson in a MHDM and the other neutral Higgs particles are assumed to be much heavier, thus, neglecting their contribution in what follows.

The decay of a neutral Higgs, for $m_{h}>2 m_{t}$, to a pair of $t \bar{t}$ will inevitably dominate the other fermionic decays of the Higgs due to the largeness of the top mass. CP violation in the reaction $\gamma \gamma \rightarrow t \bar{t}$ was investigated within a MHDM in 2231 for unpolarized incoming photons, where the effects of the s-channel Higgs were included. In 232] polarized laser beams were considered for the s-channel neutral Higgs production, $\gamma \gamma \rightarrow h$.

In [231] the complete set of CP-nonconserving contributions to $\gamma \gamma \rightarrow t \bar{t}$, at the 1-loop order, were considered within a $2 \mathrm{HDM}$ of type II. Recall that, in the SM, CP violation cannot occur in this process at least to 2-loop order. This set of 1-loop Feynman diagrams is depicted in Figs. 65(b)-65(h) and Fig. 65(a) represents the only tree-level diagram for this process; this tree-level diagram and its permuted one are also shown in Fig. 63 .

We define $\mathcal{M}_{i}$ to be the amplitude for a diagram $i$ in Fig. 65 (i.e., $i=a, \ldots, h$ ) where we further decompose $\mathcal{M}_{i}$ to its CP-odd part $\mathcal{M}_{i}^{\text {odd }}$ and CP-even part $\mathcal{M}_{i}^{\text {even }}: \mathcal{M}_{i}=\mathcal{M}_{i}^{\text {odd }}+\mathcal{M}_{i}^{\text {even }}$. Then, to leading order, the CP-odd $\left(d \sigma^{\text {odd }}\right)$ and the CP-even $\left(d \sigma^{\text {even }}\right)$ parts of the differential cross-section are given by

$$
\begin{aligned}
& d \sigma^{\text {odd }}=2 \Re \mathrm{e} \sum_{\gamma \text { pol. }} \sum_{i=b}^{h} \mathcal{M}_{a} \mathcal{M}_{i}^{\text {odd } \dagger}, \\
& d \sigma^{\text {even }}=\sum_{\gamma \text { pol. }}\left[\mathcal{M}_{a} \mathcal{M}_{a}^{\dagger}+\sum_{i=f}^{h} 2 \Re \mathrm{e} \mathcal{M}_{a} \mathcal{M}_{i}^{\text {even }} \dagger\right] .
\end{aligned}
$$

Note that the CP-even contribution from the interference of the $s$-channel Higgs graphs Fig. 65(f)65(h) with the Born amplitude of Fig. 65(a), which is explicitly included in Eq. 9.26, can become important and was taken into account in 2231] because of the non-negligible width of the Higgs. Also, the CP-odd interference in Eq. 9.25 will give rise to the simple form of $d \sigma^{\text {odd }}$ in Eq. 9.3.

Again, to efficiently trace the CP-odd spin correlations in Eq. 9.3, one defines a $t \bar{t}$ decay scenario where the $t$ decays leptonically and the $\bar{t}$ decays hadronically and vice versa. As in Eqs. 6.79 and 6.80 in section 6.2 .3 , we denote by $\mathcal{A}$ the decay sample in the case that the top decays leptonically and the anti-top decays hadronically, and by $\overline{\mathcal{A}}$ the charged conjugate decay sample [175, 231].

With these decay scenarios one can evaluate a few CP-odd asymmetries of both the $T_{N^{-}}$ odd and $T_{N^{-}}$even type which may acquire a non-vanishing value only if $\delta_{-} \neq 0$ and $\delta_{+} \neq 0$, respectively (see Eq. 9.3). To do so, let us define for sample $\mathcal{A}$, i.e., $t \rightarrow W^{+} b \rightarrow \ell^{+} \nu_{\ell} b$ and $\bar{t} \rightarrow W^{-} \bar{b} \rightarrow q \bar{q} \bar{b}$, the following operators

$$
\begin{aligned}
& \mathcal{O}_{1}=\left(\hat{\mathbf{q}}_{\ell^{+}} \times \hat{\mathbf{q}}_{W^{-}}^{*}\right) \cdot \hat{\mathbf{p}}_{\bar{t}}, \\
& \mathcal{O}_{2}=E_{\ell^{+}}, \\
& \mathcal{O}_{3}=\hat{\mathbf{q}}_{\ell^{+}} \cdot \hat{\mathbf{p}}_{\bar{t}},
\end{aligned}
$$

where the asterisk denotes the $t(\bar{t})$ rest frame. The corresponding ones for the sample $\overline{\mathcal{A}}$ are

$$
\begin{aligned}
& \overline{\mathcal{O}}_{1}=\left(\hat{\mathbf{q}}_{\ell^{-}} \times \hat{\mathbf{q}}_{W^{+}}^{*}\right) \cdot \hat{\mathbf{p}}_{t}, \\
& \overline{\mathcal{O}}_{2}=E_{\ell^{-}}, \\
& \overline{\mathcal{O}}_{3}=\hat{\mathbf{q}}_{\ell^{-}} \cdot \hat{\mathbf{p}}_{t} .
\end{aligned}
$$


Thus, the CP-odd asymmetries are constructed as [175, 231]:

$$
\begin{aligned}
& T_{N} \text { - odd : } \quad \alpha_{1}=<\mathcal{O}_{1}>+<\overline{\mathcal{O}}_{1}>, \\
& T_{N} \text { - even }: \quad \alpha_{2,3}=<\mathcal{O}_{2,3}>-<\overline{\mathcal{O}}_{2,3}>.
\end{aligned}
$$

To calculate the above asymmetries one has to fold in the distribution functions of the backscattered laser photons (for more details see [175, 231]). Also, one has to choose a definite scheme for the type II 2HDM couplings $a_{t}^{h}$ and $b_{t}^{h}$ of a neutral Higgs particles to a pair of $t \bar{t}$ in Eq. 3.70. Recall that the scalar $a_{h}^{t}$ and pseudoscalar $b_{h}^{t}$ couplings are functions of the neutral Higgs mixing matrix $R_{j i}$ in Eq. 3.73 and of the ratio between the two VEV's $\tan \beta$ (see section 3.2.3). In particular, as in [175, 231], assuming that the other two neutral Higgs of the model are much heavier than $h$ such that their mass lies above the $\gamma \gamma$ c.m. energy, their contribution is neglected. Also, the mass of the charged Higgs-boson was taken as $m_{H^{ \pm}}=500 \mathrm{GeV}$ and $R_{j 1}=1 / \sqrt{3}$ for $j=1,2,3$ was assumed (see Eq. 3.73).

Let us present a sample of the results that were obtained in [175, 231]. The $T_{N}$-odd asymmetry, i.e., the signal to noise ratio $\alpha_{1} / \Delta \alpha_{1}$, is shown in Fig. 66 and the $T_{N}$-even asymmetry ratio $\alpha_{3} / \Delta \alpha_{3}$ is depicted in Fig. 67. The asymmetries are plotted for various values of $\tan \beta$. In Fig. $66 \tan \beta=0.5$ (dashed line) and $\tan \beta=1$ (solid line) were used, while in Fig. $67 \tan \beta=0.3$ (dashed line) and $\tan \beta=0.627$ (solid line) were chosen. In those figures an $e^{+} e^{-}$collider energy of $\sqrt{s}=500 \mathrm{GeV}$ was taken, and the asymmetries were plotted for the above values of the 2HDM free parameters and as a function of the lightest Higgs mass.

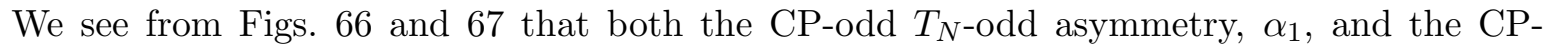
odd $T_{N}$-even asymmetry, $\alpha_{3}$, peak twice. First when the mass of the Higgs is close to the $t \bar{t}$ threshold and then when it is close to the maximal $\gamma \gamma$ energy. With $m_{h} \approx 350$ or $400 \mathrm{GeV}$ these asymmetries can reach above $10 \%$. In particular, $\alpha_{3}$ would lead to a somewhat higher CP-violating signal and we see from Fig. 67 that, for $\tan \beta=0.3,\left|\alpha_{3}\right| / \Delta \alpha_{3}$ is above the $10 \%$ level in almost the entire mass range $100 \mathrm{GeV}<m_{h}<500 \mathrm{GeV}$, and peaks around $m_{h} \approx 2 m_{t}$ at $50 \%$. Recall that the statistical significance $N_{S D}^{i}$ of the CP-violating signal that can be measured with a CP-odd asymmetry $\alpha_{i}$ is given by

$$
N_{S D}^{i}=\frac{\left|\alpha_{i}\right|}{\Delta \alpha_{i}} \sqrt{N_{\exp }}
$$

where $N_{\exp }=R_{\mathcal{A}, \overline{\mathcal{A}}} \times \mathcal{L} \sigma_{0}$ is the number of expected events, with $R_{\mathcal{A}, \overline{\mathcal{A}}}$ the branching ratios for the decay scenarios $\mathcal{A}, \overline{\mathcal{A}}$, respectively, and assuming a reconstruction efficiency of 1 . Furthermore, $\mathcal{L}$ is the collider integrated luminosity and $\sigma_{0}$ is the cross-section which, in the leading order, is calculated from $d \sigma^{\text {even }}$ in Eq. 9.26. With $\mathcal{L}=\mathcal{O}\left(10^{2}\right) \mathrm{fb}^{-1}$ the expected number of $\gamma \gamma \rightarrow t \bar{t}$ events is of the order of few $\times 10^{3}$ for collider c.m. energies of $500-700 \mathrm{GeV}$. Thus, for example, if we take $R_{\mathcal{A}, \overline{\mathcal{A}}}=4 / 27$ such that only leptonic top decays into electrons and muons are considered, then an asymmetry larger than $10 \%$ will correspond to a signal-to-noise ratio above the 3- $\sigma$ level [175].

As previously discussed, if the polarization of the backscattered photons is adjustable then $\mathrm{CP}$ asymmetries involving these polarizations can be constructed and they, in turn, can serve as an efficient tool for investigating the CP properties of the neutral Higgs-boson. Three such polarization asymmetries were suggested in [232]:

$$
\begin{aligned}
\mathcal{P}_{1} & \equiv \frac{\left|\mathcal{M}_{++}\right|^{2}-\left|\mathcal{M}_{--}\right|^{2}}{\left|\mathcal{M}_{++}\right|^{2}+\left|\mathcal{M}_{--}\right|^{2}}, \\
\mathcal{P}_{2} & \equiv \frac{2 \Im \mathrm{m}\left(\mathcal{M}_{---}^{*} \mathcal{M}_{++}\right)}{\left|\mathcal{M}_{++}\right|^{2}+\left|\mathcal{M}_{--}\right|^{2}}, \\
\mathcal{P}_{3} & \equiv \frac{2 \Re \mathrm{e}\left(\mathcal{M}_{---}^{*} \mathcal{M}_{++}\right)}{\left|\mathcal{M}_{++}\right|^{2}+\left|\mathcal{M}_{--}\right|^{2}} .
\end{aligned}
$$


In the helicity basis, one can choose the polarization of $\gamma_{1}$ (moving in the $+z$ direction) as: $\epsilon_{1}^{ \pm}=$ $\mp 2^{-1 / 2}(0,1, \pm i, 0)$, and that of $\gamma_{2}$ (moving in the $-z$ direction) as: $\epsilon_{2}^{ \pm}=\mp 2^{-1 / 2}(0,-1, \pm i, 0$ ). To understand how the above polarization asymmetries $\left(\mathcal{P}_{1,2,3}\right)$ trace the CP properties of the neutral Higgs, note that a CP-even (CP-odd) scalar couples to two photons via $F_{\mu \nu} F^{\mu \nu}\left(F_{\mu \nu} \tilde{F}^{\mu \nu}\right)$ (see [232] and references therein). Therefore, as implied from Eq. 9.12, in the c.m. of the two photons this will yield a coupling proportional to $\vec{\epsilon}_{1} \cdot \vec{\epsilon}_{2}\left(\left(\vec{\epsilon}_{1} \times \vec{\epsilon}_{2}\right)_{z}\right)$ for a CP-even (CP-odd) neutral Higgs to a $\gamma_{1} \gamma_{2}$ pair.

For the above convention of the polarizations one finds

$$
\overrightarrow{\epsilon_{1}} \cdot \vec{\epsilon}_{2}=-\frac{1}{2}\left(1+\lambda_{1} \lambda_{2}\right) \quad, \quad\left(\vec{\epsilon}_{1} \times \overrightarrow{\epsilon_{2}}\right)_{z}=\frac{i}{2} \lambda_{1}\left(1+\lambda_{1} \lambda_{2}\right),
$$

where $\lambda_{1}, \lambda_{2}= \pm 1$ are the helicities of $\gamma_{1}, \gamma_{2}$, respectively. Now, for a mixed CP state, the general amplitude to couple to $\gamma_{1} \gamma_{2}$ will have both the CP-even and the CP-odd pieces in Eq. 9.12 and it can be written as

$$
\mathcal{M}=\delta_{\text {even }} \vec{\epsilon}_{1} \cdot \vec{\epsilon}_{2}+\delta_{\text {odd }}\left(\vec{\epsilon}_{1} \times \vec{\epsilon}_{2}\right)_{z},
$$

where $\delta_{\text {even }}\left(\delta_{\text {odd }}\right)$ is the CP-even (CP-odd) coupling strength of the neutral Higgs to the two photons. Using Eq. 9.39, the squares of the helicity amplitudes which appear in $\mathcal{P}_{1,2,3}$ can be readily calculated [232]:

$$
\begin{gathered}
\left|\mathcal{M}_{++}\right|^{2}+\left|\mathcal{M}_{--}\right|^{2}=2\left(\left|\delta_{\text {even }}\right|^{2}+\left|\delta_{\text {odd }}\right|^{2}\right), \\
2 \Re \mathrm{e}\left(\mathcal{M}_{--}^{*} \mathcal{M}_{++}\right)=2\left(\left|\delta_{\text {even }}\right|^{2}-\left|\delta_{\text {odd }}\right|^{2}\right), \\
\left|\mathcal{M}_{++}\right|^{2}-\left|\mathcal{M}_{--}\right|^{2}=-4 \Im \mathrm{m}\left(\delta_{\text {even }} \delta_{\text {odd }}^{*}\right), \\
2 \Im \mathrm{m}\left(\mathcal{M}_{--}^{*} \mathcal{M}_{++}\right)=-4 \Re \mathrm{e}\left(\delta_{\text {even }} \delta_{\text {odd }}^{*}\right),
\end{gathered}
$$

where $\delta_{\text {even }}$ and $\delta_{\text {odd }}$ are given in 232] for a 2HDM with scalar and pseudoscalar couplings of a neutral Higgs to a pair of fermions. It is then evident that $\mathcal{P}_{1}, \mathcal{P}_{2} \neq 0$ and $\left|\mathcal{P}_{3}\right|<1$ only if both $\delta_{\text {even }}, \delta_{\text {odd }} \neq 0$. That is, only if both the CP-even and the CP-odd couplings are present.

Using Eq. 9.11, for the Higgs-boson production of our interest, one gets [232]:

$$
\begin{aligned}
d N=\quad & d \mathcal{L}_{\gamma \gamma} d \Gamma \frac{1}{4}\left(\left|\mathcal{M}_{++}\right|^{2}+\left|\mathcal{M}_{--}\right|^{2}\right) \\
& \times\left[\left(1+<\xi_{1}^{(2)} \xi_{2}^{(2)}>\right)+\left(<\xi_{1}^{(2)}>+<\xi_{2}^{(2)}>\right) \mathcal{P}_{1}\right. \\
& +\left(<\xi_{1}^{(3)} \xi_{2}^{(1)}>+<\xi_{1}^{(1)} \xi_{2}^{(3)}>\right) \mathcal{P}_{2} \\
& \left.+\left(<\xi_{1}^{(3)} \xi_{2}^{(3)}>-<\xi_{1}^{(1)} \xi_{2}^{(1)}>\right) \mathcal{P}_{3}\right]
\end{aligned}
$$

where $d \Gamma$ is the appropriate element of the final state phase space including the initial state flux factor. Note that the properties of $d \mathcal{L}_{\gamma \gamma}$ and of the various $\xi$ 's (appearing in Eq. 9.45) as a function of the c.m. energy of the two photons are very important for this discussion as they depend strongly on the polarization of the incoming electrons and associated photons. Instead of presenting a detailed analysis of those parameters and the numerical results, we refer the reader to 232. We will only give their summary for a general $2 \mathrm{HDM}$ in which the CP properties of a single neutral Higgs have to be determined. In particular, it was found in [232] that out of the three polarization asymmetries defined in Eqs. 9.36- 9.38, $\mathcal{P}_{1}$ provides the best statistical significance for the task at hand. A non-zero value for $\mathcal{P}_{1}$ requires that the $h \gamma \gamma$ coupling has an imaginary part, as well as both CP-even and CP-odd contributions. For a mixed CP Higgsboson with $m_{h} \lesssim 2 m_{W}$, a measurement of $\mathcal{P}_{1}$ will be easiest if $\tan \beta$ is large since the $b$-quark 
loop, which makes the only large contribution to the imaginary part for such $m_{h}$ values, will be enhanced. For $m_{h}>2 m_{W}$, the required imaginary part is dominated by the $W$-boson loop (or $t$-quark loop if $m_{h}$ is also $>2 m_{t}$ ); large $\tan \beta$ makes detection more difficult since the dominant $\mathrm{CP}$-odd contribution originates from the $t$-quark loop, which will be suppressed.

To summarize, the production of a neutral Higgs-boson by fusion of backscattered laser beams can provide a systematic analysis of the CP properties of the Higgs particle. In particular, $\gamma \gamma \rightarrow h \rightarrow t \bar{t}$ would be a promising channel for exploring CP-violating effects that can arise from an extended Higgs sector, as for quite a large range of the 2HDM parameter space this reaction can exhibit statistically significant CP-nonconserving signals in a high energy $\gamma \gamma$ collider running at c.m. energy of $\sqrt{s} \simeq 500 \mathrm{GeV}$. Moreover, if the polarizations of the incoming photons are controlled, then detailed information on both the scalar and the pseudoscalar couplings of the neutral Higgs to a pair of fermions may be extracted by considering polarization asymmetries of the two colliding photons. If the neutral Higgs is a pure CP eigenstate, the polarization asymmetries $\mathcal{P}_{1}$ and $\mathcal{P}_{2}$ in Eqs. 9.36 and 9.37 will vanish, while, in Eq. 9.38, $\mathcal{P}_{3}=1(-1)$ for a CP-even (odd) neutral Higgs. Therefore, a non-vanishing value for $\mathcal{P}_{1}$ and $\mathcal{P}_{2}$ and $\mathcal{P}_{3}<1$ will imply the existence of an extended Higgs sector beyond the SM and of CP violation in the scalar potential. 


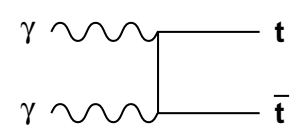

(a)

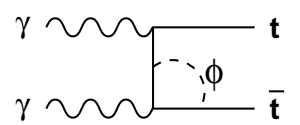

(c)

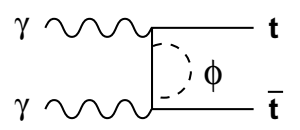

(e)

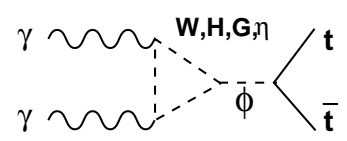

(g)

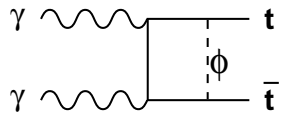

(b)

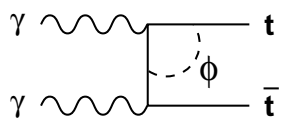

(d)

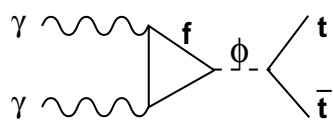

(f)

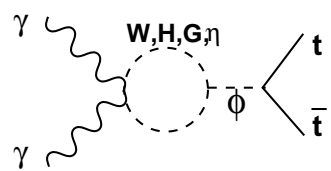

(h)

Figure 65: Feynman diagrams for $\gamma \gamma \rightarrow t \bar{t}$. In (a) the Born diagram is shown (see also Fig. 63), and in (b)-(h) the complete set of 1-loop diagrams that can violate CP are depicted. Diagrams with crossed lines are not shown. 


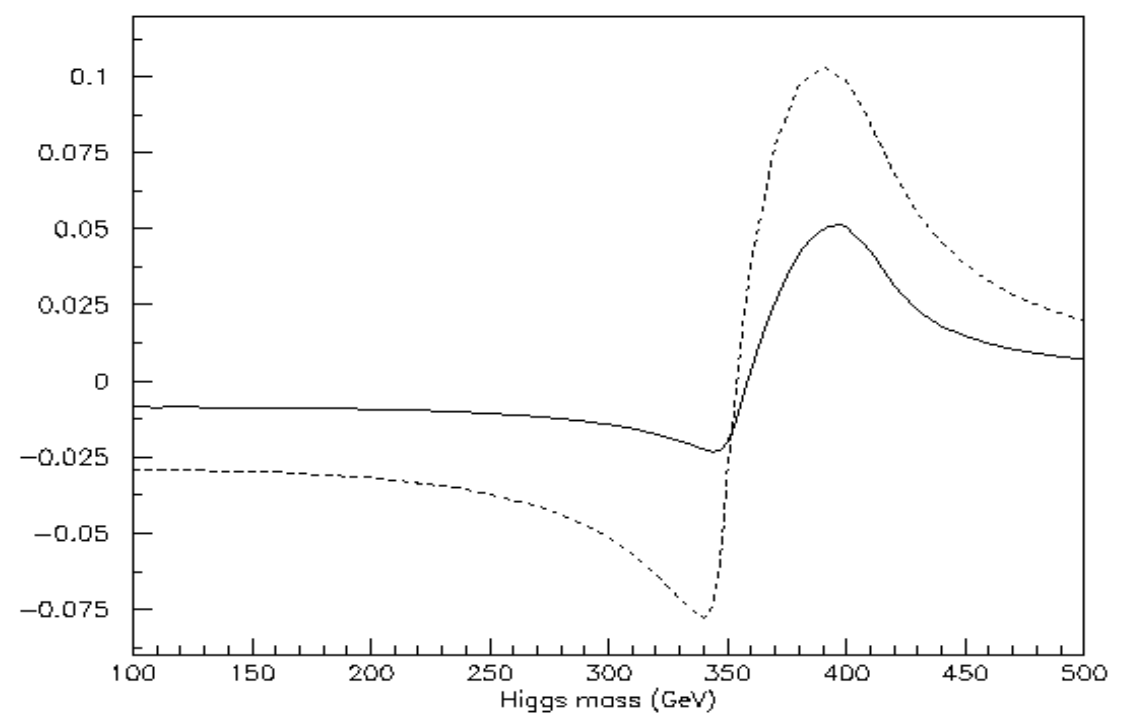

Figure 66: The ratio $\alpha_{1} / \Delta \alpha_{1}$ as a function of the lightest Higgs-boson mass, $m_{h}$, at $\sqrt{s}=0.5$ $\mathrm{TeV}$. The dashed line corresponds to $\tan \beta=0.5$ and the solid line to $\tan \beta=1 ; m_{t}=175 \mathrm{GeV}$. Figure taken from [231. 


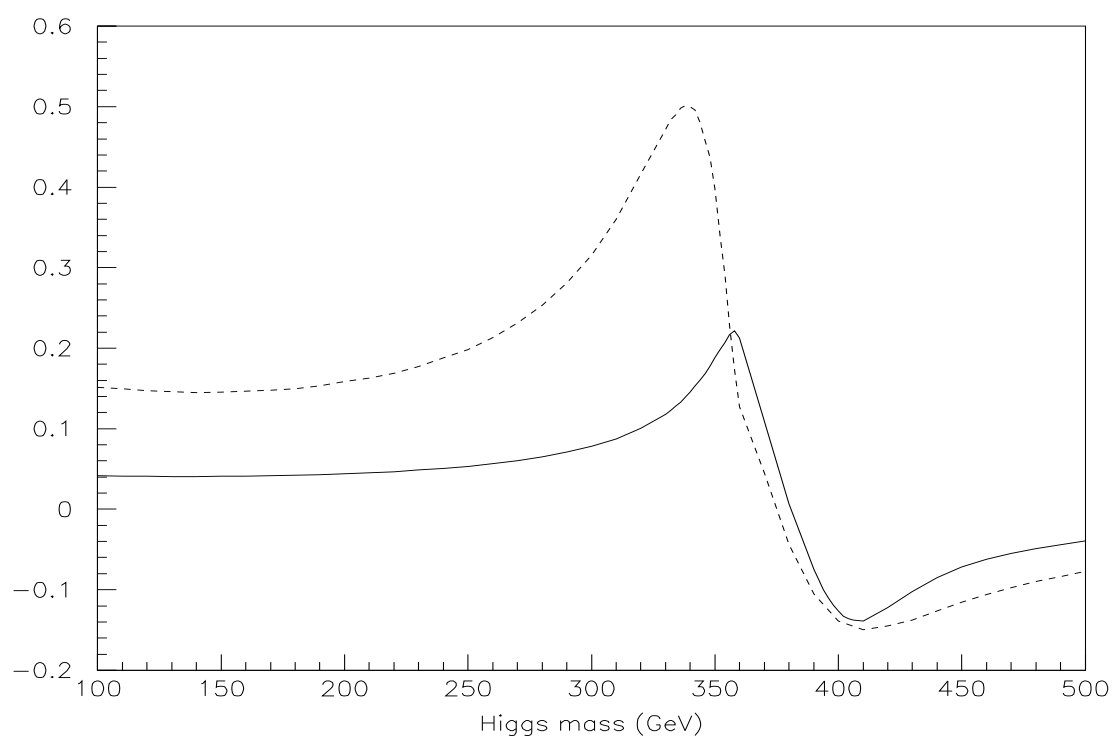

Figure 67: The ratio $\alpha_{3} / \Delta \alpha_{3}$ as a function of the lightest Higgs-boson mass, $m_{h}$, at $\sqrt{s}=0.5$ $\mathrm{TeV}$. The dashed line corresponds to $\tan \beta=0.3$ and the solid line to $\tan \beta=0.627 ; m_{t}=180$ GeV. Figure taken from [175]. 


\section{$10 \mathrm{CP}$ violation in $\mu^{+} \mu^{-}$collider experiments}

The idea to build a high energy $\mu^{+} \mu^{-}$collider is more than 25 years old [235]. It has recently gained interest in part due to the interesting possibility of using it as an " $s$-channel Higgs factory". Of course, it may also be suitable for tackling some other physics issues, e.g., SUSY. For recent reviews see [236]. The c.m. energies considered range from $100 \mathrm{GeV}$ to $4 \mathrm{TeV}$ or even more, with luminosity comparable or higher than in linear $e^{+} e^{-}$colliders. The subject is still in its infancy compared with the more established technologies of linear $e^{+} e^{-}$colliders, and of hadronic colliders such as the Tevatron or the LHC.

\section{$10.1 \mu^{+} \mu^{-} \rightarrow t \bar{t}$}

If there exists a Higgs-boson with mass of a few hundred $\mathrm{GeV}$, a muon collider running at the Higgs resonance can provide the fascinating and unique possibility of an in-depth study of the Higgs particle in the $s$-channel. In particular, the CP-violating properties of its coupling to $t \bar{t}$ may be studied via the reaction

$$
\mu^{+} \mu^{-} \rightarrow \mathcal{H} \rightarrow t \bar{t}
$$

where we have generically denoted the neutral Higgs resonance under study by $\mathcal{H}$.

It is perhaps surprising that Higgs-bosons can be produced at an appreciable rate at a muon collider. Indeed, the $\mathcal{H} \mu \mu$ coupling is very small since it is proportional to the mass of the muon, $m_{\mu}$. On the other hand, if the c.m. energy of the accelerator can be tuned to be at $s=m_{\mathcal{H}}^{2}$, then the cross-section receives appreciable enhancement due to the resonant production of the Higgs. To see how this works, consider a collider tuned precisely at the Higgs resonance, $s=m_{\mathcal{H}}^{2}$, then the cross-section $\sigma_{\mathcal{H}} \equiv \sigma\left(\mu^{+} \mu^{-} \rightarrow \mathcal{H}\right)$ for neutral Higgs-boson production is given by

$$
\sigma_{\mathcal{H}}=\frac{4 \pi}{m_{\mathcal{H}}^{2}} B_{\mu}
$$

where $B_{\mu}$ is the branching ratio of $\mathcal{H} \rightarrow \mu^{+} \mu^{-}$. It is useful to compare this with the point cross-section

$$
\sigma_{0}=\sigma\left(\mu^{+} \mu^{-} \rightarrow \gamma^{*} \rightarrow e^{+} e^{-}\right)
$$

Thus

$$
R(\mathcal{H})=\frac{\sigma_{\mathcal{H}}}{\sigma_{0}}=\frac{3}{\alpha^{2}} B_{\mu},
$$

where $\alpha$ is the fine-structure constant. Therefore, $\sigma_{\mathcal{H}}$ and $R(\mathcal{H})$ are enhanced if the neutral Higgs has a narrow width, i.e., a relatively large $B_{\mu}=\Gamma\left(\mathcal{H} \rightarrow \mu^{+} \mu^{-}\right) / \Gamma \mathcal{H}$.

One simple way to study $\mathrm{CP}$ violation at a muon collider is via the decays $\mathcal{H} \rightarrow t \bar{t}$. $\mathrm{CP}$ violating correlations can be studied in the decays of the produced $t \bar{t}$ pair. Again, this is possible due to the fact that the weak decays of the top quark are very effective in analyzing the top spin (see section 2.8).

\subsubsection{A general model for the Higgs couplings}

To keep the discussion completely general we will assume that a single neutral Higgs-boson, $\mathcal{H}$, is under study although the underlying model may contain several Higgs doublets. In practice, of course, the muon collider will only be tuned to one Higgs resonance at a time.

In section 3.2 .3 we have written an example of a useful parameterization for the $\mathcal{H} f \bar{f}$ ( $f=$ fermion) interaction (see Eq. 3.70), taking into account possible CP violation in this vertex due to an extended Higgs sector. It is, however, also instructive to introduce a different notation - somewhat 
more compact - which is useful for the investigation at hand. Let us therefore parameterize the coupling of $\mathcal{H}$ to fermions with the Feynman rule [237]:

$$
C_{\mathcal{H} f f}=i C_{f f}^{0} \chi_{f} e^{i \gamma_{5} \lambda_{f}},
$$

where $C_{f f}^{0}=-\left(g_{W} / 2\right)\left(m_{f} / m_{W}\right)$ is the coupling in the SM and $\chi_{f}$ for each fermion $f$ (e.g. $f=l$ (i.e. a charged lepton), $u, d$ ) is a real constant which gives the magnitude of the coupling in relation to the standard model. The CP nature of the coupling is determined by the value of $\lambda_{f}$. In particular, $\lambda_{f}$ which is not a multiple of $\pi / 2$ is indicative of $\mathrm{CP}$ violation since the coupling will then contain both scalar and pseudoscalar components. $\mathrm{CP}$ violation is thus essential in any scalar coupling which is not either pure scalar or pseudoscalar and so learning about $\lambda$ is equivalent to investigating $\mathrm{CP}$ violation in $\mathcal{H} \rightarrow f \bar{f}$.

Moreover, in models with an extended Higgs sector the coupling of $\mathcal{H}$ to the boson sector of the theory may be characterized as either scalar, $H$, or pseudoscalar, $A$. If $\mathcal{H}=A$ then it cannot couple to gauge-bosons while if $\mathcal{H}=H$ we can parameterize its coupling to two vector-bosons as

$$
C_{\mathcal{H V V}}=C_{V} \cos \tilde{\alpha}
$$

where, again, $C_{V}$ is the coupling in the SM, $V=Z$ or $W$, given by (see also Eq. 3.71)

$$
C_{W}=m_{W} g_{W}, C_{Z}=m_{Z} g_{W} / \cos \theta_{W},
$$

and $\tilde{\alpha}$ is the angle between the observed Higgs-boson $\mathcal{H}$ and the orientation of the vacuum in the Higgs space.

The mass eigenstate could also be a mixture of $H$ and $A$ which again would violate $\mathrm{CP}$. This aspect of $\mathrm{CP}$ violation in the Higgs sector can lead to enhanced $\mathrm{CP}$ violation if the two masses are close together as discussed in section 10.1.3. Such mixing could also lead to a CP-violating coupling to fermions (Eq. 10.5) whose implications will be discussed in the following pages.

In the following sections we will consider a number of methods to investigate CP-violating couplings of the s-channel neutral Higgs-boson to top quarks at a muon collider. We first consider the reaction $\mu^{+} \mu^{-} \rightarrow t \bar{t}$. Note that in this reaction both initial and final states are CP eigenstates. Furthermore, there is no CP-odd observable that one can construct out of the total cross-section (such as a partial rate asymmetry); if one considers angular distributions of the final $t$-quark, such distributions depend only on the angle $\theta_{\mu t}$, i.e., the angle between the $\mu^{-}$and the $t$-quark momenta in the c.m. frame. Since $\cos \theta_{\mu t}$ is a C-even P-even quantity we clearly need more information if we are to observe $\mathrm{CP}$ violation. Indeed, if the dominant amplitude is mediated by scalar exchange the angular distribution will be isotropic in any case.

To construct CP-violating observables we therefore need information about the polarization of the fermions: either the final state $t \bar{t}$ or the initial state $\mu^{+} \mu^{-}$. In section 10.1.2 we consider the use of correlations in the top polarization in $\mu^{+} \mu^{-} \rightarrow \mathcal{H} \rightarrow t \bar{t}$ to measure the CP-violating parameter of $\mathcal{H} t \bar{t}$ coupling, i.e., $\sin 2 \lambda_{t}$, where $\lambda_{t}$ is the angle in Eq. 10.5.

In section 10.1.3 we consider measurement of $\mathrm{CP}$ violation in the same reaction $\left(\mu^{+} \mu^{-} \rightarrow\right.$ $\mathcal{H} \rightarrow t \bar{t}$ ) except this time the asymmetry we construct is based on polarized muons. Clearly, to perform such experiments it is necessary to have a muon collider capable of producing muons with a significant polarization.

Finally in section 10.2 we consider the possibility of flavor changing neutral Higgs couplings which could give rise to $\mathrm{CP}$ violation in the reaction $\mu^{+} \mu^{-} \rightarrow t \bar{c}$ versus $\mu^{+} \mu^{-} \rightarrow \bar{t} c$. Large couplings of this sort may be expected in $2 \mathrm{HDM}$ of type III which is described in some detail in section 3.2.2. Here again the use of top and/or muon polarization is essential to obtain CPviolating signals.

\footnotetext{
${ }^{34}$ We note that in the language of the interaction Lagrangian in Eq. 3.70, $\lambda_{f} \neq \pi / 2$ corresponds to having $a_{t}^{\mathcal{H}}, b_{t}^{\mathcal{H}} \neq 0$, where $a_{t}^{\mathcal{H}}\left(b_{t}^{\mathcal{H}}\right)$ is the scalar(pseudoscalar) $\mathcal{H}$ coupling to a $t \bar{t}$ pair.
} 


\subsubsection{Decay correlation asymmetry}

Let us now consider the case of a muon collider where unpolarized muons produce $t \bar{t}$ through $\mu^{+} \mu^{-} \rightarrow \mathcal{H} \rightarrow t \bar{t}$. Thus, in order to learn about the coupling in Eq. 10.5, we can observe the polarization of the top quarks through their decays [237], which is discussed extensively in section 2.8 .

Here we will just consider the determination of top polarization by its correlation with the momentum of a particle in various decay modes. Thus, if $X$ is a decay product of a top decay, we define the "analyzing power":

$$
\epsilon_{X}^{t} \equiv 3\left\langle\cos \theta_{X}\right\rangle
$$

where $\theta_{X}$ is the angle between $\vec{p}_{X}$ and the spin of the top in the top rest frame.

Let us now extend this idea to study the correlations of the polarizations of the top quarks where the polarizations are indicated by the momenta of specific decay particles. As discussed in section 2.8, for the case of a single polarized top, some further optimization may follow from going beyond this which we do not consider here.

Following [237], we work in the rest frame of the Higgs-boson with the $t$ momentum along the $z$ axis. Let each of the $t$-quarks undergo a decay which can analyze the top polarization. Let $x_{i}$ and $x_{j}$ be the outgoing particles which we wish to correlate with the $t$ and $\bar{t}$ polarization respectively; for instance, the lepton or $W^{ \pm}$. Also let $y_{i}$ and $y_{j}$ denote the rest of the decay products. Thus the two decays are $t \rightarrow x_{i} y_{i}$ and $\bar{t} \rightarrow \bar{x}_{j} \bar{y}_{j}$. We can then define the azimuthal angle between $\vec{p}_{x_{i}}$ and $\vec{p}_{\bar{x}_{j}}$ projected on to the $x, y$ plane as:

$$
\sin \left(\phi_{i j}\right)=\frac{\left(\vec{p}_{x_{i}} \times \vec{p}_{\bar{x}_{j}}\right) \cdot \vec{p}_{t}}{\left|\vec{p}_{x_{i}}\right|\left|\vec{p}_{\bar{x}_{j}}\right|\left|\vec{p}_{t}\right|} .
$$

The azimuthal differential distribution of $t$ and $\bar{t}$ events is then given by

$$
\frac{d \Gamma}{\Gamma d \phi_{i j}}=1-\frac{\pi^{2}}{16} \epsilon_{i}^{t} \epsilon_{j}^{t} \rho_{t} \cos 2 \lambda_{t} \cos \phi_{i j}+\frac{\pi^{2}}{16} \epsilon_{i}^{t} \epsilon_{j}^{t} \eta_{t} \sin 2 \lambda_{t} \sin \phi_{i j}
$$

The coupling $\lambda_{t}$ is defined in Eq. 10.5 for $f=t$ and $\epsilon_{i}^{t}, \epsilon_{j}^{t}$ are the analyzing power of the decays (defined by Eq. 10.8). Also, $\rho_{t}, \eta_{t}$ are phase space factors which approach 1 as $m_{\mathcal{H}}>>2 m_{t}$. They are given by

$$
\begin{aligned}
\rho_{t} & =-\frac{1-\beta_{t}^{2}-\left(1+\beta_{t}^{2}\right) \cos 2 \lambda_{t}}{\cos 2 \lambda_{t}\left[1+\beta_{t}^{2}-\left(1-\beta_{t}^{2}\right) \cos 2 \lambda_{t}\right]}, \\
\eta_{t} & =\frac{\beta_{t}}{1-\left(1-\beta_{t}^{2}\right) \cos ^{2} \lambda_{t}},
\end{aligned}
$$

where $\beta_{t}=\sqrt{1-4 m_{t}^{2} / m_{\mathcal{H}}^{2}}$.

We may now define the following $\mathrm{CP}$-violating, $T_{N}$-odd, $P$-odd, azimuthal asymmetry by

$$
A_{i j}^{t}=\frac{\Gamma\left(\sin \phi_{i j}>0\right)-\Gamma\left(\sin \phi_{i j}<0\right)}{\Gamma\left(\sin \phi_{i j}>0\right)+\Gamma\left(\sin \phi_{i j}<0\right)} .
$$

From the distribution above, the value for this observable will be

$$
A_{i j}^{t}=\frac{\pi}{8} \epsilon_{i}^{t} \epsilon_{j}^{t} \eta_{t} \sin 2 \lambda_{t} .
$$

For each pair $i, j$ of top decays that can be used to analyze the polarization of the $t$ and $\bar{t}$, one will obtain an experimental value of $A_{i j}^{t}$ from which one can infer the value of the quantity 
$\sin 2 \lambda_{t}$. Clearly we would like to combine the information from all of the modes together in order to obtain better statistical significance for the determination of $\sin 2 \lambda_{t}$. To combine the asymmetries from different pairs of modes $\{i, j\}$ in an optimal fashion we form a weighted average of the asymmetries defined by a set of weights $w_{i j}$ with the normalization defined by:

$$
\sum w_{i j} B_{i} B_{j}=\sum B_{i} B_{j}
$$

where the summation is over the modes under observation and $B_{i}$ is the branching ratio of mode $i$.

The total weighted asymmetry is thus defined as

$$
A^{t}=\sum w_{i j} A_{i j}^{t} B_{i} B_{j},
$$

which is maximized by taking $w_{i j} \propto A_{i j}^{t}$ [16]. With these weights, then, the maximal asymmetry is

$$
A^{t}=\frac{\pi}{8}\left(\epsilon^{t}\right)^{2} \eta_{t} \sin 2 \lambda_{t}
$$

where

$$
\epsilon^{t}=\left[\sum B_{i}\left(\epsilon_{i}^{t}\right)^{2}\right]^{1 / 2}
$$

Now, for the top quark, the branching ratio into electron or muon, $B_{e}=B_{\mu} \sim \frac{1}{9}$ and the analyzing power is $\epsilon_{e}^{t} \sim \epsilon_{\mu}^{t}=1$. For decays (of $W$ ) into hadrons (i.e., jets), $B_{h}=\frac{2}{3}$ with $\epsilon_{h}^{t}=.39$. The decays into $\tau$ have, $B_{\tau}=\frac{1}{9}$ and the analyzing power is taken to be the same as into the jet modes, $\epsilon_{\tau}^{t}=.39$. Using these we can then deduce, via Eq. 10.18

$$
\epsilon^{t} \simeq .58
$$

In order to quantify how well such experiments might detect $\mathrm{CP}$ violation, let us define $\hat{y}_{j}^{(3 \sigma)}$ to be the number of years needed to accumulate a $3 \sigma$ signal for the $\mathrm{CP}$ asymmetry, $A_{j}$, in the final state $j$. Then

$$
\hat{y}_{j}^{(3 \sigma)}=9 \frac{R_{j}^{0}+R_{\mathcal{H}} B(\mathcal{H} \rightarrow j)}{\left(A_{j}\right)^{2} R_{\mathcal{H}}^{2} B(\mathcal{H} \rightarrow j)^{2} \sigma_{0} \mathcal{L}},
$$

where $\mathcal{L}$ is the integrated luminosity per year, $\sigma_{0}$ is the cross-section for $\mu^{+} \mu^{-} \rightarrow \gamma^{*} \rightarrow e^{+} e^{-}$ and for a final state $X, R_{X}=\sigma\left(\mu^{+} \mu^{-} \rightarrow X\right) / \sigma_{0}$. In the above $R_{j}^{0}$ is $R_{j}$ from SM processes only, which needs to be included as it contributes to the background.

Note that the interference between the SM and the Higgs exchange will be negligible; from helicity considerations, such an interference term will be suppressed by $m_{\mu} / m_{\mathcal{H}}$. The SM does however contribute as a background, hence the term $R_{j}^{0}$ in the numerator of Eq. 10.20. We must also consider the effect of all of the other decay modes of the Higgs taken together since $R_{\mathcal{H}}$ is proportional to $B_{\mu}$ and hence inversely proportional to the total width (see Eq. 10.2). To get an idea of how large the CP-violating effects can be, we consider $\hat{y}_{j}^{(3 \sigma)}$ as a function of $m_{\mathcal{H}} \equiv \sqrt{s}$ in fig. 68 in a number of different scenarios:

1. $\mathcal{H}=H$ with $\chi_{f}=1$ and $\lambda_{f}=45^{\circ}$ for all fermions and $\tilde{\alpha}=45^{\circ}$.

2. $\mathcal{H}=A$ with $\chi_{f}=1$ and $\lambda_{f}=45^{\circ}$ for all fermions.

3. $\mathcal{H}=A$ with $\chi_{l}=\chi_{d}=5$ and $\chi_{u}=1 / 5, \lambda_{f}=45^{\circ}$ for all fermions. 


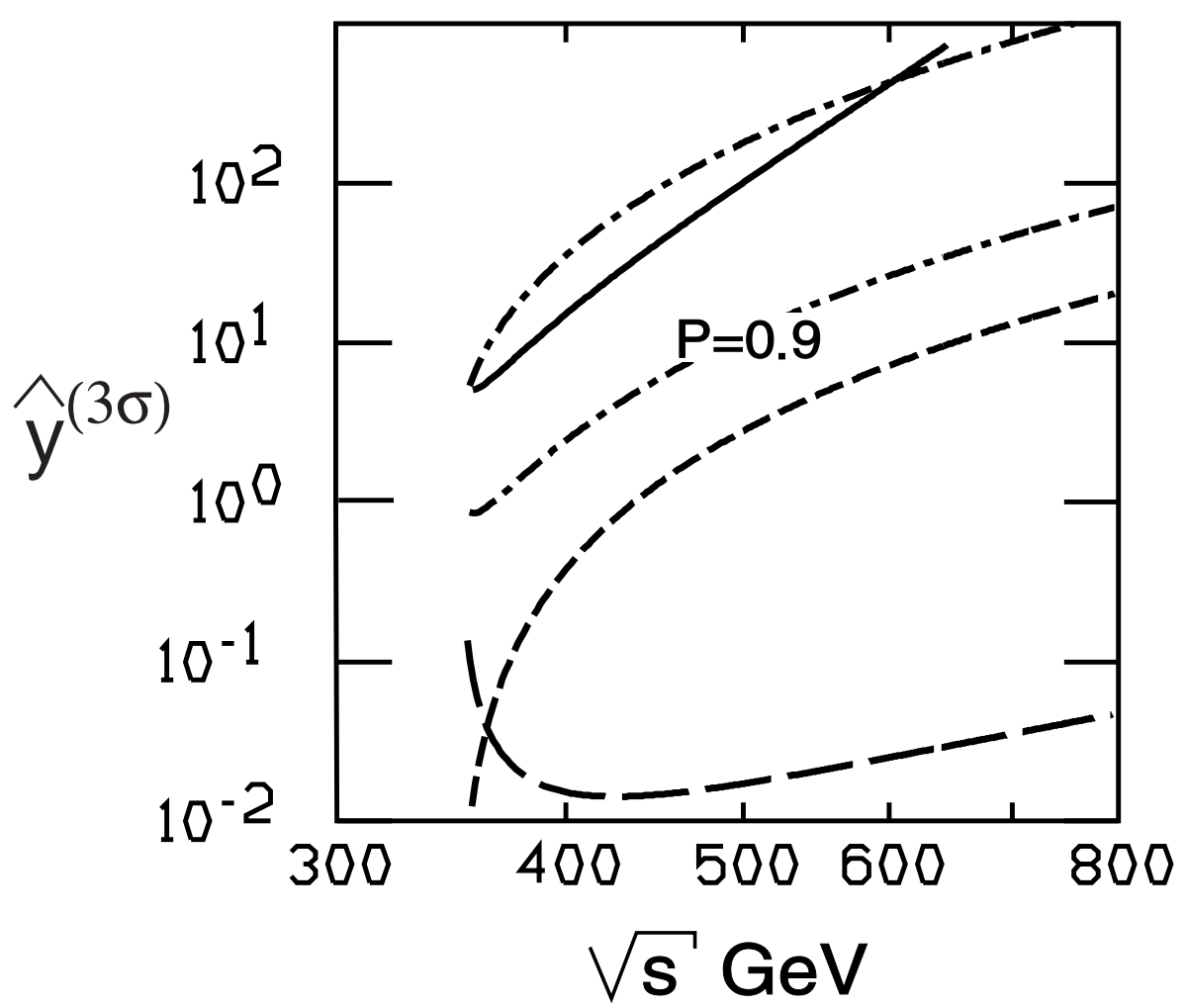

Figure 68: The values of $\hat{y}^{(3 \sigma)}$ (i.e., the number of years required for a $3 \sigma$ effect) for the three scenarios discussed in the text obtained in [23犭]. The solid line is $\hat{y}_{t \bar{t}}^{(3 \sigma)}$ for scenario (1) using top polarization correlation. The upper dash-dot line is $\hat{y}_{t \bar{t}}^{(3 \sigma)}$ for scenario (2) using top polarization correlation while the lower dash-dot line (also in scenario (2))is for the case where the initial muon beams have a longitudinal polarization $P=0.9$. The short dashed line is $\hat{y}_{\text {init }}^{(3 \sigma)}$ obtained in scenario (2) using transverse polarization of the initial muon beams. The long dash line is $\hat{y}_{t \bar{t}}^{(3 \sigma)}$ for scenario (3) using top polarization correlation. Here we take $\mathcal{L}=10^{34} \mathrm{~cm}^{-2} \mathrm{~s}^{-1}$.

We assume that $\chi_{u}=\chi_{c}=\chi_{t}$ and $\chi_{d}=\chi_{s}=\chi_{b}$. The significance of $\mathcal{H}=H$ or $A$, as discussed above, is that only if $\mathcal{H}=H$ does $\mathcal{H} \rightarrow W W, Z Z$ contribute to the total width. Thus, in particular, the value of $\tilde{\alpha}$ is not relevant to cases (2) and (3), since if $\mathcal{H}=A$, then no boson pairs are produced by the resonance at tree-level.

In Fig. 68 which shows the results from [237] we take a nominal luminosity of $10^{34} \mathrm{~cm}^{-2} \mathrm{~s}^{-1}$ and a year of $10^{7}$ sec. (i.e., with running efficiency of $1 / 3$ ). The solid line gives the result in the case of scenario (1) while the upper dot-dash line is scenario (2). In both of these cases, $\hat{y}_{t \bar{t}}^{3 \sigma}$ starts at about 5 years near threshold and increases thereafter. The result for scenario (3) is shown with the long dashed line and is considerably smaller, .01 - .1 years, due to the narrow width of the neutral Higgs $\mathcal{H}$ in this case.

One can enhance the signal with respect to the SM through the use of longitudinally polarized beams. If both of the $\mu^{+}$and $\mu^{-}$beams are left polarized with polarization $P$, then the Higgs production is multiplied by $\left(1+P^{2}\right)$ thus enhanced while the SM backgrounds are multiplied by $\left(1-P^{2}\right)$ and thus reduced. More generally if the $\mu^{+}$has polarization $P^{+}$and the $\mu^{-}$beam has polarization $P^{-}$then the Higgs cross-section gets multiplied by $\left(1+P^{+} P^{-}\right)$while the SM background gets multiplied by $\left(1-P^{+} P^{-}\right)$. In the lower dash-dot curve of fig. 68 we consider the results for scenario (2) where we have taken $P=0.9$ which gives a reduction of nearly an order of magnitude. 


\subsubsection{Production asymmetry in $\mu^{+} \mu^{-} \rightarrow t \bar{t}$ via polarized muons}

As discussed above, some knowledge about fermion polarization is required if information about $\mathrm{CP}$ violation in $\mu^{+} \mu^{-} \rightarrow t \bar{t}$ is to be obtained. Above we considered the case where we used the polarization of the top quarks to learn about $\sin 2 \lambda_{t}$. Here we consider the case where the muons are polarized.

The initial production of muons results in a substantial longitudinal polarization since the weak decay $\pi \rightarrow \mu \nu$ produces predominantly left handed $\mu^{-}$(and right handed $\mu^{+}$). If one constructed a single pass colliding beam machine, it should not be too difficult to preserve this polarization. On the other hand, in the case of muon storage rings the polarization would have to be manipulated in some way since a longitudinally polarized beam will precess at a rate proportional to $g-2$. In a recent paper Grzadkowski et al [238] discuss the details of how the polarization of muons in a storage ring may be used to make measurements on the Higgs resonance of the type considered in the following sections. Here we will assume that it is possible to prepare muons in a given initial state of transverse or longitudinal polarization.

Let us first consider an experiment where the muon beams are polarized transversely to the beam axis. The cross-section is then measured as a function of the angle between the polarizations. We can take the $z$-axis in the c.m. frame to be in the direction of the $\mu^{-}$beam and the $x$-axis to be its polarization while the $\mu^{+}$beam is polarized at an angle of $\phi_{\mu}$ to the $x$-axis, that is in the direction $\left(\cos \phi_{\mu}, \sin \phi_{\mu}, 0\right)$.

If the $\mu^{ \pm}$beams have polarization $P_{ \pm}$then the cross-section (e.g., for $\mu^{+} \mu^{-} \rightarrow t \bar{t}$ ) as a function of $\phi_{\mu}$ is 237]:

$$
\sigma\left(\phi_{\mu}\right)=\left(1-P_{+} P_{-} \cos 2 \lambda_{\mu} \cos \phi_{\mu}+P_{+} P_{-} \sin 2 \lambda_{\mu} \sin \phi_{\mu}\right) \sigma_{0},
$$

where $\sigma_{0}$ is the corresponding unpolarized cross-section. We could therefore look for the presence of $\mathrm{CP}$ violation by comparing $\sigma\left(\phi_{\mu}=+90^{\circ}\right)$ with $\sigma\left(\phi_{\mu}=-90^{\circ}\right)$. Thus, we define the CP-odd

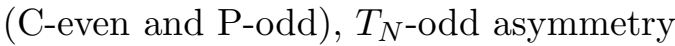

$$
A_{\mu}=\frac{\sigma\left(+90^{\circ}\right)-\sigma\left(-90^{\circ}\right)}{\sigma\left(+90^{\circ}\right)+\sigma\left(-90^{\circ}\right)}=P_{+} P_{-} \sin 2 \lambda_{\mu} .
$$

Clearly if appreciable polarizations are available and $\sin 2 \lambda_{\mu} \approx 1$ these effects are dramatic.

In this experiment, we are simply observing a change in Higgs production as a function of $\phi_{\mu}$, so in the approximation that the Higgs resonance is dominant, it would not matter in fact what the final state is.

In practice, the SM effects will also produce the same final states. Again using this asymmetry we can quantify the amount of run time required to see a signal through Eq. 10.20. In fig. 68 we show with the short dashed line the value from [237] of $\hat{y}_{\text {init }}^{(3 \sigma)}$, which is the number of years required to obtain a $3 \sigma$ signal using the initial polarizations for this asymmetry in scenario 2 taking $P_{+}=P_{-}=1$.

It is also useful in some cases to consider asymmetries which make use of longitudinally

polarized muons in the initial state. Such an asymmetry which is $T_{N}$-even, C-even and P-odd was considered in 239]:

$$
A_{C P}=\frac{\sigma\left(\mu_{L}^{-} \mu_{L}^{+} \rightarrow t \bar{t}\right)-\sigma\left(\mu_{R}^{-} \mu_{R}^{+} \rightarrow t \bar{t}\right)}{\sigma\left(\mu_{L}^{-} \mu_{L}^{+} \rightarrow t \bar{t}\right)+\sigma\left(\mu_{R}^{-} \mu_{R}^{+} \rightarrow t \bar{t}\right)} .
$$

This asymmetry in the pair production cross-section clearly requires longitudinally polarized muon beams. In principle, a similar asymmetry is possible for other fermions as well. Since this asymmetry is $T_{N}$-even, some absorptive phase is required. If the collider is running near the Higgs resonance, this will be naturally provided by the complex phase in the Higgs propagator. Thus, a mechanism for generating this asymmetry is the $\mathrm{CP}$ violation originating from the mixing between $H$ and $Z$ and/or between the scalar $(H)$ and pseudoscalar $(A)$ Higgs that can occur in 
extended models. In particular, the CP invariance of the Higgs sector may be broken by the presence of heavy Majorana fermions. Such a scenario can occur in the minimal SUSY model, in which heavy neutralinos are Majorana fermions. E6 inspired theoretical scenarios offer another possibility for heavy Majorana neutrinos at the TeV mass scale.

The most interesting situation occurs when a CP-even $H$ mixes with a CP-odd Higgs scalar, $A$, and, as is natural in SUSY models, for $M_{A}^{2}>>M_{Z}^{2}$, the two states are roughly degenerate, $M_{H} \simeq M_{A}$. In particular, if $M_{A, H}>2 M_{Z}$ the broadening of the $H$ due to the two vector decay channel can allow significant mixing between the two states. Consequently, Pilaftsis [239] finds

$$
A_{C P} \sim \frac{-2 \hat{\Pi}^{A H}\left[\Im \mathrm{m} \hat{\Pi}^{H H}\left(m_{H}^{2}\right)+\Im \mathrm{m} \hat{\Pi}^{A A}\left(m_{H}^{2}\right)\right]}{\left(m_{H}^{2}-m_{A}^{2}\right)^{2}+\left[\Im \mathrm{m} \hat{\Pi}^{A A}\left(m_{H}^{2}\right)\right]^{2}+\left[\Im \mathrm{m} \hat{\Pi}^{H H}\left(m_{H}^{2}\right)\right]^{2}}
$$

where $\Pi^{i j}$ are coupled channel propagators derived in that paper. Note, in particular, the proportionality to the imaginary parts as expected since the asymmetry is $\mathrm{CP} T_{N}$-even.

Fig. 69 shows the results from [239] in a model where $\Pi^{H A}$ is generated by heavy Majorana neutrinos with masses $M_{N}=0.5,1.0$ and $1.5 \mathrm{TeV}$. It is assumed that $\sqrt{s}=m_{H}$. Two scenarios for the masses and couplings of the Higgs-bosons are considered:

a) $M_{A}=170 \mathrm{GeV}$ and $\cos ^{2} \tilde{\alpha}=1, \chi_{d}=2=1 / \chi_{u}$ and the asymmetry is observed with a $b \bar{b}$ final state.

b) $M_{A}=400 \mathrm{GeV}$ and $\cos ^{2} \tilde{\alpha}=0.1, \chi_{d}=2=1 / \chi_{u}$ and the asymmetry is observed with a $t \bar{t}$ final state.

The cross-section is shown with solid curves while the asymmetry is shown with dotted curves. Scenario (a) is shown with the curves in the region around $\sqrt{s}=170 \mathrm{GeV}$ where the final state is $b \bar{b}$ while scenario (b) corresponds to the curves in the region $\sqrt{s}=400 \mathrm{GeV}$, with a $t \bar{t}$ final state. The enhancement of the asymmetry from the imaginary part of the scalar propagators is apparent in the case where the $A$ and $H$ masses are close together, within about $10 \%$ of each other. 


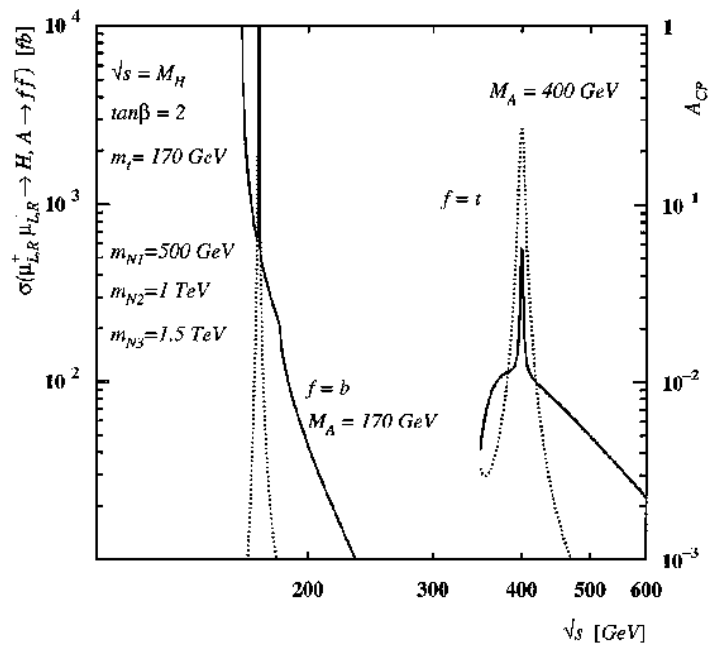

Figure 69: This figure shows the cross-section (solid curves) and $A_{C P}$ in Eq. 10.24 (dotted curves) as a function of $\sqrt{s}=m_{H}$ in a model with $A-H$ mixing induced by heavy Majorana neutrinos with masses $M_{N}=.5,1.0$, and $1.5 \mathrm{TeV}$ and with $\chi_{d}=2=1 / \chi_{u}$. The two curves at the left are for case (a) where $M_{A}=170 \mathrm{GeV}$ and $A_{C P}$ is observed in the bb channel (see text). The curves at the right are for case (b) where $M_{A}=400 \mathrm{GeV}$ and $A_{C P}$ is observed in the t $\bar{t}$ channel (see text). Note that in both cases the curves are shown in the vicinity of $M_{A} \sim M_{H}$ where the mixing effects are likely to be most prominent. Figure taken from [239]. 


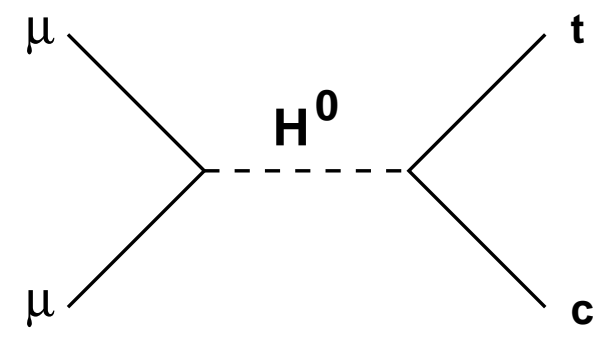

(a)

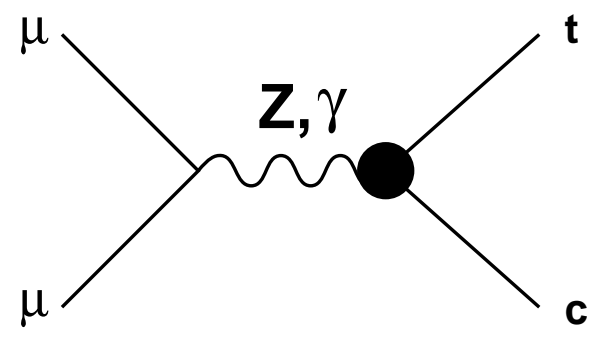

(b)

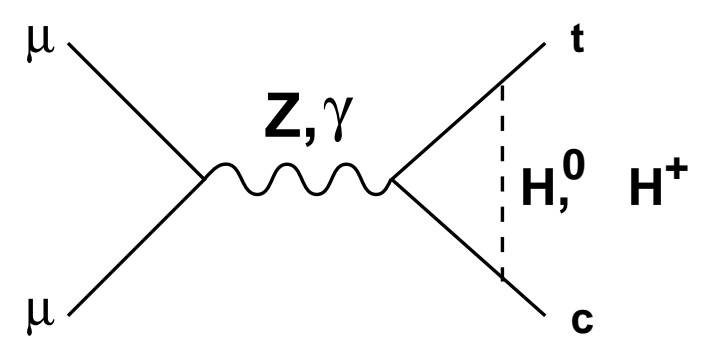

(c)

Figure 70: (a) Feynman diagram for $\mu^{+} \mu^{-} \rightarrow$ tc through s-channel neutral Higgs exchange. (b) Feynman diagram for $\mu^{+} \mu^{-} \rightarrow$ tc through virtual $Z$ and $\gamma$ exchanges, where the circle indicates a vertex correction. (c) An example of a vertex correction contributing to $\mu^{+} \mu^{-} \rightarrow t c$, where $H^{0}$ is a neutral Higgs with flavor changing interactions to fermions and $H^{ \pm}$is a charged Higgs.

\section{2 $\mathrm{CP}$ violation in the flavor changing reaction $\mu^{+} \mu^{-} \rightarrow t \bar{c}$}

As mentioned before, one of the unique properties of a muon collider is that, under favorable conditions, it may produce neutral Higgs states in the $s$-channel. If the Higgs sector contains two or more doublets, then the Higgs couplings may be FC (Flavor Changing) [84, 240] (see also section 3.2.2). This can lead to a dramatic tree-level signature of $\mu^{+} \mu^{-} \rightarrow t \bar{c}$ (or $c \bar{t}$ ) due to the neutral Higgs resonance [241]. At the same time FC processes do occur at the loop level in the SM and in practically all of its extensions, even if they are forbidden at the tree-level. Thus a continuum of FCNC reactions of the form $\mu^{+} \mu^{-} \rightarrow Z^{*}, \gamma^{*} \rightarrow t \bar{c}, \bar{t} c$ are expected. Indeed such couplings with CP-violating phases may also naturally arise in R-parity violating SUSY models [242]. Of course such processes are GIM suppressed in the SM but for the purpose of this discussion we are assuming that there is a FC Higgs sector as in section 3.2.2, thus for the reactions $Z^{*}, \gamma^{*} \rightarrow t \bar{c}, \bar{t} c$, rates appreciably larger than the SM may be expected [84, 243]. Since many such extensions of the SM contain a large number of unconstrained Yukawa couplings, they will, in general, also contain CP-violating phases. Therefore the interference between the resonant and the continuum processes can lead to CP-odd observables; it is this possibility which we wish to study in this section.

Consider now the two processes shown in Fig. 70(a) and 70(b). Since the Higgs flips the helicity of the $\mu$ while the $Z$ does not, for unpolarized or longitudinally polarized beams the interference will be proportional to the mass of the muon and consequently exceedingly small and uninteresting. Such a suppression will not occur if the beams are transversely polarized whence a large interference signal may be produced, especially if the resonant (Fig. 70(a)) and 
the continuum (Fig. [0(b)) processes are of similar strength.

Bearing all this in mind we will thus proceed as follows: first we will consider the general case of the resonance production of $t \bar{c}$ interfering with the continuum and then we will consider, more specifically, what signals are produced in models similar to the ones discussed by [84].

The process which produces $t c$ via s-channel Higgs exchange is controlled by the terms in the Lagrangian (for more details see section 3.2.2)

$$
\mathcal{L}_{H}=\left[\bar{\mu} \phi_{\mu} \mu+\bar{t} \phi_{t c} c+\cdots+\text { h.c. }\right] H^{0}
$$

where with the parameterization defined in Eq. 10.5

$$
\phi_{\mu}=C_{\mu \mu}^{0} \chi_{\mu} e^{i \gamma_{5} \lambda_{\mu}} \equiv \alpha_{\mu}+\beta_{\mu} \gamma_{5}
$$

Similarly, for $\phi_{t c}$ we can write

$$
\phi_{t c} \equiv \alpha_{t c}+\beta_{t c} \gamma_{5}
$$

Note that unitarity implies that $\alpha_{\mu}$ is real while $\beta_{\mu}$ is imaginary.

$\mathrm{CP}$ violation will then occur if there exists another mechanism for producing $t c$ which the Higgs may interfere with. Here we take this process to be $\mu^{+} \mu^{-} \rightarrow \gamma^{*} \rightarrow t \bar{c}$ and/or $\mu^{+} \mu^{-} \rightarrow$ $Z^{*} \rightarrow t \bar{c}$ with the amplitude

$$
\mathcal{M}_{Z, \gamma}=e^{2} \Pi_{Z, \gamma}\left(\bar{\mu} \gamma_{\rho} \eta_{\mu} \mu\right) \cdot\left(\bar{t} \gamma^{\rho} \eta_{t c} c\right)
$$

where $\bar{\mu}, \mu, \bar{t}$ and $c$ above are Dirac spinors and

$$
\begin{array}{r}
\Pi_{Z}=s\left[\left(s-m_{Z}^{2}\right) s_{W}^{2} c_{W}^{2}\right]^{-1}, \quad \Pi_{\gamma}=1, \\
\eta_{\mu} \equiv A_{\mu}^{Z, \gamma}+B_{\mu}^{Z, \gamma} \gamma_{5} \quad, \quad \eta_{t c} \equiv A_{t c}^{Z, \gamma}+B_{t c}^{Z, \gamma} \gamma_{5} .
\end{array}
$$

Here $s_{W}=\sin \theta_{W}, c_{W}=\cos \theta_{W}$, where $\theta_{W}$ is the weak mixing angle. $A_{\mu}^{Z, \gamma}, B_{\mu}^{Z, \gamma}$ are real and they, of course, occur in the tree-level SM Lagrangian. $A_{t c}^{Z, \gamma}, B_{t c}^{Z, \gamma}$ are form factors which are induced at the loop level. As mentioned previously, although small in the SM, they may be generated at reasonable levels in some extensions of the SM, for instance, in multi Higgs scenarios which give $H^{0} \rightarrow t \bar{c}$ [240, 241].

Since we are interfering a vector continuum with a scalar resonance, this interference is naturally suppressed by $m_{\mu}$. This suppression, however, does not apply if at least one of the beams is transversely polarized. To construct a quantity which is CP odd, we consider the case where the $\mu^{-}$beam is polarized in the $+x$ direction and add it to the result where the $\mu^{+}$is also polarized in the $+x$ direction. We will also consider the case where the continuum is dominated by the $Z$ exchange.

Combining the transversely polarized $\mu^{+}$and transversely polarized $\mu^{-}$data as suggested above, we now consider some angular distributions of this combined data which have specific properties under $C P$ and $T_{N}$. Let us define the polar coordinates $(\theta, \phi)$ of $\vec{p}_{c}$; in particular, $\phi$ is the azimuthal separation between the beam polarization and $\vec{p}_{c}$. For each event of the form $\mu^{+} \mu^{-} \rightarrow t \bar{c}$ or $\bar{t} c$, let us also define, $L_{t}$ to be +1 for the $t \bar{c}$ final state and -1 for the $\bar{t} c$ final state. It is natural, therefore, to consider the following possible asymmetries

$$
\begin{array}{ll}
x_{1}=\langle\sigma(\cos \phi)\rangle & x_{2}=\left\langle L_{t} \sigma(\cos \phi)\right\rangle \\
x_{3}=\langle\sigma(\sin \phi)\rangle & x_{4}=\left\langle L_{t} \sigma(\sin \phi)\right\rangle
\end{array}
$$


where $\sigma(x)=+1$ if $x \geq 0$ and -1 if $x<0$.

These expectation values can be characterized in terms of their symmetry properties. Thus $x_{2}, x_{4}$ are CP-odd; $x_{2}$ is $T_{N}$-even and $x_{4}$ is $T_{N}$-odd. $x_{1}$ and $x_{3}$ are CP-even; $x_{1}$ is $T_{N}$-even and $x_{3}$ is $T_{N}$-odd, indicating that $x_{2}$ and $x_{3}$ require complex Feynman amplitudes, i.e., FSI phase(s). In the process at hand one source of this is the Higgs propagator (see Fig. 70(a)). In fact, since the Higgs is close to resonance, in the experiments being envisioned here, these ( $\mathrm{CP} T_{N}$-odd) observables (i.e., $x_{2}$ and $x_{3}$ ) are likely to be the most prominent of the observables since they are enhanced by the resonant phase of the Higgs propagator.

In order to observe the signals suggested above one first requires a muon collider which is able to deliver beams with a large transverse polarization as well as an energy spread for the beam which is comparable to or smaller than the Higgs peak.

Clearly, theories which produce detectable signals should, of course, have fairly large FC tc couplings. As a specific example let us consider 2HDM of types III [84, 240], also discussed in section 3.2.2. In these scenarios a $2 \mathrm{HDM}$ is considered where the second doublet has arbitrary Yukawa couplings. The popular Cheng-Sher Ansatz [82]:

$$
\left|\phi_{t c}\right| \simeq g_{W} \frac{\sqrt{m_{t} m_{c}}}{m_{W}} \lambda
$$

for $\phi_{t c}$ in Eq. 10.25 is then imposed where $\lambda$ is a parameter that needs to be extracted from experiment. It is perhaps natural to expect $\lambda$ to be of $\mathcal{O}(1)$. It is clear that the first obstacle to a large signal is having the $Z^{*}$-exchange continuum in Fig. 70(c), generated by loop corrections, to be sizable.

Let us define

$$
\begin{aligned}
R_{H} & =\sigma\left(\mu^{+} \mu^{-} \rightarrow H^{0} \rightarrow t \bar{c}, \bar{t} c\right) / \sigma\left(\mu^{+} \mu^{-} \rightarrow \gamma^{*} \rightarrow e^{+} e^{-}\right) \\
R_{Z} & =\sigma\left(\mu^{+} \mu^{-} \rightarrow Z^{*} \rightarrow t \bar{c}, \bar{t} c\right) / \sigma\left(\mu^{+} \mu^{-} \rightarrow \gamma^{*} \rightarrow e^{+} e^{-}\right)
\end{aligned}
$$

Clearly then, a necessary condition for there to be large $\mathcal{O}(1)$ asymmetries is that $R_{H} \approx R_{Z}$. For $m_{H^{0}} \sim 150-350 \mathrm{GeV}$ range, typically $R_{Z} \sim 10^{-3}-10^{-4}$ [243]. Such a signal would have a marginal chance at a $\mathcal{L}=10^{34} \mathrm{~cm}^{-2} \mathrm{~s}^{-1}$ luminosity machine. The Higgs signal in this case can also be of order $R_{H} \sim 10^{-3}-10^{-4}$ in the scenario where the Higgs decay to two vectorbosons is allowed. In that case, $\Gamma_{H} \sim \mathcal{O}(1 \mathrm{GeV})$, so the spread in beam energy needed to be in the resonance region of the Higgs should be achievable at a muon collider. This could allow asymmetries of a few tens of percents provided that the CP-odd weak phase difference were large. Since $R_{H} \sim R_{Z} \sim 10^{-3}$, observing the asymmetry would still require a $10^{34} \mathrm{~cm}^{-2} \mathrm{~s}^{-1}$ collider. The situation, of course, would improve considerably if the continuum were larger; for example, this happens if $\lambda>1$ in Eq. 10.31. Since the continuum scales as $\lambda^{4}$ the integrated luminosity required to observe these asymmetries scales as $\lambda^{-4}$. 


\section{Summary and outlook}

There are only two known systems which have been shown to violate the CP symmetry: the neutral kaon through the parameters $\epsilon$ and $\epsilon^{\prime}$ and the entire universe through the dominance of matter over antimatter. It would be of great significance to understand the relation between these two effects or trace them to a common origin. Although experiments in the near term are likely to clarify the source of CP violation in the kaon system, the mechanisms of baryogenesis remain in the realm of theoretical speculation and may not be directly tested in the lab for some time. The top quark, however, offers a unique system where new CP-violating effects could be discovered which could, in time, shed light on the processes which were important in the early universe.

The immediate source of CP violation in the kaon is thought to be the CKM phase in the SM. This will be tested in detail in the next few years through the study of the $B$ meson. Ironically, although the exchange of virtual top quarks generates the large $\mathrm{CP}$ violation in the $B$ and $K$ mesons, the CKM phase will not produce any signal in top quark systems that is large enough to be of experimental interest. Instead, if a CP-violating signal is seen in the top quark, it must be due to some inherently large, non-standard, CP-odd phase which becomes manifest only at high energy scales. Since the effect of the CKM phase is also thought to be too weak to explain baryogenesis, the required phase for this process is likely to show up in top quark physics as well. Thus, the observation of CP violation in top quark reactions is an unambiguous signal of physics beyond the SM which may well shed light on baryogenesis.

In this review, we have considered a number of laboratory tests of $\mathrm{CP}$ violation in the top quark in the context of various non-standard models for physics beyond the SM. In particular, we focus on two classes of models which are described in some detail in Chapter 3 ;

1. Multi Higgs models containing at least two Higgs doublets with phases in the Yukawa couplings. Here CP violation manifests either in the neutral or in charged Higgs sectors.

2. SUSY models wherein we consider in detail the MSSM with the Yukawa couplings given by $N=1$ minimal SUGRA models. One manifestation of SUSY CP violation is through mixing in the sfermion sector.

We chose to focus on these models because they seem to be representative of models for physics beyond the SM which could give rise to $\mathrm{CP}$ violation and are most often considered in the literature. It is likely that the ability of a particular signal to detect $\mathrm{CP}$ violation in one of these models is a good indication of its general utility.

In this review we highlight some notable CP-violating phenomena which follow from these models:

- The transverse polarization of the $\tau$ in $t \rightarrow b \tau \nu$ which follow from $\mathrm{CP}$ violation in the charged Higgs sector (see Chapter 5)

- CP-violating correlations in $e^{+} e^{-} \rightarrow t \bar{t} H^{0}, t \bar{t} Z$ and $e^{+} e^{-} \rightarrow t \bar{t} \nu_{e} \bar{\nu}_{e}$ at high energy $e^{+} e^{-}$ colliders generated by CP violation in the neutral Higgs sector. Since these effects arise through the interference of two tree-level graphs the resulting correlations can be very large indeed. (see Chapter 6)

- CP-violating correlations in hadronic top pair and single top production which can originate from by CP-odd phase(s) in the neutral Higgs sector or in the squark sector of SUSY models. (see Chapters 7 and 8 )

- CP-violating top polarizations may arise in top production at muon and/or photon colliders. In particular at such colliders the neutral Higgs(es) can be produced in the $s$-channel, giving rise to a distinct resonant enhancement which in turn may magnify the $\mathrm{CP}$-violating effect in 
reactions such as $\mu^{+} \mu^{-}, \gamma \gamma \rightarrow t \bar{t}$ or even in the flavor changing channels $\mu^{+} \mu^{-}, \gamma \gamma \rightarrow t \bar{c}+\bar{t} c$. (Chapters 9 and 10)

- CP-violating moments of the top analogous to the electric dipole moment which may be observed at an NLC from top polarimetry in the reaction $e^{+} e^{-} \rightarrow t \bar{t}$. Such moments can be generated in SUSY models as well as models with an extended neutral Higgs sector. (Chapters 6 and 10)

- $\mathrm{CP}$ violation in the main top decay $t \rightarrow b W$. In this case a CP-odd phase in the stop sector of the MSSM can cause a partial rate asymmetry in $t \rightarrow b W^{+}$at the level of a few $\times 0.1 \%$. (Chapter 5)

A common feature of both the SM and the models mentioned throughout this review is that $\mathrm{CP}$ violation is driven directly or indirectly by Yukawa couplings in the scalar sector of the theory. In the SM the CKM matrix which contains the CP-violating parameter results from the Higgs-fermion coupling while with multi Higgs models additional CP violation may result from the couplings between the various Higgs fields, either from explicit CP violation in the Higgs potential (e.g., Model II) and/or in the Yukawa interaction terms (Model III), or CP can be violated spontaneously if there are more than two Higgs doublets. In SUSY models, phases may be associated with the scalar Lagrangian as well, for instance, from squarks and sleptons mixing. As in the SM, the amount of CP violation is proportional to the non-degeneracy of the mass spectrum. For instance, in MHDM's the non-degeneracy of the Higgs particles is required while in SUSY it is the non-degeneracy of squarks or sleptons of different helicities.

It is important to emphasize that, in many ways, the phenomena of $\mathrm{CP}$ violation in top quark systems strongly relies on the large mass of the top which, therefore, becomes the key property of the top as far as $\mathrm{CP}$ violation is concerned:

- The large mass of the top quark allows its polarization to be determined by its weak decays because, unlike the other 5 quark flavors, it decays before it hadronizes and so the information carried by its spin is not diluted. As discussed in Chapter 2, this allows experiments to consider CP-odd observables involving polarization (i.e., top spin correlations) which is crucial since in many settings no CP-violating observables could be constructed without this information. For example, the CP-violating transverse top polarization asymmetry, suggested in Chapter 8, may be used to probe tree-level CP violation in $p \bar{p} \rightarrow t \bar{b}$ which otherwise (i.e., without the use of top spins) cannot be observed.

- In MHDM's, it is the enhancement in the Yukawa coupling of a neutral Higgs to the top quark that is responsible for the enhanced CP-violating effect. As discussed in Chapter 6, this clearly manifests in e.g., $e^{+} e^{-} \rightarrow t \bar{t} Z$, where the only CP-violating diagram present, i.e., the one with a virtual neutral Higgs exchange, is comparable in size to the CP-even SM diagrams that contribute to the same final state, due to the fact that the CP-odd, $H^{0} t \bar{t}$ Yukawa coupling may be as large as the gauge coupling.

- As mentioned above, in the CP-nonconserving effect associated with SUSY particles exchanges, it is the large mass splitting between the two stop mass eigenstates of the theory that may be the cause for an enhanced CP-violating effect, again, due the corresponding large mass of their SM partner - the top quark. As discussed in Chapters 7 and 8, this is the case for example in $p p \rightarrow t \bar{t}$ and $p \bar{p} \rightarrow t \bar{b}$ where the effect arises from CP-violating loop exchanges of stop particles.

- Large $m_{t}$ enables the study of $\mathrm{CP}$ violation in cases where the CP-odd effect is driven by new thresholds (i.e., absorptive cuts across heavy particles of the underlying theory). As discussed in Chapter 5, this is the case in e.g., PRA in $t \rightarrow b W$ within supersymmetry, where it is only viable if $m_{t}>m_{\tilde{t}}+m_{\tilde{\chi}^{0}}-$ still allowed by present experimental data, basically, because of the heaviness of the top. 
- The cases where the CP-odd effect is enhanced to the detectable level due only to an intermediate resonance are also a clear manifestation of the important role played by the large $m_{t}$ in $\mathrm{CP}$ violation studies. Such is the case in e.g., CP violation in the decay $t \rightarrow b \tau \nu_{\tau}$, as discussed in Chapter 5 , where the intermediate $W$-boson resonance provides the necessary enhancement, of course, since $m_{t}>m_{W}$.

It is therefore evident that, due to its large mass, the top is very sensitive to new effects from possible new short distance theories. This sensitivity of the top quark to short distance effects from many models leads one to consider a more general approach for such studies. For instance, by parameterizing $\mathrm{CP}$ violation in a model independent way using CP-violating form factors. Such form factors which contain the information of the dynamics of some new physics scenarios at higher energy scales are expected to be more pronounced in top quark interactions. This technique is a useful prescription for extracting limits on various CP-violating couplings that may arise in new physics. Examples of such effective form factors are the top dipole moments and the CP-violating form factors in the top decays which were separately discussed in Chapters 4 and 5 respectively.

In Chapter 1 we find that in models with extra Higgs doublets as well as in SUSY models, one can expect an EDM and ZEDM of the top on the order of $\sim 10^{-19} \mathrm{e}-\mathrm{cm}$ and, likewise, a CEDM of $\sim 10^{-19} g_{s}$-cm. These values are many orders of magnitude larger than the SM prediction for these quantities. Thus, a discovery of such an effect in $t \bar{t}$ production in leptonic or hadronic colliders and perhaps also in photon and muon colliders, would be a clear signal of beyond the SM dynamics. In Chapter 6 we discuss the sensitivity of an $e^{+} e^{-}$NLC collider to these EDM and ZEDM. We find that optimal observable techniques seem to indicate that high energy $e^{+} e^{-}$ colliders will be sensitive, at best, to a top dipole moment at the level of $\sim 10^{-17}-10^{-18} \mathrm{e}-\mathrm{cm}$, about one to two orders of magnitude larger than what is expected in the models mentioned above.

In Chapter 9 we find that a similar statement is true at $\gamma \gamma$ colliders based on the backscattered laser light from an NLC. On the other hand, hadron colliders are expected to be more sensitive to the CEDM, in particular, in Chapter 7 we find that a CEDM at the level of $10^{-19}-10^{-20} \mathrm{~g}_{s}-\mathrm{cm}$ might be observable at the LHC. Although this sensitivity of a NLC to the top electric dipole moment may seem a little discouraging, there is still very strong motivation to look for this effect; the observation of a top dipole moment with this magnitude (i.e., $\sim 10^{-17}$ e-cm), will clearly be a surprise, since such a large dipole moment cannot be accounted for in the popular models such as SUSY and MHDM's.

Thus, in spite of the very large enhancements expected in such beyond the SM scenarios for the top dipole moments, it is evident that this type of signal is useful only if the dipole moments are on the very large side of the theoretical range. That being the case, one would like to search for other alternatives for the observation of CP violation in top quark systems. It may, for example, be more promising to look for specific signals of $\mathrm{CP}$ violation in the production or decay of top quarks which are not related to the dipole moments.

In Chapter 5 we consider the CP-violating effects which might be present in the decay of top quarks. The simplest kind of signal is a PRA in the decay $t \rightarrow b W$ (mentioned above) which in SUSY can have an asymmetry of $\sim 10^{-3}$ and thus may be detectable at the LHC. Another promising signal which is particularly applicable to $3 \mathrm{HDM}$ or other models with charged scalars is $\tau$ polarization asymmetries in the decay $t \rightarrow \tau \nu b$ which arises from the interference of the $\mathrm{W}$ pole with the charged Higgs propagator and could result in asymmetries on the order of a few tens of percents.

While CP-violating effects in the decay of top quarks may be searched for at any experiment where top quarks are produced, there are a broader range of signals where the CP violation occurs in the production of the top quark. In this case one must consider each kind of top quark production mechanism separately. CP violation in the production mechanism of the top was discussed in the context of an $e^{+} e^{-}$collider (in Chapter 6), hadronic colliders (in Chapters 7 and 
8), photon collider (in Chapter 9) and muon collider (in Chapter 10).

Each of these machines has its own characteristics and so special attention needs to be given in constructing appropriate CP-violating observables. For example, lepton and photon colliders have the advantage of their relative cleanliness as far as background is concerned; it should be easier to reconstruct the top quark in such colliders even when it decays via purely hadronic modes. On the other hand, it may be quite challenging for such colliders, e.g., the NLC, to posses the necessary luminosity for studying rare phenomena in top physics such as loop induced CP-violating effects. Hadron colliders such as the LHC may have an advantage in this context, since top quarks will be more readily produced there. However, the hadronic environment requires more effort in disentangling the CP-violating signal both from the experimental and the theoretical points of view. In addition for hadron colliders, it should be noted that one would prefer, in principle, to always use a $p \bar{p}$ collider (such as the Tevatron) for CP studies since then the initial state is a CP eigenstate. Unfortunately, the LHC which is expected to produce a very large number of top pairs is a $p p$ collider. It turns out, however, that the initial state at the LHC may not effect the $\mathrm{CP}$ studies considered here greatly, primarily because the dominating $t \bar{t}$ production mechanism there is in fact a CP eigenstate, i.e., gluon-gluon fusion.

Nonetheless, in such colliders it is important to use clean CP-violating observables that can reduce the backgrounds. One such useful observable for the LHC, that was suggested by Schmidt and Peskin was discussed in Chapter 7 . Their CP-odd signal uses the difference between the energy of the positrons from $t \rightarrow b e^{+} \nu_{e}$ and electrons from $\bar{t} \rightarrow \bar{b} e^{-} \bar{\nu}_{e}$ in the overall rather complicated reaction, $g g \rightarrow t \bar{t} \rightarrow b W^{+} \bar{b} W^{-} \rightarrow b e^{+} \nu_{e} \bar{b} e^{-} \bar{\nu}_{e}$. Unfortunately, the expected asymmetry is unlikely to be larger than a few times $10^{-3}$. However, Bernreuther, Brandenburg and Flesch have shown that a considerable improvement may be achieved in this reaction by employing clever cuts on the $t \bar{t}$ invariant mass. By doing that they were able to isolate the possible CP-violating contribution from an $s$-channel Higgs exchange in $g g \rightarrow t \bar{t}$. Thus, in their analysis, asymmetries at the level of a few percent may arise leading to a CP-odd signal well above the $3-\sigma$ level in $p p \rightarrow t \bar{t}+X$ at the LHC.

Another useful CP-violating signal designed for the Tevatron setting was discussed in Chapter 8. Specifically, it uses an apparent advantage of $p \bar{p}$ colliders: that there should be a high rate of virtual $W$ production via $u \bar{d}$ annihilation. In this case a number of asymmetries involving the transverse and longitudinal components of the top spin may be constructed. In both MHDM's and SUSY models we find that asymmetries around 1\% may thus occur in single top production at the Tevatron.

Loop induced CP-violating effects such as that of Schmidt and Peskin as well as dipole moments tend to give asymmetries at the level of $\sim 0.1-1 \%$. Thus experimental detection of rare $\mathrm{CP}$ violation effects in top physics, both in hadronic and leptonic colliders, leads to at least two important challenges. 1) Can detector systematics be controlled to the point that a CP asymmetry of $\mathcal{O}(0.1 \%)$ can be observed? 2) Can CP violation be studied with purely hadronic decay modes of the $t \bar{t}$ pair. That is, to what extent will the experimentalists be able to distinguish between the top and the anti-top via purely hadronic modes; if that can be done to a significant level, then the increased statistics will improve the prospects for the observability of such rare CP-odd signals.

The small CP-asymmetries which arise from phenomena that occur at one-loop may make most of those signals too small to be of great use in putting bounds on models of new physics. On the other hand, signals which arise from the interference of tree graphs only are likely to give rise to larger asymmetries. In Chapter 6 we discuss some candidate signals of this type such as $e^{+} e^{-} \rightarrow t \bar{t} H^{0}, t \bar{t} Z$, and $t \bar{t} \nu_{e} \bar{\nu}_{e}$. In addition, the decay discussed in Chapter $5, t \rightarrow b \tau \nu_{\tau}$ falls into this category. In these cases one finds that $\mathrm{CP}$-asymmetries at the level of tens of percents are possible in models with CP-odd phase(s) in the Higgs sector. This makes that type of CPviolating mechanism quite robust, requiring about a few thousands $t \bar{t}$ events per year in order to be detected; such a number may well be within the reach of the future colliders presently under 
consideration.

The two other exotic technologies which may be used in the future in this context (i.e., treelevel $\mathrm{CP}$ violation) are $\gamma \gamma$ colliders and muon storage rings. In Chapter 9 we discuss reactions which can take place at a $\gamma \gamma$ collider constructed from an $e^{+} e^{-}$NLC collider by backscattering laser light from the $e^{ \pm}$beams. As mentioned above, these machines can be used to produce $t \bar{t}$ pairs through an intermediate Higgs state and interfere it with the born cross-section for top pair production. In this case, observables constructed by considering the top polarization can give asymmetries of up to $\sim 10 \%$ in $2 \mathrm{HDM}$. In Chapter 10 we further discuss experiments at muon colliders. Clearly any experiment which can be performed at an $e^{+} e^{-}$collider may also be performed at a muon collider. In addition, however, the larger mass of the muon allows us to contemplate the production of Higgs bosons in the s-channel. In such scenarios one can analyze the scalar versus pseudoscalar couplings of the Higgs to $t \bar{t}$ by studying the polarization correlations of the tops produced which, again, could give $\sim 10 \%$ asymmetries.

Now, since a large portion of the experimental effort in these future colliders will be devoted to the search of supersymmetry, it is particularly gratifying that for such studies of tree-level CP violation, supersymmetry may play an important role in our understanding of the underlying mechanism for CP violation. In particular, once SUSY is discovered and SUSY particles are readily produced in high energy collider experiments, the next step would clearly be to start scrutinizing the basic ingredients of the SUSY Lagrangian, e.g., its CP-violating sector. Indeed, due to the potential richness of CP-odd phases in SUSY theories, tree-level CP violation can easily occur in production and decay of SM+SUSY particles. A promising venue to investigate such tree-level SUSY CP violation may be to search for reactions involving associated top production in final states which contain additional SUSY particles and to probe the CP-violating effect through top polarimetry, i.e., bypassing the missing energy limitation (typical to SUSY signatures) by using the top spins. A simple example may be $\mathrm{CP}$ violation in $e^{+} e^{-} \rightarrow t+X+$ missing energy vs. $e^{+} e^{-} \rightarrow \bar{t}+\bar{X}+$ missing energy, where $X$ is some non-SUSY hadronic final state.

Another interesting related venue in the context of tree-level CP violation within SUSY models is to search for CP-odd signals in reactions where, although involving SUSY particles, only SM particles are produced in the final state. Indeed, if SUSY theories posses $R$-parity violating interactions, CP may be violated at tree-level even in $2 \rightarrow 2$ processes in which the initial and final states consist of SM particles only. In particular, through SUSY scalar exchanges in which the CP-odd phases are carried by the $R$-parity violating couplings in the interaction vertices of a pair of SM particles to squarks and/or sleptons. Again, such tree-level CP violation may be probed even in a $2 \rightarrow 2$ process if one uses top spin asymmetries. Consider, for example, single top production at the Tevatron, $p \bar{p} \rightarrow t \bar{b}+\bar{t} b$. As was discussed in Chapter 8 , a transverse top polarization asymmetry can probe potentially tree-level $\mathrm{CP}$ violation in this process. Indeed, since $s$-channel exchanges of charged sleptons can mediate $p \bar{p} \rightarrow t \bar{b}+\bar{t} b$ in $R$-parity violating SUSY, this transverse top polarization asymmetry can potentially lead to large tree-level SUSY $\mathrm{CP}$-violating signal in this reaction.

These types of tree-level CP violation in SUSY models were not discussed in this review or anywhere else in the literature to date and could be useful to examine in the future, especially once SUSY is directly observed. More generally, the subject of tree-level CP violation seems promising and requires additional effort from the theoretical point of view.

In parting, the study of $\mathrm{CP}$ violation in top quark physics deserves to be one of the main issues on the agenda of the future high energy colliders. The expected high production rate of top quarks in these colliders turns these machines into practically top factories enabling the examination of what is presently considered rare phenomena in top physics. In particular, these colliders provide a unique opportunity for the study of CP violation - a phenomenon that till now seems to be essentially confined only to the kaon system - and its relation to top quark dynamics. The manifestation of $\mathrm{CP}$ violation in heavy particles systems in general and in the top quark system in particular, can shed light on new aspects of this phenomena due to the high 
Table 16: The underlying source of CP-odd phase and the mechanism responsible for CP violation in the processes indicated. Note that: $\tilde{t}$ CP means $C \mathrm{P}$ from $\tilde{t}_{L}-\tilde{t}_{R}$ mixing, $H^{0} \mathrm{CP}$ means CP from scalar - pseudo-scalar mixing in the $H^{0} t t$ vertex and $H^{+}$CP means a $C \mathrm{P}$ phase in the $H^{+} t b$ and/or $H^{+} \tau \nu_{\tau}$ vertices. See also Chapter 3 .

\begin{tabular}{|c|c|c|}
\hline process & CP source & mechanism \\
\hline $\bar{t} t \rightarrow b W$ & 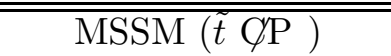 & 1-loop \\
\hline$t \rightarrow b \tau \nu_{\tau}$ & $\operatorname{MHDM}\left(H^{+} \not \mathrm{P}\right)$ & $\begin{array}{c}W^{+} \text {-resonance } \\
\text { in tree-level } \\
W^{+}-H^{+} \text {interference }\end{array}$ \\
\hline$p \bar{p} \rightarrow t \bar{b}$ & $\begin{array}{c}\text { MSSM }(\tilde{t} C Y \mathrm{P}) \\
\& \\
\operatorname{MHDM}\left(H^{0} C \mathrm{P}\right)\end{array}$ & 1-loop \\
\hline$p \bar{p} \rightarrow t \bar{t}$ & $\begin{array}{c}\text { MSSM }(\tilde{t} C \mathrm{P}) \\
\& \\
\operatorname{MHDM}\left(H^{0} \not \mathrm{P}\right)\end{array}$ & top - CEDM (1-loop) \\
\hline$p p \rightarrow t \bar{t}$ & $\begin{array}{c}\text { MSSM }(\tilde{t} \phi \mathrm{P}) \Longrightarrow \\
\& \\
\operatorname{MHDM}\left(H^{0} \phi \mathrm{P}\right) \Longrightarrow\end{array}$ & $\begin{array}{c}\text { 1-loop } \\
s \text {-channel } H^{0} \& \text { 1-loop }\end{array}$ \\
\hline$e^{+} e^{-} \rightarrow t \bar{t}$ & $\begin{array}{c}\text { MSSM }(\tilde{t} \not \mathrm{P}) \\
\& \\
\operatorname{MHDM}\left(H^{0} \not \mathrm{P}\right)\end{array}$ & top - EDM,ZEDM (1-loop) \\
\hline$e^{+} e^{-} \rightarrow t \bar{t} H^{0}, t \bar{t} Z$ & MHDM $\left(H^{0} C \mathrm{P}\right)$ & tree-level interference \\
\hline$e^{+} e^{-} \rightarrow t \bar{t} \nu_{e} \bar{\nu}_{e}$ & $\operatorname{MHDM}\left(H^{0} \not \mathrm{P}\right)$ & $\begin{array}{c}\text { s-channel } H^{0} \\
\text { in } \\
\text { tree-level interference }\end{array}$ \\
\hline$\mu^{+} \mu^{-} \rightarrow t \bar{t}$ & $\operatorname{MHDM}\left(H^{0} C \mathrm{P}\right)$ & $H^{0}$-resonance \\
\hline$\gamma \gamma \rightarrow t \bar{t}$ & $\operatorname{MHDM}\left(H^{0} C \mathrm{P}\right)$ & $s$-channel $H^{0} \&$ 1-loop \\
\hline
\end{tabular}

energy scales involved, possibly on new physics related to the dynamics of our universe in its very early stages.

One, of course, should not forget the importance of the up coming CP measurements in the $B$ system. On the other hand, it is also important to note that there is a very interesting interplay between $\mathrm{CP}$ violation in $b$ physics and in $t$ physics. In $b$ physics, one expects large CP-violating signals due to the CKM phase alone. Therefore, non-observation of $\mathrm{CP}$ violation in $B$ decays would, in fact, stand out as a signal of new physics. This is, of course, in complete contrast to the situation in the top system in which one does not expect any CP-odd signal with the CKM phase of the SM. Therefore, any signal of $\mathrm{CP}$ violation in top reactions will unambiguously prove the existence of new physics. Moreover, in order to disentangle effects of new physics in the $B$ system, one will need precision measurements and cross-checking of the different available CP-violating $B$ decay channels. In top systems the advantage is that no significant effort is needed in order to establish the existence of new physics phenomena in CP-odd top correlations - any measured CP-nonconserving effect in top systems will suffice.

Finally, in Tables 16 and 17 we summarize the main features of some of the most interesting $\mathrm{CP}$-violating signals that were discussed in this review. 
Table 17: The asymmetries that can probe $\mathrm{CP}$ violation in the processes indicated and their expected size. Also indicated is the place in which each asymmetry was discussed in the review, i.e., its equation number. The size of the asymmetry given tends to be optimistic, i.e., on the large side of its theoretical range.

\begin{tabular}{|c|c|c|}
\hline process & type of asymmetry & size \\
\hline$t \rightarrow b W$ & PRA $-\mathcal{A}_{3}$ (Eq. 5.4) & $0.1 \%$ \\
\hline$t \rightarrow b \tau \nu_{\tau}$ & $\tau$ pol. - e.g., (transverse) $\mathcal{A}_{z}^{\prime}$ (Eq. 5.85) & $10 \%$ \\
\hline$p \bar{p} \rightarrow t \bar{b}$ & $\begin{array}{c}\text { cross-section - } A_{0} \text { (Eq. 8.19) } \\
\text { top pol. - e.g., (transverse) } A(\hat{y})(\text { Eq. 8.21) }\end{array}$ & $1 \%$ \\
\hline$p \bar{p} \rightarrow t \bar{t}$ & lepton energy - e.g., (transverse) $A_{T}$ (Eq. 8.7) & $0.1 \%$ \\
\hline$p p \rightarrow t \bar{t}$ & $\begin{array}{l}\text { optimal observable - e.g., } \mathcal{O}^{\prime}(\mathrm{Eq} \cdot \mathrm{7.4}) \\
\text { top pol./lepton momenta - e.g., } \Delta N_{L R} \text { (Eq. 7.17) } \\
\text { lepton energy - e.g., (transverse) } \Delta N\left(E_{T}\right) \text { (Eq. 7.21) }\end{array}$ & $0.1-1 \%$ \\
\hline$e^{+} e^{-} \rightarrow t \bar{t}$ & $\begin{array}{c}\text { optimal observable - e.g., } \mathcal{O}_{R}(\text { Eq. 6.8) } \\
\text { top pol./lepton momenta - e.g., } \hat{T}_{i j}(\text { Eq. 6.24) } \\
\text { angular distributions - e.g., } A_{u d}(\theta)(\text { Eq. 6.43) } \\
\text { energy distributions - e.g., } A_{\ell \ell}(\text { Eq. 6.50) }\end{array}$ & $0.1 \%$ \\
\hline$e^{+} e^{-} \rightarrow t \bar{t} H^{0}, t \bar{t} Z$ & top momenta, optimal observable - $\mathcal{O}, \mathcal{O}_{\text {opt }}$ (Eq. 6.69) & $10 \%$ \\
\hline$e^{+} e^{-} \rightarrow t \bar{t} \nu_{e} \bar{\nu}_{e}$ & top pol./lepton momenta - e.g., $A_{y}$ (Eq. 6.114) & $10 \%$ \\
\hline$\mu^{+} \mu^{-} \rightarrow t \bar{t}$ & $\begin{array}{l}\text { top pol./lepton momenta - } A^{t} \text { (Eq. 10.13) } \\
\text { muon beam pol. - e.g., } A_{\mu} \text { (Eq. 10.22) }\end{array}$ & $10 \%$ \\
\hline$\gamma \gamma \rightarrow t \bar{t}$ & $\begin{array}{c}\text { top pol./lepton momenta - e.g., } \alpha_{1} \text { (Eq. 9.33) } \\
\text { photon pol. - e.g., } \mathcal{P}_{1} \text { (Eq. 9.36) }\end{array}$ & $10 \%$ \\
\hline
\end{tabular}




\section{Notes}

\section{Note on literature survey}

The literature survey for this review was primarily completed in Dec. 1999.

\section{Acknowledgments}

Two of us (GE and AS) are most grateful to the US-Israel Binational Science Foundation for its support that proved very valuable during the four year period that took to write this review. This work was also supported in part by US DOE Contract Nos. DE-FG02-94ER40817 (ISU), DE-AC02-98CH10886 (BNL) and DE-FG03-94ER40837 (UCR). 


\section{References}

[1] G. C. Branco, L. Lavoura and J. P. Silva, CP Violation, Oxford Science Publications, (1999); I. I. Bigi and A. I. Sanda, CP Violation, Cambridge University Press (2000).

[2] J.H. Christenson J.W. Cronin, V.L. Fitch and R. Turlay, Phys. Rev. Lett. 13, (1964) 138.

[3] Review of Particle Physics, C. Caso et al., Eur. Phys. J. C3, (1998) 1.

[4] N. Cabibbo, Phys. Rev. Lett. 10, (1963) 531; M. Kobayashi and T. Maskawa, Prog. Theor. Phys. 49, (1973) 652.

[5] See e.g., M. Ciuchini, E. Franco, G. Martinelli and L. Reina, Phys. Lett. B301, (1993) 263; M. Ciuchini, E. Franco, G. Martinelli and L. Reina hep-ph/9503277, in 2nd DAPHNE Physics Handbook, page 27; A.J. Buras, M. Jamin and M.E. Lautenbacher, Phys. Lett. B389, (1996) 749; S. Bertolini, J. O. Eeg, M. Fabbrichesi and E. I. Lashin, Nucl. Phys. B514, (1998) 93; Y.-Y. Keum, U. Nierste and A.I. Sanda, Phys. Lett. B457 (1999) 157; S. Bosch et al., Nucl. Phys. B565 (2000) 3; for a recent review see also A.J. Buras, hep-ph/9806471 in Probing the Standard Model of Particle Interactions, F. David and R. Gupta, eds., (Elsevier Science 1998); numerical results of the last reference are updated in hep-ph/9901409 to appear in Rescent Devlopments in Quantum Field Theory, P. Breitenlohner, D. Maison and J. Wess eds., (Springer Verlag 2000).

[6] See e.g., B. Winstein and L. Wolfenstein, Rev. of Mod. Phys. 65, (1993) 1113.

[7] G.D. Barr et al., (NA31 Collaboration), Phys. Lett. B317, (1993) 233.

[8] L.K. Gibbons et al., (E731 Collaboration), Phys. Rev. Lett. 70, (1993) 1203; for the combined result of NA31 and E731 given here, see Ref.[3].

[9] A. Alavi-Harati et al., (KTeV Collaboration), Phys. Rev. Lett. 83, (1999) 22.

[10] L. Wolfenstien, Phys. Rev. Lett. 13, (1964) 562.

[11] See e.g., F.J. Gilman, K. Kleinknecht and B. Renk, in Ref.[3], page 103; see also The BaBar Physics Book: Physics at an Asymmetric B Factory, SLAC-Report SLAC-R-504 (1998), P. F. Harrison and H. R. Quinn, eds.

[12] T. Affolder et. al. (CDF Collab.), Phys. Rev. D61 (2000) 072005.

[13] F. Abe et al., (CDF Collaboration), Phys. Rev. Lett. 74, (1995) 2626; S. Abachi et al., (D0 Collaboration), Phys. Rev. Lett. 74, (1995) 2632.

[14] For the most recent published values of the top quark mass see B. Abbott et al., (D0 Collaboration), Phys. Rev. D60, (1999) 0521001; F. Abe et al., (CDF Collaboration), Phys. Rev. Lett. 82, (1999) 271.

[15] I. I. Bigi et. al., Phys. Lett. B181 (1986) 157.

[16] D. Atwood and A. Soni, Phys. Rev. D45 (1992) 2405.

[17] W. Bernreuther, T. Schröder and T.N. Pham, Phys. Lett. B279, (1992) 389.

[18] A. Soni and R.M. Xu, Phys. Rev. Lett. 69, (1992) 33.

[19] D. Chang, W.-Y. Keung and I. Phillips, Nucl. Phys. B408, (1993) 286; Erratum-ibid. B429, (1994) 255.

[20] C.R. Schmidt and M. Peskin, Phys. Rev. Lett. 69, (1992) 410. 
[21] G.L. Kane, G.A. Ladinsky and C.-P. Yuan, Phys. Rev. D45, (1992) 124.

[22] D. Atwood, A. Aeppli and A. Soni, Phys. Rev. Lett. 69, (1992) 2754.

[23] S.L. Glashow, J. Iliopoulos and L. Maiani, Phys. Rev. D2, (1970) 1285.

[24] G. Eilam, J.L. Hewett and A. Soni, Phys. Rev. D44, (1991) 1473; Erratum-ibid., D59, (1999) 039901.

[25] B. Grządkowski, J.F. Gunion and P. Krawczyk, Phys. Lett. B268, (1991) 106.

[26] A.D. Sakharov, JETP Lett. 5, (1967) 24.

[27] See e.g., A.G. Cohen, D.B. Kaplan and A.E. Nelson, Ann. Rev. Nucl. Part. Sci. 43, (1993) 27; for a recent review on baryogenesis see A. Riotto and M. Trodden, Ann. Rev. Nucl. Part. Sci. 49 (1999) 35.

[28] See e.g., A.E. Nelson, D.B. Kaplan and A.G. Cohen, Nucl. Phys. B373, (1992) 453.

[29] M. Aoki, A. Sugamoto and N. Oshimo, Prog. Theor. Phys. 98, (1997) 1325.

[30] For a recent review see Buras in Ref. [5].

[31] For a review see Y. Grossman, Y. Nir and R. Rattazzi, in Heavy Flavors II, page 755, eds. A.J. Buras and M. Lindner (World Scientific, Singapore, 1997).

[32] See e.g., W. Bernreuther, hep-ph/9701357 and references therein, in Heidelberg 1996, NonPerturbative Particle Theory and Experimental Tests, page 141, eds. M. Jamin et al., (World Scientific, Singapore, 1997).

[33] J. Schwinger, Phys. Rev. 82, (1951) 914; J. Schwinger, Phys. Rev. 91, (1953) 713; G. Lüders, Kgl. Dansk. Vidensk. Selsk. Mat.-Fys. Medd. 28, \#5 (1954); W. Pauli, in Niels Bohr and the Development of Physics, (McGraw-Hill, New York, Pergamon, London 1955).

[34] W. Bernreuther, O. Nachtmann, P. Overmann and T. Schroder, Nucl. Phys. B388, (1992) 53; Erratum-ibid. B406, (1993) 516.

[35] C.-P. Yuan, Mod. Phys. Lett. A10, (1995) 627; see also Ref.[19].

[36] J. Bernabéu and M.B. Gavela, in CP Violation, page 269, ed. C. Jarlskog, (World Scientific, Singapore, 1989).

[37] See, e.g., C. Itzykson and J.-B. Zuber, Quantum Field Theory, (McGraw Hill 1980), in particular Chapter 5.

[38] See e.g., S. Weinberg, The Quantum Theory of Fields, Vol. 1, in particular Chapter 3, (Cambridge University Press, New York 1995).

[39] H. Simma, G. Eilam and D. Wyler, Nucl. Phys. B352, (1991) 367.

[40] J.-M. Gérard and W.-S. Hou, Phys. Rev. Lett. 62, (1989) 855 and ibid. Phys. Rev. D43, (1991) 2909.

[41] L. Wolfenstein, Phys. Rev. D43, (1991) 151.

[42] D. Atwood et al., Phys. Rev. Lett. 70, (1993) 1364.

[43] M. Bander, S. Silverman and A. Soni, Phys. Rev. Lett. 43, (1979) 242.

[44] J.M. Soares, Phys. Rev. Lett. 68, (1992) 2102. 
[45] S. Weinberg, Phys. Rev. Lett. 37, (1976) 657.

[46] T. Arens and L.M. Sehgal, Phys. Rev. D51, (1995) 3525.

[47] G. Eilam, J.L. Hewett and A. Soni, Phys. Rev. Lett. 67, (1991) 1979 and ibid. 68, (1992) 2103.

[48] For early references to resonance enhancement of CP violation in scattering processes, see M. Nowakowski and A. Pilaftsis, Phys. Lett. B245, (1990) 185, ibid. Mod. Phys. Lett. 6A, (1991) 1933. For later references see A. Pilaftsis, Nucl. Phys. B504, (1997) 61. For subtelties resulting from non-gauge-invariance of Breit-Wigner form of the $W$ propagator, see S. Dittmaier, hep-ph/9811434 Proceesings of 4'th international Symposium on Radiative Corrections, Barcelona, Spain (1998); J. Papavassiliou and A. Pilaftsis, Phys. Rev. D58, (1998) 053002, and references therein.

[49] See e.g., C.P. Burgess and J.A. Robinson, in BNL Summer Study on CP Violation, page 205, eds. S. Dawson and A. Soni, (World Scientific, Singapore, 1990).

[50] D. Atwood et al., Phys. Lett. B256, (1991) 471.

[51] J.F. Gunion, B. Grzạdkowski and X.-G. He, Phys. Rev. Lett. 77, (1996) 5172.

[52] See e.g., M. Davier et. al., Phys. Lett. B306 (1993) 411; K. Ackerstaff et al., (OPAL Collaboration), Zeit. f. Phys. C74, (1997) 403.

[53] M. Diehl and O. Nachtmann, Zeit. f. Phys. C62, (1994) 397.

[54] See e.g., D. Atwood and A. Soni, Phys. Rev. Lett. 74, (1995) 220.

[55] D. Atwood et al., Phys. Rev. D53, (1996) 1162.

[56] J. F. Donoghue, Phys. Rev. D18, (1978) 1632; E. P. Shabalin, Sov. J. Nucl. Phys. 28, (1978) 75; A. Czarnecki and B. Krause, Phys. Rev. Lett. 78, (1997) 4339.

[57] B. Grzạdkowski and J.F. Gunion, Phys. Lett. B350, (1995) 218.

[58] For some recent reviews on CP violation in the SM, see X.-G. He, hep-ph/9710551; H. R. Quinn, in Ref. [3], page 558; Y. Nir, hep-ph/9810520, Proceedings of 1998 European School of High-Energy Physics, St. Andrews, Scotland, August (1998); I. I. Bigi, hep-ph/9901366.

[59] C. Jarlskog, in CP Violation, page 3, ed. C. Jarlskog, (World Scientific, Singapore, 1989).

[60] C. Jarlskog, Zeit. f. Phys. C29, (1985) 491, ibid. Phys. Rev. Lett. 55, (1985) 1039; see also L.-L. Chau and W.-Y. Keung, Phys. Rev. Lett. 53, (1984) 1802.

[61] See e.g., V. D. Barger and R. J. N. Phillips, Collider Physics, (Addison-Wesley, New York, 1987).

[62] E. Fernandez et al., Phys. Rev. Lett. 51, (1983) 1022; N. S. Lockyer et al., Phys. Rev. Lett. 51, (1983) 1316.

[63] L. Wolfenstein, Phys. Rev. Lett. 51, (1983) 1945.

[64] See e.g., P. Paganini, F. Parodi, P. Roudeau and A. Stocchi, Phys. Scripta. 58, (1998) 556; A. Stocchi, hep-ex/9902004 proceedings of 4'th Conference on Heavy Quarks at Fixed Target Batavia, IL, October (1998); M. Artuso, hep-ph/9911347 in proceedings of International Europhysics Conference On High-Energy Physics, July, (1999), Tampere, Finland, and references therein. 
[65] A.J. Buras, M.E. Lautenbacher and G. Ostermaier, Phys. Rev. D50, (1994) 3433.

[66] C. Jarlskog and R. Stora, Phys. Lett. B208, (1988) 268.

[67] L.-L. Chau and W. Y.-Keung, Phys. Rev. Lett. 53, (1984) 1802; J. D. Bjorken, Phys. Rev. D39, (1989) 1396; C. Hamzaoui, J. L. Rosner and A. I. Sanda, in Proceedings of the Workshop on High Sensitivity Beauty Physics at Fermilab, page 215, eds. A. J. Slaughter, N. Lockyer and M. Schmidt (Fermilab, Batavia, 1988); J. L. Rosner, A. I. Sanda and M. P. Schmidt, ibid. page 165.

[68] N. Isgur and M. Wise, Phys. Rev. D42 (1990) 2388; M. Neubert, Phys. Rep. 245 (1994) 259.

[69] For recent reviews see Y. Kuramashi, hep-lat/9910032 proceedings of 17th International Symposium on Lattice Field Theory, Pisa, Italy, July (1999); G. Martinelli Nucl. Phys. Proc. Suppl. 73 (1999) 58; A. Soni, hep-ph/0003092, proceedings of 3'rd International Conference on B Physics and CP Violation, Taipei, Taiwan, Dec 1999; S. Hashimoto, heplat/9909136, proceedings of 17th International Symposium on Lattice Field Theory, Pisa, Italy, July (1999).

[70] T. Inami and C. S. Lim, Prog. Theor. Phys. 65, (1981) 297.

[71] C. Bernard, T. Blum and A. Soni, Phys. Rev. D58, (1998) 014501; see also L. Lellouch et. al., hep-ph/9912322 proceedings of 8'th International Symposium on Heavy Flavor Physics, Southampton, England, July (1999).

[72] See e.g., M. Feindt, hep-ph/9802380, in Proceedings of the International Europhysics Conference on High-Energy Physics, Jerusalem, Israel, August (1997).

[73] See the contributions by J. Alexander, D. Karlen, and P. Rosnet in the proceedings of The International Conference on High Energy Physics, Vancouver, Canada, July, (1998).

[74] http://www-cdf.fnal.gov/physics/new/bottom/cdf4855/cdf4855.html.

[75] T.D. Lee, Phys. Rev. D8, (1973) 1226 and Phys. Rep. 9, (1974) 143.

[76] H. E. Haber in Perspectives on Higgs Physics II, page 23, ed. G. L. Kane (World Scientific, Singapore, 1997); J.F. Gunion, H.E. Haber, G. Kane and S. Dawson, The Higgs Hunter's Guide, Addison-Wesley, New-York, 1990.

[77] S. Weinberg, Phys. Rev. D42, (1990) 860.

[78] Y.-L. Wu, hep-ph/9404241 (unpublished), and hep-ph/9404271 (unpablished); Y.-L. Wu and L. Wolfenstein, Phys. Rev. Lett. 73, (1994) 1762.

[79] S. Glashow and S. Weinberg, Phys. Rev. D15, (1977) 1958.

[80] C.D. Froggatt, R.G. Moorhouse and I.G. Knowles, Nucl. Phys. B386, (1992) 63.

[81] W. Bernreuther, hep-ph/9808453, Proceedings of 37'th International University School of Nuclear and Particle Physics: Broken Symmetries, Schladming, Austria, March (1998).

[82] T.P. Cheng and M. Sher, Phys. Rev. D35, (1987) 3484.

[83] M. Sher and Y. Yuan, Phys. Rev. D44, (1991) 1461.

[84] D. Atwood, L. Reina and A. Soni, Phys. Rev. D55, (1997) 3156

[85] L. Wolfenstein and Y.-L. Wu, Phys. Rev. Lett. 73, (1994) 2809. 
[86] J.F. Gunion and B. Grzạdkowski, Phys. Lett. B243, (1990) 301.

[87] V. Barger, J.L. Hewett and R.J.N. Phillips, Phys. Rev. D41, (1990) 3421.

[88] For a review see J. L. Hewett, hep-ph/9406302, in Proceedings of the SLAC Summer Inst. on Particle Physics: Spin Structure in High Energy Processes, Stanford, CA (1993).

[89] G. Cvetic, hep-ph/9411436, in Proceedings of the Workshop on Electroweak Symmetry Breaking, Budapest, Hungary, (World Scientific, Singapore, 1995).

[90] D. Chakraverty and A. Kundu, Mod. Phys. Lett. A11, (1996) 675.

[91] A. Coarasa, R.A. Jimenez and J. Sola, Phys. Lett. B406, (1997) 337.

[92] K. Kiers and A. Soni, Phys. Rev. D56, (1997) 5786.

[93] M. Krawczyk and J. Zochowski, Phys. Rev. D55, (1997) 6968.

[94] J. F. Gunion, B. Grządkowski, H. E. Haber and J. Kalinowski, Phys. Rev. Lett. 79, (1997) 982.

[95] J.L. Hewett, T. Takeuchi and S. Thomas, hep-ph/9603391, in Electroweak Symmetry Breaking and New Physics at TeV Scale, page 548, eds. T. Barklow et al., (World Scientific, Singapore, 1996).

[96] D. A. Dicus, D. J. Muller and S. Nandi, Phys. Rev. D59, (1999) 055007.

[97] F. Abe et al., (CDF Collaboration), Phys. Rev. Lett. 79, (1997) 357; P. Abreu et al., (DELPHI Collaboration), Phys. Lett. B420, (1998) 140; M. Acciarri et al., (L3 Collaboration), Phys. Lett. B446, (1999) 368; G. Abbiendi et al., (OPAL Collaboration), Eur. Phys. J. C7, (1999) 407; R. Barate et al., (ALEPH Collaboration), hep-ex/9902031; B. Abbott et al., (D0 Collaboration), Phys. Rev. Lett. 82 (1999) 4975; see also, K. Desch, Proceedings of the International Confference on High Energy Phsics Vancouver, Canada, July, (1998).

[98] A. Sopczak, Mod. Phys. Lett. A10, (1995) 1057.

[99] M. Krawczyk, hep-ph/9607268, in Les Arcs 1996, Electroweak Interactions and Unified Theories, page 221, ed. Tran Thanh Van (Edition Frontiérs, 1996); M. Krawczyk, J. Zochowski and P. Mattig, Eur. Phys. J. C8 (1999) 495.

[100] We thank JoAnne Hewett for providing us with updates of figures from Ref. 88].

[101] P. Krawczyk and S. Pokorski, Phys. Rev. Lett. 60, (1988) 182.

[102] Y. Grossman, H. Haber and Y. Nir, Phys. Lett. B357, (1995) 630.

[103] J.L. Hewett and J.D. Wells, Phys. Rev. D55, (1997) 5549.

[104] M.S. Alam et al., (CLEO Collaboration), Phys. Rev. Lett. 74, (1995) 2885.

[105] S. Glenn et al., (CLEO Collaboration), ICHEP-98-1011, Proceedings of The International Conference on High Energy Physics, Vancouver, Canada, July, (1998).

[106] G. Eigen, hep-ex/9901005, Proceedings of The 4'th International Symposium on Radiative Corrections: Applications of Quantum Field Theory to Phenomenology, Barcelona, Catalonia, Spain, September (1998).

[107] R. Barate et al., (ALEPH Collaboration), Phys. Lett. B429, (1998) 169. 
[108] G.C. Branco, A.J. Buras and J.M. Gérard, Nucl. Phys. B259, (1985) 306.

[109] H.-Y. Cheng, Int. J. Mod. Phys. A7, (1992) 1059.

[110] C.H. Albright, J. Smith and S.-H.H. Tye, Phys. Rev. D21, (1980) 711; K. Shizuya and S.-H.H. Tye, Phys. Rev. D23, (1981) 1613.

[111] G.C. Branco, Phys. Rev. Lett. 44, (1980) 504.

[112] G.C. Branco, Phys. Rev. D22, (1980) 2901.

[113] Y. Grossman and Y. Nir, Phys. Lett. B313, (1993) 126.

[114] D. Atwood, G. Eilam and A. Soni, Phys. Rev. Lett. 71, (1993) 492.

[115] Y. Grossman, Nucl. Phys. B426, (1994) 355.

[116] A.J. Buras, P. Krawczyk, M. E. Lautenbacher and C. Salazar, Nucl. Phys. B337, (1990) 284.

[117] G. Isidori, Phys. Lett. B298, (1993) 409.

[118] P. Krawczyk and S. Pokorski, Nucl. Phys. B364, (1991) 10.

[119] Y. Nir, in Proceedings of the SLAC Summer Inst. on Particle Physics: The Third Family and the Physics of Flavor, page 81, ed. L. Vassilian (SLAC-Report-412, 1993).

[120] For reviews on supersymmetry see e.g., H.P. Nilles, Phys. Rep. 110, (1984) 1; H.E. Haber and G. L. Kane, Phys. Rep. 117, (1985) 75; Articles in Perspectives on Supersymmetry, ed. G. L. Kane (World Scientific, Singapore, 1998); H. E. Haber in Ref. [3], page 743; S. Pokorski, Acta Phys. Polon. B30 (1999) 1759; H. Dreiner, hep-ph/9902347, Proceedings of Zuoz Summer School on Hidden Symmetries and Higgs Phenomena, Zuoz, Switzerland, August (1998).

[121] M. Dugan, B. Grinstein and L. Hall, Nucl. Phys. B255, (1985) 413.

[122] K. S. Babu, C. Kolda, J. March-Russel and F. Wilczek, Phys. Rev. D59, (1999) 016004.

[123] R. Garisto and J.D. Wells, Phys. Rev. D55, (1997) 1611.

[124] The most recent Review of Particle Physics [3] gives $\left|d_{n}\right|<0.97 \times 10^{-25}$ e-cm (see P. G. Harris et al., Phys. Rev. Lett. 82, (1999) 904. Both results are at 90\% C.L.

[125] L. Alvarez-Gaume, J. Polchinski and M.B. Wise, Nucl. Phys. B221, (1983) 495.

[126] J. Rosiek, Phys. Rev. D41, (1990) 3464, and hep-ph/9511250 (unpublished erratum).

[127] M. Kuroda, hep-ph/9902340.

[128] See, e.g., S. Dimopoulos and S. Thomas, Nucl. Phys. B465, (1996) 23.

[129] J.F. Gunion and H.E. Haber, Nucl. Phys. B272, (1986) 1.

[130] Y. Kizukuri and N. Oshimo, Phys. Rev. D46, (1992) 3025; ibid. D45, (1992) 1806.

[131] V. Barger, M.S. Berger and P. Ohmann, Phys. Rev. D49, (1994) 4908.

[132] P. Nath, Phys. Rev. Lett. 66, (1991) 2565.

[133] M.M. El Kheishen, A.A. Shafik and A.A. Aboshousha, Phys. Rev. D45, (1992) 4345. 
[134] T. Ibrahim and P. Nath, Phys. Rev. D57, (1998) 478, Erratum-ibid. D58, (1998) 019901; T. Ibrahim and P. Nath, Phys. Rev. D58, (1998) 111301; M. Brhlik, G.J. Good and G.L. Kane, Phys. Rev. D59, (1999) 115004.

[135] For reviews on fermion electric dipole moments see W. Marciano, in Proceedings of the Summer Study on CP Violation, Upton, New York, 1990, eds. S. Dawson and A. Soni, (World Scientific, Singapore, 1990); S. Barr and W. Marciano, in CP Violation, page 455, ed. C. Jarlskog, (World Scientific, Singapore, 1989); W. Bernreuther and M. Suzuki, Rev. Mod. Phys. 63, (1991) 313; N.F. Ramsey, Annu. Rev. Nucl. Part. Sci. 40, (1991) 1; E. Shabalin, in Electroweak Interactions and Unified Thories, page 483, ed. Tran Thanh Van (Edition Frontiérs, 1997).

[136] T. Falk, Nucl. Phys. Proc. Suppl. 52A, (1997) 78.

[137] See e.g., J. Ellis, S. Ferrara and D.V. Nanopoulos, Phys. Lett. B114, (1982) 231; W. Buchmüller and D. Wyler, Phys. Lett. B121, (1983) 321; J. Polchinski and M. B. Wise, Phys. Lett. B125, (1983) 393; F.del Aguila, M. B. Gavela, J. A. Grifols and A. Mendez, Phys. Lett. B126, (1983) 71; J.M. Gérard, W. Grimus and A. Raychaudhauri, Phys. Lett. B145, (1984); E. Franco and M. Mangano, Phys. Lett. B135, (1984) 445; J.M. Gérard et al., Nucl. Phys. B253, (1985) 93. A.I. Sanda, Phys. Rev. D32, (1985) 2992; T. Kurimoto, Prog. Theor. Phys. 73, (1985) 209; W. Fischler, S. Paban and S. Thomas, Phys. Lett. B289, (1992) 373; Y. Nir and R. Rattazzi, Phys. Lett. B382, (1996) 363; T. Moroi, Phys. Lett. B447, (1999) 75 and references therein.

[138] S. Bar-Shalom, D. Atwood and A. Soni, Phys. Rev. D57, (1998) 1495.

[139] For recent phenomenological reviews of supersymmetry and reported limits obtained and expected in existing and future experiments, see e.g., S. Dawson, TASI 97 lectures, hep-ph/9712464; X. Tata, hep-ph/9706307, in Proceedings of the 9th Jorge Andre Swieca Summer School: Particles and Fields, Sao Paulo, Brazil, 1997, page 404, eds. J. C. A. Barata, A. P. C. Malbouisson and S. F. Novaes, (World Scientific, Singapore, 1998); M. Carena et al., hep-ex/9712022; S. Katsanevas, J. Phys. G24, (1998) 337; D. P. Roy, hep-ph/9902298 Proceedings of 13th Topical Conference on Hadron Collider Physics, Mumbai, India, January (1999); M. Dittmar, hep-ex/9901004 Proceedings of Zuoz Summer School on Hidden Symmetries and Higgs Phenomena, Zuoz, Switzerland, August (1998); E. L. Berger, B. Harris, M. Klasen and T. Tait, hep-ph/9903237; talke given at Physics at Run II: Workshop on Supersymmetry/Higgs: Summary Meeting, Batavia, IL, November 1998; J. Erler and D. M. Pierce, Nucl. Phys. B526, (1998) 53, updated in J. Erler, hep-ph/9903449.

[140] J. Ellis and R. A. Flores, Phys. Lett. B377, (1996) 83.

[141] D. Atwood, S. Bar-Shalom and A. Soni, Phys. Rev. D51, (1995) 1034.

[142] E. Christova and M. Fabbrichesi, Phys. Lett. B315, (1993) 338.

[143] W. Bernreuther and P. Overmann, Z. Phys. C61, (1994) 599.

[144] B. Grządkowski, Phys. Lett. B305, (1993) 384.

[145] A. Bartl, E. Christova and W. Majerotto, Nucl. Phys. B460, (1996) 235; Erratum-ibid. Nucl. Phys. B465, (1996) 365.

[146] A. Bartl, E. Christova, T. Gajdosik and W. Majerotto, Nucl. Phys. B507, (1997) 35; Erratum-ibid., B531, (1998) 653.

[147] C.R. Schmidt, Phys. Lett. B293, (1992) 111. 
[148] J.P. Ma and A. Brandenburg, Z. Phys. C56, (1992) 97.

[149] C. Nelson, B. T. Kress, M. Lopez and T. P. McCauley, Phys. Rev. D56, (1997) 5928.

[150] J.M. Yang and B.-L. Young, Phys. Rev. D56, (1997) 5907.

[151] B. Grządkowski and W.-Y. Keung, Phys. Lett. B319, (1993) 526.

[152] T. Hasuike, T. Hattori and S. Wakaizumi, Phys. Rev. D58 (1998) 095008.

[153] B. Dutta-Roy et al., Phys. Rev. Lett. 65, (1990) 827.

[154] B. Dutta-Roy et al., Phys. Rev. D43, (1991) 268.

[155] N.G. Deshpande et al., Phys. Rev. D43, (1991) 3591.

[156] N.G. Deshpande, B. Margolis and H.D. Trottier, Phys. Rev. D45, (1992) 178.

[157] B. Grządkowski, J.F. Gunion and P. Krawczyk, Phys. Lett. B286, (1991) 106.

[158] D. Atwood, G. Eilam and A. Soni Phys. Rev. D49, (1994) 289.

[159] J. Papavassiliou, in proceedings of Beyond the Standard Model IV, page 509, eds. J. Gunion, T. Han and J. Ohnemus, (World Scientific, 1995), Lake Tahoe, Dec. 1994.

[160] J. Liu, Phys. Rev. D47, (1993) 1741, ibid. in proceedings of the 5th Workshop on Heavy Flavors Physics, page 639, eds. D.I. Britton, D.B. MacFarlane and P.M. Patel, (Editions Frontiers, 1994), Montreal, July 1993.

[161] G. López Castro, J.L. Lucio and J. Pestieau, Int. J. Mod. Phys. A11, (1996) 563, and references therein.

[162] E. Christova and M. Fabbrichesi, Phys. Lett. B320, (1994) 299.

[163] X. Bi and Y. Dai, Eur. Phys. J. C12 (2000) 125.

[164] M. Aoki and N. Oshimo, Nucl. Phys. B531, (1998) 49.

[165] W. Bernreuther, O. Nachtmann, P. Overmann and T. Schroder, Nucl. Phys. B388, (1992) 53; Erratum-ibid. B406, (1993) 516.

[166] B. Grządkowski and J.F. Gunion, Phys. Lett. B287, (1992) 237.

[167] B. Grzạdkowski and W.-Y. Keung, Phys. Lett. B316, (1993) 137.

[168] R. Cruz, B. Grządkowski and J.F. Gunion, Phys. Lett. B289, (1992) 440.

[169] E. Accomando et al., (ECFA/DESY LC Physics Working Group), Phys. Rep. 299, (1998) 1; S. Kuhlman et al., (NLC ZDR Design Group and NLC Physics Working Group), hep-ex/9605011; H. Murayama and M. E. Peskin, Ann. Rev. Nucl. Part. Sci. 49, (1996) 513.

[170] See e.g., R. Frey, hep-ph/9606201, Workshop on Physics and Experiments With Linear Colliders: Proceedings, P. 144, A. Miyamoto et al., (World Scientific, Singapore, 1996).

[171] M.S. Baek, S.Y. Choi and C.S. Kim, Phys. Rev. D56, (1997) 6835.

[172] D. Atwood, S. Bar-Shalom, G. Eilam and A. Soni, Phys. Rev. D54, (1996) 5412.

[173] W. Hollik et al., Nucl. Phys. B551, (1999) 3. 
[174] W. Bernreuther and P. Overmann, Z. Phys. C72, (1996) 461.

[175] W. Bernreuther, A. Brandenburg and P. Overmann, hep-ph/9602273, in $e^{+} e^{-}$Linear Collisions: The Physics Potential, P. 49, P. M. Zerwas ed. (1995) page 49.

[176] B. Grządkowski and Z. Hioki, Nucl. Phys. B484, (1997) 17.

[177] B. Grzạdkowski and Z. Hioki, Phys. Lett B391, (1997) 172; L. Brzezinski, B. Grzạdkowski and Z. Hioki, Int. J. Mod. Phys. A14, (1999) 1261.

[178] A. Bartl, E. Christova, T. Gajdosik and W. Majerotto, Phys. Rev. D58, (1998) 074007.

[179] A. Bartl, E. Christova, T. Gajdosik and W. Majerotto, hep-ph/9803426.

[180] A. Bartl, E. Christova, T. Gajdosik and W. Majerotto, Int. J. Mod. Phys. A14, (1999) 1.

[181] F. Cuypers and S.D. Rindani, Phys. Lett B343, (1995) 333.

[182] P. Poulose and S.D. Rindani, Phys. Lett B349, (1995) 379; ibid. Phys. Rev. D54, (1996) 4326.

[183] T. Arens and L.M. Sehgal, Phys. Rev. D50, (1994) 4372.

[184] S. Bar-Shalom, D. Atwood and A. Soni, Phys. Lett B419, (1998) 340.

[185] S. Bar-Shalom, hep-ph/9710355 (expanded version of a talk given at International Europhysics Conference on High-Energy Physics (HEP 97), Jerusalem, Israel, 1997), in International Europhysics Conference on High-Energy Physics, page 1182, eds. D. Lellouch, G. Mikenberg and E. Rabinovici, (Springer-Verlag Berlin Heidelberg, Germany, 1999).

[186] B. Grzadkowski and J. Pliszka, Phys. Rev. D60 (1999) 115018.

[187] A. Djouadi, J. Kalinowski and P.M. Zerwas, Mod. Phys. Lett. A7, (1992) 1765; ibid. Z. Phys. C54, (1992) 255.

[188] X. Zhang, S. K. Lee, K. Whisnant and B. L. Young, Phys. Rev. D50, (1994) 7042.

[189] J.F. Gunion and X.-G. He, hep-ph/9609453, in the proceedings of the 1996 DPF/DPB Summer Study for New Directions in High Energy Physics, Snowmass, Colorado, June (1996).

[190] K. Hagiwara, H. Murayama and I. Watanabe, Nucl. Phys. B367 (1991) 257; see also K. Fujii in the Proceedings of the 4th KEK Topical Conference on Flavor Physics, 1996, and references therein.

[191] T. Han, T. Huang, Z. H. Lin, J. X. Wang and X. Zhang, Phys. Rev. D61 (2000) 015006.

[192] Proceedings of the Workshop on Physics and Experiments with Linear $e^{+} e^{-}$Colliders, eds. F. Harris et al., (World Scientific, Singapore, 1993); Proceedings of the Workshop on Physics and Experiments with Linear $e^{+} e^{-}$Colliders, eds. A. Miyamoto and Y. Fujii, (World Scientific, Singapore, 1996), See also Ref. [169].

[193] H. Baer, S. Dawson and L. Reina, Phys. Rev. D61 (2000) 013002; S. Dawson and L. Reina, hep-ph/9910228, Proceenings of 4'th International Workshop on Linear Colliders, Barcelona, Spain, April (1999).

[194] W. Bernreuther, A. Brandenburg and M. Flesch, Phys. Rev. D56, (1997) 90.

[195] B. Grzạdkowski, Phys. Lett. B338, (1994) 71. 
[196] W. Bernreuther and A. Brandenburg, Phys. Lett. B314, (1993) 104.

[197] D. Chang, W.-Y. Keung, and I. Phillips, Phys. Rev. D48, (1993) 3225.

[198] W. Bernreuther and A. Brandenburg, Phys. Rev. D49, (1994) 4481.

[199] X.-G. He, J.P. Ma, and B.H.J. McKellar, Phys. Rev. D49, (1994) 4548.

[200] X.-G. He, J.P. Ma, and B.H.J. McKellar, Mod. Phys. Lett. A9, (1994) 205.

[201] M. Krämer, J. Kuhn, M. L. Strong and P. M. Zerwas, Z. Phys. C64, (1994) 21.

[202] T. Arens, U. Gieseler, and L.M. Sehgal, Phys. Lett. B339, (1994) 127.

[203] S. Bar-Shalom, D. Atwood, G. Eilam and A. Soni, Z. Phys. C72 (1996) 79.

[204] T. Rizzo, hep-ph/9610373, talk given at the 1996 DPF/DPB Summer Study on New Directions for High-energy Physics, Snowmass, CO (1996).

[205] S.D. Rindani and M.M. Tung, Phys. Lett. B424, (1998) 125.

[206] D. Atwood, and A. Soni, hep-ph/9607481.

[207] R. Cahn and S. Dawson, Phys. Lett. 136B, (1984) 196; Erratum-ibid. 136B, (1984) 464; S. Dawson, Nucl. Phys. B249, (1985) 42; M. Chanowitz and M.K. Gaillard, Phys. Lett. 142B, (1984) 85; G.L. Kane, W. W. Repko and W. B. Rolnick, Phys. Lett. 148B, (1984) 367; R. Kauffman, Phys. Rev. D41, (1989) 3343; P. W. Johnson, F. I. Olness and W.-K. Tung, Phys. Rev. D36, (1987) 291.

[208] L.R. Evans, CERN LHC Project Report 101, March 1997, talk given at the Particle Accelerator Conference (PAC 97), Vancouver, May 1997.

[209] N.V. Krasnikov and V.A. Matveev, Phys. Part. Nucl. 28, (1997) 441.

[210] I. Hinchliffe, Precision Physics at the LHC, in Future High Energy Colliders, page 129, ed. Z. Parsa, (American Inst. of Phys. pub. 1996).

[211] H.-Y. Zhou, Phys. Rev. D58, (1998) 114002.

[212] S.Y. Choi, C.S. Kim and J. Lee, Phys. Lett. b415, (1997) 67.

[213] J.F. Gunion, T.C. Yuan and B. Grządkowski, Phys. Rev. Lett 71, (1993) 488; Erratum-ibid. 71 (1997) 2681.

[214] W. Bernreuther, A. Brandenburg and M. Flesch, hep-ph/9812387.

[215] M. Diemoz et al., Zeit. f. Phys. C39, (1988) 21.

[216] R. Brown et al., Phys. Lett. B43, (1973) 403.

[217] D. Amidei et al, (TeV-2000 Study Group), FERMILAB-PUB-96/082 (April 1996).

[218] K. Cheung, Phys. Rev. D53, (1996) 3604.

[219] P. Harbel, O. Nachtmann and A. Wilch, Phys. Rev. D53, (1996) 4875.

[220] T. Rizzo, Phys. Rev. D53, (1996) 6218.

[221] B. Grządkowski, B. Lampe and K.J. Abraham, Phys. Lett. B415, (1997) 193.

[222] R. Frey et al., hep-ph/9704243. 
[223] J.-M. Yang et al., hep-ph/9802305 and references therein, in Physics at the first muon collider, page 783, talk given at Workshop on Physics at the First Muon Collider and at the Front End of the Muon Collider, Batavia, IL, 6-9 Nov 1997.

[224] T. Stelzer and S. Willenbrock, Phys. Lett. B357, (1995) 125; D.O. Carlson and C.-P. Yuan, hep-ph/9509208 proceedings of The Workshop on Physics of the Top Quark, Ames, IA, May, (1995); A.P. Heinson, A.S. Belyaev and E.E. Boos, Phys. Rev. D56, (1996) 3114; T. Stelzer, Z. Sullivan and S. Willenbrock, Phys. Rev. D56, (1997) 5919; T. Tait and C.-P. Yuan, hep-ph/9710372; A. Belyaev, E.E. Boos and L. Dudko, hep-ph/9804328 Proceedings of 32'nd Rencontres de Moriond: Electroweak Interactions and Unified Theories, Les Arcs, France, March (1997); A. Belyaev, E.E. Boos and L. Dudko, Phys. Rev. D59 (1999) 075001; T. Stelzer, Z. Sullivan and S. Willenbrock, Phys. Rev. D58, (1998) 094021. See also Ref. [217.

[225] See Shaouly Bar-Shalom, Heavy Quark Physics and CP-Violation, Ph.D Thesis, (TechnionIIT, Israel, 1996).

[226] G. Mahlon, hep-ph/9811219.

[227] I. Ginzburg et al., Pizma ZhETF 34, (1981) 514; ibid. JETP Lett. 34, (1982) 491.

[228] I. Ginzburg et al., Nucl. Instr. \& Meth. 205, (1983) 47; I. Ginzburg et al., Nucl. Instr. \& Meth. 219, (1984) 5.

[229] V. Telnov, Turk. J. Phys. 22, (1998) 541; R. Brinkman et al., Nucl. Instrum. Meth. A406, (1998) 13.

[230] G. Baur, K. Hencken and Trautmann, J. Phys. G24, (1998) 1657.

[231] H. Anlauf, W. Bernreuther and A. Brandenburg, Phys. Rev. D52, (1995) 3803; Erratumibid. D53, (1996) 1725.

[232] B. Grządkowski and J.F. Gunion, Phys. Lett. B294, (1992) 361.

[233] S.Y. Choi and K. Hagiwara, Phys. Lett. B359, (1995) 369.

[234] P. Poulose and S.D. Rindani, Phys. Rev. D57, (1998) 5444.

[235] D. Neuffer, FERMILAB-FN (1979) 319; D. Neuffer, Particle Accelerators, 14, (1983) 75, in Proceedings of the 12th International Conference on High Energy Accelerators, page 481, eds. F. T. Cole and R. Donaldson, Batavia, August 1983.

[236] Articles in Proceedings of the 3rd Symposium on Physics Potential and Development of $\mu^{+} \mu^{-}$Colliders, ed. D. B. Cline, San Francisco, Dec. 1995 (Nucl. Phys. Proc. Supp. 51A (1996)); V. Barger et al., hep-ph/9704290, in proceedings of ITP Conference on Future High-energy Colliders, Santa Barbara, CA, October (1996), ibid. Phys. Rep. 286, (1997) 1; D.B. Cline, in Proceedings of The Workshop on Beyond the Standard Model IV, page 164, eds. J. Gunion, T. Han and J. Ohnemus, (1994); C. M. Ankenbrandt et al, Phys. Rev. ST Accel. Beams 2, (1999) 081001; Prospective Study of Muon Storage Rings at CERN, B. Austin, A. Blondel and J. Ellis eds., CERN 99-02 (1999).

[237] D. Atwood, A. Soni, Phys. Rev. D52, (1995) 6271.

[238] B. Grzadkowski, J. F. Gunion and J. Pliszka, hep-ph/0003091.

[239] A. Pilaftsis, Phys. Rev. Lett. 77, (1996) 4996. 
[240] See e.g., W.-S. Hou, Phys. Lett. B296, (1992) 179; M. Luke and M.J. Savage, Phys. Lett. B307, (1993) 387.

[241] D. Atwood, L. Reina and A. Soni, Phys. Rev. Lett. 75, (1995) 3800.

[242] M. Chemtob and G. Moreau, hep-ph/9910543.

[243] D. Atwood, L. Reina and A. Soni, Phys. Rev. D53, (1996) 1199.

[244] For the FF-package that was used for numerical evaluation of loop integrals see G.J. van Oldenborgh, Comput. Phys. Commun. 66, (1991) 1. For the algorithms used in the FFpackage see G.J. van Oldenborgh and J.A.M. Vermaseren, Z. Phys. C46, (1990) 425.

[245] G. Passarino and M. Veltman, Nucl. Phys. B160, (1979) 151. 


\section{Appendix A}

In this appendix we define the coefficients $\mathrm{C}_{x},(x=0,11,12,21,22,23,24$, see below $)$ corresponding to one-loop integrals with three internal propagators in the loop, i.e. "triangle-like" one loop diagrams. In the review they appear in section 4.3 (Eqs. 4.16 - 4.17), in section 4.4 (Eqs. 4.35 and 4.35), in section 4.5 (Eqs. 4.46, 4.53, 4.54, 4.62 and 4.63), in section 5.1.4 (Eqs. 5.38 - 5.45), in section 8.2.1 (Eqs. 8.32 and 8.33) and in section 8.2 .2 (Eqs. 8.41 and 8.42).

These three-point loop from factors which are functions of masses and momenta are defined by the one-loop momentum integrals as follows [244]:

$$
\mathrm{C}_{0} ; \mathrm{C}_{\mu} ; \tilde{\mathrm{C}}_{\mu} ; \mathrm{C}_{\mu \nu}\left(m_{1}^{2}, m_{2}^{2}, m_{3}^{2}, p_{1}^{2}, p_{2}^{2}, p_{3}^{2}\right) \equiv \int \frac{d^{4} k}{i \pi^{2}} \frac{1 ; k_{\mu} ; k^{2} k_{\mu} ; k_{\mu} k_{\nu}}{\mathcal{D}_{1} \mathcal{D}_{2} \mathcal{D}_{3}}
$$

where:

$$
\begin{aligned}
& \mathcal{D}_{1} \equiv k^{2}-m_{1}^{2}, \\
& \mathcal{D}_{2} \equiv\left(k+p_{1}\right)^{2}-m_{2}^{2}, \\
& \mathcal{D}_{3} \equiv\left(k-p_{3}\right)^{2}-m_{3}^{2},
\end{aligned}
$$

and $\sum_{i} p_{i}=0, i=1-3$, is to be understood above.

The three-point loop from factors are then given through the following relations [245]:

$$
\begin{aligned}
& \mathrm{C}_{\mu}=p_{1 \mu} \mathrm{C}_{11}+p_{2 \mu} \mathrm{C}_{12}, \\
& \tilde{\mathrm{C}}_{\mu}=p_{1 \mu} \tilde{\mathrm{C}}_{11}+p_{2 \mu} \tilde{\mathrm{C}}_{12}, \\
& \mathrm{C}_{\mu \nu}=p_{1 \mu} p_{1 \nu} \mathrm{C}_{21}+p_{2 \mu} p_{2 \nu} \mathrm{C}_{22}+\left\{p_{1} p_{2}\right\}_{\mu \nu} \mathrm{C}_{23}+g_{\mu \nu} \mathrm{C}_{24},
\end{aligned}
$$

where $\{a b\}_{\mu \nu} \equiv a_{\mu} b_{\nu}+a_{\nu} b_{\mu}$. The numerical evaluation of the above form factors can be performed using the algorithm developed in [244]. 


\section{Appendix B}

We list in this appendix all the abbreviations used throughout this review:

\begin{tabular}{ll} 
SM & Standard Model \\
CKM & Cabibbo-Kobayashi-Maskawa \\
GIM & Glashow-Iliopoulos-Maiani \\
NLC & Next Linear Collider \\
LHC & Large Hadron Collider \\
PRA & Partial Rate Asymmetry \\
FSI & Final State Interactions \\
PIRA & Partially Integrated Rate Asymmetry \\
MHDM & Multi Higgs Doublet Models \\
SUSY & SUperSYmmetry or SUperSYmmetric \\
SSB & Spontaneous Symmetry Breaking \\
VEV & Vacuum Expectation Value \\
NLO & Next-to-Leading Order \\
2HDM & Two Higgs Doublet Model \\
3HDM & Three Higgs Doublet Model \\
MSSM & Minimal Supersymmetric Standard Model \\
FCNC & Flavor Changing Neutral Currents \\
FC & Flavor Changing \\
NFC & Natural Flavor Conservation \\
REWSB & Radiative ElectroWeak Symmetry Breaking \\
NEDM & Neutron Electric Dipole Moment \\
EDM & Electric Dipole Moment \\
RGE & Renormalization Group Equations \\
SUGRA & SUperGRAvity \\
EW & ElectroWeak \\
TDM & Top Dipole Moment \\
ZEDM & weak( $Z$ - Dipole Moment \\
CEDM & Chromo - Electric Dipole Moment \\
FF & Form Factor \\
LSP & Lightest Supersymmetric Particle \\
DCS & Differential Cross-Section \\
& \\
\hline SEM
\end{tabular}

